\title{
The phylogenetic system of Mantodea (Insecta: Dictyoptera)
}

\author{
Dissertation \\ zur Erlangung des Doktorgrades \\ der Mathematisch-Naturwissenschaftlichen Fakultäten \\ der Georg-August-Universität zu Göttingen
}

vorgelegt von

Frank Wieland

aus Oldenburg

Göttingen 2010 
Tag der mündlichen Prüfung: 
"In order to arrive at a rational classification for Mantodea, it would be helpful, first, to take an inventory of various useful morphological characteristics and evaluate their relative importance and, then, to determine for each which is primitive (plesiomorph) and which is derived (apomorph) by calculating which changes (probably) arose only once and which could have arisen several times.

It is also necessary to consider the fact that major variations do not necessarily mean a distant phylogenetic relationship and that striking resemblances do not necessarily translate into a close relationship."

Roger Roy, 1999

"Da es in dieser Gruppe äußerst schwierig ist, Primitives, Einfaches von Vereinfachtem zu unterscheiden, und da sicher eine Menge von Konvergenzen vorliegen, bedarf es noch weiterer sorgfältiger morphologischer Untersuchungen."

Anton Handirsch, 1930a

"[Morphology] is the most interesting department of natural history, and may be said to be its very soul."

Charles Darwin, 1859

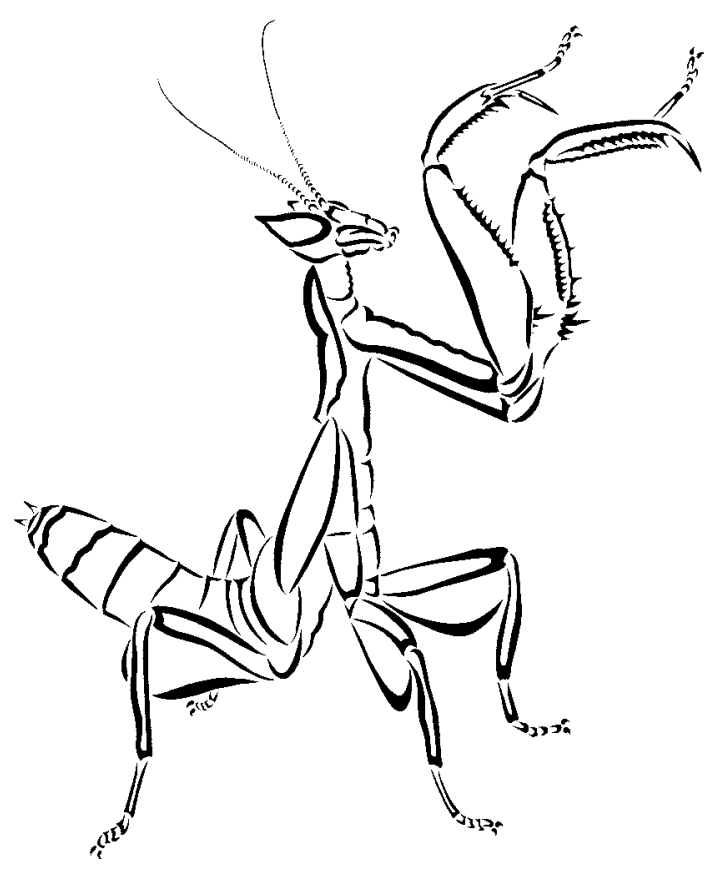





\section{Abstract}

The first reconstruction of mantodean phylogeny using a large morphological dataset of the entire group is presented. 152 morphological characters were encoded for 122 species of Mantodea, encompassing all 15 currently recognized families, 34 of 48 subfamilies (71\%) and 33 of 46 tribus (72\%). Structures from the entire exoskeleton were studied, including characters that have been stated to be convergent developments before without data-based evidence. Fossils, behaviour and ontogenetic observations were used for the interpretation of structures and the discussion of evolutionary scenarios.

Calculations resulted in 888 equally parsimonious trees (analysis I). Many characters were found to be highly homoplastic, resulting in consensus cladograms with low resolution except for many smaller distal clades. Characters were automatically reweighted (based on the Rescaled Consistency Index). Subsequent calculation (analysis II) resulted in 10 equally parsimonious trees, and the consensus cladogram was almost fully resolved. Most of the small monophyletic groups found in analysis I were recovered in analysis II.

The resulting phylogenetic reconstruction supported the monophyly of five traditional families (Acanthopidae, Empusidae, Eremiaphilidae, Thespidae, and Toxoderidae; $33 \%$ ), of 11 subfamilies (Amorphoscelinae, Angelinae, Chroicopterinae, Empusinae, Haaniinae, Hymenopodinae, Oxypilinae, Paraoxypilinae, Perlamantinae, Toxoderinae, and Tropidomantinae; $32 \%$ of the subfamilies studied) and of six tribus (Angelini, Chroicopterini, Idolomorphini, Oxypilini, Polyspilotini, and Rivetinini; $18 \%$ of the tribus studied). The subgroups of Amorphoscelidae in the traditional sense clustered together in analysis II (not in analysis I), however the group encompassed Compsothespis (which is usually assumed to belong to Mantidae) as the sistergroup of Amorphoscelinae + Perlamantinae within Amorphoscelidae. The potential likeliness of this scenario was discussed in detail.

Many structures have been shown to have evolved many times independently in Mantodea, most likely due to comparable selective pressures in the respective habitats. Among them are the head processes, lamellar expansions of the pronota, and lobes on the legs and on the abdomen. Furthermore, the metathoracic hearing organ ("cyclopean ear") evolved several times independently and possibly separately in males and females in some cases. Molecular studies have instead found evidence for a monophyletic neotropical earless taxon in the basal part of the phylogenetic tree. Thespidae was found to be monophyletic and to include Oligonicinae as well as Haaniinae, nested among the latter. The monophyly of Oligonicinae including Haaniinae is supported by their unique fore tibial morphology. This phylogenetic relationship implicates that the ear in Haaniinae originated independently form other taxa, as the group is nested among the earless Oligonicinae.

Congruence between the morphological reconstruction of the phylogeny and the traditional classification was higher than that between the latter and the results of molecular analyses. This was probably at least partly caused by the choice of characters that are often also used in taxonomy, which in turn was always meant to reflect phylogeny, at least to a certain degree. Another possibility is that the taxon sample was smaller in the present analysis than in recent molecular studies.

Congruence with molecular data was comparatively low. Relationships that are also supported (with reservations) by morphological data are the basal dichotomy between Chaeteessa and the remaining Mantodea and the monophyly of Artimantodea (with reservations regarding the position of Metallyticus). Furthermore, Paraoxypilinae, Amorphoscelinae, Ciulfina + Stenomantis, Hestiasula + Oxypilinae, Oxypilinae, Pyrgomantis + (Tarachodes + Tarachodula), Chroicopterinae (except for Chroicoptera), 
Rivetinini, Leptocola + Stenopyga, Empusidae, Empusinae, Polyspilotini, Oxyothespis + Toxoderidae, Toxoderidae, part of Hymenopodinae including Hymenopus + Theopropus, Photina $+($ Orthoderella + Acanthopidae), and Acanthopidae were found to be monophyletic in both molecular and the present studies.

Study of first instar nymphs yielded new interpretation of structures found in the adults of several species.

Chaeteessa, Metallyticus and Mantoida were available for SEM studies for the first time. First observations of living Mantoida and Metallyticus and first pictures of living and freshly deceased Chaeteessa specimens in combination with the scarce literature data allowed hypotheses regarding their lifestyles and interpretations of the corresponding morphological adaptations. The tibial spur in Chaeteessa is still present, albeit strongly reduced. Discoidal spines are still present in first instar nymphs of Metallyticus and a single discoidal spine persists in the adults. Observations of living specimens in combination with literature data led to the tentative hypothesis that a bark-dwelling lifestyle may represent the plesiomorphic condition for Mantodea.

The hypothesis of distinct mantodean ecomorphs suggested by Svenson \& Whiting (2009) was corroborated. As in the molecular studies, the Australasian and the South-East Asian species of the treedwelling Liturgusidae were found to be unrelated. Details in the morphology of the head processes suggest that the Indian Didymocorypha and the African Pyrgomantis, both superficially nearly identical grass dwellers, gained their distinctive body shape independently when they adapted to a life in the grasslands.

Female morphological digging adaptations for laying the ootheca into the soil were compared. At least four distinct types of digging devices evolved independently: The Eremiaphilidae-type (spines on sternite 6, sternite 6 partly covering sternite 7), the Ligaria-type (distal bipartite hooks on the gonapophyses VIII), the Chroicoptera-type (spines on the distal part of sternite 7) and the Rivetina-type (spines on the proximal part of sternite 7). There are possibly two more types that have not been studied in the present dataset.

The morphology of many structures was discussed with regard to the fossil record. Besides the discussion of several fossils that were described previously, a fossil first instar nymph from Baltic amber was reconstructed threedimensionally from $\mu$-CT data. Comparison of fossils and first instar nymphs and adults of several extant species made it possible to discuss so-believed unusual characters in fossils. For example, a strongly elongated distal postero-ventral fore tibial spine that was described to be special for some fossils is present in the first instar nymphs of many extant species and also in the adults of some species. Four cercomeres are present in the first instar nymphs of extant Mantodea. This character may help to assign the ontogenetic age of mantodean nymphs in amber.

Observations on the evolution of head processes, male antennomeres, pronotal lobes, foreleg morphology of Chaeteessa and Metallyticus, tarsal morphology of Heteronutarsus, postembryonic development, sexual dimorphism, female digging devices, trends in leg morphology, sound production, and on the metathoracic hearing organ were summarized and discussed in detail. A discussion of the early mantodean lifestyle and an outlook to future research topics were provided. 


\section{Table of contents}

1 Introduction $\quad 5$

2 State of knowledge $\quad 7$

$\begin{array}{ll}2.1 \text { Systematics } & 7\end{array}$

2.1.1 Phylogenetic relationships of Dictyoptera $\quad 7$

2.1.2 Systematic position of Dictyoptera within Polyneoptera 9

$\begin{array}{ll}\text { 2.1.3 Monophyly of Mantodea } & 10\end{array}$

2.1.4 Relationships within Mantodea and review of the main taxa of the current classification $\quad 11$

$\begin{array}{ll}2.1 .5 \text { Further systematic hypotheses } & 40\end{array}$

$\begin{array}{ll}2.2 \text { Palaeontological record } & 43\end{array}$

$\begin{array}{ll}2.3 \text { Biogeography } & 47\end{array}$

3 Material and methods $\quad 50$

$\begin{array}{ll}3.1 \text { Techniques } & 50\end{array}$

$\begin{array}{ll}3.2 \text { Phylogenetic methods } & 50\end{array}$

3.3 Morphological terminology, definition of orientations and measuring, character encoding 51

$\begin{array}{ll}3.4 \text { Material } & 55\end{array}$

4 Results and discussion $\quad 56$

$\begin{array}{lr}4.1 \text { Characters } & 56\end{array}$

4.1.1 Head and appendages $\quad 56$

$\begin{array}{ll}\text { 4.1.2 Thorax and appendages } & 70\end{array}$

$\begin{array}{ll}\text { 4.1.3 Abdomen and appendages } & 168\end{array}$

$\begin{array}{lr}4.2 \text { Calculations } & 183\end{array}$

$\begin{array}{ll}\text { 4.2.1 Analysis I } & 183\end{array}$

4.2.2 Analysis II $\quad 184$

$\begin{array}{ll}4.2 .3 \text { Bootstrap } & 184\end{array}$

$\begin{array}{ll}4.3 \text { Phylogeny } & 185\end{array}$

4.3.1 - 4.3.105 Intra-ordinal phylogenetic relationships of Mantodea $\quad 185$

$\begin{array}{ll}4.4 \text { Congruence with taxonomy and molecular data } & 229\end{array}$

$\begin{array}{ll}\text { 4.4.1 Congruence with taxonomy } & 229\end{array}$

4.4.2 Congruence with molecular data 230

$\begin{array}{ll}4.5 \text { Homoplasy } & 230\end{array}$

$\begin{array}{ll}4.6 \text { Observations on selected characters } & 231\end{array}$

$\begin{array}{ll}\text { 4.6.1 Head processes } & 232\end{array}$

4.6.2 Male antennomeres $\quad 234$

4.6.3 Lamellar expansions of the pronotum 236

4.6.4 The "missing" discoidal spines of Metallyticus in view of its behaviour 238

4.6.5 The "missing" tibial spur of Chaeteessa 244

4.6.6 The reduced number of tarsomeres in Heteronutarsus 248

$\begin{array}{ll}\text { 4.6.7 Ontogenetic observations } & 250\end{array}$

$\begin{array}{ll}\text { 4.6.8 Sexual dimorphism } & 259\end{array}$

4.6.9 Evolution of female digging devices $\quad 261$

4.6.10 Evolutionary trends in the morphology of the raptorial foreleg 265

4.6.11 Morphology of the meso- and metathoracic legs 269

$\begin{array}{ll}\text { 4.6.12 Early evolution of lifestyle } & 271\end{array}$

4.6.13 Sound production $\quad 273$

$\begin{array}{ll}\text { 4.6.14 Metathoracic hearing organ } & 274\end{array}$

$\begin{array}{ll}4.7 \text { Conclusions } & 274\end{array}$

$\begin{array}{ll}4.8 \text { Future work } & 275\end{array}$

$\begin{array}{lr}5 \text { References } & 279\end{array}$

6 Acknowledgements $\quad 305$

7 Appendix (Tabs. 2-8) $\quad$ i-xxix 



\section{Introduction}

Mantodea (praying mantises or praying mantids) are a group of predatory polyneopteran insects encompassing little more than 2.400 species (Ehrmann 2002; Otte \& Spearman 2005; Svenson \& Whiting 2009). They are thermophilic and therefore are most diverse in the tropical and subtropical regions of the world (Beier 1939a; Balderson 1991; Klass \& Ehrmann 2003). Mantodea usually do not occur farther north or south than $45-46^{\circ}$ latitude on either hemisphere (Beier 1939a, 1964a, 1968a; Klass \& Ehrmann 2003). Only few species exceed 50 latitude in the northern hemisphere, for instance Mantis religiosa (the original authors of species used in this study are listed in Tab. 2 and will not be repeated in the text) in Europe and Empusa pennicornis (Pallas, 1773) in Russia (Beier 1939a, 1964a, 1968a). The southernmost distribution of the group is probably reached by Orthodera novaezealandiae on the South Island of New Zealand (Ramsay 1990: map 1).

Together with Blattodea (cockroaches with the termites being nested among them as the sistergroup of Cryptocercus Scudder, 1862, see for instance Hennig 1969, 1981; Inward et al. 2007; Klass et al. 2008; Ware et al. 2008), Mantodea form the Dictyoptera, a subordinate taxon of Polyneoptera sensu Martynov (1925: 497; the monophyly of Polyneoptera is still debated, see Kristensen 1975: 10 ff., 1981: 142 ff., 1991: 132 ff.; Willmann 2003a: 32 ff., 2003b: 25 ff., 2004: 336 ff.; Klass 2007: 435 ff., 2009: 13 ff.). Polyneoptera also comprises Plecoptera (stoneflies), Embioptera (webspinners), Phasmatodea (stick insects, walking leaves), Orthoptera sensu stricto [i.e. Caelifera (grasshoppers) and Ensifera (crickets, katydids, weta and allies)], Grylloblattodea (= Notoptera: rock crawlers or ice crawlers), Mantophasmatodea (heelwalkers or gladiators), and Dermaptera (earwigs).

The biology of Mantodea as a whole or of single taxa was treated, among many others, by Shelford (1903, 1916), Handlirsch (1925, 1930a), Beier \& Jaus (1933), Roberts (1937a, b), Chopard (1938, 1949a), Beebe et al. (1952), Lieftinck (1953), Kaltenbach (1963), Beier (1964a, 1968a), Brown (1982), Ramsay (1990), Balderson (1991), Rentz (1996), Prete et al. (1999), Ehrmann (2001a, b, 2002), Neumann (2006), Schwarz et al. (2006, 2007), Delfosse et al. (2007), Heßler et al. (2008) and Wieland (2008a). Morphological and anatomical studies or synopses were published, for instance, by Levereault (1936, 1938), Strenger (1942), Chopard (1949a), La Greca \& Rainone (1949), La Greca \& Raucci (1949), Smart (1956), Beier (1964a, 1968a), Slifer (1968), Matsuda (1970), Loxton \& Nicholls (1979), Yager \& Hoy (1986, 1987), Ramsay (1990), Klass (1995, 1997, 1998a, 1999), Yager (1996b, 2005), Frantsevich (1998), Jantsch (1999), Prete et al. (1999), Roy (1999), Wieland (2003, 2006, 2008a, b), Klass \& Meier (2006), Klass \& Eulitz (2007), Yager \& Svenson (2008), Béthoux \& Wieland (2009), and Klass et al. (2009).

Monographs on Mantodea, catalogues and comprehensive taxonomic works treating the entire group were published by Olivier (1792), Audinet-Serville (1839), de Haan (1842), Saussure (1869, 1870a, b, 1871, 1872), Stål (1873, 1877), Westwood (1889), Wood-Mason (1889, 1891), Giglio-Tos (1919, 1927), Beier (1964a, 1968a), Ehrmann (2002), Otte \& Spearman (2005), 
and in the Genera Insectorum by Rehn (1911), Giglio-Tos (1913, 1921), and Beier (1934a-c, 1935a-c). Taxonomic and systematic studies or reviews on single mantodean subgroups were published, among many others, by Audinet-Serville (1831, 1839), Stål (1873, 1877), Chopard (1941), Travassos Filho (1945), Beier (1952, 1954, 1957a, b, 1976), Roy (1976, 1996, 2001, 2004a, b, 2006, 2008a, b, 2009a), Milledge (1990, 1997, 2005), Lombardo (1995b), Battiston \& Fontana (2005), Roy \& Svenson (2007), Ge \& Chen (2008), Wieland (2008a), and Vyjayandi et al. (2009).

A vast number of checklists, revisions and studies of local Mantodea faunae have been published, for instance, by Saussure \& Zehntner (1894, 1895), Werner (e.g. 1907, 1921-1925, 1928), Rehn \& Hebard (1908, 1909a, b), Blatchley (1920), Hebard (1920, 1923, 1935), Tindale (1923, 1924), Beier (1933, 1939b, 1940, 1954, 1956, 1965a, b, 1968b, 1972), Rehn (e.g. 1913, 1915, 1935, 1949), Chopard (e.g. 1911, 1941-1943, 1951), Gurney (1951), Beebe et al. (1952), Paulian (1957), Kaltenbach (1963, 1965, 1967, 1976, 1979, 1982, 1984, 1991, 1996, 1998), Gillon \& Roy (1968), Roy \& Leston (1975), Balderson (1984, 1991), Roy (1961, 1963b, 1969, 1973, 1987b, c,), Cerdá (1993, 1996a, b, 1997a, b), Mukherjee et al. (1995), Terra (1995), Çiplak \& Demirsoy (1997), Lombardo (1997, 2000b), Balderson et al. (1998), Jantsch (1999), Lombardo \& Agabiti (2001), García Becerra et al. (2001), Agudelo Rondón \& Chica (2002), Agudelo Rondón (2004), Lombardo \& Ippolito (2004), Lombardo \& Perez-Gelabert (2004), Vyjayandi (2004, 2007), Abu-Dannoun \& Katbeh-Bader (2007), Agudelo Rondón et al. (2007), Helmkampf et al. (2007), Battiston \& Massa (2008), Battiston \& Picciau (2008), Ehrmann \& Koçak (2009), and Battiston et al. (2010). The greater part of the faunistic publications until 2005 was listed by Ehrmann (2005, summarized under "Faunistik" on pp. 394-409).

The classification by Ehrmann (2002: 374 ff; in collaboration with Roger Roy) which is generally used as a reference, originated from the works of Beier (1964a, 1968a) with only small changes based on taxonomical studies by several authors (for a brief summary see Ehrmann 1997). The classifications by Beier (1964a, 1968a), Ehrmann (2002) and Otte \& Spearman (2005) are typological and do not follow the phylogenetic methods established by Hennig (e.g. 1950, 1965, 1969, 1981). Nonetheless, the classification by Ehrmann (2002) is used as a working hypothesis for the phylogenetic reconstruction of Mantodea in the present work. The catalogue by Ehrmann (2002) is widely accepted, therefore it will be used as the main basis for this study (see Tab. 1).

Mantodea have evolved a plethora of unique behavioural and morphological traits, often directly linked to their raptorial lifestyle or to crypsis or the mimesis of plant matter (phytomimesis). Many of these characters have been considered in taxonomic classifications and identification keys, and some of them may be of value for the phylogenetic reconstruction of the group. Some structures, such as the elongations of the vertex, the lateral lamellar expansions of the pronotum, and the lobe-like dilations on the legs, have been stated to represent convergences (e.g. Handlirsch 1930a; Beier \& Jaus 1933; Beier 1964a, 1968a; Roy 1999). Although being absolutely plausible considering the versatility of the mantodean body, this question has never been addressed by conducting an extensive morphological analysis including such characters. Therefore, this discussion so far lacks convincing data. 
The main objective of the present study is to analyse the phylogeny of Mantodea by generating a large dataset of morphological characters from the entire exoskeleton for a representative selection of mantodean taxa. This will also include those structures that have been assumed by previous authors to have evolved convergently. The results are compared with the most comprehensive genetic analyses (mainly Svenson \& Whiting 2009, but also Svenson \& Whiting 2004a and Yager \& Svenson 2008), and with the typological classification by Ehrmann (2002). Morphological observations on extant and fossil Blattodea as well as nymphal and fossil Mantodea are considered for comparison to ascertain homology states and polarity of the characters investigated. Apomorphic characters for monophyletic taxa within Mantodea are discussed.

\section{State of knowledge}

\subsection{Systematics}

\subsubsection{Phylogenetic relationships of Dictyoptera}

The assumed monophyly of Dictyoptera has found strong support in many studies (e.g. Hennig 1969, 1981; Kristensen 1975, 1981, 1991, 1995; Klass 1995, 1997, 1998a, b, 2003a; Wheeler 1998; Maekawa et al. 1999; Beutel \& Gorb 2001, 2006; Wheeler et al. 2001a, b; Deitz et al. 2003; Grimaldi 2003; Whiting et al. 2003; Willmann 2003a, b, 2004; Kjer 2004; Grimaldi \& Engel 2005; Jarvis et al. 2005; Terry \& Whiting 2005; Kjer et al. 2006; Klass \& Meier 2006; Wieland 2006; Inward et al. 2007; Lo et al. 2007; Fenn et al. 2008; Grimaldi 2008; Ware et al. 2008; Klass et al. 2009) and is undoubted.

Dictyopteran monophyly is well supported by the deposition of eggs in packages (oothecae), covered by a secretion from the accessory glands of the ninth abdominal segment that differ from each other in morphology and composition of the secretion. This situation is distinct from Acridoidea and Mantophasmatodea (e.g. Baccetti 1967). Oothecae are still produced by Mastotermes darwiniensis Froggatt, 1897, the adelphotaxon of all remaining extant termites which lay single eggs (see, for instance, Nalepa \& Lenz 2000, Courrent et al. 2008, and Klass et al. 2008). Monophyly also finds support in the perforated corpotentorium through which the ganglionic connectives pass. $\mathrm{CuP}$ in the forewing is strongly curved. The opener muscles of the abdominal spiracles insert on the paratergites. The females have a subgenital plate (sternite 7) carrying moveable terminal lobes distally and a vestibular sclerite dorsally. Furthermore, the joint between the gonangulum and the paratergite of the ninth segment is hinge-like. All these traits have been mentioned, for instance, by Bohn \& Klass (2003), Grimaldi (2003) and Klass (2003a). Additionally, Wieland (2006) found the transverse orientation of the intercervical sclerites and indentations on the lateral cervical sclerites and intercervical sclerites to be apomorphic for Dictyoptera, as is the presence of in inter-tibio-tarsal sclerite in the membrane between the tibia and the basitarsomere of the legs, found by Klass et al. (2009). 
Tab. 1: Current taxonomical classification (Ehrmann 2002: $374 \mathrm{ff}$.) used as a working hypothesis in this study.

\begin{tabular}{|c|c|}
\hline Mantoididae & Mantidae \\
\hline Chaeteessidae & Orthoderinae \\
\hline Metallyticidae & Oxyothespinae \\
\hline Amorphoscelidae & Paraseveriniini \\
\hline Amorphoscelinae & Oxyothespini \\
\hline Perlamantinae & Angelinae \\
\hline Paraoxypilinae & Angelini \\
\hline Eremiaphilidae & Schizocephalinae \\
\hline Acanthopidae & Compsothespinae \\
\hline Acanthopinae & Chropicopterinae \\
\hline Acanthopini & Chroicopterini \\
\hline Acontistinae & Dystactinae \\
\hline Acontistini & Dystactini \\
\hline Stenophyllinae & Amelinae \\
\hline Stenophyllini & Amelini \\
\hline Hymenopodidae & Mantinae \\
\hline Epaphroditinae & Polyspilotini \\
\hline Phyllocranini & Paramantini ${ }^{1}$ \\
\hline Acromantinae & Archimantini \\
\hline Acromantini & Mantini \\
\hline Oxypilinae & Miomantinae \\
\hline Oxypilini & Miomantini \\
\hline Hymenopodinae & Rivetinini \\
\hline Hymenopodini & Stagmomantinae \\
\hline Liturgusidae & Stagmomantini \\
\hline Liturgusinae & Mellierinae \\
\hline Liturgusini & Mellierini \\
\hline Tarachodidae & Stagmatopterinae \\
\hline Tarachodinae & Stagmatopterini \\
\hline Caliridinae & Vatinae \\
\hline Thespidae & Vatini \\
\hline Pseudomiopteriginae & Heterochaetini \\
\hline Pseudomiopterigini & Danuriini \\
\hline Miopteryginae & Antemninae \\
\hline Miopterygini & Antemnini \\
\hline Thespinae & Photinainae $^{2}$ \\
\hline Thespini & Photinaini $^{2}$ \\
\hline Parathespini & Choeradodinae \\
\hline Hoplocoryphinae & Deroplatyinae \\
\hline Hoplocoryphini & Phyllotheliinae \\
\hline Oligonicinae & Toxoderidae \\
\hline Oligonicini & Toxoderinae \\
\hline Pogonogasterini & Toxoderopsini \\
\hline Haaniinae & Aethalochroaini \\
\hline Iridopterygidae & Toxoderini \\
\hline Hapalomantinae & Sibyllidae \\
\hline Hapalomantini & Sibyllinae \\
\hline Iridopteryginae & Empusidae \\
\hline Iridopterygini & Empusinae \\
\hline Nanomantinae & Idolomorphini \\
\hline Nanomantini & Empusini \\
\hline Fulcinini & Blepharodinae \\
\hline Nilomantinae & Blepharodini \\
\hline Nilomantini & Idolomantini \\
\hline Tropidomantinae & \\
\hline Tropidomantini & \\
\hline
\end{tabular}

${ }^{1}$ erroneously also listed as Paramantinae in Ehrmann 2002 (Ehrmann, pers. comm.)

${ }^{2}$ see Svenson \& Branham 2007

The relationships among the Dictyopteran subgroups as well as their sistergroup have been discussed for a long time. Several phylogenetic scenarios have been suggested throughout the recent decades.

The first one, Isoptera being the sistergroup of Blattaria + Mantodea, was found in the analysis by Thorne \& Carpenter (1993), Kambhampati (1995), and Wheeler (1998) and was earlier proposed by Boudreaux (1979: 220). 
The second, Mantodea forming the sistergroup of Blattaria + Isoptera, was found by Whiting et al. 2003 (possibly a result of the small taxon sample of Dictyoptera; the focus of the study was on Phasmatodea).

The third scenario is Mantodea being the sistergroup of Blattodea (including the termites). The termites have repeatedly been found to be nested among the cockroaches. Within the cockroaches they are the sistergroup of Cryptocercus, a sub-social, lignivorous cockroach. The latter two taxa share several putative synapomorphic characters, such as symbionts in the gut supporting the digestion of cellulose, and life in a sub-social community (see, for instance, Cleveland et al. 1934; Ahmad 1950; Weidner 1966; Hennig 1969, 1981; Seelinger \& Seelinger 1983; Kambhampati 1995). This third hypothesis has gained strong support by numerous morphological and molecular studies throughout the recent years (e.g. Klass 1995, 1997, 1998b, 2003a; Lo et al. 2000; Eggleton 2001; Deitz et al. 2003; Svenson \& Whiting 2004a, 2009; Terry \& Whiting 2005; Grimaldi \& Engel 2005; Kjer et al. 2006; Klass \& Meier 2006; Inward et al. 2007; Grimaldi 2008; Klass et al. 2008, 2009; Ware et al. 2008). Few recent studies showed conflicting results, mainly because they defined termites as outgroup thus forcing it into the sistergroup relationship with cockroaches (e.g. Grandcolas \& D’Haese 1996; Maekawa \& Matsumoto 2000).

A fourth scenario was suggested by Lo et al. (2007) who found both Mantodea and Isoptera to be derived cockroaches in their molecular analysis. While the termites clustered with Cryptocercus and formed the sistergroup of the remaining cockroaches including the mantids therein, Mantodea were found to be the sistergroup of the cockroach genus Nocticola Bolivar, 1892 in the distal part of the phylogenetic tree. The origin of Mantodea as a subgroup of Blattaria was previously assumed by Vršanský (2002) and Vršanský et al. (2002).

I herein follow Klass et al. (2008: 809) in Dictyopteran nomenclature: The term Blattodea is used for cockroaches including the termites (in congruence with Hennig 1969, 1981). The name Blattaria is used for the cockroaches excluding the termites. Isoptera has repeatedly been supported to be the sistergroup of Cryptocercus. I am aware that the term Blattaria thus represents a paraphyletic assemblage. However, it encompasses all "typical" cockroaches and will therefore be used for descriptive convenience. The term Blattodea represents a monophyletic group.

\subsubsection{Systematic position of Dictyoptera within Polyneoptera}

The search for the adelphotaxon of Dictyoptera within Polyneoptera is still going on and most of the polyneopteran lineages have so far been suggested to hold this position (for an overview see Klass 2007: figs. 1-14). Whiting et al. (2003: fig. 2), Jarvis et al. (2005: fig. 3) and Grimaldi \& Engel (2005: fig. 7.3) found Dictyoptera to be the adelphotaxon of the remaining Polyneoptera. Some authors (e.g. Terry \& Whiting 2005; Inward et al. 2007) proposed a monophyletic grouping Mantophasmatodea + Grylloblattodea (Xenonomia of Terry \& Whiting $2005=$ Chimaeraptera of Uchifune \& Machida 2005) to be the sistergroup of Dictyoptera.

Wheeler et al. (2001a, b) found support for a sistergroup relationship between Dictyoptera 
and Zoraptera, however, this relationship was not supported by any of the constituent molecular and morphological analyses alone (Wheeler et al. 2001a: 137). A sistergroup relationship between the two groups was assumed before by Boudreaux (1979: 216), briefly discussed by Smithers (1991: 410), and found by Kukalová-Peck \& Peck (1993) based on wing morphology. Yoshizawa \& Johnson (2005) found further support for this relationship based on an 18S rDNA phylogeny of a large arthropod dataset. However, the $18 \mathrm{~S}$ data appeared to be problematic due to "unusual characteristics" of the zorapteran sequences (Yoshizawa \& Johnson 2005: 574; Yoshizawa 2007: 197) and a later morphological analysis of the zorapteran wing base (Yoshizawa 2007) implied Zoraptera to be the sistergroup of Embioptera rather than of Dictyoptera.

Hennig (1969, 1981) and Haas \& Kukalová-Peck (2001: fig. 23) discussed Dermaptera as the putative adelphotaxon of Dictyoptera (also recovered in the strict consensus of the morphological data by Wheeler et al. 2001a: fig. 10). However, Klass (2003b: 219) found only weak support for this hypothesis. While the position of Dictyoptera was unresolved in the analysis by Kjer (2004), a later study by Kjer et al. (2006) found Dictyoptera to be the sistergroup of (Grylloblattodea + Mantophasmatodea) + (Phasmatodea + Embioptera). Jarvis et al. 2005 (fig. 3) found Dictyoptera to be the sistergroup of Orthoptera + (Embioptera + (Phasmatodea + $($ Notoptera $+($ Zoraptera + Dermaptera $))))$ in their analysis which combined morphological and molecular data.

Dictyoptera and Phasmatodea formed sistergroups in the morphological analyses of insects by Beutel \& Gorb (2001: fig. 10) and Gorb \& Beutel (2001: fig. 1), whereas Beutel \& Gorb (2006: fig. 6) found support for Dictyoptera + (Orthoptera + Phasmatodea).

Fenn et al. (2008) found Dictyoptera to be the sistergroup of Orthoptera based on mitochondrial genome data.

Willmann (2003a, 2004) favoured Dictyoptera in a polytomy with Saltatoria + Phasmatodea and Embioptera.

The relationships of Dictyoptera were left unresolved by Kristensen (1981, 1991, 1995).

The internal relationships of Dictyoptera and those among its subgroups are beyond the goal of the present study and will therefore not be discussed in further detail.

\subsubsection{Monophyly of Mantodea}

The assumed monophyly of Mantodea is well supported by morphological characters (Hennig 1969, 1981; Ax 1999; Klass 1995, 1997; Deitz et al. 2003; Klass \& Meier 2006; Klass \& Eulitz 2007; Ware et al. 2008; Klass et al. 2009) and molecular data (Svenson \& Whiting 2003, 2004a, 2009; Terry \& Whiting 2005; Kjer et al. 2006; Inward et al. 2007; Lo et al. 2007; Fenn et al. 2008; Ware et al. 2008; Yager \& Svenson 2008).

Klass \& Ehrmann (2003: 196) listed the following autapomorphies for Mantodea. The forelegs are raptorial. The abdominal ganglia 2 and 3 are fused to the metathoracic ganglion 
(sic! Abdominal ganglia 1-3 are fused to the metathoracic ganglion, see Nesbitt 1941: 64 and fig. 37; Gebauer et al. 1987: 66; Hevers \& Liske 1991: 48; Köchy 1991: 97). The proventriculus morphology shows several special characters (including the lack of a sclerotized area and tooth on one of the main plicae of the proventriculus, see also Klass 1998b: 39). The fore femora have a specialized grooming device ("femoral brush"). A supracoxal sulcus is subdividing the prothorax into prozona and metazona and paraproct and paratergite 10 are fused.

Boudreaux (1979: 221) additionally mentioned the loss of segmental arteries in the thorax and the presence of calcium oxalate in the foamy secretion of the mantodean ootheca (and therefore the hardening of the ootheca only after deposition) as apomorphies for the group.

Klass \& Eulitz (2007: 231) listed several putative autapomorphies for Mantodea they derived from the head sulci and the tentorium. Among them is the presence of an interantennal sulcus bordering the characteristic scutellum of the mantodean frons (see characters 5-10).

A recent analysis of mantodean fore wings furthermore showed that the fusion of RP and M in the tegmina (i.e. forming a composite stem from the wing base) is apomorphic for Mantodea (Béthoux \& Wieland 2009: 83).

Another character that was debated to be of autapomorphic status for Mantodea is the free mobility of the head (e.g. Ax 1999: 288). However, this situation cannot be found in all Mantodea. In Chaeteessa Burmeister, 1838, Mantoida Newman, 1838, Metallyticus Westwood, 1835, Amorphoscelinae, Perlamantinae and Eremiaphilidae, the cervix is rather short and the head is much less mobile than in "higher" Mantodea. Although in these taxa the head is well able to revolve around the longitudinal axis, movement in the other planes is quite restricted (pers. obs. in Metallyticus, Mantoida, Perlamantis Guérin-Méneville, 1843, Amorphoscelis Stål, 1871, and Eremiaphila Lefèbvre, 1835). Furthermore, there are several cockroaches with highly moveable heads (see also characters 27, 28). Grimaldi (2008: 235) mentioned that in the Mantodea genera listed above the head movement is restricted due to the shortness of the pronotum ["The basal genera of mantises (i.e. Chaeteessa, Mantoida, Metallyticus, Amorphoscelis and Eremiaphila) actually have a short prothorax, and consequently the head is more sessile."]. Such coherence is ambiguous because there are many "higher" Mantodea with quite short prothoraces that are very well capable of moving their heads freely. In conclusion, the free mobility of the head cannot be interpreted as an apomorphy for Mantodea but for a group within Mantodea at most.

The praying mantids have retained several plesiomorphic traits, for instance the presence of three ocelli, the five-segmented tarsi, and the multi-segmented cerci. Furthermore, the long subcosta in the wings may be a plesiomorphic character (Karny 1921; Hennig 1969, 1981; but see Béthoux et al. 2009: 150 and discussions of characters 112 and 127 herein).

\subsubsection{Relationships within Mantodea and review of the main taxa of the current classifica- tion}

The greater part of attempts to elucidate the internal mantodean relationships have been typological, and most of the studies do not follow the principles of phylogenetic systematics sensu 
Hennig (1950) consistently. This is true for the works by Beier (1964a, 1968a) as well as the most recent ones by Ehrmann (2002) and Otte \& Spearman (2005). Roy (1987a) gave an overview of mantodean classification until the date of its publication.

Beier (1968a) maintained his previously established classification of 1964a almost unchanged in his synopsis of Mantodea in the Handbuch der Zoologie. He subdivided Mantodea into 8 families comprising 28 subfamilies and 27 tribus. Ehrmann (2002) categorized the group into 15 families with 48 subfamilies and 46 tribus (see Tab. 1). He raised several of Beier's (1968a) subfamilies to family rank (Liturgusidae, Tarachodidae, Iridopterygidae, Thespidae, Toxoderidae, Sibyllidae) and several former tribus to subfamily level. A new subfamily (Dystactinae) was erected and others were reinstated or taken over from earlier publications (e.g. Perlamantinae, Epaphroditinae, Acontiothespinae (= Acontistinae, see Roy 2004d, 2006), Pseudomiopteryginae, Hoplocoryphinae, Antemninae, Stagmatopterinae).

Several studies have addressed and discussed the phylogeny of mantodean taxa by applying phylogenetic systematics [among them Klass 1995 (Fig. 1A), 1997, 2003a; Jantsch 1999; Agabiti 2001; Wheeler et al. 2001a, b; Grimaldi 2003; Lombardo \& Ippolito 2004; Svenson \& Whiting 2004a, b, 2009; Grimaldi \& Engel 2005; Jarvis et al. 2005; Terry \& Whiting 2005; Klass \& Meier 2006 (Fig. 1B); Lo et al. 2007; Wieland 2003, 2006; Ware et al. 2008; Yager \& Svenson 2008; Béthoux \& Wieland 2009; Klass et al. 2009). However, they often considered only a small sample of Mantodea (e.g. Klass 1995, 1997, 2003a; Wheeler et al. 2001a, b; Jarvis et al. 2005; Klass \& Meier 2006; Inward et al. 2007; Lo et a. 2007; Klass et al. 2009) or focused on selected groups within Mantodea (e.g. Agabiti 2001; Lombardo \& Ippolito 2004). Only few studies used a larger taxon sample (Jantsch 1999; Svenson \& Whiting 2004a, 2009; Wieland 2006; Ware et al. 2008; Yager \& Svenson 2008; Béthoux \& Wieland 2009).

Jantsch (1999) studied the morphology of 78 genera (Fig. 2). He included mostly Neotropical species with only few Nearctic taxa (from North and Central America), thus being unable to address the phylogenetic relationships between the mantodean subgroups by omitting most of the phylogenetically important taxa (including Metallyticus, Amorphoscelidae, Eremiaphilidae, Hymenopodidae, Toxoderidae, Sibyllidae, Tarachodidae and Empusidae) which are all exclusively distributed throughout the Old World.

Wieland (2006) studied 41 dictyopteran species (30 species of Mantodea) but focused on a small number of characters from the cervical region, and did not calculate a phylogeny based on his data.

Another extensive taxon sample and at the same time the first molecular analysis of a large sample of Mantodea was published by Svenson \& Whiting (2004a, b; see Fig. 3). They studied the genetic data of 55 mantodean species from 8 out of 15 families and 20 out of 48 subfamilies sensu Ehrmann (2002). However, many important taxa were missing (for instance Chaeteessa, Metallyticus, Eremiaphilidae, Amorphoscelinae, Perlamantinae, Blepharodinae).

Yager \& Svenson (2008) studied the molecular phylogeny of more than 150 species. They additionally analysed morphological characters of the mantodean auditory system. Many key lineages that had been missing in the study by Svenson \& Whiting (2004a, b) were present in this analysis. 
Ware et al. (2008) generated a total evidence analysis based on several genes and morphology. They studied 23 Mantodea including Chaeteessa, Mantoida, Metallyticus, and Gyromantis Giglio-Tos, 1913. Their dataset lacked Eremiaphilidae, Amorphoscelinae, Perlamantinae, Toxoderidae, and Blepharodinae, among others.

Svenson \& Whiting (2009; see Fig. 4a-c) studied the most extensive dataset to date. They analysed nine genes (approximately 9.800 bp, see Svenson \& Whiting 2009: 475) for 288 mantodean species (329 specimens), encompassing members of all families and 45 out of 48 currently recognized subfamilies.

Mantoididae, Chaeteessidae, Metallyticidae. These three taxa will be treated as a unit because the discussion of the basal dichotomies of mantodean phylogeny usually revolves around them.

Mantoida shows several plesiomorphic traits of the male genital organs that it shares with cockroaches (Klass 1995, 1997; Klass \& Meier 2006). Autapomorphic characters for Mantoida (Figs. 7, 8, 412, 413) have not been mentioned.

Klass (1995: 192) stated several special morphological characters of the genital system to be autapomorphic for Chaeteessa (Figs. 5, 6).

Metallyticus (Figs. 9, 409-411) shows several characters that may be autapomorphic for the genus, for instance the iridescent body colouration (which is most elaborate in M. splendidus, see Wieland 2008a; Fig. 9) and the strongly enlarged proximal postero-ventral spine on the fore femora (Klass \& Ehrmann 2003; Wieland 2008a; Fig. 226).

Together, Mantoida, Chaeteessa and Metallyticus currently encompass 20 described extant species (see Ehr-
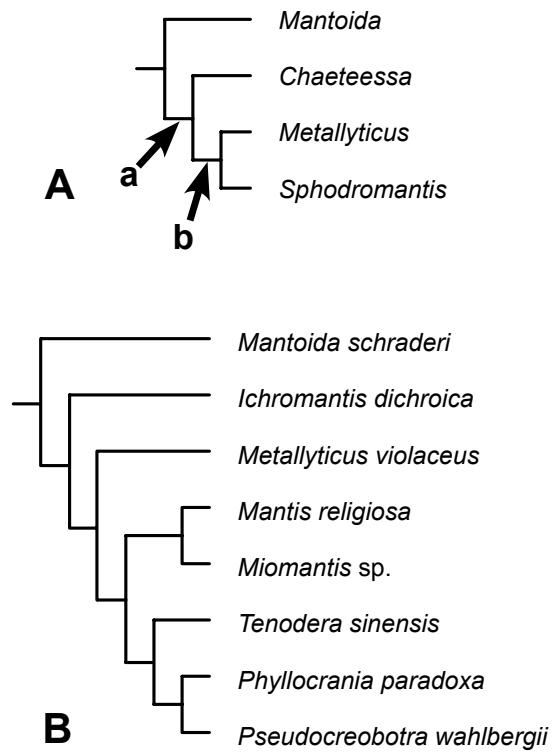

Fig. 1: Phylogenies of Mantodea. A: Klass $(1995,1997)$ and Klass \& Meier (2006); $\mathbf{a}=$ Mantomorpha; $\mathbf{b}=$ Mantidea. B: Inward et al. (2007). mann 2002; Salazar 2004b, 2005; Wieland 2008a; Roy 2010). Depending on the individual opinion of each author, one of the three genera is favoured to be the sistergroup of all remaining extant Mantodea. Only few phylogenetic studies included all three taxa in their datasets, among them Klass (1995, 1997; Fig. 1A), Klass \& Meier (2006), Wieland (2006), Ware et al. (2008), Yager \& Svenson (2008), and Svenson \& Whiting (2009). The studies by Svenson \& Whiting (2004a) and Lo et al. (2007) lacked Chaeteessa and Metallyticus, Inward et al. (2007; fig. 1B) did not include Chaeteessa, and Jantsch (1999) did not study Metallyticus. Jarvis et al. (2005) focused on Dermaptera, they had Mantoida in their analysis but only one further species of Mantodea, whereas Terry \& Whiting (2005) and Kjer et al. (2006) included none of the three taxa in their analyses.

It has been conjectured in the past that Chaeteessa might represent the sistergroup of the remaining extant mantodeans due to the lack of an enlarged apical antero-ventral spine (tibial claw or tibial spur) on the fore tibia and the slender ("setae-like") spines on the forelegs, which 
have been assumed to represent the plesiomorphic condition (e.g. Handlirsch 1930a; Beier 1964a, 1968a; Zherikhin 2002; but see character 68 and chapter 4.6.5). Furthermore, the wing venation has been stated to be most primitive in Chaeteessa (Smart 1956: 552). However, Klass (1995) and Klass \& Meier (2006) discussed and rejected the value of most of these characters. Instead, the missing tibial spur in Chaeteessa may represent a secondary loss instead of a primary lacking, as Klass \& Meier (2006) argued with regard to the Cretaceous †Jersimantis luzzii Grimaldi, 1997 (see also Grimaldi 2003; Fig. 31 herein). This fossil species had a distinct tibial spur while any spines on the forelegs were missing and only thin bristles were present (Grimaldi 1997: fig. 2, 2003: fig. 14). This is supported by the presence of a setigerous tubercle in the position of the tibial spur in extant Chaeteessa (Roy 1999: 38) (see character 68, discussion in 4.6.5 and Figs. 418-422). The position of Chaeteessa as the sistergroup of the remaining Mantodea was not supported in the analysis by Yager \& Svenson (2008: fig. 6) but was found in the molecular analysis by Svenson \& Whiting (2009: fig. 3) with high support after the extraction of tissue from a fresh specimen (see Yager \& Svenson 2008: 561; Svenson \& Whiting 2009: fig. 3). It was also assumed in the mental analysis published by Grimaldi (2003: fig. 27) and Grimaldi \& Engel (2005: fig. 7.60).

Roy (1999: 37) argued that "the Metallyticidae with only Metallyticus in the eastern region are probably the most primitive of mantids today" (implicating a sistergroup relationship between Metallyticus and the remaining extant Mantodea). He deduced this mainly from the primitive characters of the wing venation $(\mathrm{CuA}$ exhibiting the highest number of branches in Mantodea, thus strongly resembling the situation in cockroach wings) and the lack of discoidal spines, which he

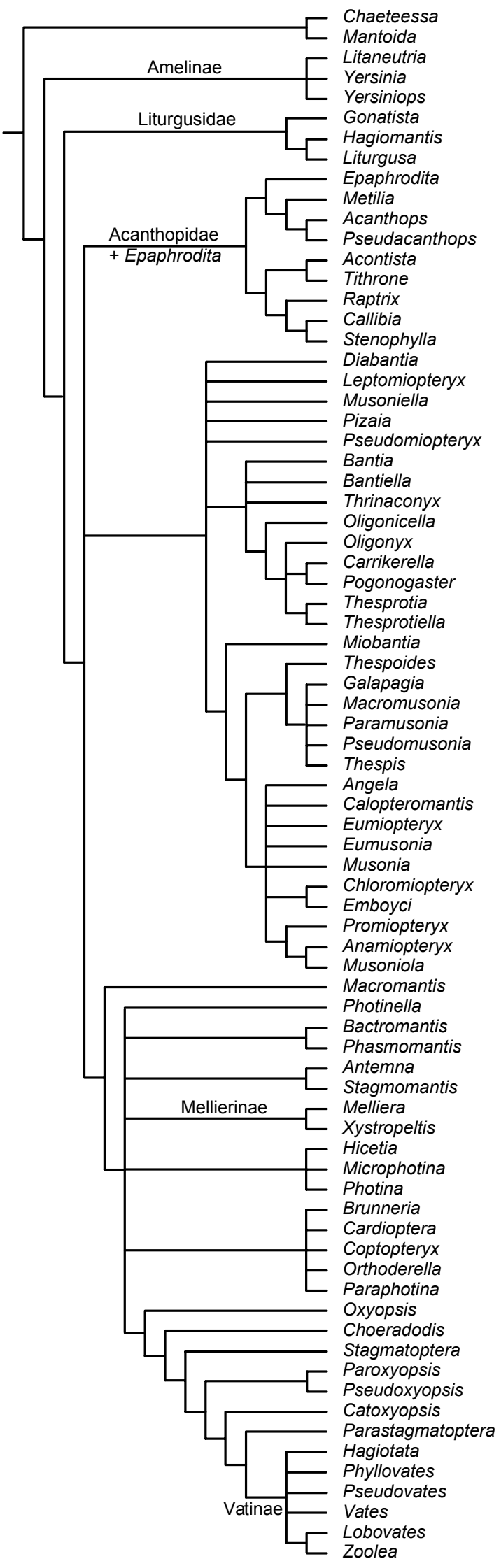

Fig. 2: Phylogeny of neotropical Mantodea found by Jantsch (1999: fig. 8). 
considered to be missing primarily (see also Wieland 2008a; but see characters 54, 55 and chapter 4.6.4).

Morphological and several molecular datasets have repeatedly found Mantoida to be the sistergroup of the remaining extant Mantodea (Klass 1995, 1997; Svenson \& Whiting 2004a; Klass \& Meier 2006; Inward et al. 2007; Lo et al. 2007; Ware et al. 2008; Yager \& Svenson 2008). Jantsch (1999) found a basal split between a monophyletic Mantoida + Chaeteessa and the remaining (predominantly Neotropical) Mantodea in his morphological analysis. Aclose relationship between the two genera had been assumed earlier, for instance by Giglio-Tos (1921, 1927) who, however, did not provide any arguments for this hypothesis.

The morphological data gained from the male genitalia (Klass 1995, 1997; Klass \& Meier 2006) unambiguously support Mantoida + (Chaeteessa + (Metallyticus + “higher" Mantodea)).

Inward et al. (2007), who did not include Chaeteessa in their analysis, found the second dichotomy after Mantoida between Ichromantis dichroica Paulian, 1957, a Madagascan Iridopterygidae (see Paulian 1957: figs. 20, 21), and the remaining Mantodea. Only then followed Metallyticus at the next dichotomy. Similar results were found in the

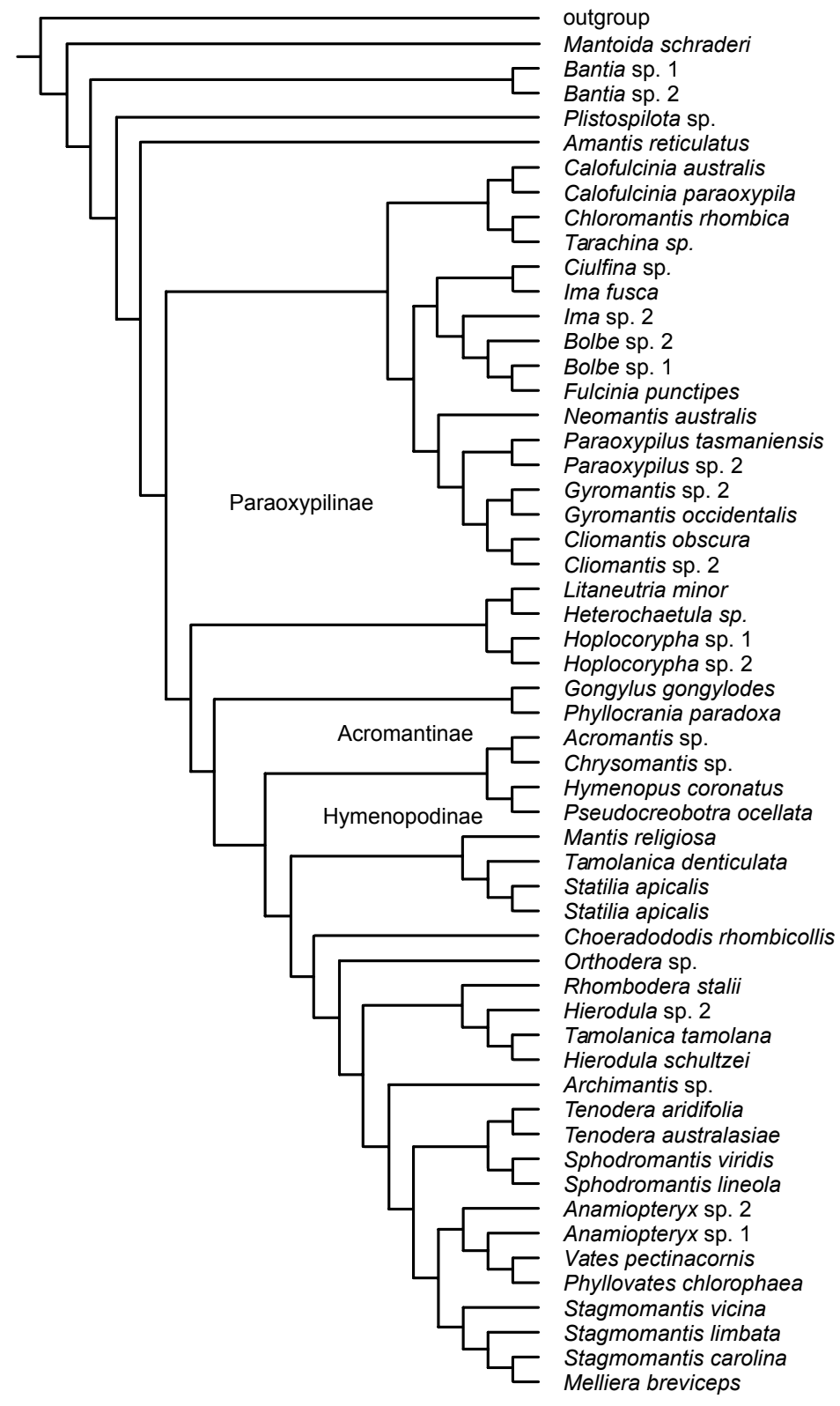

Fig. 3: Phylogeny found by Svenson \& Whiting (2004a: fig. 1). (2008). Therein, Metallyticus was found in a polytomy also comprising Chaeteessa and several "higher" taxa (the Thespidae Bantia Stål, 1877 from the Neotropics and Hoplocorypha Stål, 1871 from Africa) at the second dichotomy in the parsimony analysis (Ware et al. 2008: figs. 2A, B). In their Bayesian analysis, Metallyticus was recovered in a polytomy together with all remaining Mantodea at the fourth or, alternatively, third dichotomy (Ware et al. 2008: figs. 3A, B, respectively). This is an indication of Metallyticus possibly being positioned more apically in the phylogenetic tree than previously estimated. 
Svenson \& Whiting (2009: 492 and tab. 6) found that the support values for Chaeteessa being the sistergroup of all remaining extant Mantodea were very high but resulted mainly from the NADH hydrogenase subunit 4 (ND4) and 16S rRNA, whereas other genes, namely COI, COII, H3 and Wingless yielded contradicting signals. The remaining loci, $12 \mathrm{~S}, 18 \mathrm{~S}$ and $28 \mathrm{~S}$ rRNA, gave rather neutral signals. The second split was found between Mantoida and the remaining Mantodea with high support. The position of Metallyticus, however, was ambiguous. Parsimony analysis resulted in a position of the genus at the third dichotomy in the phylogenetic tree (following Chaeteessa and Mantoida; as sistergroup of Artimantodea, see discussion of further phylogenetic hypotheses below), whereas the maximum likelihood (ML) and mixed model Bayesian (MMB) analyses showed Metallyticus to be in a more apical position inside Artimantodea, as sistergroup of Cernomantodea. The low nodal supports found in both analyses indicated that the genetic evidence does not support either position (Svenson \& Whiting 2009: 495).

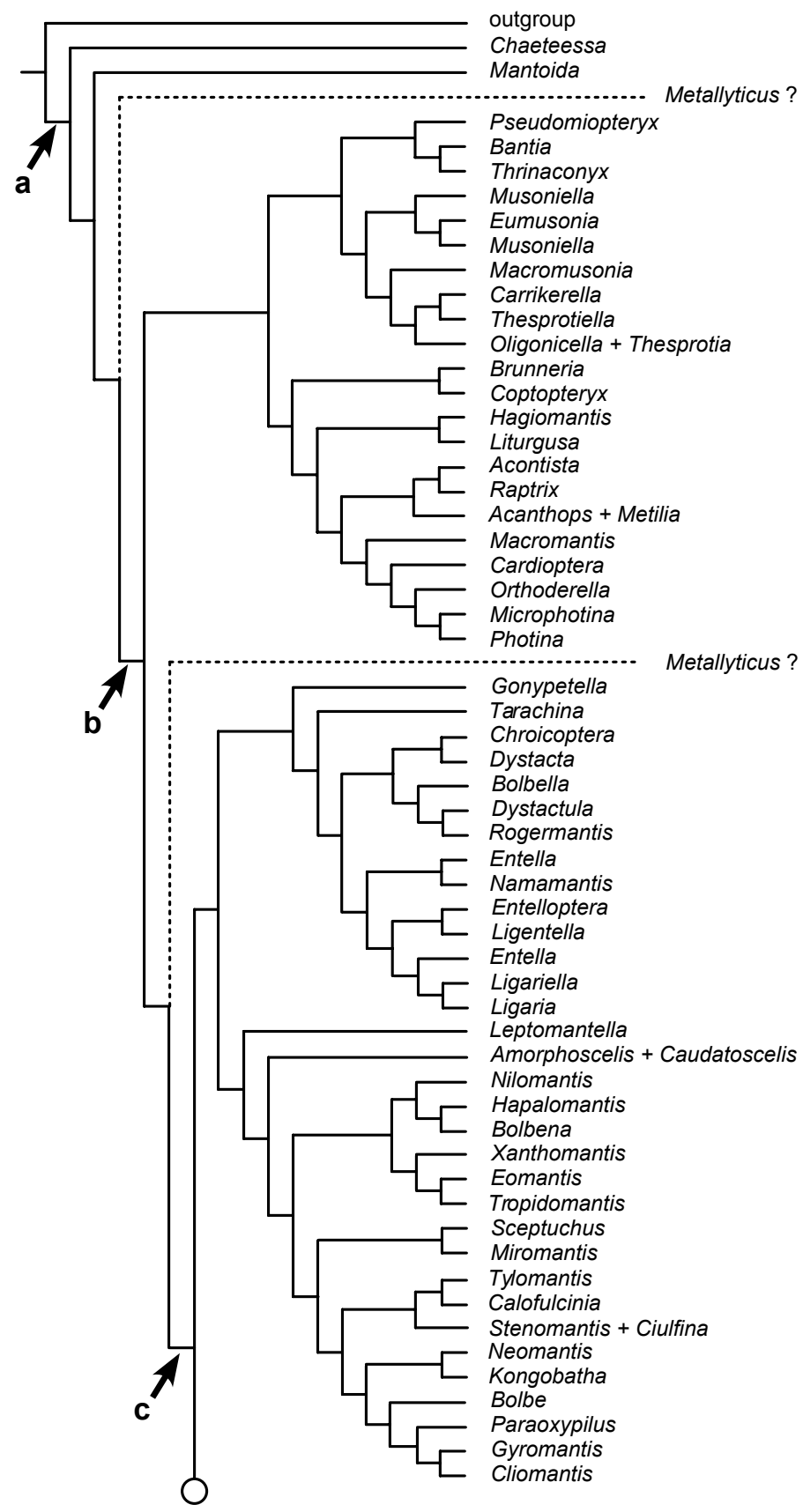

Fig. 4A: Mantodean phylogeny found in the analysis by Svenson \& Whiting (2009), slightly simplified. $\mathbf{a}=$ Eumantodea; $\mathbf{b}=$ Artimantodea; $\mathbf{c}=$ Cernomantodea. However, preliminary results gained from the analysis of the entire mitochondrial genome of representative taxa support the apical position of Metallyticus inside Artimantodea (Svenson, pers. comm. 2009).

Further analysis (SH-test) showed that none of the six possible hypotheses for the three taxa regarding their position on the branches of the lowest nodes of the mantodean phylogeny could be rejected (Svenson \& Whiting 2009: 495).

Béthoux \& Wieland (2009) stated that crowngroup-Mantodea are morphologically characterized by the fusion of the RP and M from the wing base in the tegmina (Béthoux \& Wieland 2009: 79, 101). Metallyticus and Chaeteessa on the other hand exhibit several plesiomorphic 
characters regarding their forewing venation (RA with distal anterior branches; M branched; see Béthoux \& Wieland 2009: 101). Furthermore, all extant Mantodea except for Chaeteessa, Mantoida and Metallyticus and (some) Amorphoscelidae exhibit a secondary fusion of an anterior branch of RP + M with RA (Béthoux $\&$ Wieland 2009: 94). From the angle of wing morphology these data indicate a position of these three groups at the lower dichotomies of the phylogenetic tree.

Obviously, many studies (including the molecular ones) show strongly differing results as far as the basal dichotomies of the mantodean phylogeny are concerned.

Amorphoscelidae. This group currently comprises 15 genera and is traditionally subdivided into three subgroups: Perlamantinae (two genera; Fig. 12), Amorphoscelinae (five genera; Fig. 11) and Paraoxypilinae (eight genera; Fig. 219) (Ehrmann 2002). The former two subgroups have their main distributions in southern Europe and Northern Africa (Perlamantinae) and subsaharan Africa and Southeast Asia (Amorphoscelinae). Paraoxypilinae is distributed throughout in Australia and New Guinea with a single species (Exparoxypilus africanus Beier, 1929) having been described from Tanzania.

Roy (1999: 38) stated with respect to the subgroups of Amorphoscelidae that "none of these subfamilies would be considered primitive in relation to the others, but the first two (Amorphoscelinae and Perlamantinae) have more in common with each other than with the third". Furthermore, Roy stated that Amorphoscelinae and Perlamantinae "do

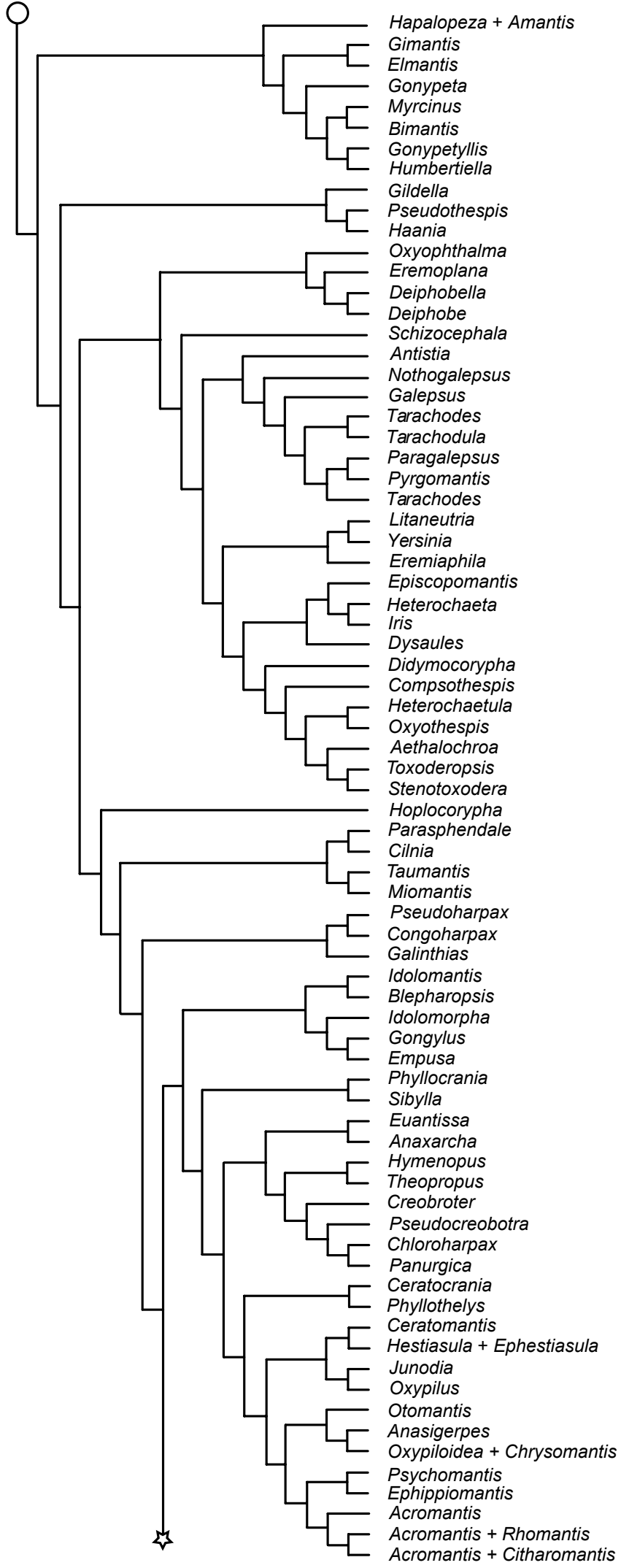

Fig. 4B: Mantodean phylogeny found in the analysis by Svenson \& Whiting (2009), slightly simplified (continued). 
not seem to have more points in common than do the Chaeteessidae and Mantoididae" (Roy 1999: 38).

Chopard (1949a: 398) assumed that Amorphoscelidae may be polyphyletic because it was created mainly based on the reduced armature of the raptorial forelegs. The forelegs probably represent a highly derived condition that may have evolved convergently in either lineage (Smart 1956: 546). A detailed comparative morphological study of the raptorial legs of the three subgroups has not been conducted to date, therefore this discussion lacks convincing support from foreleg morphology.

Phylogenetic data on Amorphoscelidae are scarce. Wieland (2003) found support for the group being paraphyletic with respect to Paraoxypilinae based on morphological characters of the ventral prothorax (see characters 25 and 26). In his study of the cervical sclerites Wieland (2006) included Paraoxypilinae and

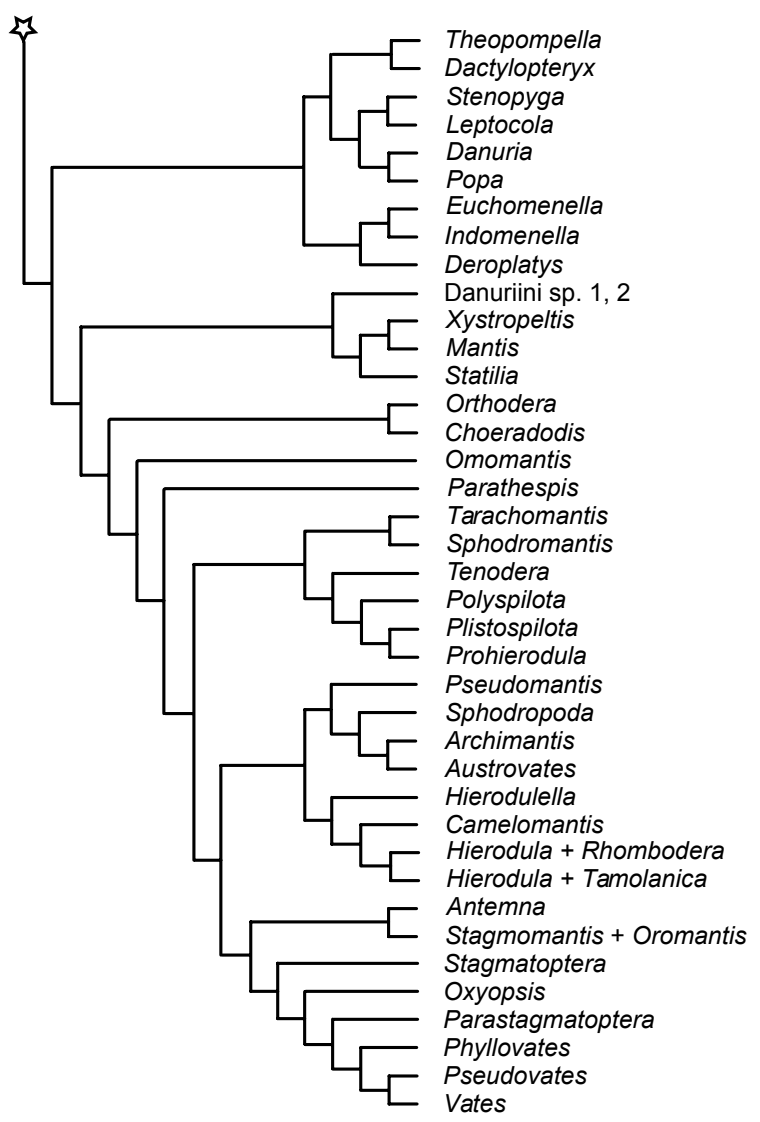

Fig. 4C: Mantodean phylogeny found in the analysis by Svenson \& Whiting (2009), slightly simplified (continued). Perlamantinae. Both have a common symplesiomorphic trait (intercervicalia separated), but no synapomorphies could be pointed out. However, the data were not analysed by means of a phylogenetic reconstruction based on the characters of the cervical sclerites. Instead, the characters were mapped onto a synoptic cladogram gathered from literature data which showed Amorphoscelidae as a monophyletic group.

Svenson \& Whiting (2004a) provided molecular data for three genera (Cliomantis GiglioTos, 1913, Gyromantis, and Paraoxypilus Saussure, 1870), all being assigned to Paraoxypilinae. In the resulting phylogenetic tree they came out monophyletic with the Australian Neomantis australis (Saussure \& Zehntner, 1895) (Iridopterygidae: Tropidomantinae) as their sister taxon. Although the analysis supported the assumed monophyly of Paraoxypilinae, their relationships with regard to Amorphoscelinae and Perlamantinae could not be addressed because neither of the two subgroups were sampled in the study. Paraoxypilinae was found in an apical position in the phylogenetic cladogram, thus leading Svenson \& Whiting (2004a) to the hypothesis that the short prothorax of Paraoxypilinae is likely to be a secondary trait. This contradicts the view that short prothoraces generally represent the plesiomorphic state in Mantodea (e.g. Roy 1999: 35; see character 28).

Kjer et al. (2006) only included one species of Paraoxypilinae (Paraoxypilus sp.), which clustered with the Australian Kongobatha Hebard, 1920 (Iridopterygidae: Tropidomantinae). 

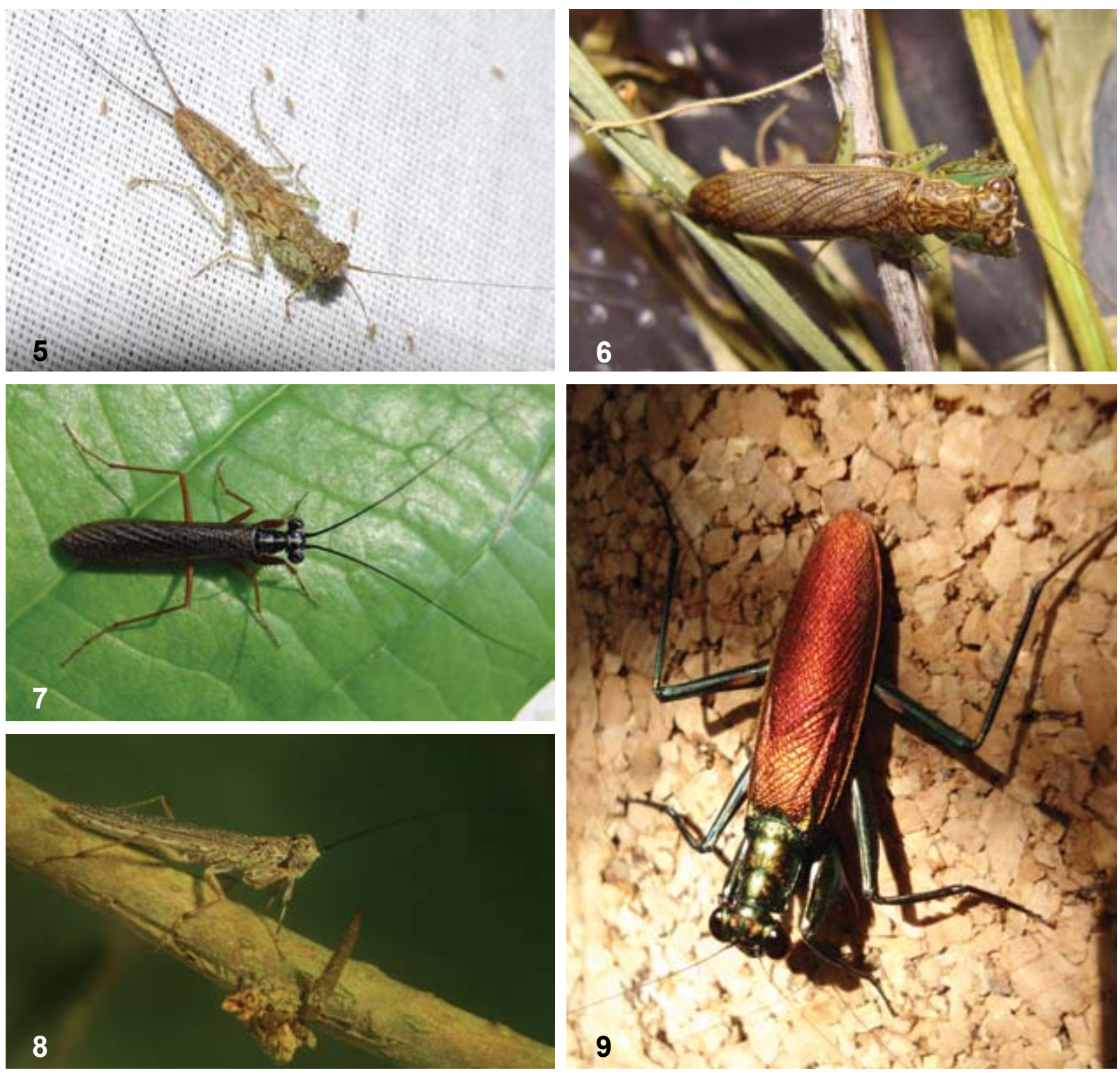

Figs. 5-9. The three lineages branching off from the basal dichotomies of the mantodean tree. 5: Chaeteessa sp., living late instar nymph from Ecuador (picture courtesy of Geoffrey Gallice, 2009). 6: Chaeteessa sp., freshly deceased adult specimen from Costa Rica (picture courtesy of Karin Sieber; length of specimen about $2 \mathrm{~cm}$ ). 7: Mantoida maya, living adult from Florida (picture courtesy of Machele White; length of specimen about $2 \mathrm{~cm}$ ). 8: Mantoida sp., living adult, from Venezuela (picture courtesy of Sören Materna and Tobias Schulze; length of specimen about $2 \mathrm{~cm}$ ). 9: Metallyticus splendidus, living female, captive bred; length of specimen about $3 \mathrm{~cm}$.

Even though this is interesting due to Svenson \& Whiting's (2004a) findings of Tropidomantinae (partim) being the sistergroup of Paraoxypilinae, the sample of Mantodea used by Kjer et al. (2006) was far too small to derive any phylogenetic conclusions from it.

Yager \& Svenson (2008) analysed basically the same set of Amorphoscelidae as Svenson \& Whiting (2009; listed below). Paraoxypilinae was therein found to be monophyletic, with the Australian Neomantis as their sistergroup. This further supported a close relationship between Paraoxypilinae and Tropidomantinae (partim). Amorphoscelinae was found to be monophyletic and nested within a group of several genera (most of them being assigned to Tropidomantinae). Although Amorphoscelidae was found to be paraphyletic, both subgroups were positioned in the same larger clade (marked as clade A in Yager \& Svenson 2008: fig. 6). 
Svenson \& Whiting (2009) included a considerable number of Amorphoscelidae in their analysis. Among them were species of two genera of Amorphoscelinae (Amorphoscelis, Caudatoscelis Roy, 1973) and three of Paraoxypilinae (Paraoxypilus, Gyromantis, Cliomantis). Perlamantinae were not represented. Both Amorphoscelinae and Paraoxypilinae came out monophyletic. The two groups were found in the same larger clade with Amorphoscelinae being the sistergroup of a clade containing several Nanomantinae, Hapalomantinae, Tropidomantinae, Liturgusinae, and Paraoxypilinae. Paraoxypilinae was therein nested in a larger monophyletic Australian clade (Svenson \& Whiting 2009: fig. 3b, clade 102) which also included the Australian Iridopterygidae and Liturgusidae.

Similar results of Paraoxypilinae (represented by Gyromantis) being part of an Australasian clade encompassing Australian Iridopterygidae were found by Ware et al. (2008: figs. 2, 3) in both their parsimony and Bayesian analyses.

Many taxonomic works on Amorphoscelidae have been published (e.g. Werner 1907; GiglioTos 1913; Beier 1929; Roy 1962, 1963a, 1964, 1965a, b, 1966, 1973, 1984, 2007, 2009b; Roy \& Leston 1975; Milledge 1990; Roy \& Stiewe 2009). Most of them focus on few species or single genera. None of the larger studies, neither molecular nor morphological, encompassed representatives of all three traditional subgroups of Amorphoscelidae. Only Yager \& Svenson (2008) included Paramorphoscelis gondokorensis Werner, 1907 (monotypic, one of the two genera of Perlamantinae, the other one being Perlamantis Guérin-Méneville, 1843) in the morphological analysis of their study, but not in the molecular analysis (Yager \& Svenson 2008: appendix 1). Therefore, neither the monophyly of Perlamantinae nor its phylogenetic affinities have so far been addressed.

Béthoux \& Wieland (2009) studied the forewing morphology of several species of Amorphoscelinae and found a high degree of variability in the venation pattern. However, their findings could not contribute to the phylogenetic relationships of the three subgroups of Amorphoscelidae.

Eremiaphilidae. This group was established as Eremophilidae by Stål (1877: 6; later emended as Eremiaphilidae by Wood-Mason 1889: 9). Stål (1877), Wood-Mason (1889) and Giglio-Tos (1921) included several heterogeneous taxa in their Eremiaphilinae or Eremiaphilidae (e.g. Metallyticus, Chaeteessa, Mantoida, Liturgusidae, Orthoderinae, Tarachodidae). Today only two genera, Eremiaphila (Fig. 10) and Heteronutarsus Lefèbvre, 1835 (Fig. 276), are included in Eremiaphilidae (Ehrmann 2002). They dwell the stony and sandy deserts from northern Africa and Turkey throughout the Middle East up to India (e.g. Kaltenbach 1980, 1982; Roy 1999; Ehrmann 2002). Their colouration strongly resembles the colour of the underground of their habitat (homochromy; e.g. Innes Bey 1909: 35; Edmunds \& Brunner 1999: 281). Both genera show similar morphological traits that are correlated with their deserticolous lifestyle: Their cursorial legs are exceptionally long (Figs. 10, 276; typical for diurnal desert dwelling insects, see Crawford 1981: 74), and they are extremely vivid runners (e.g. Chopard 1938: 60; Kral \& Prete 2004: 76; Battiston et al. 2010: 169). Furthermore, they carry strongly elongated apical spines 

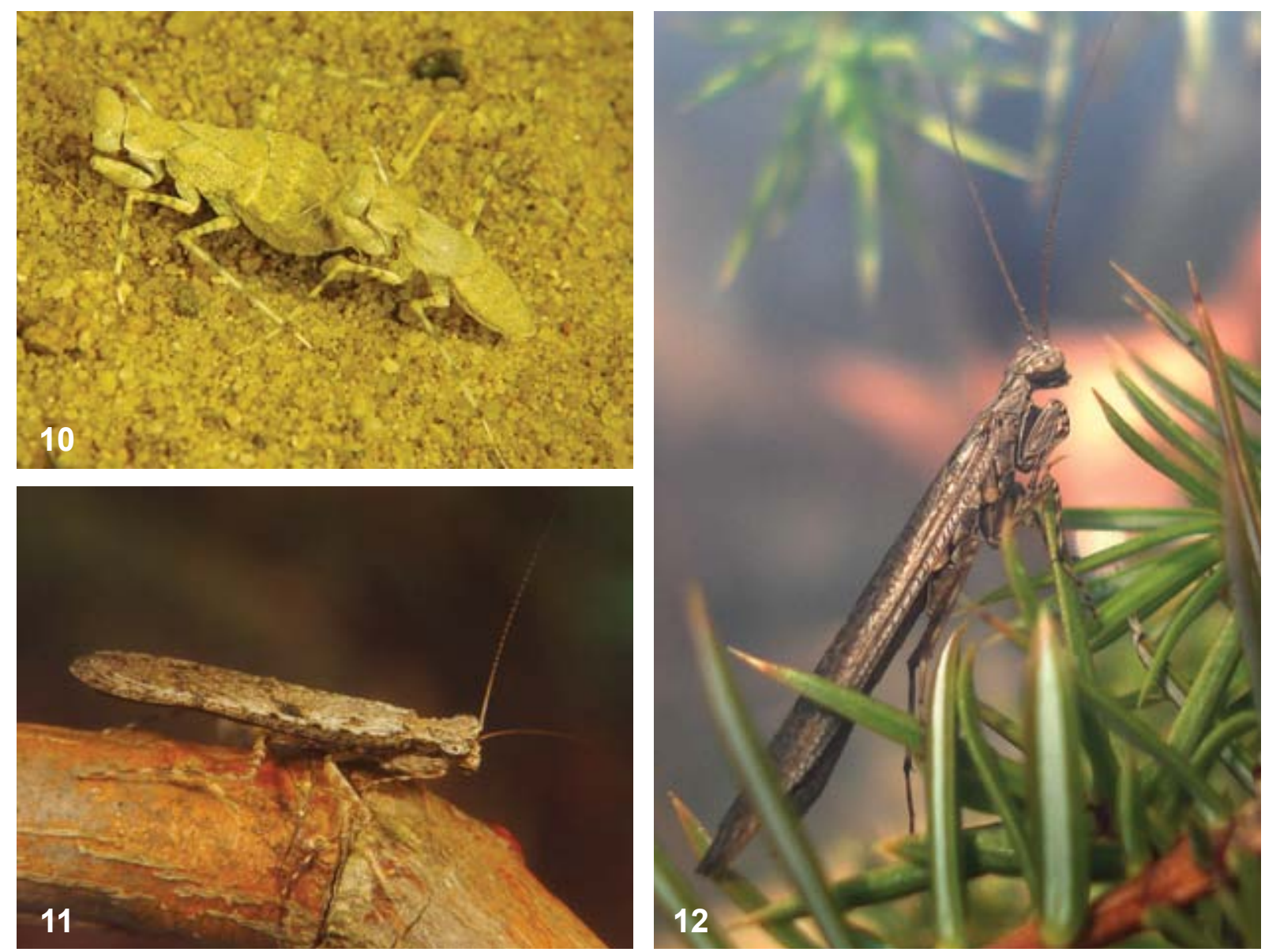

Figs. 10-12: Pictures of living Eremiaphilidae and Amorphoscelidae. 10: Eremiaphila sp. 1 from Pakistan, pair ( + left; captive bred; courtesy of Sören Materna); size of $q$ approximately $3 \mathrm{~cm}$. 11: Amorphoscelis $\mathrm{sp}$. from Malaysia (courtesy of Sören Materna); size approximately $2 \mathrm{~cm} .12$ : Perlamantis allibertii, $\widehat{\jmath}$, from southern France; size of specimen about $18 \mathrm{~mm}$.

on the meso- and metathoracic tibiae (Beier 1964a), as well as elongated ungues (Figs. 276, $305,332,333)$, both helping to drive the body forward when running on sand. Their bodies are stout and both sexes are micropterous. The most important similarity is the presence of two sturdy ventral spines that originate from the sixth sternite of the females (Lefèbvre 1835; Chopard 1941; Roy 1999; Klass \& Ehrmann 2003; Wieland 2008b) - not from the subgenital plate as erroneously stated, for instance, by Chopard 1949a ("7e sternite"), Mukherjee et al. 1995 (“last sternum”), Ehrmann 2002 (“Subgenitalplatte”), Çiplak \& Demirsoy 1997 ("subgenital plate"), Doganlar 2007 ("subgenital plate"), and Battiston et al. 2010 ("last ventral segment"). This type of female digging device is autapomorphic for Eremiaphilidae (Wieland 2008b, named "Eremiaphilidae-type" therein; see character 141, chapters 4.3 .8 and 4.6 .9 and Figs. 265-268).

Heteronutarsus is the only representative of Mantodea that does not exhibit pentamerous tarsi, which therefore probably represents an autapomorphic character for the genus. Instead, it has four tarsomeres on the prothoracic and three on the meso- and metathoracic legs (Chopard 1941, 1949a; Beier 1968a; Roy 1999; Ehrmann 2002; Klass \& Ehrmann 2003; see chapter 4.6 .6 and Figs. 423-425 for a detailed discussion). However, one eremiaphilid species, Eremiaphila zolotarevskyi Chopard 1940 (b: 17), has five tarsomeres on the fore tarsi and four on each of the 
meso- and metathoracic tarsi. Chopard himself suspected that this species might actually belong to Heteronutarsus (Chopard 1941: 34) but it is also possible that it actually is an Eremiaphila in which the four posterior tarsi were autotomized during postembryonic development and then regenerated with only four tarsomeres (which is typical for re-grown tarsi in Mantodea, see for instance Przibram 1907: 612 and Beier 1964a: 860).

Phylogenetic studies involving Eremiaphilidae are scarce. The group has taxonomically been placed between the "lower" (Chaeteessa, Mantoida, Metallyticus) and the "higher" Mantodea or Mantoidea (Roy 1999: 39). Mantoidea are assumed to include the Mantodean taxa apart from those discussed before (but see discussion of further phylogenetic hypotheses in chapter 2.1.5). Grimaldi (2003: fig. 27) and Grimaldi \& Engel (2005: fig. 7.60) found Eremiaphilidae in a polytomy together with Amorphoscelidae and Mantoidea, following the basal splits between Chaeteessa (first dichotomy) and Metallyticus and Mantoida (second dichotomy, unresolved) (Fig. 28).

The only molecular datasets including representatives of Eremiaphila were analysed by Yager \& Svenson (2008) and Svenson \& Whiting (2009). The latter found Eremiaphila in a monophyletic group with Yersinia mexicana (Saussure, 1859) and Litaneutria minor (Scudder, 1872) (both Nearctic, ground-dwelling Amelinae), although without strong support (ML bootstrap value $<50$ for nodes 149, 161 and 162).

In the study by Yager \& Svenson (2008), Eremiaphila was found in a monophyletic group together with Deiphobella Giglio-Tos, 1916 and Deiphobe Stål, 1877 (Miomantinae, both mainly distributed in India) within in a larger clade containing many Tarachodidae, Amelinae, Oxyothespinae and Toxoderidae. Although Litaneutria and Yersinia were in the same larger clade, they did not form a monophyletic group with Eremiaphila therein.

In their morphological analysis of Dictyoptera Klass \& Meier (2006: 17) briefly mentioned a similarity of the genital structures of Eremiaphilidae, Metallyticus, and Sphodromantis Stål, 1871 from a comparison with literature data, but did not raise new data for Eremiaphila. Wieland (2003: 26, 2006: 58) included Eremiaphila in his morphological studies of the ventral prothorax and the cervical sclerites. In these structures, Eremiaphilidae shows several plesiomorphic traits, such as the slender anterior part of the T-shaped sclerite (see character 26) and the medially separated intercervical sclerites (Wieland 2006: 70; see character 23). The prothorax may be secondarily shortened in Eremiaphilidae (as in Paraoxypilinae, see discussion of Amorphoscelidae above), as they have been found to be nested among taxa with long prothoraces in the molecular analyses.

Acanthopidae. The first author who established Acanthopidae (mentioned as Acanthopsidae for Acanthops Audinet-Serville, 1831 and Schizocephala Audinet-Serville, 1831) was Burmeister (1838: 551). Giglio-Tos (1919: 71) included several of the genera today assigned to Acanthopidae in his Acontistinae and Epaphroditinae. Travassos Filho (1945: 162) emended Burmeister's name of the group to Acanthopidae and included mainly the taxa that are today still assumed to belong to it (see, for instance, Terra 1995: 23 and Ehrmann 2002: 374). Beier 
(1964a: 938, 1968a: 6) placed these taxa in Hymenopodidae: Acromantinae: Epaphroditini. Terra (1995: 23) reinstated Acanthopinae to family status (independent of Hymenopodidae), following Burmeister (1838) and Travassos Filho (1945), and regarded Stenophylla Westwood, 1843 as a subgroup of Acanthopidae.

Acanthopidae is currently subdivided into three subgroups: Acanthopinae (six genera), Acontistinae (six genera), and Stenophyllinae (monogeneric) (Terra 1995; Ehrmann 2002: 374 and tab. 13; Roy 2002a, 2004c, d, 2006; Ehrmann \& Koçak 2009). As the latter subfamily is monogeneric, it is herein considered redundant and will be referred to by its generic name Stenophylla.

While the genera of Acanthopinae and Acontistinae have formerly typologically been placed in Hymenopodidae subgroups Acromantinae and Hymenopodinae (Beier 1968a: 6), Stenophylla was previously assigned to Toxoderidae (Giglio-Tos 1914a: 5, 1919: 74, 1927: 577; Beier 1934c: 8, 1968a: 12; see also discussion of Toxoderidae in this chapter).

Autapomorphic characters for Acanthopidae have not been stated yet. Its species are earless, which likely represents the plesiomorphic condition (Yager \& Svenson 2008).

Jantsch (1999: 39) included 8 genera of Acanthopidae sensu Ehrmann (2002) and found support for the monophyly of Acanthopinae, and of Acontistinae + Stenophylla. Epaphrodita Audinet-Serville, 1831 from the Greater Antilles formed the sistergroup of Acanthopinae, thus rendering Acanthopidae as whole paraphyletic. In the classification by Ehrmann (2002: 374), Epaphrodita is listed as a member of Hymenopodidae: Epaphroditinae. However, the assumed close relationship of Epaphrodita with members of Hymenopodidae is questionable as it would be the only New World representative of Hymenopodidae (see Ehrmann 2002: 374). Unfortunately, Jantsch (1999) was not able to elucidate this problem from a phylogenetic point of view due to the lack of Hymenopodidae (especially African Epaphroditinae) in his analysis.

The interest in the often bizarrely shaped species of Acanthopidae has risen considerably throughout the recent decades. Many, mostly taxonomical studies on the group have been published in the last twenty years by Lombardo (1996, 2000a, b), La Greca \& Lombardo (1997), Barabás \& Hancock (1999), Roy (2002a, b, 2004c, d, 2005a, 2006), Salazar (2003, 2004a, 2007), Ippolito \& Lombardo (2004), Lombardo \& Ippolito (2004), Lombardo \& Marletta (2004), Rivera (2005) and Ippolito (2007). A list of the species currently assigned to Acanthopidae can be found in Ehrmann \& Koçak (2009).

The assumption of monophyly of Acanthopinae has been mentioned in the literature (Rivera 2005: 255) without, however, providing apomorphic characters to support it. Lombardo \& Ippolito (2004: fig. 1) provided a phylogenetic analysis of the genus Acanthops based on morphology with one species of Pseudacanthops Saussure, 1870 as outgroup. The study revealed no information on to the phylogenetic relationships of Acanthops with respect to other Mantodea or to the putative subgroups of Acanthopidae.

Svenson \& Whiting (2009) included eight species of Acanthopidae (four of both Acontistinae and Acanthopinae) in their analysis. Therein, both subgroups were found to be monophyletic (nodes 38 and 41, ML bootstrap values of 100 each) and came out as sistergroups, thus 
rendering Acanthopidae monophyletic with high statistical support (node 37, ML bootstrap value of 100). Stenophylla was not included, therefore its affinities with respect to the other subgroups and the putative monophyly of the entire traditional Acanthopidae remain obscure. Interestingly, Acanthopidae formed the sistergroup of a clade containing all South American Liturgusidae and Mantidae: Photininae in the study. The resulting taxon was the sistergroup of a clade containing all South American Thespidae of the taxon sample. These groups together formed a major monophyletic Neotropical taxon near the base of the phylogenetic tree (node 8, although with an ML bootstrap value of $<50$ ). It included all Neotropical taxa except for three distant clades in the apical part (nodes 271, 282 and 307). The latter were interpreted to represent independent later invasions into South America (Svenson \& Whiting 2009).

Similar results for the relationships of Acanthopidae were found by Yager \& Svenson (2008: fig. 6).

Hymenopodidae. 44 genera in four subgroups are currently assigned to Hymenopodidae: Hymenopodinae (14 genera), Acromantinae (20 genera), Epaphroditinae (four genera) and Oxypilinae (six genera) (Ehrmann 2002: 374 and tab. 13). They are distributed mainly throughout Africa and Southeast Asia. A single species (Acromantis australis Saussure, 1871; Acromantinae) has been mentioned for Australia (Balderson 1991: 355; Rentz 1996: 240; Balderson et al. 1998: 261 ff.; vs. Roy 1999: 39). Saussure (1871: 450) had described the species from "Les Moluques, île de Waigiou". Waigeo is an island belonging to Western New Guinea (Indonesia). The presence of Acromantis australis in North-Eastern Australia was confirmed by Rentz (1996: 240) and others.

A single genus, Epaphrodita (if actually related to Hymenopodidae, see discussion of Acanthopidae in this chapter), occurs exclusively in the Greater Antilles (Caribbean).

Only one character is a putative apomorphy for the strongly heterogeneous Hymenopodidae. The postero-ventral spines of the fore tibiae are laid down almost horizontally and are positioned very closely together (Beier 1964a: 938, 1968a: 5). However, this character is not distinct throughout the group. Roy (1999: 39) stated that Hymenopodidae is "widely accepted but it should be noted that its boundaries are uncertain and it is not obviously monophyletic" due to these inconsistencies. Also, the postero-ventral tibial spines are organized in a very similar characteristic manner in the Madagascan Brancsikia Saussure \& Zehntner, 1895 (Mantidae: Deroplatyinae) which in the current classification is not considered to be closely related to Hymenopodidae.

Roy (1999: 39) stated that one subgroup (Hymenopodinae) is "homogenous", but that the classification of Hymenopodidae still requires many reviews. His taxonomic assumptions were taken into account in the latest classification by Ehrmann (2002).

Yager \& Hoy $(1986,1987)$ were the first to describe a metathoracic tympanal organ ("cyclopean ear") for "higher" Mantodea. Later, Yager (1996a) discovered a serially homologous second (mesothoracic) tympanal organ in several genera of Hymenopodidae (Creobroter Audinet-Serville, 1839, Pseudocreobotra Saussure, 1870, Hestiasula Saussure, 1871). All genera 
he studied showed distinct responses to two different frequency ranges, in contrast to all other hearing Mantodea, which respond to only one. The metathoracic reception resembled that of non-hymenopodid species. The metathoracic ear has been shown to form an effective evasion system against echolocating bats (Yager \& Hoy 1989; Yager et al. 1990; Yager 1996a: 472; but see "metathoracic hearing organ" in chapter 4.1.2 for a discussion). The mesothoracic sound perception, only found in certain species of Hymenopodidae, differs from the mesothoracic one. Yager (1996a: 471) concluded that different behavioural functions would be imaginable, because of the independent neuronal operation and the two non-overlapping frequency ranges perceived by the two ears. He proposed intraspecific communication as one possible answer (Yager 1996a: 472), perhaps due to the extremely small size of the males in some Hymenopodidae (e.g. as little as 40 per cent of the female length in Hymenopus Audinet-Serville, 1831 and Theopropus Saussure, 1898). However, such intraspecific acoustical communication has not been documented so far.

Nonetheless, the mesothoracic ear may represent an autapomorphy at least for a group within Hymenopodidae. The genera tested by Yager (1996a: 464) are assigned to two of the hymenopodid subgroups, Hymenopodinae and Acromantinae. The two other subgroups, Epaphroditinae and Oxypilinae, were not studied.

Svenson \& Whiting (2004a: fig. 1) included five species of Hymenopodidae assigned to Acromantinae, Hymenopodinae and Epaphroditinae in their molecular analysis. According to their results, the South-East Asian Hymenopus (Fig. 15) and the African Pseudocreobotra (Fig. 24) form a monophyletic group (i.e. Hymenopodinae) which came out as the sistergroup to Acromantinae. The latter comprises the South-East Asian Acromantis Saussure, 1870 and the African Chrysomantis Giglio-Tos, 1915. The African Phyllocrania Burmeister, 1838, however, traditionally a member of Epaphroditinae, came out as the sistergorup of the Indian Gongylus Thunberg, 1815 (Empusidae), thus rendering the traditional Hymenopodidae paraphyletic (Svenson \& Whiting 2004a: 367). Although Hymenopodinae + Acromantinae, and the monophyly of both subgroups was supported therein, too few genera were studied to be representative. A putative monophyly of Hymenopodinae + Acromantinae was also carefully discussed as one possibility of the origin of the mesothoracic ear in Hymenopodidae by Yager (1996a: 473). Svenson \& Whiting (2004a: 367) stated that Bayesian analysis of their dataset showed a monophyletic Hymenopodidae + Empusidae with Gongylus being nested among Acromantinae.

Inward et al. (2007: 332) included Phyllocrania and Pseudocreobotra in their molecular analysis, which formed a monophyletic group.

Yager \& Svenson (2008) found 12 species of Acromantinae, two species of Oxypilinae, six species of Hymenopodinae, and Phyllocrania in the crowngroup of their phylogenetic tree, as sistergroup to Empusidae. This clade also contained Sibyllidae and Phyllotheliinae. The African genera Galinthias Stål, 1877, Pseudoharpax Saussure, 1870, and Congoharpax La Greca, 1954 on the other hand formed a monophyletic group that came out more proximally in the cladogram than the remaining Hymenopodidae. None of the traditional hymenopodid subfamilies and tribus (Ehrmann 2002) were found to be monophyletic. Phyllocrania came out 

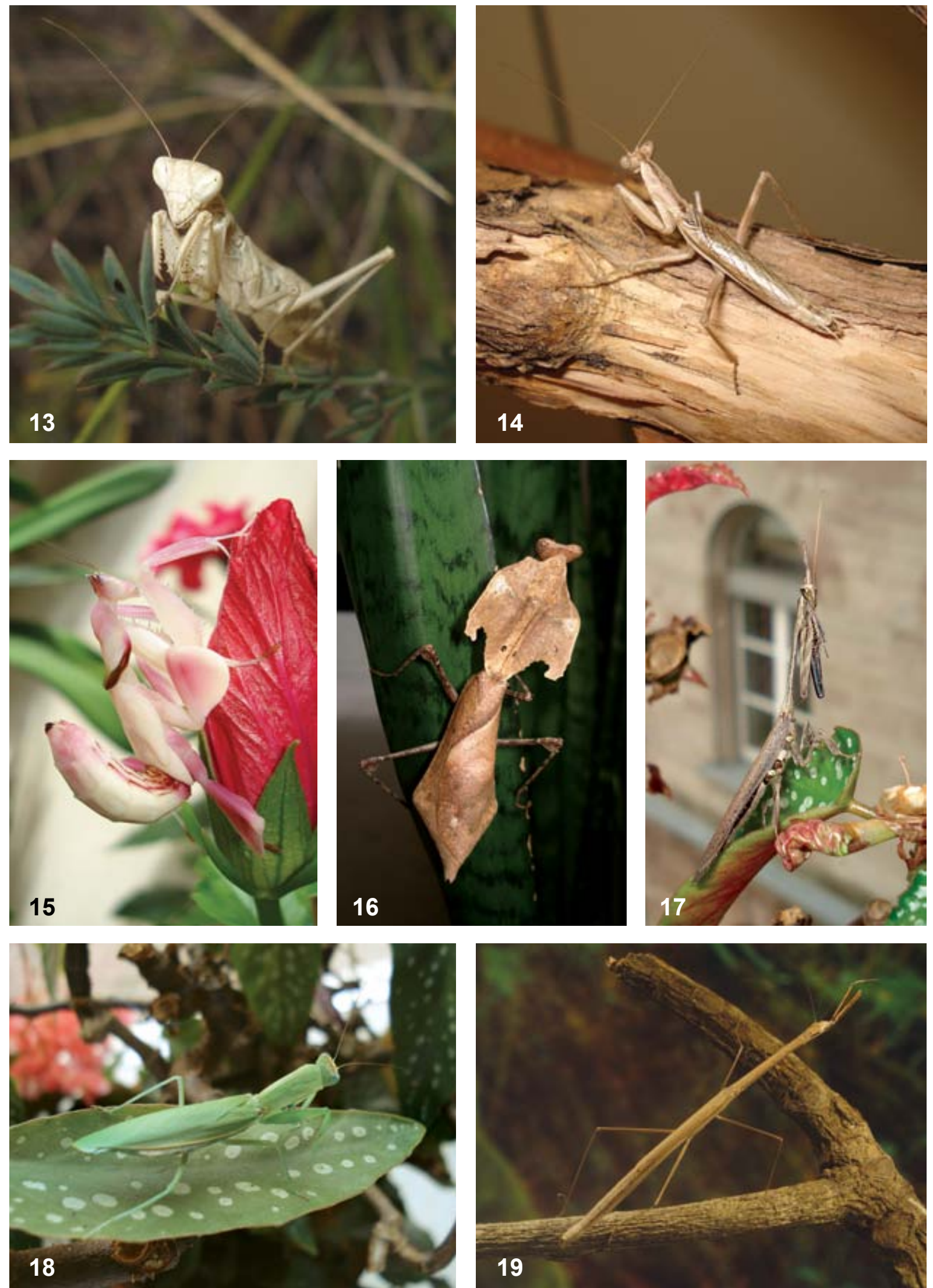

Figs. 13-19: Pictures of various living Mantodea. 13: Ameles decolor, $q$ (France: Provence). 14: Ameles gracilis, $\widehat{\jmath}$ (studio photograph). 15: Hymenopus coronatus, subadult $q$ (studio photograph); note the colouration cryptic to white flowers and large lobes on the femora of the cursorial legs. 16: Deroplatys desiccata, $q$ (studio photograph); note enlarged pronotum and cryptic colouration and body shape. 17: Ceratocrania sp., $\widehat{\sigma}$ (studio photograph). 18: Orthodera novaezealandiae, + (studio photograph). 19: Compsothespis sp., $q$ (courtesy of Sören Materna; studio photograph). 
as the sistergroup of the African Sibyllidae. These results were very similar to those found by Svenson \& Whiting (2009, see below).

Ware et al. (2008) included three species of Hymenopodidae in their analysis. Hymenopodidae was not supported as a monophyletic group therein, either. In both the parsimony and the Bayesian analysis a taxon comprising Gongylus, Pseudocreobotra and Phyllocrania was found (Ware et al. 2008: figs. 2a, b, 3a), thus supporting a close relationship of Empusidae and Hymenopodidae (partim). However, Chrysomantis (Acromantinae) did never cluster with the remaining Hymenopodidae studied, thus rendering the latter paraphyletic.

Svenson \& Whiting (2009) included 13 species of Hymenopodinae, 25 species of Acromantinae, eight species of Oxypilinae, and Phyllocrania as the only representative of Epaphroditinae. None of the traditional subfamilies or tribus were found to be monophyletic, nor was Hymenopodidae as a whole. All Hymenopodidae except for three genera clustered in a larger clade (also containing Phyllotheliinae and Sibyllidae; node 200) the sistergroup of which was Empusidae. The remaining three genera, Pseudoharpax, Congoharpax, and Galinthias, however, formed a monophyletic group (node 190) in a more basal position of the cladogram. Phyllocrania was recovered as the sistergroup of Sibyllidae (node 201), as had been found earlier by Yager \& Svenson (2008).

None of these studies confirmed the assumed monophyly of Hymenopodidae or of any of its subgroups. However, they recurrently presented evidence for a close relationship between Empusidae and Hymenopodidae (partim). This had been proposed earlier, for instance by Roy (2004a: 14) and Wieland (2006: 52, 74). The most comprehensive datasets (Yager \& Svenson 2008; Svenson \& Whiting 2009) suggest that Empusidae is the sistergroup of the greater part of Hymenopodidae (also including Sibyllidae and Mantidae: Phyllotheliinae) with the exception of Pseudoharpax, Congoharpax and Galinthias.

Liturgusidae. Liturgusidae comprises 17 genera from all zoogeographical regions with exception of the Palaearctic region (Ehrmann 2002: 375 and tab. 13; Bragg 2010: 21). The subfamily name was given by Giglio-Tos (1919). The subfamily was later assigned to Mantidae, including several more genera than contained in Giglio-Tos's Liturgusinae (Beier 1964a: 942, 1968a: 8). It was raised to family rank by Ehrmann (1997).

Liturgusidae are bark dwelling mantodeans (e.g. Beier 1964a: 942, 1968a: 8; Hill et al. 2004: 2; Svenson \& Whiting 2009: 503). Their body is flattened and often well camouflaged against the moss- and lichen-speckled bark (e.g. Hingston 1932: 275; Rehn 1935: 201; Edmunds 1972: 20, 1976: 22; Figs. 20, 21), sometimes even resembling the structure of the bark itself, as mentioned for the South-East Asian Theopompa Stål, 1877 by Chopard (1938: 417) and Edmunds (1972: 20).

Beier (1964a: 943, 1968a: 8) mentioned a morphological character that has been stated to be characteristic for Liturgusidae: A deep groove is present between the first and second posteroventral spines of the fore femora in which the distal postero-ventral tibial spine comes to rest when the fore tibia is folded against the femur. However, this character was also described for 
Caliridinae (Giglio-Tos 1927: 302; Beier 1935c: 15) and can be found in many different subtaxa of Mantodea, as will be discussed in detail in the morphological descriptions (see also Roy 1973: 275 and character 53 in chapter 4.1.2).

In his taxon sample of Neotropical Mantodea Jantsch (1999: fig. 8) included three genera of Liturgusidae. In his morphological analysis, the latter formed a monophyletic group in a relatively basal position on the phylogenetic tree. It was found to be the sistergroup to Mantidae (excluding Amelinae) + Thespidae + Acanthopidae. Although this provided support for the Neotropical Liturgusidae to be monophyletic, the relationships between them and their Afrotropical, Indomalayan and Australasian counterparts could not be addressed.

Svenson \& Whiting (2004a: fig. 1) included a single genus of Liturgusidae (Ciulfina GiglioTos, 1915 from Australasia) in their molecular analysis, therefore the potential monophyly of Liturgusidae could not be addressed. Ciulfina therein formed a monophyletic group with Ima fusca Tindale, 1924, an Australian Iridopterygidae. This taxon was nested deeply within the remaining (though paraphyletic) Iridopterygidae. Beier (1964a, 1968a) had assigned Ciulfina to Liturgusidae. Balderson $(1978,1984,1991)$ placed the genus within Iridopterygidae instead, as it "lacks the characters of the Liturgusinae as defined by Beier" (Balderson 1984: 1). Ehrmann (2002: 375) listed Ciulfina in Liturgusidae. This taxonomic position was also preferred by Holwell $(2007,2008)$ and Holwell et al. (2007a).

The genetic analysis by Svenson \& Whiting (2004a: fig. 1) supported a close phylogenetic relationship between Ciulfina and the members of Iridopterygidae (see above). Furthermore, Ciulfina was therein recovered in a large clade consisting exclusively of Australasian taxa.

Yager \& Svenson (2008) included six liturgusid genera in their study (Liturgusa Saussure, 1869, Hagiomantis Saussure \& Zehntner, 1894, Stenomantis Saussure, 1871, Theopompella Giglio-Tos, 1917, Humbertiella Saussure, 1869, and Dactylopteryx Karsch, 1892). The resulting phylogenetic tree showed Liturgusidae to be polyphyletic. The Neotropical Liturgusa (Figs. 20, 21) and Hagiomantis clustered among the Neotropical Mantodea near the base of the cladogram. The Australian Stenomantis was found in a clade of Australasian taxa, Humbertiella from South-East Asia came out in a clade of Indomalayan Amelinae, which also contained Hyalomantis Giglio-Tos, 1915 from Madagascar and Chaeteessa (probably erroneous due to old tissue samples or contamination; see Yager \& Svenson 2008: 561). Theopompella and Dactylopteryx from Africa formed a taxon among the Afrotropical Mantodea in a comparatively distal position in the phylogenetic tree.

Svenson \& Whiting (2009) were able to corroborate the results of Yager \& Svenson (2008). They included nine species of the genera Liturgusa, Hagiomantis, Ciulfina, Stenomantis, Humbertiella, Theopompella and Dactylopteryx in their analysis. Liturgusidae was therein again shown to be polyphyletic. And again, its different genera were nested in four distinct biogeographical clades. One of the four groups, at a basal node in the phylogenetic tree, were the Neotropical Liturgusidae (Liturgusa, Hagiomantis), in congruence with Jantsch (1999). Further apical in the cladogram the Australasian Liturgusidae (Ciulfina, Stenomantis) formed a monophylum clustering in a large Australasian clade (in congruence with Svenson \& Whiting 

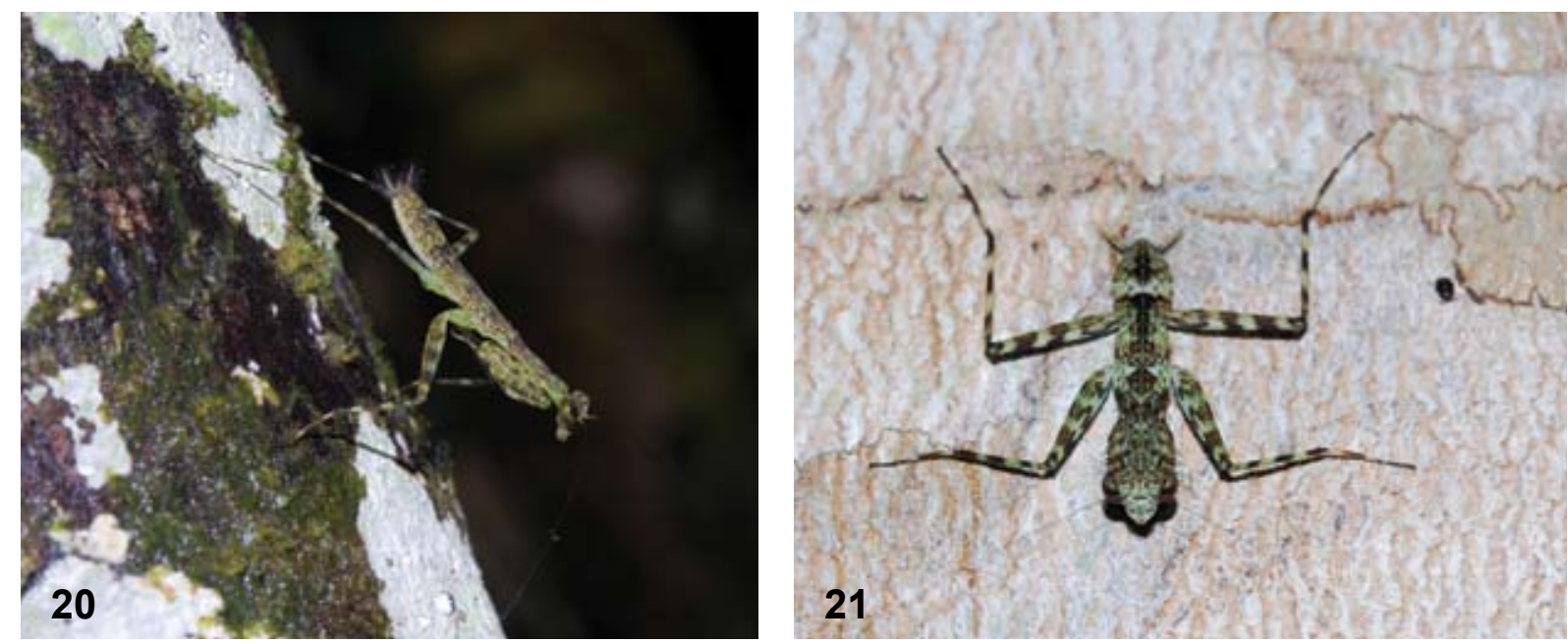

Figs. 20-21: Liturgusa sp., nymph (courtesy of Geoffrey Gallice). 20: Lateral view. 21: Oblique antero-dorsal view. Note the flattened body and the long legs typical for bark dwelling species and the forelegs which are held folded against the prosternum.

2004a and Yager \& Svenson 2008). The Indomalayan Humbertiella clustered with Indomalayan Amelinae and the Afrotropical Theopompella and Dactylopteryx clustered with Afrotropical Hymenopodinae in the apical part of the phylogenetic tree (Svenson \& Whiting 2009: fig. 6). These findings led Svenson \& Whiting (2009: 503) to the hypothesis that "Liturgusidae" as currently classified actually represent specialized, bark dwelling ecomorphs that evolved independently in these four biogeographical regions. According to these data, "Liturgusidae" provides an impressive example of convergent evolution among Mantodea due to similar ecological selective forces.

Tarachodidae. Tarachodidae currently comprises 40 genera in two subgroups, Tarachodinae (33 genera) and Caliridinae (seven genera) (Ehrmann 2002: 375 and tab. 13; Roy 2003a, 2008a; Stiewe 2009). They often have a slightly flattened habitus, flattened cerci and shortened meso- and metathoracic legs. The head is comparatively large with respect to the often slender body and mostly held in a hypognathous position in terms of camouflage (e.g. Tarachodes Burmeister, 1838, Tarachodula Giglio-Tos, 1917, Pseudogalepsus Beier, 1954, Pyrgomantis Gerstaecker, 1869, Didymocorypha Wood-Mason, 1877; see Edmunds 1972: 4 and fig. 3b, c, 1974: fig. 12.12, 1976: 23; Gillon 1983: fig. 11.5; Fig. 25). The males are mostly fully winged, whereas the females often have shortened wings (Giglio-Tos 1927: 74; Beier 1964a: 942, 1968a: 8; Ehrmann 2002: 373; Fig. 25). Nearly all genera are distributed in the Afrotropical and Oriental regions, ranging from Africa to East Asia. Only Iris Saussure, 1869 is also distributed in the Neotropical region with a single species, (Ehrmann 2002: 195). Although it does not resemble the other members of Tarachodidae closely at first sight, it was assigned to the group by Roy (1970: 1024) based on its high similarity to Paroxyophthalmus Wood-Mason, 1889 with respect to morphology, colouration pattern, and genital apparatus. Tarachodidae have not been recorded for the Australian region (compare Tindale 1923, 1924; Werner 1928; Beier 1965a, b; Balderson 1984, 1991; Balderson et al. 1998; Ehrmann 2002: 375 vs. 373). 
Several taxonomical studies are dealing with single genera or species of the group, among them those by Giglio-Tos (1911: Tarachodes, Galepsus Stål, 1877), Beier (1957a: Pyrgomantis; 1957b: Tarachodes, Galepsus), Roy (1972: Paragalepsus Beier, 1930, Metagalepsus Roy, 1971; 2003a, b: Tarachodella Giglio-Tos, 1917, Alfredistia Roy, 2003; 2005b: Galepsus; 2008a: Paralygdamia Saussure \& Zehntner, 1895, Nesogalepsus Beier, 1954, Tuberculepsus Roy, 2008), Kaltenbach (1996, 1998: Tarachodidae of South Africa), Lombardo (1997: Tarachodidae of South Africa), and Stiewe (2009: Dysaulophthalma Stiewe, 2009).

Beier (1968a: 8) mentioned that Caliridinae have a groove on the ventral surface of the fore femora, between the proximal two postero-ventral fore femoral spines. This resembles the situation found in Liturgusidae (see also discussion of Liturgusidae in this chapter). A detailed evaluation of this character can be found in the morphological descriptions (see chapter 4.1.2, character 53).

Svenson \& Whiting (2009) included two genera of Caliridinae (Leptomantella and Gildella) and 12 genera of Tarachodinae (Oxyophthalma Saussure, 1861, Antistia Stål, 1876, Nothogalepsus Beier, 1969, Galepsus, Tarachodes, Tarachodula, Pyrgomantis, Paragalepsus, Episcopomantis Uvarov, 1940, Iris, Dysaules Stål, 1877, Didymocorypha) in their analysis. Neither Tarachodidae nor any of its two subgroups were recovered monophyletic therein. Their members were found far apart on the phylogenetic tree (Svenson \& Whiting 2009: figs. 3b, c). The largest monophyletic group among Tarachodidae was formed by the Afrotropical Nothogalepsus, Galepsus, Tarachodes, Tarachodula, Pyrgomantis, Paragalepsus, and Antistia. The remaining Afrotropical Tarachodinae (Episcopomantis and Iris) formed a monophyletic group with Heterochaeta Westwood, 1843 (Vatinae), altogether being the sistergroup of the Indomalayan Dysaules (Tarachodinae). The Indian Didymocorypha was found to be the sistergroup of a taxon comprising Compsothespis Saussure, 1872 (Mantidae), Oxyothespinae (Mantidae) and Toxoderidae.

Yager \& Svenson (2008) used nearly the same taxon sample in their analysis. The results were quite similar. Therein, Didymocorypha clustered with the larger Afrotropical clade mentioned for Svenson \& Whiting (2009), and Heterochaeta was found in a more apical position in the phylogenetic tree.

Thespidae. The traditional Thespidae are distributed throughout all biogeographical regions (Beier 1939a, 1968a; Ehrmann 2002). The group comprises 43 genera categorized in six subgroups: Pseudomiopteryginae (seven genera), Miopteryginae (five genera), Thespinae (ten genera), Hoplocoryphinae (three genera), Oligonicinae (16 genera), and Haaniinae (two genera) (Ehrmann 2002: 375 and tab. 13). Many of the species are slender (Beier 1968a: 8; Ehrmann 2002: 373). Some have a stouter and sometimes bizarrely shaped body, e.g. Pogonogaster Rehn, 1918 and Calopteromantis Terra, 1982 from South America or Haania Saussure, 1871 and Astape Stål, 1877, the only members of the South-East Asian Haaniinae (Chopard 1920: figs. 1-3; Beier 1952: figs. 1-9, 1968a: 9 and fig. 9; Lieftinck 1953: figs. 1-3; Lombardo \& Ayala 1999: pl. 1: fig. 2, pl. 2: fig. 2; Ayala \& Onore 2001: figs. 3, 4). 
Beier (1968a: 8) listed several characteristic traits for Thespidae, for instance the basitarsomere of the hind legs being longer than the remaining tarsomeres taken together and the elongated supra-anal plate. These characters are also present in several other groups: The basitarsomere of the metathoracic legs is also strongly elongated in Metallyticus (e.g. Wieland 2008a: fig. 11; Fig. 292), many Mantidae: Mantinae (e.g. Archimantis gracilis Milledge, 1997 and kin, see for instance Milledge 1997: figs. 148, 149), Sibyllidae (Roy 1996), Empusidae (Gongylus, e.g. Beier 1968a: fig. 14) and others. An elongated supra-anal plate, as mentioned for Thespidae by Beier (1968a: 8) is also present in Schizocephala (Beier 1935c: 71; Ehrmann 2002: 315), Rivetina Berland \& Chopard, 1922 (Kaltenbach 1963: fig. 16a), Ischnomantis Stål, 1871 (Werner 1907: tab. 1, fig. 1; Beier 1968a: 23; Kaltenbach 1998: fig. 104; Fig. 360), Solygia Stål, 1877 (Werner 1907: tab. 1, fig. 2; Kaltenbach 1998: fig. 102), Parasphendale Schulthess-Schindler, 1898 (Kaltenbach 1998: fig. 93) and others. Another potential apomorphy are the reduced number of postero-ventral tibial spines as mentioned by Beier (1968a: 8; see character 64). A character that potentially represents an apomorphy for a taxon within Thespidae are the dorsally located spines on the fore tibiae of Haaniinae and many Oligonicinae (Roy 1999: 37; see characters 65, 66 and Figs. 247-254, 274-275).

In the morphological analysis of Neotropical Mantodea by Jantsch (1999: fig. 8) all Thespidae clustered together except for a single member of Oligonicinae (Bactromantis Scudder, 1896) which was nested among Mantidae. Furthermore, the only two members of Mantidae: Angelinae studied by Jantsch (Angela Audinet-Serville, 1839 and Thespoides Chopard, 1916) were found separated within Thespidae. All subgroups of Thespidae represented in the analysis (Oligonicinae, Thespinae, Miopteryginae, Pseudomiopteryginae) were rendered para- or polyphyletic. Hoplocoryphinae and Haaniinae were not included.

Svenson \& Whiting (2004a: fig. 1) studied species of Oligonicinae, Pseudomiopteryginae, and Hoplocoryphinae. They were represented by two species of the same genus each. Each of the genera Bantia (Neotropical) and Hoplocorypha (Afrotropical) was found to be monophyletic. The two species of Anamiopteryx Giglio-Tos, 1915, however, came out paraphyletic, with Anamiopteryx sp. 1 being the sistergroup of Mantidae: Vatinae (partim) and Anamiopteryx sp. 2 being the sistergroup to the latter group. Thespidae as a whole was rendered polyphyletic as the three taxa studied were widely distributed across the entire tree. Haaniinae, Thespinae, and Miopteryginae were not represented in the study.

Later, Svenson \& Whiting (2009) included 14 genera of Thespidae in their analysis (Svenson $\&$ Whiting 2009: tab. 2, fig. 3). Among them were six genera of the Neotropical Oligonicinae (Bantia, Carrikerella Hebard, 1922, Thrinaconyx Saussure, 1892, Thesprotiella Giglio-Tos, 1915, Oligonicella Giglio-Tos, 1915, Thesprotia Stål, 1877), one genus of the Neotropical Pseudomiopteryginae (Pseudomiopteryx Saussure, 1870), five genera of Thespinae (the Neotropical Musoniella Giglio-Tos, 1916, Eumusonia Giglio-Tos, 1916, Macromusonia Hebard, 1922, and the Indian Pseudothespis Mukherjee, 1995 and Parathespis Saussure, 1869), one genus of Hoplocoryphinae (the African Hoplocorypha) and one species of the South-East Asian Haaniinae (Haania lobiceps). Neither Thespidae, nor Thespinae or Oligonicinae were therein found to 

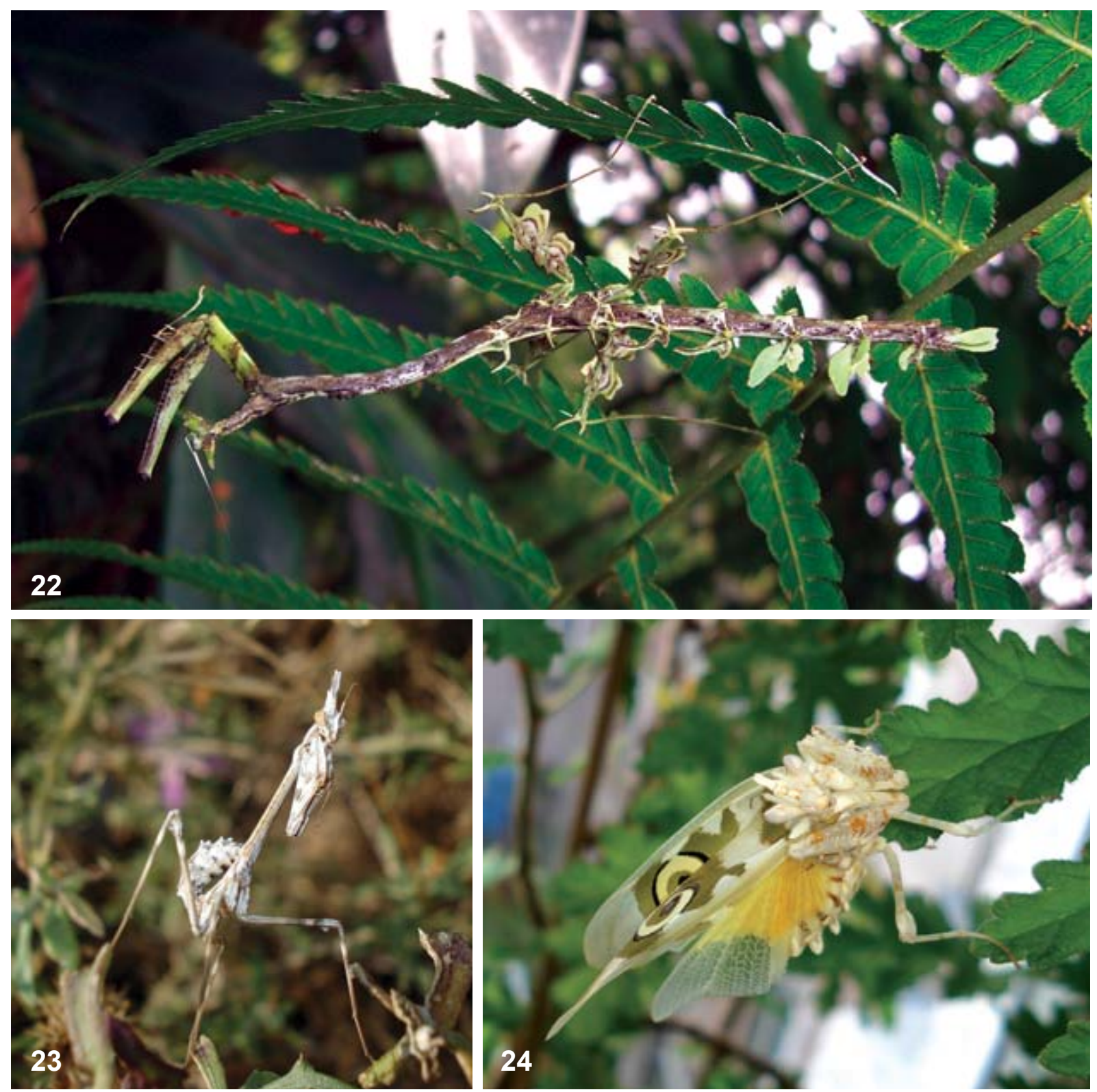

Figs. 22-25: Pictures of various living Mantodea. 22: Toxodera beieri, nymph (courtesy of Sören Materna; Malaysia; same specimen as in Roy 2009a: fig. 15); note the lobes on legs and body and the flattened cerci. 23: Empusa pennata, nymph (France: Provence); note the head process and the lobes on legs and abdomen. 24: Pseudocreobotra wahlbergii, $\bigcirc$, beginning deimatic display (studio photograph); note the eye spots on the wings, the lobes on legs and abdomen and the head process. 25: Pyrgomantis jonesi, + (courtesy of Sören Materna; studio photograph); note the head process, the short legs and the elongated body typical for grass dwellers.

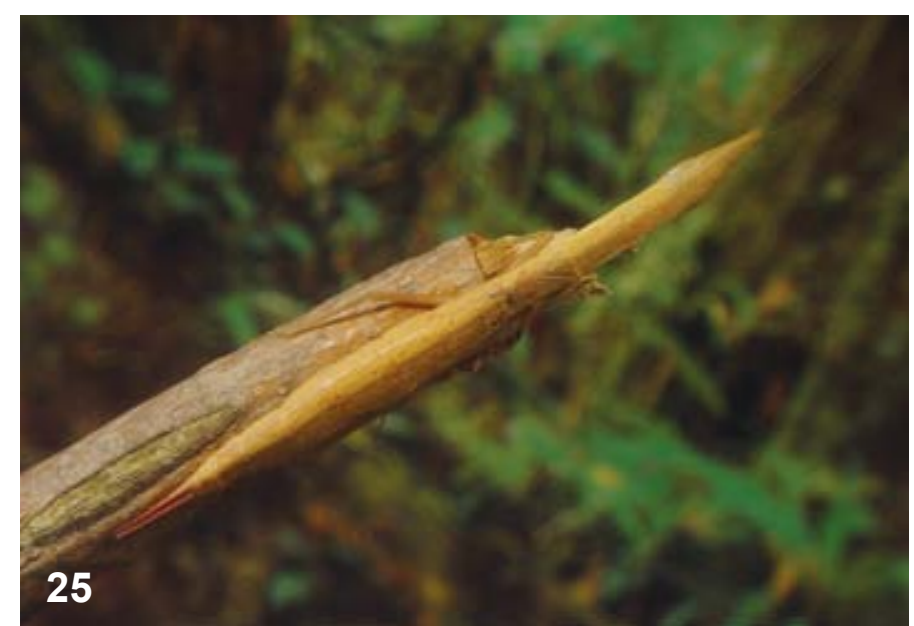

be monophyletic. As Hoplocoryphinae, Pseudomiopteryginae and Haaniinae were represented by a single genus each, the assumption of their respective monophyly could not be addressed. All Neotropical (and Nearctic) Thespidae formed a monophyletic group which appeared as the sistergroup to a clade containing all Neotropical Acanthopidae, Photininae, and Liturgusidae. 
Of the remaining Thespidae Pseudothespis and Haania formed a monophyletic group more distally in the phylogenetic tree. Hoplocorypha and Parathespis came out widely separated further distally in the tree.

Yager \& Svenson (2008) used almost the same taxa as Svenson \& Whiting (2009). Therein, Hoplocorypha formed the sistergroup of a clade distally in the cladogram, which also contained three African Miomantinae. The Indian Parathespis came out as the sistergroup of Heterochaeta (Mantidae: Vatinae), near the group including Hoplocorypha. The remaining (Neotropical) Thespidae [Pseudomiopteryx, Thrinaconyx, Bantia, Eumusonia, Musoniella, Carrikerella, Eumionyx Giglio-Tos, 1927 (synonym of Pseudomusonia Werner, 1909, see Ehrmann 2002: 296), Thesprotiella, Oligonicella, Thesprotia] formed a monophyletic group in a basal position on the phylogenetic tree. Again, neither Thespidae nor any of its subgroups were found to be monophyletic.

Ware et al. (2008) studied three Thespidae which did not show a close relationship to each other in any of the constituent analyses.

Iridopterygidae. Iridopterygidae comprises 53 genera in five subgroups: Hapalomantinae (nine genera), Iridopteryginae (eight genera), Nanomantinae (14 genera), Nilomantinae (five genera), and Tropidomantinae (17 genera) (Ehrmann 2002: tab. 13 and 376). They are distributed in the Afrotropical, Indomalayan and Australasian regions (Beier 1968a: 10; Ehrmann 2002: 373). Autapomorphic characters for the group have not been stated. Morphological traits usually mentioned to characterize the group can also be found in other Mantodea (e.g. the head being distinctly wider than long and the short pronotum, as stated by Beier 1968a: 10). Ehrmann (2002: 373) stated that most species have long wings, whereas Beier (1968a: 10) mentioned that the females often have the wings strongly shortened or entirely reduced. The latter is true for many Iridopterygidae genera (compare generic diagnoses in Ehrmann 2002). For many others (e.g. the Afrotropical Hapalogymnes Kaltenbach, 1996, the Australasian Ima Tindale, 1924 and Machairima Beier, 1965, and the Indomalayan Sceptuchus Hebard, 1920 and Parananomantis Mukherjee, 1995) the females have not been found, therefore their wing length is unknown. Thus, none of the characters mentioned in the literature represents a potential autapomorphy for Iridopterygidae.

Svenson \& Whiting (2004a) included ten species from eight genera of Nanomantinae, Tropidomantinae, and Hapalomantinae in their analysis. Their results did not support any of the three groups to be potentially monophyletic. However, they all came out in the same larger clade, which also contained Paraoxypilinae and the only member of Liturgusidae in the analysis (Ciulfina, see also discussion of Liturgusidae in this chapter). Within this taxon, one member of Tropidomantinae (Neomantis australis) formed the sistergroup of Paraoxypilinae (Svenson \& Whiting 2004a: fig. 1).

Kjer et al. (2006) only included the Australian Kongobatha in their analysis. In their majority rule consensus tree (fig. 1; but not in the weighted parsimony analysis, fig. 2), Kongobatha clustered with Paraoxypilus. 
Inward et al. (2007: fig. 1) included the Madagascan Ichromantis dichroica in their analysis of Dictyoptera. This Tropidomantinae species was found at the second dichotomy of the phylogeny, directly following the basal split between Mantoida and the remaining Mantodea. Only the third dichotomy was between Metallyticus and the other species of the analysis (see also discussion of Mantoida, Chaeteessa, and Metallyticus in this chapter).

Yager \& Svenson (2008) studied African Hapalomantinae (Tarachina Werner, 1907, Bolbena Giglio-Tos, 1915), Indomalayan Iridopteryginae (Hapalopeza Stål, 1877), African Nilomantinae (Nilomantis Werner, 1907), Nanomantinae (the Indomalayan Sceptuchus, the Australasian Tylomantis Giglio-Tos, 1915 and Calofulcinia Giglio-Tos, 1915), and Tropidomantinae [the Indomalayan Neomantis Giglio-Tos, 1915, Xanthomantis Giglio-Tos, 1915, Eomantis Giglio-Tos 1915 (currently assigned as a subgenus to Tropidomantis, see Ehrmann 2002: 376), and Tropidomantis Stål, 1877, the Afrotropical Chloromantis Kaltenbach, 1998, and the Madagascan Hyalomantis Giglio-Tos, 1915)]. Hapalomantinae, Tropidomantinae and Nanomantinae were found to be polyphyletic.

In their analysis, the Indomalayan Hapalopeza clustered with Indomalayan Amelinae. Tarachina came out in a clade containing Afrotropical Amelinae, Dystactinae, and Chroicopterinae. The Australasian Bolbe Stål, 1877, Neomantis and Tylomantis were found in a clade containing the Australasian Paraoxypilinae and Liturgusidae (Stenomantis). A clade encompassing Indomalayan and Afrotropical Iridopterygidae (Sceptuchus, Xanthomantis Giglio-Tos, 1915, Eomantis, Tropidomantis, Nilomantis, Bolbena, and Chloromantis) was found, which also included the Indomalayan Amorphoscelinae. The Madagascan Hyalomantis was found in a clade together with the Indian Mantidae: Amelinae Gonypetyllis Wood-Mason, 1891 and Elmantis Giglio-Tos, 1915 and the Indomalayan Gonypeta Saussure, 1869 (Amelinae) and Humbertiella Saussure, 1869 (Liturgusidae). Chaeteessa was also found in this clade, but see discussion of Chaeteessa, Mantoida and Metallyticus above for discussion.

Ware et al. (2008) included three Iridopterygidae in their study, all of which were from Australia. The parsimony analyses showed the three taxa to be monophyletic with Paraoxypilinae (Gyromantis) as their sistergroup. In the Bayesian analyses, Gyromantis nested within the Australian Iridopterygidae, hence rendering Iridopterygidae paraphyletic with respect to Paraoxypilinae. These results supported the relationships of the Australian Iridopterygidae (and Paraoxypilinae) found by the other authors.

Svenson \& Whiting (2009) studied 15 genera of Iridopterygidae, encompassing Nilomantinae (Nilomantis), Hapalomantinae (Hapalomantis Stål, 1871, Bolbena, Bolbe, Tarachina), Tropidomantinae (Miromantis Giglio-Tos, 1915, Eomantis, Tropidomantis, Kongobatha, Xanthomantis, Neomantis), Nanomantinae (Sceptuchus, Tylomantis, Calofulcinia), and Iridopteryginae (Hapalopeza) (Svenson \& Whiting 2009: tab. 2). The African Tarachina came out within a highly supported clade encompassing the African Amelinae, Chroicopterinae, and Dystactinae (node 50, ML bootstrap value of 100). The two Indomalayan species of Hapalopeza formed a monophyletic group with the Indomalayan Amelinae (node 120), although the genus itself was not rendered monophyletic. The Australian Bolbe was found to be the sistergroup of the 
Australian Paraoxypilinae (node 111). The remaining Iridopterygidae formed a larger clade also containing the aforementioned Bolbe + Paraoxypilinae as well as Australian members of Liturgusidae (node 84). The sistergroup of this larger clade was found to be the Amorphoscelinae which was monophyletic therein. Nanomantinae, Tropidomantinae and Hapalomantinae were not found to be monophyletic. As for Tropidomantinae, most Indomalayan species clustered in a larger clade (node 92) that was the sistergroup of the Australasian clade encompassing Hapalomantinae and Nanomantinae (node 56).

Mantidae. Mantidae represents the largest conglomerate of mantodean species in the typological classification. It currently comprises more than 1140 species (nearly fifty per cent of all described species) in little more than 180 genera that are classified in 20 subfamilies (Ehrmann 2002: tab. 13). Mantidae has been conjectured to be a paraphyletic assembly (e.g. Klass \& Ehrmann 2003: 197). This has been corroborated by the molecular analyses by Svenson \& Whiting (2004a: 367; 2009: 484) and Yager \& Svenson (2008). The members of this group are extremely heterogeneous in size and morphological appearance. Some of the traditional subfamilies of the Mantidae in Beier's classification (1968a) (Liturgusinae, Tarachodinae, Thespinae, Iridopteryginae, Toxoderinae, Sibyllinae) were raised to family-rank by Ehrmann (2002). This reduced the number of subtaxa of Mantidae but had no further effect on the understanding of their phylogenetic relations.

Although the members of many of the subtaxa of Mantidae show typological similarities, autapomorphic characters for single monophyla have not been mentioned so far. Several of the subfamilies have been established for only two or three genera [Choeradodinae (Fig. 142), Deroplatyinae (Fig. 16), Mellierinae, Orthoderinae (Fig. 18), Phyllotheliinae (Fig. 17), Stagmomantinae], others are even monogeneric [Antemninae, Compsothespinae (Fig. 19), and Schizocephalinae], and are therefore redundant. Such monogeneric subgroups will be referred to by their generic names throughout the text.

The molecular analysis by Svenson \& Whiting (2004a: 365) included several subfamilies of Mantidae (Amelinae, Choeradodinae, Mantinae, Mellierinae, Orthoderinae, Stagmomantinae, and Vatinae). "Paramantinae" as mentioned by Svenson \& Whiting (2004a, 2009) and Yager \& Svenson (2008) are actually not regarded as a separate subfamily but as a tribus Paramantini among Mantinae in the current classification. "Paramantinae" in Ehrmann (2002: 377) is a misprint (Ehrmann, pers. comm. 2007), and all taxa labelled as "Paramantinae" in these publications are consequently referring to Mantinae. In Svenson \& Whiting (2004a: fig. 1) Mantinae was found to be polyphyletic, as were Amelinae and Vatinae. Mellierinae and Stagmomantinae were only represented by a single genus each. Together, they formed a monophyletic group. Choeradodinae and Orthoderinae were represented by only one genus each.

Yager \& Svenson (2008) studied a slightly smaller taxon sample than Svenson \& Whiting (2009, see below for details), lacking Deroplatyinae and Compsothespis, and used fewer species. None of the subfamilies of which they included more than a single genus were found to be monophyletic. The results suggested several interesting relationships among the Mantidae, 
for instance a sistergroup relationship between the Australian Orthoderinae and Choeradodinae. The latter today show a disjunctive distribution in South America and South-East Asia (India, Sri Lanka, Myanmar, China and Thailand) and have a putative fossil representative in the Paleocene of Menat in France (†Prochaeradodis enigmaticus Piton, 1940). The relationship between the two groups had been proposed earlier, for instance by Beier (1935b: 1, 3), and was also found by Svenson \& Whiting (2009: node 280). Both Choeradodinae and Orthoderinae have been stated to be "comparatively primitive forms" (e.g. Beier 1935b: 1, 3, 1968a: 8). However, in the molecular studies they were repeatedly recovered distally in the phylogenetic tree.

Ware et al. (2008) studied nine Mantidae species. Again, a putative monophyly of Mantidae and of its subfamilies did not gain any support.

Svenson \& Whiting (2009) included all traditional subfamilies [Amelinae (Figs. 13, 14), Angelinae, Antemna Stål, 1877, Choeradodinae, Chroicopterinae, Compsothespis, Deroplatyinae, Dystactinae, Mantinae (including "Paramantinae" = Paramantini, see above), Mellierinae, Miomantinae, Orthoderinae, Oxyothespinae, Photininae, Phyllotheliinae, Schizocephala, Stagmatopterinae, Stagmomantinae, and Vatinae]. The taxon sample of Phyllotheliinae this time included Ceratocrania Westwood, 1889 and the group was found to be monophyletic therein (node 221). It came out as the sistergroup of a clade containing the greater part of the Acromantinae and all Oxypilinae studied (node 224). The two genera of Oxyothespinae were also found to form a monophylum (node 174) and to be the sistergroup of a monophyletic Toxoderidae (see discussion of Toxoderidae below). None of the remaining subfamilies were found to be monophyletic. Some subfamilies comprising two or more genera were represented by a single genus (Orthoderinae, Choeradodinae), therefore their monophyly could not be addressed. Nonetheless, the sistergroup relationship between the latter two groups found further support (see also discussion of Yager \& Svenson 2008 above).

Toxoderidae. 17 genera from the Afrotropical and Oriental regions are currently assigned to Toxoderidae (Ehrmann 2002: 373; Roy 2009a: 94 ff). Many of them are large and exhibit bizarrely shaped bodies (Beier 1968a: 11; Mukherjee et al. 1995: 317; Vyjayandi 2004: 568; Roy 2009a: figs. 1, 5-7, 12, 13, 15, 18, 26, 31, 35, 40, 45, 49, 54, 55, 58, 62, 66; Fig. 22). The prothorax is generally very long, often being nearly half as long as the entire body. The metazona is laterally compressed and often carries a dorsal ridge (Beier 1934c: 1, 1964a: 953, 1968a: 11, 1976: 393; Roy 2009a: 94; Fig. 22). Additionally, the prothorax may be strongly arched dorsad in lateral view, whereas the prozona is more or less strongly bent dorsad. This is the case, for instance, in Toxodera Audinet-Serville, 1837 (Beier 1964a: fig. 38, 1968a: fig. 13, 1976: figs. 1-4; Fig. 22), Protoxodera Werner, 1930 (Roy 2009a: figs. 38, 39a) and Stenotoxodera Roy, 2009 (Roy 2009a: figs. 64a, b, 66, 68A). The distal abdominal tergites often carry large lobes (e.g. Beier 1964a: fig. 38, 1968a: fig. 13, 1976: figs. 1-6; Roy 2009a: 3d, 9d, 16b, 21, 24, 29, 33c, d, 36e, 39b, 43, 47c, g, 52c, d, 56b, 57c, 61a, 64c, 68b; Fig. 22), as do the meso- and metathoracic legs in many species (Roy 2009a: figs. 3a-c, 10, 16e, 20, 23e-h, 27b-d, 32f, g, 36c, 39c, 42, 46b, c, 50d, e, 52e, f, 56c, 57e, 60a-d, 63b, c, 68c, d; Fig. 22). 
The morphological traits characterizing the group are also shared by other Mantodea. The non-visual spines on the eyes (Figs. 22, 86) are also present in some Mantidae (e.g. Heterochaeta, Oxyothespis Saussure, 1870), Tarachodidae (e.g. Episcopomantis, vs. Kaltenbach 1998: fig. 22) and Hymenopodidae (e.g. Pseudoharpax; Figs. 83, 84). Lobes on the femora of the cursorial legs (Figs. 22, 298) can also be found in some Hymenopodidae (Figs. 15, 24, 297, 299, 300), Mantidae (Figs. 16, 294), Sibyllidae (Fig. 295), and Empusidae (Figs. 23, 296). Dorsal lobes on the tergites are also present in Iridopterygidae (Calofulcinia: Beier 1965a: 484; Ehrmann 2002: 87), Thespidae (Pogonogaster tristani: Rehn 1918: pl. 18, fig. 5; Calopteromantis: Lombardo \& Ayala 1999: pl. 1, fig. 2, pl. 2, fig. 2; Ayala \& Onore 2001: figs. 3, 4; Pseudopogonogaster iguaquensis Salazar \& Carrejo 2002 (figs. 4, 6 therein). The cerci of most Toxoderidae are strongly flattened and widened [e.g. Beier 1934c: figs. 1 (Aethalochroa Wood-Mason, 1877), 3 (Paratoxodera Wood-Mason, 1889), 4 (Toxodera), 1964a: fig. 38 (Toxodera), 1968a: fig. 13 (Toxodera); Chopard 1940a: fig. 3 (Calamothespis Werner, 1907); Kaltenbach 1998: fig. 39 (Calamothespis); Vyjayandi 2004: figs. 1 (Aethalochroa), 3 (Toxoderopsis Wood-Mason, 1889); Roy 2009a: figs. 4a, 11a, 17b, c, 22a, c, 25a, 30a-c, 34a, 37a, 39d, 44a, d, 48a, b, 51a, 53a, 56d, 57d, 61c, 65a, 69a (Toxoderini); Fig. 386]. This character can also be found in other taxa, for instance in Angela (Beier 1935c: 70) and Stenophylla (Beier 1934c: 8). The terminal cercomere is often terminally notched (Fig. 386), as has been shown, for instance, by Kaltenbach (1998: fig. 39), Beier (1934c: fig. 4, 1964a: fig. 38, 1968a: fig. 13), and Roy (2009a: figs. 4a, 11a, b, 17b, c, 22a, c, 25a, 30a-c, 34a, 37a, 61c, 65a, 69a). However, this character varies even among Toxoderini (sensu Roy 2009a) some of which exhibit rounded distal cercomeres [Roy 2009a: figs. 39d (Protoxodera), 44a, d, 48a, b, 51a, 53a, 56d, 57d (all Paratoxodera)].

Stenophylla was formerly assigned to Toxoderidae, mainly due to the strongly flattened terminal cercomeres (Giglio-Tos 1914a: 5, 16, 1927: 577; Beier 1934c: 1, 8, 1968a: 12). In the current classification it forms the monogeneric subgroup Stenophyllinae within Acanthopidae (Terra 1995: 26; Lombardo 2000a: 35; Ehrmann 2002: 374; Roy 2005a: 226; Ehrmann \& Koçak 2009: 5; see also Acanthopidae in this chapter).

Svenson \& Whiting (2009) included Aethalochroa, Toxoderopsis and Stenotoxodera (Indomalayan genera) in their analysis. The three taxa formed a monophyletic group (Aethalochroa + (Toxoderopsis + Stenotoxodera) that came out as the sistergroup of Oxyothespinae (Heterochaetula Wood-Mason, 1889 + Oxyothespis) (node 173). Interestingly, Oxyothespinae and Toxoderidae share several remarkable morphological characteristics. Among them are, for instance, the flattened and elongated cerci (see Chopard 1941: figs. 8-14), the long and slender habitus, and non-visual spines on the eyes in several species (e.g. Chopard 1941: 34 and subsequent generic diagnoses; Beier 1935c: 62; Ehrmann 2002: 253).

Yager \& Svenson (2008) had earlier studied the same taxa except for Oxyothespis. The phylogenetic relationships among Toxoderidae were identical to those found by Svenson $\&$ Whiting (2009).

In his detailed revision of Toxoderini Roy (2009a: fig. 70; Fig. 26B herein) presented an attempt to reconstruct the phylogenetic relationships among the Toxoderini (sensu novo; encompassing 
Stenotoxodera, Metatoxodera Roy, 2009, Paratoxodera, Protoxodera, and Toxodera) without, however, naming apomorphic characters for the corresponding so-assumed monophyletic subgroups.

Sibyllidae. Sibyllidae is a small taxon comprising no more than three genera (Leptosibylla Roy, 1996, Presibylla Bolivar, 1908, and Sibylla Stål, 1856; with the latter being subdivided into two subgenera; Ehrmann 2002: 373). They are exclusively distributed in tropical Africa, south of the Sahara (Beier 1968a: 11; Roy 1996: 74: map 1). The external appearance closely resembles members of Empusidae, which may have led to the tentative assumption of a close relationship to the latter group by Beier (1934b: 1). Like Empusidae and some Hymenopodidae, Sibyllidae have characteristic head processes, a pointed scutellum and clypeus, and lobes on the meso- and metathoracic femora, as well as a strongly elongated prothorax. However, later workers (e.g. Roy 1996: 132) stated that the resemblance between the two taxa is merely superficial and that the structures probably have evolved convergently. This may also find support in the fact that Sibylla, unlike Empusidae, is not a grassland dweller but mostly spends its life on the bark of rainforest trees (e.g. Preston-Mafham 1990: pl. 49; Picker et al. 2002: 66), although apparently some specimens have been collected from savannah habitats (see Roy 1996: 133).

Roy (1996: fig. 53; Fig. 26A herein) presented a
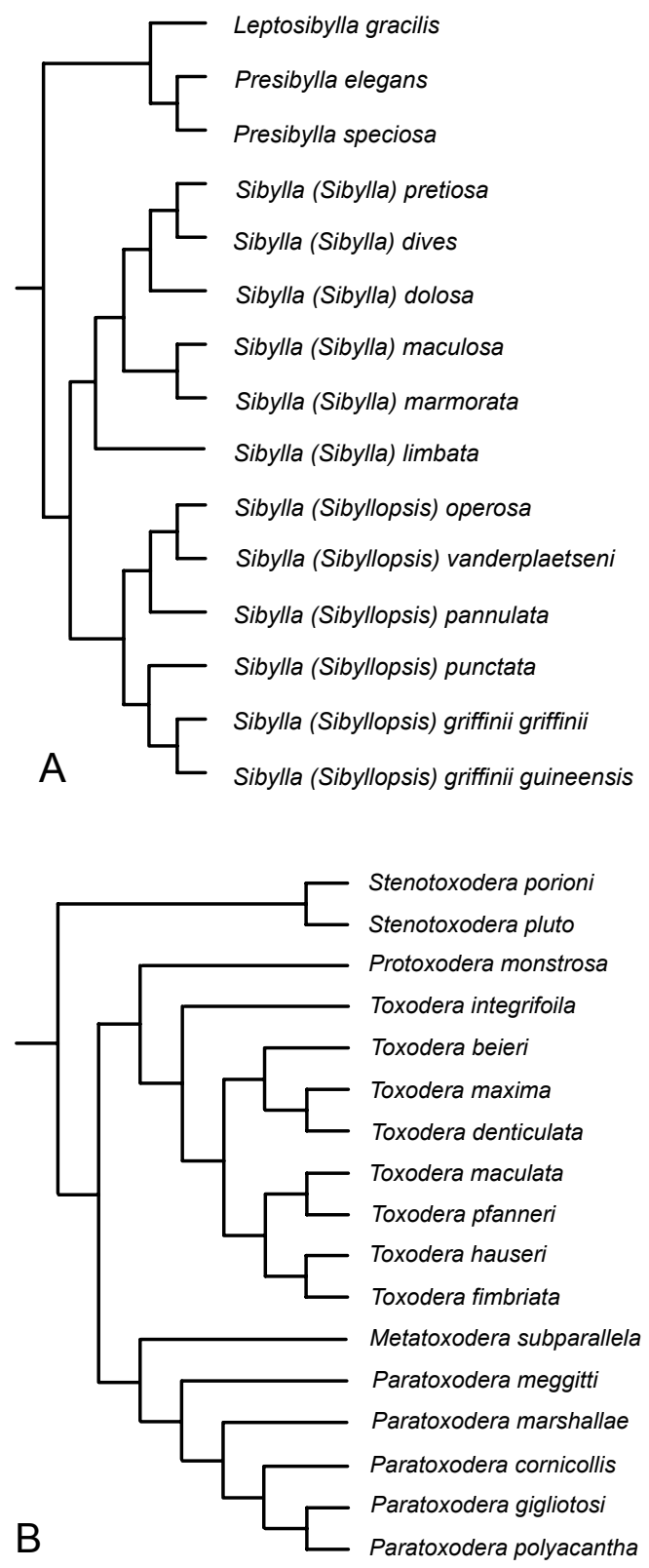

Fig. 26: Phylogeny of Sibyllidae and Toxoderini. A: Phylogeny of Sibyllidae proposed by Roy (1996: fig. 53). B: Phylogeny of Toxoderini proposed by Roy (2009a: fig. 70). mentally created phylogenetic hypothesis without, however, addressing the apomorphic characters for so-assumed monophyletic groups.

Sibyllidae were studied by Yager \& Svenson (2008) and Svenson \& Whiting (2009). Both analyses found Sibyllidae to be monophyletic, and in both studies Phyllocrania came out as the sistergroup of Sibyllidae.

Empusidae. This taxon currently comprises ten genera (Roy 2004a: 6 ff.) and is traditionally subdivided into two subgroups: Blepharodinae (Blepharodes Bolivar, 1890, Blepharopsis Rehn, 1902 and Idolomantis Uvarov, 1940) and Empusinae [Empusa Illiger, 1798, Hemiempusa Saussure \& Zehntner, 1895, Chopardempusa Paulian, 1958 (see Roy 2004a: 12), Dilatempusa Roy, 
2004, Idolomorpha Burmeister, 1838, Hypsicorypha Krauss, 1892, Gongylus] (Beier 1934b: 3; Ehrmann 2002: 378; Roy 2004a: 6 ff.). Empusinae is generally stated to exhibit the more derived character states (Beier 1934b; Roy 2004a).

Roy (2004a) published a revision of Empusidae, discussed the morphological traits that are characteristic for the group (Roy 2004a: 13), and provided a mentally created phylogeny (Roy 2004a: fig. 24; Fig. 27 herein). Important characters are the antero-ventral spination pattern of the fore femora (one long and three short spines in an alternating pattern) and the pectinate antennae of the males, both of which are likely apomorphic for Empusidae (Roy 2004a: 14). The latter character was discussed early by Wood-Mason (1878a). In two genera (Hemiempusa and Idolomorpha), each antennomere carries a single elongated process (Roy 2004a: 2; vs. Beier 1934b: 4 and Ehrmann 2002: 170, 193). The processes of two neighbouring antennomeres are bent in the same direction with opposite points of inflection, thus giving the impression of a bipectinate antenna (Wood-Mason 1878a: 261; Roy 2004a: 2; Figs. 90, 91, 97, 98). In other Empusidae the antennae of the males carry two slender processes on each antennomere, thus they are truly bipectinate (Wood-Mason 1878a: 261; Roy 2004a: 2; Figs. 87-89). Serrate or unipectinate antennae can also be found in some Vatinae (Roy 2004a: 14; see chapter 4.1.1: character 20 and discussion in chapter 4.6.2; Fig. 95).

Further characters that were mentioned by Roy (2004a: 13) are the pointed dorsal edge of the scutellum and the head process. These characters can also be found in other mantodeans, e.g. in some Hymenopodidae (e.g. Ceratomantis Wood-Mason, 1876, see for instance Roy \& Svenson 2007: figs. 1-13; Fig. 58) and Sibyllidae (Roy 2004a: 14). Therefore they are possibly not autapomorphic for Empusidae (see chapter 4.1.1, characters 1, 7; see chapter 4.6.2). The asymmetrical subgenital plate of the males has been stated to be characteristic for Empusinae (Roy 2004a: 1, 14). However, this character is probably plesiomorphic, perhaps even for Mantodea as a whole (Wieland 2006: 52, 74). Strongly asymmetrical subgenital plates can be found in male Metallyticus (Fig. 361), as well as in many other male Mantodea and Blattaria (Wieland 2006: 52, 74, 2008a: fig. 16; see chapter 4.1.3: character 137). Roy (2004a: 1 and fig. 2) mentioned an additional character of the male subgenital plate. Its right dorsal edge is thickened and carries a process in Empusinae. Such a spine is missing in Blepharodinae and is therefore likely apomorphic for Empusinae (see chapter 4.1.3, character 138; Figs. 363-364). A further characteristic of Empusinae is the strongly elongated prothorax (Fig. 124), which is much shorter in Blepharodinae (Beier 1934b: 72; Kaltenbach 1982: fig. 96; Roy 2004a: 1). In contrast to Blepharodinae, species of Empusinae have the ventral antero-distal lobe of the fore coxae elongated and lanceolate (Roy 2004a: 1; see chapter 4.1.2, character 34; Figs. 134, 135).

Members of the empusid subgroups were studied by Wieland (2006: Gongylus, Empusa, Blepharopsis). However, the morphological characters of the cervical sclerites were not suitable to yield arguments for or against the assumed monophyly of Empusidae.

Svenson \& Whiting (2004a) included a single species of Empusinae (Gongylus gongylodes), in their analysis. In their phylogenetic tree, the species clustered with Phyllocrania, which raised the question of a putative paraphyletic Hymenopodidae with respect to certain 
of its species being more closely related to Empusidae (see also discussion of Hymenopodidae in this chapter).

Svenson \& Whiting (2009) included members of both traditional subgroups of Empusidae in their analysis (Blepharopsis, Idolomantis, Idolomorpha, Gongylus, and Empusa). The assumed monophyly of Empusidae and of both subgroups found support therein. Blepharopsis and Idolomantis formed the traditional Blepharodinae as the sistergroup of Idolomorpha $+($ Gongylus + Empusa), which represented the traditional Empusinae. These results perfectly reflected and supported the discussion and cladogram provided by Roy (2004a: fig. 24; Fig. 27). Empusidae was nested in a larger clade (node

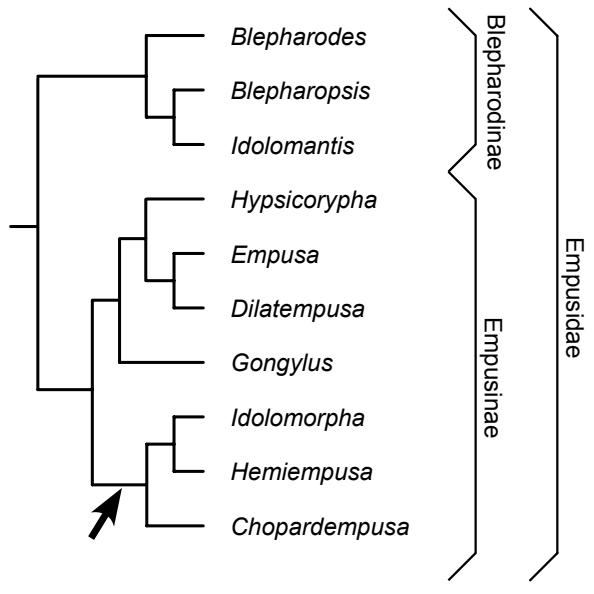

Fig. 27: Phylogeny of Empusidae proposed by Roy (2004a: fig. 24). Arrow indicates Idolomorphini sensu Roy 2004a, see Tab. 1 for Idolomorphini sensu Ehrmann 2002 (see chapter 4.3.74). 194) containing most of the Hymenopodidae, as well as Sibyllidae and Phyllotheliinae.

Yager \& Svenson (2008) used nearly the same taxon sample in their analysis (only lacking Blepharopsis). Therein, the same topology was recovered (Idolomantis + (Idolomorpha + $($ Gongylus + Empusa))) but the putative monophyly of Blepharodinae could not be addressed. The close relationship between Empusidae and Hymenopodidae was recovered therein.

Ware et al. (2008) included only a single species (Gongylus gongylodes), in their analyses. The parsimony analyses (Ware et al. 2008: fig. 2) resulted in Gongylus + (Pseudocreobotra + Phyllocrania), whereas the Bayesian analyses showed a clade Pseudocreobotra $+($ Gongylus + Phyllocrania) (Ware et al. 2008: fig. 3a) and Gongylus + Phyllocrania excluding Pseudocreobotra (Ware et al. 2008: fig. 3b). However, as none of the other Empusidae were included, Ware et al. (2008) were not able to contribute to the understanding of empusid phylogeny.

Empusidae is a rare example of a family in the traditional classification of Mantodea that has been exactly recovered by the analysis of molecular data (Yager \& Svenson 2008; Svenson \& Whiting 2009). The putative close relationship between Empusidae and parts of Hymenopodidae has been discussed above (see discussion of Hymenopodidae in this chapter).

\subsubsection{Further systematic hypotheses}

Neomantodea. Grimaldi (2003: 44 and fig. 27; Fig. 28) introduced the name Neomantodea for a taxon comprising all extant Mantodea including $\uparrow$ Ambermantis from New Jersey Cretaceous amber as their sistergroup. He named the following diagnostic characters for this grouping: "Midfemur lacking spines; claval furrow straight or only slightly arched; fore tibia with long apical spur; fore femur with patch of small scales on mesal surface" (Grimaldi 2003: 44).

However, the claval furrow is strongly arched at least in Metallyticus (e.g. Wieland 2008a: fig. 14; Béthoux \& Wieland 2009: figs. 1a, 2a, 6a; Fig. 336), but also in some "higher" Manto- 
dea, for instance in Deroplatys lobata (see Béthoux \& Wieland 2009: fig. 12e, f). Furthermore, there are small but distinct articulated spines on the mid- and hind femora of Chaeteessa (see characters 83, 84; Figs. 277-284). Additionally, the current state of knowledge is that Chaetees$s a$ does not have a tibial spur (e.g. Beier 1968a: 4; Salazar 2005: 269; but see character 68 and discussion in chapter 4.6.5). Therefore possibly none of these characters represents an autapomorphy supporting the Neomantodea hypothesis. The scale-like nature of the grooming setae may be a synapomorphic character. However, as fossil species were not included in any other phylogenetic study and are not included in the present analysis except for comparative reasons, this hypothesis cannot be examined in further detail.

Eumantodea. Grimaldi (2003: 44 and fig. 27; Fig. 28) named the taxon containing all extant mantodeans Eumantodea, for which he listed the following characteristics: "All living mantises, which have fore femur with discoidal spines". Although the current state of knowledge is that Metallyticus does not exhibit discoidal spines, Grimaldi (2003: tab. 3) encoded the discoidal spines as present for Metallyticus. However, the results herein (characters 54, 55 and discussion in chapter 4.6.4) indicate the maintenance of at least one discoidal spine in adult Metallyticus. The lack of discoidal spines was encoded as "missing" or "unknown" for the fossil taxa in Grimaldi (2003). However, several of the specimens are (possibly early instar) nymphs that may exhibit much more slender and fragile spines than the adults (see also chapter 4.6 .7 for postembryonic changes in the foreleg morphology).

The term Eumantodea was adopted by Svenson \& Whiting (2009; Fig. 4A) and is certainly suitable to distinguish the extant Mantodea from their stemgroup ancestors.

Mantoidea. Mantoidea has initially been used as a synonym for Mantodea, for instance by Handlirsch (1903a, b). Vickery \& Kevan (1983: 218, 219) introduced the superfamily Mantoidea comprising all Mantodea except for Chaeteessa and Metallyticus.

Roy (1999: 39) used Mantoidea in a more restricted sense which has been adopted by subsequent workers, encompassing the traditional Acanthopidae, Hymenopodidae, Tarachodidae, Liturgusidae, Mantidae, Toxoderidae, Thespidae, Iridopterygidae, Sibyllidae, and Empusidae of the current classification sensu Ehrmann (2002: 372 ff.). Grimaldi (2003: fig. 27; character labels 22 and 23 addressed incorrectly, corrected in Grimaldi \& Engel 2005: fig. 7.60; Fig. 28 herein) and Grimaldi \& Engel (2005: fig. 7.60), used Beier's (1968a) classification as a working hypothesis. They listed several putative autapomorphies for this grouping. Among them are the metathoracic hearing organ ("cyclopean ear"; see chapter 4.1.2.: introduction of "metathoracic hearing organ" and characters 130 and 131 for further detail; Yager \& Svenson 2008) and the prothorax being "long" (Grimaldi \& Engel 2005: tab. 7.3) or "2-20 times as long as wide" (Grimaldi 2003: 40). However, as established by Yager \& Svenson (2008: fig. 6), there are plenty of mainly Neotropical genera currently assigned to Mantoidea that exhibit the non-auditory MSMT-morphology of the metathoracic hearing organ (see Yager \& Svenson 2008 and "metathoracic hearing organ" in chapter 4.1.2), i.e. that are (primarily) deaf (Yager \& Svenson 
2008: 557). This would contradict the assumed single origin of tympanal hearing in Mantodea as found by Yager \& Svenson (2008) and Svenson \& Whiting (2009).

As far as the second potential apomorphy for Mantoidea is concerned, Svenson \& Whiting (2004a, 2009) and Yager \& Svenson (2008) found support for a secondarily shortened prothorax in several groups, among them Paraoxypilinae, Amorphoscelinae and Eremiaphilidae. As it has been generally assumed that short prothoraces in Mantodea represent the plesiomorphic state, this further contradicts the Mantoidea-hypothesis. Therefore, the two autapomorphies suggested by Grimaldi (2003) and Grimaldi \& Engel (2005) are doubtful (see also character 28 ).

Ware et al. (2008: figs. 2, 3) did not find support for Mantoidea in their analyses.

None of the other studies allowed an approach to the putative monophyly of Mantoidea, mostly due to a small taxon sample (e.g.

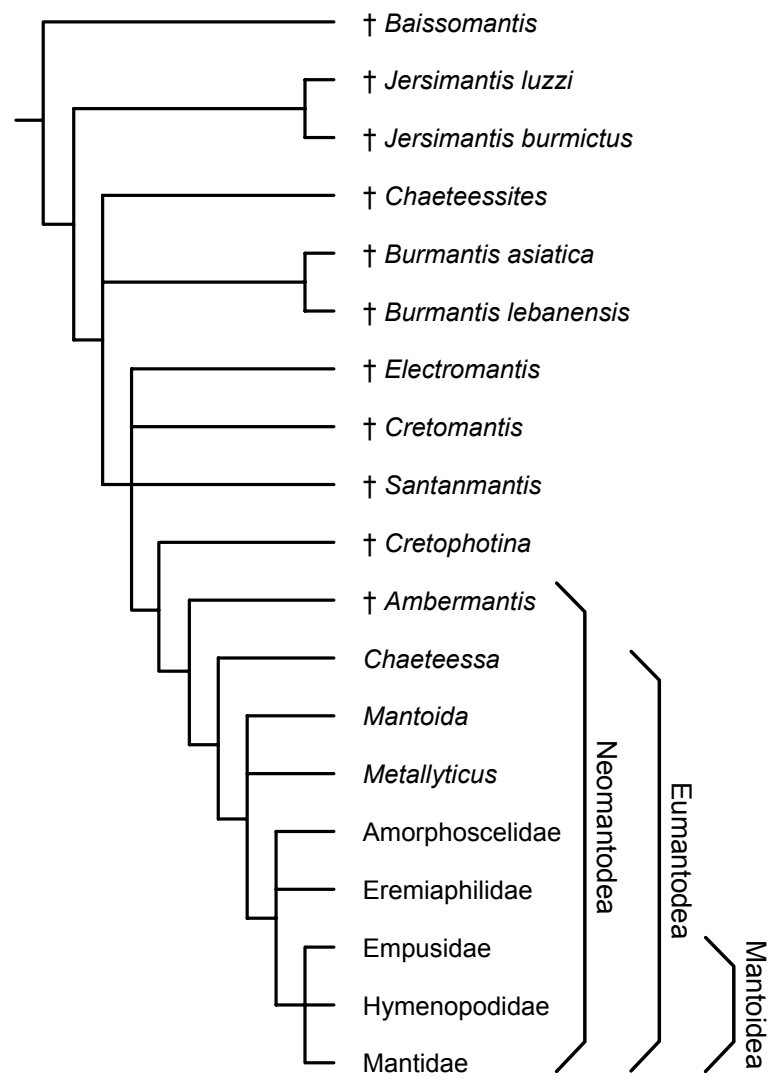

Fig. 28: Phylogeny of Mantodea including fossils found by Grimaldi (2003: fig. 27). Taxon names of extant Mantodea refer to classification of Beier (1968a), see chapter 2.1.4 for details.

Klass 1995, 1997; Klass \& Meier 2006), due to biogeographical restriction of the taxon sample (Jantsch 1999) or the lack of a subsequent phylogenetic analysis of the generated morphological data (Wieland 2006).

Mantomorpha. Klass (1995: 186; Fig. 1A) named the group containing all extant Mantodea except for Mantoida Mantomorpha. He listed 11 apomorphic morphological characters of membranous and sclerotized structures of the male genital apparatus for the group (Klass 1995: 187, 189). This was supported in several morphological studies (Klass 1995, 1997; Klass \& Meier 2006). A basal dichotomy between Mantoida and the remaining Mantodea was also found in several molecular studies (e.g. Svenson \& Whiting 2004a; Inward et al. 2007; Lo et al. 2007; Ware et al. 2008; Yager \& Svenson 2008). The molecular studies often did not include Chaeteessa and/ or Metallyticus or they did not have suitable tissue for gaining optimal DNA sequences (Yager \& Svenson 2008). Ware et al. (2008) included all three taxa and found Mantoida to be the sistergroup of all remaining Mantodea. Svenson \& Whiting (2009) who were able to gain better sequences from the DNA of Chaeteessa found the latter to be the sistergroup of the remaining Mantodea.

Mantidea. Klass (1995: 186; Fig. 1A) named all extant Mantodea except for Mantoida and Chaeteessa Mantidea. Six apomorphic morphological characters were listed (Klass 1995: 187, 189). 
Artimantodea. Svenson \& Whiting (2009: node 7; Fig. 4A) introduced the name Artimantodea for all extant Mantodea excluding Chaeteessa and Mantoida (and possibly Metallyticus). This name would be synonymous with Mantidea sensu Klass (1995: 186) if Metallyticus is included in Artimantodea (situation unclear, see discussion of Mantoida, Chaeteessa and Metallyticus in this chapter 2.1.4; Fig. 4A). Autapomorphic characters for Artimantodea (if Metallyticus falls outside of Artimantodea) have not been mentioned.

Cernomantodea. Svenson \& Whiting (2009: node 48; Fig. 4A) recovered many of the phylogenetic affinities found by Yager \& Svenson (2008). One of the most important morphological traits is the metathoracic hearing organ (see also discussion of Hymenopodidae in this chapter, characters 130 and 131 in chapter 4.1.2 and the corresponding introduction). All Cernomantodea ("perceptive Mantodea") have one or two ears on the meta- and mesothorax, respectively.

In conclusion it can be stated that the monophyly of many of the families and subfamilies of the traditional classification is not supported by the molecular and morphological studies. So far, the monophyly of Empusidae, Toxoderidae, Sibyllidae, Amorphoscelinae, Paraoxypilinae, Acanthopidae (Stenophylla not studied), including its subgroups Acontistinae and Acanthopinae, Oxyothespinae, and Phyllotheliinae, has been corroborated. The basal split in Mantodea is currently still under discussion.

\subsection{Palaeontological record}

Many authors have studied, described, re-described, discussed or reviewed fossil Mantodea. Among them are Giebel (1862), Handlirsch (1906-1908, 1937, 1938), Cockerell (1908, 1955), Klebs (1910), Zeuner (1931), Sharov (1962), Hennig (1966, 1969), Beier (1967), Harz (1980), Gratshev \& Zherikhin (1993), Nel \& Roy (1996), Grimaldi (1997, 2003, 2008), Ehrmann (1999, 2002), Vršanský (2002, 2005), Zherikhin (2002), Grimaldi \& Engel (2005), Zompro (2005), Gorochov (2006), Béthoux \& Wieland (2009) and Béthoux et al. (2010). The mantodean fossil record was poor when Beier (1964a, 1968a) published his synoptic works. Since then, a variety of fossils from several epochs was described, mainly during the recent two decades. They are preserved in copal, amber and as compression fossils. Mantodean fossils in amber were briefly discussed by Ehrmann (1999; see also Ehrmann 2002: tab. 10). The 88 fossils listed in Ehrmann (2002: tab. 10) have been assigned to Chaeteessidae, Mantoididae, Liturgusidae, Tarachodidae, and Mantidae.

The fossil record of Cretaceous Mantodea was reviewed and summarized by Grimaldi (2003: tab. 1).

The latest described fossil mantodean is †Mantoida matthiasglinki Zompro, 2005 (p. 93), which is an adult specimen preserved in Baltic amber (Eocene).

Svenson \& Whiting (2009) dated back the origin of Mantodea to the beginning of the Jurassic based on molecular clock estimations. This was also assumed earlier by Grimaldi (1997: 5). 
Béthoux \& Wieland (2009), however, found support for an even earlier origin of Mantodea in the Upper Carboniferous, approximately 310 million years ago, based on the homologization of mantodean wing venation and subsequent assignment of $\uparrow$ Mesoptilus dolloi Lameere, 1917 and its relatives [most of which were previously assigned to $†$ Strephocladidae by Carpenter (1992: $124 \mathrm{ff}$.)] to Mantodea. See also Béthoux et al. (2010) for further data on the wing venation of the $\uparrow$ Homcladus grandis Carpenter, 1966, another putative stemgroup-mantodean that was studied re-investigated based on new specimens.

A detailed account of the fossil record is out of the scope of this study. However, several fossils [†Cretomantis larvalis Gratshev \& Zherikhin, 1993 (Figs. 29, 30); † Jersimantis luzzii (Fig. 31); $\dagger$ Santanmantis axelrodi Grimaldi, 2003 (Fig. 32); and an unidentified first instar nymph from Baltic amber (Figs. 33-40)] will be referred to several times in order to discuss the putative origin or evolutionary history of morphological traits. Selected structures, partly redrawn from the original publications in order to focus on selected structures, will be provided.

When describing $\uparrow$ Cretomantis larvalis, Gratshev \& Zherikhin (1993) created a new family $\uparrow$ Cretomantidae and genus $\uparrow$ Cretomantis based on one nymphal specimen, impressed in shale. The specimen (probably a nymphal exuvia, see Grimaldi 2003: 20) is from the Lower Cretaceous of Baysa Creek, Russia. Gratshev \& Zherikhin (1993: 161) gave some comments in the differential diagnosis: "Differs from Chaeteessidae in elongated prothorax, short cerci, strongly incrassate fore femora with strong inequal spines, strong and clearly differentiated spines of fore tibiae; from other mantid families by articulated outer apical spine of fore tibiae." The authors stated that the specimen of $\uparrow C$. larvalis is a mid to late instar nymph (Gratshev \& Zherikhin 1993: 161). However, this is almost impossible to evaluate based solely on its size of $7.6 \mathrm{~mm}$. There are extant Mantodea that are small as adults but hatch as quite large first instars, for instance Amorphoscelis tigrina Giglio-Tos, 1913. In this species, newly hatched nymphs measure approximately $7 \mathrm{~mm}$, whereas the adult females measure about $24 \mathrm{~mm}$ (Schulze, pers. comm. 2009). The body size of hatchlings correspondingly already is approximately 25 per cent with respect to the adults! Other species that become very large as adults hatch as comparatively small nymphs, among them Solygia sulcatifrons (Audinet-Serville, 1839). This species reaches enormous body lengths of up to $17 \mathrm{~cm}$ (including the elongated subgenital plate, about $13 \mathrm{~cm}$ without it). Freshly hatched nymphs of this species are 6-7 $\mathrm{mm}$ (Schulze, pers. comm. 2009). Their body length thus measures no more than about 4-5 per cent of the adult body length.

These data indicate that the $\dagger C$. larvalis specimen may well represent an early instar nymph. This is corroborated by the fact that it apparently has 4-6 cercomeres (see interpretative drawing in Grimaldi 2003: fig. 13 and p. 20) which is typical for first or second instar Mantodea (see chapter 4.6.7 and Figs. 35, 392, 453, 455, 456, 458, 460). The presence of a large distal posteroventral spine on the tibia, additionally to the tibial spur (also mentioned by Grimaldi 1997 for $\dagger$ Jersimantis luzzii), is also a widespread trait in early nymphs of extant Mantodea even when inconspicuous in the adults (e.g. in Metallyticus, Mantoida, Humbertiella, pers. obs.; see Figs. 434-436). It may be correlated to the demands of capturing very small prey in the early 

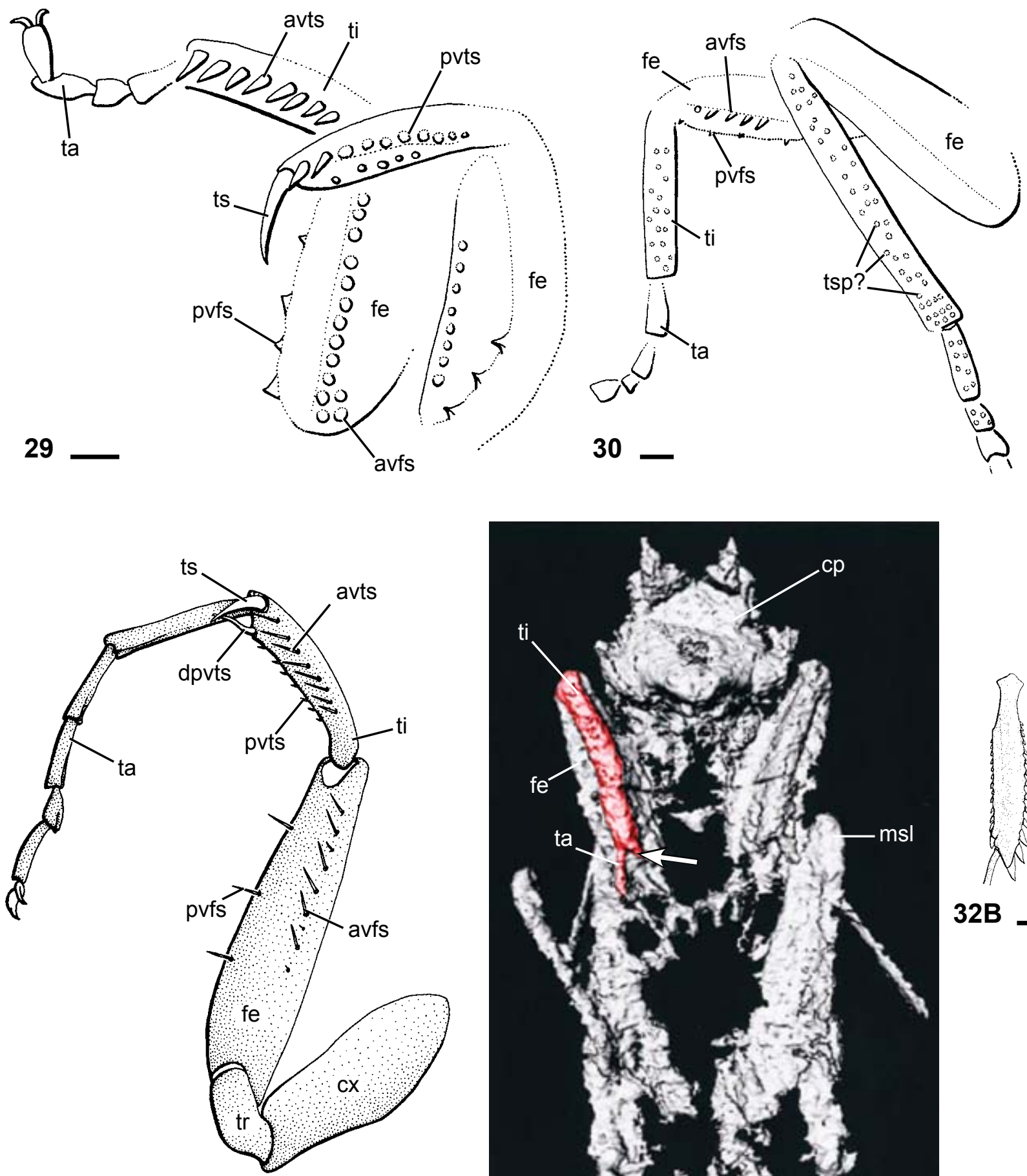

31
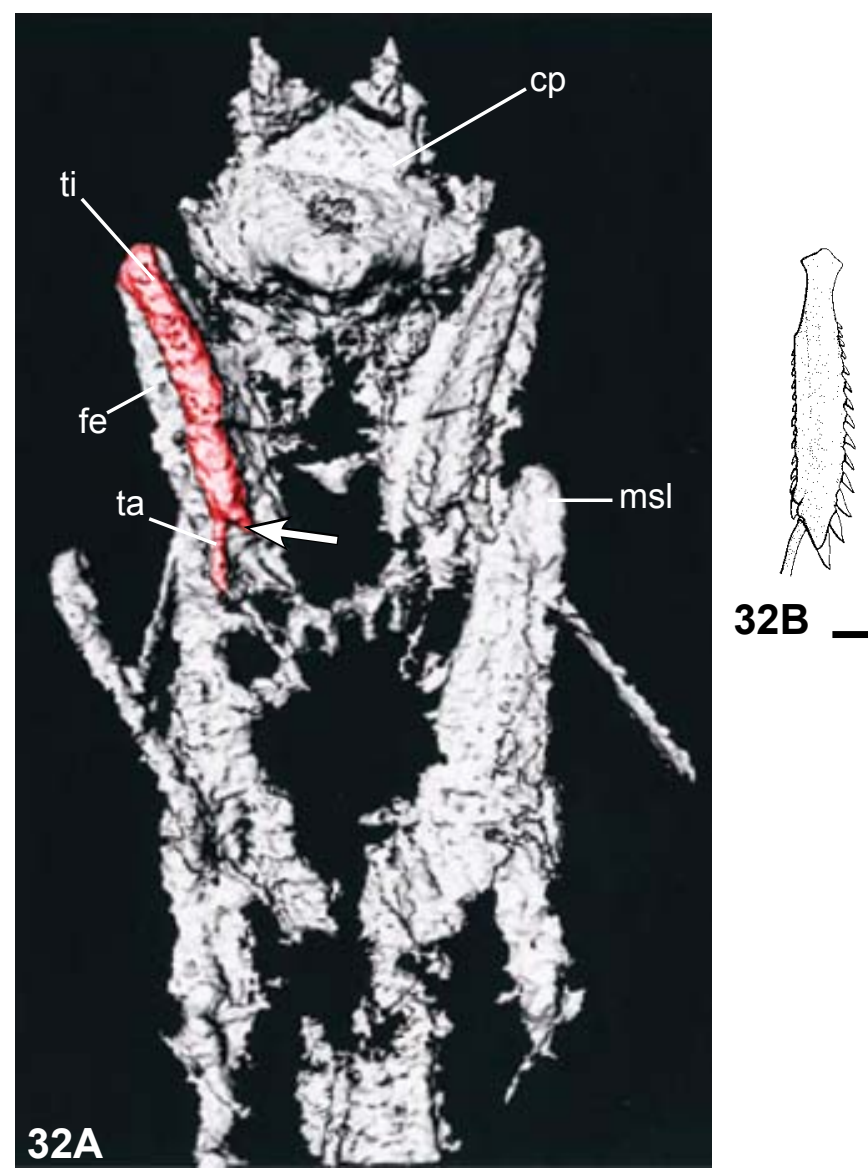

32B

Figs. 29-32: Fossil Mantodea, selected structures. 29: $\uparrow$ Cretomantis larvalis, forelegs. Redrawn and slightly altered from Grimaldi 2003: fig. 13. 30: $†$ Cretomantis larvalis, meso- and metathoracic legs (right body side). Redrawn and slightly altered from Grimaldi (2003: fig. 13). $31 \dagger$ Jersimantis luzzii, right foreleg. Redrawn and slightly altered from Grimaldi (1997: fig. 3). Fine bristles omitted. 32A: $\uparrow$ Santanmantis axelrodi, ventral view. $\mu-C T$ reconstruction. Published by Grimaldi (2003: fig. 19, right hand side), slightly altered. Red: tibia of raptorial foreleg folded against femur; arrow indicates putative distal lobe with tibial spur. 32B: Pseudocreobotra wahlbergii, ㅇ, left fore tibia (mirrored), dorsal view. - Scale bar =0.2 mm (Figs. 29-32A); $1.0 \mathrm{~mm}$ (Fig. 31B). Scale bars of Figs. 29 and 30 reconstructed from Gratshev \& Zherikhin (1993: fig. 4a, b).

Abbreviatons: avfs = antero-ventral fore femoral spine; avts = antero-ventral fore tibial spine; $\mathbf{c p}=\mathbf{c a p u t} ; \mathbf{c x}=$ coxa; dpvts = distal postero-ventral fore tibial spine; $\mathbf{f e}=$ femur; $\mathbf{m s l}=$ mesothoracic leg; $\mathbf{p v f s}=$ postero-ventral fore femoral spine; $\mathbf{p v t s}=$ postero-ventral fore tibial spine; $\mathbf{t a}=$ tarsus; $\mathbf{t i}=$ tibia; $\mathbf{t s p}=$ tibial spines. 
instars. This character is also very well recognizable in the putative fossil Mantoididae specimen shown in Weitschat \& Wichard (2002: pl. 33, fig. b) and in the unidentified nymph from Baltic amber (Figs. 34, 37, 39, 40).

As the first instar nymphs of many Mantodea (especially the three key taxa Chaeteessa, Mantoida and Metallyticus, but also Amorphoscelidae, Eremiaphilidae and many others) are very poorly studied or even unknown to date, it is impossible to use the relative length of the cerci in instars as a diagnostic character. While the cerci are very short in first instar Mantoida (and many other Mantodea), those of Metallyticus and, for instance, Didymocorypha, are comparatively long (pers. obs.; see chapter 4.6.7 for discussion; see Figs. 392 and 453 for Metallyticus and Fig. 432 for Didymocorypha). Furthermore, Chaeteessa has enormously elongated cerci as a late instar (see Figs. 5, 390), and it is unknown if they are already long in first instar nymphs. Therefore, the reasons for assigning the $\dagger C$. larvalis specimen to a new family are debatable. Grimaldi (2003: 20) reviewed the specimen and provided detailed morphological data including a high resolution photograph and an interpretive drawing of the specimen (Grimaldi 2003: figs. 12,13). The forelegs and cursorial legs were herein redrawn and slightly simplified in order to focus on certain morphological traits that are of interest for the discussion of the morphology of extant Mantodea (Figs. 29, 30). Among them are spines on the femora (and possibly tibiae) of the cursorial legs (see characters 83,84 ), and the spination pattern of the forelegs (see characters 45,54 , and chapter 4.6.5).

$\dagger$ Jersimantis luzzii was described by Grimaldi (1997) based on a complete nymphal specimen preserved in mid-Cretaceous New Jersey amber. Again, an additional large postero-ventral tibial spine was found, which was argued to be a plesiomorphic trait (Grimaldi 1997: 8). However, this character is also present in many extant Mantodea (see above). The remaining spination of the foreleg (Grimaldi 1997: fig. 3; Fig. 31 herein) is very delicate. Adult $\dagger$ Jersimantis are unknown, therefore it is unclear how much the structure of the spines changes throughout postembryonic development. This would be a highly interesting problem to address because the changes in foreleg morphology between first instar and adult Metallyticus splendidus, for instance, are drastic (see character 54 and chapter 4.6.4). † Jersimantis luzzii was mentioned by Klass \& Meier (2006: 17) because of the presence of a well-developed tibial spur. Herein, too, it is woven into the discussion of the foreleg morphology (e.g. characters 54, 86, and chapter 4.6.5). Therefore, the foreleg (Grimaldi 1997: fig. 3) was redrawn herein to emphasize the structures of interest (Fig. 31).

Another important fossil species is $\dagger$ Santanmantis axelrodi. The genus and species were described based on series of well-preserved specimens from the Crato Formation in Brazil (ca. 115 My old, see Grimaldi 2003: tab. 1). This tiny species (about $10 \mathrm{~mm}$, see Grimaldi 2008: 237) bears characters of the wings and both forelegs and cursorial legs that are referred to in the discussion of the morphology of extant taxa (e.g. characters 83, 84, 86, 104, 112 and chapter 4.6.5). Fig. 32A shows a $\mu$-CT image of $\uparrow$ Santanmantis axelrodi in ventral view (slightly altered from Grimaldi 2003: fig. 19, right hand side). 


\subsection{Biogeography}

Mantodea are generally assumed to be poor dispersers ("non-migrants", e.g. Johnson 1969: 579) as most species are relatively stationary for long periods of time (e.g. von Rebeur-Paschwitz 1895: 267; Beier 1968a: 12; Johnson 1969: 196). The females are usually not capable of flying after a brief time interval between the final moult and gravidity (about three weeks) and in a short interval between egg depositions (e.g. Werner 1909: 71; Beier 1968a: 12; Ehrmann 2002: 30). It has been shown, however, that the oothecae are resistant to all kinds of harsh conditions (frost or boiling water; e.g. Fritze 1915; James 1943; Salt \& James 1947; Ehrmann 2002: 26). This allows for an easy distribution via floating driftwood or human dispersal, for instance when an ootheca is fixed to a car or is fixed to plant matter that is being shipped for wood export (e.g. Werner 1909, 1915; Ehrmann 2002: 30). A case of dispersal of viable oothecae of Statilia maculata (Thunberg, 1784) from Japan to New Zealand via used cars was published only recently (Harris 2007; see also Toy \& Newfield 2010: tab. 1).

There are several interesting disjunctive distributions among extant and fossil Mantodea. For instance, Choeradodinae, which are today distributed in the Neotropics and in India, Sri Lanka, Myanmar, China and Thailand (e.g. Badenoch 1899: 31; Beier 1939a: 10, 1968a: 13; Roy 2004b) are probably present in the Eocene of Menat in France (Piton 1940; Nel \& Roy 1996). Likewise, Chaeteessa and Mantoida, which are both restricted to the Neotropics today, have potentially related species in the fossil record of Europe and, in case of Chaeteessa, in North America (Cockerell 1908; Sharov 1962; Nel \& Roy 1996; Ehrmann 1999, 2002; Roy 1999: 38; Weitschat \& Wichard 2002; Zompro 2005). Furthermore, a single species, Exparoxypilus africanus, has been described from Tanzania by Beier (1929: 135; see also Beier 1939a: 11), representing the otherwise exclusively Australian Paraoxypilinae. In contrast, the African Compsothespis has been recorded with one species from Australia [C. australiensis Wood-Mason, 1889 (p. 7 and fig. 4); see also Westwood 1889: 32 and pl. XII, fig. 11]. Such findings have to be treated carefully and will have to be thoroughly researched. Their validity has, for instance in the case of C. australiensis, been doubted in the literature (see Balderson 1984: 1, 5, 1991: 253).

Reviews treating the global distribution of the taxa recognized at the time were published by Werner (1909) and Beier (1939a). They were briefly summarized, for instance, by Beier (1968a: $12 \mathrm{ff}$.). There have been several publications dealing with the biogeography of single taxa or areas since then, among them Chopard (1938, 1942), Kaltenbach (1963, 1964, 1996, 1998), Roy (1987b), Agabiti (2001), Lombardo \& Agabiti (2001), Delfosse et al. (2007), and Battiston et al. (2010).

The latest and most comprehensive study on the biogeography of Mantodea was published by Svenson \& Whiting (2009) who included 288 species from all major biogeographical regions in their molecular study. Based on their analyses, Svenson \& Whiting (2009) suggested a Jurassic origin of Mantodea ( $200 \mathrm{My}$ ago). Considering the distribution of the fossil record of Cretaceous Mantodea (Gratshev \& Zherikhin 1993; Grimaldi 1997, 2003, 2008; Zherikhin 2002) it is likely that pre-Eumantodean lineages were globally distributed (on both Laurasia 

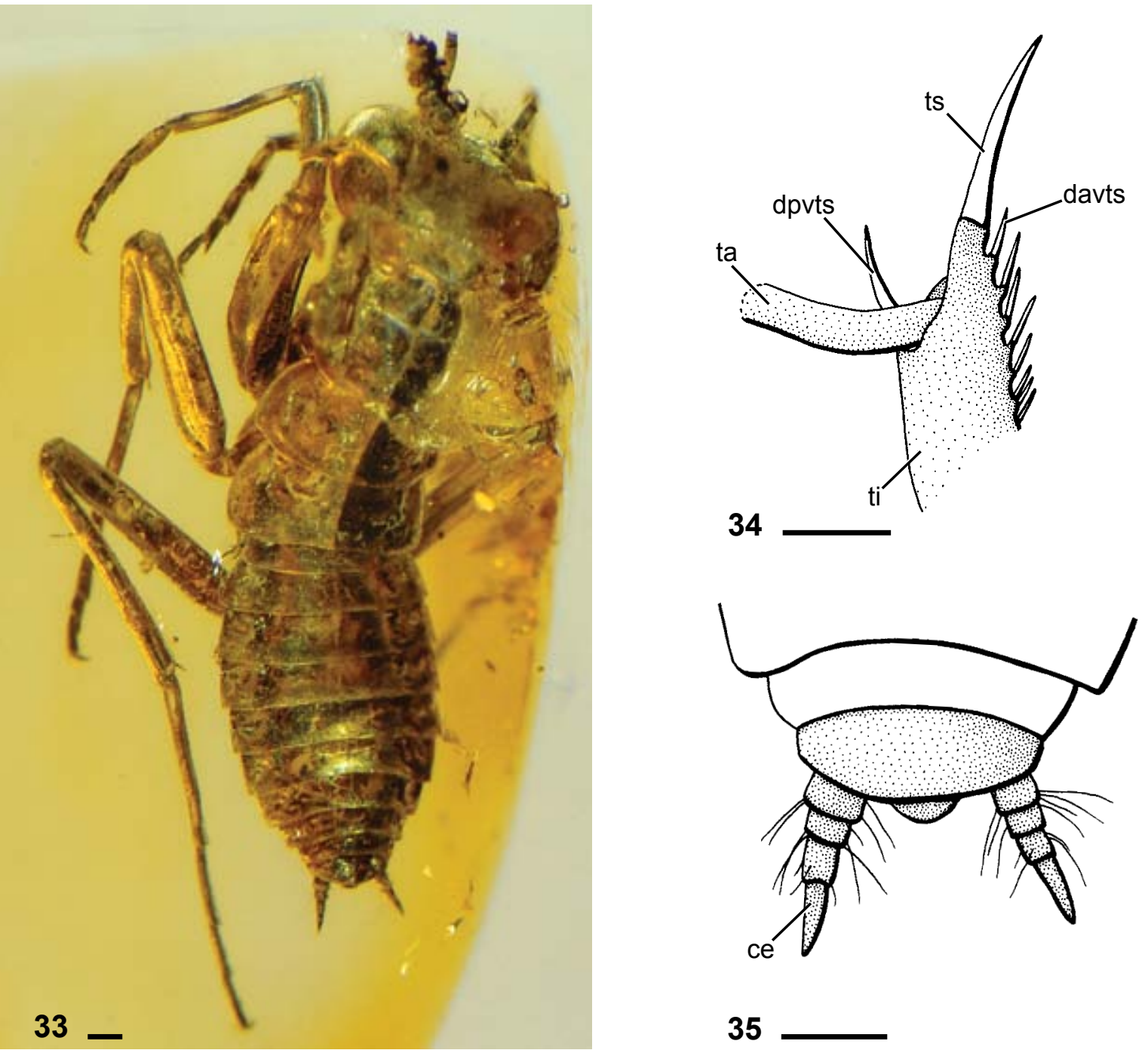

35

Figs. 33-35: Undetermined first instar Mantodea nymph from Baltic amber (collection Carsten Gröhn no. 7054 / GPIH 4502). 33: Original fossil, focus stacking image (courtesy of Carsten Gröhn); dorsal view (structures are labelled in Figs. 36-40). 34: Left fore tibia, distal end in dorsal view, drawn from original piece in amber; note the strongly enlarged distal postero-ventral fore tibial spine which is typical for many early instar Mantodea. 35: Abdominal tip, dorsal view, drawn from original piece in amber; note the number of four cercomeres which is typical for first instar Mantodea. The specimen has a large opening in the dorsal head region which was possibly caused by scavengers or decay. Internal structures were not preserved. - Scale bar $=0.25 \mathrm{~mm}$.

Abbreviations: $\mathbf{c e}=$ cercus; davts $=$ distal antero-ventral fore tibial spine; $\mathbf{d p v t s ~}=$ distal postero-ventral fore tibial spine; ta $=$ tarsus; $\mathbf{t i}=$ tibia; $\mathbf{t s}=$ tibial spur.

and Gondwana) prior to the breakup of Gondwana (Svenson \& Whiting 2009: 504). The two earliest dichotomies in the phylogenetic tree found by Svenson \& Whiting (2009) were between Neotropical (Chaeteessa and Mantoida) and the remaining Mantodea. This, the authors argued, may indicate that Eumantodea arose in Gondwana, or even in South America. The unresolved phylogenetic position of the Southeast Asian Metallyticus (Fig. 4A), however, provides a second scenario implicating that all three lineages are relics of an ancient global distribution, thus indicating that Eumantodea could have evolved anywhere on Pangaea (Svenson \& Whiting 2009: 505). Wherever Eumantodea may have originated, many of the modern Mantodea have been shown to have originated from sequential independent radiation events following the breakup of Gondwana in the Cretaceous (Svenson \& Whiting 2009). The authors concluded 

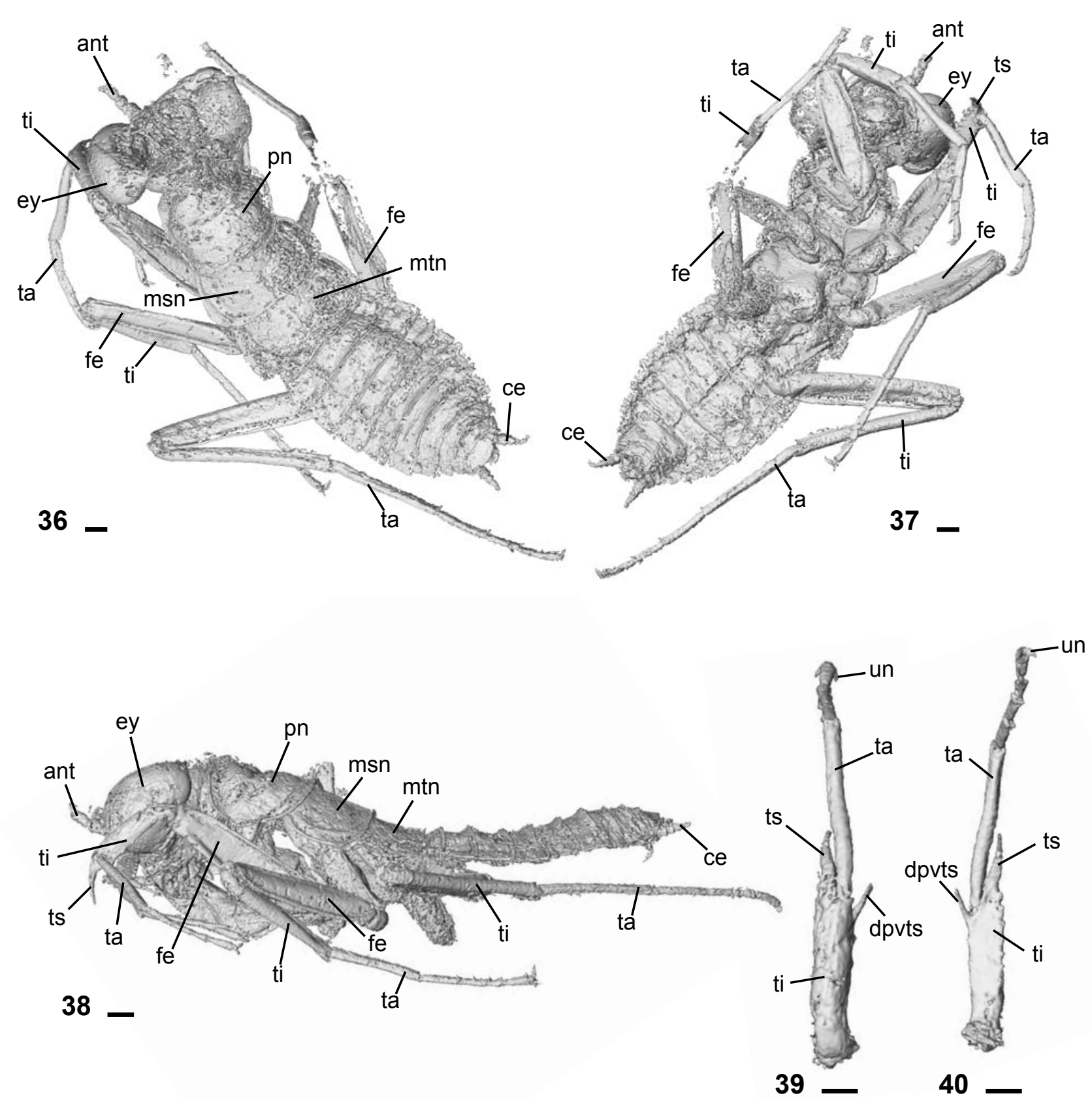

Figs. 36-40: Undetermined first instar Mantodea nymph from Baltic amber (collection Carsten Gröhn no. 7054 / GPIH 4502), $\mu$-CT reconstruction. 36: Dorsal view. 37: Ventral view. 38: Lateral view. 39: Right fore tibia, dorsal view. 40: Right fore tibia, ventral view. Note the strongly enlarged distal postero-ventral fore tibial spine which is typical for many early instar Mantodea. The specimen has a large opening in the dorsal head region which was possibly caused by scavengers or decay. Internal structures were not preserved. - Scale bar $=0.25 \mathrm{~mm}$. Abbreviations: $\mathbf{a n t}=$ antenna; $\mathbf{c e}=$ cercus; davts $=$ distal antero-ventral fore tibial spine; $\mathbf{d p v t s}=$ distal posteroventral fore tibial spine; $\mathbf{e y}=$ compound eye; $\mathbf{f e}=$ femur; $\mathbf{m s n}=$ mesonotum; $\mathbf{m t n}=$ metanotum; $\mathbf{p n}=$ pronotum; $\mathbf{t a}=$ tarsus; $\mathbf{t i}=$ tibia; $\mathbf{t s}=$ tibial spur; $\mathbf{u n}=$ unguis.

that these events may have led to the independent evolution of adaptations to similar habitats on the different continents, in response to similar ecological factors and the corresponding selective pressures. Therefore, we today find several distinct ecomorphs on all continents that exhibit a conspicuous phenotypic similarity based on which they have been taxonomically united in the past without consideration of their biogeographical distribution. Among these groups are bark 
dwellers (e.g. Liturgusidae), stick-shaped and grass-mantids (e.g. Thespidae, Angelinae) and so-called flower-mantids (e.g. Hymenopodidae).

The biogeography of Mantodea will not be treated in further detail in this study.

\section{Material and methods}

\subsection{Techniques}

The specimens studied herein were fixed in $80 \%$ or $100 \%$ ethanol. The structures of specimens that were not available in alcohol or as living material were observed in dried specimens.

The exoskeletal characters were studied under a stereo microscope (Zeiss Stemi SV 11). Structures of interest were drawn in pencil with the help of a camera lucida (Zeiss), and transferred into ink drawings using Rapidograph pens (Rotring) of $0.35 \mathrm{~mm}$ and $0.5 \mathrm{~mm}$ diameters on tracing paper (Schleicher \& Schuell, A4 and A3, 110-115 g/ $\mathrm{m}^{2}$ ). The ink drawings were scanned as bitmap files (resolution of $600 \mathrm{dpi}$ ) and corrected and finished with the Adobe Photoshop CS3 software. Several draiwings were drawn with the help of a Wacom Bamboo Fun tablet: Figs. 109, 142, 405A-F were drawn as vector graphics in Adobe Illustrator CS3; Figs. 334, 335, 337, 345, 346, 346, and 463 were drawn in Adobe Photoshop CS3.

Specimens for SEM imaging were transferred into 100\% ethanol and dried in a Balzer CPD 030 Critical Point Dryer. SEM-pictures were taken with a LEO 438 VP microscope and finished with the Adobe Photoshop CS3 software.

The tarsi of Archimantis gracilis, Deroplatys desiccata, Eremiaphila sp., Idolomantis diabolica, Metallyticus splendidus, and Paraoxypilus sp. were cut off and macerated in $15 \% \mathrm{KOH}$ (not heated) for varying lengths of time, mostly between 30 and 240 minutes, depending on the size and degree of sclerotization of the specimens.

Figs. 36-40 and Fig. 82 were gained from $\mu$-CT data. The $\mu$-CT scans were performed in the Subatomic and Radiation Physics department of Gent University, Belgium. The spatial resolution of the datasets is not available, beam strength was $60 \mathrm{keV}$. The $3 \mathrm{D}$ reconstructions in Figs. 36-40 were made in Amira 5.0. Holes in the reconstructions and missing spines do not reflect the quality of the fossil but have technical reasons. The similar density of cuticula and amber matrix led to a similar absorption of photons and therefore to a similar brightness of the two substances in the dataset. This led to problems in separating the fossil from the amber matrix in Amira 5.0.

\subsection{Phylogenetic methods}

The reconstruction of the relationships among the mantodean taxa follows the principles of phylogenetic systematics sensu Hennig $(1950,1965,1969,1981)$. The aim is to find monophyletic groups based on shared apomorphic characters and to analyse the sistergroup 
relationships among such monophyletic groups. The names of the Mantodea taxa of the current classification are maintained herein. Such names usually refer to Linnean categories (family, subfamily, tribus etc.), indicated by their endings (-idae, -inae, -ini, respectively). Such categories are subjective and in many cases do not reflect the true phylogenetic relationships (as pointed out earlier, compare Svenson \& Whiting 2004a, 2009). Therefore, these names are not used in order to express Linnean categories but to function as rank-free names referring to the species they encompass in the current taxonomical classification as given in Ehrmann (2002).

Characters were encoded as neomorphic (present/missing) or transformational ("unordered multistate") in congruence with Sereno (2007). Characters that showed intersexual differences were encoded separately for each sex.

The character matrix was created in DELTA (Dallwitz 1980; Dallwitz et al. 1999) and converted into a Nexus file. DELTA matrices consist of character states $1-n$ instead of $0-n$. The character states in the Nexus file were not altered into the latter form in order to avoid errors due to transfer mistakes. State 1 in the character states does not necessarily refer to the assumed plesiomorphic condition.

The DELTA (Dallwitz 1980) program does not distinguish between “-” (not applicable) and "?" (missing), and the algorithms of Paup* (Swofford 2001) treat both states equally when calculating the phylogenetic relationships. Therefore, this distinction was not made in the character matrix.

Paup* (Swofford 2001) was used for the calculation of the most parsimonious topologies (for details on the settings see chapter 4.2).

\subsection{Morphological terminology, definition of orientations and measuring, character en- coding}

Both the head and the forelegs are highly moveable in Mantodea an can therefore be held in strongly varying positions (different from most other insects), i.e. the head can be turned freely in all directions and the forelegs can be held to the front and be folded. This led to several different and confusing terminologies of orientation by various authors, especially whenever spination patterns on the raptorial legs are described. To avoid this, I will define the orientation for the body structures as used herein.

Due to the high mobility of the head, many mantodeans can turn their head quite far, thus being capable of "looking over their shoulders". Regarding this, the descriptions of "prognathous" and "hypognathous" orientations of the head are irritating and incorrect. A single specimen may hold its head in a "hypognathous" or "prognathous" position when at rest or hiding (e.g. many Tarachodidae and Liturgusidae, respectively). When moving or hunting, however, the same specimen may hold its head in the "orthognathous" position as displayed by most species (e.g. many Mantinae, Hymenopodidae, Empusidae). Furthermore, these terms morphologically apply to the relative position of the mouthparts with respect to other structures of the head capsule, e.g. the occipital foramen, and not to a position the head is held in life. Regarding the definition 
given, all Mantodea exhibit the orthognathous head type, and the definition of the orientation of structures in life cannot be compared with the prognathous or hypognathous head morphology as exhibited by other insects (for instance many Coleoptera and Hemiptera, respectively). Therefore, the planes of orientation are herein defined by the plane in which the occipital foramen lies (Fig. 43) and all structures are described correspondingly.

The same problem applies to the raptorial forelegs as they are highly moveable and can be stretched out in a high variety of angles. Therefore, all foreleg structures are herein assigned as if the forelegs were orientated similar to a cursorial insect leg, i.e. stretched aside (in congruence with Gray \& Mill 1985: 480-481). Correspondingly, the "inner" or "mesal" and "outer" or "lateral" surface of both the femur and the tibia as named by other authors are herein defined as anterior and posterior surfaces, respectively (Figs. 41, 44-49). Hence, the fore-femoral grooming device ("femoral brush") is located on the antero-distal surface of the fore femora. The spines are located on the ventral surfaces of femur and tibia, therefore they are named anteroventral and postero-ventral fore femoral and fore tibial rows of spines.

The spines of the raptorial forelegs are counted from proximal to distal. The small distal spines that may be present on each of the apical lobes of the fore femur (genicular spines) are not considered (in congruence with Kaltenbach 1996: 196). Five groups of spines are distinguished. The fore femora carry an antero-ventral (avfs) and a postero-ventral (pvfs) row of spines, and one to four often oblique discoidal spines (ds) that are missing in Blattodea. The fore tibiae with few exceptions carry one row of antero-ventral (avts) and one row of posteroventral (pvts) spines and (except for Chaeteessa) a strongly enlarged distal tibial spur (ts).

In most species the pronotum (with the exception of the lamellar expansions) is not rectangular but has a more or less strongly curved outline. Therefore, measurements have to be defined. Measurements of the pronotum are made to the exclusion of the lamellar expansions (see characters 28 and 29 for details). The pronotum is subdivided into pro- and metazona by a dorsal supracoxal sulcus resulting from an internal apodeme which forms a stabilizing element for the massive musculature of the forelegs. Measurements of the prozona and the metazona are made along the longitudinal prothoracic midline. This means that the length of the prozona equals the distance between the anterior edge of the pronotum up to the supracoxal sulcus, and the metazona correspondingly reaches from the supracoxal sulcus up to the posterior edge of the pronotum (Fig. 42). The pronotal width is measured at the widest point of the pronotum (excluding the lamellar expansions). This usually is the width of the supracoxal dilation (Fig. 42).

The tarsomeres and cercomeres are counted from proximal to distal. The proximal tarsomere is termed basitarsomere.

If differences in spine numbers were found between the left and the right leg of one and the same individual or between individuals of the same sex, the largest number of spines was encoded. It was generally found that in those conspecific specimens with lesser spines, there were larger spaces between the proximal spines of the tibia or the distal spines of the femur. This indicates that the spines were not developed in these spaces or were possibly lost during nymphal development (see also Henry 1932: 3). 


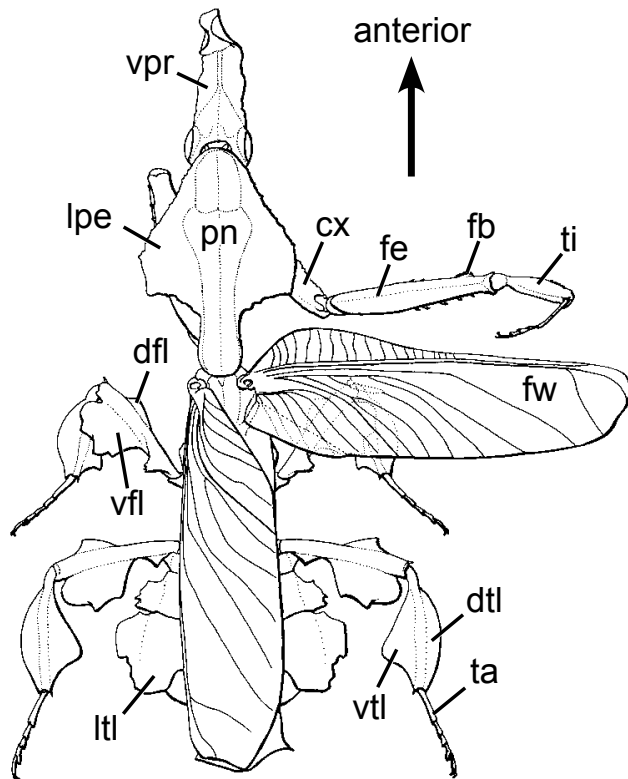

41

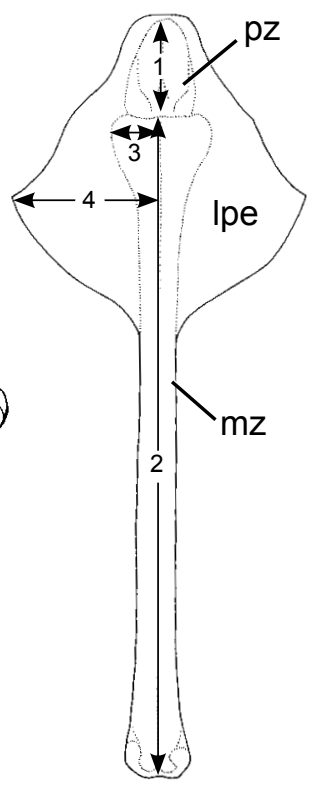

42

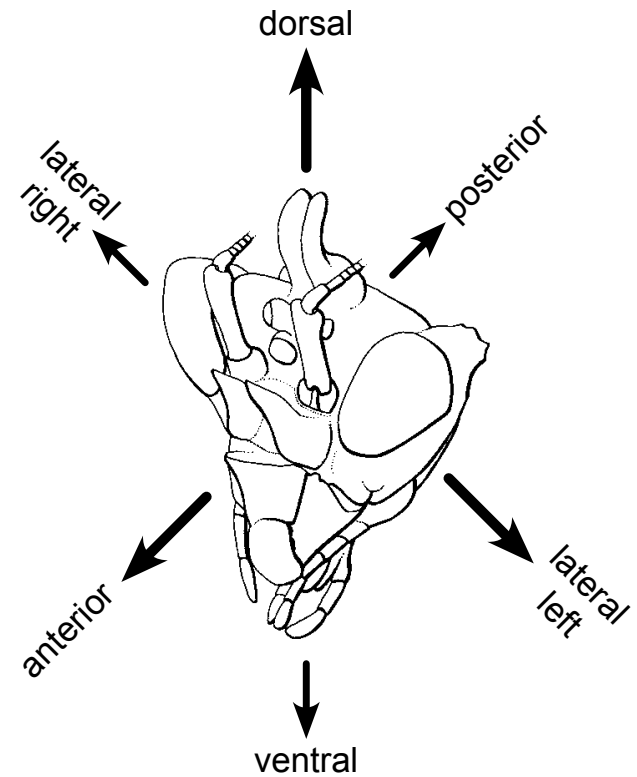

43

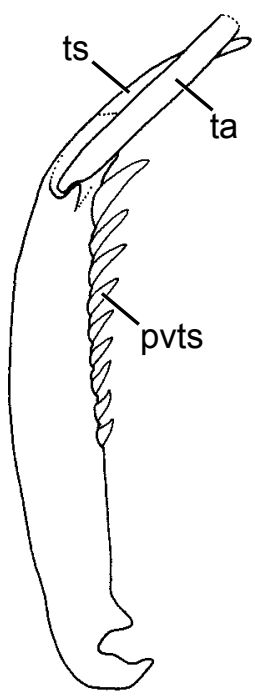

44

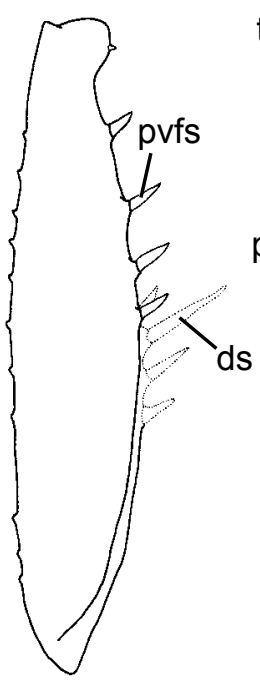

45

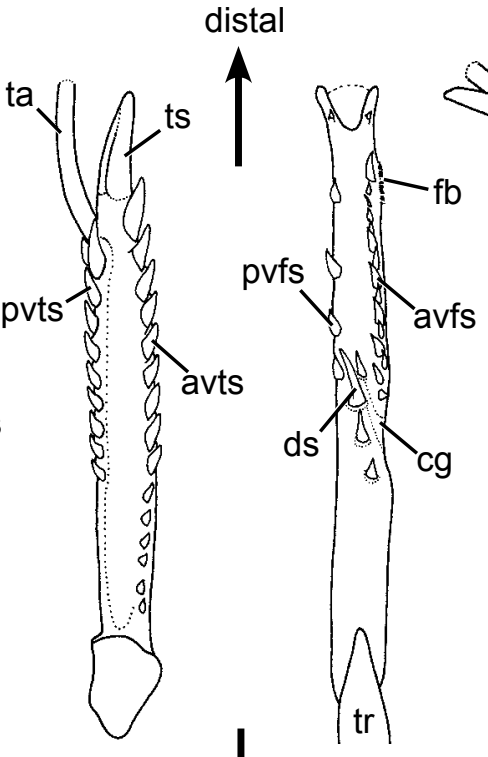

47

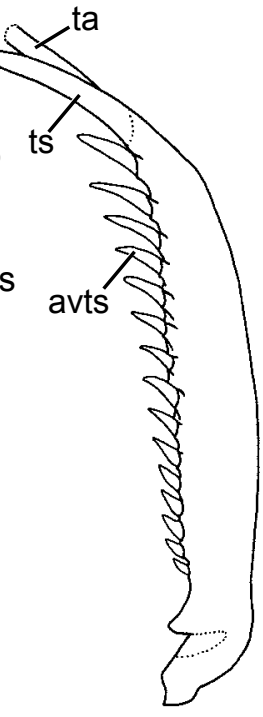

48

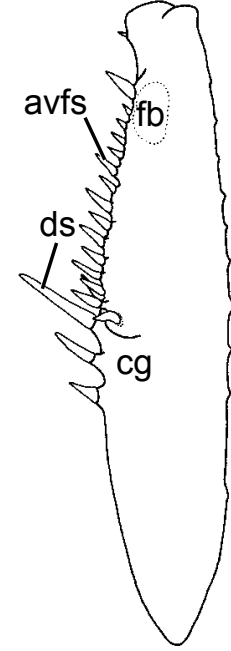

49

proximal

Figs. 41-49: Definition of orientations for the morphological structures applied in this study. 41: Phyllocrania paradoxa,, , habitus, dorsal view; structures of forelegs and wings are defined as if held laterally. 42: Gongylus gongylodes,, , pronotum, dorsal view; definition for taking measures: $1=$ length of prozona, $2=$ length of metazona, 3 = greatest width of pronotum, $4=$ greatest width of lamellar expansions. 43: Pseudocreobotra wahlbergii, $q$, head in oblique anterior view. 44-49: Deroplatys desiccata, $\partial^{\top}$, right foreleg. 44: Tibia, posterior view. 45: Femur, posterior view. 46: Tibia, ventral view. 47: Femur, ventral view. 48: Tibia, anterior view. 49: Femur, anterior view. - Scale bar $=2.0 \mathrm{~mm}$.

Abbreviations: avfs $=$ antero-ventral fore femoral spine; avts $=$ antero-ventral fore tibial spine; $\mathbf{c g}=$ claw groove; $\mathbf{c x}=$ coxa $\mathbf{d f f}=$ dorsal femoral lobe; $\mathbf{d s}=$ discoidal spine; $\mathbf{d t l}=$ dorsal tibial lobe; $\mathbf{f b}=$ femoral brush (fore femoral grooming device); $\mathbf{f w}=$ fore wing (tegmen); lpe = lateral pronotal expansion; $\mathbf{l t} \mathbf{l}=$ lateral tergal lobe; $\mathbf{m z}=$ metazona; $\mathbf{p v f s}=$ postero-ventral fore femoral spine; $\mathbf{p v t s}=$ postero-ventral fore tibial spine; $\mathbf{p z}=$ prozona; $\mathbf{t a}=$ tarsus; $\mathbf{t s}=$ tibial spur; $\mathbf{v f l}=$ ventral femoral lobe; $\mathbf{v p r}=$ vertical process $($ fastigium $) ; \mathbf{v t l}=$ ventral tibial lobe. 
The wing venation of Mantodea was discussed, for instance, by Ragge (1955), Smart (1956), Béthoux \& Wieland (2009) and Béthoux et al. (2010). The most recent homologization of mantodean forewing venation was presented by Béthoux \& Wieland (2009), which is followed herein (Fig. 50). The mantodean hind wing nomenclature as well as the wing venation nomenclature for Blattaria follows Ragge (1955) with small alterations (Ragge's R1 and $\mathrm{RS}$ are named RA and RP, his $\mathrm{Cu} 1$ and $\mathrm{Cu} 2$ are named $\mathrm{CuA}$ and $\mathrm{CuP}$ herein, in congruence with Béthoux \& Wieland 2009 among others; Fig. 51). All wing structures are described as if the wing was spread aside in dorsal view of the specimen (Fig. 41), accordingly the costa is the anteriormost

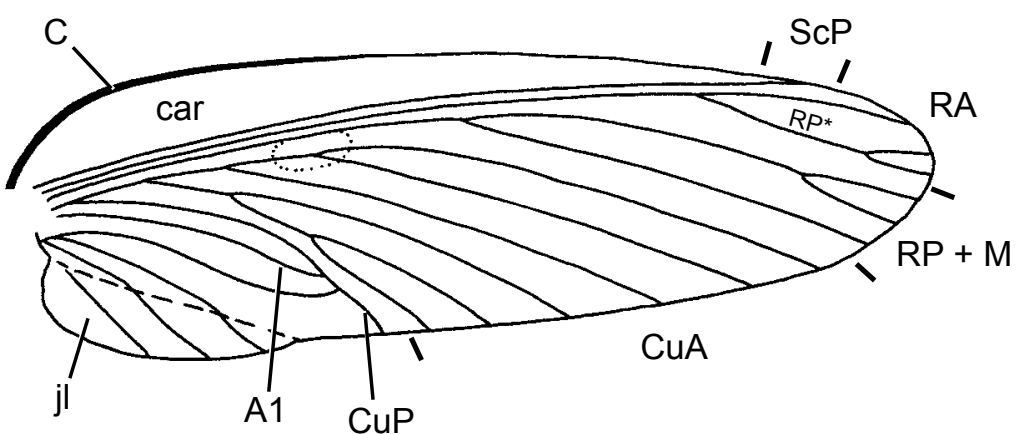

50

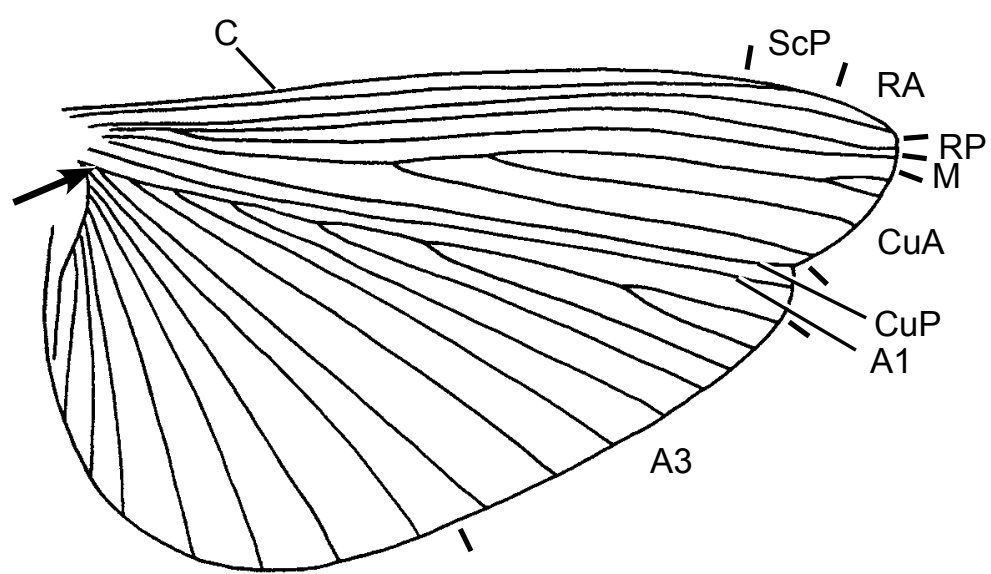

51

Figs. 50-51: Nomenclature of wing venation used in the present study. 50: Sphodromantis viridis, fore wing; redrawn from Ragge (1955: fig. 104); dotted line indicates approximate position of stigma (omitted by Ragge); forewing nomenclature follows Béthoux \& Wieland 2009. 51: Same species, hind wing; redrawn from Ragge (1955: fig. 104); hind wing nomenclature follows Ragge 1955 with slight changes. Arrow indicates proximal branching point of A3.

Abbreviations: A1, A3 = First / third Anal vein; $\mathbf{C}=$ Costa; $\mathbf{c a r}=$ costal area; $\mathbf{C u A}=$ anterior Cubitus; $\mathbf{C u P}=$ posterior Cubitus; $\mathbf{j l}=$ jugal lobe; $\mathbf{M}$ $=$ Media $; \mathbf{R A}=$ anterior Radius; $\mathbf{R P}=$ posterior Radius; $\mathbf{R P} *=$ anterior branch of RP + M that secondarily fused with RA (see Béthoux \& Wieland 2009 for discussion).

vein. Wing characters that did not show any intersexual variation in macropterous species were encoded for both sexes combined. If the numbers of branchings differed among the wings of individuals or among specimens, the highest number of branchings was encoded.

Several species were only available as a single specimen or as a pair. Comparisons of more than one specimen per species were made whenever possible. Several species were represented as fresh material from breeding colonies, therefore they were accessible in greater numbers. In dried material from museum collections it was often impossible to study character complexes that were concealed due to their position (e.g. hind wing features when specimens were dried with folded wings). Museum material was not softened in order to avoid damage to the often fragile and valuable specimens. Such missing data was encoded if literature data was available for the character states of the corresponding structures. 
Numerical characters (e.g. the number of antennomeres or spines) were counted on all specimens available that exhibited undamaged structures (e.g. antennae, cerci or forelegs). Generally, the largest number found was encoded. If the structures were obviously strongly damaged in cases where only one or few specimens were present, the characters were not encoded for the corresponding taxa.

Some specimens lacked body parts (e.g. the raptorial legs of Amphecostephanus rex, the cursorial legs of Bactromantis tolteca and Thesprotia macilenta, and the mesothoracic legs of Rivetina sp.). The characters were encoded if literature data was available.

\subsection{Material}

122 Mantodea species from the author's collection and from the collections of the Naturhistorisches Museum Wien, Vienna (NHMW), the Museum für Naturkunde of the Humboldt-University, Berlin (MNHUB), and several private collections were studied (Tab. 2 in the appendix). Furthermore, Periplaneta americana was studied as outgroup representative. The taxon sample includes all 15 families, 34 out of 48 subfamilies and 32 out of 46 tribus of Mantodea currently recognized in mantodean taxonomy (Ehrmann 2002: 374 ff.). Ethanol-preserved material was preferred in order to avoid incorrect interpretations of structures that may undergo deformation during desiccation. Rare species or such that were not available as ethanol-preserved samples were studied in dry material from museum collections. Whenever possible, both genders were encoded in the matrix. However, as many species are difficult to obtain, sometimes only one pair of a species was available for comparison. In several cases only a single specimen was available. For those taxa only one gender was encoded in the data matrix if no literature data was available. The females of Ambivia undata, Amorphoscelis sp., Amphecostephanus rex, Bactromantis tolteca, Ceratocrania macra, Choeradodis rhombicollis, Ciulfina biseriata, Epaphrodita dentifrons, Heterochaeta bernardii, Oxyothespis senegalensis, Perlamantis allibertii, Pseudogalepsus nigricoxa, Stenopyga ziela, Theopompa sp. $1 \& 2$, Thesprotia macilenta, and Thrinaconyx kirschianus, as well as the males of Acanthops tuberculata, Calamothespis condamini, Chaeteessa valida, Enicophlebia pallida, Entella sp., Eremiaphila sp. 2, Hypsicorypha gracilis, Leptocola phthisica, Ligariella trigonalis, Maculatoscelis sp., Metallyticus fallax, Paralygdamia sp., Paraoxypilus sp. 2, Paraoxypilinae sp., Photina laevis, Pogonogaster tristani, Rivetina sp., Taumantis sigiana, and Toxodera maculata were not available for the study.

Few species were not included in the phylogenetic analysis (e.g. Didymocorypha lanceolata Henry, 1932, Theopompa servillei) because they were obtained late in the study. However, their morphological traits have in some cases been used for discussion.

The original specimens of several wings presented in this study (Figs. 335, 337, 346) are part of the Insect Wing Collection (O. Béthoux, private collection; IWC-OB). They were mounted on slides in Euparal (see Béthoux 2005: 54) and scanned in high resolution. The wings were redrawn in Adobe Photoshop CS3 by using a drawing tablet (see chapter 3.1). 


\section{Results and Discussion}

The species mentioned are usually referred to by their generic names. If two or more species of the same genus were studied, the genus name represents all studied species of this genus. If different species of the same genus exhibit different character states, they are referred to specifically. Authors of the taxa studied can be found in Tab. 2, those of additional taxa discussed are given throughout the text when they are first mentioned.

\subsection{Characters}

\subsubsection{Head and appendages}

Head capsule. The mantodean head capsule is triangular and antero-posteriorly flattened to a varying degree in many taxa. It can be more globular, as, for instance, in Chaeteessa (Figs. 68, 71), Metallyticus (Figs. 69, 72), and Mantoida (Figs. 70, 73). The head capsule may have various kinds of processes and ridges and carries an autapomorphic structure of Mantodea, the so-called scutellum or frontal shield.

1: Caput, vertex, median head process: present [1]; missing [2].

Several Mantodea have a median process on the vertex of the head. Among them are all Empusidae and Sibyllidae, some Hymenopodidae [e.g. Anasigerpes Giglio-Tos, 1915, Ceratomantis (Figs. 58, 59), Hestiasula, Hymenopus (Figs. 75, 76), Junodia Schulthess-Rechberg, 1899, Oxypilus, Phyllocrania (Fig. 63), Pseudocreobotra (Fig. 53)], some Acanthopidae (e.g. Callibia, Stenophylla), some Tarachodidae (e.g. Pyrgomantis; Fig. 431), some Liturgusidae (Majangella Giglio-Tos, 1915, see Bragg 2010: figs. 6, 7), and some Mantidae, e.g. Ceratocrania (Figs. 64, 65) and Phyllothelys Wood-Mason, 1877 (synonym of Kishinouyeum Ouchi, 1938, see Ehrmann \& Roy 2009) (e.g. Wood-Mason 1889: figs. 22, 23; Chopard 1949a: 402; Beier 1968a: 5, 12, 18; Lizhong 1984: fig. 1; Roy 1999: 24; Xu \& Mao 2004: fig. 2; Zhou \& Zhou 2004: fig. 1; Ehrmann \& Roy 2009: figs. 1-3; Greven \& Brenner 2009: fig. 1; Stiewe \& Roy 2010: figs. 2 , 4-6). Such a process is lacking in the greater part of the mantodean species. The head processes have been conjectured to represent convergent developments of the different taxa displaying them (Roy 1996: 132, 2004a: 14). They have been shown to contain musculature and tracheae [Wipfler \& Hörnschemeyer 2008 (Hymenopus); Wieland pers. obs. in Phyllocrania].

This structure has been termed "fastigium" by some authors (e.g. Ehrmann 2002: 13), in congruence with the fastigium in Orthoptera, but I will refer to it as vertical process (in the sense of originating from the dorsal part of the vertex) in 
this study because a second type of head process can be found in Mantodea (see character 3 ) that should not be confused with this type.

2: Caput, vertex, median head process, shape: symmetrical, strong and massive (1); symmetrical and massive at the base, distally becoming asymmetrical, flat and lobe-like (2); symmetrical, in the shape of a small spur behind the ocelli (3).

In some taxa the head process distally has a lobe-like, asymmetrical shape (its base is usually massive and symmetrical). This probably helps to increase the mimetic effect exhibited by the entire body ("dead-leaf-appearance"). This effect is very obvious, for instance, in Phyllocrania paradoxa (Hymenopodidae; see Kaltenbach 1998: fig. 105; Ehrmann 2002: 407; Figs. 41, 63). The head process is distally also slightly asymmetrical in Stenophylla (Acanthopidae) (e.g. Terra 1995: figs. 28, 29; Lombardo 2000a: figs. 1-3; Salazar 2007: fig. 2), Ceratocrania (Figs. 64, 65) and some Phyllothelys species (both Mantidae: Phyllotheliinae; Ehrmann \& Roy 2009: figs. 1-3). Interestingly, the process is symmetrical and not lobe-like in the first instar nymph of Phyllocrania (Gillon \& Roy 1968: fig. 47; Fig. 408). This indicates a distinct morphological change of this structure during postembryonic development (see also chapter 4.6.7). The ontogenetic development from symmetrical to asymmetrical processes may reflect the phylogenetic history of asymmetrical processes.

In other taxa it is symmetrical and massive throughout its entire length. This is the case in all Empusidae (e.g. Kaltenbach 1976: fig. 467, 1998: figs. 119-121; Ehrmann 2002: figs. 7, 12; Roy 2004a: figs. 19, 20; Figs. 60, 61, 66, 67) and Sibyllidae (e.g. Roy 1996: figs. 2, 7, 12, 17, 20, 23, 26, 29, 33, 36, 39, 42, 45, 48; Kaltenbach 1998: fig. 118). It is also present in some Acanthopidae (e.g. Callibia in Terra 1995: figs. 24, 27), some Hymenopodidae, e.g. Anasigerpes, Amphecostephanus (Rehn 1912: 120; Roy 1963b: fig. 23a; Ehrmann 2002: fig. 6; Fig. 62), Junodia (Stiewe \& Roy 2010: figs. 2, 4-6) and Ceratomantis (Roy \& Svenson 2007: figs. 1-13; see also discussion by Roy 2004a: 14; Figs. 58, 59), some Phyllothelys species (Ehrmann \& Roy 2009: fig. 1 and 3a), and some Tarachodidae, e.g. Pyrgomantis (Wood-Mason 1889: figs. 22, 23; Beier 1957a: figs. 1, 4, 6; Gillon \& Roy 1968: fig. 17; Kaltenbach 1996: figs. 45-47; Ehrmann 2002: figs. 4, 5; Fig. 431).

The vertical process may be long or just a small spur as exhibited, for instance, by Hymenopus (Figs. 75-76), Creobroter, and Pseudocreobotra (Fig. 53). This also seems to be the case in the Bornean liturgusid Majangella (Bragg 2010: figs. 6, 7).

In some species, for instance in Pyrgomantis (Fig. 431) and Amphecostephanus (Fig. 62), the base of the process takes up the entire width of the vertex. 
Vertical processes show a disjunctive distribution throughout the traditional mantodean classification. Therefore, it is generally assumed that they have evolved convergently several times. Neither "Blattaria" nor Isoptera have comparable structures.

Edmunds (1972: 17) stated that predators may be able to recognize cryptic prey by conspicuous structures, for instance the legs (also stated by Robinson 1969: 297), and that, consequently, any modification breaking up the outline of a cryptic insect may be advantageous. He pointed out that the "praying" outline is concealed in several ways by morphological modifications (Edmunds 1972: 19). One of them is the modification of the head-prothorax-forelegs complex. Edmunds (1972) hypothesized that the elongated vertical process in combination with the "hypognathous" head position and the close fitting of the forelegs against the head breaks up the outline of the praying posture of several species [he referred to Oxypilus, Anasigerpes, Pseudoharpax (albeit missing the process), Hemiempusa and Idolomorpha]. This is also very obvious in Pyrgomantis (e.g. Gillon 1983: fig. 11.5).

3: Caput, vertex, area of lateral ocelli, processes or spines: present [1]; missing [2].

Some Mantodea have processes or spines arising from the region of the lateral ocelli, that morphologically is part of the vertex (Strenger 1942: 56; Beier 1964a: 865, 1968a: 18; Roy 1999: 24) but is located anterior to the area from which the aforementioned vertical processes usually arise. They are present, for instance, in some Vatinae [Zoolea (Fig. 55), Vates (Fig. 54), Pseudovates Saussure, 1869; Rehn 1911: fig. 3; Hebard 1935: pl. 4, fig. 5; Beier 1964a: 865; Terra 1995: figs. 101, 105; Rivera 2001: fig. 1a, b; Roy \& Ehrmann 2009: figs. 1, 2, 9, 11, 12, 20], some Hymenopodidae [e.g. Theopropus, Ceratomantis (Figs. 58, 59), Pseudoharpax], some Paraoxypilinae (Gyromantis; Fig. 78) and Haaniinae [e.g. Haania (Fig. 52); Beier 1964a: 865; Anisyutkin 2005: figs. 1, 2; Anisyutkin \& Gorochov 2005: figs. 1, 2]. This type of process originates from a different part of the vertex than the one being found in Empusidae, some Hymenopodidae and others (see character 2). Therefore, although looking very similar at first glance, for instance when comparing Empusidae and Zoolea, these two types are clearly not homologous. This is also supported by the fact that both types of processes may be present at the same time, for instance in Oxypilinae (Figs. 58, 59), and Theopropus.

Processes arising from the lateral ocelli generally consist of two spines or lobes. They can be short and spiniform, as for instance in Gyromantis (Fig. 78) and Haania (Fig. 52). They can be longer and more sturdy, as in Vates (Fig. 54), or may form strongly enlarged lobes that medially closely adjoin, thus gaining 


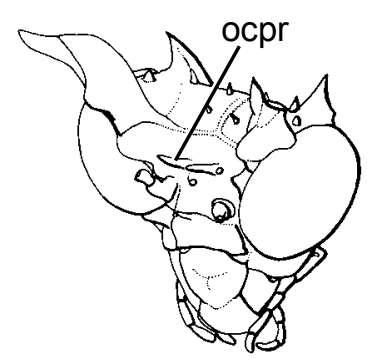

52

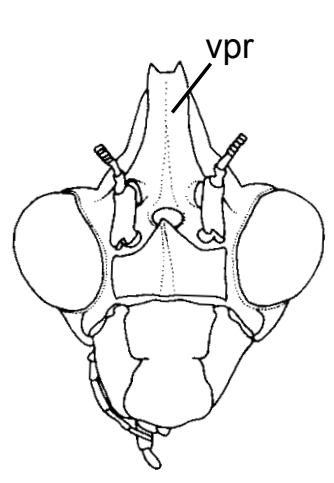

56

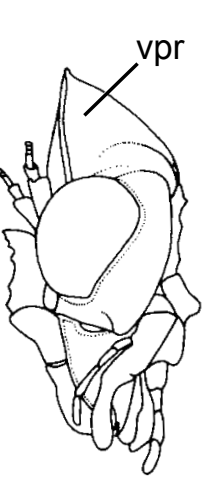

57

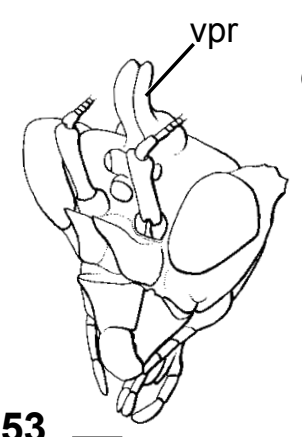

53

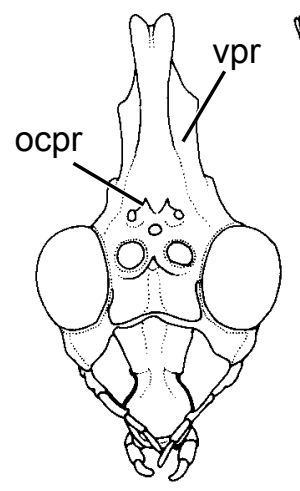

58

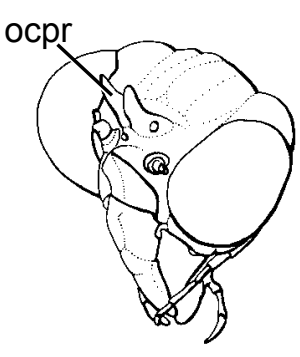

54

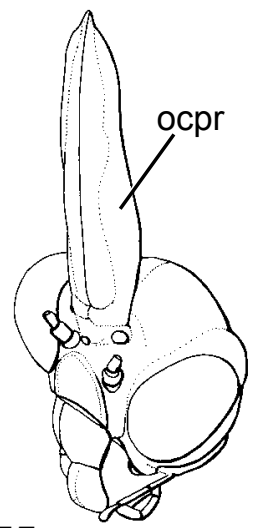

55
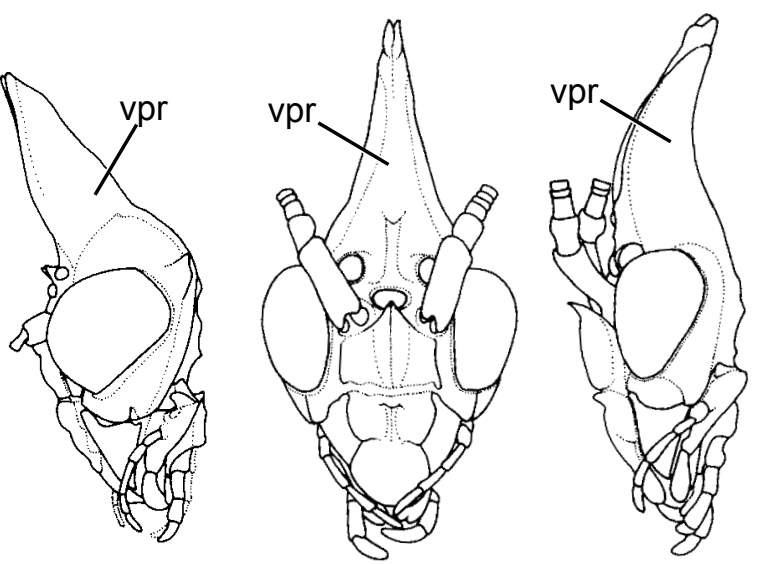

61
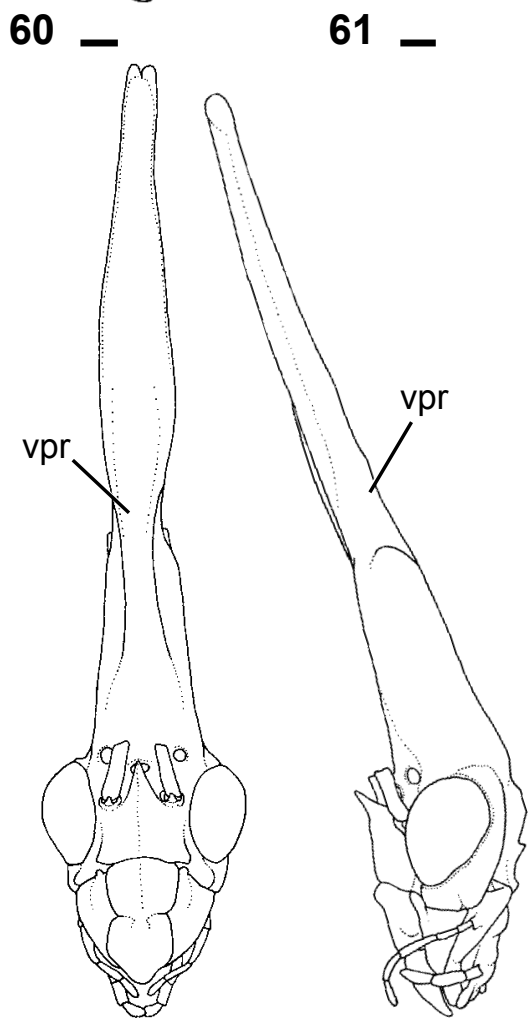

62

63

64

65

66

67

Figs. 52-67: Head processes. 52-55: Oblique anterior views; 56, 58, 60, 62-64, 66: Anterior views; 57, 59, 61, 65, 67: Lateral views (left). 52: Haania lobiceps, ․ 53: Pseudocreobotra wahlbergii, ㅇ. 54: Vates weyrauchi, $ᄋ$.

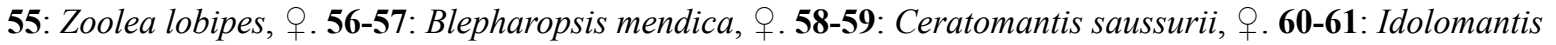

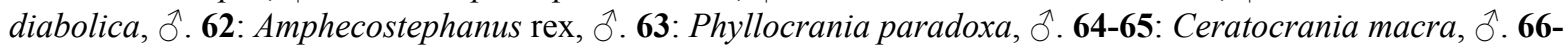
67: Hypsicorypha gracilis,, . - Scale bar $=1.0 \mathrm{~mm}$.

Abbreviations: $\mathbf{v p r}=$ vertical process (fastigium); $\mathbf{o c p r}=$ process arising from ocellar hill (posterior ocelli). 
the appearance of a single complete head process at a superficial glance. The latter state has been found in Zoolea (Fig. 55) in this study (see Roy \& Ehrmann 2009: fig. 2), but is also present, for instance, in Pseudovates (Hebard 1935: pl. 4, fig. 5) and Phyllovates Kirby, 1904 (Orofino et al. 2006: figs. 1, 2).

4: Ocelli, number: 3 [1]; 2, the anterior one reduced [2].

The insect ground plan probably includes the presence of three pairs of ocelli (Kristensen 1998: 283; Willmann 2003a: 10), a situation still present in some Collembola (Dunger 2003: 76). Most extant insect taxa have two or three ocelli (Boudreaux 1979: 137). Three ocelli (an unpaired anterior one and two lateral posterior ones) can plesiomorphically be found in all Mantodea (Boudreaux 1979: 221; Roy 1999: 24; Ehrmann 2002: 12; Klass \& Ehrmann 2003: 183). The unpaired anterior ocellus is missing in Blattodea (Bohn 2003: 197; Bohn \& Klass 2003: 181; Fig. 81).

5: Caput, frons, scutellum (bordered by system of sulci): present [1]; missing [2].

The scutellum is an area of the frons of Mantodea that is bordered by a system of external sutures and internal sulci. It is no true sclerite, unlike proposed by some authors (e.g. Roy 1999: 25), but the name is well-established in the mantodean literature and will therefore be maintained herein for terminological convenience.

The organization of the anterior head sulci of Mantodea was discussed by Klass \& Eulitz (2007: figs. 10-12). The internal dorsal border of the scutellum is dorso-medially formed by the interantennal sulcus (ias in Klass \& Eulitz 2007: figs 10-12) and dorso-laterally by the circumantennal sulci (cas in Klass \& Eulitz 2007: figs 10-12). Ventrally, it is bordered by the epistomal sulcus (ess in Klass \& Eulitz 2007: figs 10-12; externally visible as the epistomal suture, separating the clypeus from the frons). The lateral borders of the scutellum are formed by the subantennal sulci (sas in Klass \& Eulitz 2007: figs 10-12) which partially merge with the circumocular sulci (cos in Klass \& Eulitz 2007: figs 10-12).

The internal sulci probably function as stabilizing elements for the head capsule (Levereault 1936: 209), most likely against the forces of the strong mandibles. The interantennal sulcus bears the insertions of several labral muscles (Levereault 1938; Klass \& Eulitz 2007).

The interantennal sulcus is a putative autapomorphy of Mantodea. It is lacking in Blattodea (Fig. 81) or other Polyneoptera, while a similar structure is present in Odonata (the "antefrons" which spatially corresponds to the interantennal sulcus; see Klass \& Eulitz 2007: 225). However, as Klass \& Eulitz (2007: 226) stated, there is a structure in the zygentoman Thermobia domestica (Packard, 1873) 

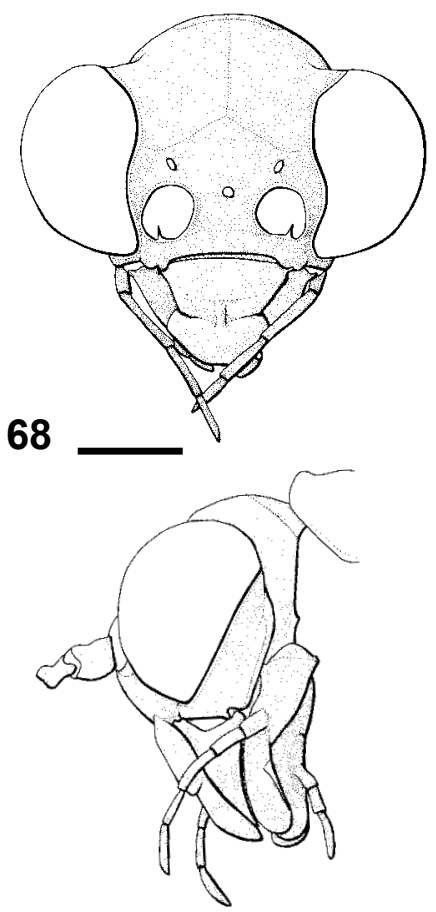

71

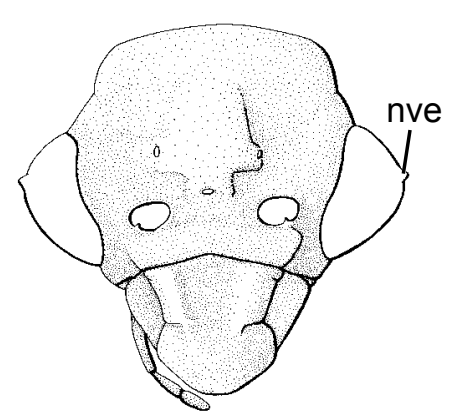

74

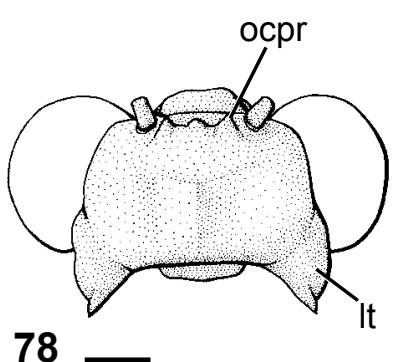

78

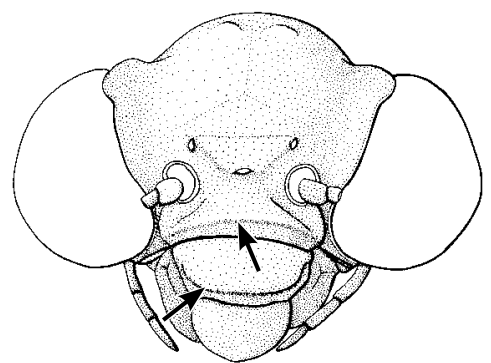

69

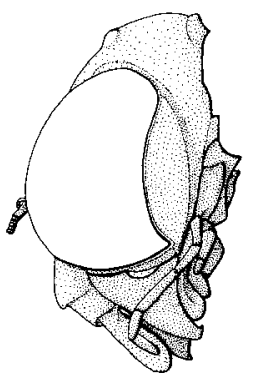

72
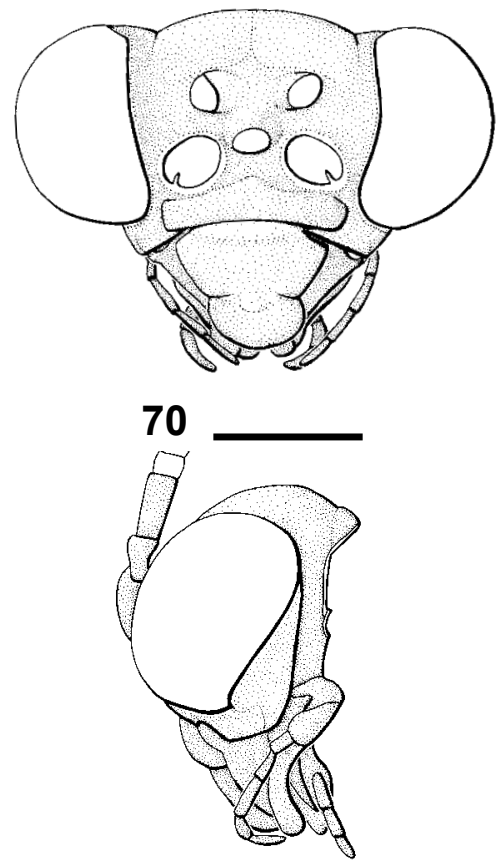

73

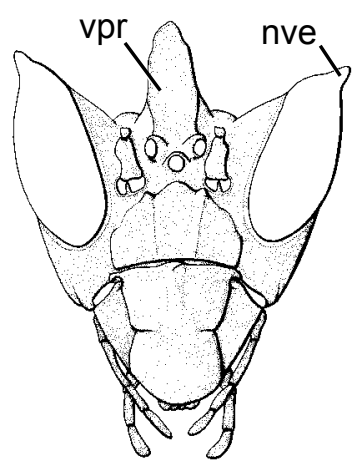

75

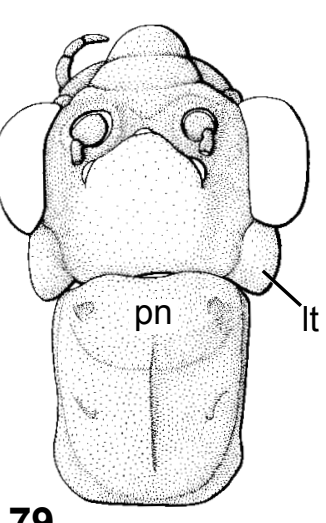

79

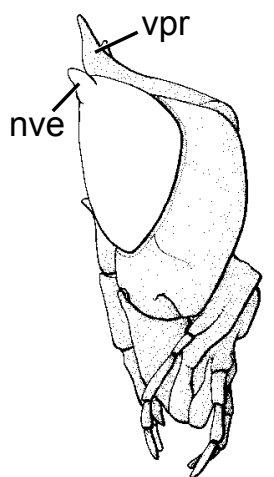

76

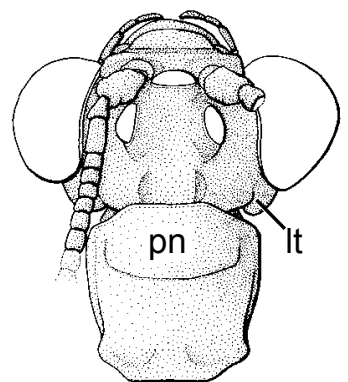

80

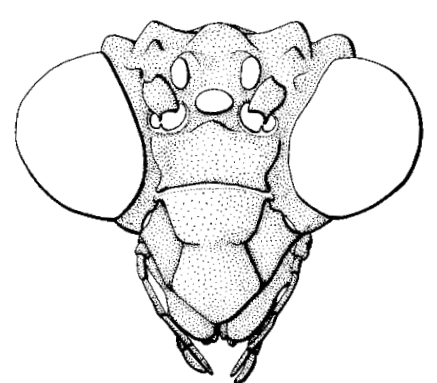

77

Figs. 68-81: Heads. 68: Chaeteessa cf. valida, sex unknown, anterior view. 69: Metallyticus splendidus, ㅇ, anterior view; arrows indicate transverse folding of scutellum and clypeus (,step-like structure“). 70: Mantoida maya, ô, anterior view. 71: Chaeteessa cf. valida, sex unknown, lateral view. 72: Metallyticus splendidus, ㅇ, lateral view. 73: Mantoida maya, ô, lateral view. 74: Compsothespis anomala,, , anterior view. 75: Hymenopus coronatus,, , anterior view. 76: Same specimen, lateral view. 77: Popa spurca crassa, ô, anterior view. 78: Gyromantis sp.,, , dorsal view; note the pointed lateral tubercles. 79: Paramorphoscelis gondokorensis, $q$, head and pronotum, dorsal view. 80: Perlamantis allibertii, $\hat{\jmath}$, head and pronotum, dorsal view. 81: Periplaneta americana, $\hat{0}$, anterior view. - Scale bar $=1.0 \mathrm{~mm}$.

Abbreviations: $\mathbf{l t}=$ lateral tubercle; $\mathbf{n v e}=$ non-visual elongation; $\mathbf{o c p r}=$ process arising from ocellar hill (posterior ocelli); $\mathbf{p n}=$ pronotum; $\mathbf{v p r}=$ vertical process (fastigium). 
("epistomal sulcus") that rather resembles the interantennal sulcus of Mantodea than the actual epistomal sulcus. Therefore, the assignment of the interantennal sulcus as an autapomorphic character for Mantodea can only be preliminary until a thorough investigation of the corresponding structures in several Zygentoma and "lower" Pterygota has been carried out (Klass \& Eulitz 2007: 226).

6: Caput, scutellum, shape: distinctly shorter than wide (transverse) [1]; nearly as wide as long (almost square) [2]; distinctly longer than wide [3].

The length of the scutellum is defined by the length of its lateral borders, i.e. the subantennal sulci (Klass \& Eulitz 2007: sas in figs. 10-12; see character 5). The scutellum may be transverse (wider than long), almost square, or vertical (longer than wide). Many Mantodea, for instance Deroplatys desiccata, have an antero-posteriorly flattened head capsule and a rather long scutellum, whereas several taxa, among them members of the basal dichotomies, such as Chaeteessa, Mantoida and Metallyticus, have a more globular head shape (Figs. 68-73) and a strongly transverse (= short) scutellum.

7: Caput, scutellum, shape of dorsal edge: straight or slightly rounded [1]; strongly rounded or angled [2]; forming a prominent, sometimes sturdy medial spine [3].

The dorsal edge of the scutellum bears structures that are characteristic for some taxa. It may be nearly straight or slightly angled or rounded. In Empusidae the scutellum carries a more or less strongly developed median ridge (see character 8) which, together with the dorsal edge, forms a tapering spine that prominently protrudes the scutellum anteriorly (Roy 1999: 25; Roy 2004a: 1; see Figs. 56, $57,60,61,66,67)$. This is also present in some Hymenopodidae in a very similar way, for instance in Anasigerpes (Ehrmann 2002: fig. 6) and Ceratomantis (Roy 2004a: 14; see Fig. 58, 59). No such structure can be found in the taxa displaying many plesiomorphic traits, therefore it is likely that a straight dorsal edge represents the plesiomorphic characters and an angled, rounded or pointed shape is apomorphic.

8: Caput, ridge(s) on scutellum: present [1]; missing [2].

The scutellum may carry one, two or three ridges that are sometimes very prominent (see character 7). Such ridges are present in several Acanthopidae (Acontista, Callibia, Raptrix, Tithrone), Ceratocrania (Figs. 64, 65), Empusidae (Figs. 56, 57, 60, 61, 66, 67), Enicophlebia, in several Hymenopodidae [Ceratomantis (Fig. 58, 59), Creobroter, Galinthias, Hymenopus (Fig. 75, 76), Oxypilus, Pseudocreo- 
botra (Fig. 53), Pseudoharpax, Theopropus], and in Toxodera. The ridges may be large and form bizarre crests as, for example, in Blepharopsis (Fig. 56, 57).

9: Caput, scutellum, organization of ridges: Single median ridge [1]; two parallel ridges that may be pointed [2]; three ridges [3].

In Empusidae, only a single median, more or less crest-like ridge is present (Figs. 56, 57, 60, 61, 66, 67). Some Hymenopodidae [e.g. Hymenopus (Fig. 75), Theopropus, Pseudocreobotra (Fig. 53)] and Toxodera exhibit two more or less parallel ridges on the scutellum which may anteriorly end in pointed spines. There may be up to three ridges, for instance in Oxypilus.

A scutellum is lacking in Blattodea (Fig. 81). Ridges on the scutellum are probably apomorphic for taxa within Mantodea.

10: Caput, scutellum, strong transverse folding (nearly 90 degrees), distance between clypeus and fold equals distance between fold and interantennal sulcus: present [1]; missing [2].

The scutellum of Metallyticus is horizontally folded to an angle of nearly 90 degrees in lateral view (Wieland 2008a: 158 and figs. 2, 5; Fig. 82 and arrow in Fig. $69)$. The distance between fold and interantennal sulcus nearly equals the distance between the fold and the clypeus. Some other taxa also exhibit a transverse folding of the scutellum (e.g. Dactylopteryx, pers. obs.), but the angle is distinctly wider than 90 degrees and the distance between the fold and the interantennal sulcus is much shorter than that between the fold and the clypeus. This character is likely autapomorphic for Metallyticus.

11: Caput, clypeus, ridge: present [1]; missing [2].

The clypeus may carry a more or less prominent vertical median ridge or be smooth. A ridge on the clypeus is missing in Blattodea and several Mantodea including Chaeteessa, Mantoida, Metallyticus, Amorphoscelidae and Eremiaphilidae, among others.

12: Caput, clypeus, transverse folding: present [1]; missing [2].

The clypeus may be transversely folded. In some taxa the folding angle may reach nearly 90 degrees in lateral view. This was found, for instance, in Metallyticus (Fig. 69, 72, 82). Together with the transverse folding of the scutellum (character 10) this results in a step-like structure in this genus (Wieland 2008a: 158 and figs. 2, 5; Fig. 82). 
13: Caput, lateral tubercles: present [1]; missing [2].

The head capsule may carry bulging and sometimes pointed elevations between the compound eye and the corresponding parietal furrow on either side. These elevations have been named "Nebenaugenhöcker" (Beier 1968a: 18; Ehrmann 2002: 13) or "lateral tubercles" (Roy 1999: 21). They are rather prominent in species that carry the head in a more or less prognathous position, as, for instance, Amorphoscelinae, Paraoxypilinae (Fig. 78), Perlamantinae (Figs. 79, 80), Hoplocorypha, and Orthoderella, among others. The lateral tubercles may be blunt [some Amorphoscelinae (e.g. Kaltenbach 1998: fig. 7; Roy 1999: fig. 2.10.A), Perlamantinae (e.g. Werner 1907: pl. 2: fig. 1a; Figs. 79, 80), Hoplocorypha (e.g. Rehn 1912: fig. 5), Orthoderella (e.g. Chopard 1916: fig. 1)] or pointed [e.g. some Amorphoscelis (see Wood-Mason 1889: fig. 2; Roy \& Stiewe 2009: fig. 12), Cliomantis, Gyromantis (Fig. 78), and Metoxypilus Giglio-Tos,

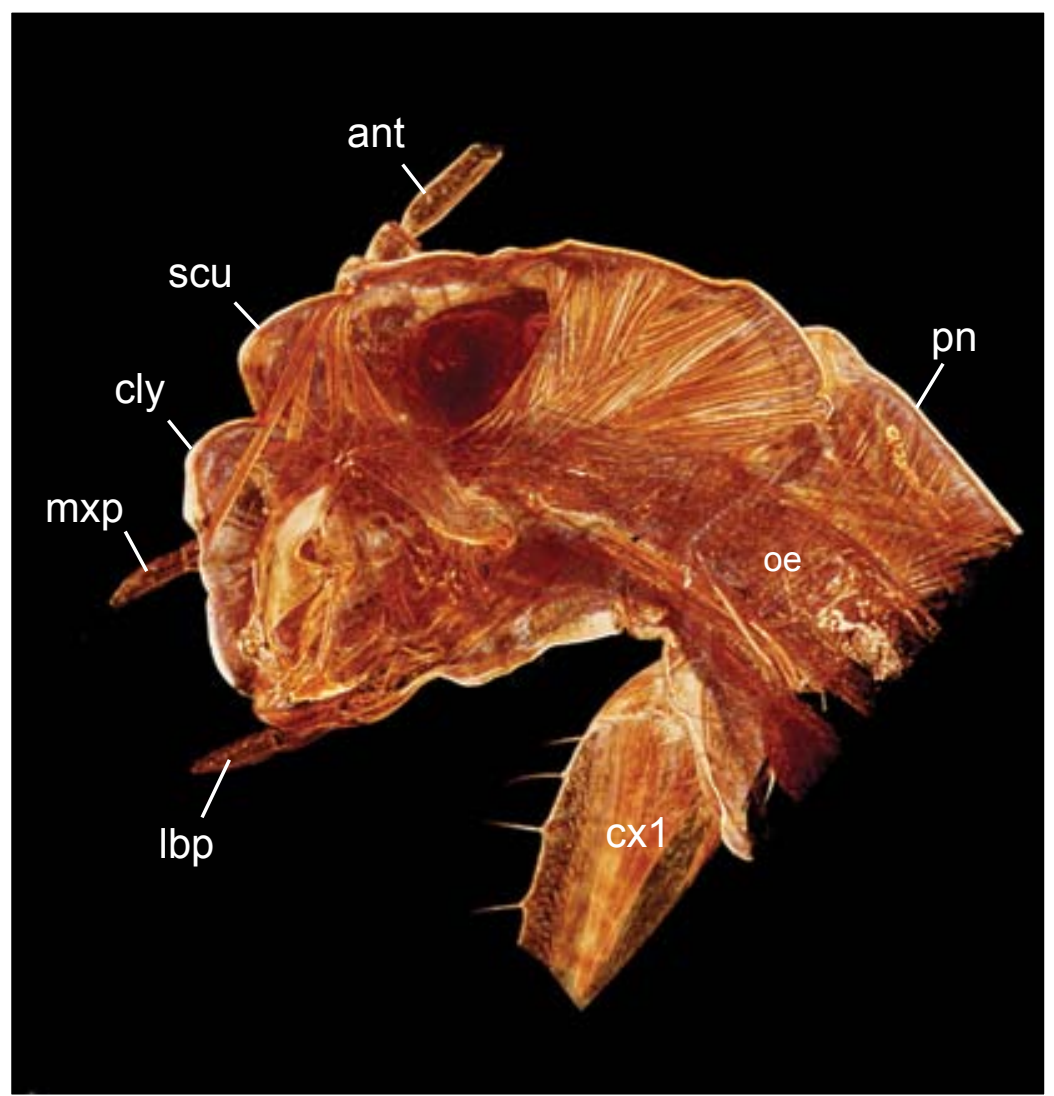

Fig. 82: Metallyticus splendidus, nymph. Median section through head ( $\mu$-CT-data; see chapter 3.1). Note the medially folded scutellum and clypeus ("step-like structure").

Abbreviations: ant = antenna; $\mathbf{c l y}=$ clypeus; $\mathbf{c x 1}=$ anterior $\operatorname{coxa} ; \mathbf{l b p}=$ labial palp; $\mathbf{m x p}=$ maxillary palp; $\mathbf{o e}=$ oesophagus; $\mathbf{p n}=$ pronotum; $\mathbf{s c u}$ $=$ scutellum. $\leftarrow$ anterior.

1913 (see Giglio-Tos

1913: figs. 3a, d, e, 12, 12a; Sjöstedt 1918: pl. 1: fig. 3c, pl. 2: figs. 1b, 2b)].

The function of the lateral tubercles has been discussed by Edmunds (1972, 1976). In dorsal view, they enhance the concealment of the neck contour thus making it more difficult for predators to recognize the mantid as such (and therefore as potential prey) by its characteristic head-prothorax outline (Edmunds 1972: 20, 1976: 5 and fig. 1; Edmunds \& Brunner 1999: 188 and fig. 13.6; see Chopard 1940a: fig. 4 and Roy 1969: fig. 8 for Calamothespis). 
Compound eyes. The compound eyes of Mantodea consist of up to 10,000 or more ommatidia (Roy 1999: 24; Ehrmann 2002: 24; Kral \& Prete 2004: 77). They often strongly protrude the head laterally (exophthalmic), which leads to an excellent stereoscopic vision (see Kral 1999 for an overview of this topic). The shape of the eyes differs considerably among the taxa.

14. Compound eyes, shape: cone-shaped, looking as if positioned on a socket in lateral view [1]; oval [2]; nearly globular [3]; kidney-shaped, partly enclosing the antennal bases [4].

Shape and position of the eyes vary considerably throughout Mantodea (Roy 1999: 24; Ehrmann 2002: 12 and figs. 2-9; Klass \& Ehrmann 2003: 183). Some lineages have globular eyes while many others may have elongated oval eyes. Some species exhibit cone-shaped eyes that are seemingly positioned on "sockets", visible in lateral view in many Hymenopodinae (e.g. Handlirsch 1930a: 804; Kaltenbach 1998: figs. 106, 112-114; Klass \& Ehrmann 2003: 183; Fig. 76).

The compound eyes of Blattaria are mostly oval to kidney-shaped (Fig. 81) and sometimes globular (e.g. in Cryptocercus, albeit probably being derived due to the wood-dwelling lifestyle).

15. Compound eyes, shape with regard to head capsule in frontal view: exophthalmic, laterally strongly bulging [1]; laterally not bulging, fitting in the head outline [2]; protruding the head dorsally [3].

In many species the eyes may strongly protrude the head capsule laterally (state 1). This is very obvious, for instance, in many Amorphoscelinae (e.g. Wood-Mason 1889: fig. 2; Roy \& Stiewe 2009: figs. 2-7, 9-14, 15-16) and $\mathrm{Me}$ tallyticus (e.g. Wieland 2008a: 158 and figs. 1-4, 7, 19-22; Fig. 69), but also in many other species.

The eye outline may laterally fit into the head contour without strongly protruding (state 2). This is the case, for instance, in Phyllocrania (Fig. 63), Pyrgomantis (Fig. 431), Acanthops, Ceratomantis (Fig. 58), Deroplatys, Tarachodula and many other genera.

In other taxa [most Hymenopodidae in this study, e.g. Hymenopus (Figs. 75, 76), but also Episcopomantis and Schizocephala], the eyes are elongated and protrude the vertex dorsally (state 3).

16. Compound eyes, non-visual elongation (ocular spine): present [1]; missing [2].

A pointed elongation on each compound eye is exhibited by several species [Acanthops (Fig. 85), Calamothespis, Compsothespis (Fig. 74), Galinthias, Heterochaeta, Hymenopus (Figs. 75, 76), Oxyothespis, Pseudoharpax (Figs. 83, 84), and Toxodera (86) in this study]. 
This character shows intrageneric variation. For example, Heterochaeta bernardii has such an elongation [also found in H. tenuipes (Westwood, 1841), see Westwood 1845a)], whereas it is missing in H. strachani (see also Roy 1976). It is present (albeit very small) in Compsothespis anomala (see Fig. 74 and Saussure 1872: 64) and C. zavattarii La Greca, 1939 (b: 257 and fig. 1.1), whereas it is missing in C. abyssinica Beier, 1961 (p. 3), C. australiensis Wood-Mason, 1889 (p. 7 and fig. 4), C. brevipennis Lombardo, 1988 (p. 103), C. cinnabarina Beier, 1955 (b: 258), C. falcifera Rehn, 1901 (p. 280), and C. occidentalis Sjöstedt, 1930 (p. 17) [not mentioned in the descriptions of C. marginipennis by Werner (1916) and C. natalica by Westwood (1889); remaining descriptions not researched].

Differences are also known to occur among species of Ameles (e.g. Kaltenbach 1964). The elongations may become gradually reduced during postembryonic development, as for instance in Ameles spallanzania (Rossi, 1792) (Kaltenbach 1963: 532 and fig. 5). They can be small and knob-like as in Compsothespis (Fig. 74), or very long and pointed [e.g. Toxodera (Roy 2009a: fig. 2a, b; Fig. 86) or Episcopomantis (Wood-Mason 1889: fig. 29; vs. Kaltenbach 1998: fig. 22, who erroneously drew the elongations as part of the vertex). They are often only slightly elongated, as in Oxyelaea stephaniae Lombardo, 1988 (fig. 1). The elongations may have different positions on the eye (Kaltenbach 1996: figs. 43-46; Kaltenbach 1998: figs. 22, 41, 45, 106, 112 114, Roy 1999: 24; Ehrmann 2002: fig. 2; Ippolito \& Lombardo 2004: fig. 1). Besides interspecific variability, there may also be a considerable intraspecific variability, as was shown for Toxodera maculata (Roy 2009a: figs 19a-e), Heterochaeta occidentalis Beier, 1963 (Roy 1976: fig. 18) and H. bernardii (Roy 1976: fig. 23).

The elongations have been stated to be non-visual (i.e. they do not show any facets of ommatidia; Westwood 1845a: 162; Roy 1999: 24). To my knowledge their structure and function has not been studied in further detail yet. SEM images of the eyes of Pseudoharpax sp. (Figs. 83, 84) confirm that there are no ommatidia present on the spines in this species (also distinctly drawn in "Harpax variegatus" in Sharp 1895: fig. 136). Instead, the elongations apparently originate from the sclerotizations between the ommatidia, which may bear setae and other structures in various insect taxa. In Zygentoma and Strepsiptera, for instance, there are large areas between the single ommatidia, which are formed by the cuticle of the head capsule. In case of the Strepsiptera these areas of cuticle bear structures such as microtricha [e.g. Kathirithamby 1989: 51 and figs. 10, 13-15, 1991: 685 and figs. 36.1B and 36.9A-C (for detail of fig. 36.9B see also dust jacket of the book); Grimaldi \& Engel 2005: fig. 10.79; Bravo et al. 2009: figs. 2-4]. 

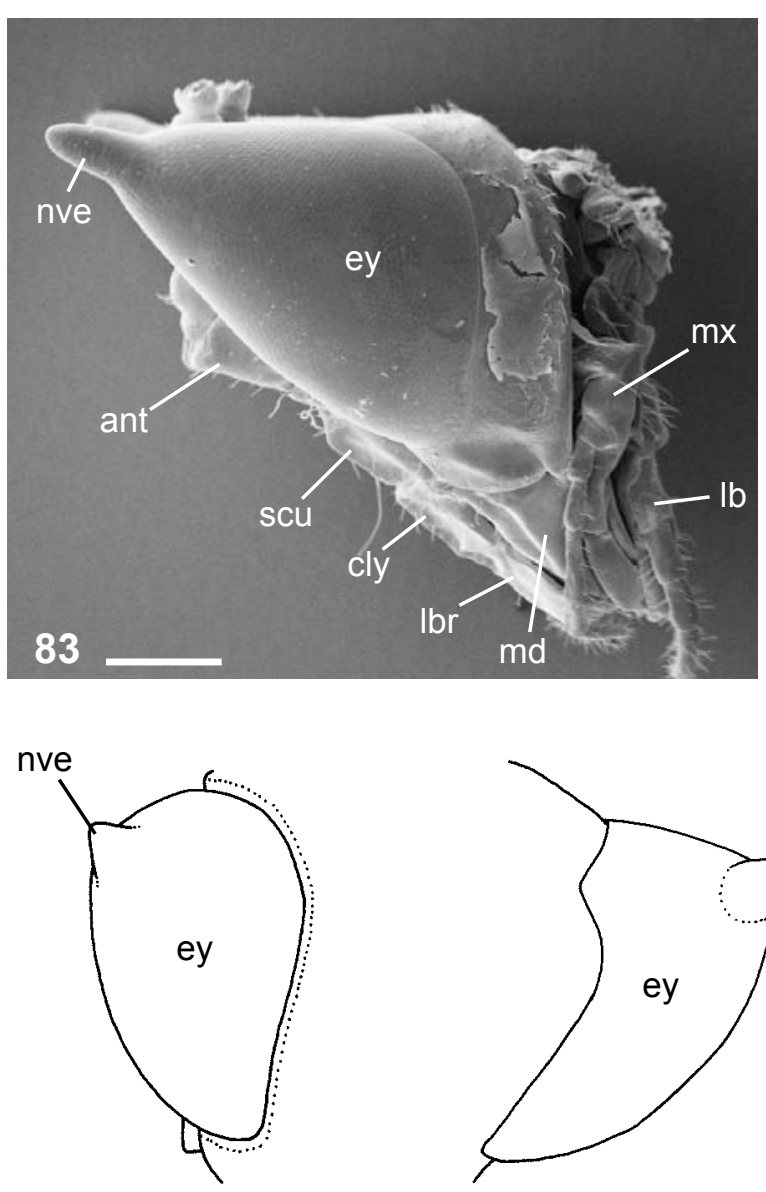

85 86

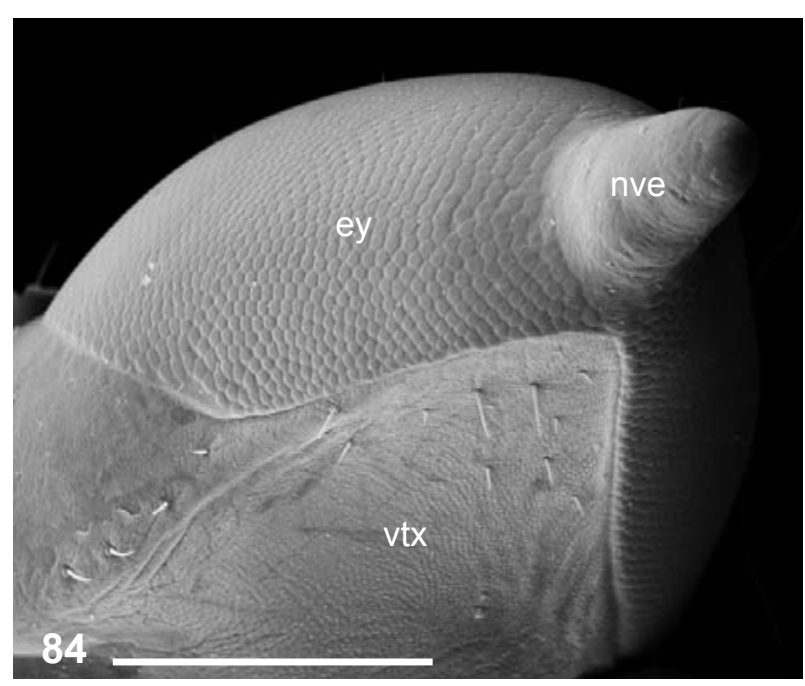

Figs. 83-86: Non-visual elongation of the compound eyes. 83, 84: Pseudoharpax sp., $\widehat{\text {, }}$, head. 83: Lateral view (left). 84: Left compound eye, oblique posterior view; note the non-visual elongation of the eye and its lack of ommatidia. 85: Acanthops tuberculata, ㅇ, detail of left eye in lateral view. 86: Toxodera

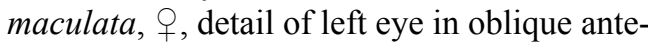
rior view. - Scale bar $=0.5 \mathrm{~mm}$.

Abbreviations: $\mathbf{a n t}=$ antenna; $\mathbf{c l y}=$ clypeus; $\mathbf{e y}=$ compound eye; $\mathbf{l b}=$ labium; lbr = labrum; $\mathbf{m d}=$ mandible $; \mathbf{m x}=$ maxilla; $\mathbf{n v e}=$ non-visual elongation; $\mathbf{s c u}=$ scutellum; $\mathbf{v t x}=$ vertex .

Non-visual elongations possibly play a role in the cryptic overall appearance of at least some species (e.g. Toxodera, Heterochaeta). Edmunds (1972: 21) mentioned a close resemblance of the widely spaced position of the eyes in $\mathrm{He}$ terochaeta strachani (although without spines) to a pair of buds at the end of a twig. Spines on the eyes certainly further enhance such an effect.

I was unable to identify any other insect group in which there are similar spines on the compound eyes, whereas in Mantodea the character is found in many species and has certainly evolved independently several times.

Antennae. The antennae are usually filiform in Mantodea. They are long in many species, but slightly longer than the entire body in only a few. Some taxa exhibit asymmetrical antennomeres carrying one or two, sometimes strongly elongated and/or flattened, processes.

17. Antennae, length with respect to body length (excluding wings): longer [1]; as long as or shorter [2].

Unlike stated by Beier (1968a: 18) and Klass \& Ehrmann (2003: 183), there are several Mantodea species with antennae being longer than the entire body. In male Mantoida maya the antennae are distinctly longer (pers. obs.; also mentioned 
by Blatchley 1920: 128), resembling those of Mantoida brunneriana (Saussure, 1871) (Chopard 1949a: fig. 84; Roy 1999: fig. 2.5a) and Mantoida sp. (Crane 1952: fig. 1). In Chaeteessa, too, the antennae exceed the body length (Burmeister 1838: 527; Jantsch 1999: encoded as char. 7 in tab. 3; Agudelo Rondón 2004: 51; Salazar-E. 2005: 269; pers. obs. in the well-preserved Chaeteessa valida female from the NHMW collection). Of the further species studied in this analysis only male Elaea marchali exhibited such long antennae, but there are other species mentioned in the literature exhibiting this character state, e.g. male Bantia simoni (Chopard, 1916) (described as Miopteryx simoni in Chopard 1916: 169 and fig. 5) and male Amorphoscelis pulchella Giglio-Tos, 1913 (see Roy 1962: Fig. 1).

There are also many cockroach species with antennae exceeding body length, among them many Blattidae, e.g. Periplaneta americana (Handlirsch 1930b: fig. 856), Periplaneta brunnea Burmeister, 1838 (Roth 1991: fig. 19.8 B), Temnelytra truncata (Brunner v. Wattenwyl, 1865) (Roth 1991: fig. 19.8 E), and Methana curvigera (Walker, 1868) (Roth 1991: fig. $19.8 \mathrm{G}$ ). Therefore it is possible that this character represents the plesiomorphic condition in Mantodea.

18. Antennae of male, largest number of antennomeres: 49 or less [1]; 50-69 [2]; 70-89 [3]; 90109 [4]; 110-129 [5]; 130 or more [6].

The number of antennomeres has been used before for phylogenetic analyses of Dictyoptera (Thorne \& Carpenter 1992; Deitz et al. 2003; Klass \& Meier 2006). It varies among the mantodean taxa (Handlirsch 1930a: 804: “[...], doch ist die Zahl der Glieder nicht fixiert”; Beier 1964a: 865: "Die Zahl der Geißelglieder scheint niemals streng fixiert zu sein.") and may show intersexual and intraspecific variation (Slifer 1968: 106: "The number of subsegments varies in different individuals"). According to the literature, mantodean antennal flagella may count as many as 134 antennomeres (Slifer 1968), whereas Blattaria reach numbers of 178 antennomeres (Guthrie \& Tindall 1968). The largest numbers of antennomeres in this study were found in two males of Archimantis gracilis (143 and 140 antennomeres, respectively) and the male of Theopompa tosta (135 antennomeres). On the lower numerical end there are species with less than 50 antennomeres, as in Compsothespis, Haaniinae, and Hoplocorypha. Males often have larger numbers of antennomeres than females.

19. Antennae of female, largest number of antennomeres: 49 or less [1]; 50-69 [2]; 70-89 [3]; 90-109 [4]; 110-129 [5]; 130 or more [6].

See character 18. The largest numbers of antennomeres (more than 110 antennomeres, states 5 and 6) are present in the females of Archimantis, Deroplatys 


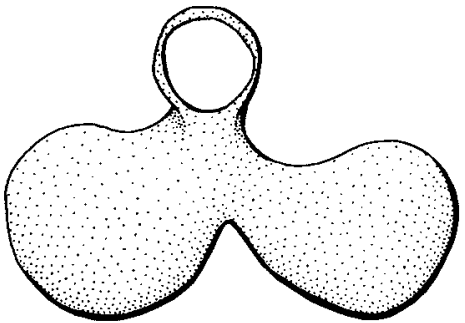

87
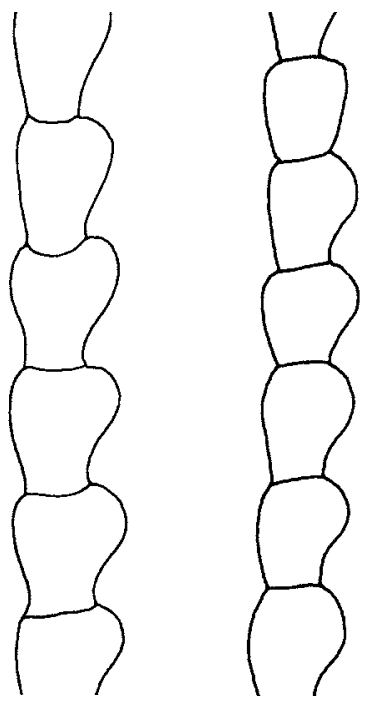

92 93

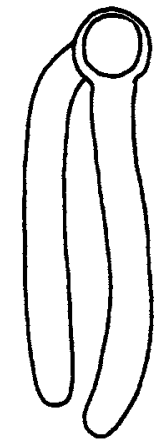

97

98

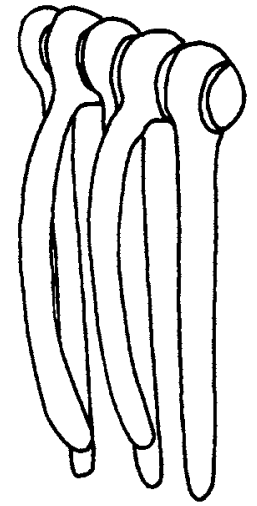

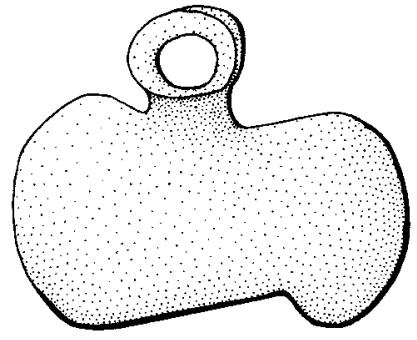

88

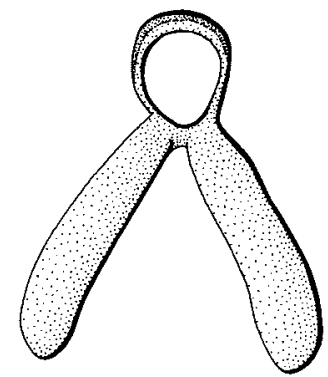

89
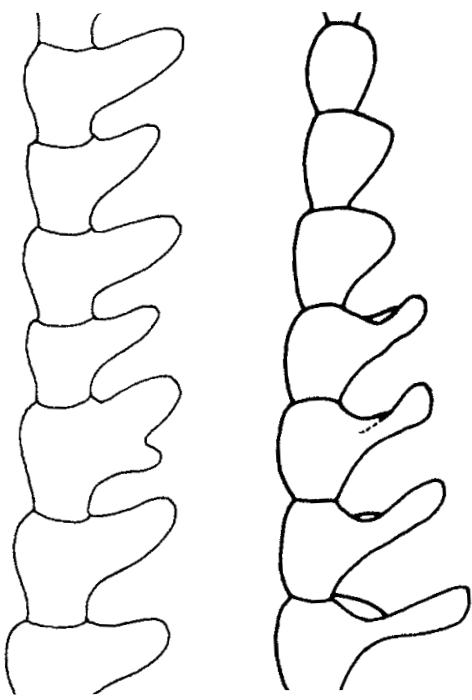

96

95

91

94
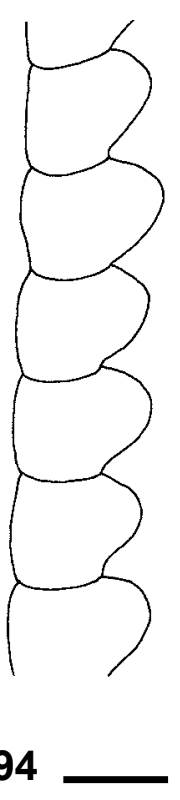

Figs. 87-98. Morphology of the male antennae. 87: Blepharopsis mendica, antennomere, dorsal view. 88: Idolomantis diabolica, antennomere, dorsal view. 89: Gongylus gongylodes, antennomere, dorsal view. 90: Idolomorpha dentifrons, antennomer, dorsal view. 91: Idolomorpha dentifrons, two successive antennomeres, lateral view. 92: Ceratocrania macra, antennomeres, lateral view. 93: Hymenopus coronatus, antennomeres, lateral view. 94: Pseudocreobotra wahlbergii, antennomeres, lateral view. 95: Vates weyrauchi, antennomeres, lateral view. 96: Idolomantis diabolica, antennomeres (distal end of antenna), lateral view. 97, 98: „Pseudo-bipectinate antenna“, redrawn from Wood-Mason (1878: fig. 1). 97: Idolomorpha capensis, successive antennomeres showing the pseudobipectinate antenna. 98: Idolomorpha capensis, two successive antennomeres in dorsal view. - Scalebar $=0.25 \mathrm{~mm}$ (scales of Figs. 97 and 98 not given in original publication).

desiccata, Humbertiella, Metallyticus, Orthoderella, Plistospilota (largest number with 142 antennomeres), Polyspilota, and Theopompa tosta. The smallest numbers (less than 50, state 1) were found in female Compsothespis, Haaniinae, Hoplocorypha, Paraoxypilus, Pogonogaster, and Pseudoharpax. 
20. Antennae of male, shape of antennomeres: symmetrical, without any processes [1]; asymmetrical, being slightly extended to one side (Antenna imbricata) [2]; each one bearing a single process which is alternately curved on successive antennomeres, therefore the antenna being "pseudobipectinate" (Antenna pectinata) [3]; bearing two processes each, therefore being truly bipectinate (Antenna bipectinata) [4]; bearing two rounded, flat, lobe-like extensions or an undivided, more or less heart-shaped lobe (for which I use the term Antenna lobata) [5].

The antennae of Dictyoptera are mostly slender and filiform or moniliform (Klass \& Meier 2006: 39). In Mantodea, the base of the flagellum may be thickened, as for instance in Schizocephala bicornis (Ehrmann 2002: 315) and Brunneria borealis Scudder, 1896 (Ehrmann 2002: 84).

The males of most mantodean species exhibit symmetrical antennomeres resembling those of the females (in which they are mostly symmetrical), whereas in the males of others they show a more or less strong lateral asymmetry. Many male Hymenopodidae and Vatinae, but also Astape have the antennomeres extended to one side at least in the proximal half of the antenna (e.g. Saussure \& Zehntner 1894: pl. 6, fig. 5; Terra 1995: fig. 102; Orofino et al. 2006: figs. 3, 51, 75, 76). This condition is also referred to as Antenna imbricata (Seifert 1995: fig. 195c) or, if more strongly asymmetrical, as Antenna serrata (Seifert 1995: fig. 195d). Male Empusidae carry either one long and slender process on each antennomere (being alternately bent to different sides, therefore herein named "pseudo-bipectinate"; only found in Idolomorpha and Hemiempusa; see Wood-Mason 1878a: 261 and fig. 1; Edmunds 1972: 16; Roy 2004a: 2 and fig. 7; Figs. 90, 91, 97, 98) or two long and slender processes on each antennomere (Antenna bipectinata; see Seifert 1995: figs. 195e, f; Fig. 89). In male Blepharodinae [Blapharodes, Blapharopsis (Fig. 87), Idolomantis (Fig. 88)] the antennomeres carry a strongly flattened and enlarged process (simple or medially distinctly notched), thus having a lobe-like appearance. The bipectinate and "pseudo-bipectinate" antennae of male Empusidae have been discussed by Wood-Mason (1878a: 259-262) and re-evaluated by Roy (2004: 2). For a detailed discussion of the male antennomeres and an interpretation of their function see, for instance, Greven \& Brenner (2009) and chapter 4.6.2.

\subsubsection{Thorax and appendages}

Cervix. The cervix is the membranous area connecting the postoccipital foramen of the head with the prothorax. While it is short and more or less folded and invaginated within the prothorax in many insects, it is long and often protrudes from the prothorax in most Mantodea. Several cervical sclerites are embedded in the cervical membrane. Most of them function as insertion points for the massive musculature of the freely moveable head (see Wieland 2006: 57 for an overview). 
21. Cervix, ventral cervical sclerites: present [1]; missing [2].

The ventral cervical sclerites are one or two slender sclerotizations (Levereault 1936: vCv and vCv2 in pl. 11, fig. 7; Beier 1968a: Po in fig. 20; Wieland 2006) without muscular connections. They are located in the ventro-median part of the cervical membrane in Mantodea and Blattaria (Wieland 2006; Figs. 99, 100, 104-108). As many of the so-believed "basal" taxa [Mantoida (Fig. 101), Metallyticus (Fig. 102), Amorphoscelidae, Eremiaphilidae (Fig. 103)] do not have ventral cervical sclerites (Wieland 2006: figs. 3, 5-8, 45) and Chaetees$s a$ has a single one (Wieland 2006: fig. 4; Fig. 100), one possible explanation would be a lack of ventral cervical sclerites in the ground plan of Mantodea with a convergent gain in Chaeteessa and "higher" Mantodea ("Mantoidea" in Wieland 2006, but see chapter 2.1.5). However, with respect to their presence in several Blattaria (Fig. 99), it is possible that ventral cervical sclerites were already present in the last common ancestor of Mantodea and were reduced independently several times. Chaeteessa was found to be the sistergroup of all remaining Mantodea in the molecular analysis by Svenson \& Whiting (2009). Metallyticus, Amorphoscelidae and Eremiaphilidae, however, were found more distally in the mantodean phylogeny. This supports the view that ventral cervical sclerites were already present in the last common ancestor of Mantodea. For a definite assessment of the evolution of this character a significantly larger number of Polyneoptera including an overview of the variability within the different groups needs to be studied.

One or both ventral cervical sclerites may be split into several smaller parts, for instance in Acanthops, Brunneria, Gongylus, Humbertiella (Fig. 104), Orthoderella and Phyllocrania (Fig. 105; see also Wieland 2006: figs. 9, 10, 14, 27, 28, 30, 31).

22. Cervix, ventral cervical sclerites, number: 1 [1]; 2 [2].

Also see character 21. The number of ventral cervical sclerites varies among taxa but is intraspecifically constant with one exception. In Gongylus, a single ventral cervical sclerite is present in the male whereas the female has two. As the female has both sclerites (albeit the anterior one is split into four parts and is only weakly sclerotized; see Wieland 2006: figs. 30,31) it can be assumed that the species originally had two and that in the male the anterior sclerite was reduced. The species is encoded as state 2 . 
23. Cervix, intercervical sclerites, median connection: present [1]; missing [2].

Wieland (2006: 69-70) discussed the intercervical sclerites of Dictyoptera. A transverse position of the sclerites can be assumed for the dictyopteran ground plan. The intercervicalia are medially separated in all Blattaria and Isoptera studied by Wieland (2006: figs. 34-44), a condition that appears to be plesiomorphic for Dictyoptera as it is the same as in the other polyneopteran taxa. Separated intercervicalia (state 1) are also present in several Mantodea [Mantoida (Fig. 101), Chaeteessa (Fig. 100), Metallyticus (Fig. 102), Eremiaphilidae (Fig. 103), Perlamantinae, Paraoxypilinae, Amorphoscelinae, and Tarachodidae; see also Wieland 2006: 69-70). In most species, however, the sclerites are medially adjoining or probably fused. This condition has been assumed to be autapomorphic for "Mantoidea" (Wieland 2006: 70; but see chapter 2.1.5 for a discussion of Mantoidea).

24. Cervix, intercervical sclerites, torus intercervicalis: present [1]; missing [2].

The intercervicalia of most Mantodea and many Blattaria exhibit a transverse median indentation (intercervical medial groove, icmg in Wieland 2006). The edges of the groove are more or less bulging. The posterior rim may additionally protrude and may carry more or less long setae, thus forming the so-called torus intercervicalis (Wieland 2006: 57; Figs. 100, 102, 105, 106). The torus intercervicalis is unique to certain Mantodea and is entirely missing in Blattaria and Isoptera. Wieland (2006: 68) described two equally parsimonious scenarios for the evolution of this character. It is either autapomorphic for Mantodea except Mantoida with a loss in several lineages, or it has evolved convergently in Chaeteessa, Metallyticus and "Mantoidea" (but see discussion of "Mantoidea" in chapter 2.1.5) with subsequent losses within the latter.

The function of this structure has not been studied so far. It is likely that it increases the stability of the ventral cervix of Mantodea, as do, for instance, ridges and apodemes in certain structures of the insect body (thorax, head etc.).

In contrast to the statements of Wieland (2006), Oxyopsis and Euchomenella do have a torus intercervicalis.

Thoracic segments. While the prothorax is strongly elongated in many Mantodea, the mesoand metathorax are short and morphologically quite similar to each other (e.g. Levereault 1936: figs. 11-13). In Cernomantodea, they carry the ventral auditory structures (see Yager \& Svenson 2008, characters 130 and 131 and chapter 4.6.14 for details).

The elongation of the prothorax in combination with an elongation of the forelegs probably allows an increased range of action for the latter during prey capture. Apart from this functional 

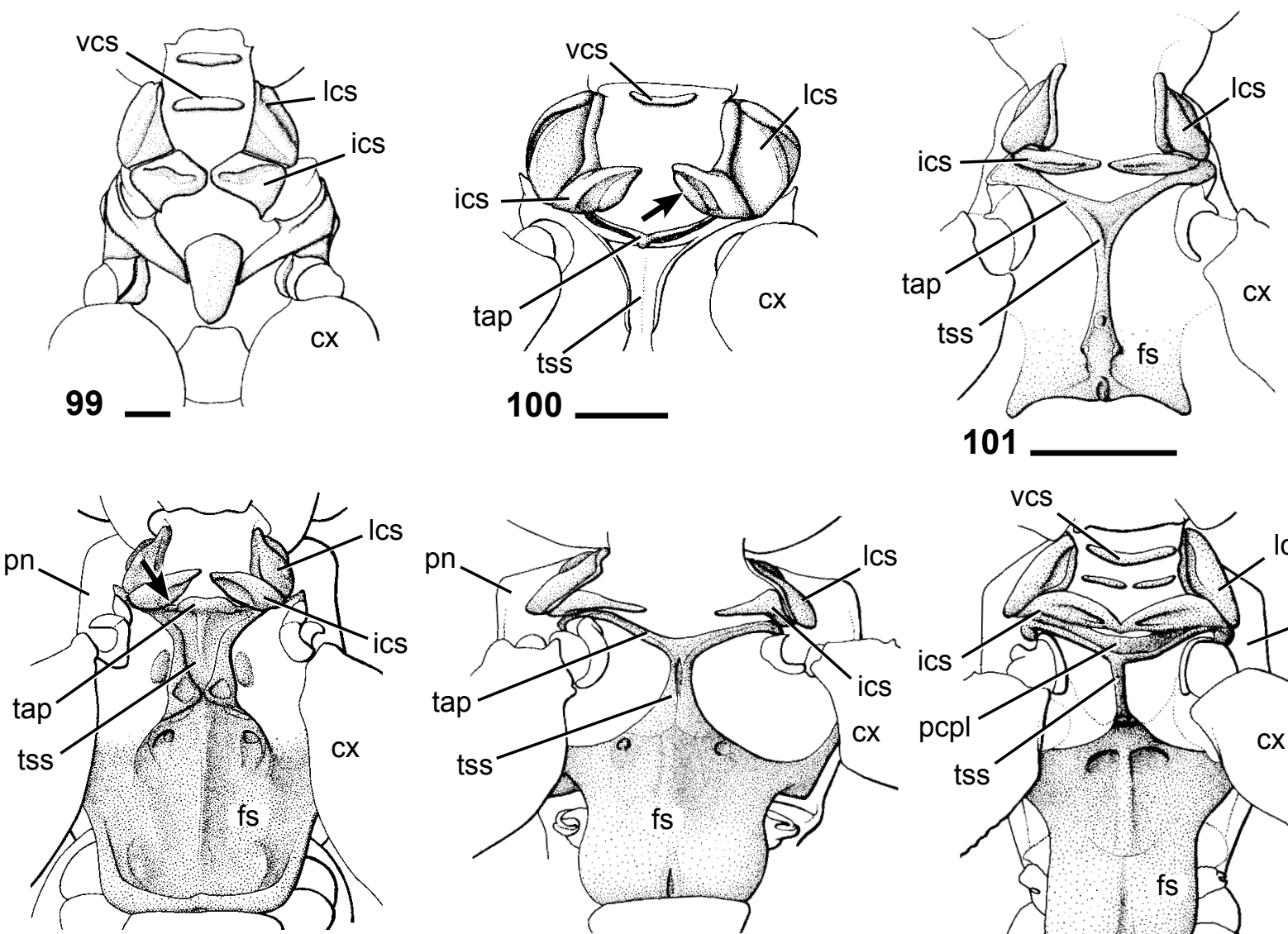

102
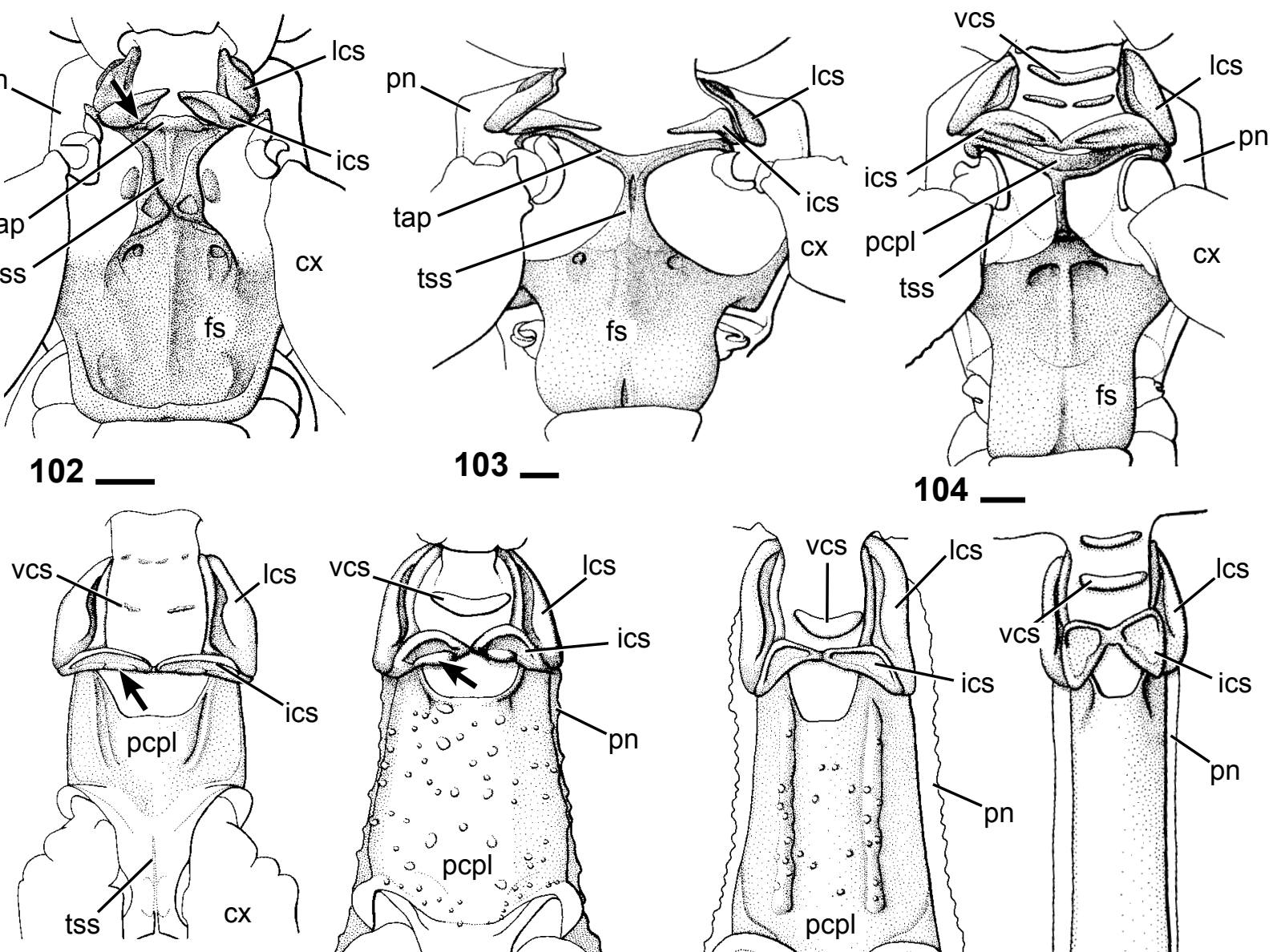

103

104

105
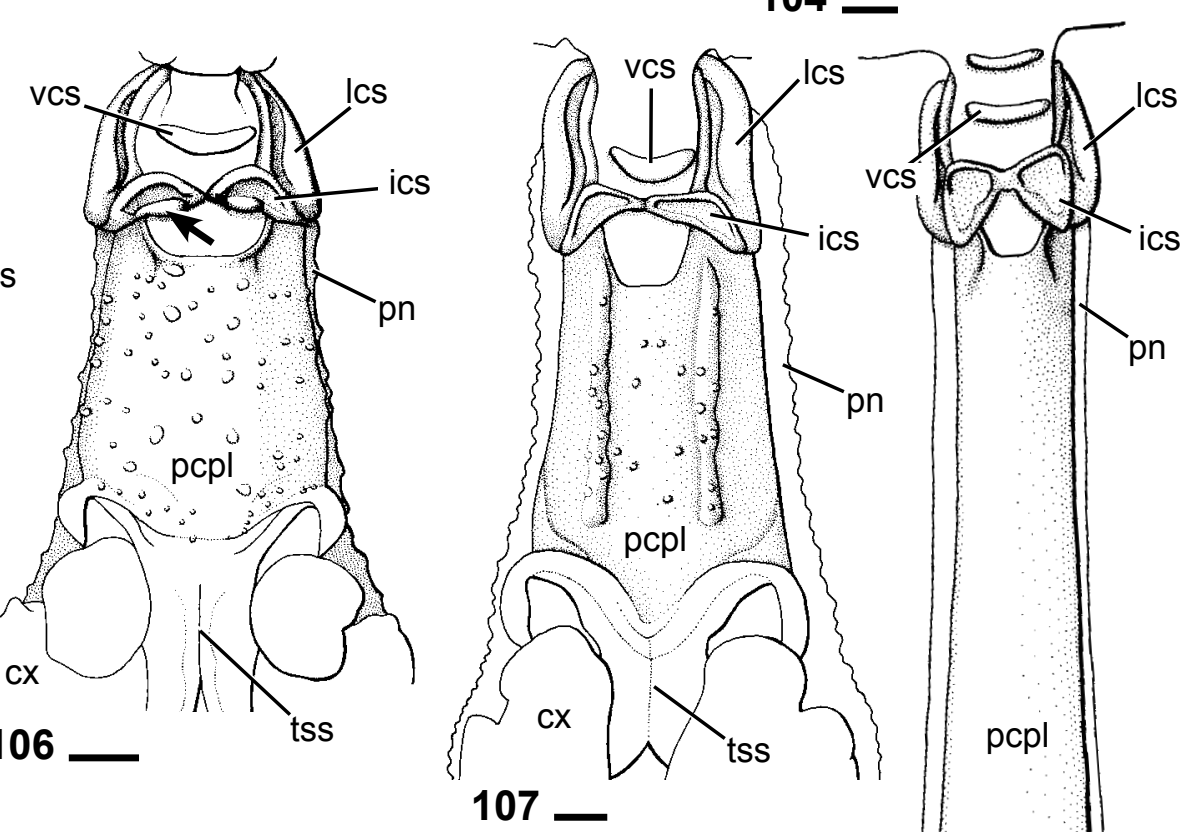

Figs. 99-108: Ventral prothorax and cervical region of Dictyoptera. 99: Periplaneta americana, +. 100: Chaeteessa caudata, ठ (NHMW, see Wieland 2006). 101: Mantoida maya, ô. 102: Metallyticus splendidus, ${ }_{+}$. 103: Eremiaphila sp. 1, ${ }_{+}$104: Humbertiella sp., ${ }_{+} .105$ : Phyllocrania paradoxa, ô. 106: Heterocha-

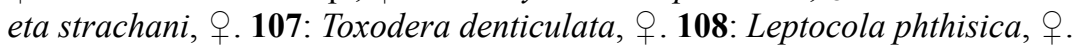
Arrows indicate torus intercervicalis on the posterior edges of the intercervical sclerites. - Scale bar $=1.0 \mathrm{~mm}$.

Abbreviations: $\mathbf{c x}=$ coxa; $\mathbf{f s}=$ furcasternite; $\mathbf{i c s}=$ intercervical sclerite; $\mathbf{l} \mathbf{c s}=$ lateral cervical sclerite; $\mathbf{p c p l}=$ postcervical plate $(=$ elongated transverse anterior part tap of T-shaped sclerite tss); $\mathbf{p n}=$ pronotum; tap $=$ transverse anterior part of T-shaped sclerite; $\mathbf{t s s}=\mathrm{T}$-shaped sclerite; $\mathbf{v c s}=$ ventral cervical sclerite.

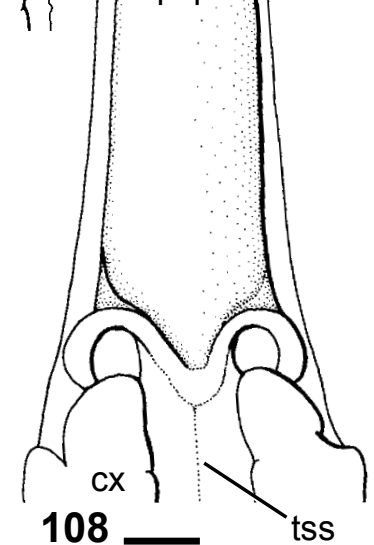


angle, the long prothorax may also have had a major influence on the morphological versatility of mantodean body shape. There is a variety of cryptic and mimetic mantids. In most of them the prothorax plays an important part in the overall appearance. Therefore, its elongation may have been a key factor that provided new possibilities for Mantodea to develop a wide range of additional external morphological alterations of the prothorax (different lengths, additional large lobes, ridges etc.), thus allowing for the impressive range of body shapes from stick- to leafshaped forms found today.

25. Prosternum, T-shaped sclerite formed by fusion of basisternite and preepisternites: present [1]; missing [2].

In Mantodea the prothorax is elongated in most extant species with exception of Mantoida, Chaeteessa, Metallyticus, Amorphoscelinae, and Perlamantinae, in which the pronotum is almost square. There are few exceptions among the "higher" Mantodea in which the prothorax is only slightly longer than wide (see also character 28).

The coxae of the raptorial legs are generally articulated with the anterior part of the prothorax, therefore it is mainly the posterior part of the prothorax (the furcasternite and the metazona of the pronotum) that is elongated in species with long prothoraces (e.g. Beier \& Jaus 1933: 128). However, a secondary stretching anterior to the coxal insertion generally occurs in taxa with long prothoraces (see character 26).

Although Roy (1999: 26) stated that "the prosternum does not generally demonstrate particular morphological characteristics, except for the occasional spines (known as acetabular) near the insertion of the prothoracic coxae", especially the anterior area of the prosternum does, on the contrary, provide valuable morphological characters (Wieland 2003). The ventral prothorax is stabilized by the fusion of possibly several sclerites (basisternite, preepisternites; see Levereault 1936: pl. 11, figs 1, 3; Beier 1968a: figs 21a, b), that are not fused in Blattodea (e.g. Martin 1916: fig. 3; Beier 1974a: fig. 21). The fused complex of sclerites consists of a longitudinal, in "higher" Mantodea mostly slender, sclerotized part between the coxae, and a transverse anterior area that forms a stabilizing bridge across the ventral prothorax and marks the border between the coxal articulations and the cervix. The anterior, transverse part is no more than a slender bridge ("basisternal clasp" of Wieland 2003 who did not take the posterior part into account) in Mantoida (Crampton 1926: fig. 85; Fig. 101), Chaeteessa (Fig. 100), Metallyticus (Wieland 2008a: fig. 6; Fig. 102), Eremiaphilidae (vs. Wieland 2003; Fig. 103), Amorphoscelinae, Perlamantinae, and Elaea. Thus the complex of basisternite and preepisternites has a T-shaped appearance and was correspondingly named " $T$ shaped sclerite" by Wieland (2008a: 159 and fig. 6). In Mantodea with a longer 
prothorax, the transverse anterior part (tap in Wieland 2008a: fig. 6) is elongated and forms the postcervical plate ("basisternal plate" in Wieland 2003; see character 26 and Figs. 104-108).

The fusion of the sclerites into the T-shaped structure is probably apomorphic for Mantodea.

26. Prosternum, transverse anterior part of T-shaped sclerite, shape: short, not elongated into a plate [1]; cranially elongated into a post-cervical plate [2].

The longitudinal, posterior part of the T-shaped sclerite is mostly very slender in taxa exhibiting an elongation of the transverse anterior part of the T-shaped sclerite, possibly due to the growing size of the coxal insertions. The T-shaped sclerite forms a distinct plate between the coxal insertion and the cervix, which I term the post-cervical plate.

The anterior part of the T-shaped sclerite is short in Mantoida (Fig. 101), Chaeteessa (Fig. 100), Metallyticus (Fig. 102), Eremiaphilidae (Fig. 103), Amorphoscelinae and Perlamantinae (Wieland 2003, see also character 25). It is elongated (thus forming a post-cervical plate) in the remaining Mantodea. In Paraoxypilus, Elaea and Humbertiella (Fig. 104), among others, it is only slightly elongated. The former two only exhibit a slight lateral elongation, whereas the medial part of the transverse anterior part remains very short. In the greater part of the taxa the transverse anterior part is wider than long. However, it is longer than wide in mantodeans of stick-like appearance [e.g. Leptocola (Fig. 108), Heterochaeta (Fig. 106), Toxodera (Fig. 107), and Brunneria (see Ehrmann 2002: 388)]. A postcervical plate has been hypothesized to be apomorphic for a taxon containing Paraoxypilinae, Eremiaphilidae, Hymenopodidae, Mantidae and Empusidae (referring to Beier's classification of 1968a) by Wieland (2003). However, the length of the transverse anterior part of the T-shaped sclerite in Eremiaphila has been misinterpreted by Wieland (2003) because only a dried specimen of E. typhon Lefèbvre, 1835 was available. Subsequent studies of ethanol-preserved specimens from two other species have revealed the slender shape of the entire structure in Eremiaphilidae. Therefore, the elongated transverse anterior part would correspondingly be apomorphic for Paraoxypilinae + Acanthopidae + Hymenopodidae + Liturgusidae + Tarachodidae + Thespidae + Iridopterygidae + Mantidae + Toxoderidae + Sibyllidae + Empusidae (sensu Ehrmann 2002). Either way, the elongation of the transverse anterior part of the T-shaped sclerite in Paraoxypilinae would support a potential non-monophyly of the traditional Amorphoscelidae (i.e. Amorphoscelinae + Perlamantinae + Paraoxypilinae; see Wieland 2003). This has been assumed before by Handlirsch (1925: 495), Chopard (1949a: 398), and Béthoux \& Wieland (2009: 107). But see discussion in chapter 4.3.14. 
27. Pronotum, anterior duplicature, size: covering head dorsally [1]; covering caudal part of head at most [2]; not covering head [3].

The anterior duplicature of the pronotum, which is often elongated in Blattaria, is also present in all Mantodea, albeit generally very short (Klass \& Meier 2006: 40). The Mantodean pronotum usually does not cover the head due to the elongation of the cervix (Beier \& Jaus 1933: 127; Klass \& Meier 2006: 40). In some Mantodea, such as Metallyticus (Figs. 9, 82, 454), Mantoida (Fig. 7, 8, 412, 413), and Chaeteessa (Fig. 5, 6, 71) the posterior part of the head is slightly covered by the anterior duplicature of the pronotum (Klass \& Meier 2006: 40). This is also true for Perlamantinae (Figs. 12, 79, 80).

In Blattaria, the discoidal pronotum covers the head, often almost entirely, with its anterior duplicature [see, for instance, Therea nuptialis (Gerstaecker, 1861) (Handlirsch 1930b: fig. 869a) and Cryptocercus (Grimaldi \& Engel 2005: fig. 7.73.)]. However, there are several lineages of cockroaches - both fossil and extant - with smaller and sometimes more slender pronota. The anterior lamellar duplicature in these taxa covers the head only posteriorly or even not at all. These is the case in the fossil groups $†$ Raphidiomimidae (Vršanský 2002: fig. 18; Vršanský et al. 2002: fig. 372; Grimaldi \& Ross 2004: fig. 1; Grimaldi \& Engel 2005: fig. 7.69; Liang et al. 2009: fig. 1a) and †Umenocoleidae (Vršanský et al. 2002: fig. 376; Grimaldi \& Engel 2005: figs. 7.70, 7.71). Among the extant cockroaches Allacta diagrammatica (Hanitsch, 1923) has a nearly free head (Hanitsch 1923: fig. 1). Several other extant cockroaches (e.g. Supella Shelford, 1911, Euphyllodromia Shelford, 1908, Macrophyllodromia Saussure \& Zehntner, 1993, Nahublattella Bruijning, 1959, Nauphoeta Burmeister, 1838) have a short duplicature which covers the posterior part of the head only slightly (Klass \& Meier 2006: 40; Bell et al. 2007: figs. 3.3, 4.6).

28. Pronotum, length with respect to width (excluding lateral lamellar expansions): twice as long as wide or longer [1]; less than twice as long as wide or almost square [2]; wider than long ("discoidal") [3].

This character is encoded "without lamellar expansions" because many Mantodea exhibit strongly widened pronota (see also character 29) which may lead to the impression of a short or square pronotum [e.g. Brancsikia (e.g. Chopard 1949c: fig. 53; Paulian 1957: figs. 2-4, 6-8), Choeradodis (e.g. Wood-Mason 1884: figs. 6, 7, 10-15; Beier 1968a: fig. 7; Ehrmann 2002: figs. 52-54; Roy 2004b: figs. 9-11, 15, 16, 23-28, 32-34; Fig. 142), Deroplatys (e.g. Anisyutkin 1998: figs. 1, 10; Ehrmann 2002: figs. 55, 56; Fig. 445), and Idolomantis (e.g. Ehrmann 2002: fig. 51; Klass \& Ehrmann 2003: fig. 13.2. A; Fig. 117)]. The 
addition of this definition allows to assess the primordial pronotal shape (i.e. without putative secondary expansions) correctly.

Sometimes, the pronotum is saddle-shaped (e.g. in Mantoida; Figs. 7, 8, 412, 413), i.e. the lateral parts are bent ventrad. Therefore, the pronotum, if forced into a flat position, would possibly be wider than in dorsal view. As this measurement is difficult to assess with accuracy in situ, I refer to the measurement relations of the drawings in dorsal view.

An elongated prothorax has been stated to be autapomorphic for Mantodea as a whole (e.g. Hennig 1969: 182; Ax 1999: 288). This seems to find support in the fact that most Blattodea have short prothoraces. However, although the mantodean prothorax is more or less strongly elongated in most species and may take even more than half the length of the entire body [e.g. Euchomenella (Giglio-Tos 1927: 236; Beier 1935c: 65; Roy 1999: 2.2c, 2001: 84; see Fig. 109) or Phasmomantis Saussure, 1869 (Beier 1935c: pl. 3, fig. 9, therein named Tauromantis)], all sobelieved "lower" lineages of the traditional classification (Mantoida, Chaeteessa, Metallyticus, Eremiaphilidae, most Amorphoscelidae; Figs. 110-112, 114-116) as well as all Mesozoic Mantodea described so far (see Grimaldi 2003) have short prothoraces being almost square or only slightly elongated. The reconstruction of the fossil nymph from Baltic amber (Figs. 33, 36) furthermore shows that the prothorax is not significantly longer than the mesoand metathoracic segments in such taxa. Therefore, the elongated prothorax cannot be apomorphic Mantodea as a whole, but for a taxon within the group at most. Grimaldi (2003) and Grimaldi \& Engel (2005) suggested an elongated prothorax being more than twice as long as wide to be apomorphic for "Mantoidea" (Grimaldi 2003: character 23; Grimaldi \& Engel 2005: character 24 in fig. 7.60). However, contradicting evidence has been presented by recent molecular findings (Svenson \& Whiting 2004a, 2009; Ware et al. 2008; Yager \& Svenson 2008; compare discussion of "Mantoidea" in 2.1.5). The short prothorax probably represents the plesiomorphic condition in

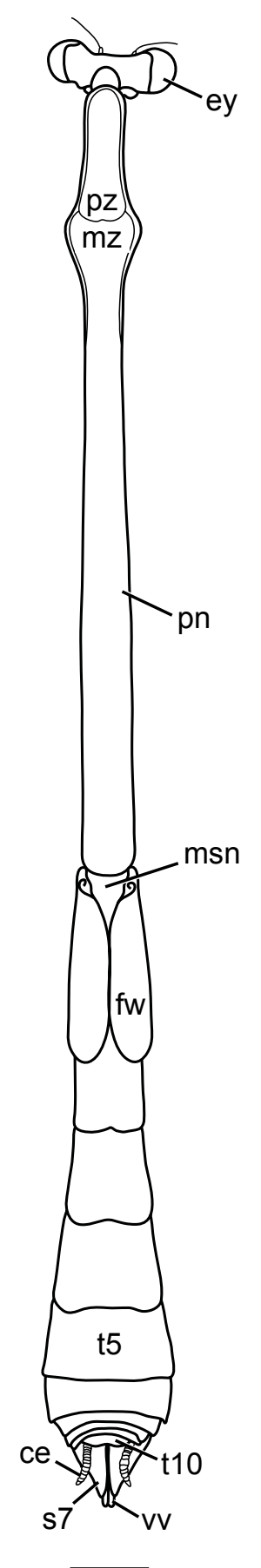

Fig. 109: Euchomenella sp., q. Habitus. Note the pronotum being distinctly longer than the remaining body. - Scale bar= $5.0 \mathrm{~mm}$.

Abbreviations: $\mathbf{c e}=$ cercus; $\mathbf{e y}=$ compound eye; $\mathbf{f w}=$ fore wing (tegmen); msn = mesonotum; $\mathbf{m z}=$ metazona; $\mathbf{p n}=$ pronotum; $\mathbf{p z}=$ prozona; $\mathbf{s} 7=$ abdominal sternite 7 (subgenital plate of + ); $\mathbf{t 5}, \mathbf{t 1 0}=$ tergite 5 , tergite 10 (= supraanal plate); $\mathbf{v v}=$ ventral valvula (gonapophysis VIII). 
some of the "lower" Mantodea (Mantoida, Chaeteessa, and possibly Metallyticus). However, Svenson \& Whiting (2004a: 364), Yager \& Svenson (2008), and Svenson \& Whiting (2009) found evidence for a secondary shortening of the prothorax in Paraoxypilinae, Amorphoscelinae, and Eremiaphilidae with respect to their distal positions in the phylogenetic trees. For Paraoxypilinae, this hypothesis gains support from the fact that Cliomantis has a pronotum being twice as long as wide (see above). The taxonomic affiliation of Cliomantis to Paraoxypilinae is unambiguous and strongly supported by the synapomorphic morphology of the characteristic paraoxypiline raptorial leg (see generic descriptions in Giglio-Tos 1913: 12; Tindale 1923: fig. 374; Ehrmann 2002: 106; see characters $42,43,45,63-65,72)$. Interestingly, Cliomantis was found to be subordinate within Paraoxypilinae in Svenson \& Whiting (2009: fig. 3b, node 113). This implies that Paraoxypilinae, itself being nested within a group of taxa that have comparatively long pronota, have secondarily reduced the length of the prothorax. Whether the elongation in Cliomantis represents a secondary elongation or if it is plesiomorphically long has to be studied in the future.

Apart from this, many "Mantoidea" in the present study, for instance Astape, Ceratomantis, Creobroter, Elaea, Entella, Gimantis, Hestiasula (Fig. 119), Humbertiella, Ligaria, Ligariella (see also Kaltenbach 1998: fig. 35), Oxypilus, Pseudocreobotra (Figs. 118, 121), and Theopompa (Fig. 122) exhibit prothoraces that are distinctly less than twice as long as wide. This further contradicts the "Mantoidea"-hypothesis by Grimaldi (2003) and Grimaldi \& Engel (2005) (see chapter 2.1.5).

Roy (1999: 25) stated that the pronotum is wider than long only in Amorphoscelinae and Eremiaphilidae. However, following the definition for measuring applied herein, the pronotum of both Eremiaphila species studied herein (Fig. 111) and of Heteronutarsus (Fig. 115) have square pronota (see also Chopard 1941: figs, 1-7). The Maculatoscelis specimen has a pronotum distinctly wider than long ("discoidal"; Fig. 112), whereas in Amorphoscelis sp. and Caudatoscelis the pronota are square (Caudatoscelis; Fig. 116) or slightly longer than wide (Amorphoscelis sp.). In Chaeteessa (Fig. 110), Mantoida (Fig. 114) and Perlamantis (Fig. 80) it is almost square, and it is slightly longer than wide in Metallyticus (Fig. 454), and Paramorphoscelis (Fig. 79). In the remaining taxa it is distinctly longer than wide (Roy 1999: 25).

In Blattaria the pronotum is mostly wider than long ("discoidal"). Only few groups have untypically slender pronota, among them the highly derived fossil $\dagger$ Raphidiomimidae and $\dagger$ Umenocoleidae (see character 27). In these groups, the pronotum is as long as wide (e.g. Grimaldi 1990: figs. 3 A and G; Vršanský 2003: fig. 15) or even longer than wide (Grimaldi \& Ross 2004: fig. 1, pp. 102, 104; Grimaldi \& Engel 2005: figs. 7.69, 7.70; Liang et al. 2009: fig. 1a). It can 


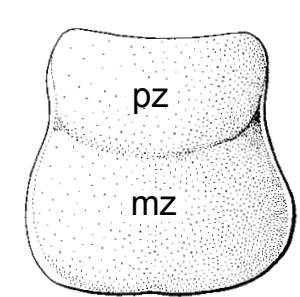

110

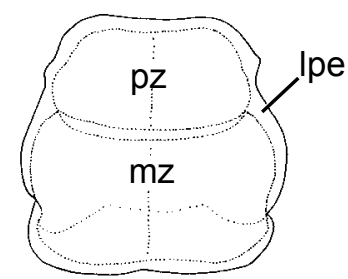

114

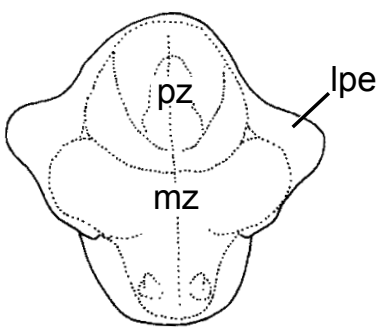

118

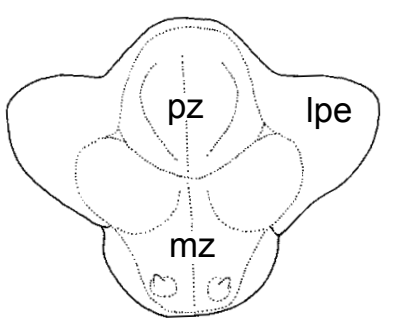

121

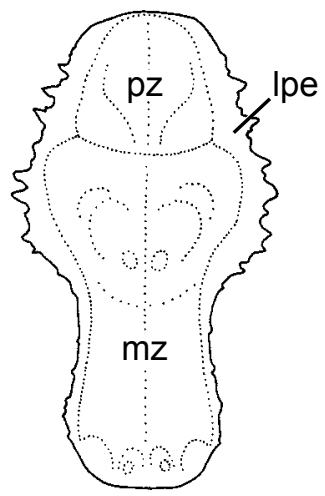

125

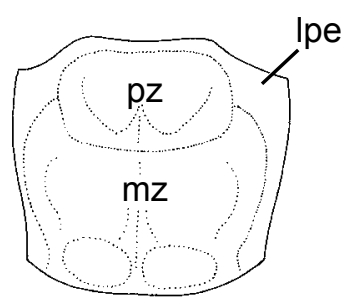

111

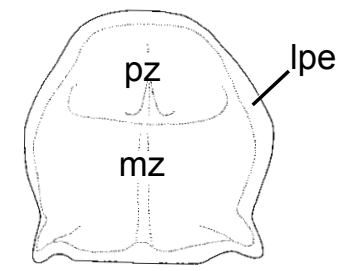

115

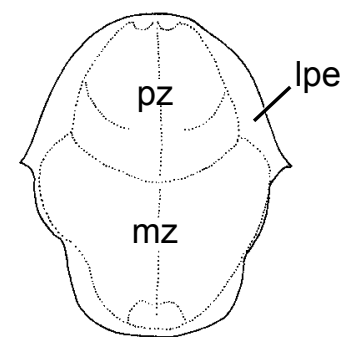

119

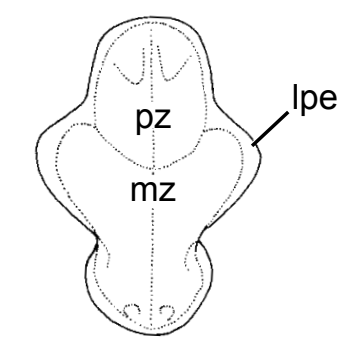

122

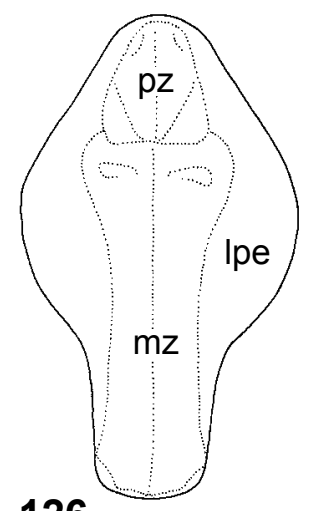

126

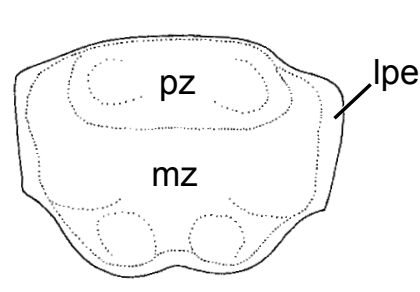

112

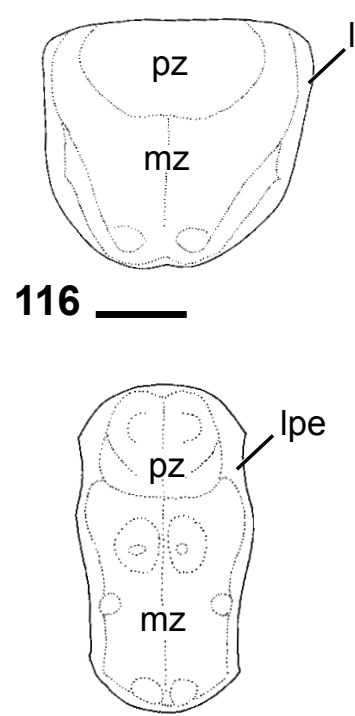

Ipe 113
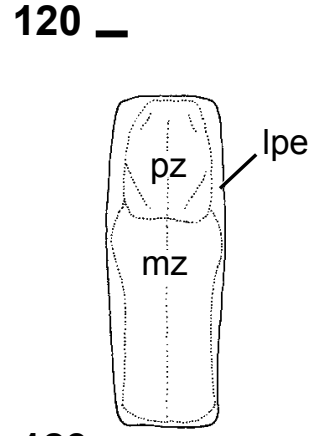

123

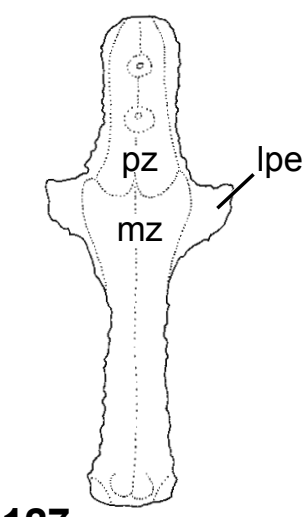

127
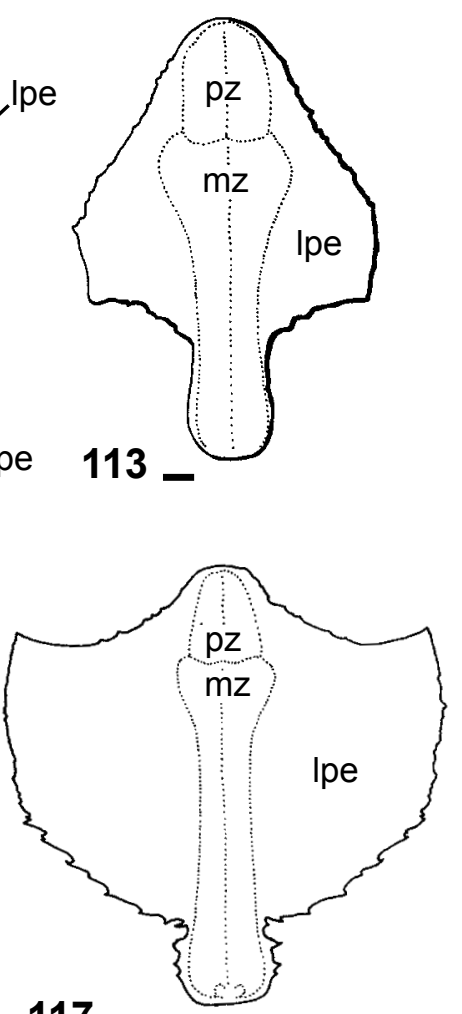

117

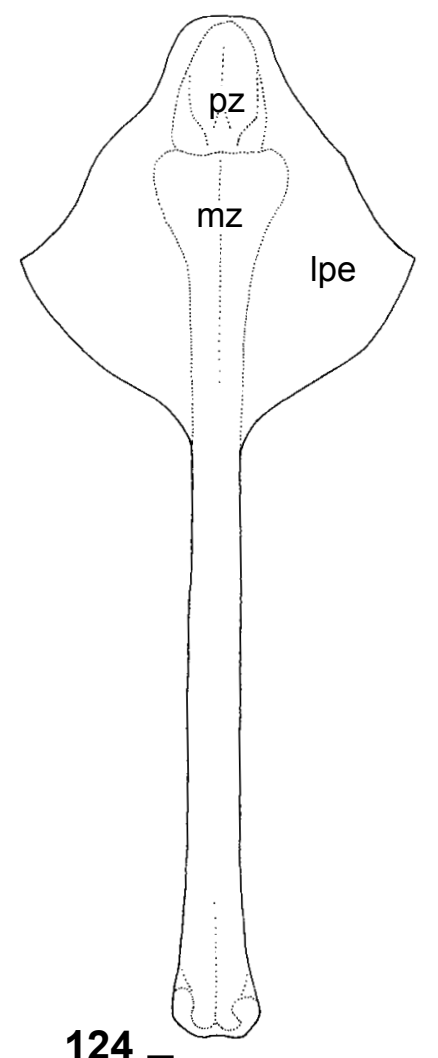

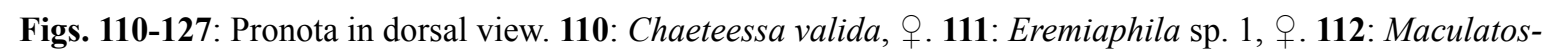
celis sp.,, . 113: Phyllocrania paradoxa,.+ 114: Mantoida maya, $\widehat{\partial}$. 115: Heteronutarsus aegyptiacus, $\widehat{\partial}$. 116: Caudatoscelis annulipes, ․ 117: Idolomantis diabolica, ô. 118: Pseudocreobotra ocellata, ․ 119: Hestiasula

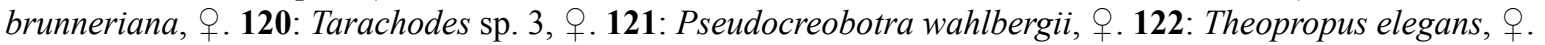
123: Pyrgomantis jonesi, + . 124: Gongylus gongylodes, ${ }_{+}$. 125: Majanga basilaris, ${ }_{+}$. 126: Tamolanica tamola$n a$, ô. 127: Pogonogaster tristani, ㅇ. - Scale bar $=1.0 \mathrm{~mm}$.

Abbreviations: Ipe $=$ lateral pronotal expansion; $\mathbf{m z}=$ metazona; $\mathbf{p z}=$ prozona. 
be assumed that the short pronotum is a groundplan feature of Mantodea and that an elongation has occurred independently in some blattarian lineages and possibly several times within Mantodea, with putative subsequent length reductions or potential secondary elongations in some taxa, as was elaborated on above.

29. Pronotum, lamellar expansions, width on either side: at widest point less wide than half the width of pronotum at supracoxal furrow (without expansions) [1]; at widest point as wide as or wider than half the width of pronotum at supracoxal furrow (without expansions) [2].

The mantodean pronotum has free anterior and lateral lamellar edges (Klass \& Ehrmann 2003: 183). The width of the lamellar expansions varies greatly among the mantodean taxa. They may be more or less strongly widened and form enormous lateral lobes [e.g. Brancsikia, Choeradodis (Fig. 142), Deroplatys (Figs. 16, 445, 446), Gongylus (Fig. 124), Idolomantis (Fig. 117), Rhombodera, Parablepharis Saussure, 1870, Phyllocrania (Fig. 113); see, for instance, Westwood 1845c: plate 9; Wood-Mason 1889: figs. 5-19; Sharp 1895: fig. 135; Ehrmann 2002: figs. 13-17, 52-56; Paulian 1957: 2-4, 6-8; see also chapter 4.6 .3 for discussion].

Lateral expansions are frequently found in cockroaches, where they are often wide. In Mantodea, they play a major role in the overall shape of the pronotum. They allow the usually slender pronotum to become rectangular [as can be found in Orthodera (Figs. 18, 461) and Pyrgomantis (Figs. 25, 123)], or very large and shield-like [as in Choeradodis (Fig. 142), Asiadodis Roy, 2004, Deroplatys (Figs. 16, 445, 446), Idolomantis (Fig. 117), Brancsikia, Rhombodera), thus adding to the special resemblance to grass blades or foliage.

Members of the basal dichotomies of the mantodean tree (Mantoida, Chaeteessa, Metallyticus, Amorphoscelidae, Eremiaphilidae) and fossil mantodeans (Grimaldi 2003: figs. 3A, 4C, 14, 15, 17; see also amber fossil in Figs. 33-38) lack strongly enlarged pronotal lobes. Hence, the presence of this character may be apomorphic for groups within Mantodea. On the other hand, lateral pronotal lobes are widespread among Blattaria. Therefore, it is also possible that the raptorial lifestyle acquired by Mantodea led to the need of reducing the size of the pronotum in order to hunt efficiently. Consequently, the enlarged pronotum in Mantodea evolved secondarily due to the advantageous cryptic effects that may have led to a positive selection of this character. In order to answer this question, further studies are indispensable.

Another potential function of the enlarged pronotum was described by Robinson (1969: 297). Therein, he mentioned that Choeradodis rubs the dorsal, tooth-bearing surface of the anterior femora (Figs. 128, 130) against the anterior edge of the pronotum, thus producing a hissing noise. If so, the pronotum may 
in this taxon also serve as an amplifying sound shield (see also Ramsay 1990: 45). Interestingly, the anterior edge of the pronotum in Choeradodis stalii appears to be hollow (Fig. 129; probably filled with haemolymph in life). To my knowledge, no further observation of this deimatic behaviour has been published. Dorsal teeth on the fore femora can also be found in several other taxa, for instance Deroplatys desiccata
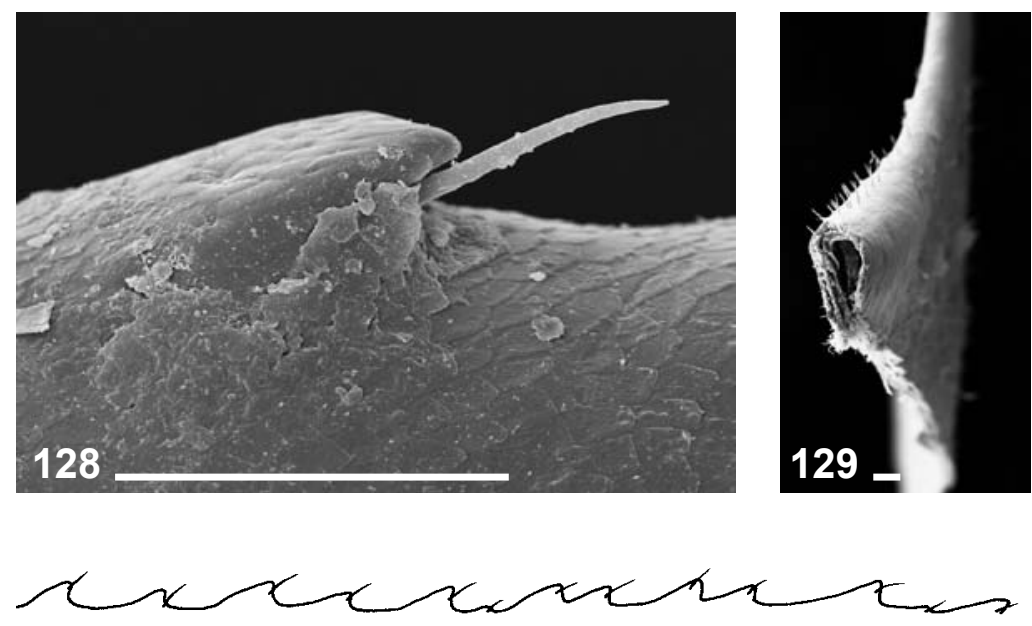
130

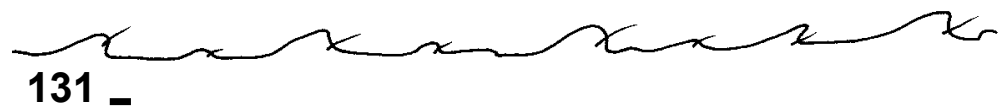

Fig. 128-131: Putative sound production in Choeradodis. 128: Choeradodis stalii, + , left raptorial leg, single tooth on dorsal edge. 129: Same specimen, part of pronotum, antero-lateral view (note hollow anterior

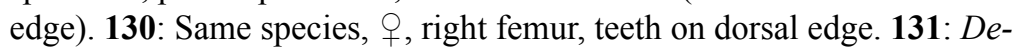
roplatys desiccata, + , right femur, teeth on dorsal edge. $\rightarrow$ distal (Figs. 128, 130, 131); - Scale bar $=0.1 \mathrm{~mm}$.

(Fig. 131).

30. Pronotum, supracoxal furrow: present [1]; missing [2].

Powerful raptorial legs and an elongated neck probably led to the requirement of stabilization for the prothorax against the forces of their massive musculature (Levereault 1936: 223; Beier 1964a: 868, 1968a: 19). This is mainly achieved by the structure of the prothoracic tergite. It is hemicylindrical (Levereault 1936: 223) and bears an internal apodeme ( 1 in Levereault 1936: fig. 2; Klass \& Ehrmann 2003: 183) which stabilizes the pronotum against the forces of the massive tergal adductors of the fore coxae (9a-c in Levereault 1938: 592 and fig. 4). Externally, the apodeme is recognizable as a transverse sulcus (supracoxal furrow, ScxF in Beier 1968a: fig. 21A; Klass \& Ehrmann 2003: 183), dividing the mantodean pronotum into prozona and metazona (e.g. Figs. 110-124). The supra-coxal furrow is lacking in Blattodea.

31. Pronotum, Prozona, length in relation to metazona: nearly as long as metazona [1]; slightly shorter to about half as long as metazona [2]; less than half as long as metazona [3].

The subdivision of the pronotum into pro- and metazona is apomorphic for Mantodea because Blattaria and Isoptera do not have a protergal apodeme and sulcus 
(see also character 30). Within Mantodea, a short prothorax can been hypothesized to represent the plesiomorphic condition (see also character 28). In the "lower" lineages of the current classification, Mantoida (Fig. 114), Chaeteessa (Fig. 110), Metallyticus (Fig. 454), Eremiaphilidae (Figs. 111, 115) and Amorphoscelidae (Figs. 79, 80, 112, 116), the prozona (measured along the prothoracic midline, see chapter 3.3) is slightly shorter than the metazona. In the greater part of the remaining taxa studied herein, the prozona is less than half as long as the metazona. Roy (1999: 25) stated that the prozona is always shorter than the prozona. However, in several taxa it is as long as the metazona or even slightly longer. This can be found in Ceratomantis (see also Roy \& Svenson 2007: figs. 3-7, 14-19), Hestiasula (Fig. 119), Parahestiasula Lombardo, 1995 (a: fig. 3), Oxypilus (Kaltenbach 1998: fig. 111), and Pseudocreobotra (Kaltenbach 1998: fig. 115, Figs. 118, 121).

Forelegs. The forelegs of Mantodea are always raptorial (autapomorphy, see chapter 2.1.3). They can be folded with the tibia closing against the femur. In most extant Mantodea the ventral surfaces of both tibia and femur carry strong spines which hold the captured prey.

32. Forelegs, function: unspecialized cursorial legs [1]; modified into raptorial legs that can be folded and held raised in front of the prothorax [2].

The primary function of the forelegs of Dictyoptera is (plesiomorphically) locomotion as in all Polyneoptera except for (most) Mantodea. This condition is exhibited by Blattodea, although the spination of the forelegs (as well as the mid- and hind legs), especially the femora, strongly resembles that of mantodeans (pers. obs.; see also Figs. 145-147, 285-287). One extinct group of cockroaches (†Raphidiomimidae) has been assumed to have raptorial legs (Grimaldi \& Ross 2004: 103; Liang et al. 2009: 17). Vršanský (2002) and Vršanský et al. (2002) proposed a monophyletic origin of $†$ Raphidiomimidae (among several other cockroach lineages) and Mantodea. However, this is probably not the case (Grimaldi \& Ross 2004: 104; Béthoux \& Wieland 2009: 107).

Raptorial forelegs together with a strong elongation of the coxae and their articulation are thus autapomorphic for Mantodea.

Interestingly, Mantoida, Metallyticus, and probably Chaeteessa (Figs. 5-9, 409-413) exhibit the plesiomorphic condition as far as the use of the forelegs as cursorial legs is concerned. Although they have true raptorial legs, the prothoracic tarsi in these taxa are put on the ground at almost all times except for few occasions [pers. obs. in living Metallyticus splendidus (Figs. 9, 409-411) and M. fallax; Deyrup 1986: fig. 1 for Mantoida maya (drawn from photograph); White, pers. comm. 2008 for living Mantoida maya; Schulze, pers. comm. 2009 
and pers. obs. 2010 for living Mantoida sp. (Figs. 412, 413]. This may also be the case in Chaeteessa, but observations of living specimens are rare and this feature has not been described for living Chaeteessa so far. Fig. 5 shows a living late instar nymph of Chaeteessa sp. from Ecuador (courtesy of G. Gallice), which exhibits the same foreleg position as observed in Metallyticus and Mantoida. As other Mantodea (Amorphoscelinae, Perlamantinae, and other, often bark dwelling taxa) also frequently use their forelegs for running (albeit mostly holding them folded against the body when at rest, see Figs. 11, 12, 20, 21), long term observations of living Chaeteessa are indispensable to support this hypothesis. For a detailed discussion of this topic see chapters 4.6.4 and 4.6.5.

33. Coxa of foreleg, antero-distal apical lobes, shape: diverging [1]; closed, medial edges not overlapping [2]; closed with overlapping medial edges [3].

The fore coxae carry a dorsal and a ventral antero-apical lobe. The notch between these lobes may be quite wide or the lobes may lie closely together. Furthermore, the medial edges may overlap (e.g. Kaltenbach 1998: figs. 61, 62; Roy 1999: 26; Figs. 132-136).

The antero-distal lobes are present in Blattaria, although the notch between them is short and the lobes are not overlapping.

Diverging lobes (state 1; Fig. 136) have been found in about 75 per cent of the species studied. State 2 is exhibited by approximately one sixth of the species examined. A problematic case, for instance, was Gongylus gongylodes (Fig. 134). In this species, the lobes do not adjoin. However, the medial edges are almost parallel, in contrast to state 1 in which the angle between them is distinctly wider (e.g. Sibylla in Fig. 136). Therefore, Gongylus was encoded as state 2.

Overlapping antero-distal apical lobes (state 3) are exhibited by Bactromantis, Blepharodes, Blepharopsis (Fig. 132), Deroplatys, Hymenopus, Idolomantis (Fig. 133), Plistospilota, Theopropus, Thesprotia, and Vates.

34. Coxa of foreleg, ventral antero-distal apical lobe, lanceolate elongation: present [1]; missing [2].

The ventral antero-apical lobe of the fore coxa may be elongated and lanceolate. This condition has been found in the Empusinae studied here (Empusa, Gongylus, Hypsicorypha, and Idolomorpha; Figs. 134, 135), but also in Idolomantis (Fig. 133), whereas it is missing in all remaining taxa studied including the remaining Empusidae: Blepharodinae [Blepharopsis (Fig. 132) and Blepharodes]. The situation in Idolomantis is somewhat ambiguous since its fore coxae carry large lobes along their entire length (Fig. 133). However, there is a distinct tip of 


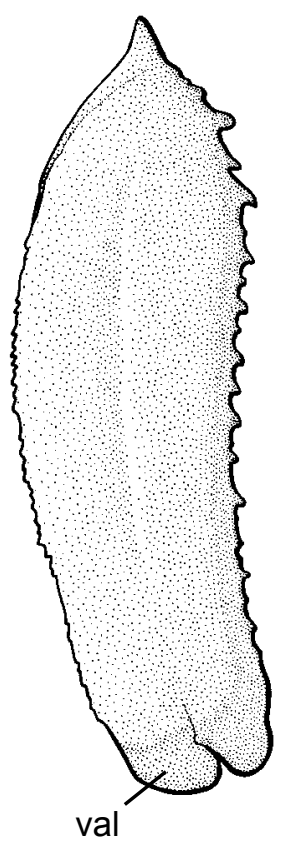

132

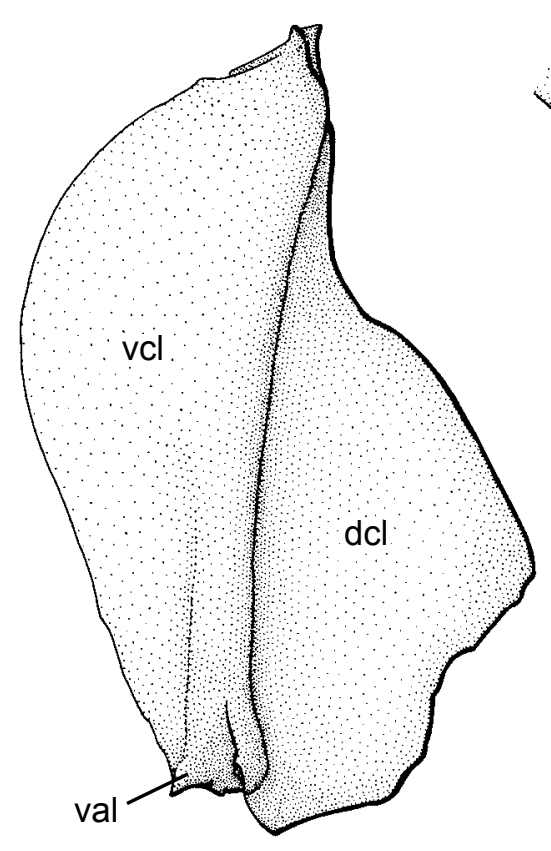

133

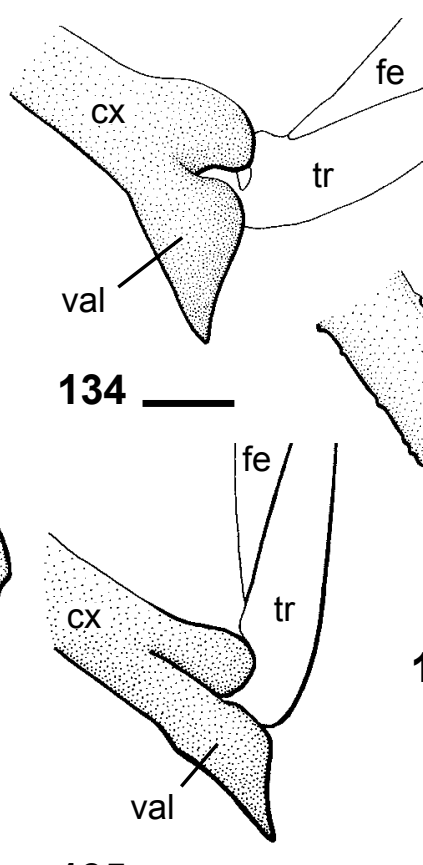

135

Figs. 132-136: Fore coxa, anterior view. 132: Blepharopsis mendica, $\uparrow$, right foreleg (mirrored). 133: Idoloman-

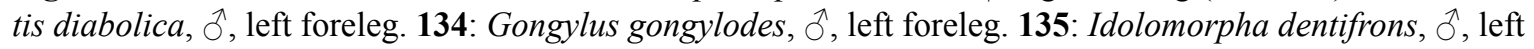
foreleg. 136: Sibylla pretiosa, $\widehat{\jmath}$, left foreleg. Note lanceolate elongation of ventral lobe in Idolomantis (concealed by the ventral lamellar expansion of the coxa), Gongylus and Idolomorpha. - Scale bar $=1.0 \mathrm{~mm}$. Abbreviations: $\mathbf{c x}=$ coxa; $\mathbf{f e}=$ femur; $\mathbf{t r}=$ trochanter; $\mathbf{v a l}=$ ventral antero-distal apical lobe of coxa; $\mathbf{v c l}=\mathbf{v e n}-$ tral coxal lobe.

a putative lanceolate structure protruding from the surrounding lobe in the area of interest in Idolomantis. Therefore, instead of being apomorphic for Empusinae (Roy 2004a: 14) it may rather be a synapomorphy for Idolomantis + Empusinae, which would render Blepharodinae paraphyletic (see also discussion of Empusidae in chapters 2.1.4, and chapter 4.3.71, and 4.6.2).

35. Edges of prothorax merging smoothly with dorsal edge of fore femur: missing [1]; present [2].

Bark dwelling mantodeans are usually well camouflaged by means of flattened bodies and/or imitation of lichen, mosses or bark. The similarity is achieved by colouration and sometimes structural adaptations [e.g. Astape, Gonatista Saussure, 1869, Haania, Humbertiella, Liturgusa, Theopompa, Theopompella, Zouza Strand, 1911 (Lieftinck 1953: 129; Beier 1964a: 861, 863; Edmunds 1974: 13)]. The habit of holding the forelegs bent aside and the dorsal edges of the femora pressed against the lateral edges of the pronotum can be found in many bark dwelling Mantodea [e.g. Gonatista, Humbertiella, Theopompa (Fig. 137), several Tarachodidae (Figs. 138-139); see Edmunds 1972: 4, 19, 20 and fig. 3C; 1974: fig. 12.12], but also in grass or twig dwellers (e.g. Pseudogalepsus or Pyr- 
gomantis, Figs. 140, 141). This behaviour helps to conceal the typical "praying" outline and to avoid lateral shadows (Edmunds 1972).

The close fitting of the dorsal fore femora against the prothorax plays an important role in camouflage. When pressed against the surface, usually a branch, the bark of a tree or a blade of grass, the dorsal edges of the fore femora of several species (Humbertiella, Tarachodidae and Theopompa in this study) perfectly merge with the ventral lateral edges of the pronotum when the fore legs are folded.

The prothorax of such species often has a nearly rectangular outline (Tarachodidae; Figs. 120, 123, 138, 139). It is often dorso-ventrally flattened, i.e. the pronotum is not arched in cross-section but rather flat (although sometimes displaying dorsal ornaments, such as pointed elevations, steep bulges etc.). The furcasternite is medially more or less strongly carinated in all Tarachodidae studied here and, to a lesser extent, in Humbertiella and Theopompa. A carinated furcasternite is also present in other Mantodea, for instance in Metallyticus (see Wieland 2008a: fig. 6; Fig. 102), although this genus does not exhibit such a concealing behaviour but puts its fore tarsi more or less constantly on the ground instead (see also chapter 4.6.4).

Posteriorly, the furcasternite of Tarachodidae is bent ventrad so that the fore coxae fit perfectly against the furcasternite, thus allowing the dorsal edges of the femora to be pressed very closely against the lateral edges of the pronotum. The latter are slightly curved in lateral view in several Tarachodidae, and the dorsal edges of the femora are correspondingly curved (Figs. 140, 141). The ventrally carinated furcasternite has been discussed by Henry (1932: 9) in some detail for Didymocorypha, Dysaules and Oxyophthalma, all of which are grass- or twigdwelling, slender Tarachodinae.

In combination with a more or less flattened body (e.g. Theopompa, Humbertiella, Tarachodes) this concealing behaviour enables such species to avoid revealing shadows on the surface (Edmunds 1972: 19, 20, 1974: figs. 2.8, 12.12). The effect is enhanced in some species by strongly widened wings, for instance in Theopompa (Chopard 1949c: fig. 37), Theopompella and Gonatista (Edmunds 1972: 29), but also in Choeradodis (mainly the females; e.g. Beier 1935b: fig. 2; Nel \& Roy 1996: fig. 7; Fig. 142). The costal area is enlarged and the costa comes to lie close to the surface when the mantid rests (see Edmunds 1972: 20, 1974: 13 and pl. 3, fig. d). This cryptic effect of bringing the edges of the body close to the substrate is widely known from invertebrates but also from vertebrates. Among the geckos (Gekkota: Gekkonidae), the genus Uroplatus Duméril, 1806 has skin frills along the body outline [U. fimbriatus (Schneider, 1797), U. henkeli Böhme \& Ibisch, 1990, and U. sikorae Boettger, 1913; see Svatek \& van Duin 2002: figs. 36, 40, 95, 122, 201-203, 210-212; Fig. 144 herein]. Another genus, 
Ptychozoon Kuhl, 1822, has large skin lobes on the sides of the head, legs, feet, body and tail. These structures are used for parachuting between tree trunks (e.g. Marcellini \& Keefer 1976). The lobes along the head and chest are usually not spread when the animals rest on trunks (Annandale 1905: 31; unlike shown in Chopard 1949c: fig. 23; pers. obs. in Ptychozoon lionotum Annandale, 1905). Therefore, they probably do not play a role in concealing the body (vs. Annandale 1905: 31). Those on the tail, however, additionally serve the purpose to avoid shadows (see Edmunds 1974: 12, 13 and fig. 2.9; see Fig. 143).

In Mantodea, the fore femora may be longer than the pronotum and protrude it anteriorly, for instance in Humbertiella and Theopompa (Fig. 137). They are as long as the pronotum in several Tarachodidae studied (Figs. 138, 139). In Paralygdamia, Pseudogalepsus (Fig. 140) and Pyrgomantis (Fig. 141) the femora do not reach the anterior end of the pronotum. Pyrgomantis is a savannah species with a high resemblance to grass blades (e.g. Gillon 1983: 293; Fig. 25). It has a very large and pointed head process (see characters 1, 2; Figs. 25, 431). When the mantids rest on blades of grass, their head is held in a hypognathous position and the process points craniad. The femora do not protrude the pronotum in cryptic posture but laterally conceal the mouthparts (e.g. Gillon \& Roy 1968: fig. 17; Gillon 1983: fig. 11.5; see Fig. 141). The pronotum and the dorsal surface of the head form a continuous shape, thus perfecting the mimetic effect (see Edmunds 1974: fig. 4.22; Fig. 25).

36. Fore femur, claw groove for containment of antero-distal tibial spur when leg is closed: present [1]; missing [2].

When the raptorial leg is folded at rest or prior to striking, the ventral surfaces of tibia and femur adjoin and their spines interlock. The antero-distal tibial spur then lies in a groove ("claw groove") on the antero-ventral surface of the fore femur. The groove is situated between the discoidal spines and the proximal antero-ventral spine of the femur (Figs. 148, 149, 150-153, 155-158, 160, 161, 163, 164, 166, 167, 169, 170, 172, 174, 180, 196, 202, 208, 209, 211, 212, 215-218, 220, 221, 227-232). A similar structure is missing in Blattodea (Figs. 145, 146, 286). The groove is apparently missing in some taxa with strongly altered tibial morphology, for instance in Amorphoscelinae and Oligonicinae. This likely represents a secondary reduction because the extant members of the basal branchings do possess such grooves [e.g. Mantoida (Figs. 150-153, 172), Chaeteessa (Figs. 148, 149, 170, 418), Metallyticus (Figs. 414-417). In the SEM images, for instance of Amorphoscelis sp. (Figs. 186, 188), the remnants of the groove can be identified between the remnants of the reduced antero-ventral spines and the strongly elevated ridge carrying the only persisting discoidal spine. The groove 


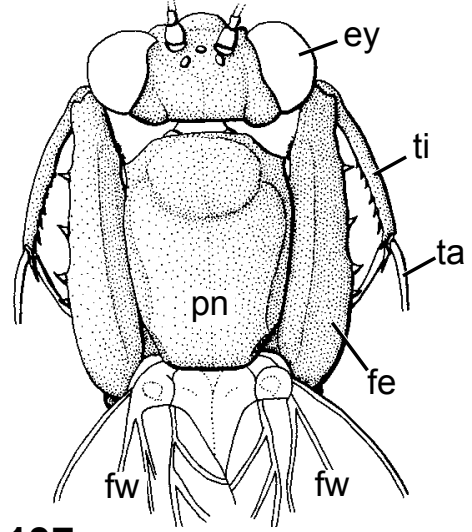

137

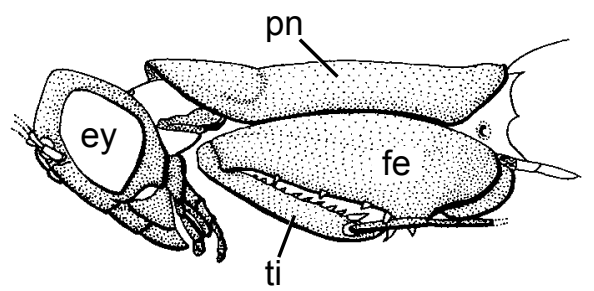

140

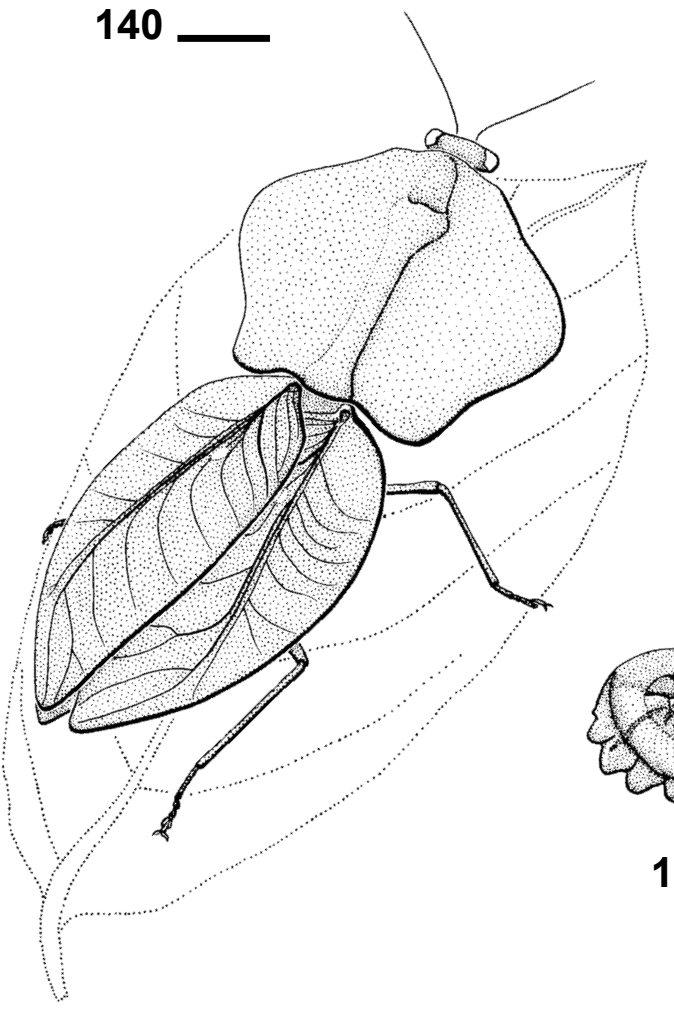

142

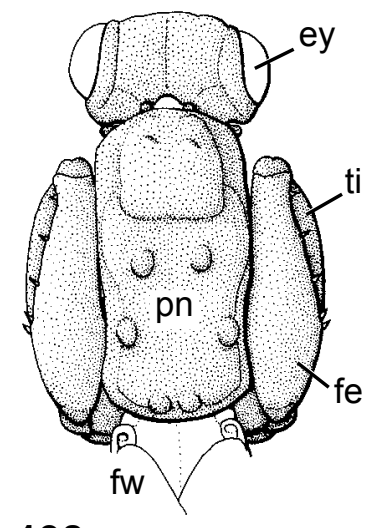

138

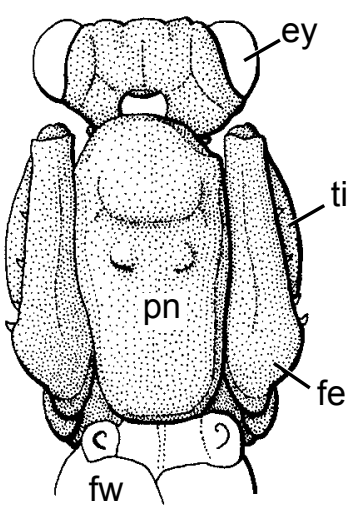

139

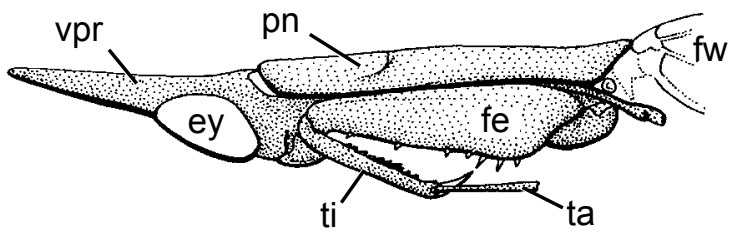

141

Figs. 137-144: Structures supporting the avoidance of lateral shadows. 137-139: Close fitting of lateral edges of pronotum and dorsal edges of fore femora, dorsal view. 137: Theopompa sp. 1, §̂. 138: Tarachodes sp. 2,, . 139: Tarachodes sp. 3, ô. 140, 141: Close fitting of lateral edges of pronotum and dorsal edges of fore femora,

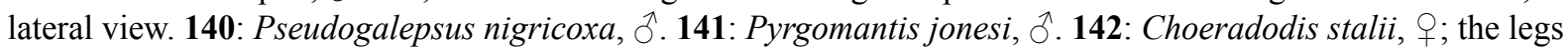
can be entirely concealed under the widened tegmina when the insect is pressing its body against the surface. 143: Ptychozoon lionotum, $\widehat{\sigma}$, habitus; note lobes on tail (arrow); the remaining skin flaps (lying close to the body, not visible in drawing) are used for parachuting from tree to tree, whereas a potential secondary function of the lobes on the tails is to avoid shadows and conceal the shape of the tail. 144: Uroplatus sp., head; note the frills along the chin (arrow). Figs. 142-144 redrawn from photographs. - Scale bar $=2.0 \mathrm{~mm}$; scales of Figs. 142144 not available.

Abbreviations: $\mathbf{e y}=$ compound eye $; \mathbf{f e}=$ femur; $\mathbf{f w}=$ fore wing $($ tegmen $) ; \mathbf{p n}=$ pronotum; $\mathbf{t a}=\operatorname{tarsus} ; \mathbf{t} \mathbf{i}=$ tibia; $\mathbf{v p r}=$ vertical process (fastigium). 
is shallow and narrow due to lateral compression of the femur, but it is nonetheless present. The same is true for Oligonicinae. In this group, the femur has become very slender, thus narrowing the area between the proximal antero-ventral spines and the discoidal spines. The SEM image (Figs. 169, 202) reveals a very slender and narrow groove in this area, in which the tibial spur rests when the leg is folded. It is much more distinct in the closely related Haania. Therefore, all Mantodea are encoded as state 1 in this analysis.

37. Fore femur, claw groove, position: at the base of the femur [1]; on the anterior surface in the proximal third or the femur [2]; medially on the anterior surface of the femur [3]; distally of the middle of the femur [4].

In extant Mantodea the position of the groove varies greatly. In the extant members of the basal branchings of the mantodean tree, such as Mantoida (Figs. 150-153, 172), Chaeteessa (Figs. 148, 149, 170, 418), and Metallyticus (Figs. 414-417), the claw groove is positioned proximally on the femur or even entirely at its proximal end, as can be seen in Metallyticus. More derived taxa including Amorphoscelidae (Figs. 178, 180, 182, 196) show a distinct apical shift of the groove. Among the few exceptions are, for instance, the Acanthopidae Callibia and Raptrix from the Neotropics which have their claw grooves in a position near the proximal end of the femur. Acanthopidae are primarily earless and have been found to branch off quite early in the molecular phylogenetic tree (Svenson $\&$ Whiting 2009). Therefore, the proximal position of the claw groove may be a plesiomorphic trait in this group (but see below).

The distal position of the claw groove could be interpreted to be correlated with a supposed shortening of the tibia in derived Mantodea, as proposed by Vršanský (2002: 14 and node 26 in fig. 27). However, this assumption is probably incorrect. The tibia is only seldom distinctly shortened [exceptions being, for instance, Thesprotia (Fig. 163, 165), Compsothespis (Fig. 19), and Amorphoscelis] (Beier 1968a: 22). It is usually the femur, proximal to the spine-bearing area, that becomes elongated, whereas the distal part of the femur and the tibia have been shifted distad as an entity. Therefore, the claw groove has seemingly moved distad. All Toxoderidae studied here show a unique distinct elongation of both femur and tibia (with the claw groove being located in a proximal position, Fig. 207). The elongation of both leg parts can also be deduced from the comparatively wide spaces between the femoral and tibial spines.

One might assume a position of the claw groove in the proximal third of the femur (not near its proximal end) in stem representatives of Mantodea, as in cockroaches the tibia of the fore legs is usually shorter than the femur. This would imply a shortening of the fore femora or an initial elongation of the tibia 

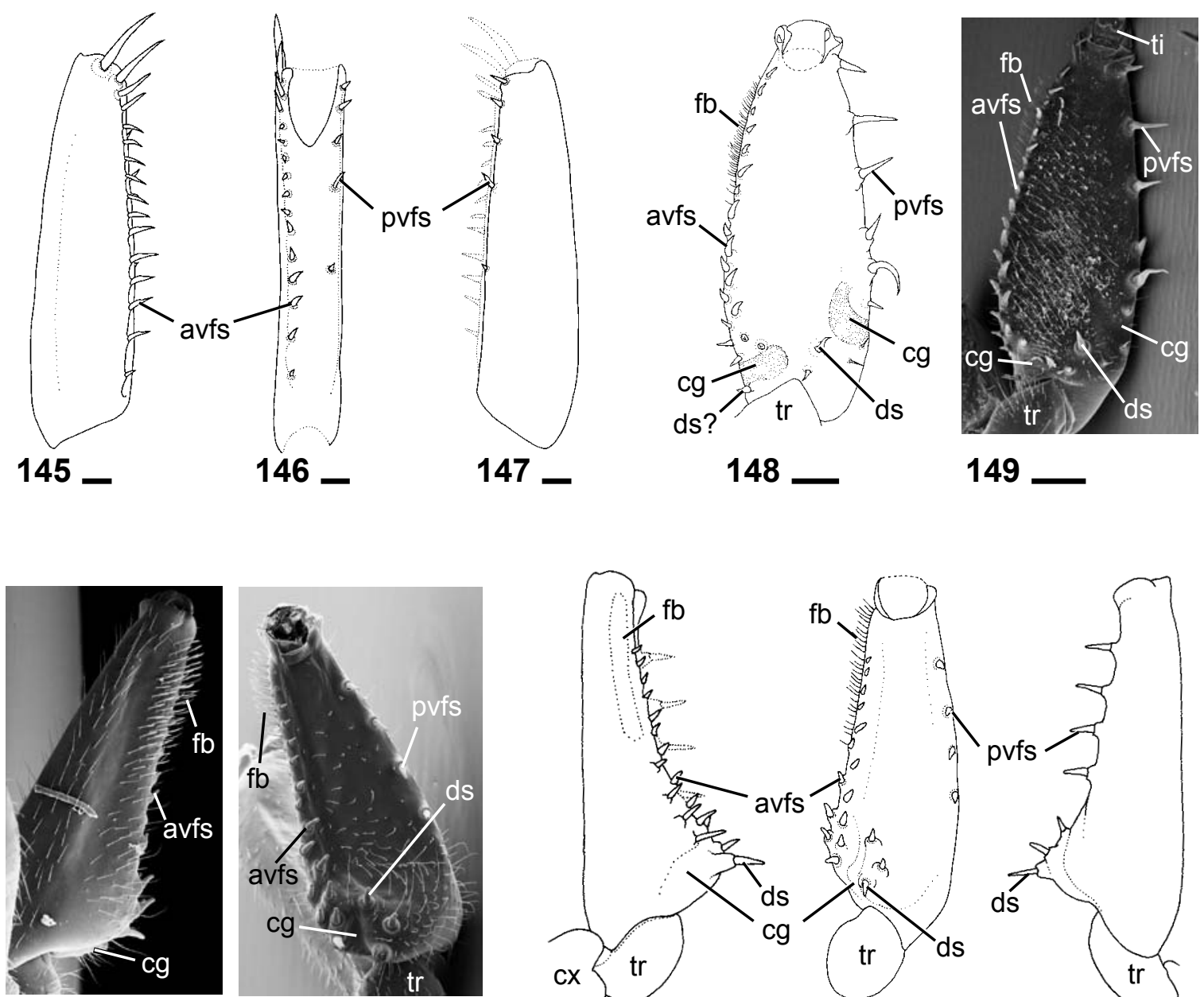

150

151

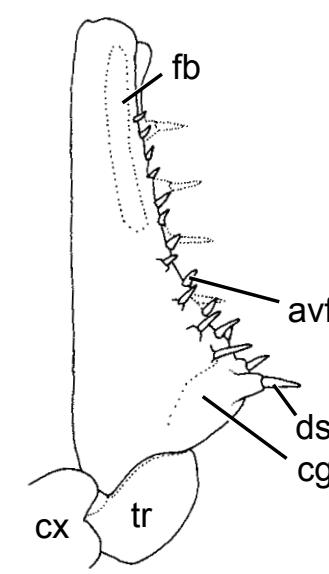

152

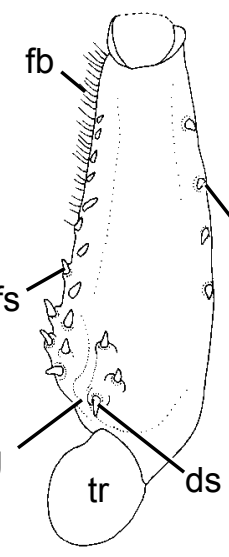

153

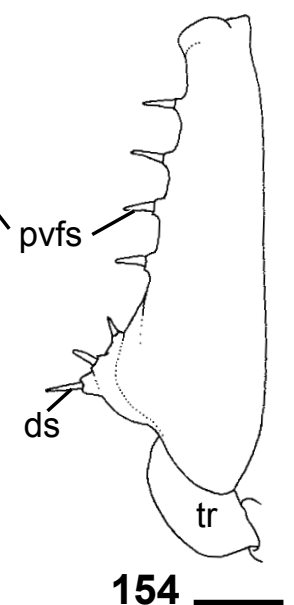

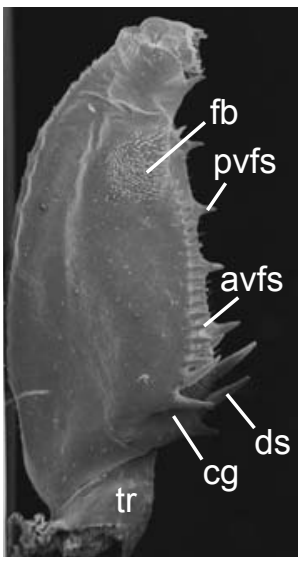

155

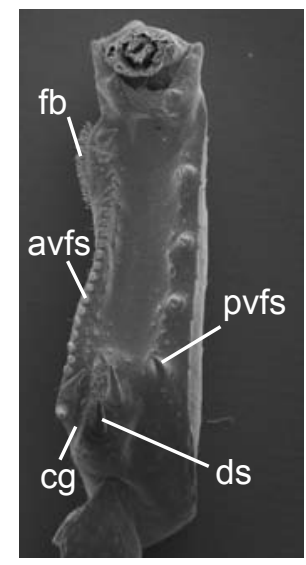

156

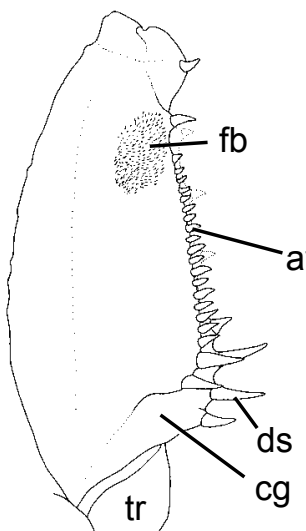

157

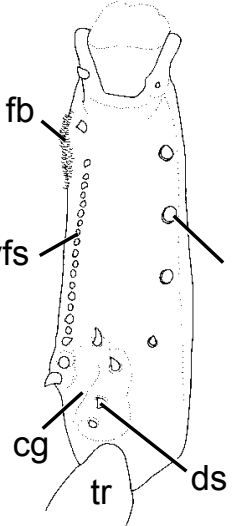

158

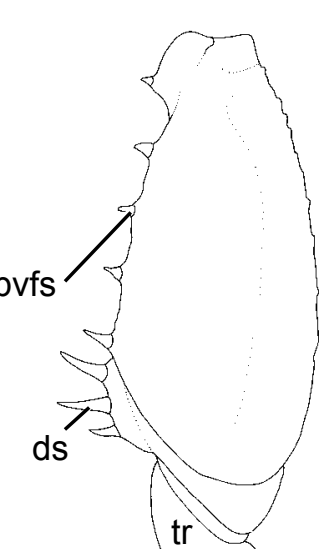

159

Figs. 145-159: Fore femoral morphology of selected taxa. 145-147: Periplaneta americana, $\bigcirc$, right fore femur (mirrored). 145: Anterior view. 146: Ventral view. 147: Posterior view. 148: Chaeteessa valida, ${ }^{\text {, }}$ left fore femur, ventral view. 149: Chaeteessa cf. valida, sex unknown, SEM picture of femur, ventral view; note the presence of the "second claw groove". 150-154: Mantoida maya, ふ̋, right fore femur (mirrored). 150: SEM image, anterior view. 151: SEM image, ventral view. 152: Anterior view. 153: Ventral view. 154: Posterior view. 155-159: Eremiaphila sp. 1,, , left fore femur. 155: SEM image, anterior view. 156: SEM image, ventral view. 157: Anterior view. 157: Ventral view. 158: Posterior view. - Scale bar $=0.5 \mathrm{~mm}$.

Abbreviations: avfs = antero-ventral fore femoral spine; $\mathbf{c g}=$ claw groove; $\mathbf{c x}=$ coxa; $\mathbf{d s}=$ discoidal $\mathbf{s p i n e} ; \mathbf{f b}=$ fore femoral grooming device (femoral brush); pvfs = postero-ventral fore femoral spine; tr = trochanter. 
(and therefore a proximal shift of the claw groove) after the split between Mantodea and Blattodea, possibly due to the beginning elongation of the fore coxae, followed by a secondary elongation of the femur in "higher" Mantodea (except Toxoderidae). In this case, state 1 in Callibia and Raptrix as mentioned above might indicate a secondary shortening of the femur or a primary elongation of the tibia. However, further extensive morphological, ontogenetic and palaeontological studies are necessary in order to investigate these hypotheses.

38. Fore femur, antero-distal grooming device ("femoral brush"): present [1]; missing [2].

Mantodea exhibit a fore femoral grooming device consisting of a patch of feathershaped setae (e.g. Figs. 170-183). They are usually located closely together in a small area on the antero-distal surface of the femur (Grimaldi 2003: characters $12,25)$. The description and function of the grooming device have been summarized by Ramsay (1990: 36). It is used for cleaning the head and the compound eyes (Beier 1968a: 22; Ramsay 1990: 36). It is present in all extant Mantodea (Ramsay 1990: 36; Grimaldi 2003: 40) and at least in some fossil specimens (Grimaldi 2003: tab. 3). Plesiomorphically, it consists of slightly thickened setae (as in †Burmantis Grimaldi, 2003, see Grimaldi 2003: 15), whereas in derived taxa the setae are scale-like and flattened, as in all extant taxa (Grimaldi 2003: character 25 and figs 25 B, D; see Figs. 170-183 for several taxa including Mantoida, Chaeteessa and Metallyticus). A structure homologous to the femoral brush is missing in Blattodea, therefore the brush is autapomorphic for Mantodea.

39. Fore femur, antero-distal grooming device ("femoral brush"), position: flat, slightly elevated at most [1]; on a distinct elevation [2].

The grooming device may lie flat on the anterior surface of the femur (in ventral view) or the setae may stand on a distinct elevation that is discernible in ventral view of the femur (see Fig. 196 for Paraoxypilus sp.).

40. Fore femur, antero-distal grooming device ("femoral brush"), grooming setae, organization: standing closely together, forming a clearly defined area of the grooming device [1]; standing far apart, area of grooming device not being clearly defined [2].

The organization of the setae of the grooming device as a small patch of narrowstanding setae is similar throughout most extant Mantodea (Figs. 170, 174, 176, 180). The setae stand further apart in some taxa, e.g. Amorphoscelinae (Fig. 178) and Perlamantinae (Fig. 182), a situation resembling the femoral brush in early instar nymphs (Figs. 437-440). 


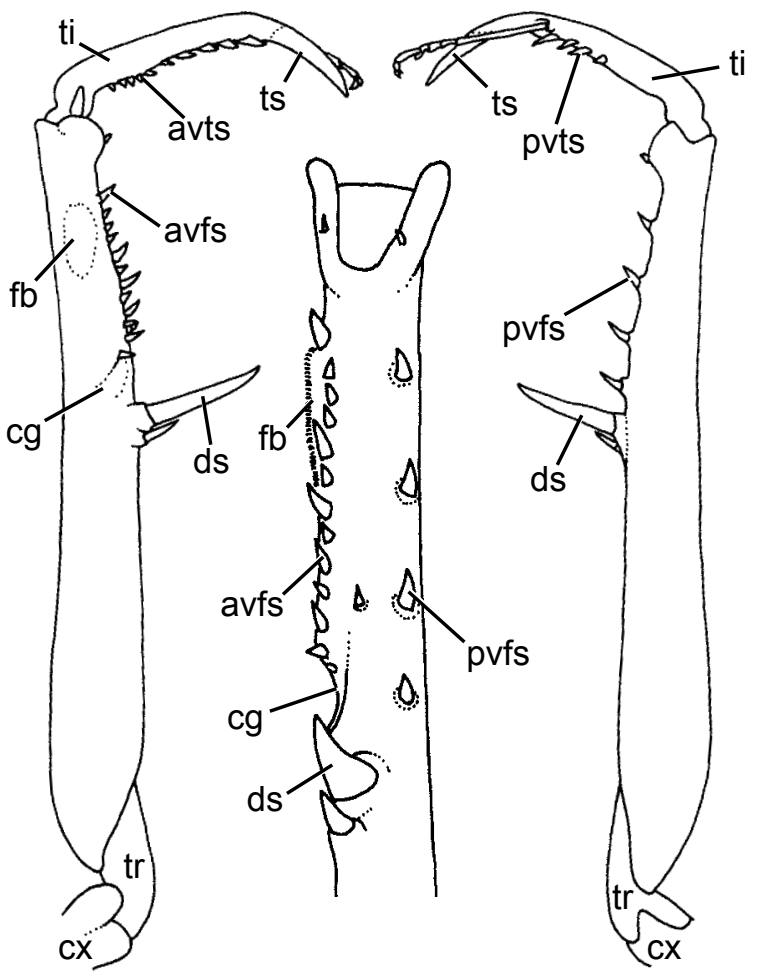

160

161

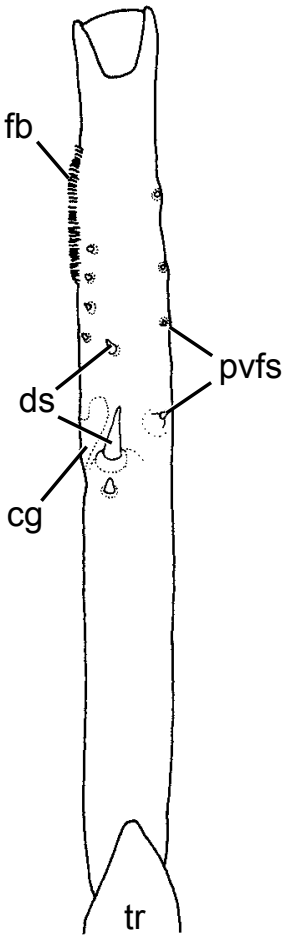

167

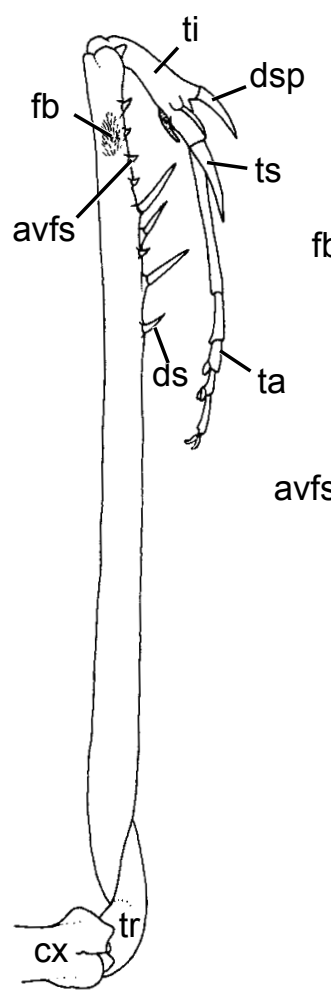

163 - $\quad 164$

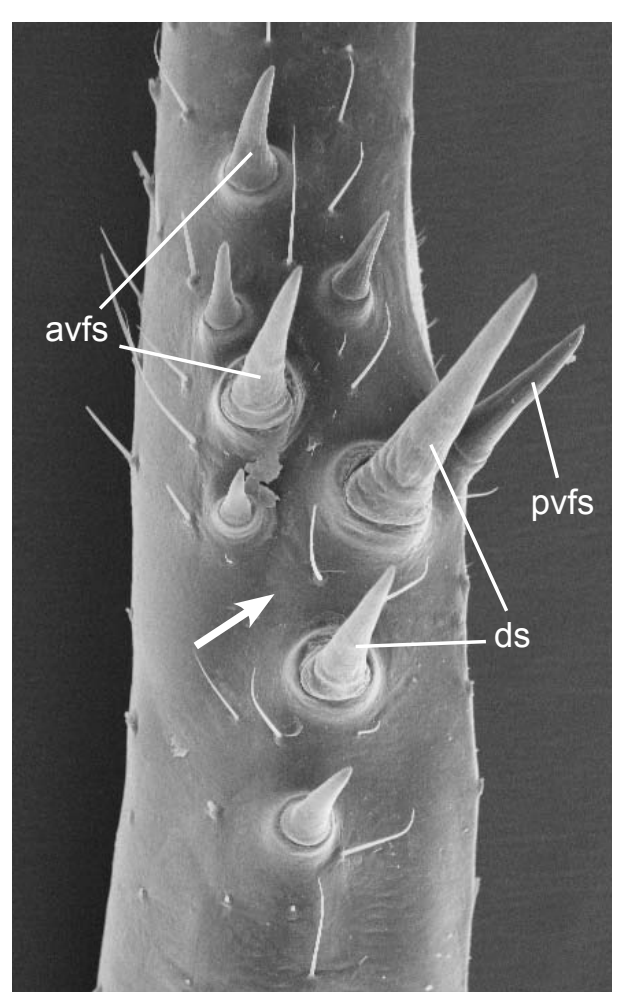

169

Figs. 160-169: Foreleg morphology of selected taxa. 160-162: Hoplocorypha sp., + , left foreleg. 160: Anterior view. 161: Detail of ventral view. 162: Posterior view. 163-165: Thesprotia graminis, $\partial^{\lambda}$, left foreleg. 163: Anterior view. 164: Detail of ventral view. 165: Posterior view. 166-168: Compsothespis anomala, $\bigcirc$, right fore femur (mirrored). 166: Anterior view. 167: Ventral view. 168: Posterior view. 169: Oligonicinae sp., §̂, right fore femur (mirrored), detail, SEM image; arrow indicates remnant of claw groove. - Scale bar $=0.5 \mathrm{~mm}$.

Abbreviations: avfs = antero-ventral fore femoral spine; $\mathbf{c g}=$ claw groove; $\mathbf{c x}=$ coxa; $\mathbf{d s}=$ discoidal spine; $\mathbf{d s p}$ $=$ dorsal spine; $\mathbf{f b}=$ fore femoral grooming device (femoral brush); $\mathbf{p v f s}=$ postero-ventral fore femoral spine; ta $=$ tarsus; $\mathbf{t i}=$ tibia; $\mathbf{t r}=$ trochanter. 

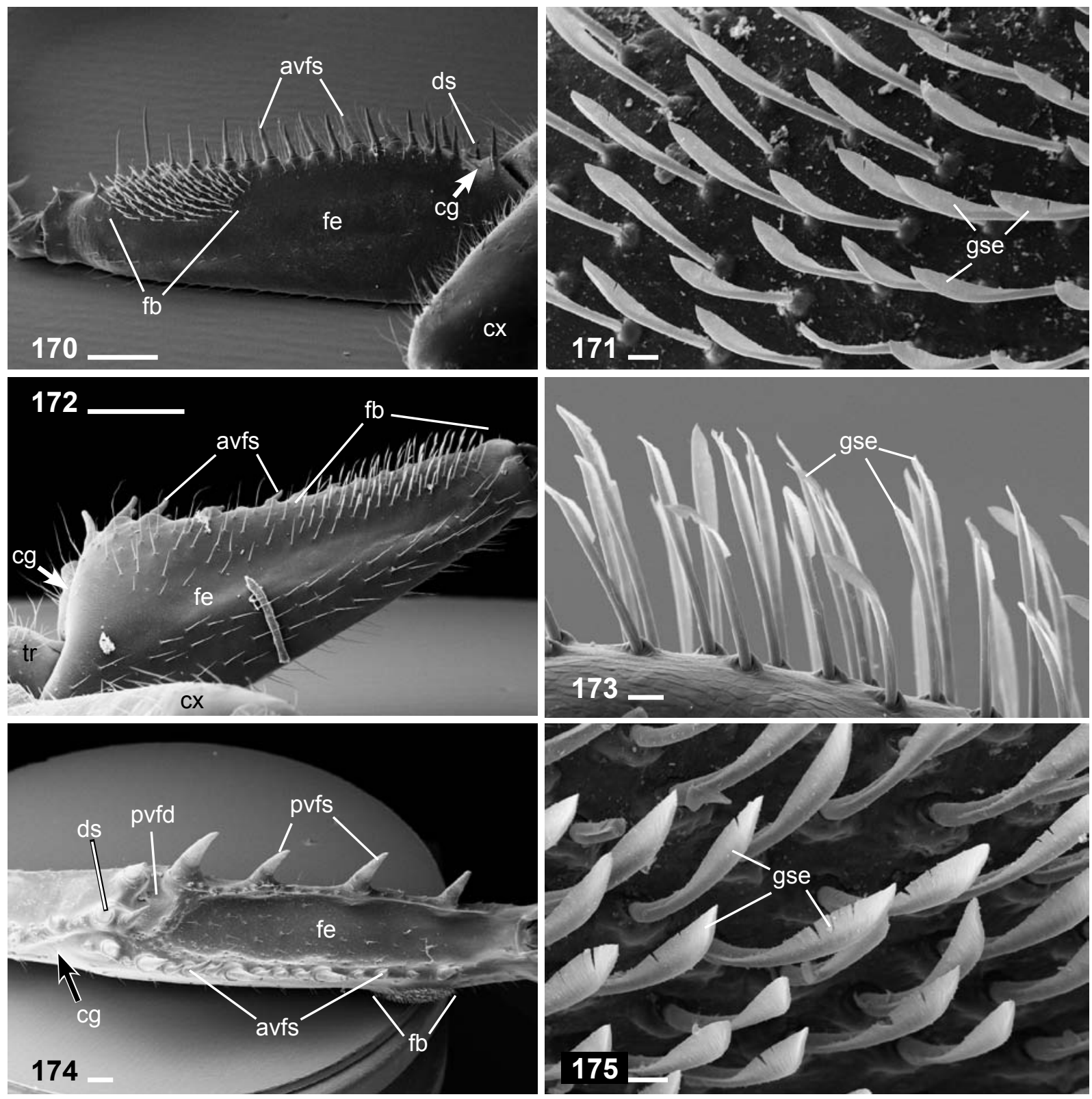

Figs. 170-175: Antero-distal fore femoral grooming devices („femoral brushes“). 170: Chaeteessa cf. valida, sex unknown, left fore femur, anterior view. 171: Same specimen, detail of femoral brush. 172: Mantoida maya, ठิ, right foreleg, anterior view. 173: Same specimen, detail of femoral brush. 174: Blepharopsis mendica, + , right fore femur, ventral view. 175: Same specimen, detail of femoral brush. - Scale bar $=0.5 \mathrm{~mm}$ (left column); 0.02 mm (right column).

Abbreviations: avfs = antero-ventral fore femoral spine; $\mathbf{c g}=$ claw groove; $\mathbf{c x}=$ coxa; $\mathbf{d s}=$ discoidal $\mathbf{s p i n e} ; \mathbf{f b}=$ fore femoral grooming device (femoral brush); fe = femur; gse = grooming setae; pvfd = proximo-ventral fore femoral depression; pvfs = postero-ventral fore femoral spine; $\mathbf{t r}=$ trochanter.

The only taxon exhibiting a strongly different organization is Mantoida, in which the entire anterior surface of the femur is equipped with long setae (Figs. $150,151,172,440)$. The feather-shaped grooming setae are not assembled in a small, antero-distal patch, but have larger spaces in between. The grooming device extends across the entire distal half of the femur and proximally merges with further, long setae, that are not flattened and feather-shaped (Figs. 150, 172). A similar group of long setae on the proximal anterior surface of the fore 

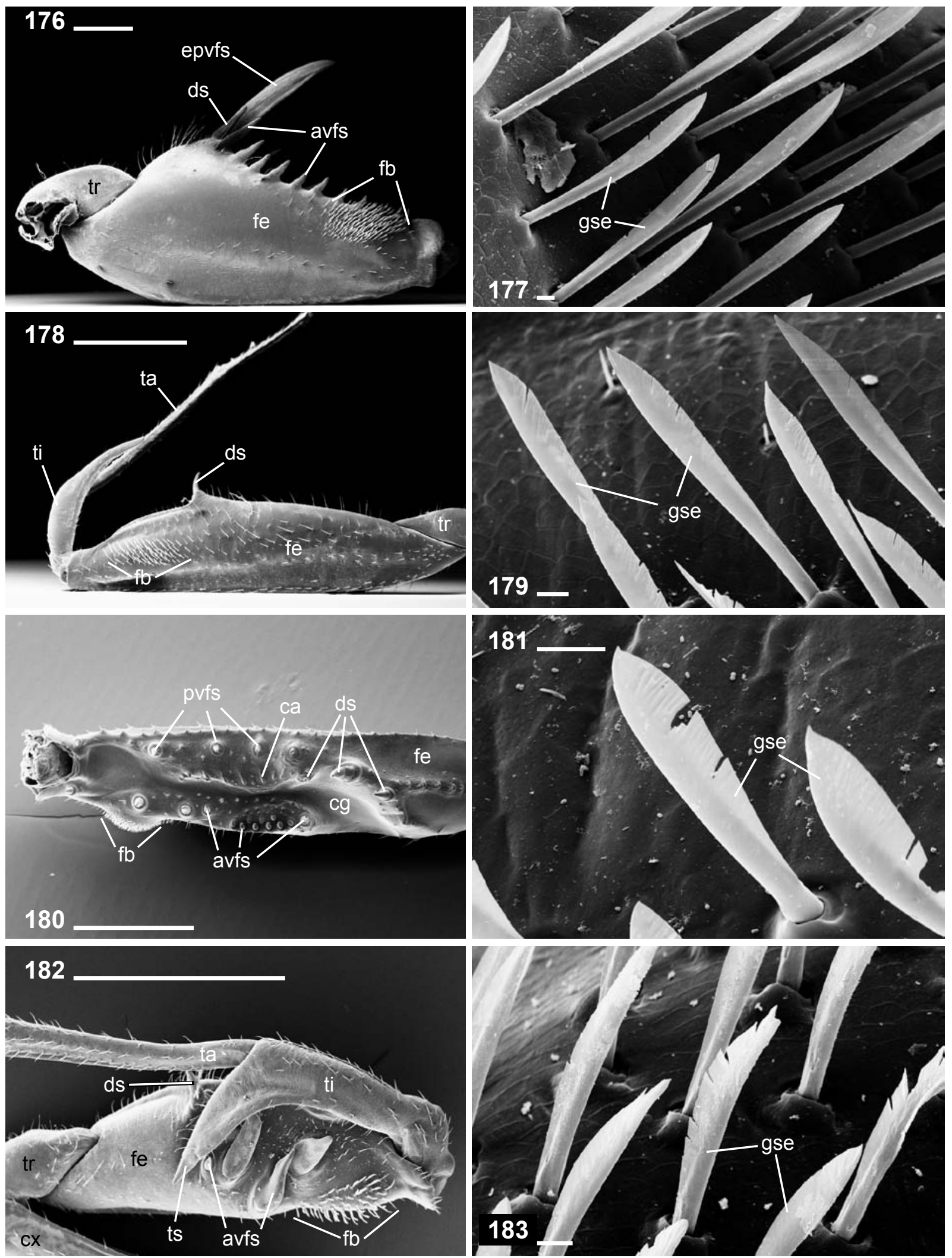

Figs. 176-183: Antero-distal fore femoral grooming devices („femoral brushes“). 176: Metallyticus splendidus, ${ }^{\circ}$, right fore femur, anterior view. 177: Same specimen, detail of femoral brush. 178: Amorphoscelis sp., $\widehat{O}$, left foreleg, anterior view. 179: Same specimen, detail of femoral brush. 180: Paraoxypilus sp. 1, $\hat{\jmath}$, left fore femur, ventral view. 181: Same specimen, detail of femoral brush. 182: Perlamantis allibertii, $\hat{\partial}$, right foreleg, antero-ventral view. 183: Same specimen, detail of femoral brush. - Scale bar $=1.0 \mathrm{~mm}$ (left column); $0.01 \mathrm{~mm}$ (right column). Abbreviations: avfs = antero-ventral fore femoral spine; $\mathbf{c a}=$,crushing area“; $\mathbf{c g}=$ claw groove; $\mathbf{c x}=\mathbf{c o x a} ; \mathbf{d s}=$ discoidal spine; $\mathbf{e p v f s}=$ elongated postero-ventral fore femoral spine; $\mathbf{f b}=$ fore femoral grooming device (femoral brush); $\mathbf{f e}=$ femur; $\mathbf{g s e}=$ grooming setae; $\mathbf{p v f s}=$ postero-ventral fore femoral spine; $\mathbf{t a}=$ tarsus; $\mathbf{t i}=\operatorname{tibia} ; \mathbf{t r}=$ trochanter; ts $=$ tibial spur. 
femora was mentioned for $\uparrow$ Ambermantis wozniaki Grimaldi, 2003. Grimaldi (2003: 10) described the grooming device in this fossil as "a patch of fine setulae (the 'brush') on the mesal surface near distal end", therefore it can be assumed that the grooming setae are probably standing distinctly closer in the fossil than in Mantoida. As the dense condition of the grooming device was already present in Cretaceous fossils, its wide extension in Mantoida may either be derived for the genus or it may be the plesiomorphic condition which would support a potentially very old age of the lineage leading to Mantoida, possibly older than the Cretaceous.

41. Fore femur, antero-ventral spines: present [1]; missing [2].

The femur of the raptorial legs carries an antero-ventral row of spines in nearly all extant Mantodea (e.g. Beier \& Jaus 1933: 130; Beier 1968a: 22; Roy 1999: 27). They are missing only in Amorphoscelinae, although remnants of the spines are visible in SEM images (see Figs. 184, 186, 187). The antero-ventral femoral spines are strongly reduced in Compsothespis (Fig. 166, 167).

A curious case is the raptorial foreleg of Oligonicinae sp. in this study. The SEM images reveal an intraindividual sequence of reduction of spines (Figs. 202-206). In the distal part of this specimen's femur three successive anteroventral femoral spines show increasing degrees of reduction (see also discussion in chapter 4.6.10). This may provide an rare insight into the reduction process in mantodean evolution.

42. Fore femur, postero-ventral spines: present [1]; missing [2].

The fore femur carries a postero-ventral row of spines in nearly all extant Mantodea (e.g. Beier \& Jaus 1933: 130; Beier 1968a: 22; Roy 1999: 27). Only in Amorphoscelinae and Perlamantinae they are missing, although remnants of the spines were identified in the SEM images of Amorphoscelis (Figs. 184, 186, 188, 189) and Perlamantis (Figs. 193-195). The situation is ambiguous in Amorphoscelis because the putative remnants of the postero-ventral spines lie in line with the discoidal spine and its preceding remnant (see Figs. 184, 186-189). However, the femur of Amorphoscelinae is laterally compressed and thereby ventrally gained a slender, blade-like ridge. The remnants lie on the posterior side of this ridge (Fig. 188). I herein provisionally interpret them as the remnants of the postero-ventral spines, but further extensive studies on the foreleg morphology and postembryonic development of Amorphoscelinae are indispensable to homologize the remnants with certainty. If they actually are reduced posteroventral spines, this might indicate that the single discoidal spine, too, may have 

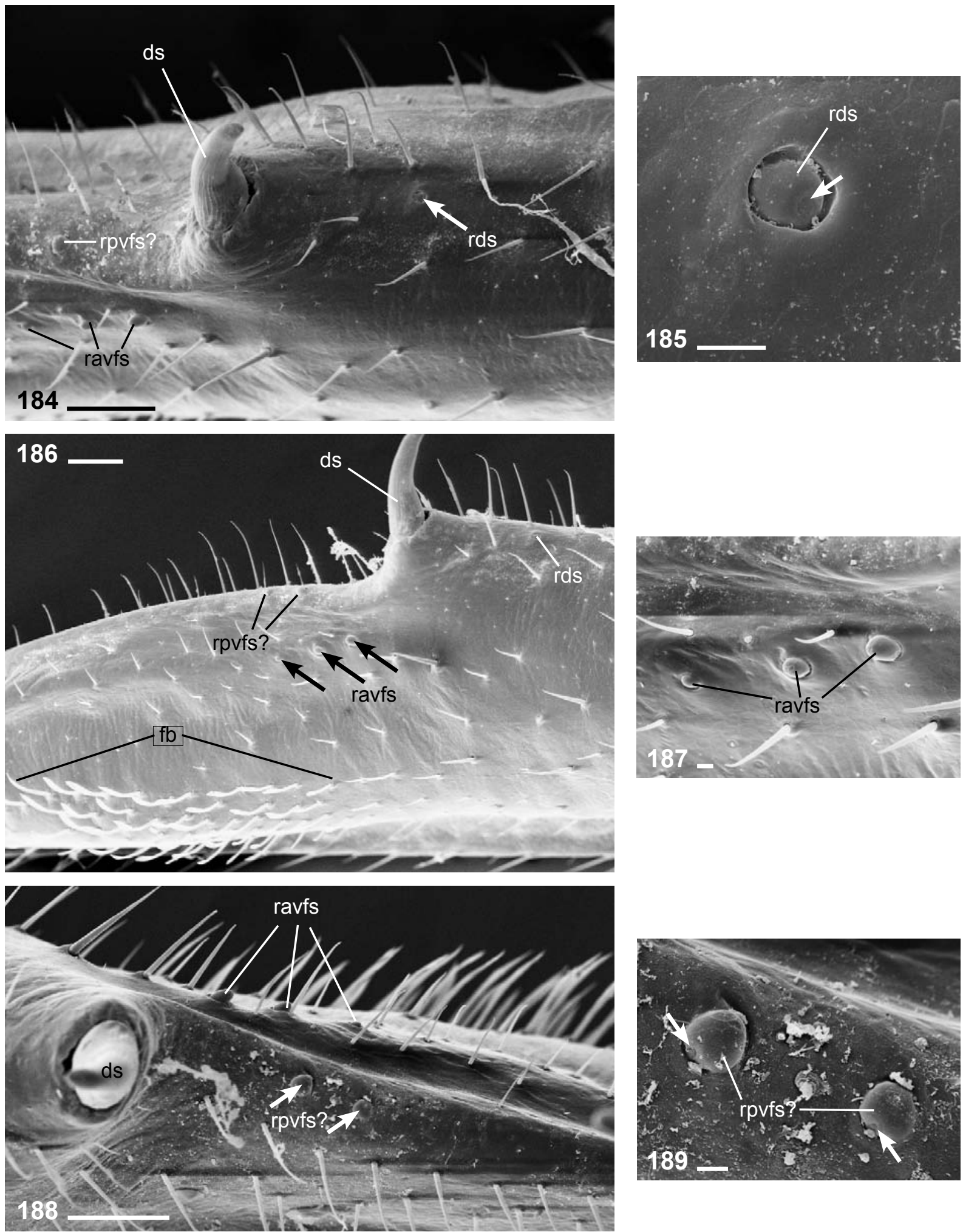

Figures 184-189: Amorphoscelis sp., $\widehat{\jmath}$, remnants of fore femoral spines on left fore femur. 184: Ventral view; arrow indicates remnant of discoidal spine shown in 185. 185: Remnant of discoidal spine, detail; arrow indicates sickle-shaped structure. 186. Anterior view; arrows indicate remnants of antero-ventral femoral spines shown in 187. 187: Remnants of antero-ventral femoral spines, detail. 188: Ventral view; remnants of posteroventral femoral spines; arrows indicate remnants of spines shown in 189. 189: Remnants of postero-ventral femoral spines, detail; arrows indicate sickle-shaped structures. $\leftarrow$ distal (184-187), $\rightarrow$ distal (188, 189). - Scale bar $=0.1 \mathrm{~mm}$ (left column); $0.01 \mathrm{~mm}$ (right column).

Abbreviations: $\mathbf{d s}=$ discoidal spine; $\mathbf{f b}=$ fore femoral grooming device (femoral brush); ravfs $=$ remnant of antero-ventral fore femoral spine; rds = remnant of discoidal spine; rpvfs? = putative remnant of postero-ventral fore femoral spine. 
originated from a postero-ventral spine, regarding its position. If, however, the remnants are the remains of discoidal spines, no traces of postero-ventral spines are left on the femora of Amorphoscelinae.

In some taxa, the spines are strongly reduced in size, among them Heteronutarsus, Paraoxypilinae (Figs. 196, 201) and Compsothespis (Figs. 167, 168). In the latter, the spines are still slightly pointed but apart from Amorphoscelinae and Perlamantinae Compsothespis shows one of the strongest overall reduction of postero-ventral spines in this study. Thesprotia graminis has a single posteroventral femoral spine left, which, however, is normally shaped while the remaining ones are completely reduced (Fig. 164).

43. Fore femur, spination, position: arranged in two rows, antero-ventral spines standing close [1]; arranged in two rows, antero-ventral spines standing widely separated from each other [2]; arranged in two rows, with the proximal antero-ventral spines being short, blunt and standing in an alternating position closely together [3]; missing except for a single discoidal spine [4]; only four antero-ventral spines present, postero-ventral spines are missing [5]; arranged in two rows of very small but still pointed and elevated remnants of spines [6].

The fore-femoral spination pattern is very similar throughout the greater part of the species studied herein. Most extant Mantodea exhibit the "typical" pattern of foreleg spination (see, for instance, Beier 1964a: fig. 8a, 1968a: fig. 25a; Roy 1999: fig. 2.11; Figs. 45, 47, 49). The spines are arranged in two parallel rows, and the antero-ventral spines are usually standing closely together (state 1).

The situation in Toxodera (Fig. 207) and Calamothespis differs from the "typical pattern" in the distance between the antero-ventral spines. The femora are long and slender, and the spines stand far apart from each other (state 2). This situation is present in all Toxoderini (sensu Roy 2009a) and may be apomorphic for Toxoderidae or a subgroup within Toxoderidae.

In Paraoxypilinae, the fore-femoral spines are arranged in two rows. However, the proximal antero-ventral spines stand very closely in two parallel rows (state 3; see also characters 43, 44 and Figs. 196, 197, 199, 201, 218). Their shape strongly differs from the remaining antero-ventral spines in that they are blunt and much shorter than the rest (Figs. 197, 199). This pattern (and the corresponding pattern of the fore tibial spines, see characters 72,63 , and 65) is characteristic and constant within the Paraoxypilinae studied herein (see also Giglio-Tos 1913: figs. 3c ( $ठ$ and ( ), 4c; Sjöstedt 1918: pl. 1: fig. 1d, pl. 2, figs. 1d, 2d, pl. 3: fig. 7; Beier 1929: fig. 5; Milledge 1990: fig. 10). Specimens of Exparoxypilus, Nesoxypilus Beier, 1965, Myrmecomantis Giglio-Tos, 1913, Cliomantis, and Metoxypilus were not available for this study but the literature provides descriptions of the forelegs of some of these genera. The raptorial leg 

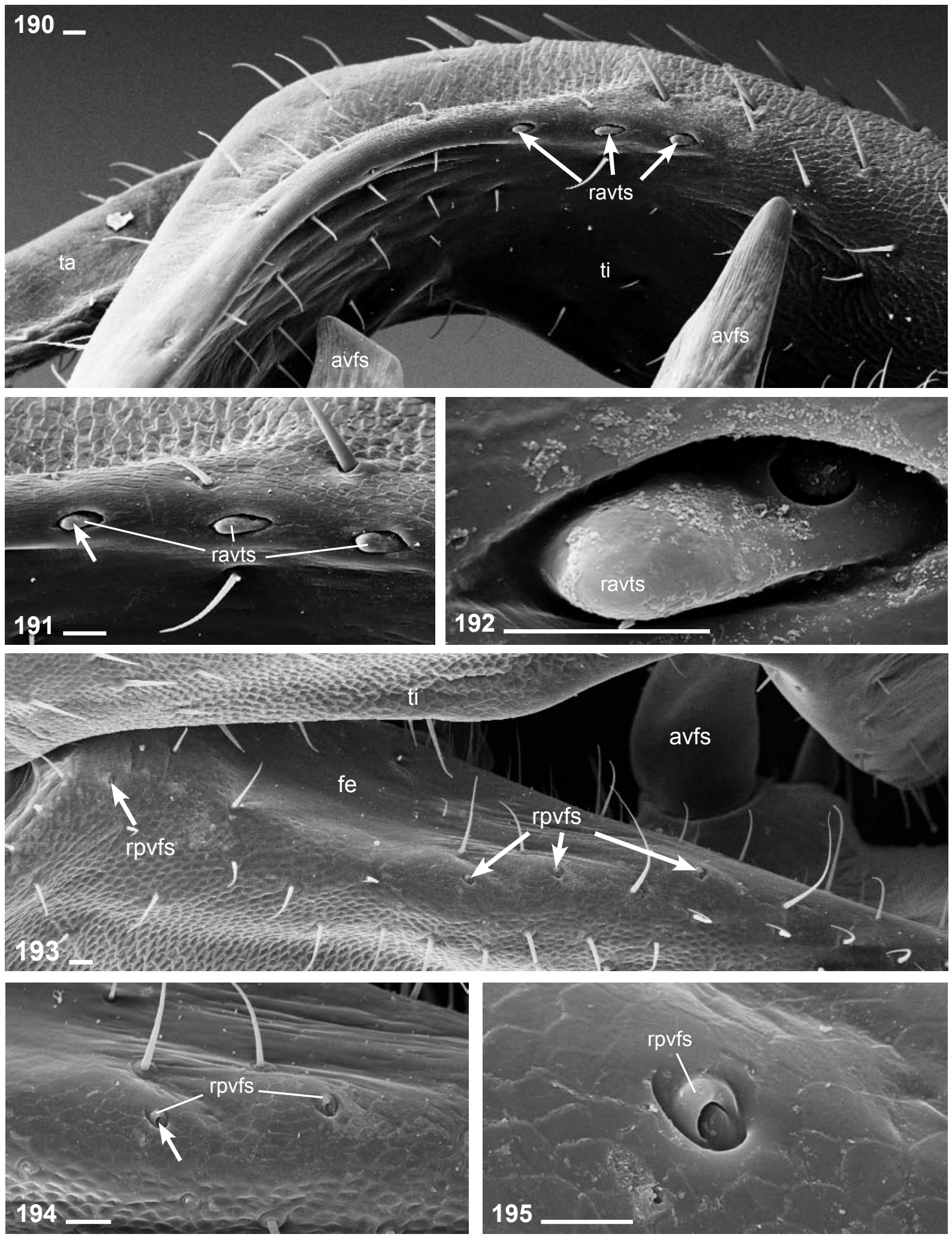

Figs. 190-195: Perlamantis allibertii, đ̂, remnants of spines on forelegs. 190: Right fore tibia, oblique anteroventral view; arrows indicate remnants of antero-ventral fore tibial spines. 191: Remnants of antero-ventral fore tibial spines, overview; arrow indicates spine shown in 192. 192: Remnant of antero-ventral fore tibial spine, detail. 193. Right fore femur, posterior view; arrows indicate remnants of postero-ventral fore femoral spines. 194: Remnants of postero-ventral femoral spines, overview; arrow indicates spine shown in 195. 195: Remnant of postero-ventral femoral spine, detail. $\leftarrow$ distal end of tibia (190-192); $\leftarrow$ distal end of femur (190, 191, 193, 194). - Scale bar =0.02 mm (190-194); $0.01 \mathrm{~mm}(192,195)$.

Abbreviations: avfs = antero-ventral fore femoral spine; $\mathbf{f e}=$ femur; ravts $=$ remnant of antero-ventral fore tibial spine; $\mathbf{r p v f s}=$ remnant of postero-ventral fore femoral spine; $\mathbf{t a}=\operatorname{tarsus} ; \mathbf{t} \mathbf{i}=$ tibia. 

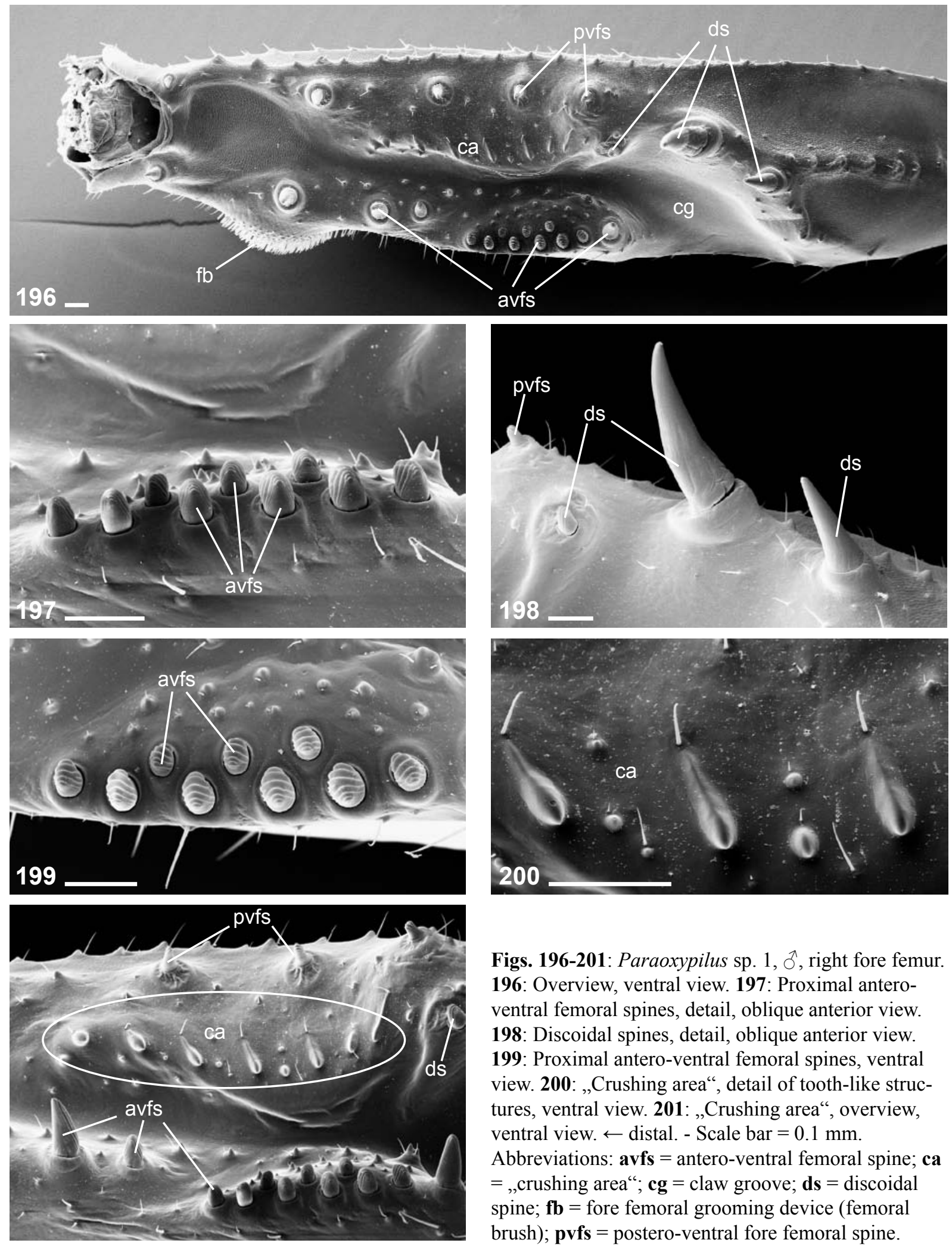

Figs. 196-201: Paraoxypilus sp. 1, $\widehat{\jmath}$, right fore femur. 196: Overview, ventral view. 197: Proximal anteroventral femoral spines, detail, oblique anterior view. 198: Discoidal spines, detail, oblique anterior view. 199: Proximal antero-ventral femoral spines, ventral view. 200: „Crushing area“, detail of tooth-like structures, ventral view. 201: „Crushing area“, overview, ventral view. $\leftarrow$ distal. - Scale bar $=0.1 \mathrm{~mm}$. Abbreviations: avfs = antero-ventral femoral spine; ca $=$, ,crushing area“; $\mathbf{c g}=$ claw groove; $\mathbf{d s}=$ discoidal spine; $\mathbf{f b}=$ fore femoral grooming device (femoral brush); pvfs = postero-ventral fore femoral spine.

of Cliomantis was drawn by Tindale (1923: fig. 374) and obviously shares the morphological peculiarities with the paraoxypiline taxa studied herein. The raptorial leg of Nesoxypilus was shown by Milledge (1990: fig. 10), whereas that of Exparoxypilus was depicted by Beier (1929: fig. 5). All three drawings leave no doubt that the forelegs are very similar to those of Paraoxypilus and Gyromantis, thus strongly supporting the spination pattern of state 3 as an autapomorphy for a monophyletic Paraoxypilinae. 

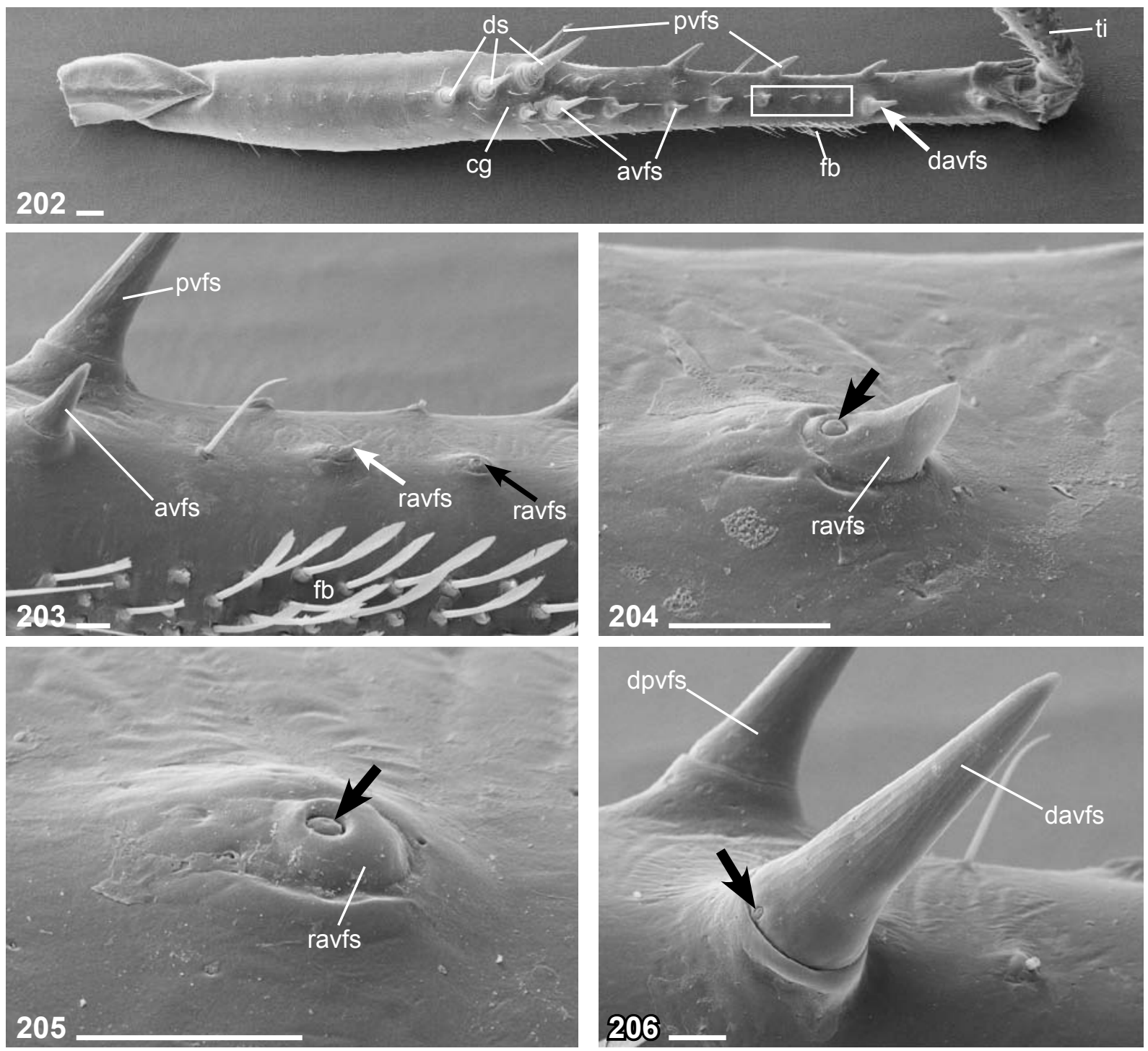

Figs. 202-206: Oligonicinae sp., $\widehat{\jmath}$, right fore femur. 202: Ventral overview; rectangle indicates area shown in 203; arrow indicates spine shown in 206. 203: Sequential reduction of distal antero-ventral fore femoral spines, overview in oblique anterior view; white arrow indicates remnant shown in 204; black arrow indicates remnant shown in 206. 204: Slightly reduced proximal spine, detail. 205: Strongly reduced distal spine, detail. 206: Distal antero-ventral femoral spine, detail. Note the indentation at the base of the spine which is also present in reduced spines (indicated by arrows in Figs. 204-206). $\rightarrow$ distal. - Scale bar $=0.1 \mathrm{~mm}(202,203), 0.02 \mathrm{~mm}(204-206)$.

Abbreviations: avfs = antero-ventral fore femoral spine; $\mathbf{c g}=$ claw groove; davts $=$ distal antero-ventral fore tibial spine; $\mathbf{d p v t s}=$ distal postero-ventral fore tibial spine; $\mathbf{d s}=$ discoidal spine; $\mathbf{f b}=$ fore femoral grooming device (femoral brush); pvfs = postero-ventral fore femoral spine; ravfs = remnant of antero-ventral fore femoral spine; $\mathbf{t i}=$ tibia.

The femoral spination is missing except for a single discoidal spine (state 4; see also character 54) in the three Amorphoscelinae species studied herein (Figs. 178, 186). This unique combination seems to be constant within Amorphoscelinae (e.g. Chopard 1938: fig. 293; Roy 1962: figs 5, 9, 13, 16a, b, 20a, b, 23, 1963a: fig. 2, 1963b: figs 6a, 8a, 1965b: fig. 4b; Gillon \& Roy 1968: fig. 5), and is probably autapomorphic for the group.

State 5 is exhibited only by Perlamantis and Paramorphoscelis and is autapomorphic for Perlamantinae. In these taxa, the antero-ventral femoral spines consist of four spines, the distal three of which are strongly enlarged and plate-like (see character 44 and Figs. 182, 220-222). 


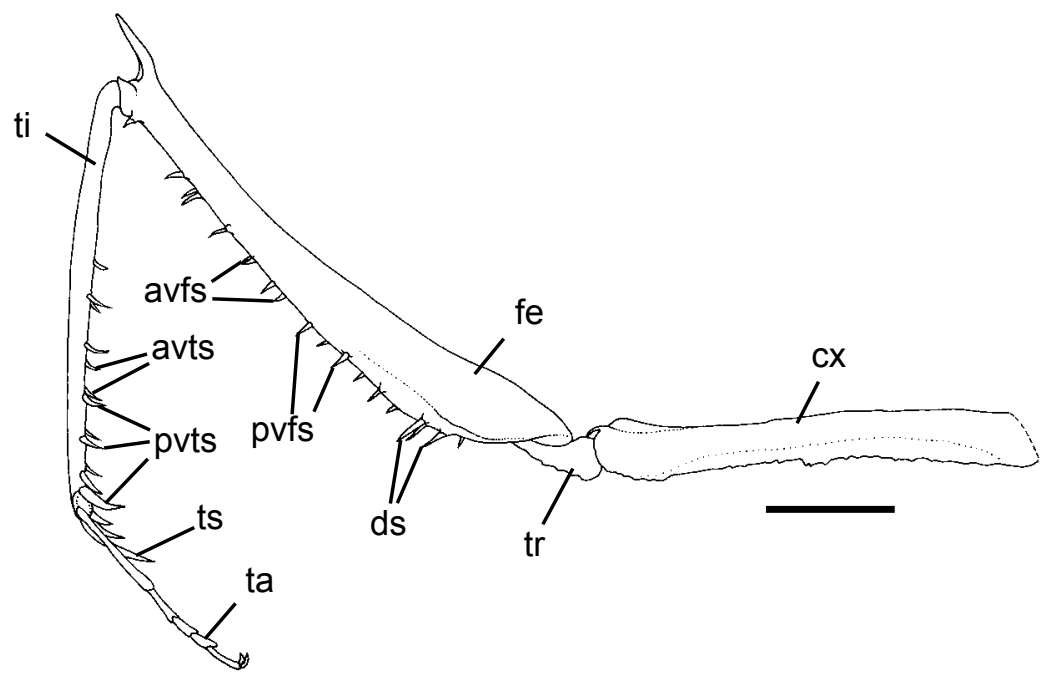

Fig. 207: Toxodera maculata,, , left foreleg, posterior view. Note the wide spaces between the spines of femur and tibia. - Scale bar $=5.0$ mm.

Abbreviations: avfs $=$ antero-ventral fore femoral spine; avts $=$ anteroventral fore tibial spine; $\mathbf{c x}=\operatorname{coxa} ; \mathbf{d s}=$ discoidal spine; $\mathbf{f e}=$ femur; $\mathbf{p v f s}=$ postero-ventral fore femoral spine; $\mathbf{p v t s}=$ postero-ventral fore tibial spine; $\mathbf{t a}=$ tarsus; $\mathbf{t i}=$ tibia; $\mathbf{t r}=$ trochanter; $\mathbf{t s}=$ tibial spur.

The situation in Compsothespis is difficult to assess. There are two rows of femoral spines, albeit strongly reduced in length (state 6; Fig. 166-168). The antero-ventral spines are reduced in number and stand together more closely than the postero-ventral spines. This type of reduction and organization is unique within Mantodea and autapomorphic for Compsothespis.

44. Fore femur of adults, antero-ventral femoral spines, length: almost equal or only slightly alternating [1]; distinctly alternating (one short spine followed by one long spine), distal spines of an alternative pattern at most [2]; alternating in a 1-3-1 pattern, one spine being long, followed by three short spines and another long one, often the medial of the three short spines being slightly longer than the other two [3]; proximally shifted together, small and blunt [4]; large and plate-like flattened [5].

The antero-ventral fore-femoral spines of most extant mantodeans show a distinct pattern of alternating length (state 2, e.g. Roy 1999: 28).

In few taxa (herein confirmed for Mantoida, Metallyticus, Eremiaphilidae, Theopompa, Tarachodes, Tarachodula and Pyrgomantis) they are equally long or differ only slightly in length (state 1).

All Empusidae studied herein show a pattern of three shorter spines between two long spines (1-3-1-pattern, state 3; Figs. 208, 209, 211, 212). This character has been discussed before and is assumed to represent an autapomorphy for Empusidae (Beier 1964a: 953, 1968a: 12; Roy 1999: 28, 2004a: 1, 14).

Perlamantinae have uniquely shaped antero-ventral spines on their fore femora 


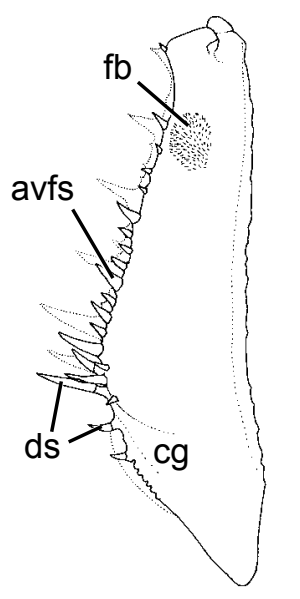

208
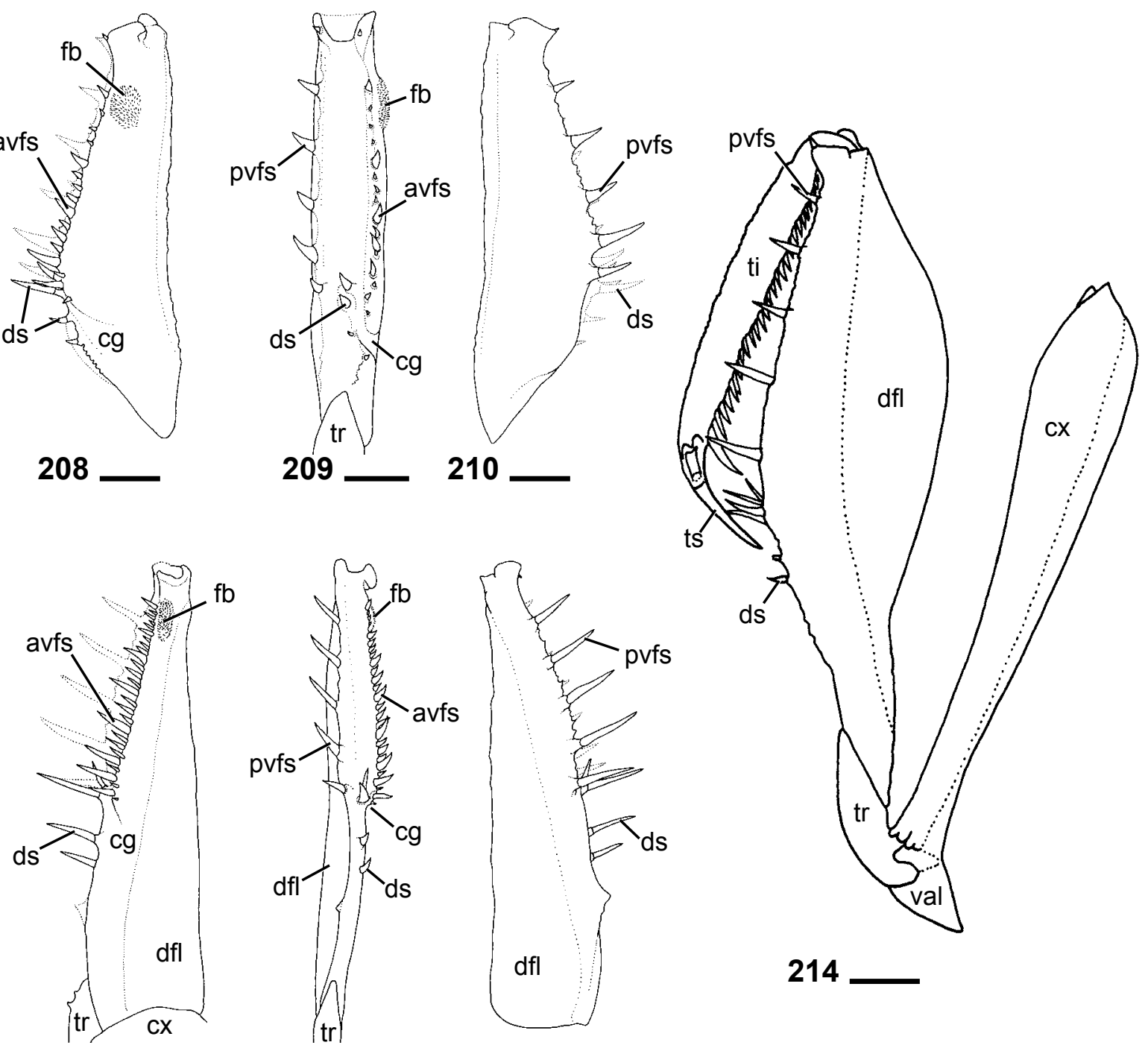

214

211

212

213

Figs. 108-214: Foreleg morphology of Empusidae. 208-210: Blepharopsis mendica, $\uparrow$, right fore femur. 208: Anterior view. 209: Ventral view. 210: Posterior view. 211-213: Idolomantis diabolica, $\widehat{\jmath}$, left fore femur (mirrored); note slender primordial shape of femur (without expansions), similar to Gongylus but differing from Blepharopsis. 211: Anterior view. 212: Ventral view. 213: Posterior view. 214: Gongylus gongylodes, ${ }^{\text {, }}$ left foreleg, posterior view. - Scale bar $=2.0 \mathrm{~mm}$.

Abbreviations: avfs = antero-ventral fore femoral spine; $\mathbf{c g}=$ claw groove; $\mathbf{c x}=$ coxa; $\mathbf{d f l}=$ dorsal femoral lobe; $\mathbf{d s}=$ discoidal spine; $\mathbf{f b}=$ fore femoral grooming device (femoral brush); $\mathbf{p v f s}=$ postero-ventral fore femoral spine; $\mathbf{t r}=$ trochanter; val $=$ ventral antero-distal apical lobe of coxa.

(state 5; see character 43 and Fig. 221). While the proximal one of the four spines is normally shaped, the three distal spines are strongly enlarged and flattened in a plate-like manner (Fig. 221, 222). The second spine is nearly rectangular with a slightly oblique tip, whereas the third and fourth spines are curved and orientated in a nearly right angle with respect to the second spine (Figs. 220, 221).

Pogonogaster and Thesprotia graminis (Fig. 163, 164) exhibit strongly reduced antero-ventral fore femoral spines, and in Compsothespis (Fig. 166-168) all femoral spines are strongly reduced in size, therefore the character was not encoded for these taxa. 
45. Fore femur, proximal 5-10 antero-ventral spines, position: distinctly alternating positions, therefore standing in two distinct rows (bases of spines not overlapping in ventral view) [1]; standing in a single row, the proximal spines alternating slightly at most (bases of spines overlapping in ventral view) or pointing alternating craniad or caudad [2].

In few Mantodea the proximal antero-ventral spines on the fore femur stand alternating, almost in two parallel rows (state 1). In extant Mantodea this has been confirmed for Acanthops (Fig. 217), Callibia, Chaeteessa (Figs. 148, 149, 216, 418), Mantoida (Figs. 151, 153, 215), and Paraoxypilinae (Figs. 196, 197, 199, 201, 218). This character state was probably also present in some fossil Mantodea as can be derived from the bases of the spines in $\uparrow$ Cretomantis larvalis, see Grimaldi (2003: fig. 13; Fig. 29). In most fossil and extant taxa described so far, however, the proximal spines are standing in a more or less straight row or alternate only slightly (state 2 ).

It is unknown why the proximal antero-ventral femoral spines are arranged in two rows in the taxa showing this trait. A potential function becomes obvious in Paraoxypilinae. A photograph of Gyromantis devouring an ant shows the manner, in which this genus uses the foreleg (Fig. 219). The abdominal tip of an ant is held between the comb-like structure. By doing this, the mantid probably ensures that it cannot be harmed by the ant's sting or its squirting acid. It is unclear if other taxa exhibiting such an arrangement of spines show a similar behaviour and if these taxa possibly prefer to hunt ants.

46. Fore femur of male, antero-ventral spines, largest number: 1-5 [1]; 6-12 [2]; 13-19 [3]; 20-25 [4]; 26 or more [5].

The number of spines on the fore femora of Mantodea varies strongly among taxa, whereas it differs only slightly (usually one or two spines at most) among individuals. However, an intersexual difference between the highest numbers of spines was found in Pyrgomantis, Paraoxypilus sp. 1, and Heterochaeta strach$a n i$. Therefore, the numeric characters were encoded individually for both sexes.

One to five antero-ventral spines (state 1) are only present in Compsothespis and Perlamantinae (Fig. 221; the lack of antero-ventral spines in Amorphoscelinae were already taken into consideration in character 41). The foreleg morphology of these two taxa (and of Amorphoscelis; Fig. 178, 184-188) is likely to be derived rather than primitive because remnants of the antero-ventral and posteroventral fore femoral spines can be recognized in SEM picture, e.g. of Amorphoscelis and Perlamantis (see character 41, 42 and Figs. 184, 186-188, 193-195).

Six to 12 spines (state 2 ) were found in about one quarter of the species examined, whereas two thirds of the taxa examined here showed a highest number of 

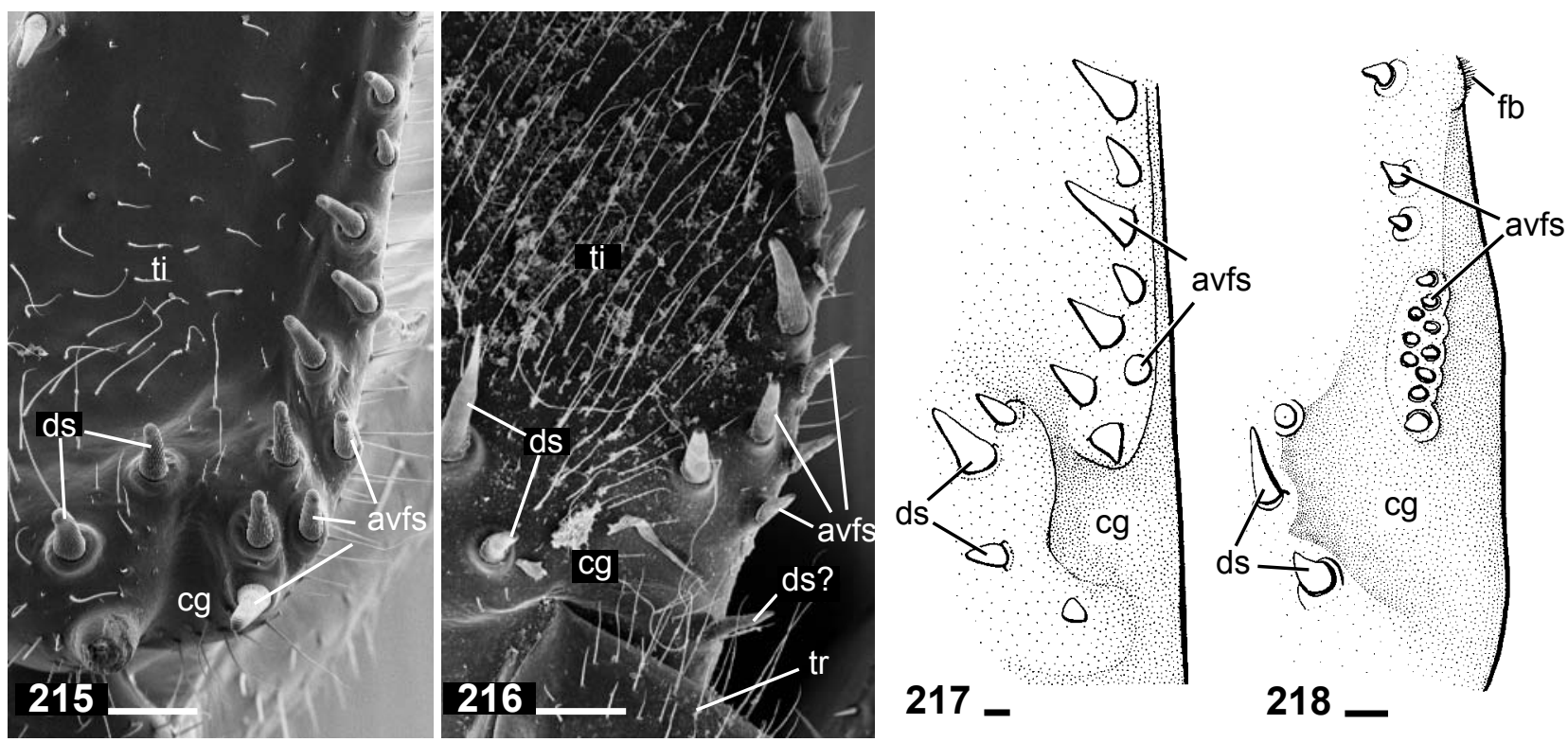

Figs. 215-218: Antero-ventral fore femoral spines being positioned in two parallel rows; ventral view. 215: Mantoida maya, $\hat{\jmath}$, right foreleg. 216: Chaeteessa cf. valida, sex unknown, left foreleg (mirrored). 217: Acanthops tuberculata,, , right foreleg. 218: Paraoxypilus sp.,, , right foreleg. - Scale bar: $0.2 \mathrm{~mm}$.

Abbreviations: avfs = antero-ventral fore femoral spine; $\mathbf{c g}=$ claw groove; $\mathbf{d s}=$ discoidal spine; $\mathbf{f b}=$ fore femoral grooming device (femoral brush); $\mathbf{t i}=$ tibia; $\mathbf{t r}=$ trochanter.

13-19 antero-ventral spines (state 3). Numbers between 20 and 25 (state 4) were only found in Eremiaphila and some Empusidae [Idolomorpha, Hypsicorypha (only in + , o was not available)], whereas numbers higher than 25 (state 5) were only found in Idolomantis (Fig. 211, 212) and Gongylus.

State 3 was also found in Chaeteessa and Mantoida (Figs. 150-153), as well as in the outgroup (Periplaneta), and is also exhibited by several fossil Mantodea $[\dagger$ Burmantis asiatica Grimaldi, 2003, †Cretomantis larvalis (Fig. 29); see Grimaldi 2003]. It can be hypothesized that this character represents the groundplan situation for Mantodea. Unfortunately, the Carboniferous fossils recently re-interpreted as stemgroupMantodea (e.g. $\uparrow$ Mesoptilus dolloi, †Homocladus grandis) have poorly preserved legs (Béthoux \& Wieland 2009: 101 ff.) and therefore

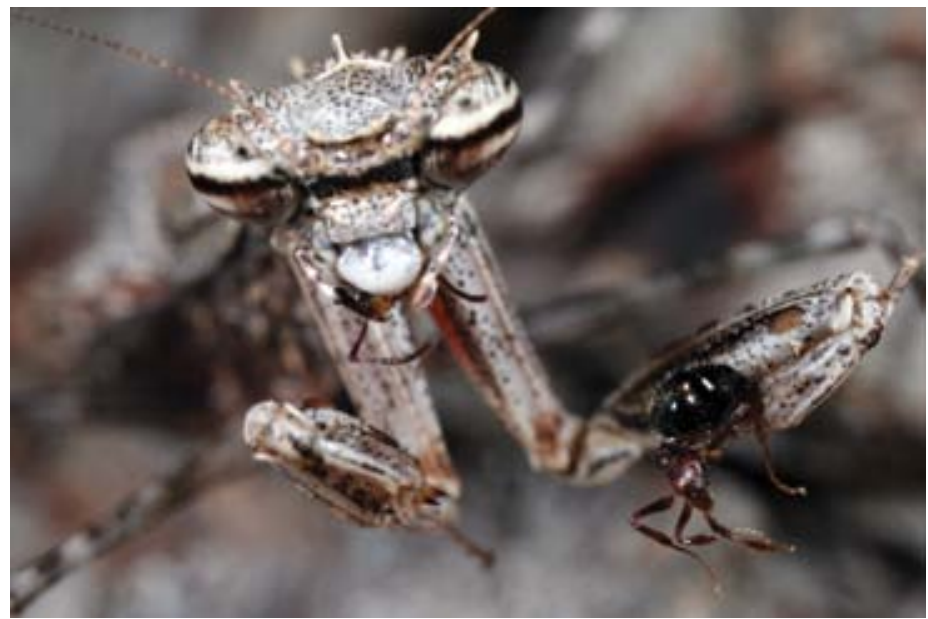

Fig. 219: Gyromantis sp., sex unknown. Specimen feeding on ant. The position shows the mantid holding the ant between the small antero-ventral femoral and tibial spines, possibly to avoid being stung by it or injured by its acid. This might be a potential function of the proximal antero-ventral femoral spines standing in two rows.

CFarhan Bokhari; myrmician@gmail.com; http://www.flickr. com/photos/myrmician/. 
do not allow an interpretation of the spination of the fore femora that would allow to support this character state as a ground plan feature for Mantodea.

47. Fore femur of female, antero-ventral spines, largest number: 1-5 [1]; 6-12 [2]; 13-19 [3]; 20-25 [4]; 26 or more [5].

See character 46. One to five spines (state 1) were found only in female Compsothespis (Figs. 166-168) and Paramorphoscelis ( $q$ of Perlamantis not available).

As in the males, six to 12 spines (state 2) were found in about one quarter of the females examined. In about half of the species studied the females showed a highest number of 13-19 antero-ventral spines (state 3), including Chaeteessa (Fig. 148), Mantoida and Periplaneta (Figs. 145, 146). Again, numbers between 20 and 25 (state 4) were only found in Eremiaphila (Figs. 155-158) and some Empusidae [Idolomorpha, Hypsicorypha], whereas numbers higher than 25 (state 5) were only found in female Idolomantis and Gongylus.

48. Fore femur, postero-ventral spines, number: 1-3 [1]; 4 [2]; 5 [3]; 6 or more [4].

The postero-ventral femoral spines may be missing (see character 42). It is likely that they are reduced because remnants of the spines can be detected in the SEM images of several species (e.g. Amorphoscelis, Perlamantis; see also character 41, 42 and Figs. 184, 186-188, 193-195). In species with postero-ventral fore femoral spines, their number is equal in both sexes. The numbers are intraspecifically constant, except for few individuals that may exhibit an additional or missing spine on one of the legs (observed in a single Orthodera novaezealandiae specimen).

One to three spines (state 1) are found only in Haania and Thesprotia (Fig. 164). A number of five spines (state 3) is exhibited only by Acanthopidae (except Acanthops; see, for instance, Callibia in Fig. 223), Amphecostephanus, Empusidae (except Gongylus; see Figs. 208-214), Epaphrodita, Heterochaeta, Iris, Orthoderella, Photina, Phyllocrania, and Periplaneta (Fig. 146, 147). The situation in Periplaneta is ambiguous, as the spination pattern of Mantodea and Blattaria has not been homologized yet. Therefore, it is unclear whether the distal postero-ventral femoral spine is homologous to the postero-ventral genicular spine or the distal postero-ventral fore femoral spine in Mantodea. Hence, the assignment of this character in Periplaneta to state 3 can only be provisional until a proper homologization is established.

Six or even more spines (state 4) are exhibited by Acanthops, Gongylus (Fig. 214), and Toxoderidae (Fig. 207). Interestingly, Chaeteessa has 4 sturdy 

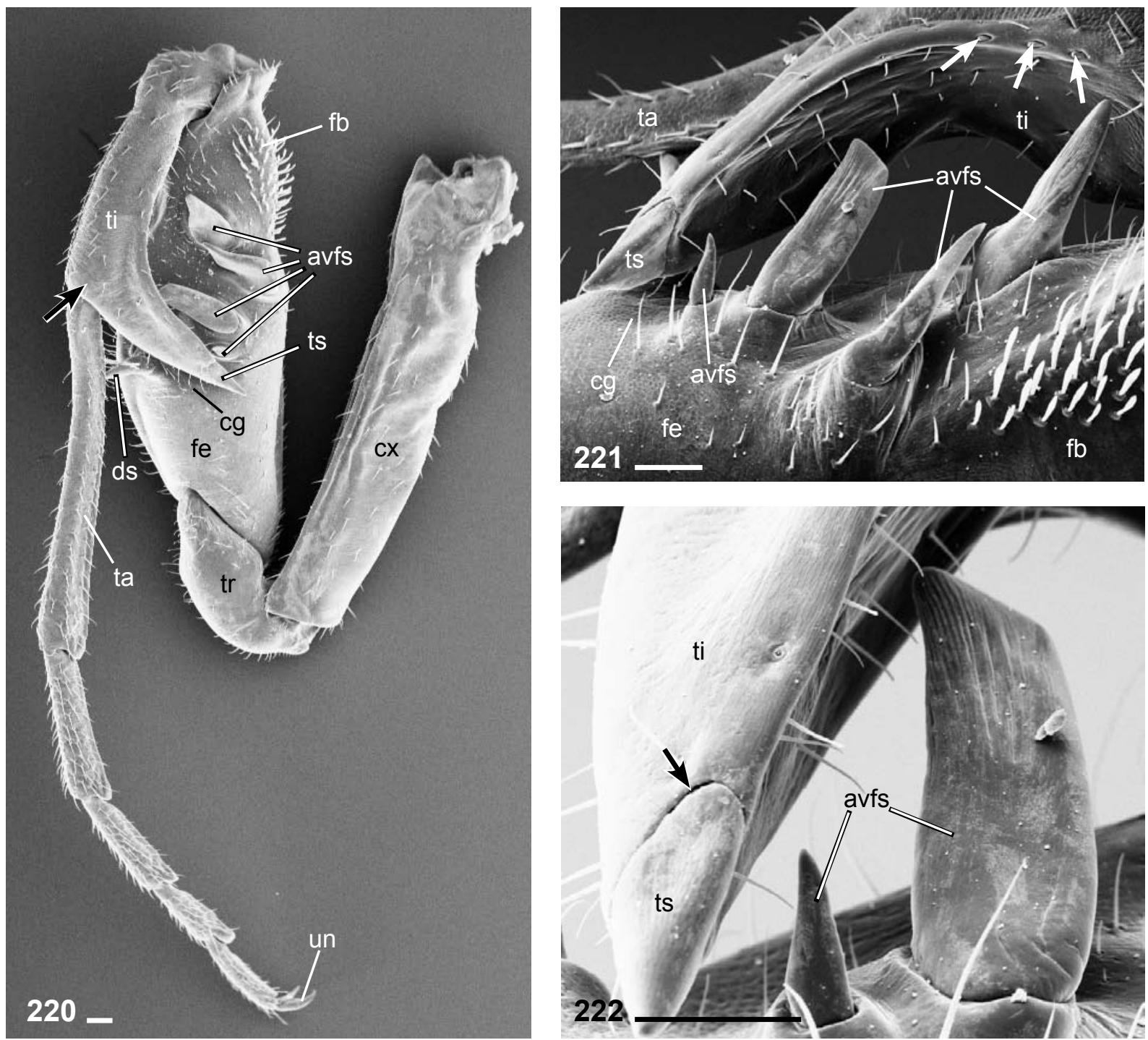

Figs. 220-222: Perlamantis allibertii, $\widehat{\jmath}$, right foreleg. 220: Overview in antero-ventral perspective; arrow indicates $90^{\circ}$ folding of tibia; note the strongly enlarged distal tibial lobe carrying the minute tibial spur. 221: Antero-ventral fore femoral spines and distal part of tibia, overview in antero-ventral perspective; arrows indicate the remnants of the antero-ventral fore tibial spines. 222: First and second proximal antero-ventral femoral spines, detail, anterior view; arrow indicates suture between the distal tibial lobe and the tibial spur. - Scale bar = $0.1 \mathrm{~mm}$.

Abbreviations: avfs = antero-ventral fore femoral spine; $\mathbf{c x}=$ coxa; $\mathbf{d s}=$ discoidal spine; $\mathbf{f b}=$ fore femoral grooming device (femoral brush); $\mathbf{f e}=$ femur; $\mathbf{t a}=$ tarsus; $\mathbf{t i}=$ tibia; $\mathbf{t r}=$ trochanter; $\mathbf{t s}=$ tibial spur; $\mathbf{u n}=$ unguis.

postero-ventral femoral spines. Proximally, there are three further tubercles carrying thick setae (Figs. 148, 149, 227, 418). Their shape that strongly differs from the remaining setae on the surface of the femur in that they are three to four times thicker than the latter and are located on distinct sockets. This suggests that these three seta-like spines are possibly remnants of regular postero-ventral femoral spines that were reduced in size. Chaeteessa is correspondingly provisionally encoded as state 4 .

All remaining taxa (two thirds of the species studied herein) have four postero-ventral femoral spines (state 2), including Compsothespis in which, however, they are strongly reduced (Fig. 167, 168). This state is apparently also present 
in several fossil taxa (e.g. †Ambermantis wozniaki, †Jersimantis burmiticus, Grimaldi, 2003; †Burmantis lebanensis Grimaldi, 2003; see Grimaldi 2003).

Cockroaches other than Periplaneta (or possibly Blattidae) show strong variations of the postero-ventral fore femoral spines. In many cases [Rhyparobia maderae (Fabricius, 1781), Blaberus craniifer Burmeister, 1838, Panchlora nivea (Linnaeus, 1758), Ectobius sylvestris (Poda, 1761)] all but the distal postero-ventral femoral spine are missing or reduced into very thin setae. In others, e.g. Blatta orientalis Linnaeus, 1758, there are four spines on the fore femora (six on the cursorial legs).

49. Fore femur, postero-ventral spines, position: positioned evenly on ventral surface of femur [1]; positioned on slightly to strongly elevated, thin ridge [2]; positioned individually on long sockets [3].

In most species (as in Blattodea, see Figs. 145-147, 185-287) the postero-ventral femoral spines are articulated low on the ventral surface of the fore femora (state 1; e.g. Figs. 152, 154, 157, 159, 160, 162, 163, 165).

They may be positioned on a more or less strongly elevated, thin ridge that runs along the edge of the ventral surface of the femur (state 2), as can be seen in Acanthops, Empusidae (Fig. 225), Hymenopus, and Theopropus.

Some other taxa have the spines located on the tips of single, elongated and sturdy sockets (state 3), as exhibited by Callibia (Fig. 223) and, much more distinctly, by Pseudocreobotra (Fig. 224; see also Beier 1974b: fig. 7; Loxton \& Nicholls 1979: fig. 6P.w.; Kaltenbach 1998: fig. 117). The presence of socketed postero-ventral femoral spines was observed and discussed by Loxton \& Nicholls for Pseudocreobotra wahlbergii (Loxton \& Nicholls 1979: 198 and fig. 6P.w.). They argued that, according to MacKinnon (1970), Pseudocreobotra nymphs show intraspecific display in order to defend the blossoms they are sitting on, and that the sturdy sockets may play a role in that display behaviour rather than in prey capture (Loxton \& Nicholls 1979: 198).

50. Fore femur, postero-ventral spines, length: not strongly elongated (except for possibly extremely elongated first postero-ventral spine), not dorsally surpassing the tibia when the leg is folded [1]; all strongly elongated, dorsally surpassing the tibia when leg is folded [2]; the proximal 2 postero-ventral spines distinctly longer than the remaining postero-ventral spines [3].

The postero-ventral spines are strongly elongated (state 2) in Chaeteessa (see Loxton \& Nicholls 1979: fig. 6 C.f.; Grimaldi \& Engel 2005: fig. 7.91), Empusidae (except Blepharodes and Blepharopsis; Figs. 210, 213, 214), Euchomenella, Sibylla, Stenomantis and Toxodera denticulata (female). 

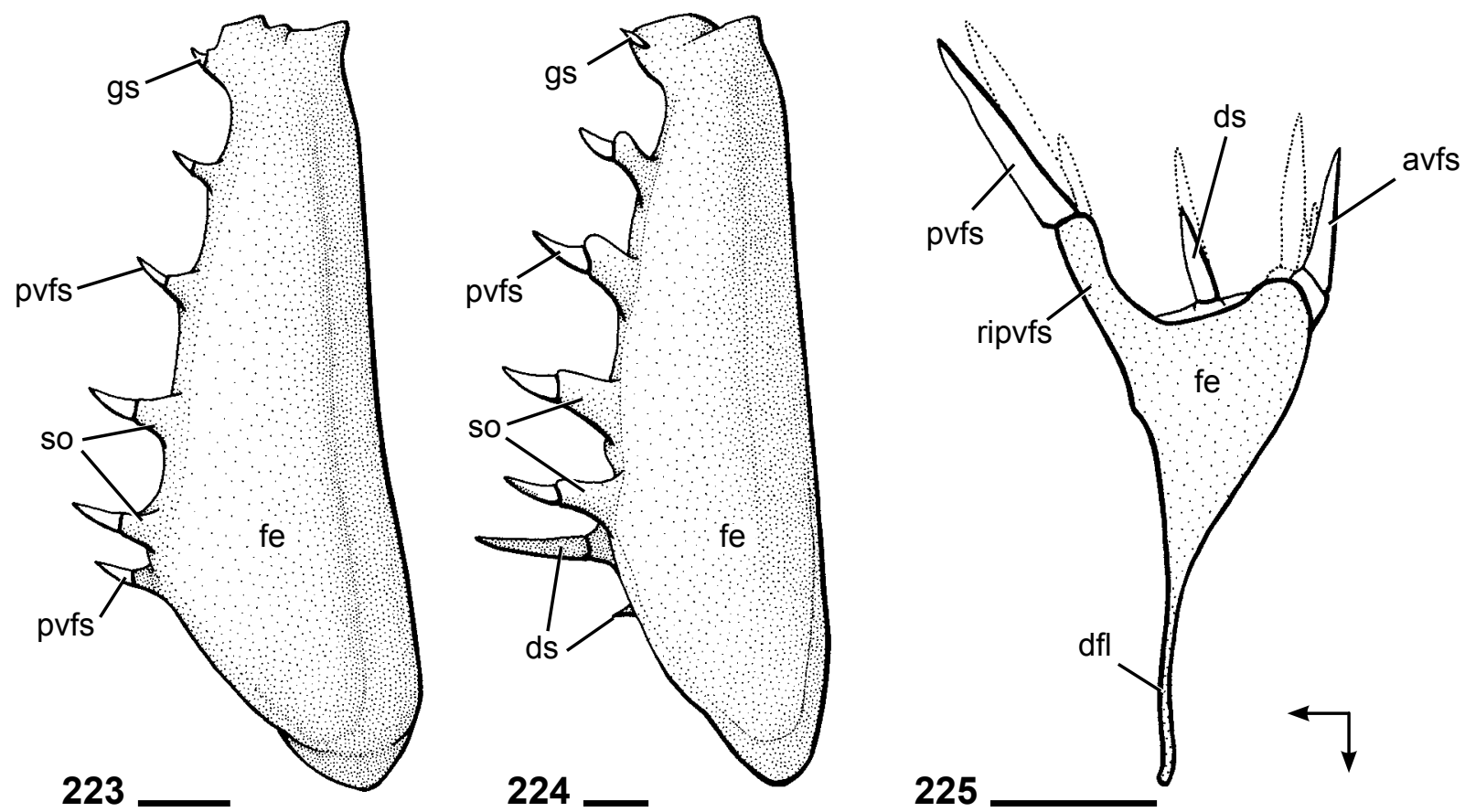

Figs. 223-225: Left fore femora showing position of postero-ventral spines. 223: Callibia diana, , $_{\text {, posterior }}$

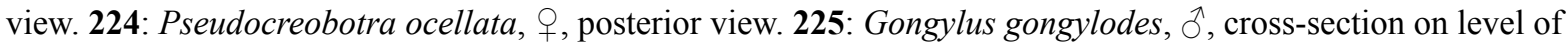
third postero-ventral femoral spine; arrows in 225 indicate $\leftarrow$ posterior and $\downarrow$ dorsal (proximad view towards the discoidal spines). - Scale bar $=1.0 \mathrm{~mm}$.

Abbreviations: avfs = antero-ventral fore femoral spine; $\mathbf{d f l}=$ dorsal femoral lobe; $\mathbf{d s}=$ discoidal spine; $\mathbf{f e}=$ femur; gs = genicular spine; pvfs = postero-ventral fore femoral spine; ripvfs = ridge on which the posteroventral fore femoral spines are located; so = sockets on which the postero-ventral fore femoral spines are located individually.

An elongation of only the proximal two postero-ventral spines (state 3) is present in Heterochaeta, Iris, and Pseudoyersinia.

The remaining taxa (over 80 per cent of the species studied) have posteroventral spines without any strong elongation (state 1).

51. Fore femur, proximal postero-ventral spine of adult, length with respect to remaining postero-ventral spines: almost equal [1]; several times longer and much thicker [2].

The proximal postero-ventral femoral spine is strongly elongated in adult Metallyticus (state 2; e.g. Loxton \& Nicholls 1979: fig. 6M.v.; Steinmann \& Zombori 1985: fig. 291; Klass \& Ehrmann 2003: fig. 13.4g; Wieland 2008a: figs 1, 7, 8-10, 22, 23-25, 28-30; Figs. 176, 226, 409, 411, 414). This is unique among Mantodea (e.g. Beier 1968a; Roy 1999) and autapomorphic for the genus (Wieland 2008a: 160). The function of the enlarged spine is unknown. Loxton \& Nicholls (1979: 198) argued that, based on the potential specialization to cockroaches as prey (e.g. Shelford 1903, 1916; Wieland 2008a), it might be used "like the prongs of a fork-lift truck" for lifting cockroaches off the bark, or maybe "act as a spear or as a pin to winkle cockroaches out of crevices" (Loxton \& Nicholls 1979: 198). For a detailed discussion of this topic see chapter 4.6.4. 
The proximal postero-ventral femoral spine may be longer than some of the remaining spines in species with irregularly long postero-ventral spines, but never several times longer as in Metallyticus.

52. Fore femur, proximal postero-ventral spines 1 and 2, position with respect to remaining postero-ventral spines: closer together, remaining postero-ventral spines farther apart [1]; not standing closer together than remaining postero-ventral spines [2].

Roy (1999: 27) stated that "the interval between the two first [postero-ventral] spines is generally smaller than between the others". However, in about one quarter of the species studied here the two proximal postero-ventral spines are not standing distinctly closer together than the remaining spines [state 2: Acontista, Bactromantis, Ceratomantis, Choeradodis stalii, Compsothespis (Fig. 167, 168), Eremiaphilidae (Fig. 158, 159), Gimantis, Haaniinae, Heterochaeta, Hoplocorypha (161, 162), Leptocola, Mantoida (Fig. 151, 153, 154), Oligonicinae sp. (Fig. 202), Oxypilus, Parentella, Pogonogaster, Pseudocreobotra (Fig. 224), Sphodromantis, Stagmatoptera, Stenomantis, Theopropus, Thrinaconyx, Toxoderidae (Fig. 207, 234), and Vates]. In about three quarters of the species, however, the proximal two spines stand distinctly closer than the remaining

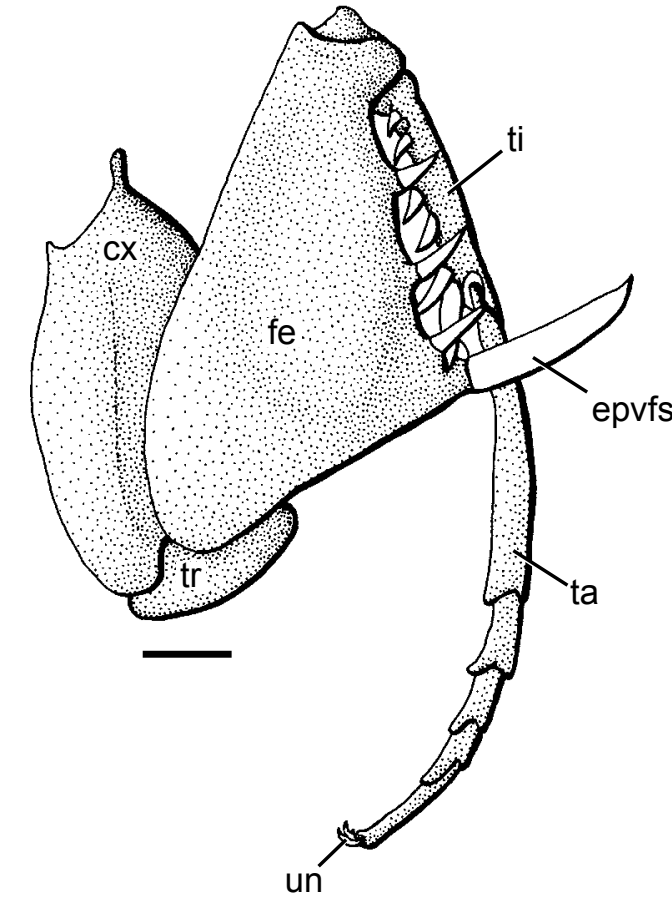

Fig. 226: Metallyticus splendidus, $\bigcirc$ : Right foreleg, posterior view. Note the strongly enlarged proximal postero-ventral fore femoral spine. Scale bar $=1.0 \mathrm{~mm}$. Abbreviations: $\mathbf{c x}=$ coxa; epvfs $=$ elongated postero-ventral fore femoral spine; fe $=$ femur; ta $=$ tarsus; $\mathbf{t i}=$ tibia; $\mathbf{t r}=$ trochanter; $\mathbf{u n}=$ unguis. spines (state 1).

Interestingly, Choeradodis stalii exhibits state 2, whereas $C$. rhombicollis exhibits state 1 . However, there may be intraspecific variation of spine position. Although the position was constant in the species studied herein, Vyjayandi et al. (2008) presented evidence for intraspecific variation of this character in Gimantis autheamon (an Indomalayan Mantidae: Amelinae). In a female specimen from Malaysia (Vyjayandi et al. 2008: fig. 4) the proximal postero-ventral spines exhibited state 2, whereas two females from India (Vyjayandi et al. 2008: figs. 3, 6) exhibited state 1 . It is possible that this is also the case in Choeradodis. Comparative studies of larger series of specimens as well as ontogenetic studies are important to address the degree of variability in such species. 
53. Fore femur, ventral depression between the proximal postero-ventral spines for accommodating the distal postero-ventral spine of the tibia when leg is folded: present [1]; missing [2].

A depression on the ventral surface of the femur has been described to be characteristic for Liturgusidae and Caliridinae (Giglio-Tos 1927: 289, 302; Beier 1935c: 5, 15, 1964a: 874, 1968a: 8; Gillon \& Roy 1968: 1066; Cerdá 1996a: 74, 75; Kaltenbach 1998: 26). Beier (1968a) and Kaltenbach (1998) stated that the depression in Liturgusidae contains the tibial spur when the leg is folded: "Zwischen dem 1. und 2. Außendorn befindet sich eine tiefe, meist dunkle Grube zur Aufnahme der Endklaue der Tibia" (Beier 1968a: 8); "Femur I zwischen den beiden proximalen Außenranddornen mit einer tiefen Mulde zur Aufnahme der Endklaue der Tibia" (Kaltenbach 1998: 26).

Beier \& Jaus (1933: 130) had stated earlier that depressions for other large spines than the tibial spur often occur: "Übrigens sind in vielen Fällen am Femur nicht nur für diese Endklaue, sondern auch für andere größere Dornen der Tibia besondere Grübchen vorhanden [...]". The same was mentioned by Beier [1964a: 874: “Auch zur Aufnahme des kürzeren apikalen Außendorns der Tibia oder für andere ihrer Dornen können auf der Ventralfläche des Femur besondere Gruben vorhanden sein (Liturgusinae)"]. Roy (1973: 275) mentioned with respect to the position of Deromantis Giglio-Tos, 1916 that "Le caractère mis en avant pour le [Deromantis] placer dans cette sous-famille [Caliridinae] consiste en la présence d'une fossette entre le première et la seconde épines externes des fémurs antérieurs, caractère qui se trouve en fait avec un développement équivalent chez divers autres genres des Mantinae, et qui à mon avis n'a donc pas à être pris en considération".

The presence of such a groove has been reported, for instance, by Henry (1932: 3, 16), who explicitly mentioned it for Dysaules uvana Henry, 1932 and Cheddikulama straminea Henry, 1932.

From a functional morphological point of view it is not plausible that the tibial spur rests in such a ventral depression because this would imply the possibility of considerable lateral movement in the femoro-tibial joint, thus impairing the stability of the raptorial leg and hence increasing the possibility of injury during prey capture due to a lack of stability. Furthermore, in Liturgusidae the "regular" claw groove on the antero-ventral surface of the femur is additionally present so the leg can be folded in the normal way.

A ventral depression in the area of the proximal two postero-ventral spines is by no means unique to Liturgusidae and Caliridinae (e.g. Roy 1973: 275), but it is present in more than half of the taxa studied herein. It may be quite shallow but nonetheless present - as exhibited by many taxa. In some taxa, for instance in Blepharopsis (Fig. 229), Hymenopus (Fig. 230), and Choeradodis (Fig. 231), 

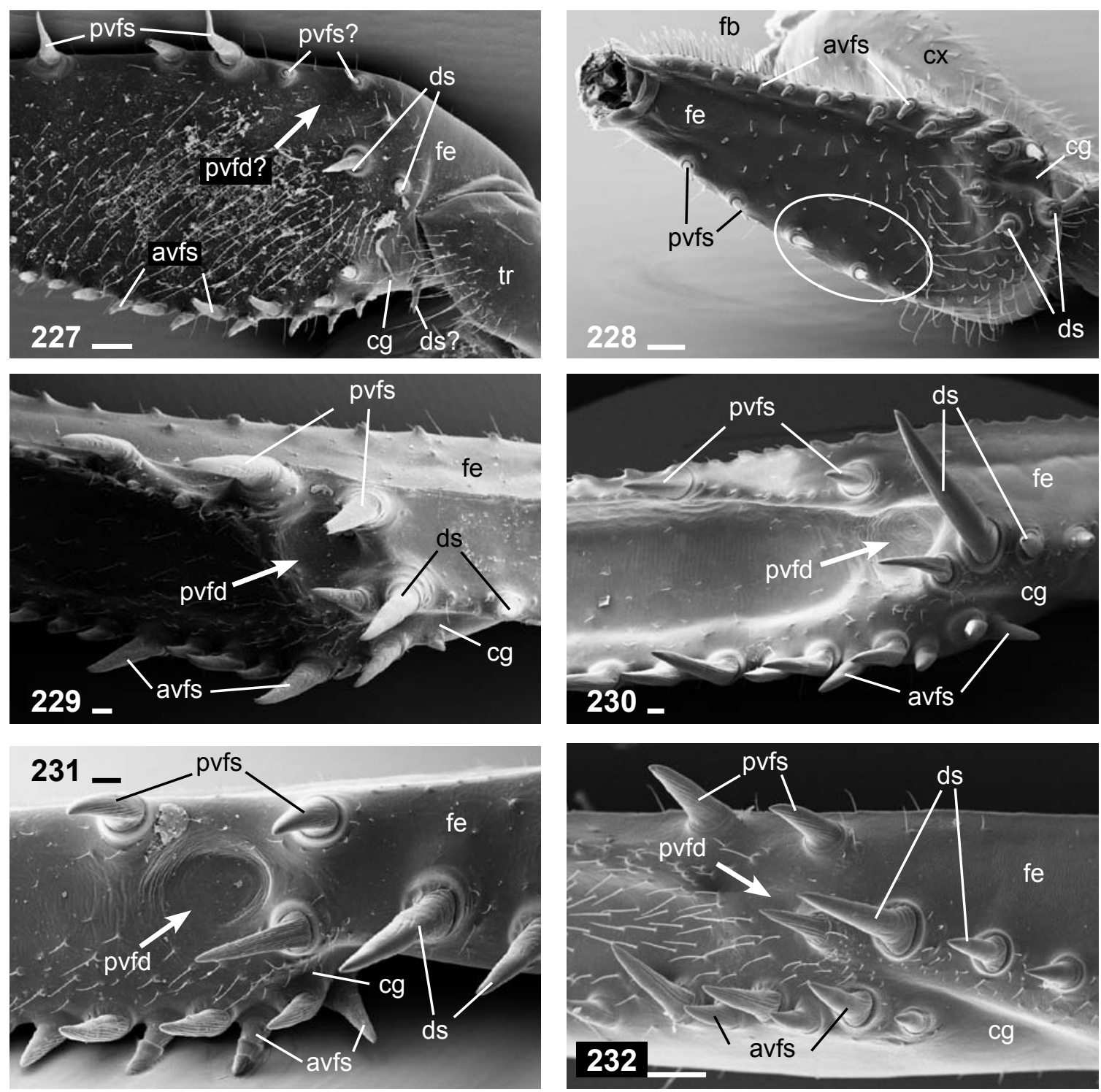

Fig. 227-232: SEM images of groove for distal postero-ventral tibial spine, located between the proximal postero-ventral fore femoral spines. 227: Chaeteessa cf. valida, sex unknown, left foreleg. 228: Mantoida maya, $\hat{0}$, right foreleg; white circle indicates area where groove can be found in many other taxa. 229: Blepharopsis mendica, + , right foreleg (mirrored). 230: Hymenopus coronatus, $q$, left foreleg. 231: Choeradodis stalii, $\hat{\sigma}$, left foreleg. 232: Pseudoharpax sp., ô, left foreleg. $\leftarrow$ distal. - Scale bar $=0.02 \mathrm{~mm}$.

Abbreviations: avfs = antero-ventral fore femoral spine; $\mathbf{c g}=$ claw groove; $\mathbf{d s}=$ discoidal spine; $\mathbf{f b}=$ fore femoral grooming device (femoral brush); fe = femur; pvfd = postero-ventral fore femoral depression; pvfs = posteroventral fore femoral spine; $\mathbf{t r}=$ trochanter.

the depression is deep and wide. Here too, the "regular" claw groove is additionally present (Figs. 229-232). When the leg is folded, it becomes evident that it is always the long apical postero-ventral tibial spine that rests in the ventral depression. This is in congruence with the statements by Beier \& Jaus (1933) and Beier (1964a).

Interestingly, a depression between two strongly reduced postero-ventral spines, holding the distal postero-ventral tibial spine (although being similar to the claw groove, i.e. open to the posterior surface of the femur, thus forming a "second claw groove"), is also present in Chaeteessa (Figs. 148, 227, 418) but 
neither in Metallyticus (Fig. 414) nor in Mantoida (Figs. 151, 228). The situation in Chaeteessa possibly represents a secondary trait due to the strong elongation of the distal postero-ventral tibial spine (see chapter 4.6.5 for discussion).

In the Toxoderidae $\mathrm{To}^{-}$ xodera and Calamothespis, the ventral depression is distinct and deep but rather narrow. The depressions in both taxa house the distal postero-ventral tibial spine when the leg is folded. Interestingly, the position of the depression in ventral view differs in the two taxa. While it is located antero-ventrally with respect to the postero-ventral spines in Calamothespis (Fig. 233), it lies posterior to these spines in Toxodera (Fig. 234).

Both taxa exhibit one ridge on the ventral surface of the proximal part of the femur (between the antero-ventral and postero-ventral femoral rows of spines) and one ridge on its postero-ventral surface (towards the posterior surface). Both ridges are indicated by arrows in Figs. 233 and 234. The ventral depression is located between these two ridges in both taxa. In Calamothespis it borders the ventral ridge (anterior to the postero-ventral femoral spines), whereas in Toxodera it borders the postero-ventral ridge (posterior to the postero-ventral femoral spines).

In both genera, as well as in the other taxa observed, the depression is located in the area between the proximal two postero-ventral femoral spines. As the position of the groove is similar and its function is always to accommodate the distal postero-ventral tibial spine, homology is supported. Apparently, a long distal postero-ventral tibial spine has an important function for effectively capturing prey. Therefore, a depression on the femur was positively selected in many taxa instead of the shortening of the spine. 
54. Fore femur, discoidal spines: present [1]; missing [2].

The fore femur in Mantodea carries an oblique row of one to five spines of varying length (Roy 1999: 27; Klass \& Ehrmann 2003: 185) (see also chapter 3.3). When the foreleg is folded, the tibial claw rests in an antero-ventral depression (claw groove; see character 36) between the discoidal spines and the proximal antero-ventral femoral spines. The only extant member of Mantodea that has hitherto been believed to be lacking the discoidal spines is Metallyticus (Roy 1999: 27; Ehrmann 2002: 221; Klass \& Ehrmann 2003: 185; Wieland 2008a: 161, 163). However, Metallyticus exhibits a single discoidal spine as can been derived from the situation in the first instar nymph under the SEM (for a detailed discussion see chapter 4.6.4 and Figs. 414-417). There are several fossil Mantodea apparently lacking these spines [e.g. Grimaldi 2003: fig. 3B: $\uparrow$ Ambermantis; figs. 8A, 9B: $\uparrow$ Burmantis; fig. 13: $\uparrow$ Cretomantis (Fig. 29 herein); fig. 14: †Jersimantis (Fig. 31 herein); see also encoding of character 14 in table 3 therein]. No such spination is present in Blattaria (Figs. 146, 286) and Isoptera. As discoidal spines are missing in cockroaches and possibly many fossil mantodeans (although uncertain due to nymphal state and preservation of the fossils and sometimes the lack of legs in the fossil record) but are present in all extant Mantodea it is possible that these spines evolved comparatively late in stemgroup-mantodeans. However, considering their mechanical significance for prey capture (e.g. Loxton \& Nicholls 1979) such a late origin of the discoidal spine has to be considered debateable and should be reinvestigated thoroughly with respect to postembryonic development.

55. Fore femur, discoidal spines, number: 1 [1]; 2 [2]; 3 [3]; 4 [4].

Most extant species have three or four discoidal spines. A single one is found in Amorphoscelinae, Perlamantinae, and Metallyticus (see character 54 and chapter 4.6.4; Roy 1999: 27), whereas Chaeteessa is the only taxon in this study exhibiting two discoidal spines (Roy 1999: 27). A single proximal antero-ventral spine lies anterior to the trochantero-femoral junction (the two discoidal spines are located posterior to it; Figs. 148, 149, 216, 418). It possibly represents a third (i.e. the proximal) discoidal spine (labelled ds? in Figs. 216, 227, 418). This hypothesis finds support in the fact that the claw groove runs between this spine and the antero-ventral femoral spines. The position of this spine, however, is highly unusual for a discoidal spine. Therefore Chaeteessa will be encoded as having two discoidal spines until further data on the origin of the single spine becomes available.

Unlike stated by Roy (1999: 27), the Astape (and Haania) specimens 
studied here have four discoidal spines, the proximal two of which are standing nearly parallel. Roy furthermore stated that two discoidal spines are present "in Chaeteessidae, Astape (Haaniinae), and in only some Paraoxypilinae, Compsothespinae, and Iridopteryginae, subfamilies for which the the normal number is three". All Paraoxypilinae, Iridopterygidae, and Compsothespis specimens studied herein exhibited three discoidal spines and were encoded correspondingly.

56. Fore femur, proximal (or only) discoidal spine, position with respect to remaining discoidal spines: standing on elevated socket [1]; not standing on elevated socket [2].

The proximal (or only) discoidal spine is standing on an elevated socket (state 1) in Amorphoscelinae (Figs. 178, 186) and Mantoida (Figs. 154, 244), whereas in all remaining taxa studied it is not (state 2).

57. Fore femur, discoidal spines, length from proximal to distal if 4 spines are present: 1 and 4 short (of equal length), 2 longer and 3 the longest [1]; 1 short, 2 and 4 longer and of equal length, 3 the longest [2]; 1 and 4 short (of equal length), 2 and 3 distinctly longer and of equal length [3]; 1 and 2 of equal length, 4 shorter and 3 the longest [4]; 2 and 4 very short, 1 longer, 3 the longest [5]; 1, 2 and 3 becoming gradually longer, 3 the longest, 4 much shorter than the rest [6]; 1 the shortest, followed by 4 and 2, 3 the longest [7].

The length relations between the discoidal spines have distinct patterns. They can be described by numeric patterns representing the order of length of the spines. The numbers (from left to right) represent the position (proximal to distal). Their value is representative of their respective length relation to the other spines with 1 being the shortest and 4 being the longest spine. Identical values represent equally long spines). For instance, the pattern 1231 (state 1) indicates the proximal and distal discoidal spines being of equal length and at the same time the shortest. The second spine is longer and the third is the longest.

State 1 (pattern 1231) is represented by Acanthops, Acromantis, Ceratomantis, Choeradodis (Fig. 235), Dystacta, Epaphrodita, Galinthias, Idolomorpha, Majanga, Parasphendale, Polyspilota, Popa, Pseudoharpax, Pyrgomantis, Stenopyga, Taumantis, and Thrinaconyx.

State 2 (pattern 1232) is present in Blepharodes, Blepharopsis, Ceratocrania, Creobroter, Empusa, Gimantis, Gongylus, Heterochaeta, Humbertiella, Hymenopus, Hypsicorypha, Iris, Orthodera, Oxyopsis, Parentella, Photina, Pseudogalepsus, Pseudoyersinia, Rhombodera, Tamolanica, and Theopompa (Fig. 236).

State 3 (pattern 1221) is present in Elaea and Eremiaphilidae (Fig. 237). 
State 4 (pattern 2231) is exhibited by Haaniinae, Plistospilota, Sphodromantis, Stagmatoptera, Tisma (Fig. 238), Vates, and Zoolea.

State 5 (pattern 2131) is autapomorphic for Archimantis (Fig. 239).

State 6 (pattern 2341) was found in Bactromantis, Bolivaria, Deroplatys, Euchomenella (Fig. 240), Hestiasula, Idolomantis, Leptocola, Mantis, Oligonicinae sp., Oxypilus, and Rivetina.

State 7 (pattern 1342) is exhibited by Ameles, Entella, Gonypeta, Ligaria, Ligariella, Odontomantis, Paralygdamia, Sibylla (Fig. 241), Tarachodes, and Tarachodula.

It is unclear why the pattern differs so strongly among taxa. The longest discoidal spine usually plays the important role of the antagonist for the tibial spur during prey capture, helping both closing the leg early and forcing the prey farther between femur and tibia in a lever-and-ratchet manner (e.g. Loxton \& Nicholls 1979: $193 \mathrm{ff}$.). Its function is vital for capturing prey. In all species exhibiting four discoidal spines the third one is the longest (except for state 3 ). Why the other spines differ in length, and why, for instance, in Archimantis the first spine is additionally elongated, remains obscure.

It is important to gather further data on intraspecific variability of this character because the differences between certain states are sometimes minimal.

58. Fore femur, discoidal spines, length from proximal to distal if 3 spines are present: 1 short, 3 longer, 2 the longest [1]; 2 distinctly longer than 1, 3 the shortest [2]; 1, 2 and 3 becoming gradually shorter [3].

A length relation pattern (see explanations in character 57) could also be found in species exhibiting only 3 discoidal spines.

State 1 (pattern 132) was found in Acontista, Callibia, Enicophlebia, Pseudocreobotra, Raptrix, Thesprotia, Tithrone, and Toxodera (Fig. 242).

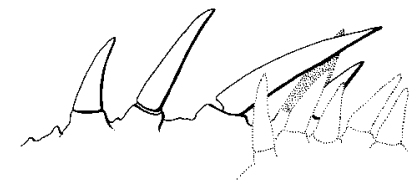

235

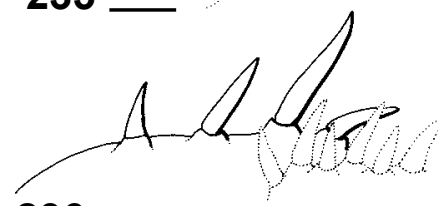

236
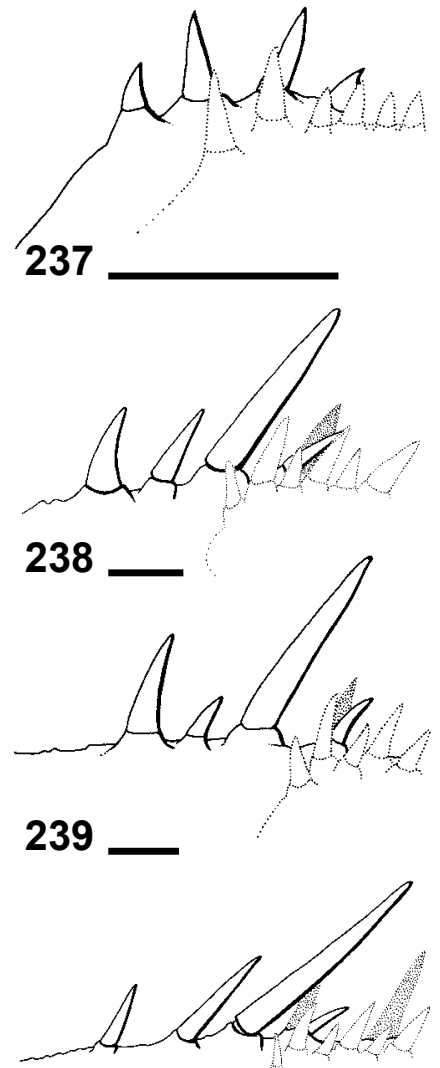

240

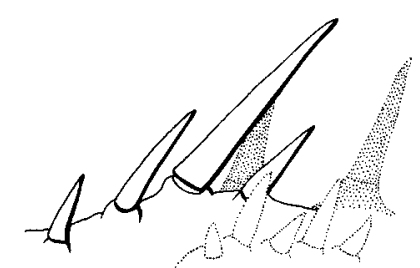

241

Figs. 235-241: Length patterns of discoidal spines, left fore femur, anterior view. 235: Choeradodis stalii, ô (state 1). 236: Theopompa sp. 2, đ̂ (state 2). 237: Eremiaphila sp. 1, ô (state 3). 238: Tisma pauliani, + (state 4). 239: Archimantis gracilis, ㅇ (state 5). 240: Euchomenella sp., + (state 6). 241: Sibylla pretiosa, ô (state 7). Discoidal spines are drawn in solid lines. $\leftarrow$ proximal. - Scale bar $=1.0 \mathrm{~mm}$. 
State 2 (pattern 231) is present in Calamothespis, Ciulfina, Compsothespis, Gyromantis, Hoplocorypha (Figs. 160, 162, 243), Orthoderella, Oxyothespis, Paraoxypilus, Pogonogaster, Stenomantis, Theopropus, and Tropidomantis.

State 3 (pattern 321) is only present in Mantoida (Figs. 152, 154, 244) and may represent an autapomorphy for the genus. The elongated proximal discoidal spine is broken off in the specimen in Figs. 150-151.

This character (as well as characters 59-62) is difficult to assess from the point of homology. Whenever three discoidal spines are present it is unclear if it is always the same spine that was reduced, or whether three discoidal spines represent an early, plesiomorphic character. If the latter is true, the presence of four or more discoidal spines would be apomorphic for a group within Mantodea. The latter hypothesis is supported by the fact that Chaeteessa, Mantoida and Metallyticus all have less than four discoidal spines (although the forelegs of Chaeteessa and Metallyticus may

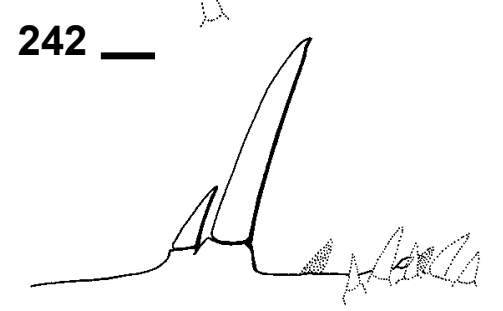

243

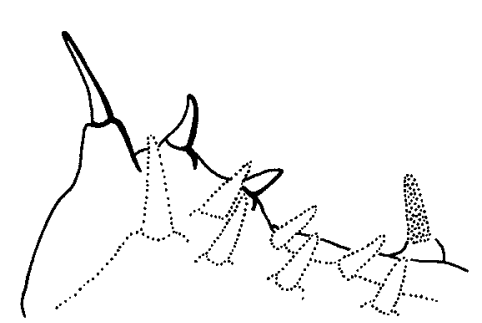

244

Figs. 242-244: Length patterns of discoidal spines, left fore femur, anterior view. 242: Toxodera maculata, $q$ (state 1). 243: Hoplocorypha sp., + (state 2). 244: Mantoida maya, ô (state 3). Discoidal spines are drawn in solid lines. $\leftarrow$ proximal. Scale bar $=0.5 \mathrm{~mm}$. as well represent highly derived conditions due to their lifestyle, see chapters 4.6.4 and 4.6.5). Furthermore, in the taxa with three discoidal spines studied here no remnants of a putative fourth spine could be detected, neither in the microscope nor under the SEM (Mantoida, Paraoxypilus, Thesprotia).

59. Fore femur, proximal 2 discoidal spines (if 4 discoidal spines are present), position: standing closely together, sometimes positioned on a common elevation [1]; not standing closer together than the remaining discoidal spines [2].

The two proximal discoidal spines are standing closer together than the remaining spines (state 1) in Ameles decolor, Bolivaria, Eremiaphila sp. 1, Gongylus, Heteronutarsus, Leptocola, Mantis, and Parentella. The remaining taxa having four discoidal spines exhibit state 2 . See also characters 60-62.

60. Fore femur, proximal 2 discoidal spines (if 3 discoidal spines are present), position: standing closely together, sometimes positioned on a common elevation [1]; not standing closer together than the remaining discoidal spines [2]. 
The two proximal discoidal spines are standing closer together than the remaining spines (state 1) in Compsothespis (Fig. 167), Hoplocorypha (Figs. 160, 162, 243), Raptrix, and Stenomantis. The remaining taxa having three discoidal spines exhibit state 2. See also characters 59, 61 and 62.

61. Fore femur, distal 2 discoidal spines (if 4 discoidal spines are present), position: standing closely together, sometimes positioned on a common elevation [1]; not standing closer together than the remaining discoidal spines [2].

The distal two discoidal spines are standing closer together than the remaining spines (state 1) in Acanthops, Acontista, Empusidae (Figs. 208-213), Epaphrodita, Gimantis, Hestiasula, Hymenopus, and Taumantis.

The remaining taxa having four discoidal spines exhibit state 2 . See also characters 59, 60 and 62 .

62. Fore femur, distal 2 discoidal spines (if 3 discoidal spines are present), position: standing closely together, sometimes positioned on a common elevation [1]; not standing closer together than the remaining discoidal spines [2].

The distal two discoidal spines are standing closer together than the remaining spines (state 1) in Acontista, Pseudocreobotra, Theopropus and Tropidomantis. The remaining taxa having three discoidal spines exhibit state 2 . See also characters 59-61.

63. Fore tibia, antero-ventral spines: present [1]; missing [2].

As far as is known from the literature, the fore tibia carries antero-ventral tibial spines in all Mantodea except for Amorphoscelinae, Perlamantinae and Compsothespis (Roy 1999: 28). This is corroborated by the present study. However, the SEM images of the Perlamantis foreleg revealed comparatively large remnants of antero-ventral tibial spines (Figs. 190-192, 221), whereas no remnants were identified in Amorphoscelis (SEM) and Compsothespis (microscope; see Fig. 266, 267). The remnants of Perlamantis are located on the antero-ventral edge of the tibial main part, near the point of the strong elongation of the distal part (see characters 69, 70; Figs. 190-192, 221). They have a curious shape and resemble sensilla rather than spines, but their size of nearly $20 \mu \mathrm{m}$ is contradictory (Fig. 192). The remnants are obliquely orientated, pointing ventro-distad, and are lowered into cavities. Furthermore, they have large openings at their bases. Their function is unknown. 
Tibial spines in two parallel rows cannot be found in Blattodea (Figs. 255257), therefore this trait is apomorphic for Mantodea. In mantodeans lacking antero-ventral spines it is likely that these have been reduced secondarily. This is supported by the fact that such spines were already present in the Cretaceous mantodeans $\uparrow$ Ambermantis, $\uparrow$ Burmantis, $\uparrow$ Cretomantis (Fig. 29), $\uparrow$ Jersimantis (Fig. 31) (see Grimaldi 2003) and also in putative Carboniferous stemgroupMantodea (Béthoux \& Wieland 2009: 103 f.).

In Paraoxypilinae, the antero-ventral tibial spines are blunt and reduced in size and form a comb-like structure in the antero-distal half of the tibia, being located on a bulging, shelf-like structure (e.g. Sjöstedt 1918: pl. 1: fig. 1d, pl. 2: figs. 1d, 2d, pl. 3: fig. 7; Beier 1929: fig. 5; Milledge 1990: fig. 10; Figs. 245, 264, 265). Apparently, Roy (1999: 28) was insecure about the identification of these small "granules" as actual tibial spines: "The situation [antero-ventral spines missing] is the same for Paraoxypilinae if one disregards a small subapical row of internal granules, which face a similar row of granules on the femora" (Roy 1999: 28). However, both their location on the tibia, their surface structure and the presence of sutures between the spines and the tibia (see chapter 4.6.10 and Figs. 245, 264, 265) are in my opinion indicative of an origin from regular antero-tibial spines. Therefore such spines are encoded as "present" for Paraoxypilinae.

64. Fore tibia, postero-ventral spines: present [1]; missing [2].

See also character 63. The postero-ventral tibial spines are missing in Amorphoscelinae (Fig. 178), Perlamantinae, Paraoxypilinae (Fig. 245), and Compsothespis (e.g. Roy 1999: 28; Fig. 266, 267). A trend of reduction can also be found in some Oligonicinae. While a single postero-ventral tibial spine is present in Thesprotia (Figs. 165, 250) and Oligonyx Saussure, 1869, these spines are completely absent in Thesprotiella (the two latter not being present in this study) (Beier 1964a: fig. 8d, 1968a: fig. 8; Roy 1999: 28). A strong reduction of the posteroventral tibial spines can also be found in Heteronutarsus. While the female still has a distinct distal postero-ventral spine and three further very small spines, the male only has the distal spine left.

It is likely that the postero-ventral tibial spines were secondarily reduced in those taxa exhibiting this character state. For instance, in Paraoxypilus there are distinct remnants of the distal postero-ventral tibial spines visible in the SEM (Figs. 245, 246). In Amorphoscelis and Perlamantis no remnants of posteroventral tibial spines could be identified with the SEM. 
65. Fore tibia, spination, position: in two ventral rows [1]; in two rows, only few spines present, distal spines shifted dorsad [2]; only antero-ventral spines present [3]; spines missing except for tibial spur [4].

The fore tibia in the greater part of the extant Mantodea carries two ventral rows of spines. The spines in both of them usually become longer distally. The apical antero-ventral spine is located on a lobe-like extension of the tibia and forms the tibial claw. This pattern (state 1) is altered in some taxa.

Some Thespidae show a strong degree of reduction of the spines combined with a shortening of the tibia and a characteristic orientation of the antero-ventral tibial spines (state 2; see also characters $64,66,73,74)$. This character state is exhibited by Oligonicinae and Haaniinae (Figs. 247-254).

In Paraoxypilinae the postero-ventral spines are reduced, whereas the antero-ventral spines are miniaturized and slightly shifted apicad (state 3; see also characters 63, 64; Figs. 245, 165). In Amorphoscelinae, Perlamantinae, and Compsothespis the tibial spines are reduced (state 4; Figs. 178, 190, 220, 266, 267). All remaining taxa in the present study show the “typical” pattern (state 1).

66. Fore tibia, distal 2-3 antero-ventral spines, orientation: inserting ventrally, pointing ventro-apicad [1]; inserting anteriorly to dorsally, distinctly pointing dorso-apicad [2].
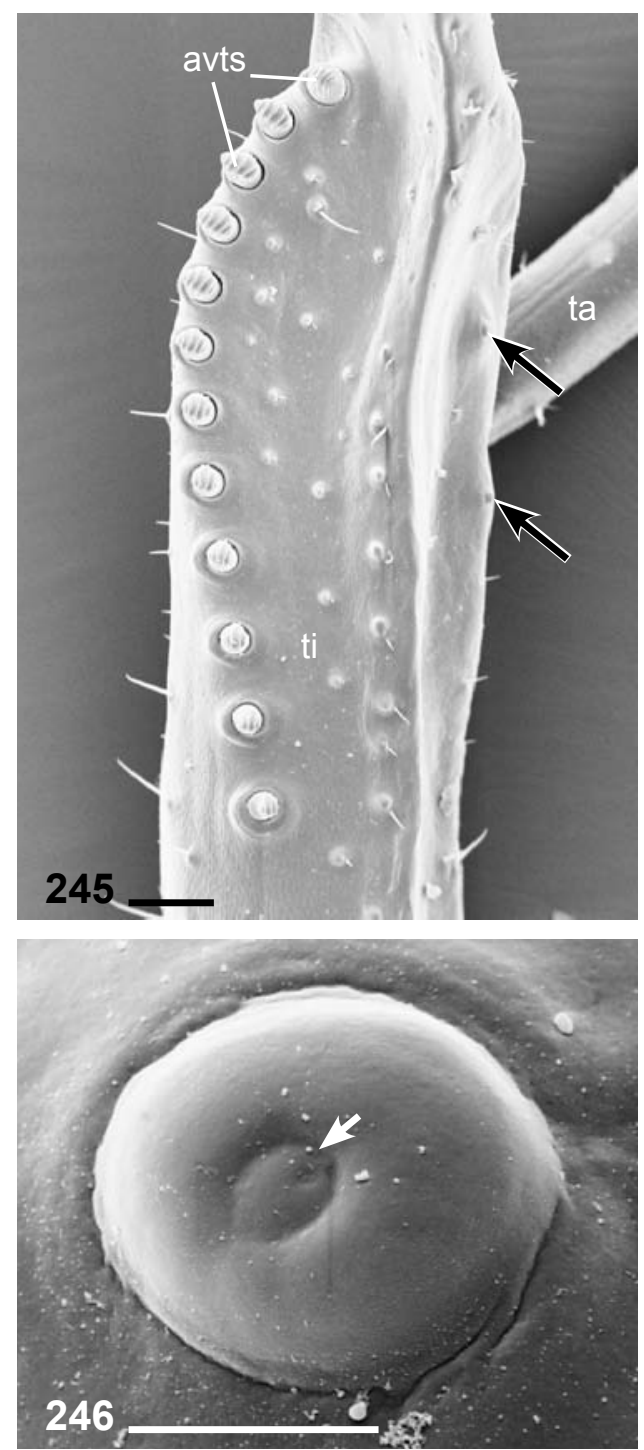

Figs. 245-246: Paraoxypilus sp., §̂, left fore tibia. 245: Ventral overview of distal part of tibia; arrows indicate remnants of spines. 246: Remnant of postero-ventral fore tibial spine, detail; arrow indicates indentation typical for spines on the forelegs. - Scale bar $=0.1 \mathrm{~mm}$ (Fig. 245); $0.01 \mathrm{~mm}$ (Fig. 246). Abbreviations: avts $=$ antero-ventral fore tibial spine; $\mathbf{t a}=$ tarsus.

A shift of the distal antero-ventral spines in antero-dorsal direction (state 2) can only be found in the Neotropical Oligonicinae (Saussure \& Zehntner 1894: 161 and pl. 9 figs. 21, 28, 29, pl. 10 figs 2, 7, 8; Chopard 1916: fig. 9; Beier 1935a: 8 and pl. 1 fig. 13a; Beier 1964a: fig. 8c, d, 1968a: fig. 8; Nickle 1987: fig. 13.2; Figs. 247, 249, 251) and the Indomalayan Haaniinae (Chopard 1920: figs. 2, 3; Beier 1952: figs. 7, 8; Anisyutkin \& Gorochov 2005: fig. 9; Fig. 253) 

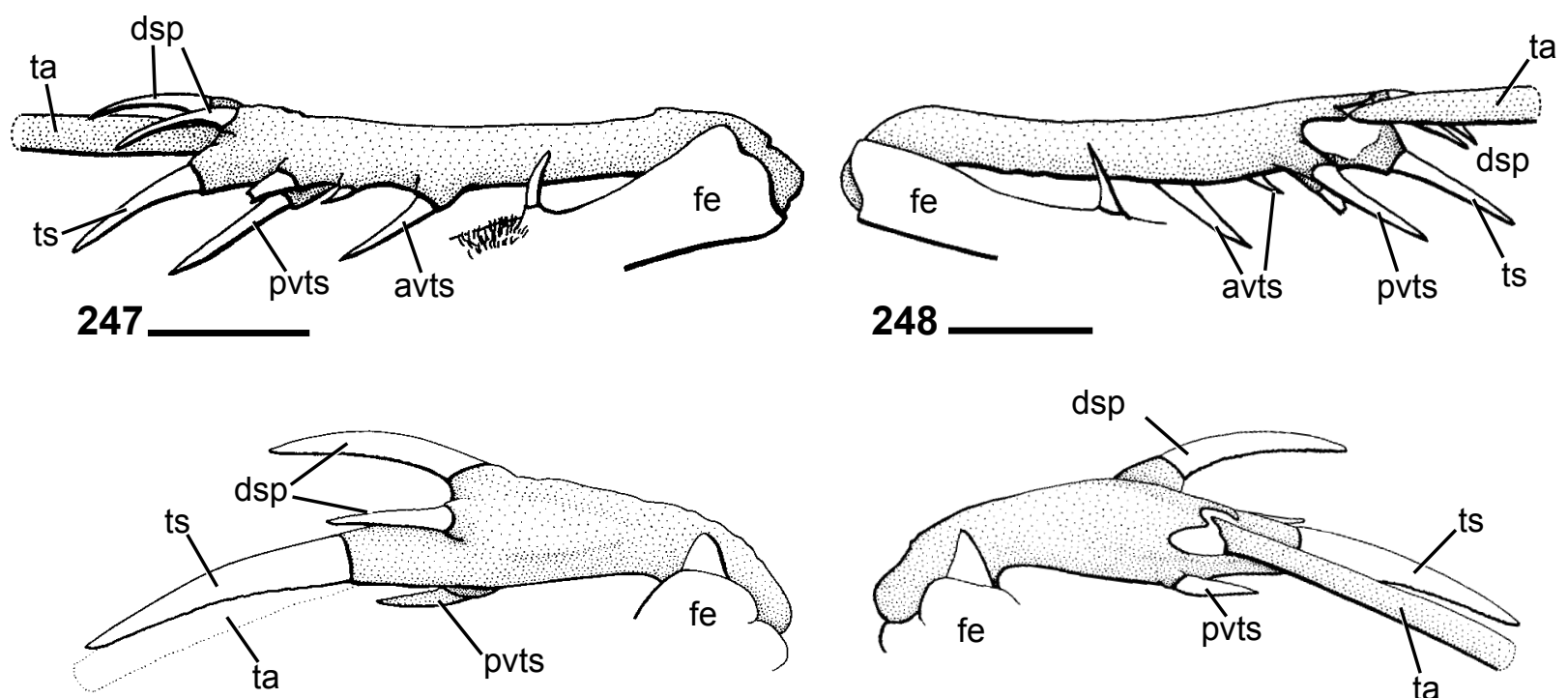

249

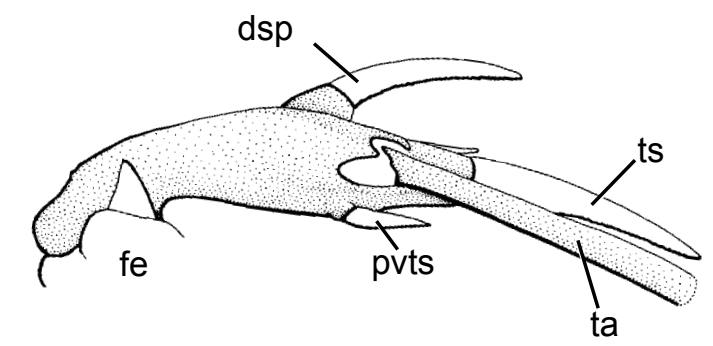

250
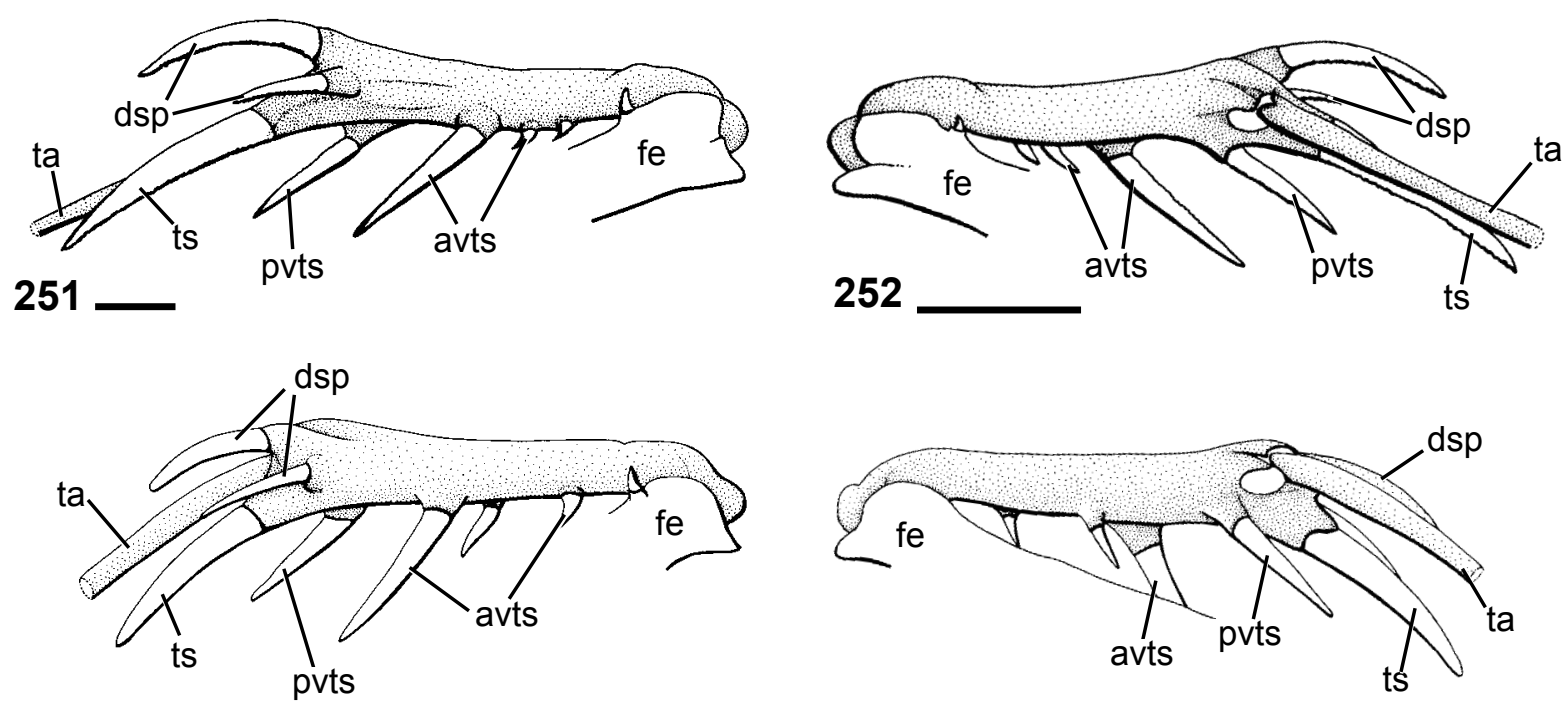

253

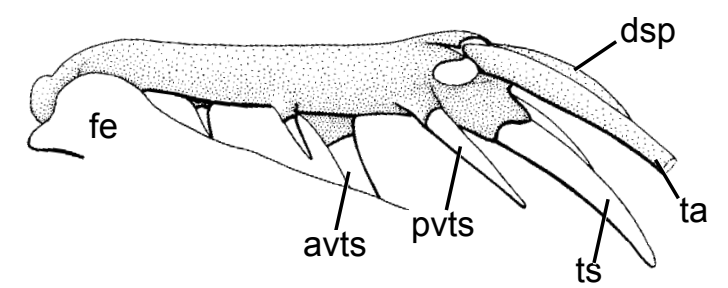

254

Figs. 247-254: Tibial morphology of Oligonicinae and Haaniinae. Left column: anterior view. Right column: posterior view. 247, 248: Thrinaconyx kirschianus, $\widehat{\partial}$, right fore tibia. 249, 250: Thesprotia graminis, $\widehat{\partial}$, left fore tibia (mirrored). 251, 252: Pogonogaster tristani, ㅇ, left fore tibia (mirrored). 253, 254: Haania lobiceps, ㅇ, right fore tibia. - Scale bar $=0.5 \mathrm{~mm}$.

Abbreviations: avts $=$ antero-ventral fore tibial spine; $\mathbf{d s p}=$ dorsal spine; $\mathbf{f e}=$ femur; $\mathbf{p v t s}=$ postero-ventral fore tibial spine; $\mathbf{t a}=$ tarsus; $\mathbf{t s}=$ tibial spur.

(see chapter 4.6.10). In all remaining Mantodea the antero-ventral spines show the regular orientation (state 1).

Roy (1999: 37) stated that "the migration of some internal spines to the dorsal position occurred only once", thus indicating the monophyly of those taxa exhibiting this character state (i.e. Haaniinae + Oligonicinae).

Svenson \& Whiting (2009: fig. 3a) found a clade comprising all Oligonicinae but among them also several taxa that do not exhibit the dorsal tibial spines $(\mathrm{Mu}$ soniella, Macromusonia, Eumusonia, Pseudomiopteryx). Therefore, this character 

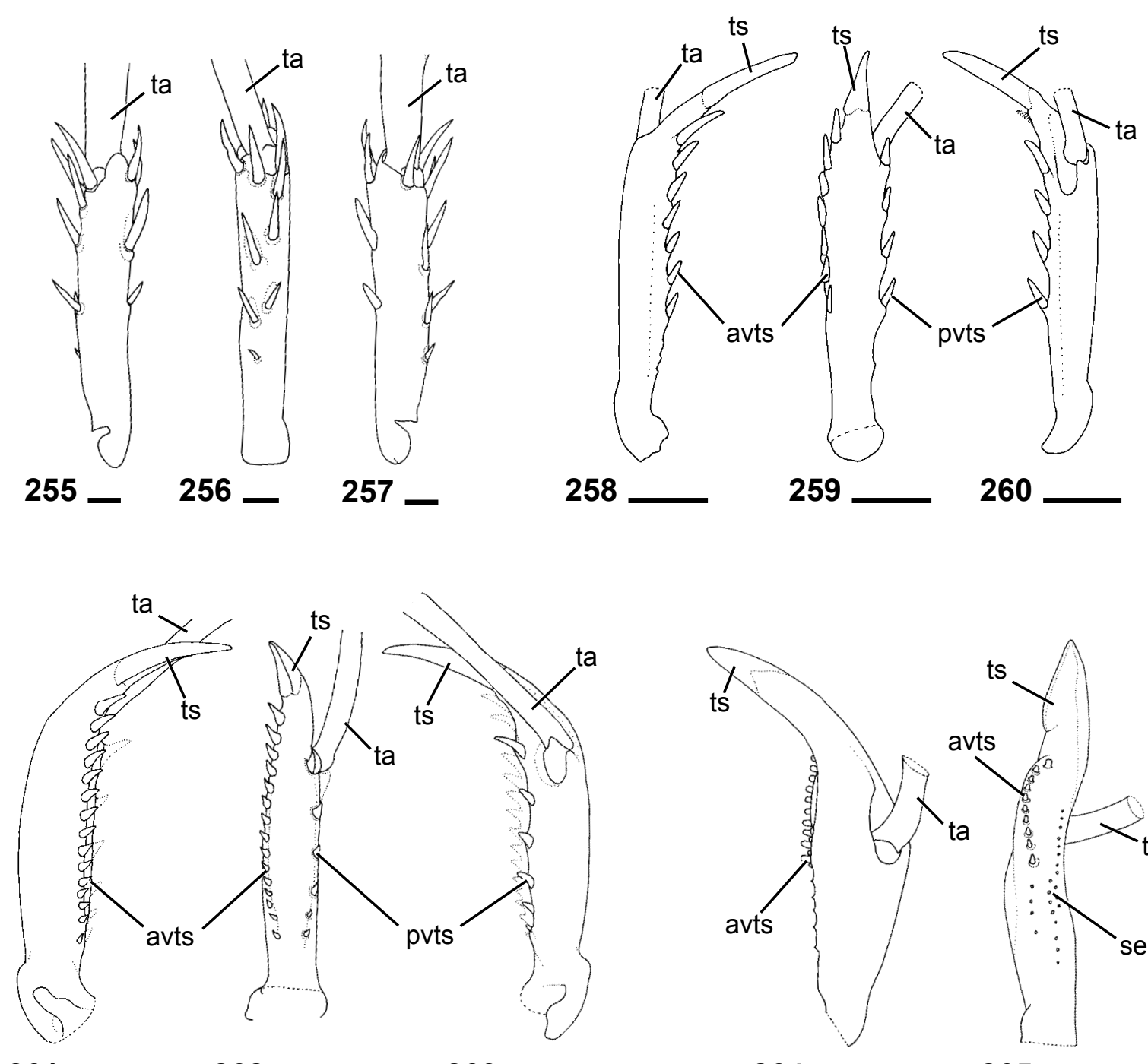

261

262

263
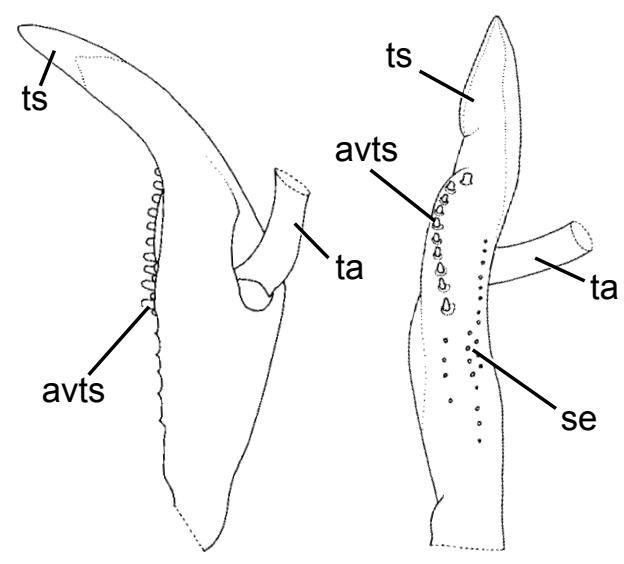

264

265
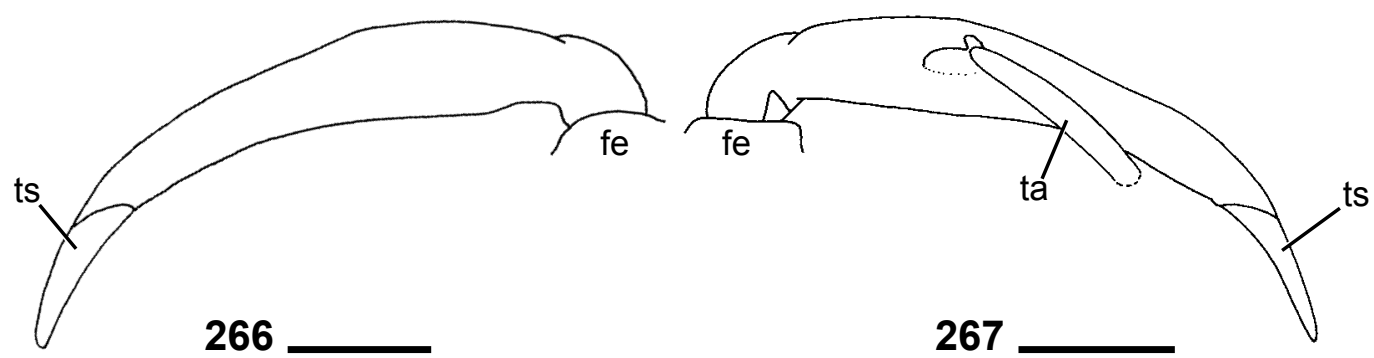

Figs. 255-267: Fore tibiae of selected taxa. 255-257: Periplaneta americana, $q$, right foreleg. 255: Anterior view. 256: Ventral view. 257: Posterior view; note that the spines of Mantodea and Blattaria have so far not been homologized, therefore antero-ventral and postero-ventral spines cannot be addressed in the cockroach. 258-260: Mantoida maya, ô, left foreleg. 258: Anterior view. 259: Ventral view. 260: Posterior view. 261-263: Eremiaphila sp. 1,, , left foreleg. 261: Anterior view. 262: Ventral view. 263: Posterior view. 264-265: Paraoxypilus sp., + , left foreleg. 264: Posterior view. 265: Ventral view. 266-267: Compsothespis anomala, + , right foreleg. 266: Anterior view. 267: Posterior view. - Scale bar $=0.5 \mathrm{~mm}$.

Abbreviations: avts $=$ antero-ventral fore tibial spine; $\mathbf{f e}=$ femur; $\mathbf{p v t s}=$ postero-ventral fore tibial spine; $\mathbf{s e}=$ seta; $\mathbf{t a}=$ tarsus $; \mathbf{t i}=$ tibia; $\mathbf{t s}=$ tibial spur. 

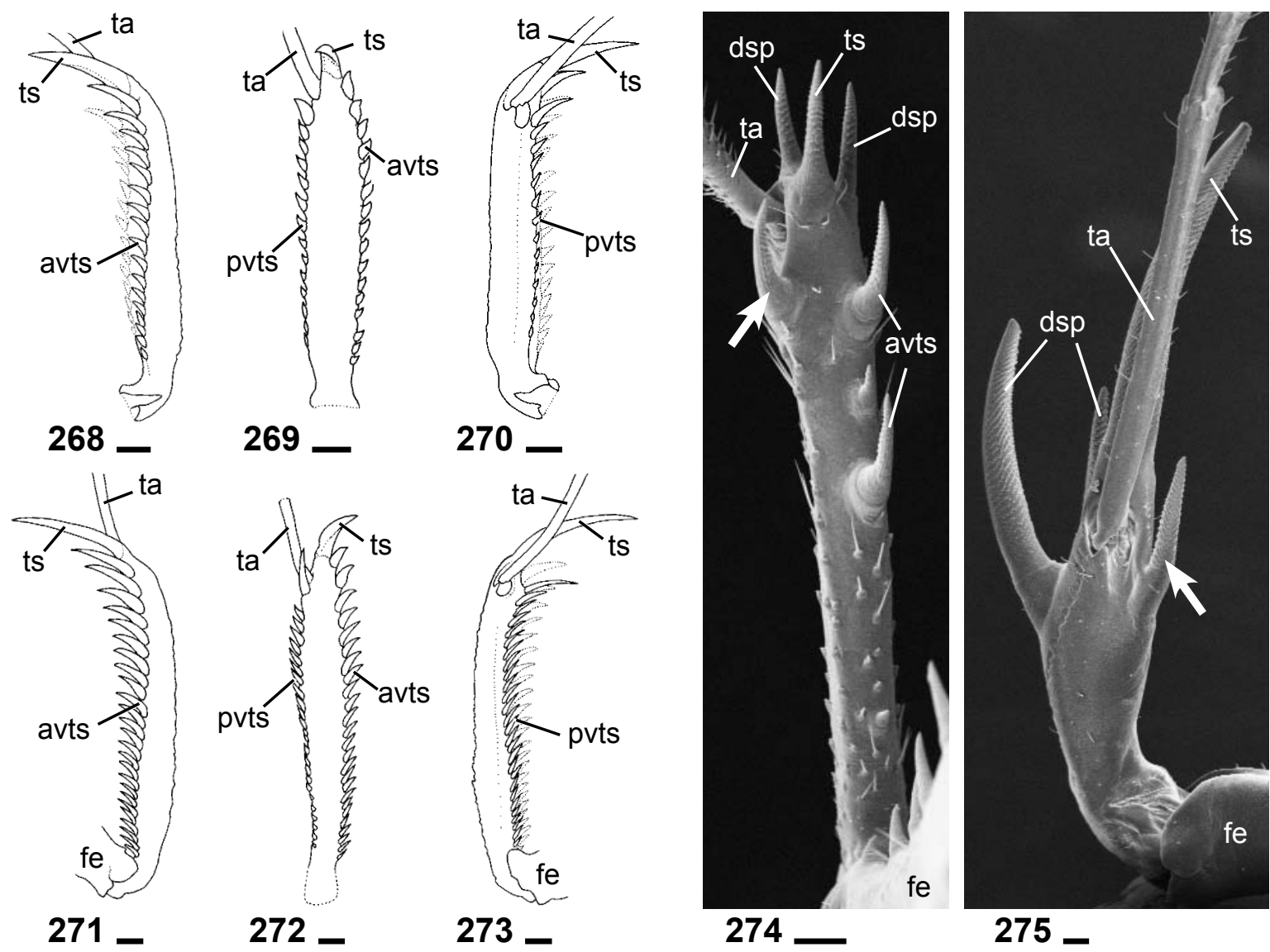

275

Figs. 268-275: Fore tibiae of selected taxa. 268-270: Blepharopsis mendica, $\uparrow$, right foreleg. 268: Anterior view. 269: Ventral view. 270: Posterior view. 271-273: Idolomantis diabolica, ڤ̂, left foreleg (mirrored). 271: Anterior view. 272: Ventral view. 273: Posterior view. 274: Oligonicinae sp., §̂, SEM image of right foreleg, ventral overview of tibia; note the strong reduction of postero-ventral spines with exception of one (arrow). 275: Thesprotia graminis, + , SEM image of left fore tibia, posterior view (mirrored); note the single remaining postero-ventral fore tibial spine (arrow). - Scale bar $=1.0 \mathrm{~mm}(268-273), 0.1 \mathrm{~mm}(274,275)$.

Abbreviations: avts $=$ antero-ventral fore tibial spine; $\mathbf{d s p}=$ dorsal spine; $\mathbf{f e}=$ femur; $\mathbf{p v t s}=$ postero-ventral fore tibial spine; $\mathbf{t a}=$ tarsus; $\mathbf{t s}=$ tibial spur.

may be apomorphic for a group within Oligonicinae. Haania was also present in the molecular dataset of Svenson \& Whiting (2009: fig. 3c). Therein, it was found far more distal in the phylogenetic tree than Oligonicinae. This is supported in the presence of the DK morphology of the metathoracic ear in Haania (see chars. 130, 131 and Yager \& Svenson 2008: 559, 566). In contrast, the ear is primarily missing in the Neotropical Oligonicinae. Therefore, a convergent gain of dorsally positioned antero-ventral spines at least in Oligonicinae and Haaniinae is indicated by both ear morphology and molecular data. Otherwise, the metathoracic ear must have evolved several times independently (see also discussion of the taxon in chapter 4.3.23 and in chapter 4.6.14).

67. Fore tibia, length with respect to length of femur: half as long or shorter [1]; more than half as long [2]. 
The prothoracic tibiae are usually shorter than the femora (Roy 1999: 28; Klass \& Ehrmann 2003: 185). They are less than half as long as the femur (state 1) in about 40 per cent of the taxa studied herein. The rest of the species studied exhibit state 2 .

It has been stated in the literature that the tibia never joins the frequent elongation of coxa and femur (e.g. Beier \& Jaus 1933: 131; Beier 1964a: 874, 1968a: 22; see also discussion of character 37). However, there is an exception: Several Toxoderidae (represented by Toxodera and Calamothespis in the present study) have strongly elongated and slender fore femora. The claw groove, however, lies near the proximal end of the femur [e.g. Beier 1934c: 1, and explicit mentioning in the generic diagnoses of Toxodanuria Uvarov, 1940 (mentioned as Paradanuria on p. 3 in Beier 1935c), Loxomantis Giglio-Tos, 1914 (p. 4), Aethalochroa (p. 5), Belomantis Giglio-Tos, 1914 (p. 5), Compsothespis (p. 6), Toxomantis Giglio-Tos, 1914 (p. 7), Paratoxodera (p. 7), and Toxodera (p. 8); see also figures in Roy 2009a]. Considering the position of the claw groove, the femoral elongation in this group has not taken place in the proximal part of the femur alone, as in all other extant Mantodea with elongated femora, but the entire leg including femur and tibia has become stretched in the area carrying the spines instead. This hypothesis finds support in the comparatively wide spaces between the femoral spines (see Figs. 22 and 207 for Toxodera maculata). The tibia also exhibits wide spaces between its spines (also mentioned by Beier 1976: 394). The tibial spur rests in the claw groove near the base of the femur (see Beier 1976: plates 1-3; Kaltenbach 1998: fig. 39; figures in Roy 2009a), therefore it is very likely that in (at least some) Toxoderidae the tibia underwent a distinct elongation, paralleling that of the femur.

Stenophylla, formerly assumed to have Toxoderidae affinities, is in the current systematization assigned to Acanthopidae. For this genus Beier (1934c: 8) also mentioned a proximal position of the claw groove. However, the fore legs of Stenophylla are much less elongated and the spines are standing much more closely together, therefore the condition in this genus differs distinctly from that in Toxoderidae.

68. Fore tibia, tibial spur: present [1]; missing [2].

The tibial spur has always been mentioned to be present in all extant Mantodea but to be missing in Chaeteessa (e.g. Beier 1968a; Roy 1999). However, it is present but probably apomorphically reduced into a small, thickened seta-like spine located on a small tubercle (mentioned by Klass 1995: 193 "Bei Chaeteessa findet man median der Tarsus-Artikulation einen kleinen Stummel mit feinen Borsten [...]"; Roy 1999: 38 "small, setigerous tubercle"; see Figs. 418-420). 
Chaeteessa is therefore encoded as state 1 in this analysis. A detailed discussion of this topic is given in chapter 4.6.5.

69. Fore tibia, tibial spur, length with respect to length of tibia: one fifth as long or longer [1]; distinctly shorter than one fifth, merely forming the tip of the apical tibial lobe [2]; shorter than one fifth of tibia but tibia very long and slender [3].

The length of the tibial spur, i.e. from its base (marked by a suture) to its tip, varies considerably within Mantodea.

In most species studied, the tibial spur measures one fifth of the tibial length or more (state 1). In Toxoderidae it is shorter than that (state 3; Figs. 22, 207). The spur in this group is large and sturdy, similar to most other Mantodea, but the tibia is strongly elongated (see character 67) thus leading to a relatively shorter tibial spur (see Roy 2009a: figs. 2E, 19G, 59H, 67C). State 2 is exhibited only by Perlamantinae in which the tibia is strongly folded (see character 70; Figs. 190, 220). The apical lobe that carries the spur is strongly enlarged and the tibial spur itself is only a small remnant on the lobe's tip (Figs. 220-222). Apart from Chaeteessa, Perlamantis is the only species with a distinctly reduced tibial spur.

This character has not been encoded for Chaeteessa because the tibial spur was probably reduced into a small seta and the plesiomorphic condition in the stem lineage of Chaeteessa is unknown (see character 68 and discussion in 4.6.5).

70. Fore tibia, shape: straight or curved, but medially not strongly folded [1]; distal part of tibia strongly folded, almost $90^{\circ}$ with respect to proximal tibia in lateral view [2].

A strongly folded tibia is autapomorphic for Perlamantinae. In both Perlamantis and Paramorphoscelis the antero-distal part of the fore tibia (i.e. the lobe carrying the tibial spur, see also character 69) is strongly widened, lengthened and folded to an angle of nearly $90^{\circ}$ with respect to the proximal part of the tibia (e.g. Morales Agacino 1947: figs 15PA, 21PA; Figs. 190, 220). The tibial spur is forming only the distal tip of this structure (see character 69; Figs. 220-222).

In all remaining Mantodea a folding of the tibia to this extent does not exist. In Chaeteessa, the entire tibia is curved (Figs. 418, 420). This possibly represents a derived condition and is autapomorphic for the genus (see 4.6 .5 for a detailed discussion).

71. Fore tibia, expansion of antero-ventral and postero-ventral edges: missing [1]; present [2].

The anterior and (to a lesser extent) posterior ventral edges of the fore tibia are widened in several taxa. The spines are thus positioned farther away from 
the centre of the tibia, and the ventral surface of the tibia is correspondingly enlarged. This character state is exhibited by several Empusidae, Hymenopodinae, and Acanthopidae.

72. Fore tibia, antero-ventral tibial spines, length: nearly equally long [1]; distally increasing in length [2]; alternating irregularly long [3]; spines irregularly positioned on tibia or only a single spine present [4]; becoming longer distally, becoming short in the distal third and increasing in length again [5].

The length pattern of the antero-ventral tibial spines is similar in most Mantodea with few exceptions. The greater part of the species studied herein exhibit state 2 with the spines becoming subsequently longer distally.

State 1 is probably autapomorphic for Paraoxypilinae. In this group, here represented by Paraoxypilus, Gyromantis and the Paraoxypilinae sp. specimen, the antero-ventral tibial spines are located on a shelf-like widening of the fore tibia. They are equally short and blunt and form a comb-like structure (e.g. Beier 1929: fig. 5; Milledge 1990: fig. 10; see characters 63, 65 and Figs. 245, 264, 265).

In some taxa the spines are irregularly long [state 3: Pogonogaster (Fig. 251), Thrinaconyx (Fig. 247, one spine broken off), Toxodera denticulata (Toxodera maculata and Calamothespis exhibit state 2)] or they are irregularly positioned on the tibia, or only a single spine is present [state 4: Astape, Haania (Fig. 253), Oligonicinae sp. (Fig. 274), and Thesprotia (Figs. 163, 249, 275)].

State 5 has in this study only been found in Chaeteessa (Figs. 418-420; see also Terra 1995: fig. 12). However, a similar condition is apparently also present in several Neotropical Thespidae, for instance in Bantiella Giglio-Tos, 1915, Bantia, and Pseudomusonia (Terra 1995: figs. 77-79). It may have been the starting point of the evolution of the odd fore tibial spination pattern found in Oligonicinae and Haaniinae.

73. Fore tibia of male, antero-ventral spines (excluding tibial spur), largest number: 1-5 [1]; 6-12 [2]; 13-15 [3]; 16-19 [4]; 20-25 [5]; 26 or more [6].

1-5 spines (state 1) have been counted in the males of Astape, Bactromantis, Haania, Oligonicinae sp., Thesprotia, and Thrinaconyx.

6-12 spines (state 2) are present in male Acromantis, Ambivia, Ameles, Bolivaria, Ceratomantis, Ciulfina, Dystacta, Elaea, Epaphrodita, Gimantis, Gonypeta, Gyromantis, Hestiasula, Hoplocorypha, Humbertiella, Ligaria, Majanga, Mantoida, Metallyticus, Odontomantis, Oxyothespis, Oxypilus, Paraoxypilus, Parentella, Popa, Pseudogalepsus, Pseudoharpax, Pseudoyersinia, Stenomantis, Theopompa, Toxodera denticulata, and Tropidomantis. 
13-15 spines (state 3) are present in male Acontista, Archimantis gracilis, Blepharodes, Callibia, Choeradodis, Creobroter, Deroplatys desiccata, Eremiaphila sp. 1, Euchomenella, Galinthias, Heteronutarsus, Iris, Mantis, Orthodera, Oxyopsis, Parasphendale, Phyllocrania, Plistospilota, Polyspilota, Pseudocreobotra, Pyrgomantis, Raptrix, Rhombodera, Sibylla, Sphodromantis, Stagmatoptera, Stenopyga, Tamolanica, Tarachodes, Tarachodula, Tisma, Tithrone, and Vates.

16-19 spines (state 4) are found in male Acanthops tuberculata, Archimantis sobrina, Ceratocrania, Deroplatys lobata, Empusa, Hymenopus, Idolomorpha, Orthoderella, Theopropus, and Zoolea.

20-25 spines (state 5) are exhibited by male Blepharopsis, Heterochaeta, and Idolomantis.

26 spines or more (state 6) are only present in male Gongylus.

74. Fore tibia of female, antero-ventral spines (excluding tibial spur), largest number: 1-5 [1]; 6-12 [2]; 13-15 [3]; 16-19 [4]; 20-25 [5]; 26 or more [6].

1-5 spines (state 1) are present in the females of Haaniinae, Oligonicinae sp., Pogonogaster, and Thesprotia graminis.

6-12 spines (state 2) are present in female Acontista, Acromantis, Ameles, Ceratomantis, Dystacta, Elaea, Entella, Gimantis, Gonypeta, Hestiasula, Hoplocorypha, Humbertiella, Ligaria, Ligariella, Majanga, Mantoida, Metallyticus, Odontomantis, Oxypilus, Paralygdamia, Paraoxypilus, Parentella, Paraoxypilinae sp., Polyspilota, Popa, Pseudoyersinia, Rivetina, Stenomantis, Taumantis, Theopompa tosta, Toxodera maculata, and Tropidomantis.

13-15 spines (state 3) are present in female Archimantis, Blepharodes, Bolivaria, Calamothespis, Chaeteessa, Choeradodis stalii, Creobroter gemmatus, Deroplatys desiccata, Enicophlebia, Eremiaphila, Euchomenella, Galinthias, Gyromantis, Iris, Leptocola, Mantis, Orthodera, Oxyopsis, Parasphendale, Photina, Plistospilota, Pseudocreobotra, Pseudoharpax, Pyrgomantis, Raptrix, Rhombodera, Sibylla, Sphodromantis, Stagmatoptera, Tamolanica, Tarachodes, Tarachodula, Tisma, Tithrone, Vates.

16-19 spines (state 4) have been found in female Acanthops, Callibia, Creobroter elongata, Deroplatys lobata, Heterochaeta strachani, Heteronutarsus, Hypsicorypha, Idolomorpha, Orthoderella, Phyllocrania, Theopropus, and Zoolea.

20-25 spines (state 5) were exhibited by female Blepharopsis, Empusa, Hymenopus, Idolomantis, and Toxodera denticulata.

26 spines or more (state 6) are only present in female Gongylus.

The antero-ventral spines are missing in Amorphoscelinae, Perlamantinae, and Compsothespis. 
Interestingly, the two Toxodera species exhibit strongly differing numbers of spines. Such differences were also mentioned by Roy (2009a). While Toxodera denticulata, T. maxima Roy, 2009, T. beieri Roy, 2009, T. fimbriata Werner, 1930 and T. integrifolia Werner, 1925 are therein listed to have up to 31 antero-ventral tibial spines (Roy 2009a: 101, 107, 113, 127, 136), T. maculata has no more than 12 (Roy 2009a: 118). The species of Paratoxodera even exhibit up to 42 antero-ventral tibial spines (Roy 2009a: 153). There seems to be a distinct sexual dimorphism in the number of antero-ventral tibial spines at least in T. denticulata (compare character 73 and chapter 4.6.8).

75. Fore tibia, postero-ventral spines, length: nearly equally long [1]; becoming longer distad [2]; becoming longer distad in the proximal third, then becoming short and increasing in length again [3]; spines irregularly positioned on tibia or only a single spine present [4].

The postero-ventral tibial spines are nearly equally long (state 1) only in Mantoida (Fig. 260).

State 3 is present in Majanga, Popa, Sibylla, Stenomantis, and Tropidomantis. This character state was also shown in the literature, for instance by Kaltenbach [1998: Hapalomantis orba (Stål, 1856) (fig. 13) and Negromantis gracillima Kaltenbach, 1996 (fig. 14)]. There are drawings in the literature that are indicative of a similar condition in Neotropical taxa, for instance in Liturgusa (Terra 1995: fig. 87), for Australian taxa, for instance Ima fusca (Tindale 1924: fig. 387c), and for Madagascan taxa, for instance Liturgusella malagassa (Saussure \& Zehntner 1895) (pl. 9, fig. 31a therein).

State 4 is present in Haaniinae and Oligonicinae (Figs. 248, 250, 252, 254).

In all remaining taxa studied herein $(\sim 75 \%$; except for those lacking tibial spines) the postero-ventral tibial spines become gradually longer distad (state 2; Figs. 162, 263, 270, 273), as is also the case in the antero-ventral tibial spines in most taxa (see character 72).

In Chaeteessa, the situation is difficult to assess (Figs. 418, 420). Four postero-ventral tibial spines are present as in Mantoida. But unlike in the latter, the spines strongly differ in length. The second spine is longer than the proximal one, the third one is distinctly shorter, whereas the distal spine is longer than the second proximal spine, thus being the longest in the row. Chaeteessa is correspondingly provisionally encoded as state 3 .

76. Fore tibia, postero-ventral spines, orientation: resembling antero-ventral tibial spines ("normal condition": standing closely together with less than the width of one spine base between them, not being laid down) [1]; reduced in size and being laid down in the proximal half of tibia at most, much smaller and more closely arranged than antero-ventral spines [2]; all spines 
smaller and more closely arranged than antero-ventral spines and being laid down (almost parallel to tibia) along the entire tibia [3]; in normal distance to each other (resembling anteroventral spines, width between spines usually less than diameter of one spine base) but being laid down [4]; oblique to a normal degree (resembling antero-ventral spines), but with wide space between the spines (usually more than width of a spine base) [5].

While the orientation of the antero-ventral tibial spines is mostly quite similar among the taxa, the postero-ventral tibial spines show distinctive interspecific differences. Several authors have stated that dense spines being laid down (i.e. being directed distad in a very low angle from the tibial longitudinal axis, state 2 and 3 ) and almost being fused are characteristic for Hymenopodidae (e.g. Beier 1964a: 938, 1968a: 5; but see Roy 1999: 39 and discussion of Hymenopodidae in chapter 2.1.4). However, the distribution of this character state throughout Mantodea has not been studied in further detail so far.

State 1 is exhibited by about $50 \%$ of the species studied.

State 2 is present in Acanthops, Ambivia, Ceratomantis, Choeradodis, Creobroter gemmatus (C. elongata exhibiting state 3), Empusidae (except for Idolomantis which exhibits state 1; see Figs. 270, 273), Enicophlebia, Epaphrodita, Hymenopus, Photina, Tarachodes sp. 3 (the two other species exhibiting state 1), and Zoolea.

State 3 is exhibited by Acontista, Acromantis, Callibia, Creobroter elongata, Galinthias, Hestiasula, Odontomantis, Phyllocrania, Pseudocreobotra, Pseudoharpax, Raptrix, and Theopropus.

State 4 has been found only in Amphecostephanus and Oxypilus.

State 5 is exhibited by Toxodera, Heterochaeta, Eremiaphilidae, and Haania.

Interestingly, there are intrageneric differences, for instance in Creobroter and Tarachodes.

77. Fore tibia of male, postero-ventral spines, largest number: 1-3 [1]; 4-7 [2]; 8-12 [3]; 13-17 [4]; 18-21 [5]; 22 or more [6].

1-3 postero-ventral tibial spines (state 1) are present in male Haaniinae, Heteronutarsus, and Oligonicinae.

4-7 postero-ventral tibial spines (state 2) are present in male Ciulfina, Eremiaphila sp. 1, Heterochaeta strachani, Hoplocorypha, Leptocola, Majanga, Mantoida, Metallyticus splendidus, Oxypilus, Parasphendale, Parentella, Sibylla, Stenomantis, and Toxodera denticulata.

8-12 postero-ventral spines (state 3) are present in male Acromantis, Ameles, Archimantis, Blepharodes, Bolivaria, Ceratomantis, Choeradodis stalii, Deroplatys, Dystacta, Elaea, Euchomenella, Gimantis, Gonypeta, Hestiasula, 
Heterochaeta bernardii, Humbertiella, Mantis, Oxyopsis, Oxyothespis, Plistospilota, Polyspilota, Popa, Pseudogalepsus, Pseudoharpax, Pseudoyersinia, Rhombodera, Sphodromantis, Stagmatoptera, Stenopyga, Tamolanica, Theopompa, Tisma, Tropidomantis, and Vatinae except Ambivia.

13-17 postero-ventral tibial spines (state 4) are present in male Acontista, Ambivia, Ceratocrania, Choeradodis rhombicollis, Creobroter, Epaphrodita, Galinthias, Iris, Odontomantis, Orthodera, Orthoderella, Phyllocrania, Pseudocreobotra ocellata, Pyrgomantis, Raptrix, Tarachodes, Tarachodula, and Tithrone.

18-21 postero-ventral tibial spines (state 5) are present in male Blepharopsis, Callibia, Empusa, Idolomorpha, Pseudocreobotra wahlbergii, and Theopropus.

22 or more postero-ventral tibial spines (state 6) are present only in male Acanthops, Gongylus, Hymenopus, and Idolomantis.

78. Fore tibia of female, postero-ventral spines, largest number: 1-3 [1]; 4-7 [2]; 8-12 [3]; 13-17 [4]; 18-21 [5]; 22 or more [6].

1-3 postero-ventral tibial spines (state 1) are present in female Haaniinae, Oligonicinae sp., Pogonogaster, and Thesprotia graminis.

4-7 postero-ventral tibial spines (state 2) are present in female Calamothespis, Chaeteessa, Entella, Eremiaphilidae, Euchomenella, Heterochaeta strachani, Hoplocorypha, Leptocola, Ligaria, Ligariella, Majanga, Mantoida, Metallyticus, Oxypilus, Parasphendale, Parentella, Sibylla, Stenomantis, and Toxodera maculata.

8-12 postero-ventral spines (state 3) are exhibited by female Acromantis, Ameles, Archimantis, Blepharodes, Bolivaria, Ceratomantis, Choeradodis stalii, Deroplatys, Dystacta, Elaea, Gimantis, Gonypeta, Humbertiella, Mantis, Oxyopsis, Paralygdamia, Plistospilota, Polyspilota, Popa, Pseudoharpax, Pseudoyersinia, Rhombodera, Rivetina, Sphodromantis, Stagmatoptera, Tamolanica, Taumantis, Theopompa tosta, Tisma, Toxodera denticulata, Tropidomantis, and Zoolea.

13-17 postero-ventral tibial spines (state 4) are present in female Acontista, Blepharopsis, Creobroter, Enicophlebia, Galinthias, Hestiasula, Iris, Odontomantis, Orthodera, Orthoderella, Photina, Phyllocrania, Pseudocreobotra wahlbergii, Pyrgomantis, Raptrix, Tarachodes, Tarachodula, Tithrone, and Vates.

18-21 postero-ventral tibial spines (state 5) are present in female Callibia, Empusa, and Pseudocreobotra ocellata.

22 or more postero-ventral tibial spines (state 6) are present only in female Acanthops, Gongylus, Hymenopus, Hypsicorypha, Idolomantis, Idolomorpha, and Theopropus. 
79. Fore tibia, postero-ventral spines, largest number in relation to largest number of anteroventral spines: less than one third smaller or equal [1]; more than one third smaller [2]; larger [3].

The number of postero-ventral tibial spines is up to one third smaller than the number of antero-ventral tibial spines (state 1) in Acontista, Ambivia, Ameles, Blepharodinae, Bolivaria, Ceratocrania, Choeradodis, Ciulfina, Creobroter, Dystacta, Galinthias, Gongylus, Gonypeta, Humbertiella, Iris, Mantis, Metallyticus, Orthodera, Orthoderella, Oxyothespis, Oxypilus, Paralygdamia, Parentella, Photina, Popa, Pseudogalepsus, Pseudoharpax, Pseudoyersinia, Pyrgomantis, Rhombodera, Sphodromantis, Stagmatoptera, Stenopyga, Tamolanica, Tarachodes, Tarachodula, Theopompa, Tisma, Tropidomantis, Vates, and Zoolea.

The number of postero-ventral tibial spines counting less than a third of the number of antero-ventral spines (state 2) is present in Archimantis, Bactromantis, Chaeteessa, Deroplatys, Elaea, Entella, Eremiaphila, Euchomenella, Haaniinae, Heterochaeta, Heteronutarsus, Hoplocorypha, Leptocola, Ligaria, Ligariella, Majanga, Mantoida, Oligonicinae sp., Oxyopsis, Paraoxypilinae, Parasphendale, Plistospilota, Pogonogaster, Polyspilota, Rivetina, Sibylla, Stenomantis, Taumantis, Thesprotia, Thrinaconyx, and Toxoderidae.

The number of postero-ventral tibial spines is larger than that of the anteroventral tibial spines (state 3) in Acanthopidae, Acromantis, Ceratomantis, Empusa, Enicophlebia, Epaphrodita, Gimantis, Hestiasula, Hymenopus, Hypsicorypha, Idolomantis, Idolomorpha, Odontomantis, Phyllocrania, Pseudocreobotra, and Theopropus.

80. Fore tibia, tarsomere 1, length with respect to remaining tarsomeres taken together: longer [1]; of equal length or shorter [2].

The proximal tarsomere is elongated in most Mantodea (Beier \& Jaus 1933: 131; Beier 1964a: 874, 875; Ehrmann 2002: 20). It is as long as the four distal tarsomeres or shorter (state 2) in Ameles decolor, Chaeteessa, Ciulfina, Elaea, Entella, Leptocola, Ligaria, Metallyticus, Oxyothespis, Paralygdamia, Perlamantinae, Pseudogalepsus, Pseudoyersinia, Stenomantis, Tarachodes, Tarachodula, and Toxoderidae.

In all remaining taxa the proximal tarsomere is longer than the remaining tarsomeres taken together (state 1).

In Blattodea the proximal tarsomere is not as strongly elongated as in Mantodea. 
81. Fore tibia, tarsomere 1, length with respect to tibia: of equal length or longer [1]; shorter [2].

The proximal tarsomere is as long as the tibia (measured from the tibio-femoral joint to the suture between the tibial spur and the tibia) or longer (state 1) in Amorphoscelinae, Astape, Ciulfina, Compsothespis, Gimantis, Mantoida [see Mantoida toulgoeti Roy, 2010 (p. 22)], Oligonicinae, Oxyothespis, Paraoxypilinae, and Perlamantinae.

In all remaining taxa and in Blattodea, the proximal tarsomere is shorter than the tibia (state 2).

Cursorial legs. The cursorial legs (i.e. the meso- and metathoracic legs) are unspecialized in the greater part of the extant species. In some taxa (e.g. Eremiaphilidae) they are very long which is an adaptation for quick running in desert habitats (e.g. Chopard 1938: 60; Beier 1964a: 861; Figs. 10, 276) or living on the bark of trees, as for instance in Liturgusa (Figs. 20, 21). There are also some species hiding in grass or bushes that resemble slender phasmatodeans and have long and slender legs (e.g. Schizocephala, Leptocola). In several other taxa showing special resemblance to sticks or grass blades (e.g. Calamothespis, Pyrgomantis, Popa and others, for examples see Chopard 1940a: fig. 3; Edmunds 1972: fig. 3B; Edmunds \& Brunner 1999: fig. 13.2B, 13.5C; Schwarz 2004: figs. 3, 5, 7, 8), the legs are often quite short in relation to body length. Especially species hiding directly on grass blades tend to cling to the grass very closely when disturbed in order to break up their conspicuous body shape, with short legs enhancing this effect (e.g. Edmunds 1972: 19; Edmunds \& Brunner 1999: 288).

Further modifications of the legs are the presence of lobes on the femora and/or tibiae, the symmetry of the tibia, and differences in the length relations between tibia and femur as well as between the tarsomeres.

82. Meso- and metathoracic femora, antero-distal apical spine (= genicular spine): present [1]; missing [2].

The meso- and metathoracic femora often carry an antero-distal spine, named genicular spine (often also present in forelegs, albeit much smaller), that may be straight or more or less strongly curved (Roy 1999: 30; state 1; Figs. 294-300). There are taxa from several lineages lacking this spine (state 2; mentioned for Mantis by Roy 1999: 30). This was found in Amorphoscelinae, Calamothespis, Choeradodis stalii, Epaphrodita, Haaniinae, Hoplocorypha, Mantis, Oligonicinae sp., Orthoderella, Oxyopsis, Paraoxypilinae, Perlamantinae, Pogonogaster, Stagmatoptera, Thesprotia graminis, Tropidomantinae, and Thrinaconyx. The genicular spine is present in the remaining taxa. It is unknown whether these spines have any special function. 
83. Meso- and metathoracic legs, femora, articulated, sturdy spines: present [1]; missing [2].

In most cockroaches, the meso- and metathoracic femora exhibit a pattern of articulated spines (with a basal suture) very similar to that of the prothoracic legs (e.g. Beier 1974a: 24; Bohn 2003: 199; see also Roth 1991: figs. 19.1, 19.2B). Beier (1968a: 22) stated that such spines are missing on the cursorial legs of most extant Mantodea ("Femur und Tibia sind fast immer unbedornt"). He had stated earlier that spines on the cursorial legs are missing entirely or may be present only on the tibia as minute spines (Beier 1964a: 875).

Distinctly articulated spines with basal sutures on the femora of the meso- and metathoracic legs could be confirmed only for Chaeteessa (Figs. 277, 278, 282, 283). This was also observed by Burmeister (1838: 527). They are located on the ventral surface of the meso- and metathoracic femora, also mentioned by Giglio-Tos (1927: 41). On the mesothoracic femur, there are five anteroventral and eight postero-ventral spines (Figs. 277, 278, 282).

Spines on the femora of the cursorial legs have been described for the fossil species

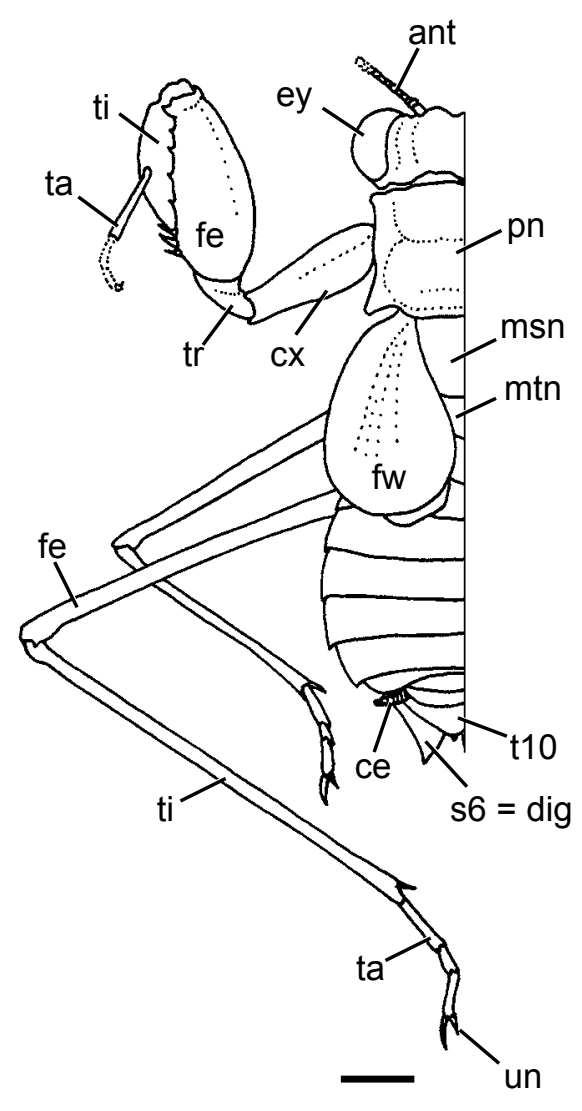

Fig. 276: Heteronutarsus albipennis,, ; redrawn and simplified from Chopard (1941: fig. 7). Note the elongated legs and the inequal length of the ungues. - Scale bar = $2.0 \mathrm{~mm}$ (reconstructed from data given in Chopard 1941: 34).

Abbreviations: ant $=$ antenna; $\mathbf{c e}=$ cercus; $\mathbf{c x}$ = coxa; dig = digging device; $\mathbf{e y}=$ compound eye; $\mathbf{f e}=$ femur; $\mathbf{f w}=$ fore wing (tegmen); $\mathbf{m s n}=$ mesonotum; $\mathbf{m t n}=$ metanotum; $\mathbf{p n}=$ pronotum; $\mathbf{s 6}=$ abdominal sternite $6 ; \mathbf{t 1 0}=$ tergite 10 (supraanal plate); $\mathbf{t a}=$ tarsus; $\mathbf{t i}=$ tibia; $\mathbf{t r}=$ trochanter; $\mathbf{u n}=$ unguis.

$\uparrow$ Cretomantis larvalis (Grimaldi 2003: 20 and figs 12, 13; Fig. 30) and $\uparrow$ Santanmantis axelrodi (Grimaldi 2003: 27 and fig. 17; Grimaldi \& Engel 2005: fig. 7.98).

Beier (1964a: 875) stated that the loss of the spines in Mantodea is probably correlated with the transition to a free lifestyle ("freie Lebensweise", Beier 1964a: 875), probably meaning a lifestyle that is not bound to hiding in crevices or in the leaf litter or the soil. To my knowledge nothing is known of the hiding habits of living Chaeteessa that could corroborate this hypothesis. The only known reference is Salazar (2005: $270 \mathrm{ff}$.), who described Chaeteessa as a bark dweller living on avocado trunks (Persea sp.; see also 4.6.5). The presence of spines on the cursorial legs has been assumed to represent the plesiomorphic state in fossil Mantodea, as it resembles the situation in cockroaches (see Grimaldi 

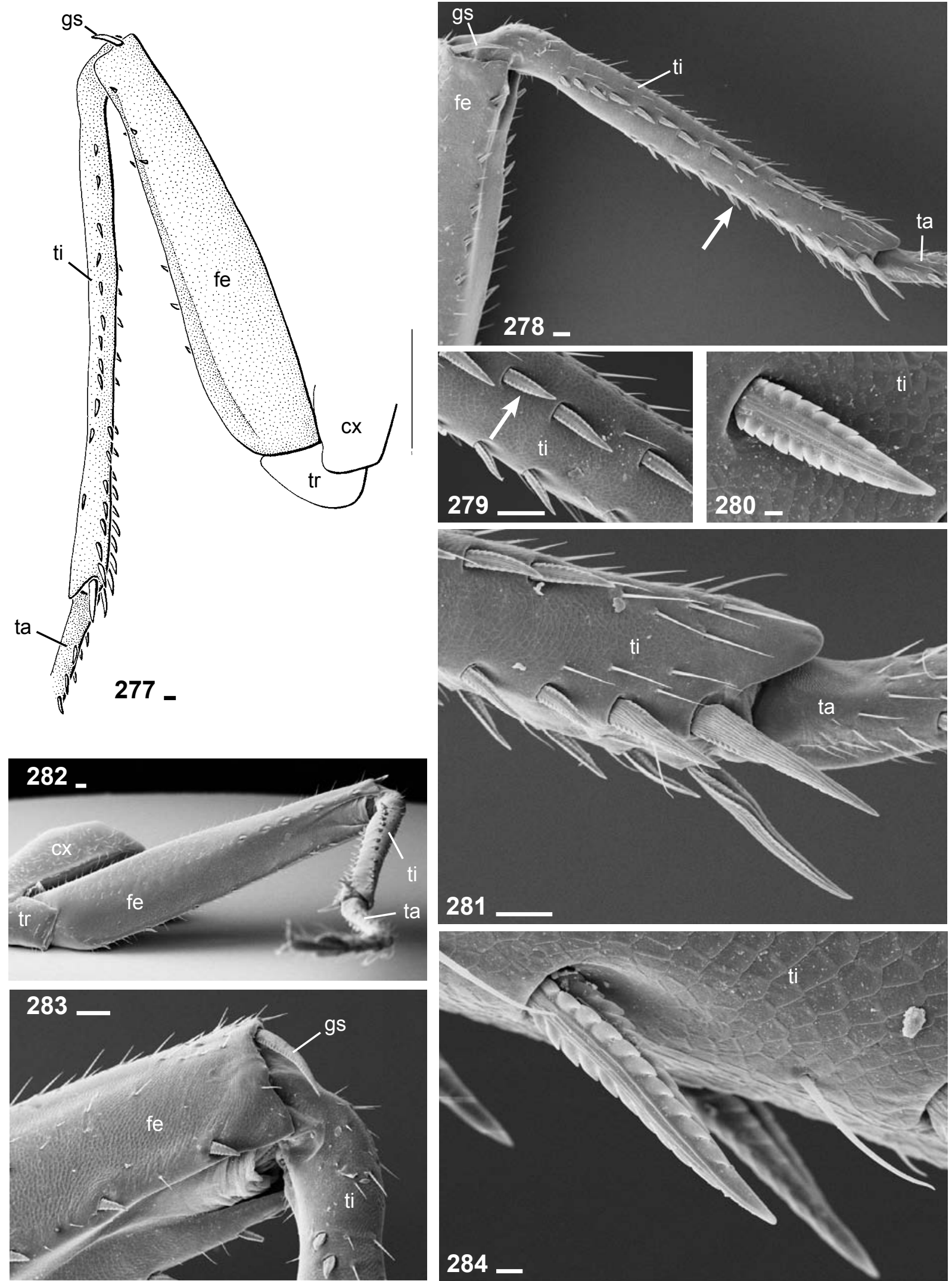

Figs. 277-284: Spination of the meso- and metathoracic legs of Chaeteessa. 277: Chaeteessa valida, 9 (NHMW), right metathoracic leg, anterior view. 278-284: Chaeteessa cf. valida, sex unknown, left mesothoracic leg. 278: Overview of tibia, anterior view (arrow indicates spine in Fig. 284). 279: Detail of tibial spines, anterior view (arrow indicates spine shown in Fig. 280). 280: Detail of single tibial spine. 281: Detail of tibial apex. 282: Overview of femur, oblique ventral view. 283: Detail of femoral apex in oblique anterior view. 284: Detail of ventral tibial spines. - Scale bar $=0.1 \mathrm{~mm}(277-279,281-283) ; 0.01 \mathrm{~mm}(280,284)$.

Abbreviations: $\mathbf{c x}=$ coxa; $\mathbf{f e}=$ femur; $\mathbf{g s}=$ genicular spine; $\mathbf{t a}=$ tarsus; $\mathbf{t i}=$ tibia; $\mathbf{t r}=$ trochanter. 

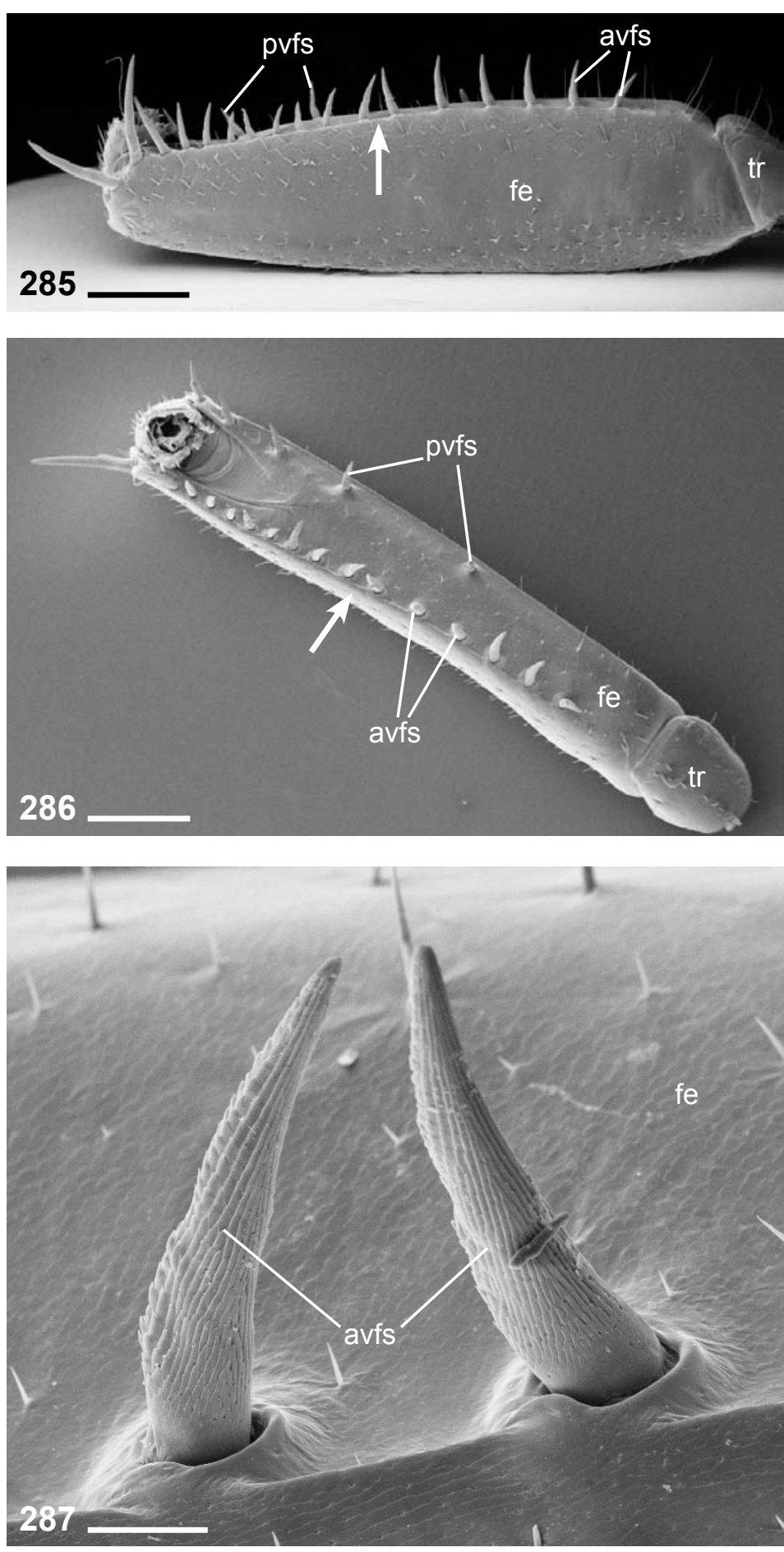

Figs. 285-287: Periplaneta americana,, , femur of left prothoracic leg. 285: Overview, anterior view. 286: Overview, ventral view; arrows indicate spines shown in Fig. 287. 287: Detail of fore femoral spines. - Scale bar $=1.0 \mathrm{~mm}(285-286) ; 0.1 \mathrm{~mm}$ (287).

Abbreviations: avfs = antero-ventral fore femoral spine; $\mathbf{f e}=$ femur; $\mathbf{p v f s}=$ postero-ventral fore femoral spine; $\mathbf{t r}=$ trochanter.
2003: character 5 on p. 38).

I accordingly assume that the situation in Chaeteessa represents the plesiomorphic state. This finds additional support in the unique shape of the spines. They have a strongly serrate surface structure both on the fore- and cursorial legs. This structure shows a close similarity to the situation found in Periplaneta (Fig. 287) and is present in many species from the different currently recognized cockroach subgroups (Klass, pers. comm. 2009). However, such a serrate surface structure could so far not be confirmed for the spines of any other Mantodea than Chaeteessa. The only at least superficial resemblance is exhibited by the tibial spines of the Oligonicinae sp. specimen (Fig. 274) and Thesprotia graminis (Fig. 275) in which, however, the surface is different. The serrate structure as found in Chaeteessa and cockroaches was probably inherited from the last common ancestor of cockroaches and mantodeans and probably represents the plesiomorphic state for Chaeteessa rather than a derived structure.

Among extant Mantodea spines on the cursorial legs can - at a superficial glance - also be observed in Eremiaphila and Astape (e.g. Lieftinck 1953: figs. 1-3). 
In Astape, they are cuticular outgrowths (Fig. 291) which certainly add to the elaborate camouflage of the species (mimesis of lichen and mosses; see Lieftinck 1953: 129). They are also mere cuticular structures without basal sutures in Eremiaphila, as becomes evident in SEM images (see Figs. 288, 289).

Some taxa (e.g. Paraoxypilus, Ciulfina and Metallyticus) have serrate ventral edges of the femora. It has been shown for Metallyticus splendidus that these are not spines originating from setae but cuticular outgrowths forming the pointed bases of small setae (Wieland 2008a: 163 and figs. 11-13; Figs. 292, 293). The same could be confirmed for Paraoxypilus and Ciulfina, and this is also the case in Eremiaphila (Fig. 289).

84. Meso- and metathoracic legs, tibiae, articulated, sturdy spines: present [1]; missing [2].

Also see character 83 . The meso- and metathoracic tibiae of cockroaches carry articulated spines in an unordered pattern, resembling the situation on the prothoracic tibiae (see Figs. 255-257). A similar situation, albeit with distinctly smaller spines, is present in Chaeteessa. The tibia of the cursorial legs may carry more than 30 articulated spines, organized in several rows on the ventral and lateral surfaces of the tibia (Figs. 277, 278, 281, 282); also mentioned by GiglioTos 1927: 41).

Spines on the meso- and metathoracic tibiae have been found in $\dagger$ Santanmantis axelrodi (Grimaldi \& Engel 2005: fig. 7.98; Grimaldi 2008: fig. 11.22). A large number of small tubercles has furthermore been indicated in the drawing of $\dagger$ Cretomantis larvalis by Grimaldi (2003: fig. 13; Fig. 30). It is unclear whether these tubercles represent the bases of small spines that broke off during fossilization or if they are cuticular ornamental structures.

Among extant Mantodea spines can also be observed, for instance, on the mid- and hind tibiae of Eremiaphila and Astape (e.g. Lieftinck 1953: figs. 1-3). In Astape, they are not of setal origin but are cuticular outgrowths (Fig. 291) which certainly adds to the elaborate camouflage of the species (see Lieftinck 1953: 129). In Eremiaphila, too, they are cuticular outgrowths without basal sutures (Fig. 290). The spines on the tibiae of Chaeteessa (as on the femora, see character 83) resemble the spines of cockroaches in their serrate structure (Figs. 279, 280, 281, 284, 287).

As stated above for the femora of the meso- and metathoracic legs (character 83), the presence of articulated spines likely represents the plesiomorphic state that can also be found in cockroaches and possibly some fossil Mantodea. 
85. Mesothoracic leg, tibia, length with respect to femur: longer [1]; of equal length or shorter [2].

Roy (1999: 30) stated that the tibiae of the cursorial legs may be longer or shorter than the corresponding femora. As differences between the meso- and the metathoracic legs have been found, the character was encoded individually for each of them.

The mesothoracic tibia is as long as or shorter than the femur in the greater part of the extant Mantodea studied herein (e.g. Fig. 276). Exceptions with mesothoracic tibiae longer than the femora are Acanthops, Compsothespis, Empusa, Idolomantis, Oxyothespis, Parasphendale, Pogonogaster, Pseudoyersinia, Thesprotia graminis, and Toxodera.

86. Mesothoracic leg, tarsomere 1, length with respect to the remaining tarsomeres taken together: longer [1]; of equal length or shorter [2].

The proximal tarsomere (basitarsomere) is strongly elongated in most Mantodea (Beier \& Jaus 1933: 131; Chopard 1949a: 389; Beier 1964a: 875; Ehrmann 2002: 20). Chopard (1949a: 389) stated that the proximal tarsomere is always longer than the other tarsomeres (i.e. than each of the latter), however, there are taxa in which it is shorter than each of the remaining
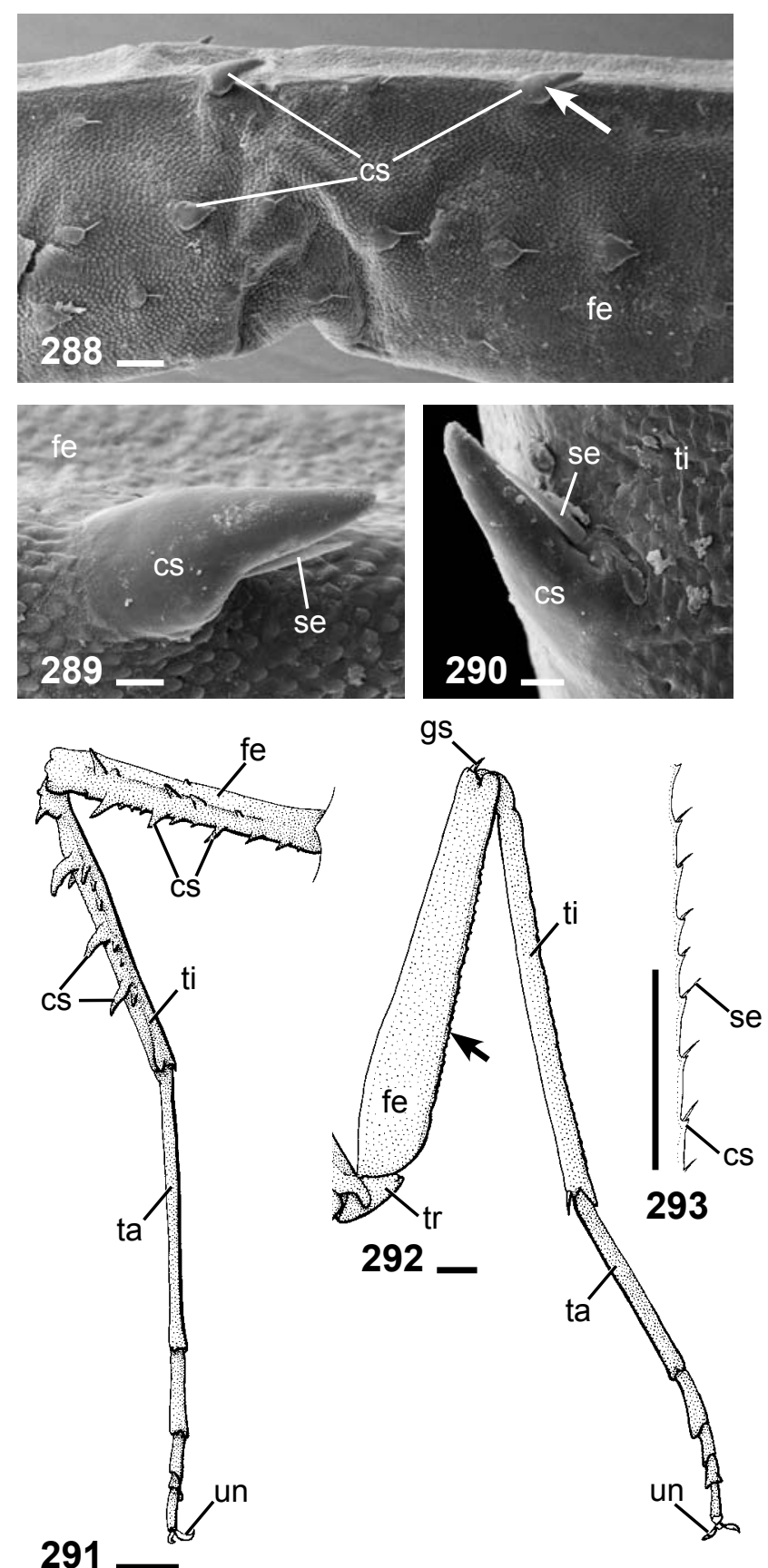

Figs. 288-293: Cuticular spines on the meso- and metathoracic legs of different taxa. 288-290: Eremiaphila sp. 1,, , left mesothoracic leg. 288: Ventral femur, spines in overview (distortion of femur is an artefact), arrow indicates cuticular spine shown in Fig. 289. 289: Detail of spine on ventral surface of mesothoracic femur. 290: Detail of spine on mesothoracic tibia. 291: Astape denticollis, $\rightarrow$, left metathoracic leg, posterior view. 292-293: Metallyticus splendidus,, . 292: Left metathoracic leg, anterior view; arrow indicates serrate posterior edge of femur shown in detail in Fig. 293. 293: Detail of serrate posterior edge of femur. Note that all spines are cuticular formations that do not exhibit a basal suture. - Scale bar $=0.1 \mathrm{~mm}$ (288); $0.02 \mathrm{~mm}(289,290) ; 1.0 \mathrm{~mm}$ (291-293). Abbreviations: $\mathbf{c s}=$ cutcular spine; $\mathbf{f e}=$ femur; $\mathbf{g s}=$ genicular spine; $\mathbf{s e}=$ seta; $\mathbf{t a}=$ tarsus; $\mathbf{t i}=$ tibia; $\mathbf{t r}=$ trochanter; $\mathbf{u n}=$ unguis. 
tarsomeres. This can be found, for instance, in Ameles (Ehrmann 2002: 58) and Pseudoyersinia.

The basitarsomere is longer than each of the other tarsomeres in cockroaches and may be as long as the latter taken together (e.g. Beier 1974a: 24; Grimaldi \& Engel 2005: fig. 7.64). Several fossil Mantodea have long basitarsomeres (e.g. $\uparrow$ Ambermantis, $\uparrow$ Burmantis, $\uparrow$ Cretomantis, $\uparrow$ Jersimantis, and $\dagger$ Santanmantis; see Grimaldi 2003). Therefore, it is likely that the proximal tarsomere is secondarily shortened in Mantodea where it is as short as each of the remaining tarsomeres. This is also supported by the fact that, albeit having very short basitarsomeres on the meso- and metathoracic tarsi, Ameles has distinctly elongated basitarsomeres on the forelegs. The basitarsomere being longer than the remaining tarsomeres taken together may represent an apomorphy for a group within Mantodea.

The basitarsomere of the mesothoracic leg is longer than the remaining tarsomeres taken together (state 1) in Amorphoscelis sp., Astape, Ceratomantis, Epaphrodita, Gimantis, Gongylus, Gyromantis, Haania, Hoplocorypha, Hypsicorypha, Leptocola, Maculatoscelis sp., Oligonicinae sp., Pogonogaster, Sibylla, Stenomantis, Thesprotia graminis, Thrinaconyx, and Zoolea.

In the remaining taxa the proximal tarsomere of the mesothoracic leg is as long as or shorter than the remaining tarsomeres taken together (state 2; Figs. 294, 297, 299).

87. Metathoracic leg, tarsomere 1, length with respect to the remaining tarsomeres taken together: longer [1]; of equal length or shorter [2].

The basitarsomere of the metathoracic legs is elongated more frequently than that of the mesothoracic legs. State 1 is represented by Amorphoscelinae, Archimantis, Bactromantis, Bolivaria, Chaeteessa, Dystacta, Empusa, Enicophlebia, Epaphrodita, Euchomenella, Gimantis, Gongylus, Haaniinae, Hoplocorypha, Hypsicorypha, Idolomorpha, Leptocola, Ligariella, Majanga, Metallyticus (Fig. 292), Odontomantis, Oligonicinae sp., Paraoxypilinae, Plistospilota, Pogonogaster, Polyspilota, Sibylla, Stenomantis, Stenopyga, Thesprotia, Thrinaconyx, Tisma, Tithrone, Tropidomantis, and Zoolea.

The metathoracic basitarsomere was as long as or shorter than the distal four tarsomeres taken together (state 2) in the remaining taxa (e.g. Figs. 300, 316).

In some species (herein confirmed for Ameles and Pseudoyersinia) the basitarsomere of the metathoracic tarsi is slightly longer than each of the remaining tarsomeres at most (Fig. 316). For a discussion see character 86. 
88. Metathoracic leg of male, tibia, length with respect to femur: longer [1]; of equal length or shorter [2].

See also characters 85 and 89 . Intraspecific differences in length ratio between the metathoracic tibia and femur were found in several species, therefore this character was encoded individually for both sexes.

The metathoracic tibia of the male is as long as or shorter than the corresponding femur (state 2) in Amorphoscelis sp., Astape, Callibia, Ceratocrania, Ciulfina, Creobroter, Galinthias, Gongylus, Haania, Hestiasula, Heteronutarsus, Hymenopus, Mantis, Odontomantis, Orthodera, Paraoxypilus sp. 1, Phyllocrania, Popa, Pseudocreobotra, Pseudoharpax, Stenomantis, Stenopyga, Theopropus, and Vates.

89. Metathoracic leg of female, tibia, length with respect to femur: longer [1]; of equal length or shorter [2].

See also characters 85 and 88 . The metathoracic tibia of the female is as long as or shorter than the corresponding femur (state 2) in Astape (in Fig. 291 the proximal part of the femur is covered by the tergite), Creobroter, Caudatoscelis, Entella, Galinthias, Gongylus, Haania, Hestiasula, Heteronutarsus, Hymenopus, Hypsicorypha, Maculatoscelis sp., Mantis, Odontomantis, Orthodera, Phyllocrania (Fig. 300), Pseudocreobotra ocellata, Pseudoharpax, Stenomantis, Theopropus, and Vates.

The females of the remaining taxa have a metathoracic tibia being longer than the femur (state 1; e.g. Fig. 292 for Metallyticus).

90. Meso- and metathoracic legs, femora, lobe-like expansions: present [1]; missing [2].

Many species carry lobes on the femora and/or the tibiae of the cursorial legs (Beier \& Jaus 1933: 131; Chopard 1949a: 389; Beier 1964a: 875; 1968a: 22; Roy 1999: 29 ff.; Ehrmann 2002: 20; Klass \& Ehrmann 2003: 185). Roy (1999: 29) stated that lobe-like expansions of the femora occur frequently in Hymenopodidae, Empusidae and several subgroups of Mantidae.

Such lobe-like expansions are likely to play an important part in the camouflage of Mantodea, especially when dead plant matter is imitated (e.g. Gongylus, Phyllocrania; see Edmunds \& Brunner 1999: 287), but also, for instance, in the mimesis of orchid blossoms as in Hymenopus (e.g. Cornet 1894: 183; MeadeWaldo 1910).

Lobe-like expansions on the femora of the cursorial legs are present (state 1) in Acromantis, Ambivia, Blepharopsis, Callibia, Ceratocrania, Creobroter, 
Deroplatys, Empusa, Epaphrodita, Galinthias, Gongylus (Fig. 296), Hestiasula, Heterochaeta, Hymenopus (Fig. 297), Idolomantis, Phyllocrania (Fig. 300), Popa (Fig. 294), Pseudocreobotra (Fig. 299), Pseudoharpax, Sibylla (Fig. 295), Theopropus, Toxodera (Fig. 298), Vates, and Zoolea.

91. Meso- and metathoracic legs, femora, lobe-like expansions, position: in the proximal third [1]; medially [2]; distally from the middle or apically [3]; along the entire femur or several lobes placed along the entire length [4].

See also character 90 . The position of the lobes on the femora of the cursorial legs varies among the mantodean subgroups.

A position in the proximal third (state 1) is a rare case. In the present taxonsample it was only found in Hestiasula. Roy (1999: 29) also mentioned this case to be rare and only to be found in Hestiasula and Pseudacanthops (see also La Greca \& Lombardo 1997: fig. 5).

The medial position (state 2) was found exclusively in the two Toxodera species (Figs. 22, 298).

The distal or apical position of the lobe (state 3) is the most common case. It is exhibited by Acromantis, Ambivia, Blepharopsis, Callibia, Creobroter, Deroplatys, Empusa, Epaphrodita, Galinthias, Gongylus (Fig. 296), Heterochaeta, Idolomantis, Pseudocreobotra (Fig. 299), Pseudoharpax, Theopropus, and Zoolea. In Pseudocreobotra, the lobe is expanded as a very slender ridge proximad (Fig. 299). It is unclear if it is part of a former larger lobe that could have resembled Hymenopus (Fig. 297). The position of the lobe in Pseudocreobotra will be provisionally encoded as state 3 until further studies regarding the evolution of the lobe in this genus can be conducted.

A large lobe along the entire length of the femur or several smaller lobes lined up along its entire length (state 4) are exhibited by Ceratocrania, Hymenopus (Fig. 297), Phyllocrania (Fig. 300), Popa (although the proximal lobe is quite small in P. spurca crassa; Fig. 294), Sibylla (Fig. 295), and Vates.

92. Meso- and metathoracic legs, tibiae, lobe-like expansions: present [1]; missing [2].

Several species exhibit lobe-like expansions on the tibiae of the cursorial legs. However, they are less common than lobes on the femora (Roy 1999: 30). Tibial lobes are present in Ceratocrania, Phyllocrania (Paulian 1957: figs. 48, 49; Fig. 300), Popa (Giglio-Tos 1914b: 83, 1927: 630; Fig. 294), Vates (Giglio-Tos 1927: 619; Chopard 1938: fig. 9), and Zoolea (e.g. Giglio-Tos 1927: 614 ff.; Roy \& Ehrmann 2009: figs. 13-15, 19, 21, 22). A tibial lobe has furthermore been described in the literature, for instance, for Pseudacanthops lobipes La Greca \& Lombardo, 1997 (fig. 6). 

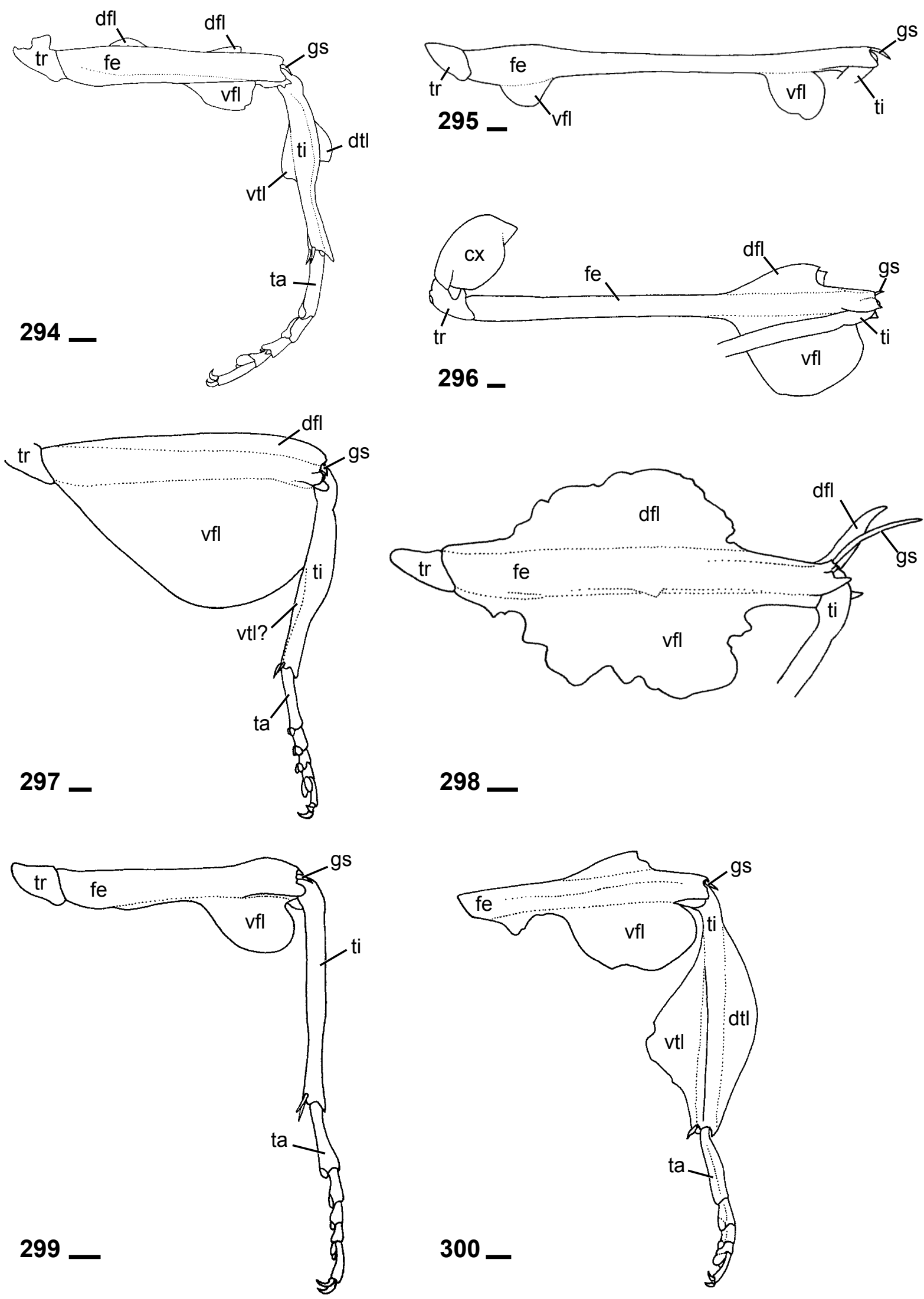

Figs. 294-300: Lobes on the meso- and metathoracic femora and tibiae. 294: Popa spurca crassa, $\widehat{\partial}$, left mesothoracic leg, anterior view; note the asymmetrical shape of the tibia. 295: Sibylla pretiosa,, , right mesothoracic femur (mirrored), anterior view. 296: Gongylus gongylodes, $q$, left mesothoracic femur, anterior view. 297:

Hymenopus coronatus, $q$, left mesothoracic leg, anterior view. 298: Toxodera maculata, $\bigcirc$, left mesothoracic femur, anterior view. 299: Pseudocreobotra wahlbergii,, , left mesothoracic leg, anterior view. 300: Phyllocrania paradoxa,, , right metathoracic leg, anterior view (mirrored). - Scale bar $=1.0 \mathrm{~mm}$.

Abbreviations: $\mathbf{c x}=$ coxa; $\mathbf{d f l}=$ dorsal femoral lobe; $\mathbf{d t l}=$ dorsal tibial lobe; $\mathbf{f e}=$ femur; $\mathbf{g s}=$ genicular spine; $\mathbf{t a}$ $=$ tarsus; $\mathbf{t i}=$ tibia; $\mathbf{t r}=$ trochanter; $\mathbf{v f l}=$ ventral femoral lobe; $\mathbf{v t l}=$ ventral tibial lobe. 
In Hymenopus, a slender ridge has been identified on the ventral tibiae (Fig. 297). Due to its inconspicuousness and the lack of studies on the postembryonic development in this species, the lobe is herein provisionally encoded as missing for Hymenopus.

93. Meso- and metathoracic legs, tibiae, longitudinal shape in anterior or posterior view: nearly parallel, not strongly asymmetrical in some areas [1]; strongly asymmetrical in some areas, having a partly swollen appearance [2].

The tibiae of the cursorial legs (excluding any lobes) of several species have a partly swollen appearance. They are asymmetrically thickened, mostly in the proximal to medial area of the tibia. This character state is exhibited by Acanthops, Callibia, Ceratocrania, Creobroter, Deroplatys lobata, Galinthias, Heterochaeta, Hymenopus (297), Popa (Fig. 294), Pseudocreobotra (299), Pseudoharpax, Theopropus, and Vates.

In the remaining taxa studied herein no such asymmetrical thickening could be found. However, it has been described in the literature for Pseudacanthops lobipes (La Greca \& Lombardo 1997: figs. 6, 7).

It is likely that asymmetrical tibiae support the effect of mimicking plant matter, as symmetrical structures, especially the long and slender legs, are often conspicuous in natural environments and may easily give away the camouflaged insect to predators (e.g. Edmunds 1972: 17).

Tarsal euplantulae, arolium and ungues. All Mantodea have euplantulae (adhesive pads) on their tarsomeres that are usually well-developed and large except for a number of taxa in which they are comparatively small. In insects, euplantulae can be membraneous ("smooth, flexible pads") or carry a large number of very small bristles, the acanthae ("hairy soles") (Beutel \& Gorb 2001: 177, 2008: 501). In Dictyoptera, they are generally membranous and often enable the insects to walk on smooth surfaces due to the adhesive interactions between the euplantulae and the surface. Interestingly, not all mantodeans are able to get a grip on smooth surfaces, such as glass or plastic. While many species are well able to climb glass, many others are not. Among the latter are for instance Metallyticus, Didymocorypha, Eremiaphila (Materna, Mehl, Schütte, pers. comm. 2009; Wieland, pers. obs.), adult Cilnia Stål, 1876 (Wieland, pers. obs. 2009) and Empusidae (Schwarz et al. 2007: 65; Schwarz, pers. comm.; Wieland, pers. obs.). The euplantulae of Metallyticus, Eremiaphilidae (in the latter possibly a secondary trait due to adaptation to quick running on bare soil) and few other taxa are small and mostly restricted to the distal ends of the tarsomeres. However, they are comparatively well-developed and proximally strongly elongated (see characters 94, 95) in Empusidae, which makes their incompetence in climbing smooth surfaces somewhat unexpected. Another remarkable fact is that, although adult Cilnia are incapable of climbing smooth surfaces, early instar nymphs are very well able to do 
so (Wieland, pers. obs. 2009). Further studies of the climbing ability of such taxa throughout their postembryonic development may yield interesting results with regard to morphology and phylogeny.

94. Tarsi, euplantulae of some or all tarsomeres ta2-ta4, proximal elongation: present [1]; missing [2].

The tarsomeres ta 1 to ta 4 of Mantodea carry smooth ventral euplantulae (e.g. Beutel \& Gorb 2001: 180). The euplantulae of tarsomeres ta2-ta4 may be restricted to the distal tip of the tarsomeres (state 2 ) or be proximally elongated along the ventral midline of the tarsomeres (state 1).

The proximal tarsomere in most Mantodea species does not have a medially elongated euplantula. Exceptions found among the taxa studied are Elaea, Humbertiella, Iris, Oxypilus, Paralygdamia, Parasphendale, Popa, Tarachodidae, Theopompa, and Toxodera. Interestingly, a medially elongated euplantula can be found on tal of several cockroaches, for instance in Simandoa conserfariam Roth \& Naskrecki, 2003 (fig. 2H). This has been confirmed for Blaberus craniifer, Blatta orientalis, and Maoriblatta novaeseelandiae (Brunner v. Wattenwyl, 1865 ) (pers. obs.). However, it is missing in Cryptocercus punctulatus, Periplaneta americana, Polyphaga aegyptiaca (Linnaeus, 1758), and Paratemnopteryx couloniana (Saussure, 1863) (Wieland, pers. obs.).

In those Mantodea that do not exhibit such a proximal elongation in the euplantulae on ta2-ta4, the midline of the tarsomeres is often discernible due to the presence of a ventro-median structure. Under the SEM, this structure appears to be a very slender ridge. It is unclear whether it has any adhesive functions. Apparently, in taxa with proximally elongated euplantulae, it is this ridge (or its area) that is considerably widened. It is unclear whether the ridge actually consists of the same membrane as the euplantulae. In Metallyticus, $\mathrm{KOH}$ maceration of the tarsus revealed that the ridge may in fact be a membrane between the edges of a ventral suture on the tarsomeres and that this suture is more or less strongly widened, thus resulting in elongated euplantulae, in those taxa that show this state. An initial distal widening of the area can be observed, for instance, in Metallyticus and Mantoida. This hypothesis is supported by the situation observed in an SEM image of tarsomere ta 4 of Paraoxypilus sp. (Fig. 331), in which the membrane of the proximal elongation is sunken in, probably due to desiccation. However, this character needs further study.

The elongation is missing (state 2) in Eremiaphilidae (Fig. 305), Haaniinae (Figs. 314, 315), and Oligonicinae sp. (Fig. 312). It is unknown whether or not the elongation is secondarily missing in Eremiaphila and Heteronutarsus due to their deserticolous lifestyle. 

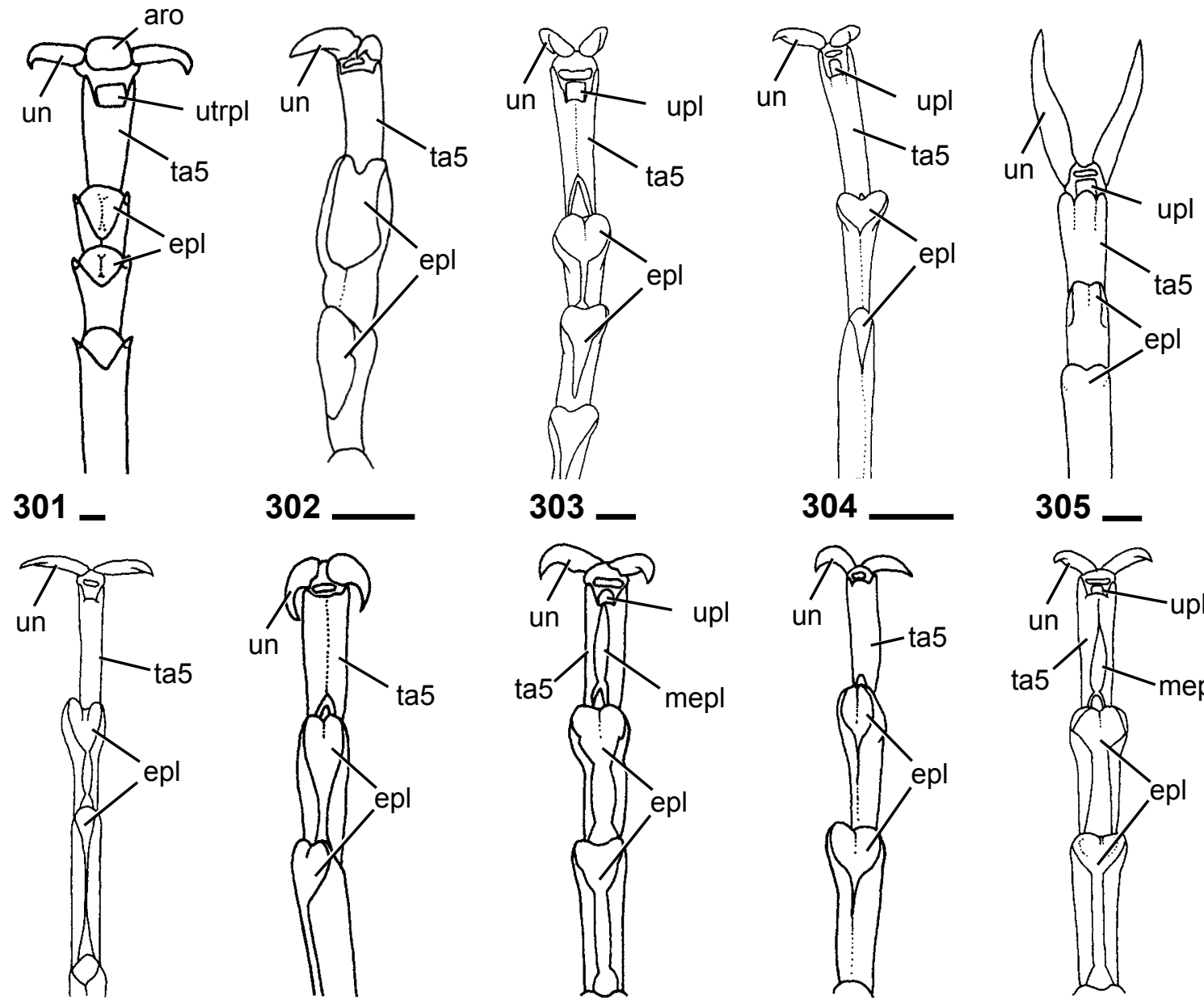

304
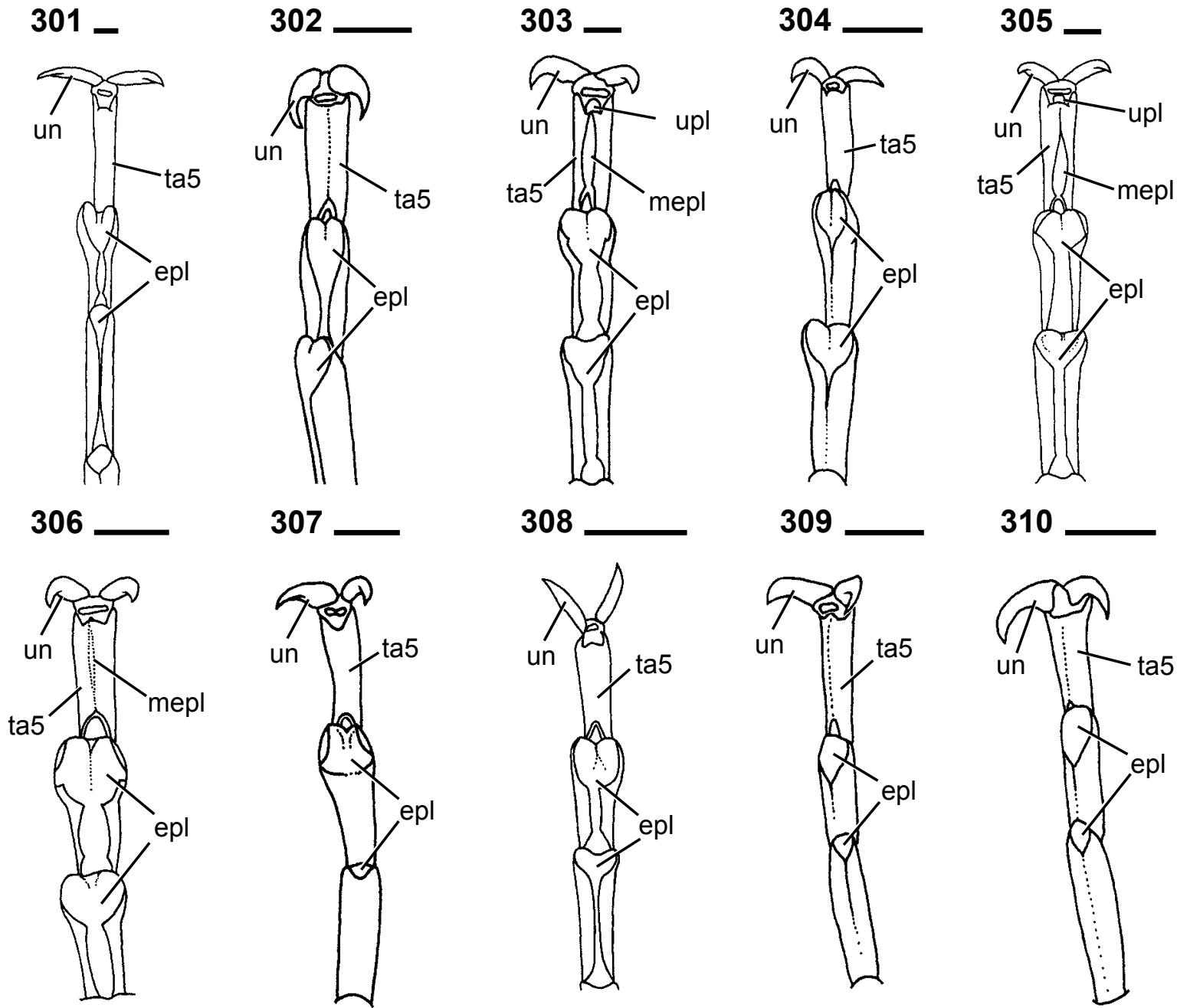

308
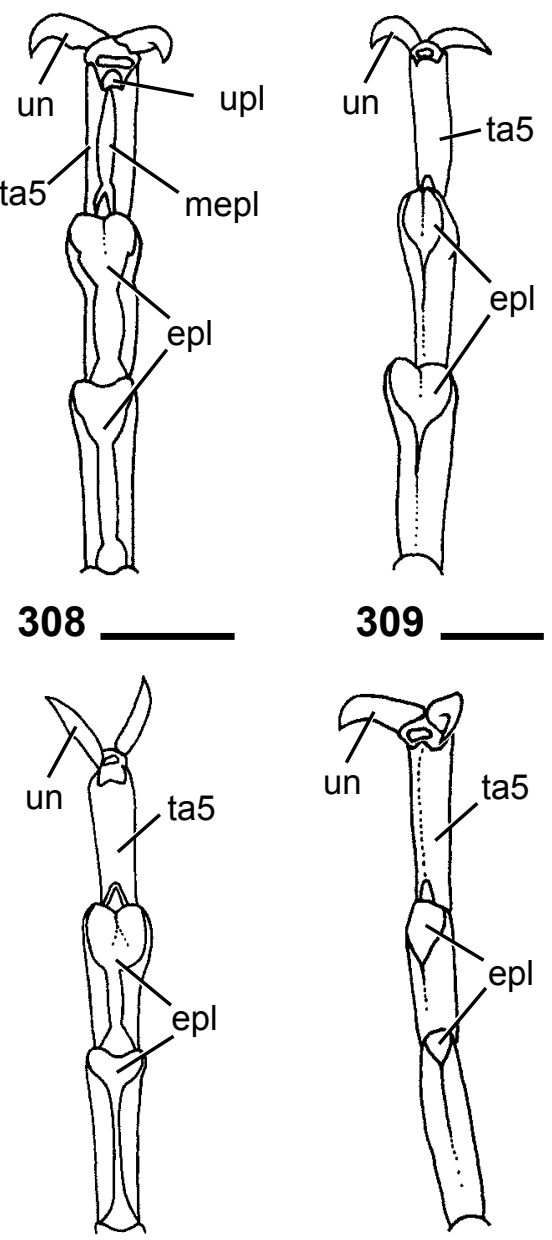

309

310
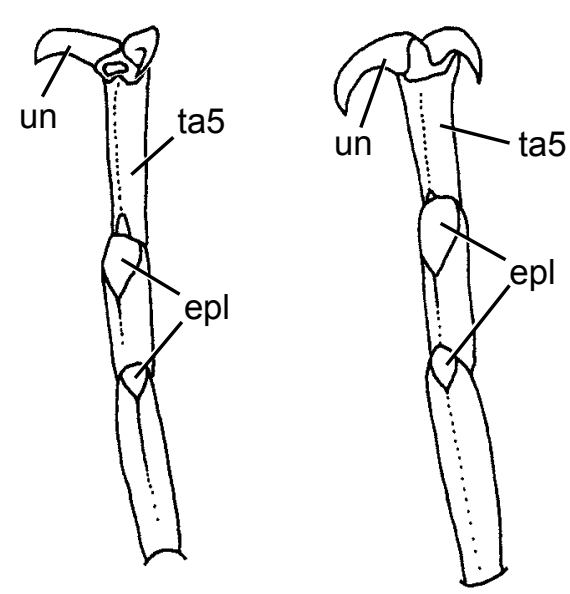

311

312

313

314

315

Figs. 301-315: Tarsomeres and euplantulae, ventral view, proximal tarsomeres omitted. 301: Periplaneta americana, + , left metathoracic leg. 302: Chaeteessa valida [NHMW], + , left metathoracic leg. 303: Metallyticus splendidus,, , right metathoracic leg. 304: Mantoida maya, ô, right metathoracic leg. 305: Eremiaphila sp. 1,, ,

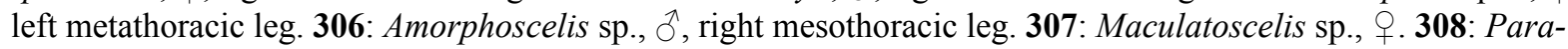
oxypilus sp. 1, ô, left metathoracic leg. 309: Paramorphoscelis gondokorensis, ${ }_{\text {, }}$, right metathoracic leg. 310: Perlamantis allibertii, ${ }_{0}$, left metathoracic leg. 311: Hoplocorypha sp.,, , left metathoracic leg. 312: Oligonicinae sp., $q$, left metathoracic leg. 313: Thesprotia graminis, ${ }_{0}$, left mesothoracic leg. 314: Astape denticollis,, , left metathoracic leg. 315: Haania lobiceps,, , left metathoracic leg. - Scale bar $=0.25 \mathrm{~mm}$.

Abbreviations: aro $=$ arolium; $\mathbf{e p l}=$ euplantula; $\mathbf{m e p l}=$ medial euplantula of ta5; ta5 $=$ tarsomere 5 ; un $=$ unguis; $\mathbf{u p l}=$ unguitractor plate. 

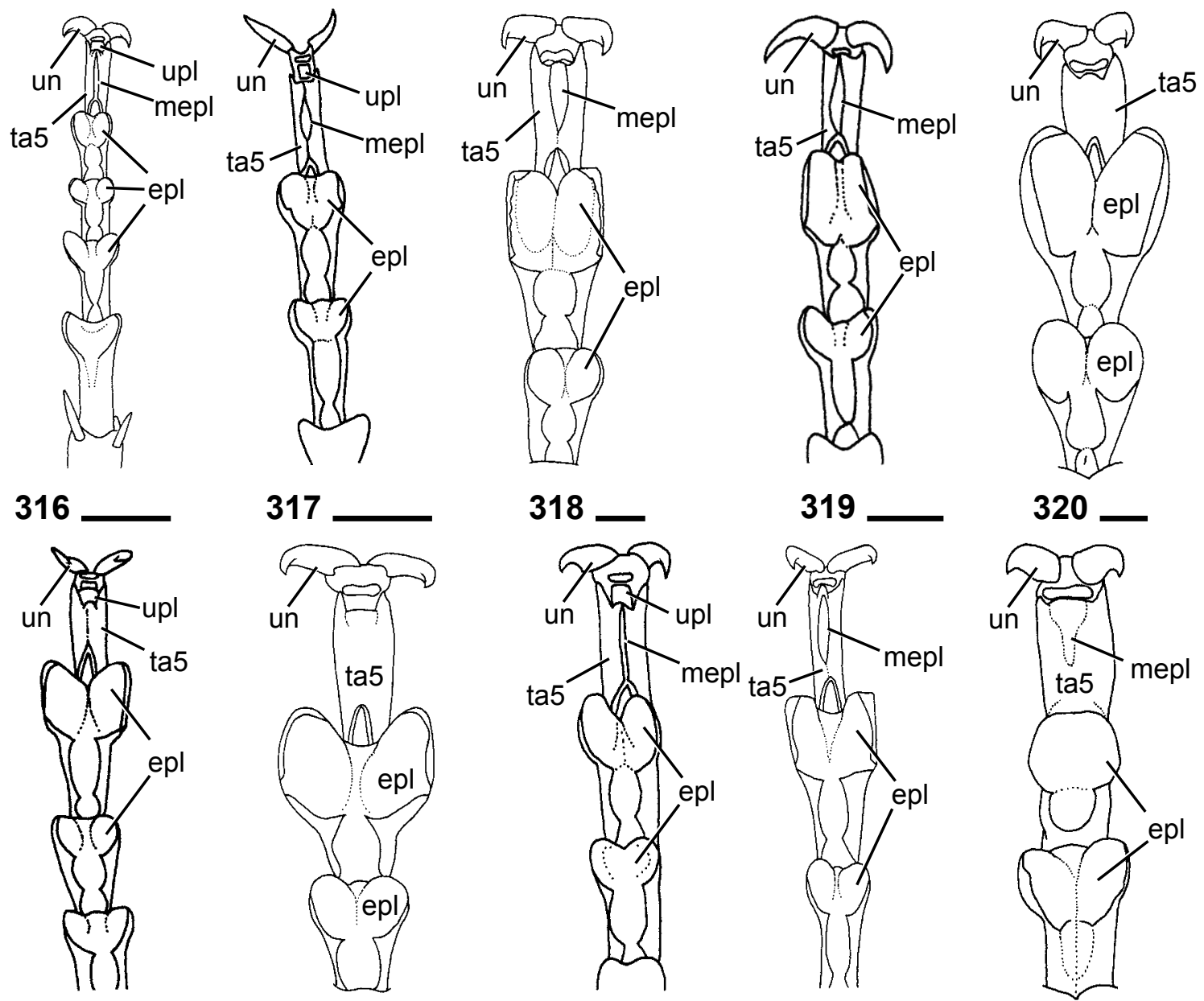

321

322

323

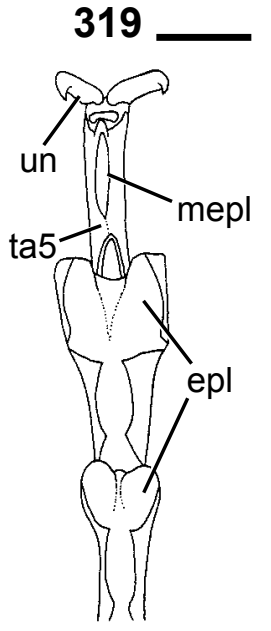

320
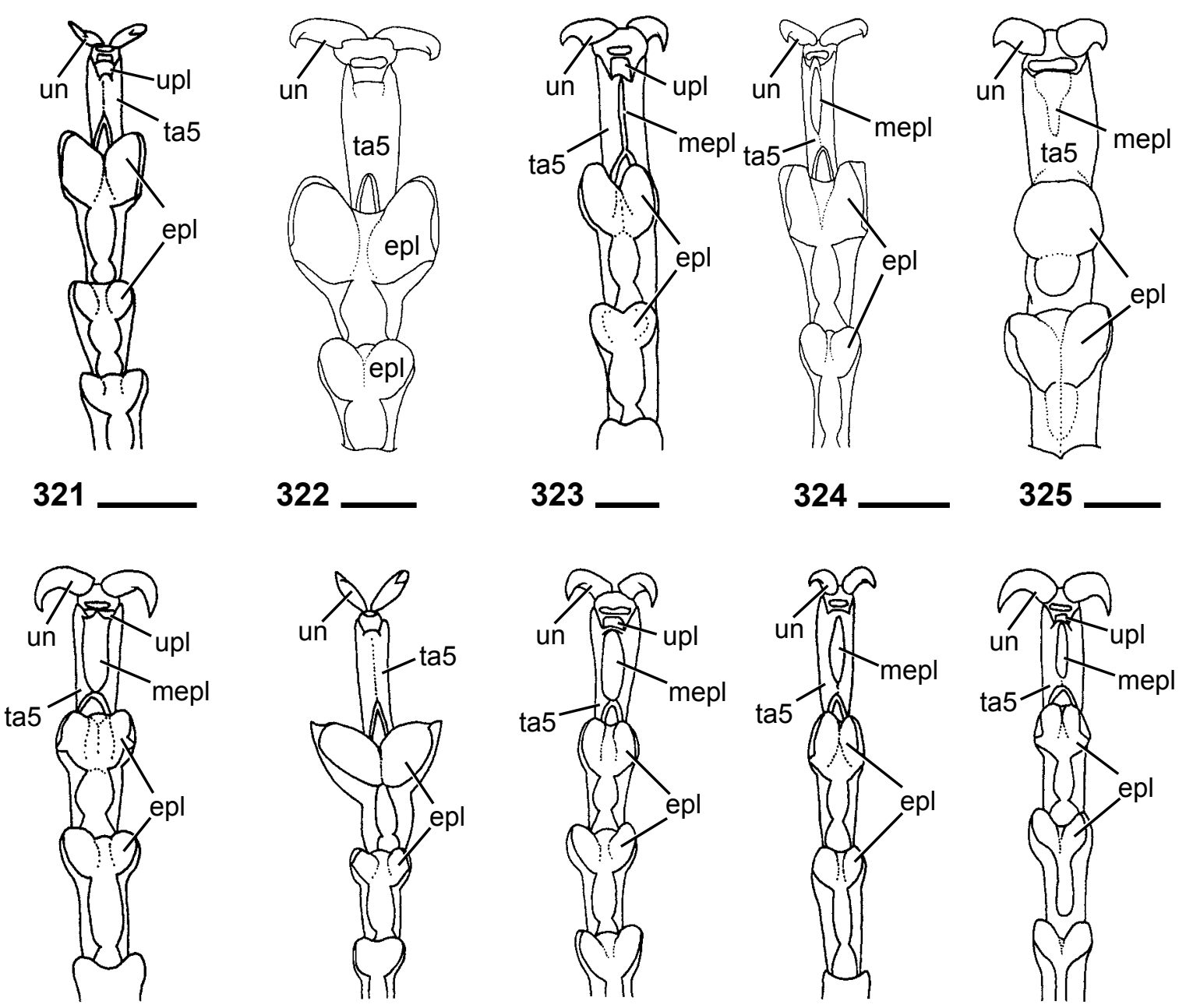

325

324

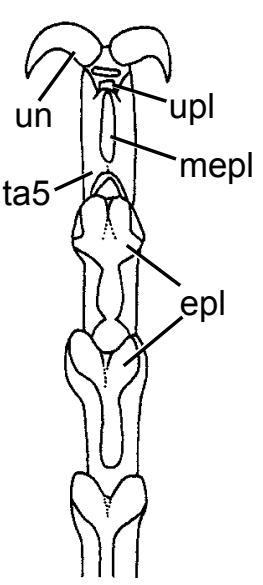

326

327

328

329

330

Figs. 316-330: Tarsomeres and euplantulae, ventral view, proximal tarsomeres omitted (except for 316). 316: Pseudoyersinia betancuriae, $\uparrow$, right metathoracic leg. 317: Ameles decolor, $\jmath^{\circ}$, left metathoracic leg. 318: Deroplatys desiccata,, , left metathoracic leg. 319: Mantis religiosa,, , right metathoracic leg. 320: Rhombodera basalis,, , left metathoracic leg. 321: Orthodera novaezealandiae, ô, right mesothoracic leg. 322: Choeradodis rhombicollis, $\hat{o}$, left metathoracic leg. 323: Humbertiella sp.,, , right mesothoracic leg. 324: Euchomenella sp., $\widehat{\partial}$, right metathoracic leg. 325: Heterochaeta strachani, + , left metathoracic leg. 326: Bolivaria sp., + , left

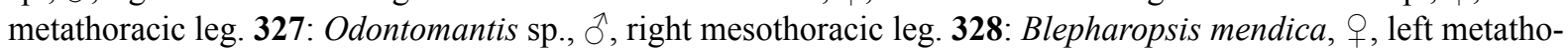
racic leg. 329: Gongylus gongylodes, ${ }_{0}$, right metathoracic leg. 330: Empusa pennata,, , right metathoracic leg. - Scale bar $=0.5 \mathrm{~mm}$.

Abbreviations: epl = euplantula; mepl = medial euplantula of ta5; ta5 = tarsomere 5; un $=$ unguis; $\mathbf{u p l}=$ unguitractor plate. 
95. Tarsi, tarsomeres ta1-ta3, euplantulae, deep notch (V-shape) and lateral spreading: present [1]; missing [2].

The euplantulae of the tarsomeres ta1-ta3 are terminally notched (sometimes only slightly, but often deeply) in most Mantodea. They thereby gain a V-shape and appear to consist of two individual pads.

A distinct V-shape of the euplantulae is missing (state 2) in Chaeteessa (Fig. 302), Ciulfina, Eremiaphilidae (Fig. 305), Haaniinae (Figs. 314, 315), Mantoida (Fig. 304), Oligonicinae sp., and Thrinaconyx.

In some taxa the notch is not very prominent but the lateral spreading and the resulting V-shape are distinct [e.g. Hoplocorypha (Fig. 311), Metal-

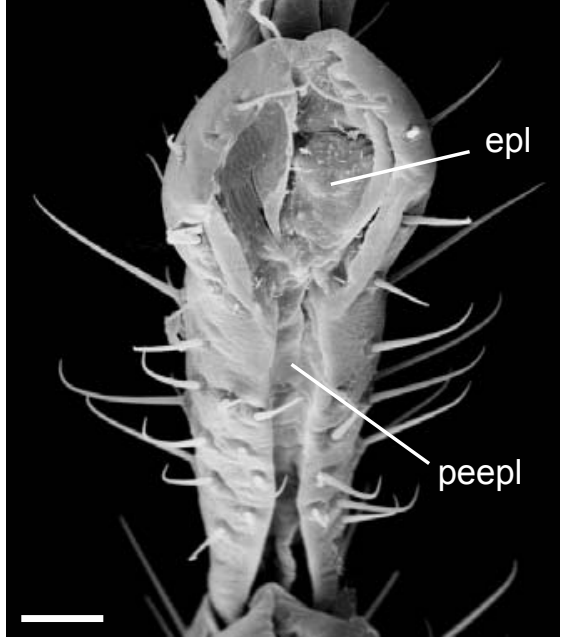

Fig. 331: Paraoxypilus sp. 1, ठ̂. Left foreleg, tarsomere ta4, ventral view. Note the posterior elongation of the euplantula being sunk in, probably due to desiccation. - Scale bar $=0.05 \mathrm{~mm}$. Abbreviations: $\mathbf{e p l}=$ euplantula; peepl $=$ posterior elongation of euplantula. lyticus (303), Perlamantinae (309, 310), Paraoxypilinae (Fig. 308)]. In these taxa the shape of the euplantulae resembles the situation found in some cockroaches (e.g. Blatta orientalis). In Periplaneta, however, the morphology of the euplantulae exhibits state 2 (Fig. 301).

96. Tarsi, cursorial legs, euplantulae of tarsomere ta4, strong enlargement in relation to euplantula of previous tarsomere (1.5 times as long or longer): present [1]; missing [2].

Tarsomere ta 4 of the cursorial legs has v-shaped euplantulae in Mantodea (see also character 95). In many species it is strongly enlarged. Enlargement (1.5 times as long as the euplantulae of the remaining tarsomeres or longer; state 1) is exhibited by 75 per cent of the species studied (Figs. 302, 312, 313, 314, 315, 318-327.

Strongly elongated euplantulae are missing (state 2) in Ameles (Fig. 317), Amorphoscelinae (Figs. 306, 307), Bolivaria (Fig. 326), Compsothespis, Elaea, Empusidae except for Gongylus (Fig. 328-330), Eremiaphilidae (Fig. 305), Hoplocorypha (Fig. 311), Ligaria, Ligariella, Mantoida (Fig. 304), Metallyticus (Fig. 303), Paraoxypilinae (Fig. 308), Parentella, Perlamantinae (Figs. 309, 310), Paralygdamia, Pseudoyersinia (Fig. 316), Tarachodes, and Zoolea.

In one case (Pseudoyersinia sp., Fig. 316) the euplantulae of the two proximal tarsomeres were larger than the distal ones.

97. Tarsi, tarsomere ta5, medial euplantula: present [1]; missing, tarsomere with a narrow longitudinal ventral suture at most [2]. 
Beutel \& Gorb (2001: 180) stated for Mantodea that the "euplantulae are well developed on the proximal four tarsomeres" and mentioned the same for Blattaria. Roth \& Naskrecki (2003) described the cave-dwelling cockroach Simandoa conserfariam from Guinea that has a median adhesive structure on the distal tarsomere (Roth \& Naskrecki 2003: 58 and figs. 2G, H). A similar structure is also present in several Mantodea. This structure is distinctly discernible by its white colour in living or alcohol preserved specimens, whereas it may be less conspicuous in dried specimens. Roth \& Naskrecki (2003: 57) mentioned that in dried specimens of $S$. conserfariam the euplantula of the fifth tarsomere may collapse and disappear.

Maceration of the tarsi makes it easier to discern the borders between the tarsomere and the median euplantula. Such a medial euplantula is also present, for instance, in Blaberus craniifer (Wieland, pers. obs.).

A distinct medial euplantula on the fifth tarsomere (state 1) has been found in Acanthops, Ambivia, Ameles (Fig. 317), Chroicopterinae (not discernible in Ligariella) Deroplatys (Fig. 318), Dystacta, Elaea, Empusidae (Figs. 328-330), Entella, Epaphrodita, Euchomenella (Fig. 324), Heterochaeta (Fig. 325), Hoplocorypha (Fig. 311), Iris, Leptocola, Ligaria, Liturgusidae (except for Stenomantis and Ciulfina; Fig. 323), Mantinae (Figs. 319; except for Rhombodera, Fig. 320), Miomantinae (Fig. 326), Paraoxypilinae (Fig. 308), Perlamantis (Fig. 310; missing in Paramorphoscelis, Fig. 309), Phyllocrania, Pseudocreobotra, Pseudoyersinia (Fig. 316), Sibylla, Stagmatopterinae, Theopropus, Toxodera, and Vatinae.

98. Tarsi, tarsomere ta5, arolium: present [1]; missing [2].

The arolium is an adhesive device on the terminal tarsomere being located between the ungues (Holway 1935: 3; Snodgrass 1935: 209 and figs. 111A, B; Seifert 1995: 158 and fig. 150; Beutel \& Gorb 2001: 179). In some insect groups, for instance in Mantophasmatodea (Beutel \& Gorb 2006: 6 and figs. 2A-D, 3A, B, 2008: fig. 1D), the arolium may be strongly enlarged. The arolium has been discussed to be a groundplan feature of Neoptera (Beutel \& Gorb 2006: 14; Klass 2007: 431). It is missing in several insect lineages, among others in Grylloblattodea and Dermaptera (Beutel \& Gorb 2006: 6). It is present, for instance in many Blattaria (Bohn 2003: 199), and the alates of several of the "lower" Isoptera (Crosland et al. 2005: tab. 1).

The arolium is missing in extant Mantodea (Holway 1935: 8; Beier 1964a: 874, 1968a: 22; Roy 1999: 29; Klass \& Ehrmann 2003: 185) and has not been described for any fossil species (e.g. Grimaldi 2003), either. It can therefore be assumed that it was already reduced in stemgroup-Mantodea. 
99. Tarsi, tarsomere ta5, ungues, length relation: equally long [1]; the anterior unguis being distinctly longer than the posterior one [2].

In almost all Mantodea the ungues (tarsal claws) are of equal length. A length difference has been reported only for some Eremiaphilidae (Lefèbvre 1835: 504 and pl. 13B fig. 9; Chopard 1940b: 17, 1941: 27, 33; Beier 1964a: 875, 1968a: 22; Roy 1999: 30). Beier (1964a: 875) stated that elongated ungues help to accelerate the mantodean body when running on sand.

Roy (1999: 30) mentioned unequal ungues for Eremiaphila typhon Lefèbvre, 1835 and E. numida Saussure, 1872. In the present taxon sample this character state could be confirmed only for Heteronutarsus aegyptiacus (Figs. 332, 424, 425; also depicted by Lefèbvre 1835: pl. 13B fig. 9), but not for the two Eremiaphila species (Fig. 305, 333). It is unknown whether in species with unequal ungues only one was elongated or if the other one was reduced after initial elongation. This character requires further study throughout Eremiaphilidae in order to get an insight into the putative phylogenetic value for this taxon.

100. Tarsi, tarsomere ta5, ungues, strong elongation (ungues longer than tarsomere ta5): missing [1]; present [2].

The ungues are usually shorter than the distal tarsomere in most Mantodea studied. Only in Eremiaphilidae they are distinctly longer (Figs. 324, 325, 332, 333), which is a potential autapomorphy for the group.

Eremiaphilidae dwell stony and sandy deserts in northern Africa and western Asia up to Pakistan and northern India (e.g. Chopard 1938: 59; Roy 1999: 38; Ranade et al. 2004: 1694). They are quick and agile runners (Chopard 1938: 60; Klass \& Ehrmann 2003: 196) and have very long legs compared to their body size (Roy 1999: 29, 39; Fig. 276). The elongation of the ungues (also see Lefèbvre 1835: pl. 11 figs. 9, 10) is probably an adaptation to running on bare soil or sand (Beier 1964a: 875; Roy 1999: 30).

Wings. Dictyoptera plesiomorphically have long wings extending the tip of the abdomen, which probably represents a ground plan feature that persists in many of the so-assumed "basal" lineages, for instance Mantoida, Chaeteessa, Metallyticus, Amorphoscelinae, and Perlamantinae (Figs. 5-9, 11, 12; but see Roy 2010 for the first brachypterous Mantoida mentioned in the literature), but also by many of the taxa exhibiting a more derived morphology. In many Mantodea the females have strongly shortened wings whereas the males usually retain full wing length (Beier 1964a: 877, 1968a: 21; Ehrmann 2002: 17). In some species both sexes have 
strongly shortened wings [e.g. Eremiaphilidae (see Battiston et al. 2010: 204; Fig. 10 herein), Bolivaria (see Battiston \& Massa 2008: fig. 10 and Battiston et al. 2010: 201 for a male)]. The wings may be entirely reduced without any trace of wing pads in both sexes [e.g. Apteromantis Werner, 1931 (p. 202; see also Battiston et al. 2010: 199), Geomantis Pantel, 1896 (p. 67; see also Battiston et al. 2010: 205), and Holaptilon Beier, 1964 (b: p. 184; Battiston et al. 2010: fig. 81)]. In other cases they may be entirely reduced only in the females while the males retain their flight ability [e.g. Nesoxypilus (e.g. Milledge 1990: figs. 1-2), Paraoxypilus, Myrmecomantis and many Thespidae).

The venation nomenclature of mantodean tegmina has been revised and homologized by Béthoux \& Wieland (2009) and is followed herein (Fig. 50). A homologization of the wing veins in mantodean alae has not been established yet, therefore their nomenclature follows Ragge (1955; Fig. 51).

101. Wings of male, length: macropterous, protruding tip of abdomen or slightly shortened (abdominal tergites t8-t10 free at most) [1]; strongly shortened, more abdominal tergites than t8-t10 free, small wings present at most [2].

The wings of males are seldom strongly reduced in length, because they usually fly around in search for females. The latter mostly become incapable of flying once they become gravid, even if they are fully winged (e.g. Beier \& Heikertinger 1952: 8; Beier 1964a: 879, 1968a: 20; see also chapter 2.3).

A length reduction of the wings (state 2) was found in male Ameles gracilis, an island species endemic to the Canary Islands (Fig. 14; García Becerra et al. 2001: 130; the males of $A$. decolor and other Ameles species are fully winged and well capable of flight, see Kaltenbach 1979: fig. 10, García Becerra et al. 2001: 129, Battiston \& Fontana 2005: fig. 13; see character 130), Bolivaria, Ciulfina, Compsothespis, Eremiaphilidae (Fig. 19), Hoplocorypha, Leptocola, Paralygdamia, Pseudoyersinia, Stenomantis, Stenopyga, Thesprotia, and Toxodera.

The macropterous state (state 1) is most common in males and was found in the remaining taxa (Figs. 7, 12, 17, 409-411). 
102. Tegmina, fusion of RP with M: present [1]; missing [2].

All extant Mantodea exhibit a fusion of RP and M from the wing base as was shown by Béthoux \& Wieland (2009: 106). This character is autapomorphic for Mantodea as Blattaria do not have such a fusion (M and RP distinct; Béthoux \& Wieland 2009: 106; Figs. 50, 334-337).

103. Tegmina, branching of RA (only posterior branchings; i.e. fusion of RP* with RA): present [1]; missing [2].

Few extant Mantodea exhibit an anteriorly branched RA (only Metallyticus and Chaeteessa; see Smart 1956: fig. 1, pl. 1, fig. 2A; Wieland 2008a: fig. 14; Béthoux \& Wieland 2009: figs. 1A, 2A, 6A, C, 20A, B; character 109; Figs. 334, 336). In most extant species the branches of RA, if present, are orientated posteriorly (Figs. 50, 337). Béthoux \& Wieland (2009) hypothesized from the evaluation of intraspecific venation variations of mantodean forewings that in all extant Mantodea, except for Chaeteessa, Mantoida and Metallyticus and few Amorphoscelinae, an anterior branch of RP + M (named RP* by Béthoux \& Wieland 2009; Figs. 50, 337) is translocated onto RA and has a common stem with RA from the wing base (see Béthoux \& Wieland 2009: 94, fig. 20D), which the authors assumed to be autapomorphic for a monophyletic group within Mantodea.

Amorphoscelis has been demonstrated to exhibit various intermediate stages between a missing and a full fusion of RP* with RA on an intraindividual to intraspecific level (Béthoux \& Wieland 2009: figs. 8, 9), therefore Amorphoscelinae are encoded as "unknown" herein. Amorphoscelinae have been assumed to represent intermediate stages between taxa without RP* being fused with RA and those exhibiting a full fusion, such as Perlamantis and Paramorphoscelis (Béthoux \& Wieland 2009: 105).

104. Tegmina of male, CuP (claval furrow), shape: curvature simple (one point of inflection) [1]; curvature sigmoid (two points of inflection) [2].

A strongly curved CuP is autapomorphic for Dictyoptera (e.g. Ragge 1955: 140; Bohn \& Klass 2003: 181; see chapter 2.1.1). The claval furrow (in which CuP runs) forms a deep impression in most Blattaria and many Mantodea. The curvature may have a single point of inflection ( $\mathrm{CuP}$ being curved) or two points of inflection ( $\mathrm{CuP}$ running in a sigmoid course). It has been assumed that the mantodean wings became stretched during mantodean evolution (at least in their distal part) with respect to cockroach wings (Hennig 1969: 183, 1981: 194). It 


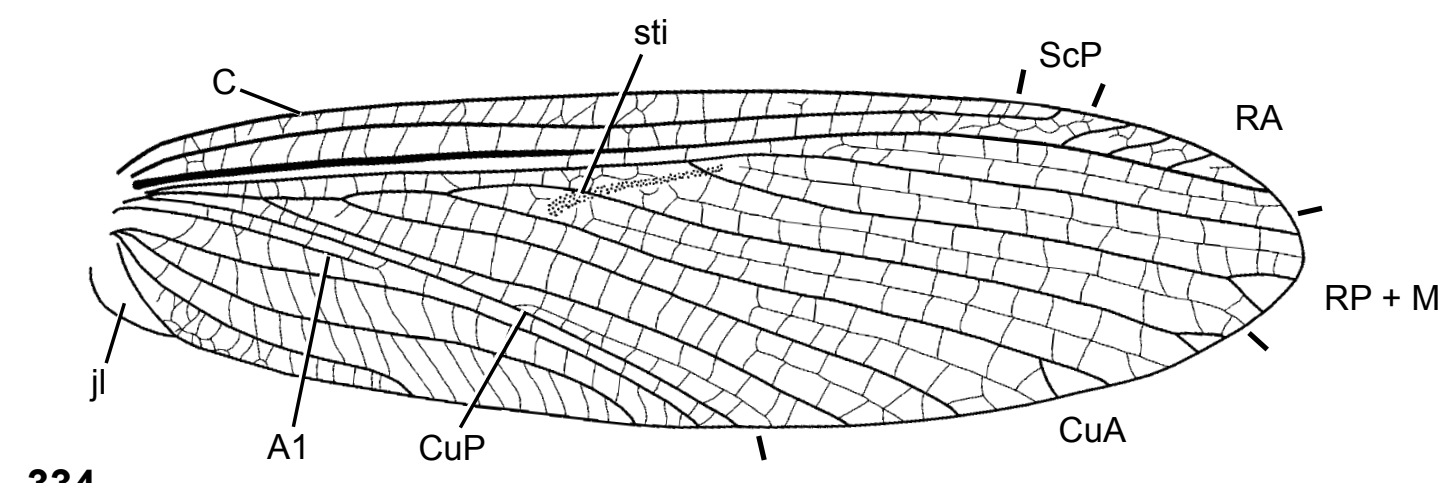

334

A1 CuP

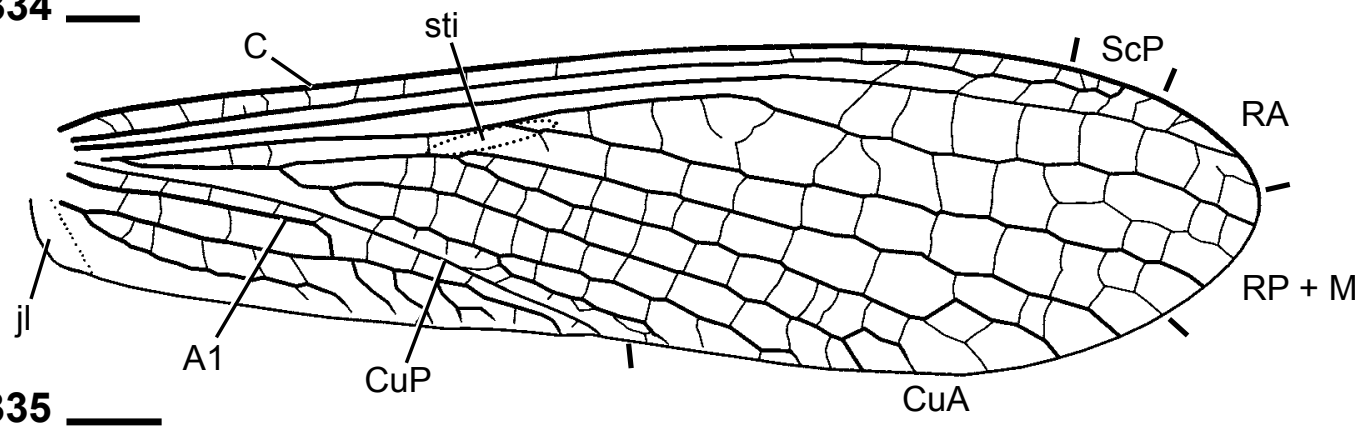

335

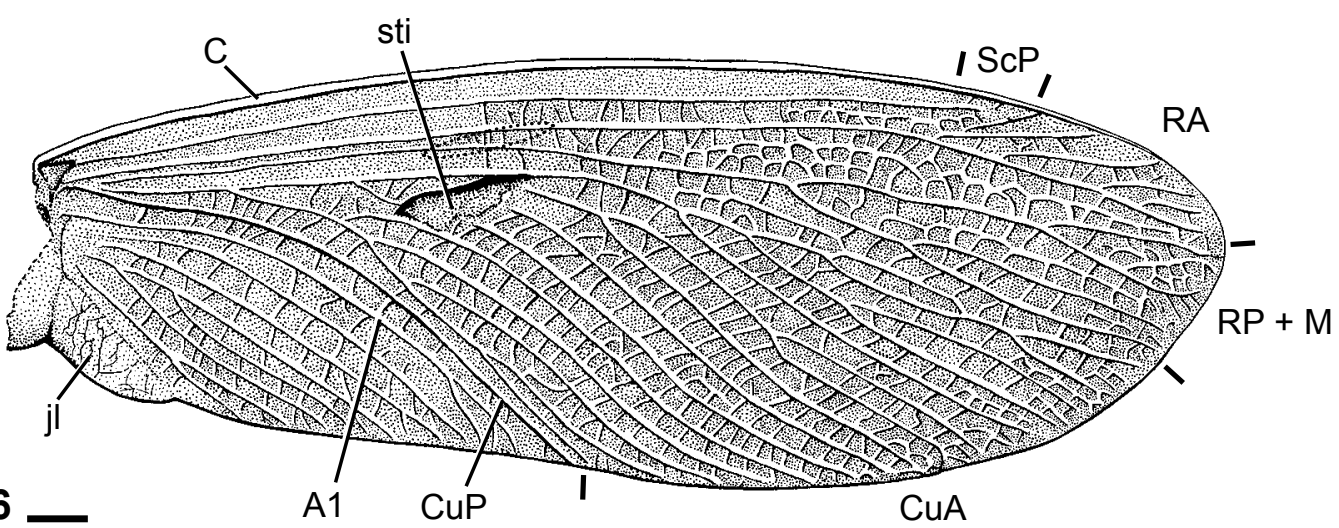

336

A1

CUA

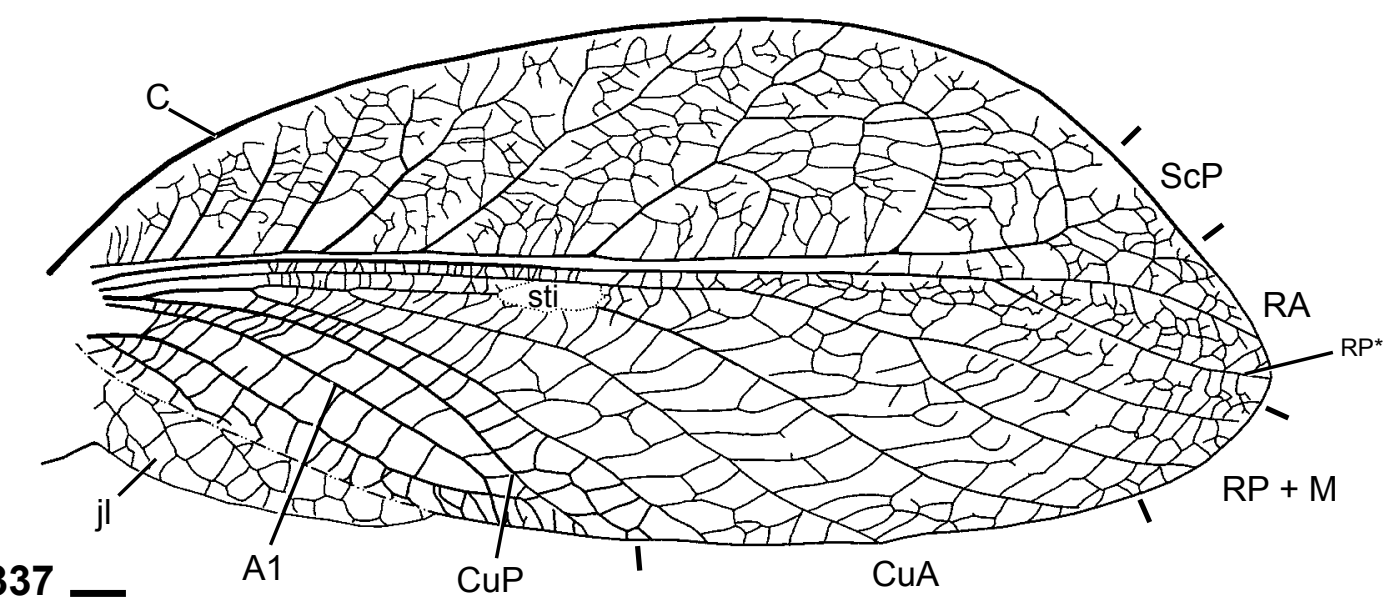

Figs. 334-337: Mantodean fore wings. 334: Chaetessa filata, right tegmen; redrawn and slightly altered from Smart (1956: fig. 1). 335: Mantoida maya, ô, right tegmen; redrawn from specimen IWC-OB 107 (O. Béthoux, private collection, see Material \& Methods). 336: Metallyticus splendidus, + , right tegmen; from Wieland (2008a: fig. 14). 337: Oxyopsis gracilis, , , right tegmen; redrawn from specimen IWC-OB 235 (O. Béthoux, private collection, see Material \& Methods); note the enlarged costal area and the conspicuous leaf-shape of the wing adding to the effect of special resemblance to plant matter in this species. - Scale bar $=1.0 \mathrm{~mm}$.

Abbreviations: $\mathbf{A 1}=$ first Anal vein; $\mathbf{C}=$ costa; $\mathbf{C u A}=$ anterior cubitus; $\mathbf{C u P}=$ posterior cubitus; $\mathbf{j} \mathbf{l}=$ jugal lobe; $\mathbf{M}=$ Media; $\mathbf{R A}=$ anterior Radius; $\mathbf{R P}=$ posterior Radius; $\mathbf{S c P}=$ posterior Subcosta; $\mathbf{s t i}=$ stigma . 
is possible that, due to an overall elongation of the wings, $\mathrm{CuP}$ became secondarily curved distad near the posterior edge of the tegmen but this would have to be studied in detail including observations on the fossil record.

Mantodea often exhibit strong sexual dimorphism, especially in wing length and -shape (see also characters 101, 106, 106), therefore this character was encoded individually for both sexes.

Interestingly, $\mathrm{CuP}$ is sigmoid (state 2) in Metallyticus (e.g. Béthoux \& Wieland 2009: fig. 6A; slightly less distinct in Fig. 336 herein), whereas in Chaeteessa (Smart 1956: figs. 1 and pl. 1, fig. 2A; Béthoux \& Wieland 2009: fig. 6C; Fig. 334 herein) and Mantoida (Smart 1956: fig. 3, incorrectly assigned to Miomantis Saussure, 1870; Béthoux \& Wieland 2009: fig. 6E; Fig. 335 herein) as well as in Amorphoscelinae (e.g. Béthoux \& Wieland 2009: fig. 9), Perlamantinae and cockroaches (e.g. Smart 1951: text-fig. 2 and pl. 1, fig. 1) CuP has a single point of inflection. It can be hypothesized that a simply curved $\mathrm{CuP}$ is a groundplan feature of Dictyoptera and represents the plesiomorphic state for extant Mantodea. This gains further support from the palaeontological record. The adult fossil Cretaceous Mantodea reviewed or described in Grimaldi (2003) have a simply curved $\mathrm{CuP}$. This is true for $\uparrow$ Cretophotina, $\uparrow$ Baissomantis, and $\uparrow$ Santanmantis (Grimaldi 2003: fig. 5b, c, 6, 23, 24), and possibly $\uparrow$ Ambermantis (Grimaldi 2003: figs. 3, 4).

There are intersexual differences in several species. It is noticeable that such differences often occur in species displaying a strong sexual wing length dimorphism, for instance Tarachodes, Tarachodula, Gyromantis, but also in several species with long wings in both sexes, among them Mantis, Zoolea, Heterochaeta, Orthodera, and Toxodera (see character 107).

105. Wings of female: present [1]; missing [2].

The reduction of the wings is a common trait in female Mantodea (e.g. Beier 1968a: 21; Klass \& Ehrmann 2003: 184). It has generally been assumed that wing reduction in female insects is a trade-off providing advantages with regard to greater fecundity and faster reproduction than in flying species (e.g. Wagner \& Liebherr 1992: 217). However, recent results from a study of a wing-polymorphic cricket indicate that this relation may have been overestimated in the past, as the differences in female anatomy (ovaries) between the short-winged and long-winged morphs, and therefore the trade-off, were eliminated after only two days, while the flight additionally increased the probability of courtship (Guerra \& Pollack 2009: 230).

In most Mantodea with shortened wings, the latter are reduced in length but still present (brachypterous forms; see character 106; e.g. Figs. 10, 19, 25), however, they are entirely missing in the females of several species (apterous forms). 
This was found in Hoplocorypha, Oligonicinae, Oxypilus, and Paraoxypilus in the present dataset. Beier (1968a: 21) furthermore named Yersinia (in addition to further Paraoxypilinae and Oligonicinae) to have wingless females. Beier (1950) also described an apterous species from Macedonia for which he erected a new genus and species (Apterameles rammei Beier, 1950) which, however, was found to be a nymphal specimen of Ameles heldreichi Brunner von Wattenwyl, 1882 (Kaltenbach 1963: 561; see also Battiston et al. 2010: fig. 57 for a habitus drawing of the holotype and p. 75 for a discussion of this topic).

It is likely that the wings in females were reduced many times independently. One example supporting this hypothesis is Paraoxypilinae. While the females of some genera are entirely wingless (e.g. Paraoxypilus, Nesoxypilus; see Milledge 1990: fig. 2), those of others are brachypterous or more or less fully winged (Metoxypilus, Gyromantis, Phthersigena; see Giglio-Tos 1913: figs. 3b, 5; Ehrmann 2002: 222, 278). The foreleg morphology is highly distinctive in Paraoxypilinae and autapomorphic for the group (see characters 42, 43, 44, 62, 63, 71, 74 and corresponding figures), supporting its monophyly. Monophyly is also indicated by molecular evidence (Svenson \& Whiting 2009). The group shows a rather restricted distribution today, occurring only in the Australian faunal region. Paraoxypilinae has phylogenetically been recovered within a group containing fully winged taxa in the molecular phylogeny (Svenson \& Whiting 2009). Therefore, it is likely that the wings of female Paraoxypilinae were reduced convergently with respect to the other Mantodea. This is also likely for other species with wingless females.

106. Wings of female, length: macropterous, protruding tip of abdomen or slightly shortened (abdominal tergites $\mathrm{t} 8$-t10 free at most) [1]; strongly shortened, more abdominal tergites than $\mathrm{t} 8$-t10 free, at least small wings present [2].

Shortened wings are common in female Mantodea. Wings are lost entirely in few species only. See also characters 101 and 105. Interestingly, Roy (2010) recently described a brachypterous female Mantoida from French Guyana, which is the first evidence for brachyptery in one of the three so-assumed earliest branches of extant Mantodea.

State 1 was exhibited by about $50 \%$ of the species (e.g. Figs. 9, 16, 18, 24, 142, 454).

107. Tegmina of female, $\mathrm{CuP}$ (claval furrow), shape: curvature simple (one point of inflection) [1]; curvature sigmoid (two points of inflection) [2].

See character 104. Differences between the sexes were found in Gyromantis

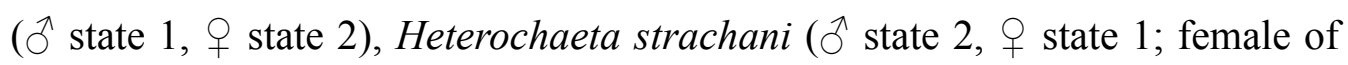




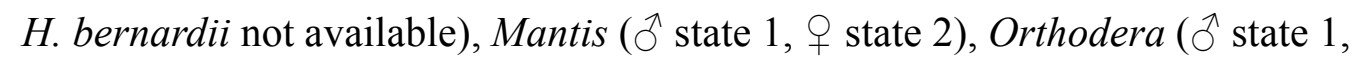
$q$ state 2), Pyrgomantis ( $\delta$ state $1, q$ state 2), Tarachodes and Tarachodula

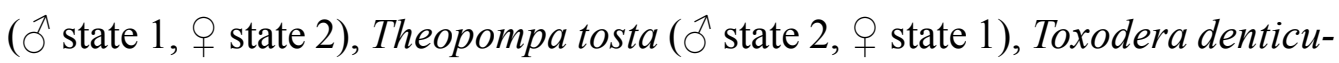

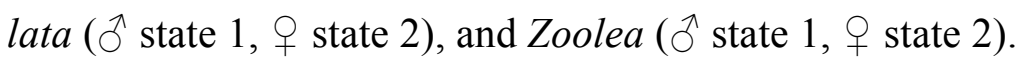

108. Tegmina, costal area between $\mathrm{C}$ and $\mathrm{ScP}$ in proximal part of wing, distance in relation to distance between ScP and RA: twice as long or longer [1]; only slightly longer or equally long [2].

The distance between $\mathrm{C}$ and $\mathrm{ScP}$ in the proximal part of the tegmina is short (insignificantly longer than the distance between ScP and RA; state 2) in several lineages of Mantodea. A short costal area is exhibited by Astape [e.g. Lieftinck 1953: fig. 1; interestingly, the area is longer in Haania (see Anisyutkin 2005: fig. 4)], Chaeteessa (e.g. Smart 1956: fig. 1 and pl. 1, fig. 2A; Klass \& Ehrmann 2003: fig. 13.3A; Béthoux \& Wieland 2009: fig. 6C; Fig. 334), Mantoida [Smart 1956: fig. 3 (incorrectly labelled as Miomantis due to confusion with fig. 4); Klass \& Ehrmann 2003: fig. 13.3B (incorrectly taken over as Miomantis from Smart 1956: fig. 3); Béthoux \& Wieland 2009: fig. 6E; Fig. 335], and Metallyticus (e.g. Wieland 2008a: fig. 14; Béthoux \& Wieland 2009: figs. 1A, 2A, 6A; Figs. 336, 454).

In the remaining taxa the costal area is twice as long or longer than the distance between ScP and RA (state 1). ScP lies closer to RA than to $\mathrm{C}$ in most extant mantodeans (e.g. Beier 1968a: 21; Roy 1999: 30), and it is always the area between $\mathrm{C}$ and ScP that is elongated (Roy 1999: 30). An enormously elongated area between $\mathrm{C}$ and $\mathrm{ScP}$ can be found, for instance, in species with a special resemblance to leaves and in bark dwellers. In the former, the enlarged wings add to the impression of green or dead leaves, albeit much more often in the females than in the males. Such enlarged costal areas are present, for instance, in several Acanthopidae, Antemna, Camelomantis Giglio-Tos, 1917, Choeradodinae (Fig. 142), Deroplatys, Rhombodera, Stagmatoptera, Oxyopsis (Fig. 337), and Taumantis (see Saussure \& Zehntner 1894: pl. 6, fig. 6, pl. 8, fig. 2; Beier 1935b: figs. 2, 5; Sharp 1895: fig. 135; figures in Ehrmann 2002; Roy 2004b: fig. 33; Ippolito 2007: fig. 9). In several bark dwelling taxa (e.g. Theopompa, Theopompella; see Giglio-Tos 1921: pl. 1, figs. 9, 10, pl. 2, fig. 11), and in some others (e.g. Choeradodis, Neomantis) elongation of this area in combination with a dorso-ventrally flattened habitus leads to the reduction of conspicuous shadows on the surface when the mantid is resting (Edmunds 1974: 13). By the elongation of the area between $\mathrm{C}$ and ScP and the additional close adjoining of the main branches of the main veins, a strong impression of a plant leaf including a perfect imitation of the median major and lateral minor 
leaf veins is accomplished. This is the case, for instance, in Choeradodis (e.g. Nel \& Roy 1996: fig. 7; Fig. 142), Oxyopsis (Fig. 337), and Deroplatys (e.g. Sharp 1895: fig. 135).

109. Tegmina, RA, distal anterior branches towards C: present [1]; missing [2].

The presence of anterior branches of RA being directed towards $\mathrm{C}$ has been hypothesized to represent the plesiomorphic state for Chaeteessa and Metallyticus (Smart 1956: 548, 550 and fig. 1; Klass \& Ehrmann 2003: fig. 13.3A; Wieland 2008a: fig. 14; Béthoux \& Wieland 2009: figs. 1A, 2A, 6A, C, 20A, B; Figs. 334, 336).

The assignment of the anterior branches of the radius in the tegmina of cockroaches is problematic (Smart 1951: 507). Beier (1974a: 27) mentioned that the anterior main branch of R (mostly assigned to RA, but ambiguous) may be branched. As the homologization of the tegminal veins in Blattaria is uncertain and the branch that has been assumed to be RA by previous authors is simple in Periplaneta, it is provisionally encoded as "unknown" herein.

110. Tegmina, costal rim, ventral teeth: present [1]; missing [2].

The anterior rim of the mantodean forewings may carry a row of pointed or blunt ventral teeth that reach their highest density near the middle of the costa (Figs. 338-344). This structure is part of a stridulating apparatus that is used for the production of a rasping sound as a secondary defence mechanism by few groups, namely all Empusidae and few Mantidae (e.g. Wood-Mason 1878b: fig. 2; Williams \& Sharp 1904: 129; Willey 1906: 226, 1918: 357; Giglio-Tos 1912: 59; Shelford 1916: 140; Hebard 1920: 51 ff.; Hingston 1932: 278; Burtt \& Carpenter 1943: 57; Schwarz et al. 2006: 69 and figures; see summary in Ramsay 1990: 44-45). The counterpart of the toothed rim, a ridge on the hind femur which is rubbed against the wing margins when stridulating, was not observed in Gongylus by Willey (1918: 359) but was mentioned for Idolomantis diabolica and other Empusidae by Schwarz et al. (2006: 69).

Teeth on the ventral edge of the anterior wing margin have been confirmed for all Empusidae (Figs. 338-341), Rhombodera (Fig. 342), Sphodromantis (Fig. 343), and Tamolanica (Fig. 344) in the present dataset. They were also described for Hierodula (Wood-Mason 1878b, c; Werner 1923: 396). But see 4.3.66 and 4.6.13 for discussion.

111. Tegmina, main veins, course: at least partially sigmoid (two points of inflection) [1]; distally bent or nearly straight (one point of inflection) [2]. 
The main veins in the Tegmen may run in a more or less strongly sigmoid course, i.e., they have two points of inflection (Figs. 336, 337). Hennig (1969: 183; 1981: 194) stated, that the mantodean wings are elongated, at least in the distal part, if compared to Blattaria (see character 104). As in the case of $\mathrm{CuP}$ (characters 104,107$)$ the sigmoid course of the veins may find an explanation in such an elongation of the tegmina.

Interestingly, the course of many of the main veins (mainly $\mathrm{CuA}$ and $\mathrm{CuP}$ ) is sigmoid in Metallyticus (e.g. Béthoux \& Wieland 2009: figs. 1A, 2A, 6A; Fig. 336). It is only slightly sigmoid in Chaeteessa (Smart 1956: figs. 1 and pl. 1, fig. 2A; Béthoux \& Wieland 2009: figs. 6C, 20B; Fig. 334), and it is straight or only has a single point of inflection in Mantoida (Smart 1956: fig. 3, incorrectly assigned to Miomantis; Béthoux \& Wieland 2009: figs. 6E, 20C; Fig. 335), Amorphoscelinae (Béthoux \& Wieland 2009: figs. 7A, B, 8A, C, 9A, B, E, F), and Paraoxypilinae.

112. Tegmina, ScP, length: ending beyond the distal third of the wing length [1]; ending proximal to the middle of the wing length [2].

$\mathrm{ScP}$ meets $\mathrm{C}$ or vanishes in the distal third of the tegmen (state 1) in all extant Mantodea (see Ragge 1955: figs. 104, 105; Smart 1956: figs. 1-4; Hennig 1969: fig. 48; figures in Béthoux \& Wieland 2009). In contrast, ScP in the tegmen of extant cockroaches always ends in the proximal half of the wing (e.g. Bohn 2003: 199; Béthoux et al. 2009: 150). Hennig (1969, 1981) stated that a long $\mathrm{ScP}$ (he was probably referring to both fore and hind wings) represents the plesiomorphic state. However, as pointed out by Béthoux et al. (2009: 150), the polarity of this character is ambiguous because it varies among the neopteran taxa which are candidates for a sistergroup of Dictyoptera.

A long ScP in the tegmen can be found in several fossil Mantodea (e.g. $\uparrow \mathrm{Am}$ bermantis and $\dagger$ Baissomantis; see Grimaldi 2003: figs. 3, 6), whereas it merely reaches the middle of the wing in †Santanmantis (Grimaldi 2003: fig. 23). In $\uparrow$ Mesoptilus, which has been found by Béthoux \& Wieland (2009) to be a putative Carboniferous stemgroup-Mantodea, ScP distinctly exceeds the middle of the wing (Béthoux \& Wieland 2009: fig. 21C).

However, there are also Carboniferous fossils of putative Blattaria with a long $\mathrm{ScP}$ in the tegmen, for instance $\dagger$ Sysciophlebia euglyptica (Germar, 1851) [p. 86 and pl. XXXI: fig. 7; see also Handlirsch (1906-1908: pl. 25: fig. 6), Tillyard (1937a: pl. 1, fig. 3) and Hennig (1969: fig. 51a)]. Furthermore, Béthoux et al. (2009) re-investigated the wing venation of $\dagger$ Protoblattinopsis stubblefieldi Laurentiaux, 1953, which also exhibits a long ScP in the tegmen (Béthoux et al. 2009: fig. 1). After evaluating the data they provisionally favoured the 
scenario of $\dagger P$. stubblefieldi being a stem-dictyopteran at some unknown level of the Dictyoptera phylogenetic tree (Béthoux et al. 2009: 151 and fig. 3C). This might indicate a long tegminal $\mathrm{ScP}$ in the early stem representatives of Dictyoptera, whereas a shortened $\mathrm{ScP}$ in the tegmen was probably present in the last common ancestor of Mantodea and Blattodea, followed by a secondary re-elongation of $\mathrm{ScP}$ in the tegmina of Mantodea. If this hypothesis is assumed, $\dagger$ Sysciophlebia might be another stemgroup-Dictyoptera.

113. Tegmina, $\mathrm{CuA}$, maximum number of branches: 0-8 [1]; 9 or more [2].

$\mathrm{CuA}$ usually has up to 8 branches in Mantodea (Roy 1999: 31), whereas in Blattaria the number of branches is usually higher (e.g. Ragge 1955: 128 and fig. 100; Bohn 2003: fig. 14.2).

The only exceptions found in the Mantodea sample herein are Metallyticus, in which CuA may reach up to 12 or more branches (Roy 1999: 31; Fig. 336), and Plistospilota with ten branches. Chaeteessa and Mantoida have about 6 branches [e.g. Smart 1956: figs. 1, 3 (incorrectly assigned to Miomantis), pl. 1, fig. 2A; Béthoux \& Wieland 2009: figs. 6C, E; Figs. 334, 335].

As such a high number of branches of $\mathrm{CuA}$ is present in Plistospilota, it is likely that this pattern is a secondary trait and not homologous to the situation in Blattaria. Whether or not this is also the case for Metallyticus, is debateable.

114. Tegmina (excluding jugal lobe), number of branches of anal veins (including A1): 2-3 [1]; $4-5$ [2]; 6 or more [3].

The anal veins have $4-5$ branches (including A1, state 2 ; see character 115; Figs. 50, $334,337)$ in most species.

State 3 is only exhibited by Callibia, Caudatoscelis, Galinthias, Metallyticus (Fig. 336), Plistospilota, and Polyspilota and has also been found in Periplaneta. 
State 1 was found in Amphecostephanus, Astape, Bactromantis, Bolivaria, Ciulfina, Deroplatys, Leptocola, Mantoida (Fig. 335), Oligonicinae sp., Oxypilus, Paraoxypilus, Perlamantinae, Popa spurca spurca (P. spurca crassa exhibits state 2), Pyrgomantis, Sibylla, Thrinaconyx, and Tropidomantis.

115. Tegmina, branching of A1: present [1]; missing [2].

A1 is simple in most Mantodea. A1 is branched only in Amorphoscelinae and Metallyticus (Fig. 336; Wieland 2008a: fig. 14; Béthoux \& Wieland 2009: figs. 1A, 2A, 3A, 7A, B, 9A, B, E, F; Roy 2009b: fig. 4) with exception of few aberrant specimens. Béthoux \& Wieland (2009: 106) stated that homologization of the simple A1 in derived Mantodea is difficult. They proposed that the short, simple A1 as found in Chaeteessa (Fig. 334), Mantoida (Fig. 335) and the fossil $\uparrow$ Arvernineura insignis Piton, 1940, might represent the plesiomorphic state, whereas the forked situation in Metallyticus (Fig. 336) and Amorphoscelinae might be derived. It furthermore cannot be ruled out that the anterior branch of A1 of Metallyticus and Amorphoscelinae becomes fused to $\mathrm{CuP}$ in derived mantodeans (Béthoux \& Wieland 2009: 106).

In the present study, no taxa except for Metallyticus and Amorphoscelinae have been confirmed to exhibit a branched A1. The situation in Amorphoscelis sp. is ambiguous (see Béthoux \& Wieland 2009: fig. 8: IWC-OB 190). This specimen apparently does not exhibit a forked A1. CuP, however, emits a posterior branch in both tegmina (Béthoux \& Wieland 2009: fig. 8A, C) which may be indicative for the anterior branch of $\mathrm{A} 1$ being translocated onto $\mathrm{CuP}$, as the latter is always simple in extant Mantodea (e.g. Ragge 1955: 132; Smart 1956: 548; Roy 1999: 31). Therefore, this character is encoded as state 1 in the matrix, as for the remaining Amorphoscelinae.

116. Tegmina, length of A1: almost or entirely reaching the posterior wing margin [1]; not reaching the posterior wing margin, vanishing early in the wing membrane or fusing with other veins [2].

Hennig (1969: 185, 1981: 195) stated that A1 in the tegmen is short in Blattaria and Mantodea and never reaches the posterior wing margin. However, A1 is long and (at least almost) reaches the posterior wing margin in a variety of taxa studied, including Metallyticus (e.g. Wieland 2008a: fig. 14; Béthoux \& Wieland 2009: figs. 1A, 2A, 6A; Fig. 336) and, for instance, Oxyopsis (Fig. 337). Chaeteessa and Mantoida (Figs. 334, 335) have a very short A1 that vanishes early in the wing membrane [see also Smart 1956; figs. 1, 3 (Mantoida, incorrectly assigned to Miomantis), pl. 1, fig. 2A; Klass \& Ehrmann 2003: fig. 13.3A, B 
(Mantoida, incorrectly assigned to Miomantis); Béthoux \& Wieland 2009: figs. $6 \mathrm{C}, \mathrm{E})]$.

A1 is short and vanishes in the wing membrane in several of the fossil Mantodea described by Grimaldi (2003, misinterpreted as CuP therein: figs. 3, 23, 24; see also character 20 therein), whereas it is long, for instance, in $\dagger$ Baissomantis (Grimaldi 2003: fig. 6). Grimaldi (2003: 40) interpreted the long A1 (his CuP), reaching the posterior wing margin, as the plesiomorphic state. This has gained support by the assignment of the Carboniferous $\uparrow$ Mesoptilus dolloi, exhibiting a long A1, to stemgroup-Mantodea (Béthoux \& Wieland 2009: fig. 21C). Therefore, the short A1 in Blattaria and extant Mantodea has possibly evolved convergently.

117. Tegmina, jugal lobe, crossing by anal veins: present [1]; missing [2].

A jugal lobe is present in the tegmina of Mantodea (Ragge 1955: 136). Its distal border is distinctly discernible by an inflection of the posterior wing margin (e.g. Ragge 1955: figs. 104, 105; Figs. 50, 336, 337). The tegminal jugal lobes are generally folded underneath the wings when the latter are folded above the abdomen (Ragge 1955: 136).

The jugal lobe may be very narrow and restricted to the proximal area of the wing [e.g. Chaeteessa (Fig. 334), Metallyticus (Fig. 336), Mantoida (Fig. 335); see Smart 1956: fig. 1, pl. 1, fig. 2A; Wieland 2008a: fig. 14; Béthoux \& Wieland 2009: figs. 6A, C, E, 20A-C]. In many taxa, however, it is wider (e.g. in $O x y$ opsis, Fig. 337) and may almost reach the middle of the wing, as, for instance, in Creobroter (see Béthoux \& Wieland 2009: figs. 12A, C; Béthoux 2010: fig. 1). Ragge (1955: 136) stated that the jugal lobe is supported by the distal parts of one or more of the anal veins (i.e. crossed by them; see Ragge 1955: figs. 104, 105; Figs. 50 and 337 herein). However, a distinct crossing of the jugal lobe by the anal veins is missing in several species, in which the lobe is narrow. This is the case in Acanthops, Chaeteessa (Fig. 334), Eremiaphila, Haania, Ligaria, Mantoida (Fig. 335), Metallyticus (Fig. 336), the undetermined Oligonicinae specimen, Oxypilus, Raptrix, Thesprotia, and Tithrone.

The jugal lobe in the tegmina is very narrow at most in Blattaria (e.g. Smart 1951: 506 for Periplaneta: "A small membraneous alula connects the vannus to the wall of the thorax and folds beneath the vannal region when the tegmen is in repose on the abdomen"). The small jugal lobe in Blattaria, as shown for Periplaneta by Smart (1951: pl. 1, fig. 1 and text-fig. 1), apparently has no branches of anal veins crossing it. It is therefore likely that the crossing of the jugal lobe by anal veins is apomorphic a group within Mantodea, and that Chaeteessa, Metallyticus and Mantoida exhibit the plesiomorphic state. 
118. Tegmina, "stigma": present [1]; missing [2].

The terms "stigma", "pterostigma", and "pseudovein" (e.g. Chopard 1949a: 389; Beier 1964a: 875, 1968a: 21; Ramsay 1990: 41; Nel \& Roy 1996: 225; Roy 1999: 31; Grimaldi 2003: 6, 38) refer to an often calloused area on the tegmina, mostly between $\mathrm{RP}+\mathrm{M}$ and $\mathrm{CuA}$ in the proximal half of the wing (Figs. 50, 142, 334-337). The veins crossing the stigma are often interrupted in this area, although their tracheae are most probably not (Béthoux, pers. comm. 2008). It may be conspicuous due to a colouration differing from the rest of the tegmen (white or yellow, e.g. Hierodula, Sphodromantis). In other mantodeans it is nearly invisible, but has been stated to be always present (Chopard 1949a: 389; Roy 1999: 31).

Ramsay (1990: $41 \mathrm{ff}$.) gave a detailed overview of the references of the stigma in the literature. He stated that it probably is a plesiomorphic trait, as it is exhibited by taxa pertaining to the early branches of the mantodean phylogeny (naming Chaeteessa; Ramsay 1990: 43; Fig. 334). However, such a structure has not been identified in any fossil or extant cockroaches or termites and it is lacking in the other neopteran taxa. Therefore it is probably apomorphic for Mantodea.

Ramsay (1990: 43 and figs. M14, M15, M18-M20) showed SEM pictures of the surface of the stigma, which is different from that of the surrounding wing membrane and exhibits a scaly structure. The function of the stigma is unknown (Ramsay 1990: 43).

Vršanský (2002: 10) assumed the presence of a "pterostigma" in the hind wing [sic!] of mantodeans and $\dagger$ Liberiblattinidae to be a potential synapomorphic character of the two groups. However, Mantodea do not possess any such structure in the hind wing. The stigma of Mantodea is certainly autapomorphic for the group and occurs exclusively in the fore wings (see above). A close relationship of $\dagger$ Liberiblattinidae and Mantodea is therefore not supported by this character (as was also elaborated by Grimaldi \& Ross 2004: 104; also discussed by Béthoux \& Wieland 2009: 107).

119. Alae of male, branching of RA: present [1]; missing [2].

While most extant Mantodea exhibit a simple RA in the hind wings (state 2), only few taxa have it branched (state 1). This was found in male Idolomantis, Metallyticus, Phyllocrania, and Theopompa tosta. In the latter, however, only one hind wing was accessible. In that wing, RA exhibited an anterior branch in the distal fifth. It is unclear, whether this is an anomaly or if such a branching occurs regularly in this species. 
The situation is ambiguous for cockroach hind wings. While Smart (1951: 504) mentioned that RA (his R1) "has a few antero-pectinate branches", Ragge (1955: 128 and fig. 100) stated that "in the hind wing [of cockroaches] the radius has a similar form [as in the fore wing]; the radial sector may be represented either by the small posterior branch, which is only developed in the more primitive wings, or by the entire vein except for the most proximal of the anterior branches, which tends to have an appearance rather distinct from the remainder". The same was mentioned by Beier (1974a: 27). As the hind wing venation of cockroaches has not been revised yet, Periplaneta has been encoded as “unknown”. See also character 123.

120. Alae of male, branching of CuA: present [1]; missing [2].

Ragge (1955) stated that in both Blattaria and Mantodea CuA (his Cu1) in the hind wings is usually branched, but that he had observed species in both groups that exhibit and undivided CuA (Ragge 1955: 128, 136).

$\mathrm{CuA}$ is simple (state 2) in male Acontista, Ameles gracilis, Galinthias, Heterochaeta, Hoplocorypha, Iris, Raptrix, and Toxodera denticulata. The remaining male Mantodea and Periplaneta exhibit a branched $\mathrm{CuA}$ in the alae (state 1).

121. Alae of male, anal veins (including A1, branches of A3 not counted individually), largest number: less than 5 [1]; between 5 and 8 [2]; 8 or more [3].

Beier (1964a: 877, 1968a: 21) mentioned that usually eight or nine anal veins occur in the hind wing of Mantodea. Handlirsch (1906-1908: 24) mentioned numerous anal veins for the hind wing ("Analadern zahlreich, ähnlich wie bei Blattiden $[. . .]^{6 ")}$ ) thus referring to the large number of anal veins in the hind wings of cockroaches.

Ragge (1955: 136) did not address the number of anal veins in the hind wing but stated that "in the adult wing it is often impossible to tell where these accessory branches [i.e. branchings of A3] are succeeded by distinct anal veins. As there is apparently nothing to be gained by applying a nomenclature to these veins, they are not named here".

Beier \& Jaus (1933: 129) assumed that the size of the hind wing fan generally increases with the degree of specialization of the taxa, i.e. they indicated the small anal area of the hind wings to be plesiomorphic.

State 1 was only found in male Eremiaphila, probably due to strong wing reduction.

The situation was not accessible for Chaeteessa valida and was therefore not 
encoded. The drawing and photograph in Smart (1956: fig. 1 and pl. 1, fig. 2B; Fig. 345), although the sex was not mentioned therein, indicate that Chaeteessa exhibits state 2. The same is true for Mantoida (Smart 1956: fig. 3, incorrectly assigned to Miomantis; Fig. 346).

The males of little more than 30 per cent of the taxa studied exhibit more than eight anal veins in the alae (state 3 ), including male Metallyticus. The same is true for Periplaneta (see Smart 1951: fig. 2; Roth 1991: fig. 19.2C). However, there are also cockroaches with smaller numbers of anal veins in the hind wing, for instance Neotemnopteryx australis (Saussure, 1863) (Roth 1991: fig. 19.9) and Alluaudellina cavernicola (Shelford, 1910) (Chopard 1949b: fig. 53B). The latter, however, belongs to a group of cavernicolous and termitophilous cockroaches (Nocticolidae) that are likely to exhibit highly specialized morphological traits in adaptation to their lifestyle.

It is unclear whether the number of 5-8 anal veins in the hind wings of Chaeteessa and Mantoida represents a plesiomorphic character with respect to Dictyoptera (as implied by Beier \& Jaus 1933) or if an anal fan with more than 8 anal veins is plesiomorphic. The latter hypothesis is supported by the fact that several polyneopteran taxa have large hind wing fans, among them Phasmatodea, Orthoptera, Dermaptera and Plecoptera (e.g. Grimaldi \& Engel 2005: fig. 7.2). Furthermore, Periplaneta has been found to be phylogenetically related to a group of Blattinae species that is assumed to form the sistergroup of all remaining Blattodea (e.g. Klass 1997: diagr. 1; Klass \& Meier 2006). This indicates that a large anal fan with many anal veins may have been present in the hind wing of last common ancestor of cockroaches and mantodeans and therefore to be plesiomorphic. In this case the smaller anal fan with lesser veins in Chaeteessa and Mantoida would represent a derived condition. However, total evidence analysis implies a different scenario (Ware et al. 2008). In the parsimony analysis (Ware et al. 2008: fig. 2A), Nocticolidae came out in a polytomy with other taxa at the basal split of the tree, whereas Periplaneta was found subordinate far more distally in the tree.

Current data are insufficient at this point but an extensive study of the hind wing anal fan throughout a large number of cockroaches in comparison with Mantodea (and other Polyneoptera) might be a promising task.

122. Alae of male, A3, branching: once or twice [1]; 3-4 times [2]; 5 times or more [3].

A1 in the hind wings of Mantodea is always simple (Roy 1999: 32; Figs. 51, 345-347). A2 (simple) has been stated to be missing in Mantodea except for Chaeteessa and Metallyticus, although this situation is still unresolved (for a discussion of this topic see character 129). 

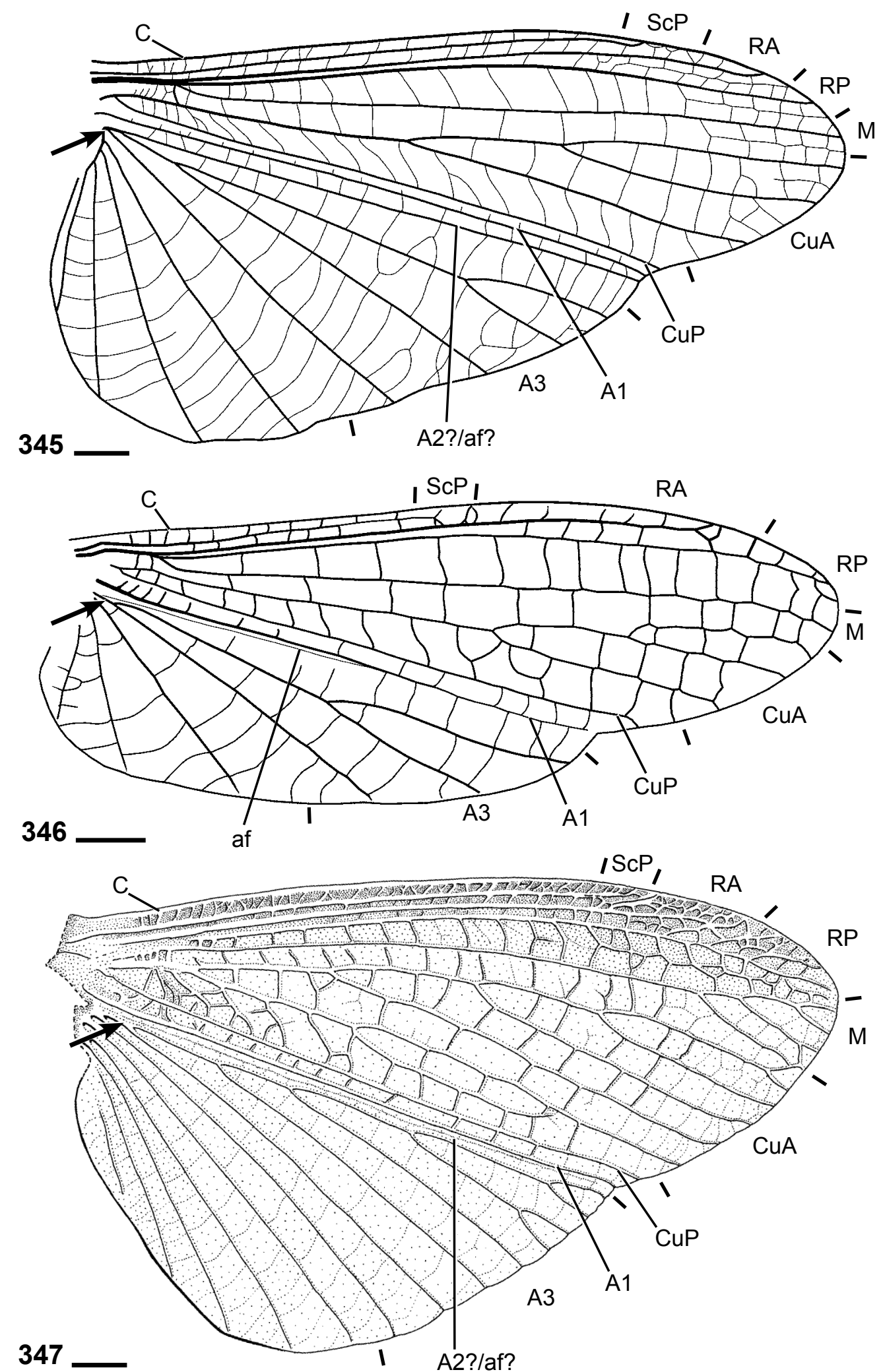

Figs. 345-347: Mantodean hind wings. 345: Chaetessa filata, right ala; redrawn and slightly altered from Smart (1956: fig. 1). 346: Mantoida maya, ô, right ala; redrawn from specimen IWC-OB 107 (O. Béthoux, private collection, see Material \& Methods). 347: Metallyticus splendidus, $\bigcirc$, left ala (mirrored); from Wieland (2008a: fig. 15). - Scale bar $=1.0 \mathrm{~mm}$.

Abbreviations: A1, A2, A3 = first, second, third Anal vein; $\mathbf{a f}=$ anal fold; $\mathbf{C}=\operatorname{costa} ; \mathbf{C u A}=$ anterior cubitus; $\mathbf{C u P}=$ posterior cubitus; $\mathbf{M}=$ Media; $\mathbf{R A}=$ anterior Radius; $\mathbf{R P}=$ posterior Radius; $\mathbf{S c P}=$ posterior Subcosta; sti $=$ stigma. 
A3 in the hind wing is branched in most Blattaria and Mantodea [Smart 1951: pl. 1, fig. 2; Ragge 1955: 136 and figs. 100, 104, 105; Smart 1956: 551 and figs. 1-4 (named 2V); Beier 1968a: 21, 1974a: 27 and fig. 24a (named A2); Klass 1995: 158; Klass \& Ehrmann 2003: 185 and fig. 13.3].

The males of about ten per cent of the species studied herein have an A3 with five branchings or more (state 3 ). About 25 per cent had it split three or four times (state 2). Nearly 30 per cent had A3 branched once or twice (state 1). In the remaining species the males were not available or the structure was not accessible.

Beier (1974a: 27) stated for cockroaches that "von den Adern des Vannus (Analfeldes) ist die vorderste (2. Analader, Axillarader) [meaning A3 because $\mathrm{A} 1$ is named $\mathrm{PCu}$ therein] fast immer verzweigt und entsendet aus seiner gemeinsamen Wurzel 3 bis 6 oder auch mehr Strahlen gegen den Rand“, indicating that A3 is usually branched twice or more. In Periplaneta americana A3 in the hind wing has five or more branchings (see Smart 1951: pl. 1, fig. 2; Ragge 1955: fig. 100; Hennig 1969: fig. 49; Beier 1974a: fig. 24a). The same is true, for instance, for Periplaneta brunnea (Roth 1991: fig. 19.2C), Panesthia australis Brunner v. Wattenwyl, 1865 (Blattidae; Roth 1991: fig. 19.10), and Megaloblatta blaberoides (Walker, 1871) ("Blattellidae"; see Bell et al. 2007: fig. 1.5). However, there may be fewer branchings in other taxa. It is unclear which state represents the plesiomorphic condition. However, since less than five branchings of A3 are found in Chaeteessa (Béthoux 2008: fig. 6.1, unidentified species; Fig. 345), Mantoida and Metallyticus (Béthoux 2008: fig. 6.3; Wieland 2008a: fig. 15; Figs. 346, 347), this is likely to be the groundplan situation for Mantodea. Wieland (2008a: 164) mentioned a number of six branchings for A3 in the hind wing of Metallyticus. However, the small terminal branchings as can be seen in Wieland (2008a: fig. 15; female, Fig. 347 herein) and Béthoux (2008: fig. 6.3) occur infrequently and are missing more often than they are present, therefore leaving Metallyticus with four branchings (correspondingly encoded as state 2).

123. Alae of female, branching of RA: present [1]; missing [2].

See also character 119. Only the females of Bolivaria and Metallyticus have a branched RA in the hind wing (state 1). The hind wing of a female Metallyticus was shown in Béthoux (2008: fig. 6) and in Wieland (2008a: fig. 15; Fig. 347 herein).

As the situation in the hind wings of cockroaches is ambiguous (see character 119), the character has been encoded as "unknown" for Periplaneta. 
124. Alae of female, branching of $\mathrm{CuA}$ : present [1]; missing [2].

See character 120. The females of Ameles, Archimantis, Entella, Euchomenella, Galinthias, Heterochaeta strachani, Iris, Oxyopsis, Paramorphoscelis, Popa spurca spurca, Pyrgomantis, Raptrix, Rivetina, Tarachodes sp. 1, Tarachodula, and Toxodera have an undivided CuA in the hind wing (state 2). The remaining female Mantodea and Periplaneta have a branched $\mathrm{CuA}$ in the alae (state 1; Figs. 51, 345-347).

125. Alae of female, anal veins (including A1, branches of A3 not counted individually), largest number: less than 5 [1]; between 5 and 8 [2]; 8 or more [3].

See character 121. The number of anal veins in the hind wing is less than 5 (state 1) more often in females than in males. Among them are Ameles, Elaea, Eremiaphila, Pyrgomantis, Tarachodes, and Tarachodula. This result is not surprising as in all these taxa the females have more or less strongly reduced wings, which is likely to coincide with a reduction of the venation.

Chaeteessa was not encoded because the character was not accessible in the dried female specimen.

However, an intersexual difference was also found in Metallyticus, in which the females exhibit state 2, whereas the males fall into state 3.

As in the males (see character 121) about 30 per cent of the females studied exhibit state 3 .

126. Alae of female, A3, branching: once or twice [1]; 3-4 times [2]; 5 times or more [3].

See character 122. About 50 per cent of the species studied have less than five branchings of A3 (states 1, 2). State 3 is present in female Hymenopus, Idolomantis, Mantis, Orthodera, Plistospilota, Polyspilota, Pseudocreobotra ocellata (P. wahlbergii exhibiting state 2), Rhombodera, Stagmatoptera, Tamolanica, Theopropus, and Vates. Intersexual differences have been found in Choeradodis, Dystacta, Hymenopus, Mantis, Pseudocreobotra ocellata, Sphodromantis, and Theopropus.

127. Alae, ScP, length with respect to wing length: ending beyond distal third of the wing length [1]; ending proximal of the middle of the wing length [2].

Most extant Mantodea have a long $\mathrm{ScP}$ in the hind wing, meeting $\mathrm{C}$ beyond the distal third of the wing (Ragge 1955: figs. 104, 105; Smart 1956: figs. 1, 2, 4, 
pl. 1: fig. 2B; Hennig 1969: fig. 48, 1981: fig. 48; Klass \& Ehrmann 2003: figs. 13.3A; Figs. 51, 345, 347). This character has been stated to represent the plesiomorphic state (Hennig 1969: 183, 1981: 194). In contrast, ScP is short and meets $\mathrm{C}$ in the proximal half of the alae in Blattodea (Ragge 1955: fig. 100; Hennig 1969: 185 and fig. 49, 1981: 195 and fig. 49) and Mantoida (Smart 1956: fig. 3, incorrectly assigned to Miomantis; Klass \& Ehrmann 2003: figs. 13.3B, incorrectly assigned to Miomantis; Fig. 346).

Putative Blattaria fossils from the Carboniferous and Permian (Handlirsch 1906-1908; Tillyard 1937a, b) have a shortened ScP in the alae. In some fossils the vein slightly exceeds the middle of the wing at most before meeting $\mathrm{C}$ [e.g. $\uparrow$ Permoblattina curta (Sellards, 1908) (Tillyard 1937a: pl. 2, fig. 13A), and $\dagger$ Pycnoblattina Sellards, 1908 (Tillyard 1937b: figs. 21A, 22)]. None of the Palaeozoic members of Blattaria presented therein has an ScP resembling modern Mantodea in length (except for Mantoida), i.e. distinctly surpassing the distal third of the wing length.

Potential Carboniferous stemgroup-Mantodea have been identified (Béthoux \& Wieland 2009; Béthoux et al. 2010). There is only a poorly preserved hind wing visible in the holotype specimen of $\uparrow$ Mesoptilus dolloi (MNHN DP R51159; see Béthoux \& Wieland 2009: fig. 21A). However, a well-preserved hind wing fragment of the Permian $\dagger$ Homocladus grandis was published by Béthoux et al. (2010: fig. 4). Although the base of the wing is not entirely preserved, the specimen clearly has a short $\mathrm{ScP}$ which probably did not exceed the middle of the wing.

The situation in Palaeozoic Blattaria, putative stemgroup-Mantodea and the extant Mantoida indicate that a short ScP in the alae (possibly slightly exceeding the middle of the hind wing) was already established in the last common ancestor of Mantodea and Blattodea. It is possible that it became secondarily elongated within Mantodea. This would consequently imply Mantoida to be the sistergroup of all extant Mantodea with regard to this character (also supported by genital morphology and some molecular analyses: Klass 1995, 1997; Ware et al. 2008; Yager \& Svenson 2008). This hypothesis contradicts the general assumption that a long ScP is plesiomorphic for Mantodea (e.g. Hennig 1969: 183; but see character 112 for a discussion of $\mathrm{ScP}$ in the tegmina) but gains support from several Cretaceous Mantodea. Grimaldi (2003) showed the hind wings of several fossil species. In the alae of $\dagger$ Cretophotina tristriata Gratshev \& Zherikhin, 1993 and $†$ Baissomantis maculata Gratshev \& Zherikhin, 1993 (Grimaldi 2003: fig. 6), ScP only slightly exceeds the middle of the wing but clearly does not reach its distal third. Unfortunately, the situation in the hind wings of $\uparrow$ Santanmantis is unclear due to poor preservation of the alae in the specimens shown in Grimaldi (2003). 
128. Alae, crossveins between $\mathrm{CuA}$ and $\mathrm{CuP}$, shape: at least partially sigmoid (two points of inflection) [1]; not sigmoid, slightly curved or straight [2].

The crossveins between $\mathrm{CuA}$ and $\mathrm{CuP}$ may be more or less strongly sigmoid, as in Chaeteessa (Fig. 345). In nearly 50 per cent of the species studied here at least a part of the crossveins is slightly sigmoid.

129. Alae, A2 (simple): present [1]; missing [2].

A simple A2 is present in the alae of Blattaria (e.g. Klass 1995: 158, 194 and fig. 345c; Bohn 2003: fig. 14.2A), where in some species it may be weak but always present (Klass 1995: 158). In Mantodea it has been assumed to be present in Chaeteessa and Metallyticus. While this structure is easily discernible in the former (Smart 1956: figs. 1 and 2B; Klass \& Ehrmann 2003: fig. 13.3A; Béthoux 2008: fig. 6.3; Fig. 345), it is weak and possibly shortened in the latter (Smart 1956: 552 and $1 \mathrm{~V}$ in fig. 1; Klass 1995: 158, 194 and fig. 345b; Klass \& Ehrmann 2003: 185 and fig. 13.3B; Wieland 2008a: 163 ff. and A2? in fig. 15; Fig. 347).

However, the situation in both taxa is ambiguous (see Wieland 2008a: 164 for Metallyticus). Smart (1956: 552) suggested that A2 (his 1V) in Metallyticus is the remnant of a true vein because he was able to identify cross-veins that reach it from A1 and A3 (his Pcu and 2V). A thin line, about as thin as the cross-veins and running parallel to A1 in the area of concern, could be identified in Metallyticus (Wieland 2008a: A2? in fig. 15; Fig. 347). It resembles a folding line rather than a true vein and almost reaches the posterior rim of the wing, but indeed inconspicuous cross veins are present as observed by Smart (1956: 552). A similar structure is present in other Mantodea in the same area: the anal fold (e.g. Klass 1995: Af in Fig. 346b for Mantis religiosa; Fig. 346), which is used during the wing folding process. Therefore it remains unclear whether the structure in Metallyticus is actually a remnant of a reduced A2 or rather a folding line - or maybe both.

Although the structure is apparently much more distinct in Chaeteessa [see Smart 1956: figs. 1, 2B (redrawn herein as Fig. 345); Klass \& Ehrmann 2003: fig. 13.3A; Béthoux 2008: fig. 6.3], it there, too, possibly represents a folding line rather than a true vein, because it lacks any trachea (Béthoux, pers. comm. 2009). The state of A2 has therefore been encoded as "unknown" for both Chaeteessa and Metallyticus.

Metathoracic hearing organ ("cyclopean ear"). The greater part of the extant Mantodea have an auditory device, the metathoracic hearing organ or "cyclopean ear", which is externally discernible as a median furrow between the corresponding coxae (Yager \& Hoy 1986, 1987, 
1989; Yager 1990, 1999a, b; Triblehorn \& Yager 2001; Yager \& Svenson 2008). Its morphology has been described in detail by Yager \& Hoy (1987), and its physiology was carefully studied by Yager \& Hoy in 1989. The postembryonic development of the hearing organ from both physiological and morphological angles were described by Yager (1996b), and the homologous structures in cockroaches were identified and discussed by Yager (2005). In 1990, Yager found evidence for distinct sexual dimorphism of the auditory system, being closely correlated with wing length dimorphism and capability of flight. Overviews on the topic were published by Yager (1999a, b).

The ear contains two tympana and is sensitive to ultrasonic sound of frequencies mostly between 25 and 50 KHz (e.g. Yager 1999a: 386, 1999b: 96; Triblehorn \& Yager 2001). However, the ear does not enable the mantids of directional hearing (Yager \& Hoy 1986, 1989; Yager 1999b: 99). Instead, it has been conjectured that it might serve as a warning-and-escape system to avoid being captured by echolocating bats (Yager \& Hoy 1986, 1989; Yager et al. 1990; Yager 1996a, 1999b; Miller \& Surlykke 2001; Triblehorn \& Yager 2001, 2002, 2005). This found support in studies on several Mantodea species that exhibit a distinct short-latency evasion behaviour to ultrasound pulses during flight - they perform a "power dive" (Yager \& May 1990; Yager et al. 1990; Cumming 1996; Yager 1999b). Interestingly, the latest analysis of mantodean hearing, based on the morphology and molecular phylogeny of a large number of species (Yager \& Svenson 2008: 555), indicated the origin of the DK-shaped mantodean ear to be a singular event dating back to the Cretaceous (approximately $120 \mathrm{My}$ ago, also found by Svenson \& Whiting 2009: fig. 8). This contradicts the assumption of the origin of hearing in Mantodea primarily as an answer to echolocating bats, because the latter probably evolved more than 60 My later (Novacek 1985; Teeling et al. 2005; Jones \& Teeling 2006; Benton 2007; Yager \& Svenson 2008; earlier assumed by Yager \& May 1990: 57). Simmons et al. (2008) described a fossil bat that shows several plesiomorphic traits of the group and was well able to fly, but probably lacked the ability to echolocate, based on morphological data of the ear region. This implies that flight evolved prior to echolocation in bats. The early origin of the hearing organ in Mantodea therefore may have been an answer to other predators than bats using ultrasound, e.g. frogs, insectivores or birds, or possibly to ultrasound produced indirectly by predator movements (Yager \& Svenson 2008: 561). Another explanation would be a different primary function of the metathoracic structures (e.g. prey location or intraspecific communication), that were lost when the ear took over its function as a bat-detecting device (Yager \& Svenson 2008: 561). These hypotheses gain support by the fact that mantodean nymphs already acquire the hearing ability during the second half of their nymphal development, not only as adults (Yager 1996b, 1999b).

In 1996, Yager described a second auditory organ on the ventral mesothorax, serially homologous to the metathoracic one, for three Hymenopodidae (Creobroter, Hestiasula, and Pseudocreobotra). The presence of the mesothoracic ear results in $\mathrm{W}$-shaped audiograms due to the sensitivity to two non-overlapping frequency-ranges (Yager 1996a: 471). 
130. Metathoracic hearing organ of male, external shape: DK [1]; DNK [2]; DO [3]; MSMT (non-auditory) [4].

The definition of the different ear-morphologies follows Yager \& Svenson (2008: tab. 2 and fig. 2). DK stands for "deep groove with prominent knobs" (Yager \& Svenson 2008: 546 and fig. 2a), DNK stands for "deep groove, no knobs" (Yager \& Svenson 2008: 547 and fig. 2b), DO stands for "deep groove, opened" (Yager \& Svenson 2008: 548 and fig. 2c), and MSMT stands for "mesothoracic and metathoracic segments similar" (Yager \& Svenson 2008: 548 and fig. 2d, e). The latter condition is non-auditory (Yager \& Svenson 2008: 557).

Yager \& Svenson (2008: tab. 3) mentioned the distribution of ear-types among the Mantodea studied as follows: $78 \%$ of the males and $45.6 \%$ of the females in their study had a DK ear; $1.3 \%$ of the males and $26.6 \%$ of the females had a DNK ear; $1 \%$ of the males and $7.2 \%$ of the females had a DO ear, $19.7 \%$ of the males and $20.1 \%$ of the females exhibited the MSMT-type. For both sexes taken together, $63.2 \%$ of the specimens studied showed a DK ear, $12.9 \%$ had a DNK ear, $3.8 \%$ had a DO ear and $20.1 \%$ corresponded to the MSMT-type.

The taxa in the present study have been encoded in correspondence with the data of Yager \& Svenson (2008: fig. 6, appendix 1 and observations mentioned throughout the text).

Eremiaphilidae have a unique metathoracic morphology that cannot be assigned to neither MSMT nor DO morphology (Yager \& Svenson 2008: 553, 558). Due to the derived position of Eremiaphila in the molecular analysis, being nested among taxa with functional ear morphologies (also recovered by Svenson \& Whiting 2009: fig. 3c), the authors assumed that the morphology exhibited by Eremiaphilidae may represent a secondary reduction (Yager \& Svenson 2008: 558). Heteronutarsus exhibits the same condition as Eremiaphila. Eremiaphilidae has correspondingly been encoded as "unknown" in this study. Why Eremiaphilidae show a different ear morphology and if it possibly has secondarily acquired another function will have to be studied in the future.

Taxa of the present dataset that were not sampled in Yager \& Svenson (2008) have been assigned to their character state and correspondingly encoded by using the morphological descriptions provided by Yager \& Svenson (2008: 552 and fig. 2).

Interestingly, several different combinations are present in Ameles. While Ameles decolor has DK males and DO females (in congruence with Yager \& Svenson 2008: appendix 1), A. gracilis exhibits the DO-type in the females and a strong DNK-type in the males. A. gracilis is endemic to the Canary Islands (recorded for La Palma, Tenerife and Gran Canaria; see Kaltenbach 1979: 526; Roy 1987c: 118; García Becerra et al. 2001: 126; Ehrmann 2002: 59; Battiston et al. 
2010: 74). Insects on islands often lose their ability to fly and/or reduce their wings, probably due to strong winds that would easily blow flying insects out to sea, leading to strong selective forces on the reduction of wing length (Darwin 1859: 135, 136; but see Roff 1990). While the females of Ameles are generally micropterous, the males are usually good flyers with long wings. The males of A. gracilis, however, have wings that leave the posterior quarter or third of the abdomen uncovered (e.g. Kaltenbach 1979: 524 and fig. 12; García Becerra et al. 2001: 124 ff. and corresponding photographs; Fig. 14). It is unknown to me whether or not the males can fly. Regardless of the flight ability, the metathoracic ear in the male is probably in the process of secondary reduction. This supports the statement by Yager \& Svenson (2008: 558) that "the high DNK/ DO incidence undoubtedly reflects patterns of mantodean evolution rather than auditory evolution per se". There are obviously intrageneric differences in ear morphology. The study of island species, often undergoing comparatively rapid morphological changes, may provide further insight into the evolutionary processes and selective pressures underlying the reduction of the DK ear.

131. Metathoracic hearing organ of female, external shape: DK [1]; DNK [2]; DO [3]; MSMT [4].

See character 130. The external morphology of the female ear-region varies more strongly than in males. Females with reduced or missing wings often show a reduction of the metathoracic ear. Males of DNK and DO females in most cases exhibit the DK-type (Yager \& Svenson 2008).

For the reasons elaborated in the discussion of character 130, Eremiaphilidae were encoded as "unknown".

\subsubsection{Abdomen and appendages}

\section{External genitalia.}

132. Abdomen, supra-anal plate (tergite 10), length: wider than long [1]; nearly as long as wide [2]; longer than wide [3].

The length of the supra-anal plate in Mantodea varies considerably. About 75 per cent of the species studied have a short supra-anal plate (state 1; Figs. 348357). A supra-anal plate that is nearly as long as wide (state 2) has been found in Ameles, Haaniinae, Heterochaeta, Iris, Oligonicinae sp., Paraoxypilinae (except Gyromantis), Photina, Stenomantis, and Taumantis. The plate is longer than wide (state 3) in Epaphrodita, Gyromantis, Hoplocorypha, Oligonicinae (except 
for Oligonicinae sp. and Thrinaconyx, which could not be studied due to the position of the wings on the dried specimen; see Pogonogaster in Fig. 358), Rivetina, and Tropidomantinae. The longest supra-anal plate is probably exhibited by Solygia and Ischnomantis (Kaltenbach 1998: figs. 102, 104; Fig. 360).

Nymphal Brancsikia also exhibit a very long supra-anal plate including a large dorsal ridge (Paulian 1957: fig. 4; Wieland, pers. obs.; Materna pers. comm. 2009; Fig. 359) which strongly adds to the appearance of a withered leaf. Interestingly, adults also have an elongated supra-anal plate, but in relation to body size it is significantly shorter than in the nymphs. The male holotype of Brancsikia simplex Beier, 1935 also has an elongated supra-anal plate (Schütte, pers. comm. 2009).

Periplaneta exhibited state 2 . However, the supra-anal plate exhibits state 1 in several other cockroaches (e.g. Blatta orientalis, Ectobius sylvestris).

133. Abdomen, supra-anal plate (tergite 10), ridge: present [1]; missing [2].

The supra-anal plate may carry a more or less prominent median ridge. It is often small and inconspicuous but may be elevated and distinct (e.g. Brancsikia, see Fig. 359). The ridge may provide stability and rigidity to the supra-anal plate, but it is unknown whether or not it has another function.

134. Abdomen of male, supra-anal plate (tergite 10), shape: almost rectangular [1]; nearly triangular [2]; rounded or almost semicircular, possibly slightly pointed [3]; notched, therefore with two rounded or pointed lobes [4].

The shape of the supra-anal plate can show intersexual differences (e.g. Bolivaria sp., Choeradodis stalii, Heteronutarsus, Hoplocorypha, Iris, and Stenomantis; see also character 136).

More than 50 per cent of the males studied have a rounded or almost semicircular tergite 10 (state 3 ). One sixth of the species have a nearly triangular supraanal plate (state 2 ). A nearly rectangular tergite 10 (state 1 ) has been only found in male Metallyticus splendidus (albeit also present in the females of several other species, see character 136).

State 4 (a supra-anal plate with two rounded or pointed lobes) is present in Acanthops, Callibia, and Periplaneta.

135. Abdomen, supra-anal plate (tergite 10), distal cut or notch: present [1]; missing [2].

The supra-anal plate (tergite 10) may be more or less deeply notched. This is present in Acanthops and Callibia in this study (Fig. 355). This character has 


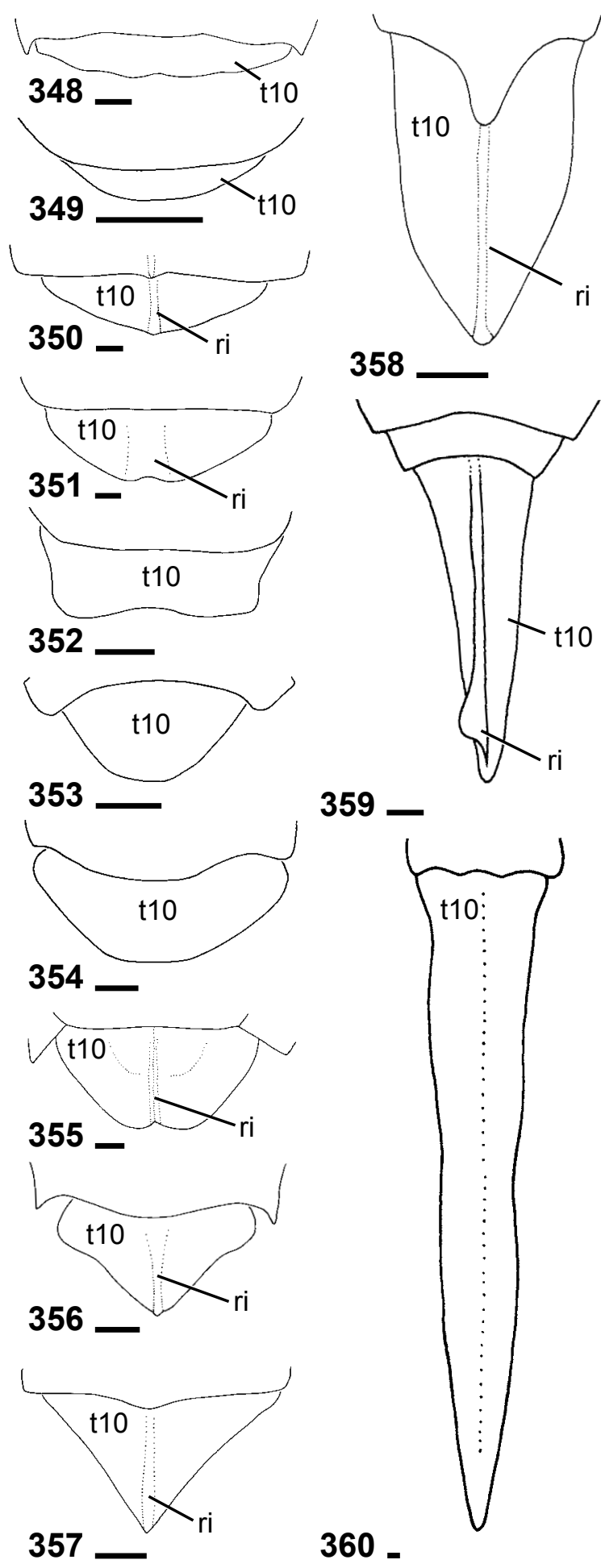

Figs. 348-360: Supraanal plates. 348: Oxyopsis gracilis, ô. 349: Mantoida maya, ô. 350: Gongylus gongylodes,, . 351: Hymenopus coronatus, ․ 352: Metallyticus splendidus, ‥ 353: Pseudoyersinia betancuriae,, . 354: Sibylla pretiosa,, . 355: Acanthops tuberculata, 9.356 Hypsicorypha gracilis, . . 357: Orthodera novaezealandiae, $q$. 358 Pogonogaster tristani, . . 359: Brancsikia sp., male nymph (ZMG). 360: Ischnomantis fatiloque, o (redrawn from Kaltenbach 1998: fig. 104). Scale bar $=0.5 \mathrm{~mm}$.

Abbreviations: $\mathbf{r i}=$ ridge; $\mathbf{t 1 0}=$ tergite $10(=$ supraanal plate). been confirmed for Acanthops (Beier 1934a: 2; Roy 2002b: 300; Lombardo \& Ippolito 2004: 1078) and has additionally been described for Miracanthops Roy, 2004 (e.g. Roy 2004c: figs. 22, 24).

A notched supra-anal plate is also exhibited by Periplaneta (drawing in Beier 1974a: fig. 26). Beier stated that the supra-anal plate may end in two pointed or rounded lobes, i.e. it may be notched in Blattaria (Beier 1974a: 29). A notched supra-anal plate is also present, for instance, in Simandoa conserfariam (Roth \& Naskrecki 2003: fig. 2D).

136. Abdomen of female, supra-anal plate (tergite 10), shape: almost rectangular [1]; nearly triangular [2]; rounded or almost semicircular, possibly slightly pointed [3]; notched, therefore with two rounded or pointed lobes [4].

See also character 134. In the females (as in the males) a rounded or nearly semicircular supra-anal plate (state 3 ) is exhibited by most species studied here (nearly two thirds of the species; e.g. Figs. 348-351, 353, 354), whereas only less than 10 per cent of the females exhibit state 2 (Figs. 356, 357). A nearly rectangular supra-anal plate (state 1) is present in female Metallyticus (see Wieland 2008a: fig. 17; Fig. 352), Heteronutarsus and Hoplocorypha. State 4 has been found in Acanthops (Fig. 355) and Periplaneta (not accessible in the female Callibia). 
137. Abdomen of male, subgenital plate (sternite 9): symmetrical [1]; asymmetrical [2].

Roy (1999: 33) stated that the male subgenital plate "is usually symmetrical, but may be asymmetrical as in Polyspilota and Chlidonoptera". In contrast, the male subgenital plates of many mantodean taxa are more or less strongly asymmetrical. Wieland (2006: 74) stated that this is the case in Metallyticus splendidus (e.g. Wieland 208: fig. 16; Fig. 361), Tarachodula pantherina, and Pseudocreobotra wahlbergii. In fact, this situation has been found in most of the taxa studied herein (e.g. Fig. 361, 362). The male of Chaeteessa valida was not available for this study, so this character has been encoded as "unknown" herein. However, Klass (1997: 266 and fig. 30) showed, that the subgenital plate is symmetrical (or nearly so) in male Chaeteessa caudata Saussure, 1871.

A nearly symmetrical subgenital plate is present in the males of Amorphoscelis sp., but it may be asymmetrical in other species of this group (see also Roy 2009b: fig. 1 for Amorphoscelis asymmetrica Ingrisch, 1999), Haaniinae (see also Anisyutkin 2005: fig. 8; Anisyutkin \& Gorochov 2005: fig. 14), Majanga, Paramorphoscelis (asymmetrical in Perlamantis: Fig. 362), and Sibylla.

The subgenital plate is more or less strongly asymmetrical in many male Blattaria (Klass 1997: 266). A symmetrical subgenital plate has been assumed to represent the primary condition for cockroaches (Bohn 2003: 201).

The male genitalia are strongly asymmetrical in the groundplan of Dictyoptera (Klass 1997: 327; homology relations ascertained by Klass 1995, 1997). It is therefore possible that an asymmetrical subgenital plate may be a dictyopteran ground plan feature and therefore might be plesiomorphic with respect to Mantodea. However, this has to be studied in a wide range of Dictyoptera before more precise answers can be given.

138. Abdomen of male, subgenital plate (sternite 9), dorsal process: present [1]; missing [2].

Roy (2004a: 1, 2, and figs. 2, 5) stated that male Empusinae carry a process on the dorsal edge of the right side of the subgenital plate (sternite 9; see Figs. 363, 364). This character could not be confirmed for any
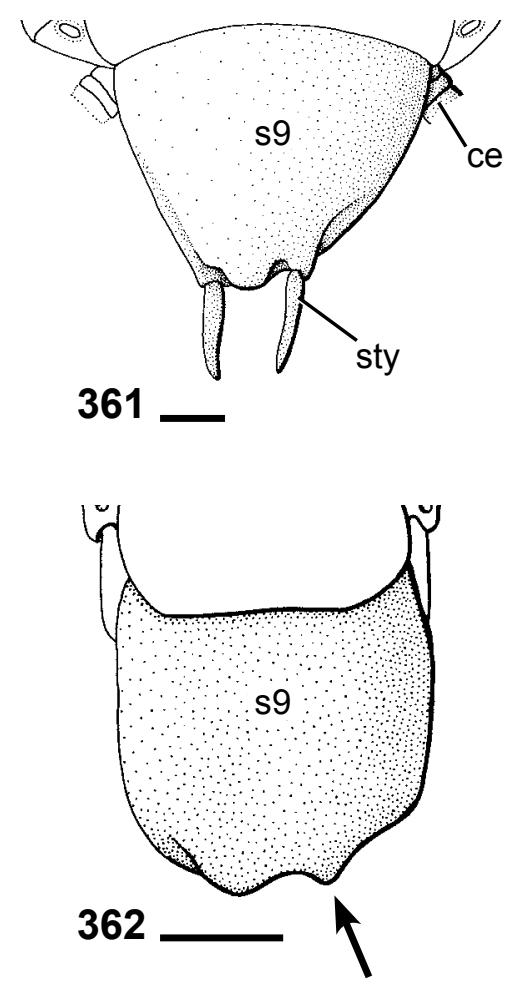

Figs. 361-362: Asymmetrical male subgenital plates (sternite 9). 361: Metallyticus splendidus. 362: Perlamantis allibertii. Arrow indicates missing styli in Perlamantis (see text for a discussion). - Scale bar = $0.5 \mathrm{~mm}$.

Abbreviations: $\mathbf{c e}=$ cercus; $\mathbf{s} 9=$ abdominal sternite 9 (subgenital plate of $\precsim)$; sty $=$ stylus . 
other taxon than for Empusinae, therefore it is most likely autapomorphic for the group. See also discussion of Empusidae in chapter 2.1.4.

139. Abdomen of female, subgenital plate (sternite 7), covering by sternite 6: present [1]; missing [2].

This character is probably autapomorphic for Eremiaphilidae. In this group, the females carry sturdy and slender (Eremiaphila, Fig. 365) or widened and shovellike digging spines (Heteronutarsus, Fig. 367) on the sixth sternite (see character 141). Sternite 6 is distally elongated and partly covers the subgenital plate (Saussure 1871: 369; Figs. 366, 368). It is possible that the elongated supra-anal plate (tergite 10) and the elongated sternite 6 can be pressed together during the process of digging, thus probably covering and protecting the subgenital plate and the delicate tips of the valvulae against rough sand and pebbles. However, anatomical studies of the musculature of the abdomen as well as intensive observations of living specimens in captivity are indispensable for providing answers to this question.

This character is more distinct in Heteronutarsus than in Eremiaphila (see Lefèbvre 1835: 502 and pl. 11, figs. 14-16, pl. 13, figs. 4, 5; Figs. 366, 368). See also chapter 4.6.9 for a detailed discussion.

140. Abdomen of female, proximal part of subgenital plate (sternite 7), strong ventral spines: present [1]; missing [2].

Sturdy spines on the proximal half of the subgenital plate are only present in Rivetina in the present dataset (Kaltenbach 1963: fig. 31h, 1982: fig. 65; Figs. 369, 370). They are probably autapomorphic for the genus or potentially for Rivetina + Rivetinula La Greca, 1977 (not present in this study; see Wieland $2008 \mathrm{~b}$ and see chapter 4.6 .9 for a detailed discussion).

141. Abdomen of female, sternite 6, strong ventral spines: present [1]; missing [2].

Spines on the posterior margin of sternite 6 are only present in female Eremiaphilidae and are autapomorphic for the group (Wieland 2008b; Figs. 365368). These structures have been described and drawn before [Lefèbvre 1835: 469, 479 (incorrectly assigned to the subgenital plate) and pl. 11, figs. 1, 14, 15, pl. 13B, figs. 3, 4; Saussure 1871: 365 ff. and pl. 7, fig. 55a; Giglio-Tos 1921: 6, 1927: 45 (both incorrectly assigned to the subgenital plate); Chopard 1941: fig. 7; Roy 1999: 32; Ehrmann 2002: 138 (incorrectly assigned to subgenital plate); Klass \& Ehrmann 2003: 188; Dongalar 2007: 1 and fig. 14 (incorrectly assigned 

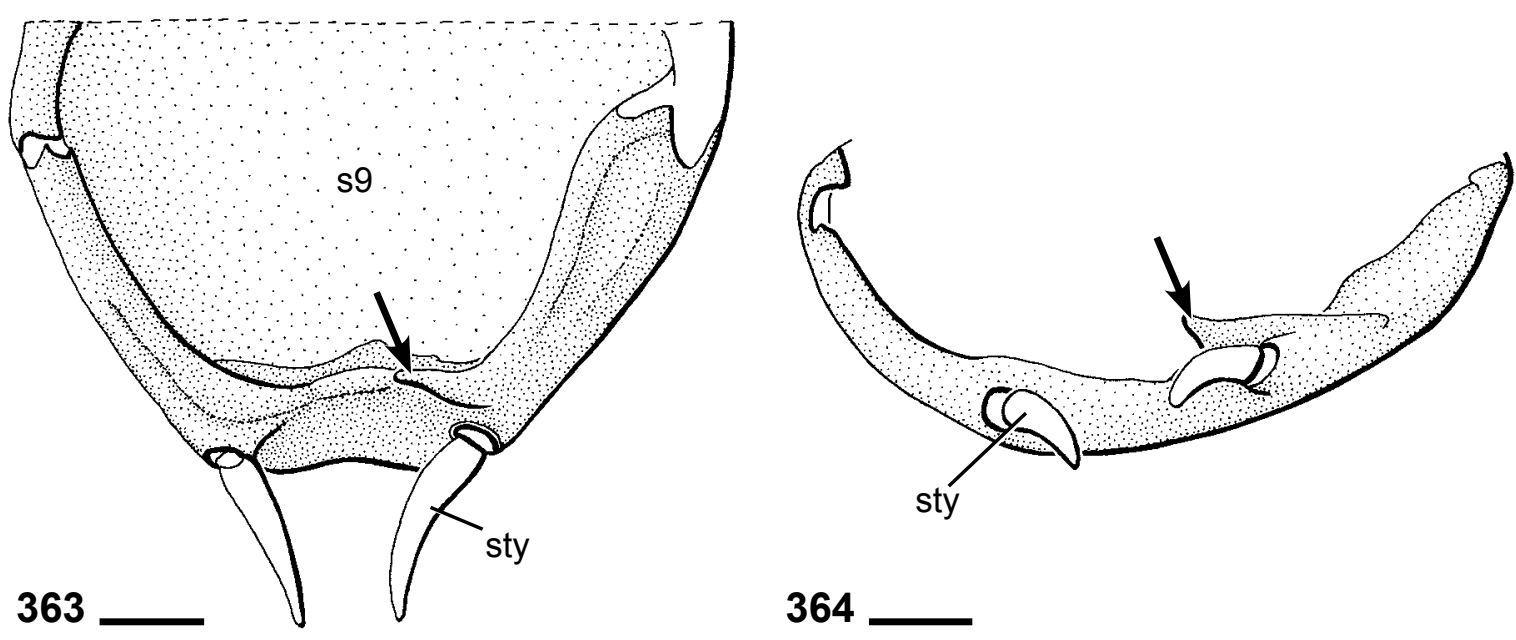

364

Fig. 362-363: Idolomorpha dentifrons, $\widehat{\jmath}$, subgenital plate. 362: Dorsal view. 363: Posterior view. Arrows indicate dorsal process. - Scale bar $=0.5 \mathrm{~mm}$.

Abbreviations: $\mathbf{s} 9=$ sternite 9 (subgenital plate of $\left.{ }^{\Uparrow}\right) ; \mathbf{s t y}=$ stylus.

to subgenital plate); Battiston et al. 2010: 169 (incorrectly assigned to subgenital plate)] but their function has not been discussed except by Wieland (2008b). This type of digging device was named Eremiaphilidae-type by Wieland (2008b: 344).

Eremiaphilidae live in sandy and rocky deserts of the Palaearctic. They deposit their oothecae in the soil (e.g. Andres 1914: 73; Chopard 1938: 205; Klass \& Ehrmann 2003: 191), probably in order to keep the eggs safe from heat and drought. The spines on sternite 6 support the female Eremiaphilidae when they dig a furrow into the ground into which they lay the ootheca. The latter is subsequently covered by sand with the help of the posterior legs. During the process of digging, the female stretches its abdomen and uses its tip (i.e. the elongated tergite 10 and the ventral spines) in a wiping movement (Andres 1914: 73; Chopard 1938: 205; Ehrmann 2001a: 31; Wieland, pers. obs. 2006). See also chapter 4.6.9 and overview of Eremiaphilidae in chapter 2.1.4.

142. Abdomen of male, subgenital plate (sternite 9), styli: present [1]; missing [2].

The male styli are missing in several species. This has been described in literature, for instance for Perlamantis by Kaltenbach (1963: 553 and fig. 17e) and Amorphoscelis asymmetrica (Roy 2009b: 209 and fig. 1).

Styli are missing (state 2) in Callibia, Heteronutarsus, Odontomantis, Perlamantis (Fig. 362), Raptrix, Theopropus, and Tithrone. Interestingly, male Paramorphoscelis do have styli while its sistergroup, Perlamantis, is devoid of them.

Styli are delicate and may break off easily when the specimens are handled, therefore these findings have to be confirmed by further specimens. The lack of styli has been confirmed for the males of Odontomantis in the collection of 
the SMNK (Ehrmann, pers. comm. 2008). Lombardo (1996) mentioned missing styli for the males of Tithrone roseipennis (the species present in this study), T. latipennis Lombardo, 1996, T. laeta Lombardo, 1996, and Paratithrone royi Lombardo, 1996. In their study of Raptrix, Lombardo and Marletta (2004) did not mention a lack of styli in this genus, neither was it mentioned in the original description of the genus by Terra (1995: 25; Terra did not include the shape of the subgenital plate or the presence of styli in his descriptions at all), or by Roy (2006) in his overview of Acontistinae. As most of the other Acontistinae studied here (except for Acontista) are devoid of styli it is possible that they are regularly lacking in Raptrix, too. Until the situation can be addressed by the study of further males, the character will provisionally be encoded as state 2 for Raptrix. Interestingly, male Acanthopinae seem to have styli, as in Acanthops [also confirmed for Decimiana bolivari (Chopard, 1916): Lombardo 2000b: 161; Ippolito \& Lombardo 2004: 374; Schütte, pers. comm. 2010].

Styli are missing in the male Heteronutarsus specimen studied, whereas they are present in Eremiaphila. However, Innes-Bey (1912: 43) mentioned that the subgenital plate in male Heteronutarsus is "armée de styles". Heteronutarsus is correspondingly encoded as "unknown" herein. A thorough investigation of further male specimens is important to settle this question.

143. Abdomen of female, ovipositor, length of valvulae with respect to opening of genital vestibulum: very short, but tips of valvulae protruding from inside the vestibulum [1]; valvulae entirely hidden within the vestibulum, tips of valvulae externally not recognizable [2].

All extant Dictyoptera have strongly reduced ovipositors (Hennig 1969: 180, 1981: 192; Kristensen 1991: 133; Grimaldi \& Engel 2005: 227). While it is entirely concealed within the subgenital plate in Blattodea, the ovipositor is still comparatively well-built in Mantodea, where it plesiomorphically protrudes from the genital chamber (Klass \& Ehrmann 2003: 188 and fig. 13.6A; e.g. Figs. 365-372, 376, 377).

144. Abdomen of female, valvulae I (gonapophyses VIII), strongly sclerotized and hook-shaped distal ends: present [1]; missing [2].

The presence of sclerotized and hook-shaped distal ends of the gonapophyses of the $8^{\text {th }}$ segment (= ventral valvulae, valvulae I) is synapomorphic for Entella, Parentella (Fig. 374), Ligaria (Figs. 371, 372, 375) and Ligariella (Fig. 373). The hooks are split in two parts. The shorter and slenderer dorsal part is straight, whereas the sturdier and longer ventral part is curved dorsad (Fig. 375). The close relationship of the species exhibiting the hooks found support in the 


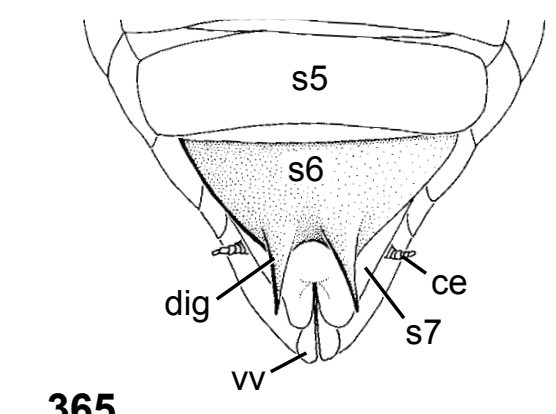

365

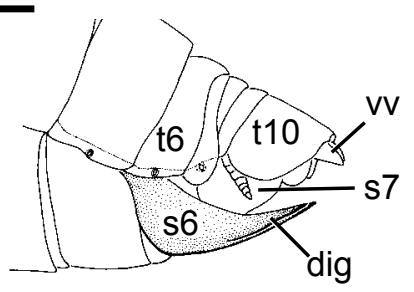

366
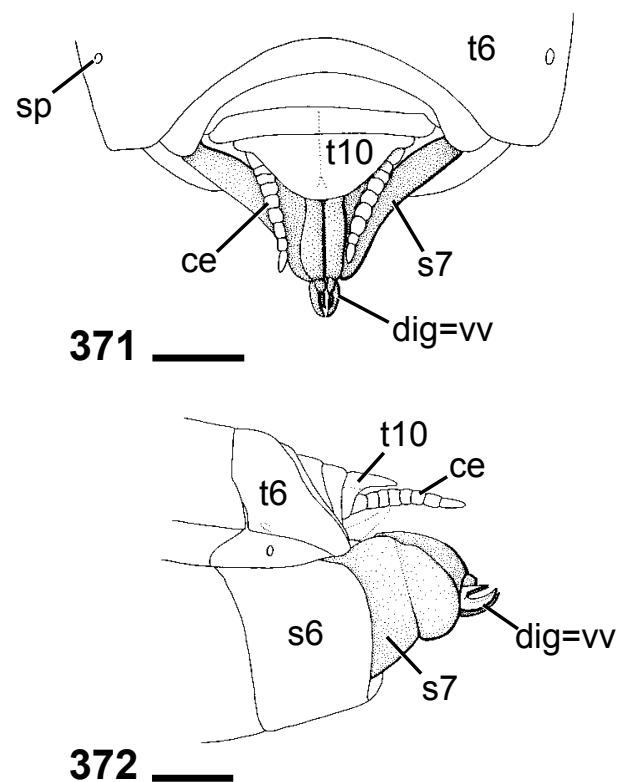

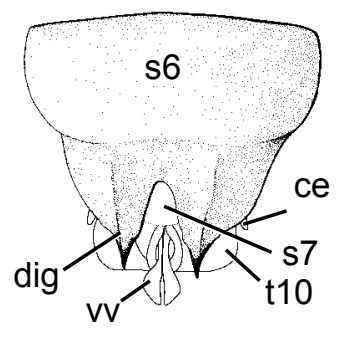

367

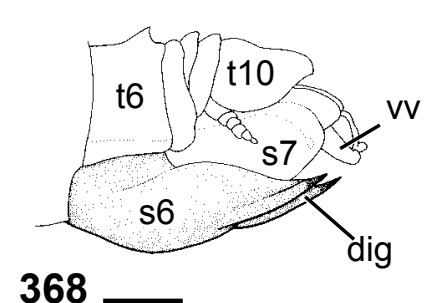

368

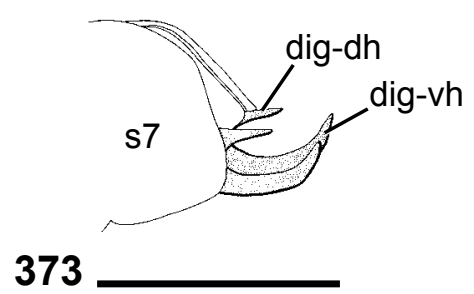

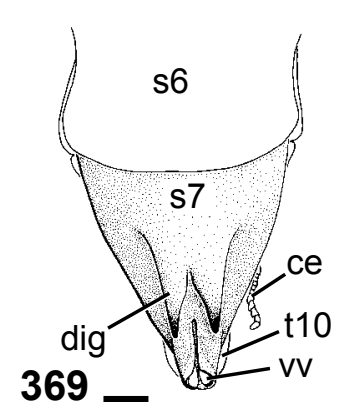

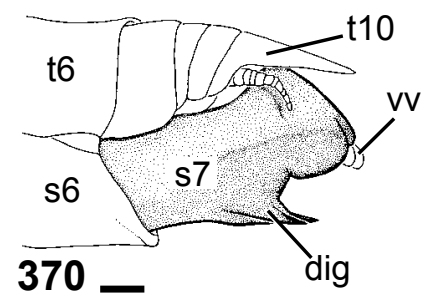

374

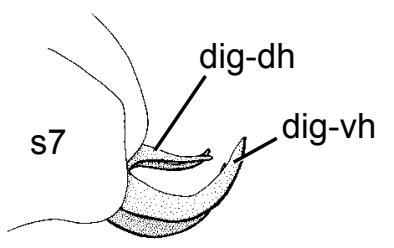

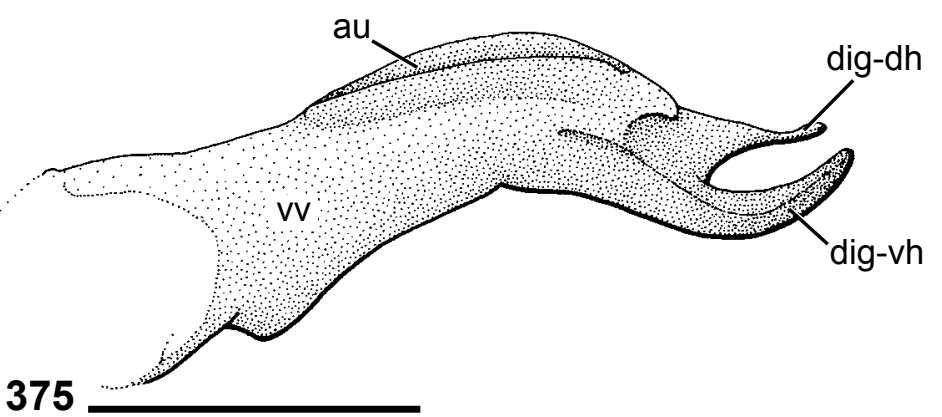

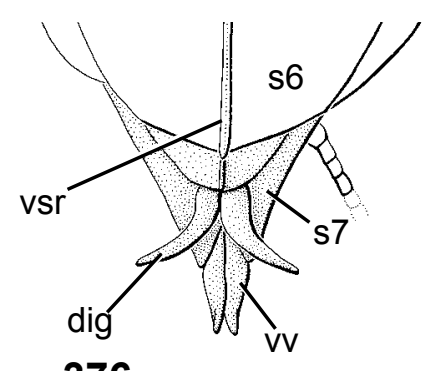

376

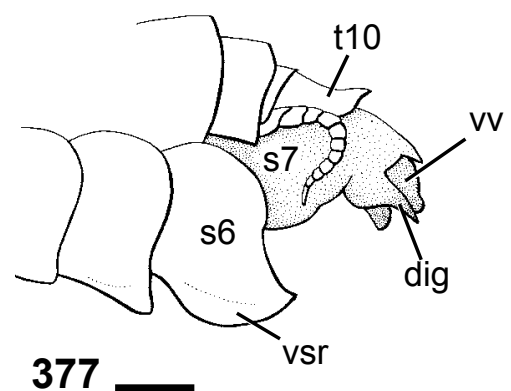

Figs. 365-377: Female digging structures. 365-368: Eremiaphilidae-type. 365-366: Eremiaphila sp. 1, abdominal tip. 365: Ventral view. 366: Lateral view (left). 367-368: Heteronutarsus aegyptiacus, abdominal tip. 367: Ventral view; note shovel-like spines. 368: Lateral view (left). 369-370: Rivetina-type; Rivetina sp., abdominal tip. 369: Ventral view. 370: Lateral view (left). 371-375: Ligaria-type; Ligaria sp., abdominal tip. 371: Dorsal view. 372: Lateral view (left). 373-376: Details of Ligaria-type. 373: Ligariella trigonalis, digging spines, lateral view (left). 374: Parentella major, digging spines, lateral view (left). 375: Ligaria sp., left ventral valvula (gonapophysis VIII), lateral view (left). 376-377: Chroicoptera-type. 376: Chroicoptera longa, $ᄋ$ holotype (MNHUB), abdominal tip, ventral view. 377: Chroicoptera saussurei, lateral view (redrawn and mirrored from Kaltenbach 1996: fig. 67); note the ventral ridges on sternites 5 and 6 . - Scale bar $=1.0 \mathrm{~mm}$.

Abbreviations: au = aulax; $\mathbf{c e}=$ cercus; $\mathbf{d i g}=$ digging device; dig-dh $=$ digging device, dorsal hook; dig-vh $=$ digging device, ventral hook; s5-s7 = sternite 5-7; t6 $/ \mathbf{t 1 0}=$ tergite $6 / 10 ; \mathbf{v s r}=$ ventral sternal ridge; $\mathbf{v v}=$ ventral valvula (= gonapophysis VIII). 
molecular data (Svenson \& Whiting 2009: fig. 3b, node 53).

This structure probably plays an important role during the deposition of oothecae below the ground in arid and hot environments, such as savannahs, semi-deserts and deserts (see Wieland 2008b and chapter 4.6.9). This type of digging device was named the Ligaria-type by Wieland (2008b: 344).

\begin{abstract}
Abdominal segments. The abdomen is often cylindrical in shape. However, it is widened in many species. The abdominal segments are often additionally widened by lobe-like expansions of the sternites and/or the tergites. Furthermore, the tergites may be posteriorly elevated or carry large dorsal lobes, and the sternites may carry medio-ventral lobes. Such lobes on the abdomen play an important role in the cryptic appearance and special resemblance to plant parts in many species. The lobes are usually also present in males but they are often more elaborate in females. I herein concentrate on the females.
\end{abstract}

145. Abdomen of female, tergites, lateral lobe-like expansions: present [1]; missing [2].

Lateral lobes on the tergites are present (state 1) in female Acanthops, Blepharopsis, Callibia, Ceratomantis, Deroplatys, Empusa, Gongylus (Figs. 379, 381), Gyromantis, Haaniinae (Fig. 378), Idolomantis, Majanga, Paraoxypilinae (except for Paraoxypilinae sp.), Phyllocrania, Pogonogaster, Pseudocreobotra (Fig. 380), Sibylla, Theopompa tosta, and Zoolea.

They have been mentioned in the literature for several taxa, among them Pseudocreobotra (Kaltenbach 1998: fig. 115), Haania (Beier 1952: 200), and Astape (Beier 1952: 209; Lieftinck 1953: fig. 3). Such lobe-like expansions are assumed to add to the cryptic effect in special resemblance to plants by breaking up the body outline (e.g. Edmunds \& Brunner 1999: 287). This effect becomes especially obvious, for instance, in Haania in which the lateral lobes are more or less frayed and thus add to the resemblance of mosses and lichen.

See also characters 146-148.

146. Abdomen of female, sternites, ventral lobe-like expansions: present [1]; missing [2].

The sternites of the female abdomen may carry ventral expansions (state 1). They are present in Blepharopsis, Empusa, Gongylus (Fig. 381), Idolomantis, Pogonogaster, Pseudocreobotra (Figs. 380, 382), Sibylla, Theopropus, and Zoolea.

Like other lobe-like expansions of the body, these lobes play an important role in the special resemblance to plant structures (see characters 145, 147 and 148). 

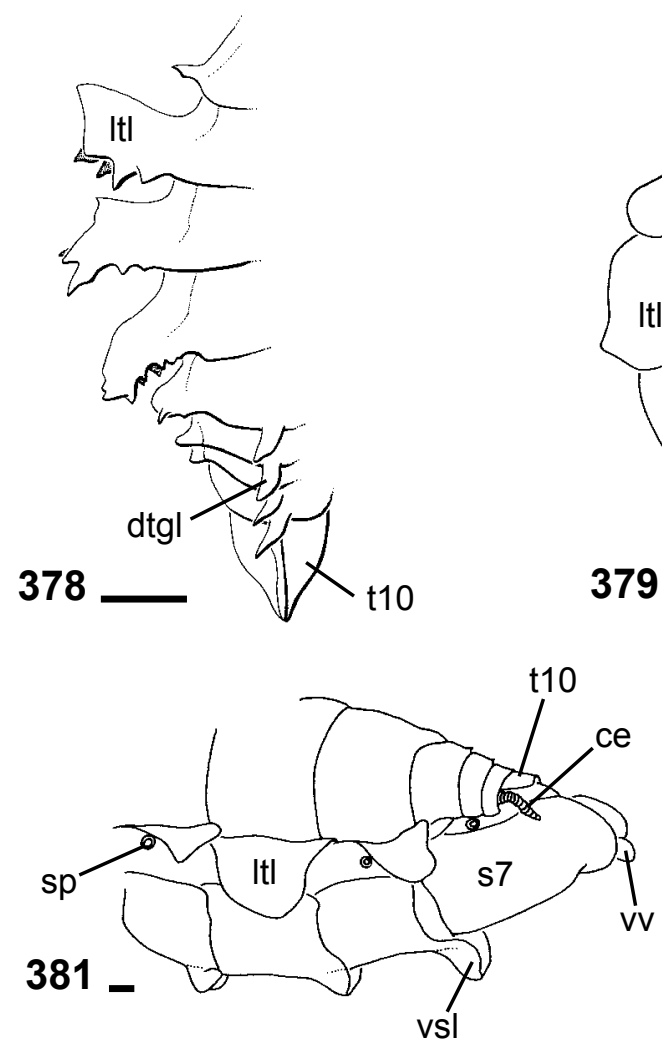
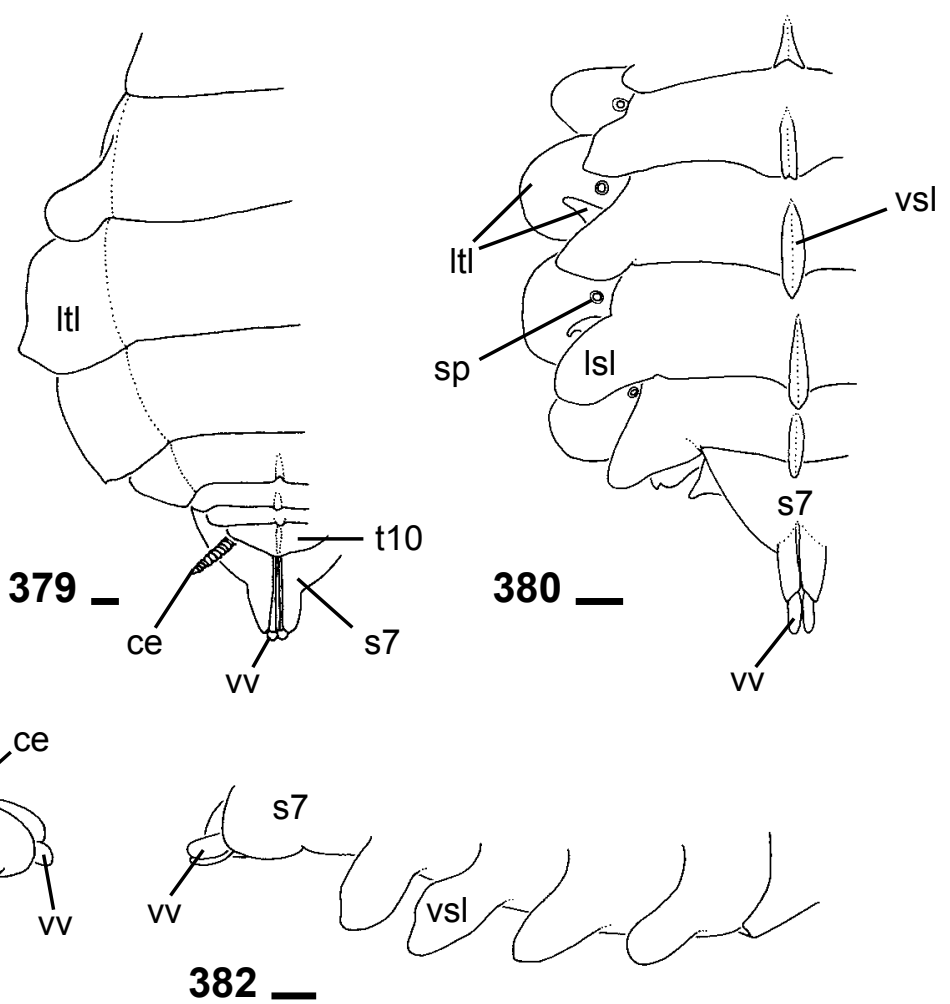

382

Figs. 378-382: Abdominal lobes exhibited by females. 378: Haania lobiceps, dorsal view. 379: Gongylus gongylodes, dorsal view. 380: Pseudocreobotra wahlbergii, ventral view. 381: Gongylus gongylodes, lateral view (left). 382: Pseudocreobotra wahlbergii, lateral view (right). - Scale bar $=1.0 \mathrm{~mm}$.

Abbreviations: $\mathbf{c e}=$ cercus; $\mathbf{d t g l}=$ dorsal tergal lobe; $\mathbf{l s l}=$ lateral sternal lobe; $\mathbf{l t l}=$ lateral tergal lobe; $\mathbf{s} 7=$ sternite 7 (= subgenital plate of + $) ; \mathbf{s p}=$ spiracle; $\mathbf{t 1 0}=$ tergite 10 (= supra-anal plate $) ; \mathbf{v s l}=$ ventral sternal lobe; $\mathbf{v v}$ = ventral valvula (= gonapophysis VIII).

147. Abdomen of female, sternites, lateral lobe-like expansions: present [1]; missing [2].

The females of some species exhibit lateral expansions of the sternites instead of or in addition to the expansions of the tergites (state 1). This is the case in Astape, Callibia, Ceratomantis, Empusa, Humbertiella, Majanga, Pseudocreobotra (Fig. 380), Sibylla, Theopompa tosta, and Zoolea.

Such lobes are assumed to add to the special resemblance to plant matter (see characters 145, 146 and 148).

148. Abdomen of female, tergites, dorsal lobe-like expansions or distinctly pulled-out posterior rims: present [1]; missing [2].

The females of many species of Mantodea have their wings more or less strongly reduced (see characters 105, 106). If this is the case, some or all abdominal tergites are free and unprotected. In several species they are therefore involved in the special resemblance to plant matter (see characters 145-147). Often only the posterior edge of the tergites is medially elevated [e.g. in Geothespis australis 
Giglio-Tos, 1916 (Kaltenbach 1998: fig. 100) and Carvilia saussurii Stål, 1876 (Kaltenbach 1996: fig. 174), among others]. The females (and in several cases also the males) of several species, however, exhibit large, lobe-like, frayed expansions on the tergites, e.g. Palaeothespis oreophilus Tinkham, 1937 (a: pl. 16, fig. 4), many Toxoderidae (e.g. Beier 1976: figs. 1b, 2b, 3b, 4b, 5b, 6b; Roy 2009a: figs. 3D, 13, 16C, 43D, 47D-G, 57C, 61A; Fig. 22), Pseudopogonogaster Beier, 1942 (e.g. Salazar \& Carrejo 2002: figs. 4, 6), Pogonogaster (e.g. Rehn 1918: pl. 18, fig. 5), Haaniinae (e.g. Lieftinck 1953: fig. 3 for Astape; Fig. 378 herein for Haania), Calofulcinia (La Greca 1966: 4), and Calopteromantis (Lombardo \& Ayala 1999: 114, 116, 117 and pl. 1, fig. 2, pl. 2, fig. 2; Ayala \& Onore 2001: figs. 3, 4). Astape, Calopteromantis, Pogonogaster and Calofulcinia females thus achieve a close resemblance to mosses and lichen (e.g. Lieftinck 1953: 129; Ayala \& Onore 2001: 249), while the processes of Toxoderidae rather evoke the impression of the remnants of leaf matter on branches (pers. obs.; see photographs in Roy 2009a; Fig. 22).

Cerci. The cerci of Mantodea carry several different types of sensilla (e.g. Ball \& Stone 1982) and have been described to function in various ways. They can be used in tactile reception, for instance when testing the underground before laying an ootheca (Kershaw 1910). They have been shown to be important for behavioural functions, for instance the coordination of the male mating movement (Davis \& Liske 1988) and intraspecific communication (Edmunds 1976: 33). Ehrmann (1984) and Karuppanan (1987) described the supportive function of the cerci in forming the ootheca. The cerci also play a role in auditory low-frequency sound reception (e.g. Triblehorn \& Yager 1997) and have wind-receptive functions for avoiding bat attacks (Boyan \& Ball 1986; Triblehorn \& Yager 2006). Furthermore, the cerci certainly add to the cryptic effect of grass- and stick mimics, for instance in Pyrgomantis (e.g. Edmunds 1972: fig. 3B; Fig. 25) or Didymocorypha (Henry 1932: pl. 4, figs. 1, 2; Fig. 433) where they are long and lanceolate and therefore add to the overall elongation of the slender body, or in Toxoderidae and many other species in which they appear like small leaves on twigs (see figures in Roy 2009a; Figs. $22,386)$.

149. Cerci, shape in cross-section: strongly flattened as a whole or in the distal half of the cerci at least [1]; circular as a whole or only slightly flattened (only terminal cercomere flattened at most) [2].

The cerci are circular in cross-section in most Mantodea (Roy 1999: 33) but can be slightly or strongly flattened (Beier \& Jaus 1933: 133; Beier 1964a: 879, 1968a: 23; Ehrmann 2002: 20).

Cerci that are distinctly flattened at least in the distal half (state 1; see Ball \& Stone 1982: fig. 1b for Archimantis) are exhibited by Amorphoscelinae (Fig. 389), 
Archimantis, Calamothespis, Chaeteessa (Figs. 5, 6), Compsothespis, Heterochaeta (Fig. 387), Orthoderella, Oxyothespis, Paramorphoscelis (but not Perlamantis! Compare Figs. 384 and 385), most Tarachodidae, and Toxodera (Fig. 386). The remaining taxa have globular, cylindrical or slightly flattened cercomeres.

Furthermore, strongly flattened cerci have been described in the literature, for instance, for Angela (e.g. Beier 1935c: 70; Cerdá 1996b), Stenophylla (e.g. Beier 1934c: 8; Salazar 2004a: 213) and many Toxoderidae (Giglio-Tos 1914a; Beier 1934c, 1976; Ehrmann 2002; Roy 2009a). Many of the taxa exhibiting such a cercal shape show special resemblance to grass blades or dead leaves or branches. Therefore, it is likely that it plays a role in the concealment of the overall body shape or in helping to avoid lateral shadows in the case of bark dwellers (e.g. Tarachodes, Tarachodula). However, it has also been described in the literature that the cerci are used for shaping the ootheca prior to its hardening (e.g. Ehrmann 1984; Karuppanan 1987). It is unclear whether enlarged and flattened cercomeres perhaps play an additional or a different role in egg-laying. To my knowledge literature data on observations of living specimens of most of these taxa have not been published.

150. Cerci, distal cercomere, shape: smaller than remaining cercomeres or less than twice as long as the preceding cercomere, sometimes slightly elongated into a cone-shape or slightly flattened [1]; strongly flattened but not forming a strongly enlarged lobe, less than twice as long as preceding cercomere [2]; strongly flattened and forming a strongly enlarged lobe, more than twice as long as preceding cercomere [3].

See also character 149. The distal cercomere of most Mantodea is smaller than each of the remaining segments and it is often more or less conical and pointed (state 1; Beier \& Jaus 1933: fig. 10; Balderson 1991: figs. 21.3A, B; Roy 1999: 33; Ehrmann 2002: figs. 31, 32; Klass \& Ehrmann 2003: fig. 13.6A; Fig. 388). However, the distal cercomere may be flattened and more or less strongly enlarged (Roy 1999: 33), sometimes being longer than the remaining cercomeres together.

State 2 (flattened but not strongly enlarged) is exhibited by Archimantis (e.g. Ball \& Stone 1982: fig. 1a, b; Milledge 1997: figs. 73, 74, 85, 86, 97, 98, 106, 107, 119, 120, 153, 154), Calamothespis (e.g. Kaltenbach 1998: fig. 39), Compsothespis (e.g. Kaltenbach 1998: fig. 40), Heterochaeta (e.g. Roy 1976: figs. 5, 11, 16, 21, 26, 33, 37; Fig. 387), Perlamantis (Fig. 385), and Tarachodidae except for Iris (e.g. Beier 1957b: fig. 19; Roy 1972: fig. 2, 2005b: figs. 5, 8).

The distal cercomeres are flattened and additionally strongly enlarged (state 3 ) in Acanthops (e.g. Roy 2002a: figs. 3, 7, 2002b: fig. 5; Salazar-E. 2004a: 215; 
Fig. 383), Amorphoscelinae (e.g. Roy 1962: figs. 2c, 3c, 6c, 7c, 10c, 14c, 17c, 18c, d, 21c, d, 24c, 1965a: figs. 2c, 5b, c, 1966: figs. 1b, 2b, e, 3c, 5c, d, 6b, 8b, 1984: fig. 2; Roy \& Stiewe 2009: fig. 18; Figs. 389, 391), Amphecostephanus (Rehn 1912: 120), Paramorphoscelis (Edmunds 1976: fig. 1; Fig. 384), and Toxodera (e.g. Beier 1934c: fig. 4, 1976: 393; Fig. 386).

The enormous enlargement of the distal cercomere has been described for other taxa as well, for instance, for Miracanthops (Roy 2004c: figs. 23, 25; Rivera 2005: figs. 8, 9, 19, 20), Pseudacanthops (La Greca \& Lombardo 1997: 52 and figs. 8, 9) and Stenophylla (Sharp 1895: fig. 147; Lombardo 2000a: 35 and figs. 11-13; Salazar-E. 2004a: 213; Roy 2005a: 226 and figs. 8, 12, 14). It apparently has several functions in the different taxa. While in Toxoderidae, Stenophylla, Amphecostephanus and Acanthops it probably plays an important role in the general "withered-leaf" or stick-appearance, it has a specialized behavioural function in Amorphoscelinae. Edmunds (1976: 33 and fig. 8) described the territorial behaviour ("tail-display") of Caudatoscelis lagrecai (Roy, 1964) (assigned to Amorphoscelis in Edmunds 1976; redrawn in Fig. 391). This species, along with many of the Amorphoscelinae described so far (Roy 1962, 1965a, 1966, 1984; Roy \& Stiewe 2009: fig. 18; among others), exhibits enormously enlarged distal cercomeres (Figs. 389, 391). In relation to body length the cerci of Amorphoscelinae are often very long. Especially the nymphs have been described to display their enlarged (and often dark) distal cercomeres in a waving movement of the cerci that is used in intraspecific encounters (Edmunds 1976: 33 and fig. 8). So far this behaviour has only been described for $C$. lagrecai but a similar behaviour has been observed in other African and Southeast Asian Amorphoscelinae, as well (Schwarz, pers. comm. 2007; Wieland pers. obs. 2007).

To my knowledge it is unknown if and how the enlarged distal cercomere plays a potential different role in the building of the ootheca than in those taxa lacking such an enlargement.

151. Cerci of male, cercomeres, largest number: 8 or less [1]; 9-11 [2]; 12-15 [3]; 16-19 [4]; $20-22$ [5]; 23 or more [6].

The counting of cercomeres is a difficult task because in the proximal part several small cercomeres appear to be fused, although in most cases they can be discerned by the corresponding rings of setae (there is usually one ring of larger setae arranged around a cercomere, distinctly discernible by their circular grooves; e.g. Figs. 392). Cercomeres were counted including these often very short proximal cercomeres

The number of cercomeres varies strongly among the mantodean taxa. The cerci have been mentioned to generally consist of between 10 and 20 cercomeres (e.g. 


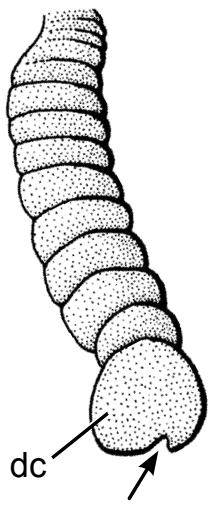

383

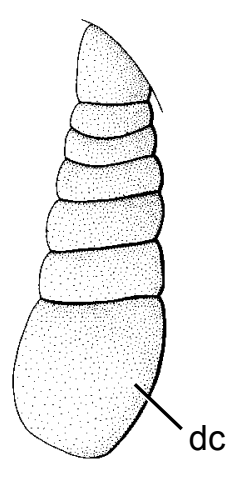

384

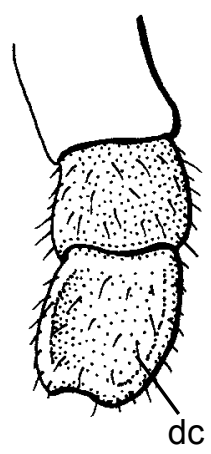

385

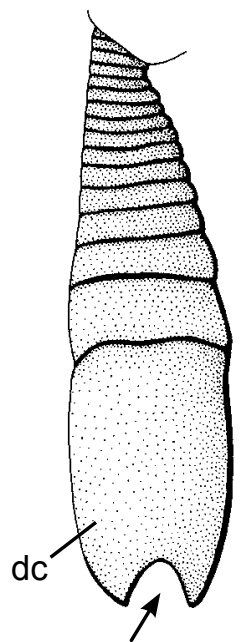

386

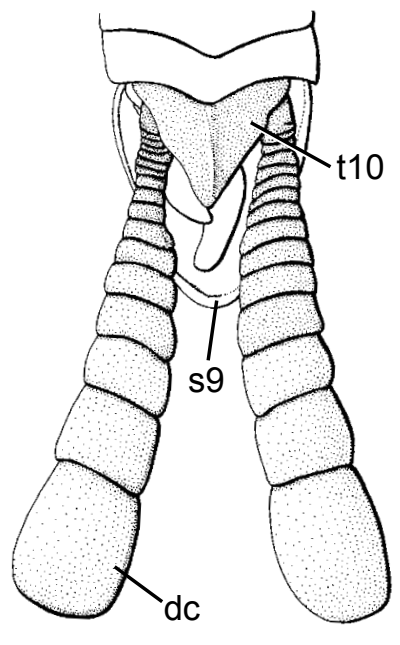

387

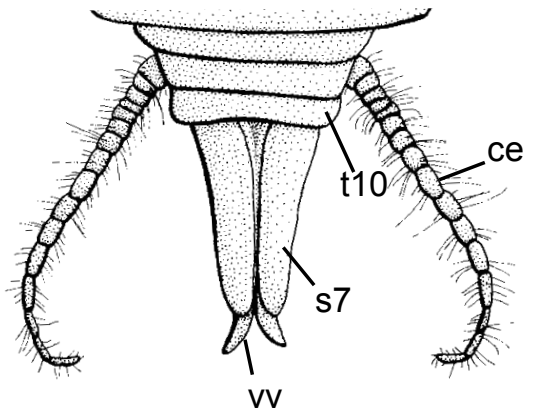

388

389

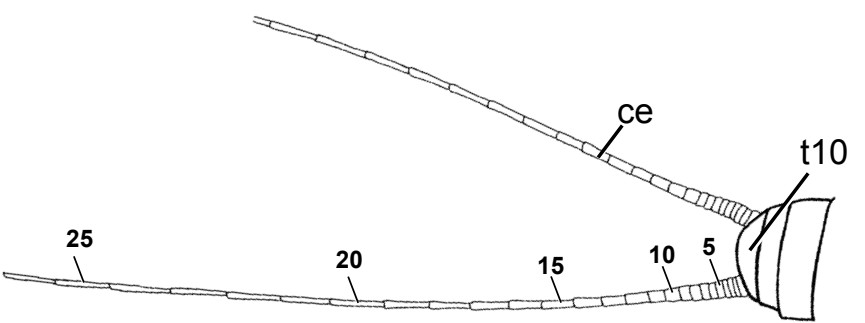

390

391

Figs. 383-391: Cerci. 383: Acanthops tuberculata,, , right cercus. 384: Paramorphoscelis gondokorensis,, , right cercus (mirrored). 385: Perlamantis allibertii, ô, right cercus (mirrored), distal cercomeres. 386: Toxodera maculata,, , left cercus (cercomeres partly covered; ventral view reveals 21 segments). 387: Heterochaeta bernardii, ô, abdominal apex in dorsal view. 388: Metallyticus splendidus,, , abdominal apex in dorsal view. 389: Amorphoscelis sp., $\widehat{o}$, adbominal apex in ventral view; left cercus omitted; note that the missing styli are probably broken off as Amorphoscelinae usually have them (see figs. in Roy 1965b for South-East Asian Amorphoscelinae). 390: Chaeteessa sp., detail of living specimen in picture by Geoffrey Gallice (Fig. 5). 391: Caudatoscelis lagrecai, nymph showing tail display by waving its abdominal tip and cerci (redrawn and slightly altered from Edmunds 1976: fig. 8; generic assignment following Roy 1973: 245). Arrows in Figs. 383 and 386 indicate notch in distal cercomere. Arrows in Fig. 391 indicate directions of the waving movement of cerci. - Scale bar $=$ 0.5 mm (Figs. 383-384, 386-389), 0.2 mm (Fig. 385), no scales available for Figs. 390 and 391.

Abbreviations: 5-25 = cercomere no. 5-25; $\mathbf{c e}=$ cercus; $\mathbf{d c}=$ distal cercomere; $\mathbf{s} 7=$ sternite 7 (subgenital plate of $\bigcirc) ; \mathbf{s} 9=$ sternite 9 (subgenital plate of $\widehat{\jmath}) ; \mathbf{t 1 0}=$ tergite 10 (supraanal plate); $\mathbf{v v}=$ ventral valvulae (gonapophyses VIII). 
Roy 1999: 33). Grimaldi (2003: 11) stated, that "in Chaeteessa [their number is] 23-25, but in most other mantises there are 8-12 [cercomeres], including the most basal fossil forms [...]". He furthermore stated that "there are species in the Mantidae that also have many cercal segments (i.e., Theopompella, 26-28; Choerododis [sic!], 20-22".

Less than eight cercomeres (state 1) were found in the males of Acanthops, Callibia, Compsothespis, and Eremiaphilidae. In the latter group the cerci are very small (e.g. Grimaldi 2003: 11), probably due to the arid and hot habitat.

The highest numbers of cercomeres (23 or more, state 6) is present only in male Theopompa, whereas state 5 (20-22 cercomeres) is exhibited by male Choeradodis, Plistospilota, and Rhombodera. Grimaldi (2003: 11) stated that Chaeteessa, too, exhibits 23-25 cercomeres. The male of $C$. valida was not available for this study and was correspondingly not encoded (the abdominal apices in the alcohol preserved specimens were missing). The female exhibited less than 23 cercomeres. However, it is likely that the distal cercomeres are broken off in this specimen. A picture of a living late instar nymph (Fig. 5, see detail of cercus in Fig. 390) reveals a number of more than 25 cercomeres in Chaeteessa. Therefore, the character was provisionally encoded as state 6 for the female (see character 152). All remaining males that could be studied and had intact cerci have between 9 and 19 cercomeres.

In cockroaches, the number of cercomeres usually varies between eight and 15 (Beier 1974a: 29) but may reach numbers of about 20 cercomeres (Bohn 2003: 201). The number of cercomeres in cockroaches increases from the first nymphal stage to the adult and is generally slightly higher in females than in males (Beier 1974a: 29). The same can be found in Mantodea (e.g. Karuppanan 1998; Wieland, pers. obs.; see also Figs. 432, 433, 453, 454, 455-457, 458, 460). The cerci of the first instar nymph of Metallyticus splendidus have no more than four cercomeres of which the distal one is strongly elongated (Fig. 392). Adult M. splendidus have 16-19 cercomeres (Fig. 366). This is also the case in Theopompa. While the first nymph of T. servillei has three cercomeres (Fig. 458), the adult female (not included in this study) and the adults of the three Theopompa species studied herein have 23 cercomeres or more. Karuppanan (1998: tab. 1) studied the cercal development in Euantissa pulchra (Fabricius, 1787). In this species the first instar nymph has three to four cercomeres, whereas the adults have 12 to 13 cercal segments.

Chopard (1949a: 390) mentioned that the cerci in Mantodea are generally longer than in Blattaria and that in the former group the cercomeres are more distinctly separated from each other (probably meaning the often more or less strongly constricted borders between the cercal segments) than in cockroaches. He therefore deduced that the mantodean cerci probably represent the 
plesiomorphic state and that the blattarian cerci with short cercomeres, forming a smooth unit, are derived within Dictyoptera.

In Periplaneta, both sexes exhibit state 5. Further cockroach species studied (Panchlora nivea, Blatta orientalis, Ectobius sylvestris) have between seven and 15 cercomeres.

See also chapter 4.6.7.

152. Cerci of female, cercomeres, largest number: 8 or less [1]; 9-11 [2]; 12-15 [3]; $16-19$ [4]; 20-22 [5]; 23 or more [6].

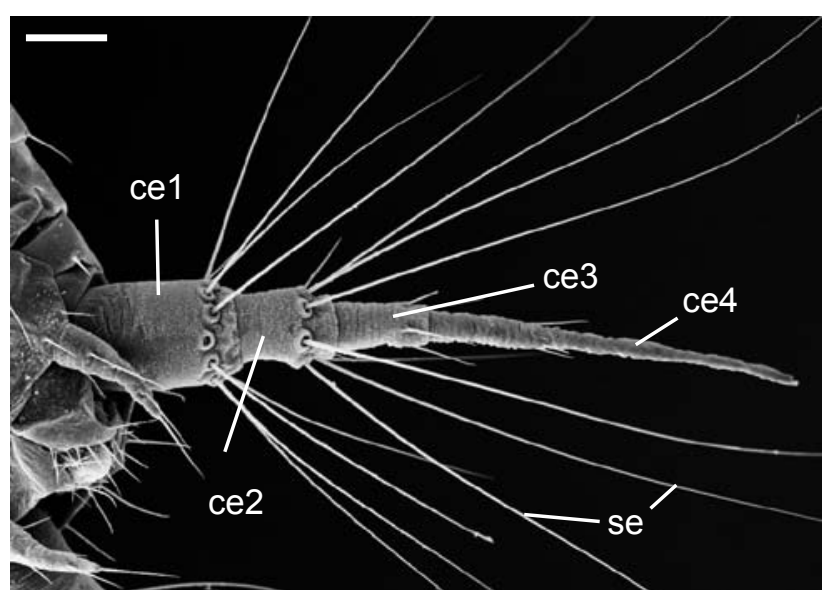

Fig. 392: Metallyticus splendidus, first instar nymph. Left cercus, ventral view. Note that each cercomere carries a ring of long setae which is helpful for identifying the number of cercomeres (especially the short, proximal ones) in adult Mantodea. - Scale bar $=0.1 \mathrm{~mm}$.

Abbreviations: ce1-ce4 $=$ cercomeres 1-4; se = seta.

Also see character 151 and chapter 4.6.7. Less than eight cercomeres (state 1) are present in female Acanthops falcataria (the only A. tuberculata female available exhibits state 3! See Fig. 383), Callibia, Compsothespis, Haania, Eremiaphilidae, and Ligariella.

The highest numbers of cercomeres (23 or more, state 6 ) are present in female Chaeteessa (see also character 152 and Fig. 390), Plistospilota and Theopompa, whereas state 5 (20-22 cercomeres) is exhibited by female Choeradodis, Deroplatys lobata (D. desiccata exhibited state 4), Humbertiella, Parasphendale, Polyspilota, Rhombodera, Sibylla, Stagmatoptera, Taumantis, Toxodera (Fig. 386; cercomeres partly covered by tergite), and Zoolea.

All remaining females that could be studied and had intact cerci have between nine and 19 cercomeres.

\subsection{Calculations}

The data matrix comprised 152 morphological characters encoded for 122 Mantodea species and Periplaneta americana. 19 characters were parsimony-uninformative. All characters were originally set as "unordered multistate" and were of equal weight $(=1.0)$ in the initial calculation (analysis I).

\subsubsection{Analysis I}

A heuristic search was conducted with the following parameters: addition sequence $=$ random; number of replicates $=100$; branch-swapping algorithm $=$ tree-bisection-reconnection (tbr); time setting $=3,600$ seconds per replicate; maximum number of trees $=$ automatically increased by 100 . 
Paup* (Swofford, 2001) found 888 equally parsimonious trees from three individual islands (CI: 0.1878; HI: 0.8122) with a tree length of 1,347 steps.

From these 888 trees the strict consensus-cladogram and the (50\%) majority rule-cladogram of analysis I were generated. The strict consensus cladogram (Fig. 393) showed many smaller monophyletic groups of taxa but most of the main nodes were not resolved. The resolution of the majority rule cladogram (Fig. 394) was better. However, although the basal and the apical dichotomies were well resolved therein, many of the nodes in between were still not.

The analysis of the data [consistency indices (ci) and homoplasy indices (hi) of the characters, see Tab. 4] indicated that the high degree of homoplastic characters was responsible for the low resolution of the cladogram. The inclusion of characters that are potentially homoplastic was intended a priori in order to demonstrate the putative multiple origin of, for instance, head processes and pronotal enlargements (see chapter 1). Therefore, a low resolution of the tree was to be expected.

\subsubsection{Analysis II}

In order to deal with the high degree of homoplasy, Paup* was used to conduct a second calculation (based on the results of analysis I) for which it was programmed to automatically reweight the characters a posteriori based on their respective rescaled consistency indices (reweight $=$ RC; see Tab. 5). Paup* left 39 characters un-weighted (weight 1) and assigned a weight other than 1.0 to 113 characters. The heuristic search of analysis II (1,000 replicates; branch swapping algorithm $=\mathrm{TBR}$ ) resulted in 10 equally parsimonious trees from a single island (ci: 0.4411 ; hi: 0.5589). The strict consensus and the majority rule cladograms of analysis II were identical and showed a full resolution of the tree with only two polytomies [Amorphoscelinae and the basal dichotomy within Tarachodidae (excluding Iris) were unresolved].

The strict consensus cladogram was used for further analysis. It was described by generating apomorphy lists for the character state optimization criteria under the assumption of delayed (DELTRAN) and accelerated (ACCTRAN) character transformation (see Tab. 6). Characters occurring as apomorphies under both criteria for the same nodes were considered as unambiguous apomorphies for the corresponding taxon (Tab. 6). In the following, such unambiguous apomorphies are used to describe the relationships reflected by the cladogram. Where no unambiguous apomorphic characters were found for a clade, the apomorphies found under ACCTRAN and DELTRAN assumptions alone are discussed separately.

\subsubsection{Bootstrap}

Bootstrap-values were calculated in Paup* by using the weights of the characters of analysis II. Paup* was set for 1000 replications with a time limit of 900 seconds per replication. The bootstrap values were rounded and applied to the cladogram (Fig. 395). 


\subsection{Phylogeny}

Tab. 6 provides an overview of the clade numbers and the respective taxa in the strict consensus tree of analysis II. It furthermore lists the apomorphic characters as well as those only recovered under the assumption of accelerated (ACCTRAN) or delayed (DELTRAN) optimization alone.

In the following, the phylogenetic relationships of selected taxa and selected apomorphies will be discussed. Square brackets refer to the clade-numbers given in the strict consensus cladogram of analysis II (Fig. 395, Tab. 6). The term OTU (operational taxonomic unit) will be used throughout the discussion as a common term for single species. Underlined names represent traditional groups of the current classification or previous phylogenetic hypotheses that were herein recovered to be monophyletic.

The discussion focuses mainly on unambiguous apomorphies (characters that occur under both ACCTRAN and DELTRAN assumptions). Where no or only few unambiguous apomorphies were found, ACCTRAN and DELTRAN data were consulted. Paup* based the calculations of analysis II on the exact tree length of 1,347 steps found in analysis I.

In some cases Paup* found apomorphic characters under both DELTRAN and ACCTRAN but interpreted them to have evolved from different ancestral states. Such characters were listed as unambiguous apomorphies (first column) because of their identical character state, and both transformations were listed with a slash separating the ACCTRAN from the DELTRAN interpretation (e.g. Heteronutarsus [10]: 74:3>4/2>4).

\subsection{1 - 4.3.105 Intra-ordinal phylogenetic relationships of Mantodea}

\subsubsection{Mantodea [1] (= Eumantodea)}

The basal dichotomies of the mantodean phylogeny were identical in analysis I (only majority rule cladogram) and analysis II (Fig. 396). However, no unambiguous apomorphies were found for Mantodea as a monophyletic group under both ACCTRAN and DELTRAN assumptions. All apomorphies were found under DELTRAN alone, whereas the identical character transformations were interpreted as convergent developments for Chaeteessa [2] and for the remaining Mantodea [3] under ACCTRAN assumptions. This probably is an artefact caused by the algorithm Paup* used for reweighting the characters based on their homoplasy indices. Nonetheless, many of these characters are undoubtedly autapomorphic for Mantodea. These encompass the following characters: Origin of the scutellum $(5: 2>1)$; fusion of the prothoracic basisternite and preepisternites, thus forming the T-shaped sclerite $(25: 2>1)$; prothorax becoming longer than the discoidal shape in cockroaches (28:3>2; convergently evolved in Humbertiella + Theopompa [65], clade [110], clade [204] within Hymenopodinae and several OTUs; with a subsequent reversal in Amorphoscelinae [28]); origin of the supracoxal furrow (30:2>1); the use of the forelegs as raptorial organs $(32: 1>2)$; origin of the claw groove $(36: 2>1)$; origin of the femoral brush (38:2>1); origin of discoidal spines (54:2>1; also present in Metallyticus, see description of character 54 in chapter 4.1.2 and discussion in chapter 4.6.4); origin of the 


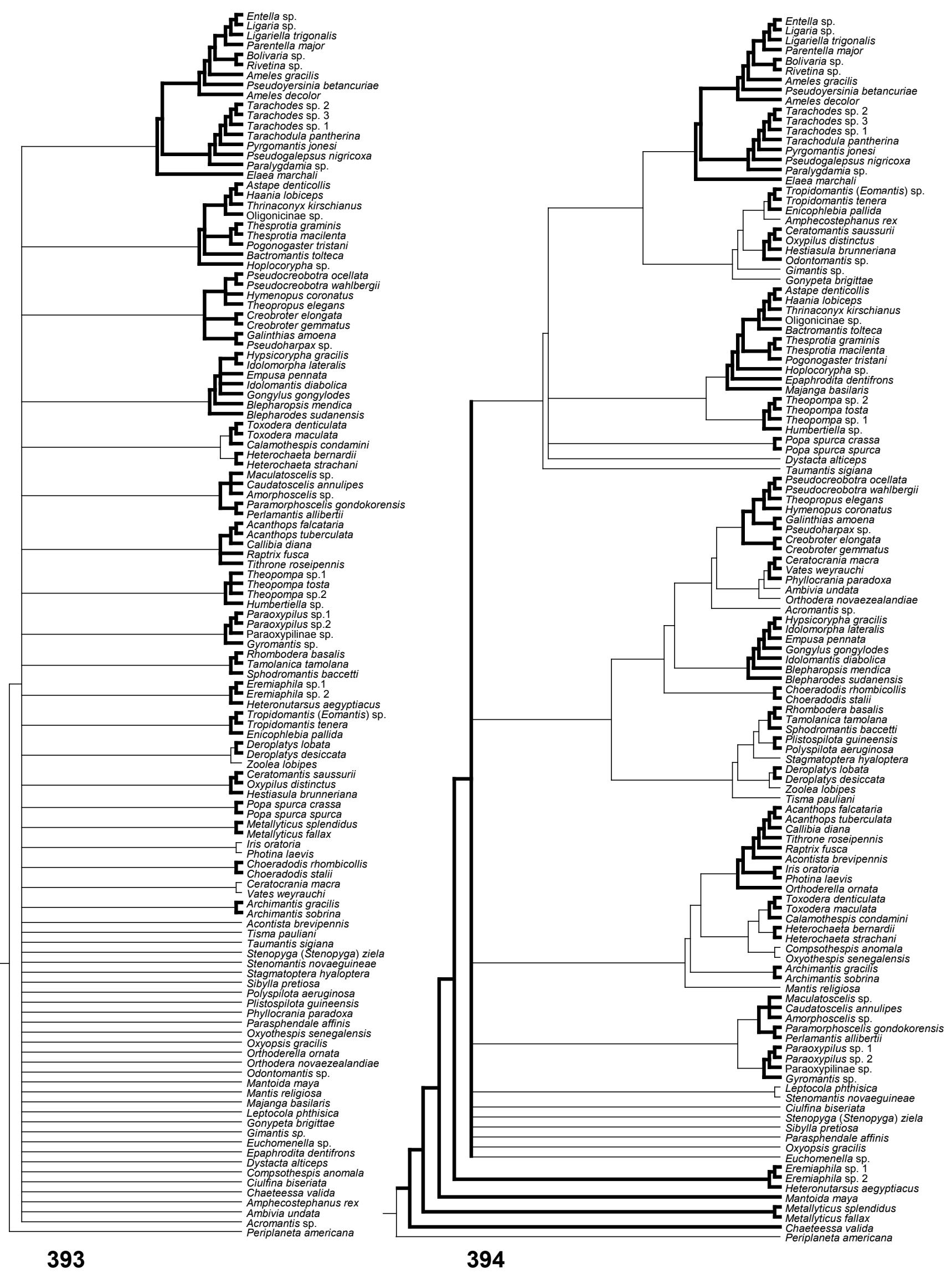

Figs. 393-394: Analysis I (all characters unweighted). Consensus cladograms resulting from 888 equally parsimonious trees from 3 islands. 393: Strict consensus cladogram. 394: Majority rule cladogram (50\%). Bold taxa were also recovered in analysis II, but not necessarily with identical internal relationships (see Tab. 6). 


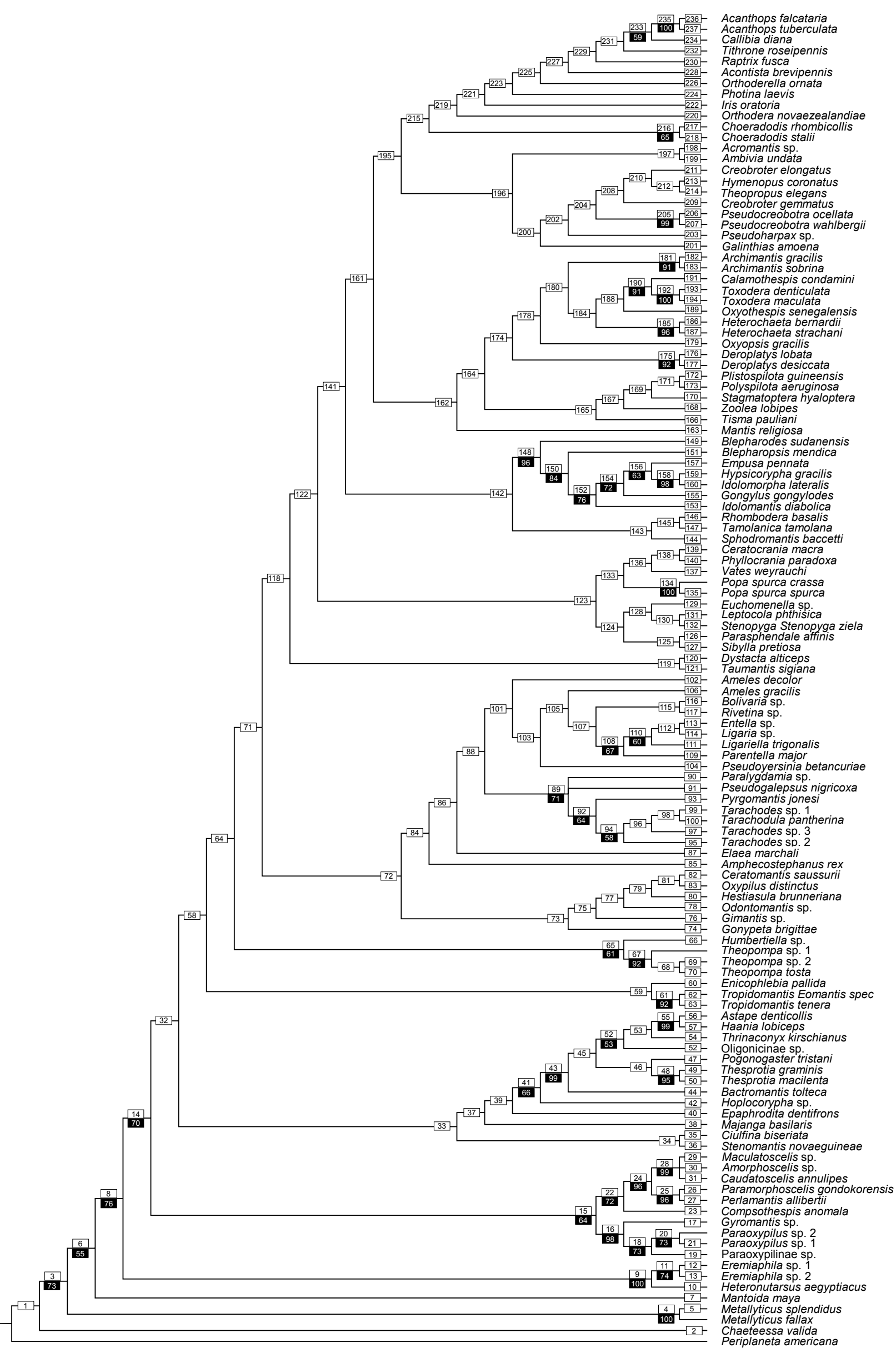

Fig. 395: Strict consensus cladogram resulting from the 10 equally parsimonious trees of analysis II. White boxes represent the node numbers referred to in the text. Black boxes are bootstrap values. 
tibial spur (68:2>1; also present in Chaeteessa, see description of character 68 in chapter 4.1.2 and discussion in chapter 4.6.5); reduction of the arolium (98:1>2); fusion of RP and $\mathrm{M}$ from the wing base in the tegmina (102:2>1; see also Béthoux \& Wieland 2009); elongation of ScP in the tegmina (112:2>1); presence of a callus in the tegmina (118: $2>1)$.

Paup* interpreted the presence of three ocelli $(4: 2>1)$ as a situation derived from the cockroaches which exhibit two. However, the polarity of this character is incorrect as becomes evident from the comparison with the situation outside Dictyoptera (see discussion of character 4 in chapter 4.1.1). The length of the ovipositor in Mantodea was interpreted to be derived from situation in Blattaria (i.e. secondarily elongated) $(143: 2>1)$ which has been assumed before, for instance by Marks \& Lawson (1962: 142). However, Klass (1998a: 76) demonstrated that ovipositor length in Mantodea likely represents the plesiomorphic condition and that Blattodea have apomorphically further reduced its length.

\subsubsection{Chaeteessa valida [2]}

Apomorphic characters for Chaeteessa have been rarely mentioned in the literature with exception of apomorphies found in the genital morphology as provided by Klass (1995: 192) and Klass \& Meier (2006: fig. 4).

Unambiguous apomorphic characters are: Rounded shape of the eyes (14:4>3; convergently evolved in clade [143] and several OTUs); number of antennomeres in the female counting 70-89 (19:6>3; convergently evolved in Mantodea except for Chaeteessa and Metallyticus [6], Tropidomantinae [59], clade [71], and several OTUs); presence of a single ventral cervical sclerite (22:2>1; convergently evolved in Tarachodinae except for Iris [89], clade [184], and sev-

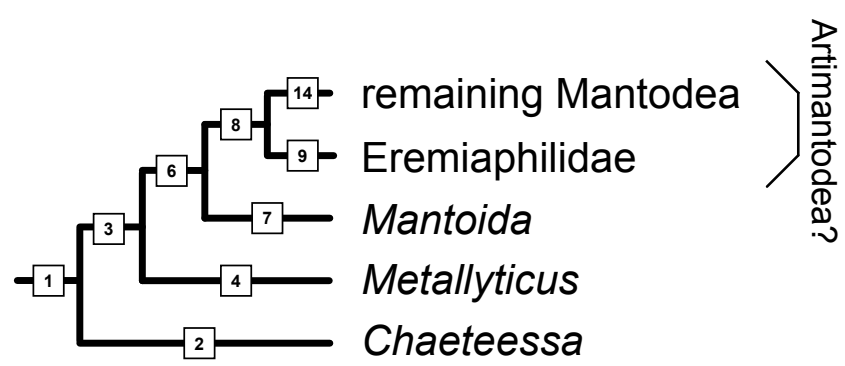

Fig. 396: Basal dichotomies in Mantodea found in analysis I (majority rule consensus cladogram) and analysis II.

For Artimantodea see discussions of the group in 2.1.5 and 4.3.7. eral OTUs); distinct alternating length pattern of the antero-ventral fore femoral spines (44:1>2); alternating position of the proximal antero-ventral femoral spines in two rows $(45: 2>1$; convergently evolved in Mantoida [7] and Paraoxypilinae [16]); number of postero-ventral fore femoral spines counting 6 or more (48:1>4; convergently evolved in Toxoderidae [190] and Gongylus [155]); elongation of the postero-ventral fore femoral spines (50:1>2; convergently evolved in clade [152] within Empusidae and in several OTUs); presence of a groove between the proximal postero-ventral femoral spines (53:2>1; also apomorphic for Ceratomantis [82]), the flattening of the cerci (149:2>1; convergently evolved in clade [22], Tarachodidae excluding Iris [89], clade [180], and several OTUs); number of cercomeres in the female counting 23 or more (152:5>6; convergently evolved in Plistospilota [172]). 


\subsubsection{Mantodea excluding Chaeteessa [3]}

This monophylum was recovered in analysis II and the majority rule cladogram of analysis I. There are no unambiguous apomorphies for this group. All apomorphies for Mantodea as a whole (exclusively found under DELTRAN, see 4.3.1) were interpreted to have evolved convergently in Chaeteessa [2] and Mantodea except for Chaeteessa [3] under ACCTRAN assumptions.

There are several further apomorphies for this taxon that were found under DELTRAN assumptions alone. Among them are: Oval shape of the compound eyes (14:4>2; convergently evolved in clade [221] and Theopropus [214]); lack of ventral cervical sclerites (21:1>2; convergently evolved in clade [88] within Tarachodidae and several OTUs); reduction of the articulated spines on the meso- and metathoracic femora and tibiae $(83: 1>2 ; 84: 1>2)$; elongation of ScP in the alae, ending beyond the distal third of the wing $(127: 2>1)$; crossveins between the Cubital veins $\mathrm{CuA}$ and $\mathrm{CuP}$ in the alae having a sigmoid shape (128:2>1; convergently evolved in Maculatoscelis [29]); short supra-anal plate (132:2>1; convergently evolved in Orthoderella + Acanthopidae [225]).

The loss of articulated spines on the cursorial legs $(83: 1>2 ; 84: 1>2)$ was found to be apomorphic for this group under DELTRAN, whereas it is assumed to have evolved convergently in Metallyticus [4] and Mantodea except Chaeteessa and Metallyticus [6] under ACCTRAN. This character, however, very likely represents a synapomorphy for all Mantodea except for Chaeteessa [3].

The basal dichotomy between Chaeteessa and the remaining Mantodea was also supported by the molecular data (Svenson \& Whiting 2009: fig. 3a, nodes 1, 2). Previous morphological studies, however, mostly did not find this dichotomy but instead found Mantoida to be the sistergroup of all remaining Mantodea (e.g. Klass 1995; Klass \& Meier 2006; see chapter 2.1.4 for a discussion). An exception are Grimaldi (2003: fig. 27) and Grimaldi \& Engel (2005: fig. 7.60) who assumed the sistergroup relationship between Chaeteessa and the remaining extant Mantodea in their mental analysis.

Note that this dichotomy was supported although for Chaeteessa the tibial spur was encoded as "present" (68:1).

\subsubsection{Metallyticus [4]}

The monophyly of Metallyticus is supported by the strong folding of the scutellum $(10: 2>1)$ and the enormously enlarged proximal postero-ventral femoral spine $(51: 1>2)$. Selected further unambiguous apomorphies are: Length reduction of the antennae $(17: 1>2)$; location of the fore femoral grooming device on an elevation (39:1 >2; convergently evolved in Eremiaphilidae [9], Paraoxypilinae [16], Perlamantinae [25], Haaniinae [55], and several OTUs); presence of a single discoidal spine (55:2>1; convergently evolved in Amorphoscelinae + Perlamantinae [24]); postero-ventral tibial spines increasing in length distad [75:3>2; convergently evolved in Artimantodea (?) [8] (when mentioned in the discussion Artimantodea will be referred to with 
a question mark, see discussion of the group in chapter 4.3.7)]; number of the postero-ventral fore tibial spines being less than a third lower than or equal to the number of antero-ventral spines (79:2>1; convergently evolved in several OTUs); sigmoid course of the tegminal main veins (111:2>1; convergently evolved in Rivetinini [115], clade [164], Hymenopus + Theopropus [212], and several OTUs); branching of the first anal vein in the tegmina $(115: 2>1$; convergently evolved in Amorphoscelinae [28]); lack of branchings of the third anal vein in the alae of the females (126:3>2; convergently evolved in Artimantodea (?) [8] and Archimantis [181]); nearly rectangular shape of the supra-anal plate (136:4>1; convergently evolved in Hoplocorypha [42]).

The monophyly of Metallyticus was also well supported by molecular data (Svenson \& Whiting 2009: fig. 3a, node 45).

\subsubsection{Mantodea excluding Chaeteessa and Metallyticus [6]}

This monophylum was recovered in analysis II and the majority rule cladogram of analysis I. Two unambiguous apomorphies support this group. These are the number of antennomeres in the female counting 70-89 (19:6>3; convergently evolved in Chaeteessa [2], Tropidomantinae [59], clade [71], and several OTUs) and the length pattern of the discoidal spines $(58: 1>3)$.

This relationship may also be supported by the reduction of the anterior branches of RA towards $C$ in the tegmina (109:1>2; found under DELTRAN; interpreted as having been convergently reduced in Mantoida [7] and clade [14] under ACCTRAN, probably because the situation in Eremiaphilidae [9] was not encoded as it is difficult to assess due to the strong reduction and the rigidity of the tegmina in this group).

The presence of the second anal vein (A2) in the alae was not encoded for Chaeteessa and Metallyticus, because the situation is uncertain (see discussion of character 129 in chapter 4.1.2). Paup* interpreted this character to be present in Chaeteessa and Metallyticus and to be missing in Mantodea except for these two [6] (129:1>2 under DELTRAN) or to have been convergently reduced in Mantoida [7] and Mantodea except for Chaeteessa, Metallyticus and Mantoida [8] (under ACCTRAN). If A2 is actually present in the alae of Chaeteessa and reduced in Metallyticus, its loss would probably be apomorphic for clade [3]. If it is missing in Chaeteessa, as well, it would likely be an autapomorphy for Mantodea as a whole.

This relationship supports the Mantoidea-hypothesis sensu Vickery \& Kevan (1983: 217219; see discussion of Mantoidea in chapter 2.1.5).

\subsubsection{Mantoida maya [7]}

An apomorphic character for Mantoida maya (and possibly the entire genus) is the wide distribution of the setae of the fore femoral grooming device along the entire distal half of the femur $(40: 1>2)$. Further unambiguous apomorphies are, for instance: Organization of the proximal antero-ventral fore femoral spines in two nearly parallel rows (45:2>1; convergently evolved in Chaeteessa [2] and Paraoxypilinae [16]); presence of 3 discoidal spines (55:2>3; convergently evolved in clade [14], Pogonogaster + Thesprotia [46], Oxyothespis + 
Toxoderidae [188], and several OTUs); proximal discoidal spine being located on an elevation (56:2>1; evolved convergently in Mantoida [7] and Amorphoscelinae [28]); posteroventral tibial spines being of equal length $(75: 3>1)$; basitarsomere of the raptorial legs being as long as or longer than the fore tibia [81:2>1; convergently evolved in Amorphoscelidae including Compsothespis [15], Oligonicinae including Haaniinae [43], and several OTUs).

Paup* interpreted the wide separation of the grooming setae $(40: 1>2)$ as an apomorphy for Mantoida. However, this hypothesis requires further extensive studies, most importantly of fossil and nymphal Mantodea. The grooming setae are fragile and may not have fossilized in many individuals, especially in early instar nymphs in which their number is generally much lower than in adults (see chapter 4.6.7). It is possible that the polarity of this character reads from few to many and, as hypothesized by Grimaldi (2003: character 25 on p. 40), from simple to feather-shaped setae. Therefore, the situation found in Mantoida may as well represent the plesiomorphic condition that originated in early Mantodea.

\subsubsection{Artimantodea (?): Mantodea excluding Chaeteessa, Metallyticus and Mantoida [8]}

This monophylum was recovered in analysis II and the majority rule cladogram of analysis I. It is supported by the length pattern of the discoidal spines $(57: 1>3)$, the postero-ventral tibial spines increasing in length distad (75:3>2; convergently evolved in Metallyticus [4]) and the third anal vein in the alae of the females being branched 3-4 times (126:3>2; convergently evolved in Metallyticus [4] and Archimantis [181]).

The position of Metallyticus within or outside Artimantodea could not be unambiguously resolved in the molecular analysis by Svenson \& Whiting (2009; Fig. 4A). The present study suggests the position of Metallyticus outside Artimantodea ("alternate placement of Metallyticus" in Svenson \& Whiting 2009: fig. 3a). Due to the uncertain position of Metallyticus in the different analyses Artimantodea is referred to with a question mark throughout the discussion.

\subsubsection{Eremiaphilidae: Eremiaphila + Heteronutarsus [9]}

Eremiaphilidae was found to be monophyletic in analysis I and II. The monophyly of Eremiaphilidae is well supported by the strong elongation of the ungues $(100: 1>2)$, by the female subgenital plate being enclosed by sternite $6(139: 2>1)$, and by the presence of ventral digging spines originating from sternite 6 in the female $(141: 2>1)$.

There are furthermore several homoplastic characters that support the monophyly of the group, among them the short wings in the males (101:1>2; convergently evolved in Ciulfina + Stenomantis [34], clade [103], Leptocola + Stenopyga [130], and several OTUs) and the low number of cercomeres in both sexes (151:5>1, 152:5>1; convergently evolved in several OTUs).

The monophyly of Eremiaphilidae could not be addressed by the molecular data (Svenson \& Whiting 2009) because Heteronutarsus was not included. 


\subsubsection{Mantodea excluding Chaeteessa, Metallyticus, Mantoida and Eremiaphilidae [14]}

This monophylum was found in analysis II and the majority rule cladogram of analysis I. It is supported by: Presence of 3 discoidal spines (55:2>3; convergently evolved in Pogonogaster + Thesprotia [46], Oxyothespis + Toxoderidae [188], and several OTUs); number of anal veins in the alae of the male counting between 5 and 8 (121:3>2; convergently evolved in several OTUs including Mantoida maya [7]); third anal vein in the alae of the male branching between 3 and 4 times (122:3>2; convergently evolved in Archimantis [181]); number of cercomeres in the males counting 9-11 (151:5>2; convergently evolved in clade [73], clade [101], Acanthopidae [227] and several OTUs) and in the females (152:5>2; convergently evolved in clade [101] and several OTUs).

\subsubsection{Amorphoscelidae (including Compsothespis) [15]}

This clade was only found in analysis II (Fig. 397). Several unambiguous autapomorphies support this relationship, among them the lack of postero-ventral fore-tibial spines $(64: 1>2)$, the fore tibia being half as long as the femur or shorter (67:2>1; convergently evolved in Angelinae [128], clade [162], and several OTUs), and the fore basitarsomere being as long as or longer than the fore tibia (81:2>1; convergently evolved in Oligonicinae including Haaniinae [43] and several OTUs including Mantoida maya [7]).

Compsothespis being nested within Amorphoscelidae was not found in either consensus cladogram of analysis I. In the majority rule cladogram it was found to be closely related to Toxoderidae, Heterochaeta and Oxyothespis. Although Otte \& Spearman (2005: 36) assigned Compsothespis to Perlamantinae, possibly taken over from Giglio-Tos (1913: 2, 1927: index), a close relationship of Compsothespis and Amorphoscelidae has never been assumed since (compare, for instance, Beier 1935c; La Greca 1939b; Roy \& Leston 1975; Lombardo 1988; Kaltenbach 1996). The molecular analysis by Svenson \& Whiting (2009: fig. 3c) showed results very similar to the majority rule cladogram of analysis I, with Compsothespis being closely related with Oxyothespinae and Toxoderidae. Therefore, it may be assumed that the relationship between Compsothespis and (parts of) Amorphoscelidae does probably not reflect their true relationship.

The results of analysis II are ambiguous with regard to the evolution of foreleg morphology (mainly characters 43 and 65) within this group. The morphology of the fore femoral spination (character 43), for instance, is assumed to have originated from state 1 under DELTRAN assumptions with a subsequent independent transformation into the characteristic femoral spination morphologies in Paraoxypilinae [16] (43:1>3), Compsothespis [23] (43:1>6; same state assumed to have evolved from a different state under ACCTRAN assumptions, therefore listed in the apomorphies column), Perlamantinae [25] (43:1 $>5$; same state assumed to have evolved from a different state under ACCTRAN, therefore listed in the apomorphies column), and Amorphoscelinae (43:1>4).

Under ACCTRAN assumptions, too, state 43:1 was assumed to represent the ancestral state. In this scenario, however, Amorphoscelidae including Compsothespis [15] evolved state 3 


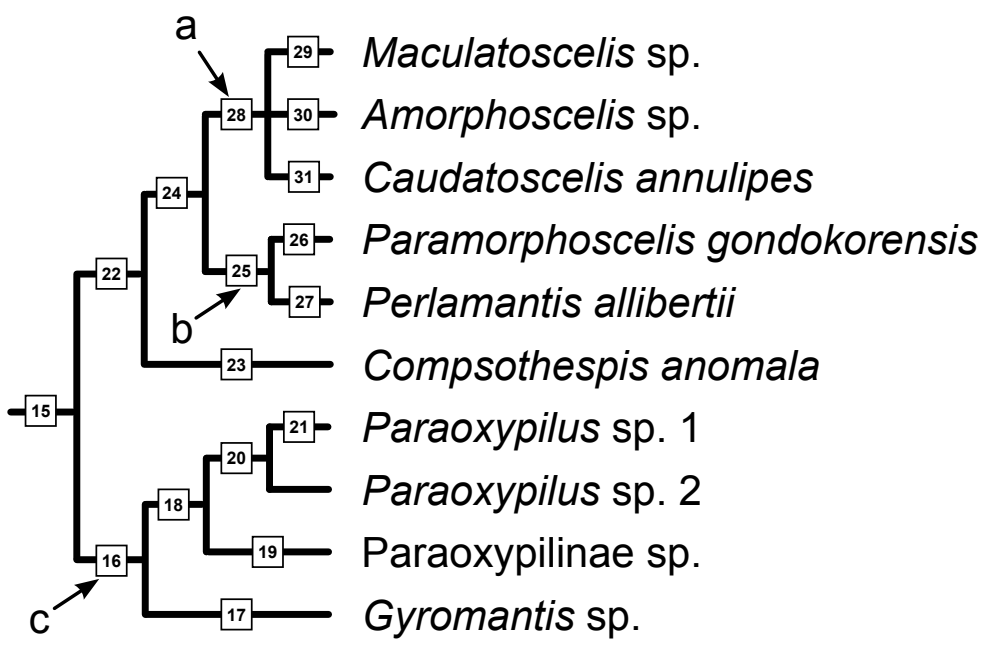

Fig. 397: Phylogeny of Amorphoscelidae including Compsothespis (clade [15]). See chapter 4.3.10 for discussion.

$\mathbf{a}=$ Amorphoscelinae $; \mathbf{b}=$ Perlamantinae; $\mathbf{c}=$ Paraoxypilinae .
$(43: 1>3=$ the characteristic Paraoxypilinae morphology) with the subsequent evolution into the different character states in the subtaxa Compsothespis + (Amorphoscelinae + Perlamantinae) [22] (43:3>4), Compsothespis [23] (43:4>6; same state assumed to have evolved from a different state under DELTRAN, therefore listed in the apomorphies column), and Perlamantinae [25] (43:4>5; same state assumed to have evolved from a different state under DELTRAN,

therefore listed in the apomorphies column). This scenario would indicate that state $43: 3$ is the plesiomorphic condition retained by Paraoxypilinae [16].

The same is true for character 65 , which encodes the fore tibial morphology. Under DELTRAN, state 65:1 is assumed to be the plesiomorphic condition from which the individual subgroups individually evolved their respective morphologies: State 65:1>3 in Paraoxypilinae [16], and state 65:1>4 in Compsothespis + (Amorphoscelinae + Perlamantinae [22] (same state assumed to have evolved from a different state under ACCTRAN, therefore listed in the apomorphies column).

Under ACCTRAN assumptions, state 65:1>3 evolved in Amorphoscelidae including Compsothespis [15] with subsequent transformation in Compsothespis + (Amorphoscelinae + Perlamantinae) [22] (65:3>4; same state assumed to have evolved from a different state under ACCTRAN, therefore listed in the apomorphies column). In this case, too, this would indicate Paraoxypilinae having retained the plesiomorphic condition of Amorphoscelidae including Compsothespis [15] (state 65:3).

In conclusion, although the character states are highly characteristic for the respective subgroups, it remains uncertain which character represents the plesiomorphic situation for the last common ancestor of Amorphoscelidae (including Compsothespis) if the relationships actually reflect the true phylogeny of the group. Specifically, it is unclear if the foreleg morphology of Paraoxypilinae reflects the plesiomorphic condition or an apomorphic state.

In the molecular analysis by Svenson \& Whiting (2009) it was found that Paraoxypilinae, although being in the same larger clade with Amorphoscelinae (Svenson \& Whiting 2009: fig. 3b, node 74), originated from an Australasian clade which encompassed the greater part of the Australasian taxa in that analysis, most of them very likely exhibiting states 43:1 and 65:1. The same is true for Amorphoscelinae. Furthermore, the entire clade 74 in Svenson \& Whiting (2009: fig. 3b) originated from mantodeans exhibiting state 43:1 and 65:1, and Compsothespis 
was found to be closely related to Oxyothespinae and Toxoderidae therein, much more distally in the cladogram (Svenson \& Whiting 2009: fig. 3c, node 172; see discussion above).

Therefore, it can be assumed that the situation found in Paraoxypilinae [16] (state 43:3, 65:3) and Amorphoscelinae [28] (state 43:4, 65:4) is apomorphic for the respective taxa and evolved independently from the ancestral state. The situation in the fore femora of Perlamantinae [25] (state 43:5) can be assumed to be autapomorphic for the taxon, whereas the situation in the fore tibiae (65:4) is uncertain and may be synapomorphic for Amorphoscelinae + Perlamantinae [24] if Compsothespis is not closely related to Amorphoscelidae (see discussion above).

The assumption of the monophyly of Amorphoscelidae has been doubted several times (e.g. Handlirsch 1925: 495; Chopard 1949a: 398) but it is still assumed in current classifications. It must be stated that, although the relationship of Amorphoscelinae and Compsothespis was neither supported in analysis I nor by the molecular data, the foreleg morphology of the two groups is intriguingly similar in several aspects. For instance, the fore tibiae are morphologically nearly identical (no remnants of spines discernible, fore tibial lobe carrying the tibial spur very long; Figs. 178, 266, 267). The fore femora of Compsothespis on the other hand exhibit a less reduced state of the spines (very small except for the discoidal spines, but distinctly discernible; see Figs. 166-168) and show an asymmetry in cross section, i.e., the antero-ventral spines are positioned on an elevation (Figs. 168), which is quite similar to the asymmetry observed in Amorphoscelinae (Fig. 184, 186, 188). Furthermore, the claw groove is located in the middle of the fore femoral length (shifted further distad in some Amorphoscelinae; see Roy 1962: figs. 9, 13, 1965b: fig. 4b), and in lateral view the ventral surface-line distally gains a sinuous shape (Fig. 166, 168), which can also be found in Amorphoscelinae (Fig. 178).

An extensive study of the postembryonic development and anatomical characters of the body and the genitalia of these enigmatic groups is likely to result in more definite data, helping to further elucidate their origins and relationships. It is also important to analyse DNA sequences of Perlamantis in order to address its relationships with the remaining Amorphoscelidae from a molecular perspective.

\subsubsection{Paraoxypilinae: Gyromantis + (Paraoxypilinae sp. + Paraoxypilus) [16]}

Paraoxypilinae was recovered monophyletic in both analyses and in both the strict consensus and the majority rule cladogram of analysis I. In all analyses, Gyromantis [17] was found to be the sistergroup of the remaining Paraoxypilinae, followed by a dichotomy between Paraoxypilinae sp. and Paraoxypilus [18].

Unambiguous apomorphic characters of Paraoxypilinae are: Elevation of the femoral brush (39:1>2; convergently evolved in Eremiaphilidae [9], Perlamantinae [25], Haaniinae [55] and many OTUs including the two species of Metallyticus [4]); alternating position of the proximal antero-ventral spines (45:2>1; convergently evolved in Chaeteessa [2], and Mantoida [7]); presence of a medial euplantula of tarsomere ta5 of the cursorial legs (97:2>1; convergently gained in clade [37] and several OTUs); abdominal tergites of the females carrying dorsal lobelike expansions or elevated posterior ridges (148:2>1; convergently evolved in several OTUs).

It is likely that the foreleg morphology (mainly character states 43:3 and 65:3) is apomorphic 
for this group but the current dataset is ambiguous about the ancestral state for Amorphoscelidae (see discussion in 4.3.10). Svenson \& Whiting (2009: fig. 3b, node 113) found a monophyletic Paraoxypilinae being nested in an Australian clade encompassing Australian Nanomantinae, Liturgusinae and Hapalomantinae. This suggests that the characteristic foreleg morphology of Paraoxypilinae evolved independently from taxa exhibiting the "typical" mantodean morphology (character states 43:1 and 65:1; see discussion in chapter 4.3.10).

\subsubsection{Paraoxypilinae sp. + Paraoxypilus [18]}

This clade was found in analysis II and in both consensus cladograms of analysis I. It is supported by the female aptery (105:1 $>2$; convergently evolved in Thespidae [41] and Oxypilus [83]) and by the of metathoracic ear exhibiting DO morphology in the female (131:2>3/1>3; convergently evolved in clade [37] and Oxypilus [83]).

\subsubsection{Compsothespis + (Amorphoscelinae + Perlamantinae) [22]}

The sistergroup relationship between Compsothespis [23] and Amorphoscelinae + Perlamantinae [24] is supported by the lack of antero-ventral tibial spines on the forelegs $(63: 1>2$; $65: 3>4 / 1>4)$. Further characters supporting this relationship are the number of antero-ventral femoral spines in both sexes counting $1-5(46: 3>1 ; 47: 3>1)$ and the flattening of the cerci (149:2>1; evolved convergently in Tarachodidae excluding Iris [89], clade [180], and several OTUs including Chaeteessa valida [2]).

The close relationship of Compsothespis with Amorphoscelinae and Perlamantinae is highly debatable, for a detailed discussion see chapter 4.3.10.

\subsubsection{Amorphoscelinae + Perlamantinae [24]}

This monophylum is supported by the following characters: Lack of postero-ventral fore femoral spines $(42: 2>1)$; reduction of the post-cervical plate into a slender transverse anterior part of the T-shaped sclerite (26:2>1); presence of a single discoidal spine (55:3>1; convergently evolved in Metallyticus [4]); elongation of the wings in the females (106:2>1; convergently evolved in Tropidomantinae [59], clade [75], clade [136] and several OTUs).

It is possible that character 65:4 is apomorphic for this group as its relationship with Compsothespis [23] is doubtful (see discussion of 4.3.10).

In contrast to the hypothesis that the elongation of the anterior part of the T-shaped sclerite has evolved once and may be an argument against the monophyly of the traditional Amorphoscelidae (see discussion of character 26 in chapter 5.2.2), Paup* assumed the elongation of the sclerite $(26: 1>2)$ to have evolved in clade [14] (under DELTRAN) or independently in Amorphoscelidae including Compsothespis [15] and clade [32] (under ACCTRAN) with a subsequent reversal (26:2>1) in Amorphoscelinae + Perlamantinae [24] in both scenarios.

This relationship could so far not be addressed by molecular approaches due to the lack of Perlamantis. However, the position of Amorphoscelinae in the molecular tree by Svenson \& Whiting (2009: fig. 3b, node 75) supports a secondary shortening of the T-shaped sclerite in this group. 


\subsubsection{Perlamantinae: Perlamantis + Paramorphoscelis [25]}

Perlamantinae is well supported by a number of unambiguous apomorphies. Among them are the characteristic antero-ventral fore-femoral spination $(43: 4>5 / 1>5)$, the length reduction of the tibial spur $(69: 1>2)$ and the elongation of the distal part of the tibia and its nearly $90^{\circ}$ folding $(70: 1>2)$. Furthermore, the monophyly of the group is supported by the position of the grooming setae on an elevation (39:1>2; convergently evolved in Eremiaphilidae [9], Paraoxypilinae [16], Haaniinae [55], and several OTUs including the species of Metallyticus [4]), and by the anal veins in the tegmina counting 2-3 (114:2>1; convergently evolved in several OTUs including Mantoida maya [7]).

\subsubsection{Amorphoscelinae: Maculatoscelis + Amorphoscelis + Caudatoscelis [28]}

An autapomorphy supporting Amorphoscelinae is the lack of antero-ventral fore femoral spines $(41: 1>2)$. The elevation of the discoidal spine on a socket $(56: 2>1)$ has evolved convergently in Mantoida [7]. The branching of the first anal vein in the tegmina $(115: 2>1)$ has evolved convergently in Metallyticus [4].

It is important to conduct a comparative study of the foreleg morphology of the African and South-East Asian Amorphoscelinae, as well as the postembryonic development of the forelegs in the group. This might shed further light on their internal relationships, which could not be resolved herein, and on their putative relationship with Compsothespis (see discussion in 4.3.10).

\subsubsection{Clade [32]}

This clade was found in analysis II alone. The only unambiguous apomorphy supporting it are the lobes on the femora of the meso- and metathoracic femora being positioned distally from the middle or apically $(91: 1>3)$. With the exception of Compsothespis [23] being nested within Amorphoscelidae [15], which is debatable (see chapters 4.3.10 and 4.3.16), this group represents the traditional Mantoidea sensu Roy (1999), Grimaldi (2003) and Grimaldi \& Engel (2005) (see Mantoidea in chapter 2.1.5).

Although the unambiguous support for this clade is weak, there are several characters that have been interpreted as having evolved in this clade under DELTRAN or ACCTRAN alone.

Apomorphies found under DELTRAN are, for instance: Presence of the ventral cervical sclerites (21:2>1; convergently evolved in clade [107]); gain of the median connection of the ventral cervical sclerites (23:2>1; convergently evolved in Compsothespis [23]); prothorax being twice as long as wide or longer (28:2>1; convergently evolved in Compsothespis [23] and Hymenopus + Theopropus [212]); alternating length pattern of the antero-ventral fore femoral spines (44:1>2; convergently evolved in Chaeteessa [2]); presence of the ventral groove between the proximal postero-ventral fore femoral spines $(53: 2>1$; convergently evolved in Chaeteessa [2] and Ceratomantis [82]).

Apomorphic characters found under ACCTRAN alone are: Presence of elongated postcervical plate (26:1>2; convergently evolved in Amorphoscelidae including Compsothespis [15]); claw groove lying in the proximal third of the antero-ventral femoral surface $(37: 1>2$; 
convergently evolved in Eremiaphilidae [9], clade [51], Polyspilotini [171], Toxoderidae [190], and Oxyopsis [179]); fore tibia being half as long as the femur or shorter (67:2>1; convergently evolved in Amorphoscelidae [15], Angelinae [128], clade [152] within Empusidae, clade [162], and several OTUs); spreading and V-shape of the euplantulae on tarsomeres 1-3 (95:2>1; convergently evolved in Amorphoscelidae including Compsothespis [15]); branching of RA in the tegmina (103:2>1; convergently evolved in Amorphoscelidae including Compsothespis [15]); origin of the DK morphology ("deep groove with knobs") of the metathoracic ear in the females (131:4>1; convergently evolved in Amorphoscelidae including Compsothespis [15], Haaniinae [55], Hestiasula + Oxypilinae [79], clade [142], clade [161], and several OTUs).

\subsubsection{8 (Stenomantis + Ciulfina $)+($ Majanga $+($ Epaphrodita $+($ Hoplocorypha $+($ Oligonici- nae including Haaniinae))) [33]}

This taxon was only found in analysis II and is supported by the prozona of the pronotum being less than half as long as the metazona (31:2>3; convergently evolved in clade [33], Tarachodidae excluding Iris [89], and several OTUs). Furthermore, the basitarsomere of the metathoracic legs are longer than the remaining tarsomeres taken together $(87: 2>1)$, which has evolved convergently in Paraoxypilinae [16], Amorphoscelinae [28], Empusinae [154], clade [165], and several OTUs.

\subsubsection{Stenomantis + Ciulfina [34] ("Australian Liturgusidae")}

The monophyly of a taxon comprising these two Australian bark dwelling species finds support in the basitarsomere of the forelegs being as long as the remaining tarsomeres or shorter (80:1>2; convergently evolved in Perlamantinae [25], Entella + Ligaria [112], Oxyothespis + Toxoderidae [188], and several OTUs), the metathoracic tibiae in the male being as long as the corresponding femur or shorter (88:1>2; convergently evolved in Haaniinae [55], clade [133], Hymenopodinae [200], and several OTUs), and the males being micropterous (101:1>2; convergently evolved in Eremiaphilidae [9], clade [103], Leptocola + Stenopyga [130], and several OTUs).

A sistergroup relationship between the species of the Australian Liturgusidae is also well supported by molecular data (Svenson \& Whiting 2009: fig. 3b, node 105). In the present dataset, a sistergroup relationship between the South-East Asian Liturgusidae taxa (Humbertiella + Theopompa [65]) finds support, which further corroborates the hypothesis of the multiple evolution of a liturgusid ecomorph as suggested by Svenson \& Whiting (2009: 471). Unfortunately, Theopompa was not present in the molecular dataset, however, the Neotropical (Hagiomantis + Liturgusa) and Afrotropical Liturgusidae (Dactylopteryx + Theopompella) (not included herein) were also found to form biogeographically distinct monophyletic groups in the molecular analysis.

Interestingly, the Madagascan Majanga [38] (not present in Svenson \& Whiting 2009), which is traditionally assigned to Liturgusidae, did not cluster with either of the liturgusid groups studied herein (clades [34] and [65]). Future molecular studies may show if Majanga 
is closely related to the monophyletic African liturgusid ecomorph in a close relationship with Dactylopteryx and Theopompella or if it perhaps represents even another tree dwelling group that evolved independently.

\subsubsection{Majanga $+($ Epaphrodita $+($ Hoplocorypha $+($ Oligonicinae including Haaniinae $))$} [37]

This clade was found in analysis II and in the majority rule consensus cladogram of analysis I, albeit with slightly differing internal relationships. Its monophyly is supported by the females having evolved a DO shape ("deep groove, opened") of the metathoracic hearing organ (131:1>3; convergently evolved in clade [18] within Paraoxypilinae and in Oxypilus [83]), by the presence of 4 discoidal spines (55:3>4; convergently evolved in Eremiaphilidae [9]), and by tarsomere ta 5 of the cursorial legs possessing a medial euplantula (97:2>1; convergently gained in Paraoxypilinae [16] and several OTUs).

\subsubsection{Epaphrodita + (Hoplocorypha + (Oligonicinae including Haaniinae) $)$ [39]}

This clade was found in analysis II and in the majority rule consensus cladogram of analysis I, albeit with slightly different internal relationships. Its monophyly is supported, for instance, by the lack of the antero-distal apical spine on the meso- and metathoracic femora (82:1>2; convergently reduced in Tropidomantinae [59] and several OTUs), and the supra-anal plate being longer than wide (132:1>3; convergently evolved in Tropidomantinae [59] and several OTUs).

\subsubsection{Thespidae: Hoplocorypha + (Oligonicinae including Haaniinae) [41]}

Thespidae was found to be monophyletic in analysis II and both consensus cladograms of analysis I, albeit in a slightly different constellation (Fig. 398). The monophyly of Thespidae is supported by the following apomorphies: Position of the claw groove in the distal half of the femur $(37: 2>4$; convergently evolved in Angelinae [128], Idolomorphini [158], and Ceratocrania [139]); lack of the groove between the two proximal postero-ventral femoral spines $(53: 1>2$; convergently evolved in Chroicopterinae [108] and several OTUs); females apterous (105:1>2; convergently evolved in clade [18] within Paraoxypilinae and Oxypilus [83]).

Hoplocorypha was not found to be in a close relationship with Oligonicinae and Haaniinae in any of the molecular analyses (e.g. Yager \& Svenson 2008: fig. 6; Svenson \& Whiting 2009: fig. 3c, node 178), which found Thespidae to be polyphyletic (see also introduction of Thespidae in chapter 2.1.4).

\subsubsection{Oligonicinae including Haaniinae: Bactromantis + ((Pogonogaster + Thesprotia $)+$} (Oligonicinae sp. + (Thrinaconyx + Haaniinae)) [43]

This clade was found in analysis II and both consensus cladograms of analysis I with slightly differing internal relationships. Its monophyly is supported by the position of the antero-distal tibial spines being orientated more or less strongly dorsad $(65: 1>2,66: 1>2)$. This character is unique among Mantodea. Further characters supporting this relationship are: Presence of 1-5 
antero- and postero-ventral spines on the fore tibiae of the males $(73: 2>1,77: 2>1$; the latter has evolved convergently in Heteronutarsus [10]); cross veins between the Cubital veins CuA and $\mathrm{CuP}$ in the alae being straight or slightly curved (128:1>2; convergently evolved in clade [101], Dystacta + Taumantis [119], clade [124], clade [178], and several OTUs); secondary gain of the MSMT morphology ("meso- and metathoracic segments similar") of the metathoracic ear in the female (131:3>4; with a reversal from MSMT to DK morphology (“deep groove with knobs") in Haaniinae [55], see Yager \& Svenson 2008: 559); supra-anal plate of the males triangular (134:3>2; convergently evolved in several OTUs).

The monophylum found in this study corroborates the statement by Roy (1999: 37) that "the migration of some internal spines to the dorsal position occurred only once". However, the analyses by Yager \& Svenson (2008) and Svenson \& Whiting (2009) recurrently imply a single origin of the metathoracic hearing organ in Mantodea. Furthermore, Svenson \& Whiting (2009: fig. 3, node 141) found Haania in a distal position of their phylogenetic tree, whereas Oligonicinae is part of a Neotropical earless clade near the base of the tree. If Oligonicinae actually encompasses Haaniinae, a convergent origin of the cyclopean ear would have to be assumed (see also characters 130 and 131 in chapter 4.1.2., and chapter 4.6.14; Fig. 398). Otherwise the dorsal shift of the fore tibial spines would have occurred twice. Considering the complicated

independent gain
of cyclopean ear?

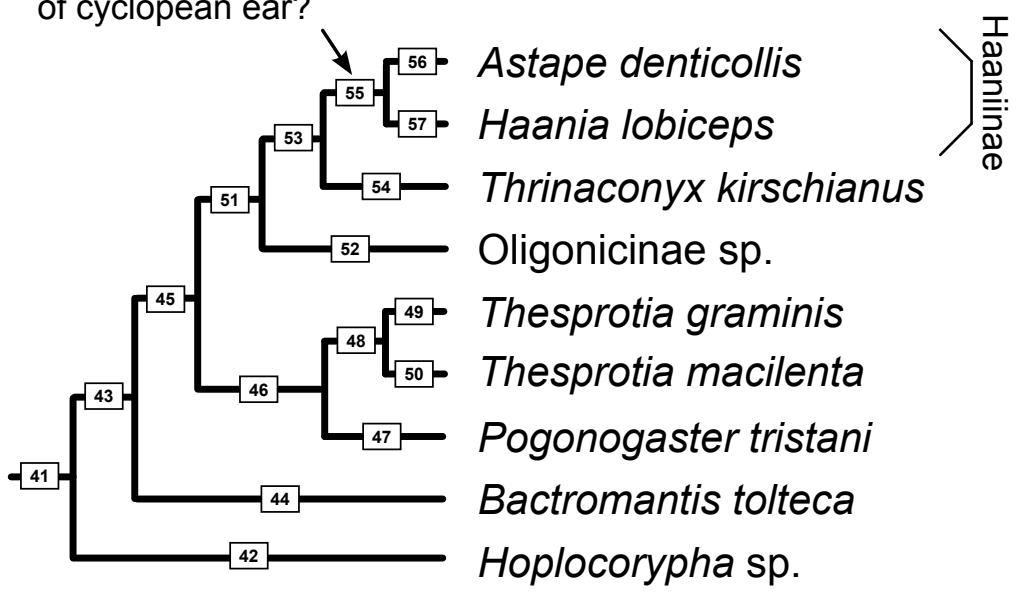

Fig. 398: Relationships of Thespidae found in analysis II. Haaniinae is nested within Thespidae. This scenario indicates a potential convergent gain of the metathoracic hearing organ (see chapters 4.2.23 and 4.6.14 for discussion). structure of the metathoracic ear and the high versatility of the foreleg morphology, a nonmonophyly of Oligonicinae and Haaniinae would be a more convincing assumption. The fore tibial morphology of Haania and Pogonogaster on the other hand is almost identical, and much more similar than the morphology among the remaining Oligonicinae (Figs. 163, 165, 247-254, 274, 275). The function of this conspicuous tibial morphology is unknown. Saussure \& Zehntner

(1894: 161) assumed that the foreleg morphology might indicate a specialization of very small prey, probably soft insects. This was also assumed by Beier (1964a: 874), who considered the forelegs in taxa such as Thesprotia functionally entirely unsuited for catching larger and strongly active prey ("größere, flüchtige Insekten") but instead assumed a specialization on small and slow prey ("kleine, wenig bewegliche Beute"), for instance aphids.

Further studies, mainly in the fields of postembryonic development, feeding ecology and habitat choice in both groups, are indispensable for providing a more detailed basis on which 
assessing putative convergences would be easier. On the other hand, a detailed anatomical and physiological study of the metathoracic hearing organ in Oligonicinae and Haaniinae may yield further support for its putative singular origin.

\subsubsection{4 (Pogonogaster + Thesprotia $)+($ Oligonicinae sp. $+($ Thrinaconyx + Haaniinae $))[45]$} This clade is was only found in analysis II (Fig. 398) and is supported by the unordered position of the antero-ventral tibial spines $(72: 2>4)$ and the lack of anal veins crossing the jugal lobe in the tegmina $(117: 1>2$; convergently evolved in few OTUs).

\subsubsection{Pogonogaster + Thesprotia [46]}

The sistergroup relationship between the two genera was found in analysis I and II. It is supported by the presence of 3 discoidal spines (55:4>3; convergently evolved in clade [14], Oxyothespis + Toxoderidae [188] and several OTUs) and the mesothoracic tibiae being longer than to the corresponding femora (85:2>1; convergently evolved in few OTUs).

\subsubsection{Oligonicinae sp. + (Thrinaconyx + Haaniinae) [51]}

This clade was found in analysis I and II. Its monophyly is supported by the lack of the proximal elongation of the euplantulae of the tarsomeres ta2-ta4 (94:1>2; convergently evolved in Eremiaphilidae [9]), the lack of the deep notch of the euplantulae of tarsomeres ta1-ta3 and the corresponding V-shape or spreading of the euplantulae $(95: 1>2$; convergently evolved in Ciulfina [35]), and the supra-anal plate being nearly as long as wide (132:3>2; convergently evolved in several OTUs).

\subsubsection{Thrinaconyx + Haaniinae [53]}

The sistergroup relationship between Thrinaconyx and Haaniinae was found in analysis I and II. It is supported by the presence of a torus intercervicalis on the posterior rim of the intercervicalia (24:2>1; convergently evolved in Metallyticus [4], Gyromantis [17] and Compsothespis [23]) and by the claw groove being located antero-ventrally in the middle of the femur length (37:2>3/4>3; convergently evolved in clade [162] and Stenopyga [132]).

\subsubsection{Haaniinae: Haania + Astape [55]}

Haaniinae was found to be monophyletic in analysis II and in both consensus cladograms of analysis I (Fig. 398). This relationship is supported by the following characters: Presence of processes arising from the area of the lateral ocelli (3:2>1; convergently evolved in Oxypilinae [81] and several OTUs); dorsal edge of the scutellum being more or less pointed (7:2>3; convergently evolved in Hestiasula + Oxypilinae [79], Empusidae [148] and Acromantis + Ambivia [197]); femoral brush being elevated (39:1 >2; convergently evolved in Eremiaphilidae [9], Paraoxypilinae [16], Perlamantinae [25] and several OTUs including the species of Metallyticus [4]); females having regained their wings (105:2>1; based on the present dataset this has only occurred in this group); both sexes having evolved the DK-type ("deep groove with knobs") of the metathoracic hearing organ from the MSMT-type ("meso- and metathoracic segments 
similar") of Oligonicinae [43] (males: 130:4>1; convergently evolved in Amorphoscelidae including Compsothespis [15], clade [58], and few OTUs; females: 131:4>1; convergently evolved in Hestiasula + Oxypilinae [79] and several OTUs); males with symmetrical subgenital plate (137:2>1; convergently evolved in Majanga [38] and Sibylla [127]).

Haaniinae is well supported by morphological data. The monophyly could not yet be addressed from a molecular perspective because Astape was not included (Svenson \& Whiting 2009). For a discussion of a potential close relationship between Haaniinae and Oligonicinae see chapters 2.1.4 (Thespidae) and 4.3.23.

\subsubsection{Clade [58]}

This clade has been found only in analysis II. Its monophyly is supported by the metathoracic hearing organ of the male originating from MSMT ("meso- and metathoracic segments similar") to DK ("deep groove with knobs") morphology (130:4>1; convergently evolved in Amorphoscelidae including Compsothespis [15], Haaniinae [55], and Stenomantis [36]).

\subsubsection{Tropidomantinae (= Tropidomantini): Enicophlebia + Tropidomantis [59]}

Tropidomantini was found to be monophyletic in analysis I and II. The following unambiguous apomorphic characters for Tropidomantinae were found: Transverse folding of the clypeus (12:1>2; convergently evolved in Eremiaphilidae [9] and several OTUs); lack of the anterodistal femoral spine on the meso- and metathoracic legs (82:1 $>2$; convergently evolved in clade [39] and several OTUs); secondary elongation of the wings in the females (106:2>1; convergently evolved in Amorphoscelinae + Perlamantinae [24], clade [75], clade [136] and several OTUs); supra-anal plate being longer than wide (132:1>3; convergently evolved in clade [39], Gyromantis [17], and Rivetina [117]); female supra-anal plate being triangular (136:3>2; convergently evolved in Idolomorphini [158] and several OTUs).

Only three species were studied in the present dataset. Svenson \& Whiting (2009: fig. 3b) sampled seven species (not including Enicophlebia) and found Tropidomantinae to be paraphyletic. For an overview see "Iridopterygidae" in chapter 2.1.4.

\subsubsection{Clade [64]}

This clade was only found in analysis II. It is not supported by any unambiguous apomorphies. Selected apomorphies found under ACCTRAN are: Presence of lateral tubercles on the head (13:2>1; convergently evolved in Amorphoscelidae including Compsothespis [15], clade [37], and several OTUs); fore tibiae being more than half as long as the femora $(67: 1>2$; convergently evolved in Tropidomantinae [59], Polyspilotini [171], Toxoderidae [190] and several OTUs); number of the postero-ventral fore tibial spines being less than one third smaller than the antero-ventral spines or equal (79:2>1; convergently evolved in Tropidomantinae [59]).

Apomorphies found under DELTRAN assumptions alone include the presence of 4 discoidal spines (55:3>4; convergently evolved in Eremiaphilidae [9], clade [37], and Acanthops [235]) and the presence of a medial euplantula on tarsomere ta5 (97:2>1; convergently evolved in Paraoxypilinae [16], clade [37], and several OTUs). 


\subsubsection{Humbertiella + Theopompa [65] ("South-East Asian Liturgusidae")}

This monophyletic group was found in analysis II and in both consensus cladograms of analysis I. In the strict consensus cladogram the relationships within Theopompa differed from the majority rule cladogram, which was identical to analysis II.

The sistergroup relationship between Humbertiella and Theopompa is supported by the prothorax being less than twice as long as wide (28:1>2; convergently evolved in a group within Chroicopterinae [110], a group within Hymenopodinae [204], and several OTUs; state 28:2 as an elongation from state 3 may be apomorphic for Mantodea as a whole; see discussion in chapter 4.3.1), by the close fitting of the dorsal edge of the fore femora with the lateral edges of the pronotum (35:1 $>2$; convergently evolved in Tarachodidae excluding Iris [89]), and the presence of lateral lobe-like expansions of the abdominal sternites in the female (147:2>1; convergently evolved in several OTUs).

Theopompa was not present in the molecular dataset of Svenson \& Whiting (2009), so this relationship could not be addressed therein. However, the relationship found herein strongly supports the evolution of a liturgusid ecomorph as hypothesized by Svenson \& Whiting (2009). See also the discussions in chapters 2.1.4 (Liturgusidae) and 4.3.19 (“Australian Liturgusidae").

\subsubsection{Clade [71]}

This clade was only found in analysis II. The only unambiguous apomorphy for this clade is the number of antennomeres in the females counting 70-89 (19:1>3; convergently evolved in Mantodea except for Chaeteessa and Metallyticus [6], Tropidomantinae [59], and several OTUs).

Among the apomorphies only found under ACCTRAN are the number of 4 discoidal spines (55:3>4; convergently evolved in Eremiaphilidae [9], clade [37], Humbertiella + Theopompa [65], and several OTUs), and the tarsomere of the metathoracic cursorial leg being as long as or shorter than the remaining tarsomeres (87:1>2; convergently evolved in Artimantodea (?) [8], Humbertiella + Theopompa [65], Hestiasula + Oxypilinae [79], and several OTUs).

Among the apomorphies found under DELTRAN alone are: Missing horizontal folding of the clypeus (12:1>2; convergently reduced in Eremiaphilidae [9], Tropidomantinae [59], and several OTUs); fitting of the eyes into the head outline (15:1>2; convergently evolved in Eremiaphilidae [9], Ceratocrania + Phyllocrania [138], and several OTUs); CuP having a sigmoid shape in the tegmina of the males (104:1>2; convergently evolved in clade [39] and several OTUs) and the females (107:1>2; convergently evolved in several OTUs); metathoracic ear of the female gaining DNK morphology (“deep groove, no knobs"; 131:1>2; convergently evolved in several OTUs).

\subsubsection{Clade [72]}

This clade was only found in analysis II. The only unambiguous apomorphy supporting it is the straight to slightly bent dorsal edge of the scutellum (7:2>1; convergently evolved in clade [39], Leptocola + Stenopyga [130], and many OTUs). 
No apomorphies were found under DELTRAN alone. Among those found under ACCTRAN alone are the following: Horizontal folding of the clypeus lacking (12:1>2; convergently reduced in Tropidomantinae [59], clade [118], and several OTUs); eyes fitting into the head outline (15:1>2; convergently evolved in Eremiaphilidae [9], clade [118], Ceratocrania + Phyllocrania [138], and several OTUs); groove between the proximal postero-ventral fore femoral spines lacking (53:1>2; convergently evolved in Thespidae [41], Chroicopterinae [108], Callibia + Acanthops [233], and several OTUs); CuP having a sigmoid shape in the tegmina of the males (104:1>2; convergently evolved in clade [39], clade [118], and several OTUs) and the females (107:1>2; convergently evolved in Paraoxypilinae [16], clade [122], Oxyothespis + Toxoderidae [188], and several OTUs); metathoracic ear of the female gaining DNK morphology (“deep groove, no knobs"; 131:1>2; convergently evolved in Paraoxypilinae [16], clade [118], and several OTUs).

\subsubsection{Gonypeta $+($ Gimantis $+($ Odontomantis $+($ Hestiasula + Oxypilinae $)))[73]$}

This clade was only found in analysis II. The only unambiguous apomorphy for this taxon is the number of cercomeres in the male counting 9-11 (151:3>2; convergently evolved in clade [14], clade [101], Acanthopidae [227], and several OTUs).

Among potential further apomorphies found only under DELTRAN are the lack of the groove between the proximal postero-ventral fore femoral spines $(53: 1>2$; convergently missing in Thespidae [41], Chroicopterinae [108], Acanthopidae [227], and several OTUs) and the lack of a median euplantula on tarsomere 5 of the cursorial legs $(97: 1>2$; convergently missing in Oligonicinae including Haaniinae [43], Tarachodidae excluding Iris [89], clade [195], and several OTUs).

\subsubsection{Gimantis + (Odontomantis + (Hestiasula + Oxypilinae)) [75]}

This clade was only found in analysis II. It is supported, for instance, by the secondary elongation of the wings in the females (106:2>1; convergently evolved in Amorphoscelinae + Perlamantinae [24], Tropidomantinae [59], clade [136] and several OTUs) and by the number of postero-ventral tibial spines being higher than that of the antero-ventral spines $(79: 1>3$; convergently evolved in Hymenopus + Theopropus [212] and several OTUs).

\subsubsection{Odontomantis $+($ Hestiasula + Oxypilinae $)[77]$}

This clade was found in analysis I and the majority rule consensus cladogram of analysis I. It is supported, among other characters, by the postero-ventral fore tibial spines being entirely laid down (76:1>3; convergently evolved in clades [77] and [196], and Phyllocrania [140]) CuP being straight in the tegmina of the male (104:2>1; convergently evolved in clade [77], clade [92] within Tarachodinae except for Iris, Acromantis + Ambivia [197], clade [208] within Hymenopodidae, and several OTUs) and the female (107:2>1; convergently evolved in clade [77], Idolomorphini [158], clade [208] within Hymenopodidae, and several OTUs). 


\subsubsection{Hestiasula + Oxypilinae [79]}

This clade was found in analysis II and in both strict consensus and majority rule cladogram of analysis I. It is also supported by molecular data (Svenson \& Whiting 2009: fig. 3d, node 225) where Hestiasula and Ephestiasula Giglio-Tos, 1915 were nested among Oxypilinae.

Among the apomorphic characters for this taxon are the following: Presence of a median vertical head process (1:2>1; with convergent evolution in Amphecostephanus [85], Pyrgomantis [93], Sibylla [127], Ceratocrania + Phyllocrania [138], Empusidae [148], Acromantis + Ambivia [197], clade [204] within Hymenopodinae, and Callibia [234] (see 4.6.1 for a discussion); presence of a ridge on the clypeus (11:2>1; convergently evolved in clade [136], Empusidae [148], and several OTUs); presence of a spine on the dorsal edge of the scutellum (7:2>3/1>3; convergently evolved in Haaniinae [55], Empusidae [148] and Acromantis + Ambivia [197]).

\subsubsection{Oxypilinae: Ceratomantis + Oxypilus [81]}

This group was also found in the molecular dataset of Svenson \& Whiting (2009: fig. 3d, node 225) where it also included Hestiasula and Ephestiasula. Its monophyly has been found in analysis I (strict and majority rule) and II. It is supported, for instance, by the presence of processes originating from the area of the lateral ocelli (3:2>1; convergently evolved in Haaniinae [55] and several OTUs), and by the presence of ridges on the scutellum (8:2>1; convergently evolved in Empusidae [148] and several OTUs).

\subsubsection{Amphecostephanus + (Elaea + (Tarachodinae except for Iris + Ameles + Pseudoyers- inia + Rivetinini + Chroicopterinae)) [84]}

This taxon was only found in analysis II. The only unambiguous apomorphy for this clade is the third anal vein in the alae of the males being branched once or twice (122:2>1; convergently evolved in clade [174], Acanthopidae [227] and several OTUs).

Further putative apomorphies were found under ACCTRAN alone: Enlarged euplantulae of tarsomere ta4 of the cursorial legs lacking (96:1>2; convergently evolved in Empusidae [148], Hoplocorypha [42], and Zoolea [168]); median euplantula on tarsomere ta5 of the legs present (97:2>1; convergently evolved in Paraoxypilinae [16], clade [37], Humbertiella + Theopompa [65], clade [118], and several OTUs); DO-shape of the metathoracic ear in the females (131:2>3; convergently evolved in clade [18] within Paraoxypilinae, clade [37], and Oxypilus [83]).

\subsubsection{Elaea + (Tarachodinae except for Iris + Ameles + Pseudoyersinia + Rivetinini + Chroicopterinae) [86]}

The group of taxa was found to be monophyletic in analysis II and in both consensus cladograms of analysis I, albeit with slightly different internal relationships.

Its monophyly is supported by the first anal vein in the tegmina not reaching the posterior wing margin (116:1>2; convergently evolved in clade [77] and several OTUs), and by the lack of a ridge on the supra-anal plate (133:1>2; convergently missing in Humbertiella + Theopompa [65], clade [77], Parasphendale + Sibylla [125], and several OTUs). 


\subsubsection{Tarachodinae except for Iris + (Ameles + Pseudoyersinia + Rivetinini + Chroicop- terinae) $[88]$}

This group of taxa was found to be monophyletic in analysis II and in both consensus cladograms of analysis I, albeit with slightly differing internal relationships.

Among the apomorphies found for this clade are the lack of lateral tubercles on the head (13:1>2; convergently evolved in several OTUs) and CuA in the alae of the females not being split (124:1>2; convergently evolved in clade [178] and several OTUs).

\subsubsection{Tarachodinae except for Iris: Paralygdamia + Pseudogalepsus + (Pyrgomantis $+($ Ta- rachodes + Tarachodula)) [89]}

Tarachodinae was found to be paraphyletic with respect to Iris in analysis I and II (Figs. 399A, B). The so-assumed monophyly of Tarachodidae could not be addressed because Caliridinae was not included in this study. The monophyly of the remaining taxa of the group was supported in the strict and majority rule consensus cladograms of analysis I, albeit the internal relationships were better resolved and therefore slightly different therein (Fig. 399A).

The following apomorphies were found for Tarachodinae except for Iris: Separate ventral cervical sclerites $(23: 1>2)$; prozona being less than half as long as the metazona $(31: 2>3$; convergently evolved in clade [33] and several OTUs); close fitting of the dorsal edge of the fore femora with the lateral edges of the pronotum (35:1>2; convergently evolved in Humbertiella + Theopompa [65]); overall flattened cerci (149:2>1; convergently evolved in clade [22], clade [180], and several OTUs including Chaeteessa valida [2]); distal cercomere strongly flattened without strong enlargement (150:1>2; convergently evolved in clade [180]).

These results do not corroborate the assignment of Iris to Tarachodidae as was proposed by Roy (1970: 1024; taken over by Ehrmann 2002: 375). Nonetheless, Iris was found to be closely related to some species of Tarachodinae (Dysaules, Episcopomantis, also comprising Heterochaeta) by Svenson \& Whiting (2009: fig. 3c, node 166; see chapter 2.1.4 on Tarachodidae).

\subsubsection{Pyrgomantis + (Tarachodes + Tarachodula $)[92]$}

This clade was found to be monophyletic in analysis II and in both consensus cladograms of analysis I, albeit with slightly differing internal relationships (Fig. 399). In the molecular analysis by Svenson \& Whiting (2009: fig. 3c, node 154), Pyrgomantis was nested among species of Tarachodes, Tarachodula and Paragalepsus (another genus of the traditional Tarachodinae), thus further supporting a potential close relationship of these genera.

The monophyly of this group is supported by the loss of the distinct alternating length pattern of the antero-ventral fore femoral spines (44:2>1; convergently reduced in Theopompa [67]), by the number of antero-ventral fore tibial spines counting 13-15 in the males (73:2>3; convergently evolved in Eremiaphilidae [9]) and in the females (74:2>3; convergently evolved in several OTUs), and the postero-ventral fore tibial spines counting 13-17 in the males (77:3>4; convergently evolved in Ceratocrania + Phyllocrania [138], clade [219], and several OTUs), and in the females (78:3>4; convergently evolved in clade [136] and several OTUs). 


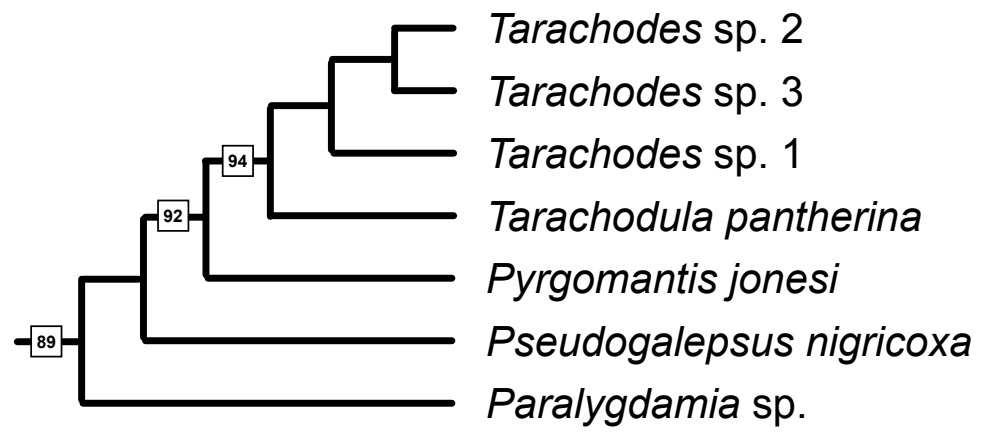

A

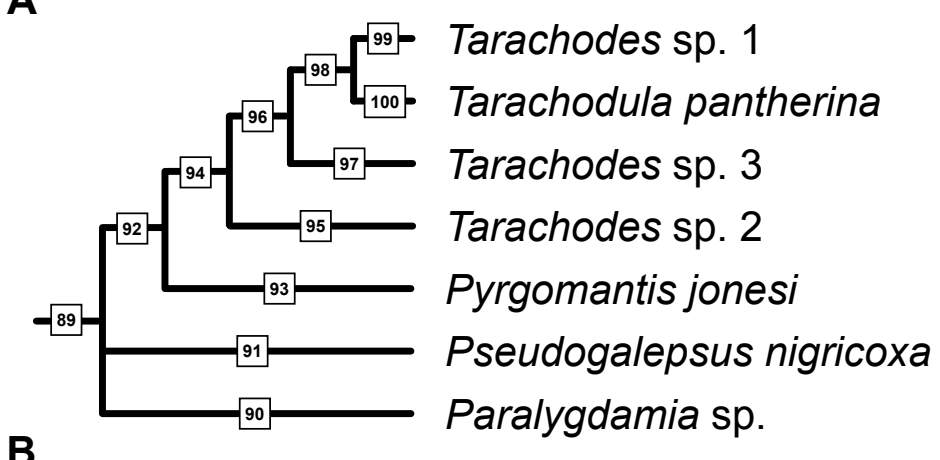

Figs. 399: Relationships found for Tarachodidae (excluding Iris). A: Relationships found in the strict and majority rule consensus cladograms of analysis I. B: Relationships found in analysis II.
4.3.45 Tarachodes sp. $2+$ (Tarachodes sp. $3+$ (Tarachodes sp. 1 + Tarachodula)) [94]

The clade was found to be monophyletic in analysis II and in both consensus cladograms of analysis I, albeit with slightly differing internal relationships (Fig. 399).

Tarachodes and Tarachodula were found in the same smaller monophyletic group in the molecular analysis (Svenson \& Whiting 2009: fig. 3c, node 154). However, two of the Tarachodes species therein were more closely related to Pyrgomantis and Paragalepsus than to a third Tarachodes species and Tarachodula. Therefore the results found herein are not identical to the molecular findings.

Apomorphic characters for this taxon are the prozona being slightly shorter up to half as long as the metazona (31:3>2; convergently evolved in clade [202] within Hymenopodidae) and the presence of a ridge on the supra-anal plate (133:2>1; convergently evolved in Rivetinini [115], Empusidae [154], clade [184], and several OTUs).

\subsubsection{Ameles decolor + (Pseudoyersinia + (Ameles gracilis + (Rivetinini + Chroicopteri- nae))) [101]}

This taxon was found with identical internal relationships in analysis I (both consensus cladograms) and analysis II (Fig. 400).

Among its apomorphic characters are the following: Number of antennomeres in the female counting 50-96 (19:3>2; convergently evolved in clade [136], Empusinae [154], clade [204] within Hymenopodinae, and several OTUs); crossveins between the Cubital veins CuA and $\mathrm{CuP}$ in the alae being straight or slightly curved (128:1>2; convergently evolved in Oligonicinae including Haaniinae [43], Dystacta + Taumantis [119], clade [124], clade [178], and several OTUs); 9-11 cercomeres present in the males (151:3>2; convergently evolved in clade [14], clade [73], Acanthopidae [227], and several OTUs) and females (152:3>2; convergently evolved in clade [14] and several OTUs).

\subsubsection{Pseudoyersinia + (Ameles gracilis + (Rivetinini + Chroicopterinae) [103]}

This taxon was found with identical internal relationships in analysis I (both consensus cladograms) and analysis II. 


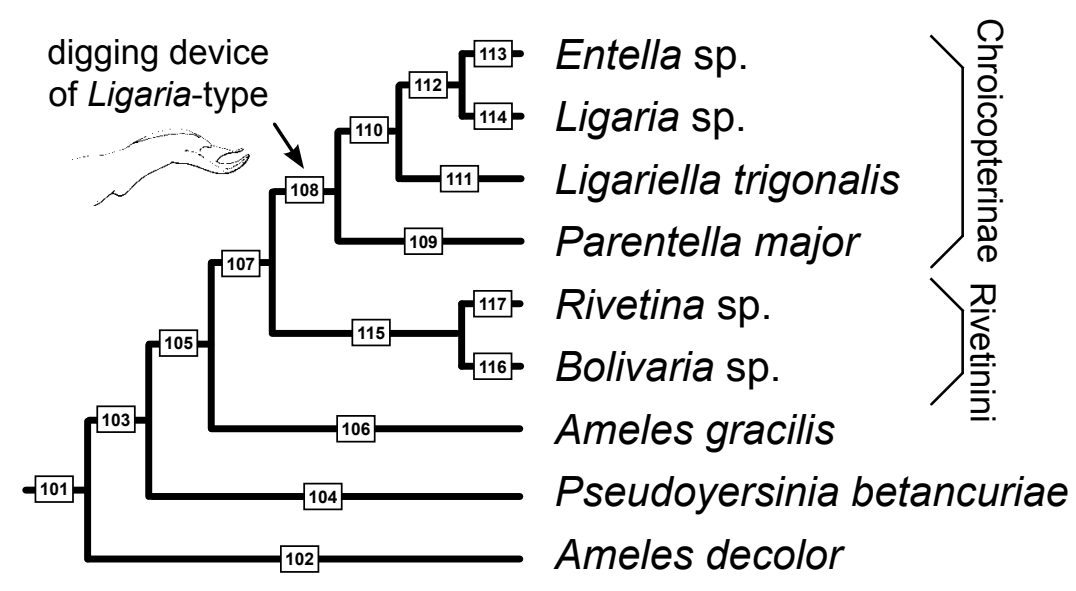

Fig. 400: Relationships of clade [101] found in analysis II. This taxon contains Amelinae (partim), Chroicopterinae and Rivetinini. See text for discussions on the evolution of female digging structures.

Apomorphic characters for this taxon are the presence of 50-69 antennomeres in the males $(18: 3>2$; convergently evolved in clade [196] and several OTUs) and the reduction of the wing length in the males (101:1>2; convergently evolved in Eremiaphilidae[9], Ciulfina+Stenomantis [34], Leptocola + Stenopyga [130], and several OTUs).

\subsubsection{Ameles gracilis + (Rivetinini + Chroicopterinae) [105]}

This taxon was found with identical internal relationships in analysis I (both consensus cladograms) and analysis II.

The only apomorphy supporting this relationship is the basitarsomere on the forelegs being longer than the remaining tarsomeres taken together (80:2>1; convergently evolved in Pyrgomantis [93]).

\subsubsection{Rivetinini + Chroicopterinae [107]}

This taxon was found with identical internal relationships in analysis I (both consensus cladograms) and analysis II.

The monophyly of this group is supported by the secondary origin of ventral cervical sclerites $(21: 2>1)$ and by the number of branches of the anal veins in the alae of the females counting between 5 and 8 (125:1>2; convergently evolved in several OTUs).

\subsubsection{Chroicopterinae (= Chroicopterini): Parentella + (Ligariella $+($ Entella + Ligaria)) [108]}

This group was found with identical internal relationships in analysis II and both consensus cladograms of analysis I (Fig. 400).

The monophyly of the Chroicopterinae studied herein is well supported by the autapomorphic bipartite distal hooks on the ventral valvulae (= gonapophyses VIII) (144:2>1). Further characters are the lack of the ventral depression between the proximal postero-ventral femoral spines (53:1>2; convergently reduced in Thespidae [41] and several OTUs), and a secondary elongation of the wings in the males $(101: 2>1)$.

The name bearing genus for Chroicopterinae is Chroicoptera. In this genus, too, the females possess a structure that probably serves for digging in the soil ("Chroicoptera-type"; see Wieland 2008 b and chapter 4.6.9; Figs. 36, 377). However, it differs strongly from the "Ligaria-type" 
exhibited by the Chroicopterinae studied herein (see description of character 144 and chapter 4.6.9). In the molecular results Chroicoptera was found to be more closely related to some Dystactinae and Amelinae than to the remaining Chroicopterinae, while the greater part of the remaining Chroicopterinae (the species with hooks on the valvulae) formed a monophyletic group (Svenson \& Whiting 2009: 3b, clades 55, 59). This indicates a potential non-monophyly of the traditional Chroicopterinae. Nonetheless, all Chroicopterinae sampled in the molecular analysis were found in the same larger Afrotropical clade together with species of Amelinae, Dystactinae and Hapalomantinae (Svenson \& Whiting 2009: fig. 3b, clade 50). Whether or not Chroicopterinae is monophyletic will have to be further studied in the future. But if they are, those species with distal hooks on the valvulae are more closely related to each other than to Chroicoptera.

\subsubsection{Ligariella $+($ Entella + Ligaria $)[110]$}

This taxon was found with identical internal relationships in analysis I (both consensus cladograms) and analysis II. It was also found in the molecular analysis by Svenson \& Whiting (2009: fig. 3b, clade 64), although with slightly different internal relationships.

The monophyly of this group is supported by the pronotum being less than twice as long as wide or square shaped (28:1>2; but possibly apomorphic for Mantodea as a whole, see 4.3.1; convergently evolved in Humbertiella + Theopompa [65], clade [204] among Hymenopodinae, and several OTUs), and by the number of the postero-ventral fore tibial spines being more than one third lower than the antero-ventral spines (79:1 $>2$; convergently evolved in clade [124], Polyspilotini [171], clade [174], and several OTUs).

\subsubsection{Entella + Ligaria [112]}

This taxon was found with identical internal relationships in analysis I (both consensus cladograms) and analysis II and is supported by the basitarsomere of the forelegs being as long as or shorter than the remaining tarsomeres taken together $(80: 1>2$; convergently evolved in Perlamantinae [25], Ciulfina + Stenomantis [34], Oxyothespis + Toxoderidae [188], and Leptocola [131]).

\subsubsection{Rivetinini: Bolivaria + Rivetina [115]}

Rivetinini was found in analysis I and II (strict and majority rule consensus).

The monophyly of Rivetinini is supported by the following characters: Horizontal folding of the clypeus (12:2>1; convergently evolved in Oxyothespis + Toxoderidae [188], clade [196], and several OTUs); course of the main veins in the tegmina sigmoid [111:2>1; convergently evolved in clade [164], Hymenopus + Theopropus [212], and many OTUs); median ridge present on the supra-anal plate (133:2>1; convergently evolved in a part of Tarachodinae except for Iris [94], Empusinae [154], clade [184], and several OTUs).

The monophyly of Rivetinini (albeit neither including Rivetina nor Bolivaria) was supported by the molecular data (Svenson \& Whiting 2009: fig. 3c). 


\subsubsection{Clade [118]}

This clade was found in analysis II alone. The only unambiguous apomorphy supporting this relationship is the length pattern of the discoidal spines ( $57: 3>1$; convergently evolved in clade [118] and several OTUs).

Among the apomorphic characters found only under ACCTRAN are the following: Eyes fitting into the head outline $(15: 1>2$; convergently evolved in Eremiaphilidae [9], Ceratocrania + Phyllocrania [138], and several OTUs); medial euplantula present on tarsomere ta5 (97:2>1; convergently evolved in Paraoxypilinae [16], clade [37], Humbertiella + Theopompa [65], clade [84], and several OTUs); CuP of the tegmina being sigmoid in the males (104:1>2; convergently evolved in clade [39], clade [72], and several OTUs); DNK morphology of the metathoracic ear in the females (131:1>2; convergently evolved in Paraoxypilinae [16], clade [72], and several OTUs).

Among those apomorphies found under DELTRAN alone are the lack of lateral tubercles on the vertex (13:1>2; convergently evolved in Ciulfina + Stenomantis [34], clade [88], and several OTUs) and the prozona being less than half as long as the metazona (31:2>3; convergently evolved in clade [33], Tarachodinae except for Iris [89], and several OTUs).

\subsubsection{Dystacta + Taumantis [119]}

This relationship was only found in analysis II. The only unambiguous apomorphy supporting this sistergroup relationship is the straight to slightly curved course of the cross veins between the Cubital veins $\mathrm{CuA}$ and $\mathrm{CuP}$ in the alae (128:1>2; convergently evolved in Oligonicinae including Haaniinae [43], clade [101], clade [124], clade [178], and several OTUs).

\subsubsection{Clade [122]}

This clade was found in analysis II alone and is not supported by any unambiguous apomorphies.

Among the apomorphies found under ACCTRAN alone are following characters: Prozona being less than half as long as the metazona (31:2>3; convergently evolved in clade [33], clade [46], Tarachodinae except for Iris [89], Dystacta + Taumantis [119] and several OTUs); $\mathrm{CuP}$ in the tegmina of the female having a sigmoid shape $(107: 1>2$; convergently evolved in Paraoxypilinae [16], clade [72], Oxyothespis + Toxoderidae [188], and several OTUs); number of anal veins in the alae of the males being higher than 8 (121:2>3; convergently evolved in Amorphoscelinae [28], and Dystacta + Taumantis [119], and several OTUs).

The apomorphies found under DELTRAN assumptions alone encompass a number of 13-15 antero-ventral fore tibial spines in the male (73:2>3; convergently evolved in Eremiaphilidae [9] and clade [92]) and in the female (74:2>3; convergently evolved in clade [92] and several OTUs), as well as the number of anal veins in the alae of the female being higher than 8 (125:2>3; convergently evolved in Amorphoscelinae [28]). 
4.3.57 $(($ Parasphendale + Sibylla $)+($ Euchomenella $+($ Leptocola + Stenopyga $)))+($ Popa + $($ Vates $+($ Ceratocrania + Phyllocrania $)))[123]$

This relationship was only found in analysis II. It is supported by the eyes protruding laterally from the head capsule (15:2>1; convergently evolved in clade [178] and several OTUs) and by the lobes on the femora of the cursorial legs being located along the entire length or forming a single large lobe (91:3>4; convergently evolved in Hymenopus [213]).

\subsubsection{8 (Parasphendale + Sibylla $)+($ Euchomenella $+($ Leptocola + Stenopyga $))[124]$}

This relationship was only found in analysis II. Among the apomorphies for this taxon are the number of postero-ventral fore tibial spines in the female counting 4-7 (78:3>2; convergently evolved in Chroicopterinae [108] and clade [184]), the number of postero-ventral fore tibial spines being more than one third smaller than that of the antero-ventral spines $(79: 1>2$; convergently evolved in clade [110] within Chroicopterinae, Polyspilotini [171], clade [174], and several OTUs), and the straight or slightly bent shape of the crossveins between the $\mathrm{Cu}$ bital veins $\mathrm{CuA}$ and $\mathrm{CuP}$ in the alae (128:1>2; convergently evolved in Oligonicinae including Haaniinae [43], clade [101], Dystacta + Taumantis [119], clade [178], and several OTUs).

\subsubsection{Parasphendale + Sibylla [125]}

The sistergroup relationship between these two genera was only found in analysis II. It is supported by the following characters: Postero-ventral fore tibial spines in the males counting 4-7 (77:3>2; convergently evolved in Chroicopterinae [108] and several OTUs); ridge on the supra-anal plate lacking (133:1>2; convergently missing in Humbertiella + Theopompa [65], clade [77], clade [86] and several OTUs); number of cercomeres counting 16-19 in the male (151:3>4; convergently evolved in clade [143], clade [162] and several OTUs) and 20-22 in the female (152: 3>5; convergently evolved in clade [167] and several OTUs).

\subsubsection{Angelinae (= Angelini): Euchomenella + (Leptocola + Stenopyga) [128]}

Angelinae was found to be monophyletic only in analysis II. It is supported by the claw groove being located distal of the middle of the femur (37:2>4; convergently evolved in Thespidae [41], Idolomorphini [158] and Ceratocrania [139]) and the fore tibia being half as long as the femur or shorter (67:2>1; convergently evolved in Amorphoscelidae including Compsothespis [15], clade [162], and several OTUs).

The molecular data supported the sistergroup relationship between Stenopyga and Leptocola [130] but showed Euchomenella (and Indomenella Roy, 2008) to be more closely related to Deroplatys than to Stenopyga and Leptocola (Svenson \& Whiting 2009: fig. 3d, clades 257, 260, 261).

\subsubsection{Leptocola + Stenopyga $[130]$}

This taxon was only found in analysis II and is supported by the straight to slightly rounded dorsal edge of the scutellum (7:2>1; convergently evolved in clade [39], clade [72] and several 
OTUs) and by the shortened wings in the males (101:1>2; convergently evolved in Eremiaphilidae [9], Ciulfina + Stenomantis [34], clade [103], and several OTUs).

The sistergroup relationship between Stenopyga and Leptocola [130] found support in the molecular analysis by Svenson \& Whiting (2009: fig. 3d, clade 257). It was not found in analysis I of the present study.

\subsubsection{Popa $+($ Vates $+($ Ceratocrania + Phyllocrania $))[133]$}

This taxon was only found in analysis II. It is supported by the following apomorphies: Tibia of the metathoracic leg of the male as long as or shorter than the femur $(88: 1>2$; convergently evolved in Ciulfina + Stenomantis [34], Haaniinae [55], Hymenopodinae [200], and several OTUs); presence of lobe-like expansions on the meso- and metathoracic femora (90:2>1; convergently evolved in clade [150] within Empusidae, clade [196], and several OTUs) and on the tibiae (92:2>1; convergently evolved in Zoolea [168]); asymmetrical tibiae (93:1>2; convergently evolved in Hymenopodinae [200] and several OTUs); A3 in the alae of the females branching once or twice (126:2>1; convergently evolved in clade [174], clade [231], and several OTUs).

\subsubsection{Vates + (Ceratocrania + Phyllocrania $)[136]$}

The monophyly of this clade was found in the majority rule consensus cladogram of analysis I, albeit with Phyllocrania as the sistergroup of the two other taxa.

It is supported by the following characters: Ridge on the clypeus present (11:2>1; convergently evolved in Hestiasula + Oxypilinae [79], Empusidae [148] and several OTUs); antennomeres slightly asymmetrical (= antenna imbricata, see description of character 20 in chapter 4.1.1) in the male (20:1>2; convergently evolved in clade [202] within Hymenopodinae and several OTUs); metathoracic tibiae being as long as the femora or shorter in the females (89:1>2; convergently evolved in Amorphoscelinae [28], Hymenopodinae [200], and several OTUs), the secondary elongation of the wings in the females (106:2>1; convergently evolved in Amorphoscelinae + Perlamantinae [24], Tropidomantinae [59], clade [75], and several OTUs); A3 in the alae of the males being branched five times or more (122:1>3/2>3; convergently evolved in clade [143], Stagmatoptera + Polyspilotini [169], Idolomantis [153] and Ambivia [199]).

\subsubsection{Ceratocrania + Phyllocrania $[138]$}

This relationship was only found in analysis II. It is supported, for instance, by the presence of a vertical head process (1:2>1; convergently evolved in Hestiasula + Oxypilinae [79], Amphecostephanus [85], Pyrgomantis [93], Sibylla [127], Empusidae [148], Acromantis + Ambivia [197], clade [204] within Hymenopodinae, and Callibia [234]) and by the eyes fitting into the head outline (i.e. not strongly protruding) (15:1>2; convergently evolved in Eremiaphilidae [9], Calamothespis [191], and Theopropus [214]). 


\subsubsection{Clade [141]}

This clade was only found in analysis II. It is not supported by any unambiguous apomorphies. Among the apomorphies found under ACCTRAN assumptions are the following: Lateral tubercles of the head lacking (13:1>2; convergently reduced in clade [88], Dystacta + Taumantis [119], clade [124], Ceratocrania + Phyllocrania [138] and several OTUs); first anal vein in the tegmina vanishing early and not reaching the posterior margin of the wing (116:1>2; convergently evolved in Oligonicinae including Haaniinae [43], clade [77], clade [86], clade [123], and several OTUs); number of anal veins in the alae of the female counting more than 8 (125:2>3; convergently evolved in Amorphoscelinae [28] and clade [123]).

Among the apomorphies found under DELTRAN alone are the short wings of the females (106:1>2; convergently evolved in Artimantodea (?) [8], clade [180], clade [221], and Gongylus [155]) and the metathoracic ear in the female exhibiting DK-morphology ("deep groove with knobs"; 131:2>1; convergently evolved in clade [14], Haaniinae [55], Hestiasula + Oxypilinae [79], and several OTUs).

\subsubsection{6 (Sphodromantis + (Rhombodera + Tamolanica $))+$ Empusidae [142]}

This taxon was only found in analysis II and is supported by the organization of the discoidal spines (57:1>2; convergently evolved in clade [178], clade [65], and several OTUs) and the presence of a serrate antero-ventral edge of the tegmina (110:2>1).

Giglio-Tos (1912: 59) applied the serration of the anterior wing margin as a discrimination character for separating the genera Sphodromantis, Hierodula and Parhierodula. This was doubted by Hebard (1920: 51-53) because the character is inconsistent within the genera or even within the same species group (Hebard 1920: 52). This led to several taxonomic changes of the genera introduced by Giglio-Tos (1912). Hebard's main argument against the value of this character was that "the tegminal features are found in the adult condition only and for that reason we feel obliged to consider them of secondary value" (Hebard 1920: 52). This statement is hardly tenable because, if consistently applied, this would imply that not a single wing character or structure of the genitalia is valuable for taxonomical or phylogenetic studies since neither of these characters is present in nymphs. This is certainly not the case. However, as intrageneric variation of the character has been stated in the literature, the sistergroup relationship of clade [143] with Empusidae is debatable. It has to be treated with care and needs further study.

\subsubsection{Sphodromantis + (Rhombodera + Tamolanica $)[143]$}

The monophyly of this clade was found identically in both consensus cladograms of analysis I and in analysis II. It is supported, for instance, by the presence of rounded compound eyes (14:2>3; convergently evolved in many OTUs including Chaeteessa valida [2]) and by the branchings of A3 in the alae of the males counting 5 or more (122:2>3; convergently evolved in clade [136], Stagmatoptera + Polyspilotini [169], Idolomantis [153], and Ambivia [199]). 


\subsubsection{Rhombodera + Tamolanica [145]}

This sistergroup relationship was found in both consensus cladograms of analysis I and in analysis II. A close relationship between the two genera is also supported by the molecular data (Svenson \& Whiting 2009: fig. 3e), although therein the two genera were nested in a clade among several Hierodula species (not included in the present dataset).

The following apomorphies support this relationship: Wide lateral lamellar expansions of the pronotum present (29:1>2; convergently evolved in clade [150] within Empusidae, Pogonogaster [47], Phyllocrania [140], Deroplatys [175], Pseudocreobotra wahlbergii [207], and Choeradodis [207]); A3 in the alae of the females with 5 or more branches (126:2>3; convergently evolved in Stagmatoptera + Polyspilotini [169], Hymenopus + Theopropus [212], and several OTUs).

\subsubsection{Empusidae: Blepharodes + (Blepharopsis + (Idolomantis + Empusinae)) [148]}

Empusidae was found to be monophyletic in analysis I and II (Fig. 401). In the strict consensus cladogram of analysis I the internal relationships were slightly different from those in the majority rule cladogram and analysis II. Interestingly, Blepharodinae was found to be paraphyletic in all cases.

The monophyly of Empusidae is supported by the following characters: Vertical head process present (1:2>1; convergently evolved in Hestiasula + Oxypilinae [79], Ceratocrania + Phyllocrania [138], Acromantis + Ambivia [197], clade [204] within Hymenopodinae, and several OTUs); spine on the dorsal edge of the scutellum present (7:2>3; convergently evolved in Haaniinae [55], Hestiasula + Oxypilinae [79], and Acromantis + Ambivia [197]); ridges on the scutellum present (8:2>1; convergently evolved in Oxypilinae [81] and several OTUs); ridges on the clypeus present (11:2>1; convergently evolved in Hestiasula + Oxypilinae [79], clade [136] and several OTUs); presence of antenna lobata in the males (20:1>5); antero-distal apical lobes of the coxa overlapping (33:2>3/1>3; convergently evolved in Hymenopus + Theopropus [212]

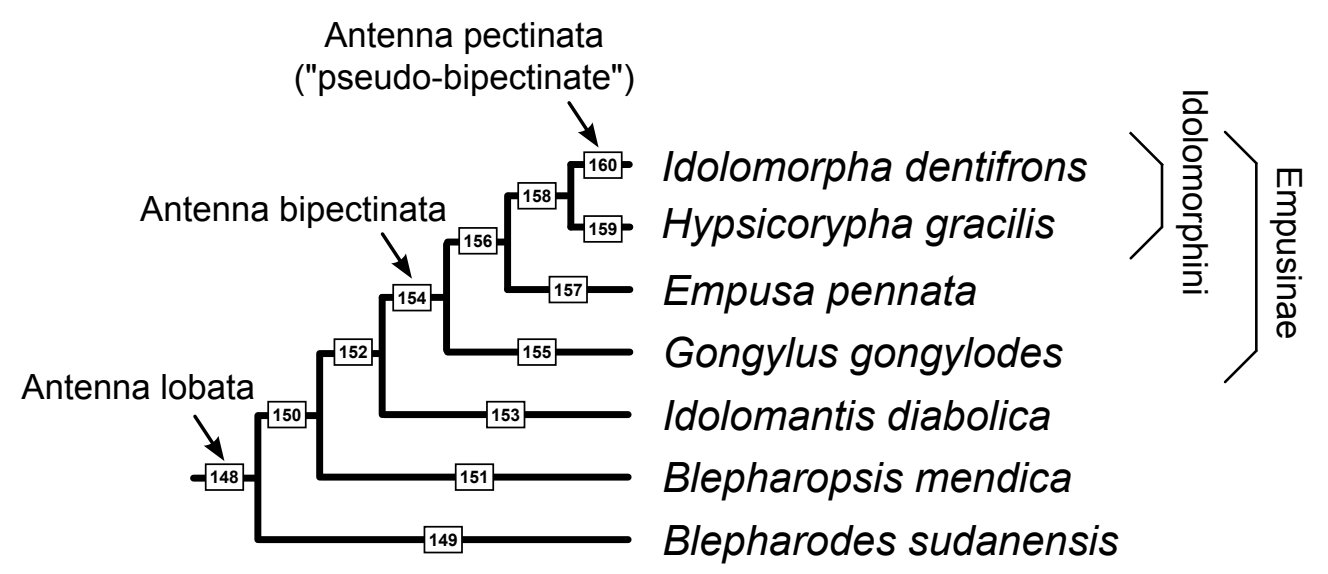

Fig. 401: Relationships of Empusidae found in analysis II. Blepharodinae is paraphyletic with respect to Idolomantis. Evolution of male antennomeres is indicated. 
and several OTUs); antero-ventral femoral spines being organized in the typical empusid 1-3-1 length pattern $(44: 2>3)$; position of the postero-ventral femoral spines on a ridge (49:1>2; convergently evolved in Hymenopus + Theopropus [121]); expansion of the antero- and posteroventral edges of the fore tibiae present (71:1>2; convergently evolved in clade [204] within Hymenopodinae).

The monophyly of Empusidae is well supported by molecular data (Svenson \& Whiting 2009: fig. 3d, clade 195). Therein, each of the subgroups, Blepharodinae and Empusinae, were found to be monophyletic (Svenson \& Whiting 2009: fig. 3d, clades 197, 197). The monophyly of each of each subgroup was also assumed by Roy (2004a: fig. 15). My findings suggest that Idolomantis is more closely related to Empusinae than to Blepharodes and Blepharopsis, thus rendering Blepharodinae paraphyletic. This allows a new hypothesis explaining the polarity of the evolution of empusid male antennomeres (see discussion in chapter 4.6.2 and Fig. 401).

\subsubsection{Blepharopsis + (Idolomantis + Empusinae) [150]}

This relationship within Empusidae was found identically in analysis II and the majority rule consensus cladogram of analysis I, whereas in the strict consensus it was monophyletic but not completely resolved.

This taxon is supported by the following characters: Lamellar expansions of the pronotum being wider than half the width of the pronotum (29:1>2; convergently evolved in Pogonogaster [47], Phyllocrania [140], Rhombodera + Tamolanica [145], Deroplatys [175], Pseudocreobotra wahlbergii [207], and Choeradodis [207]); antero-ventral fore tibial spines counting 20-25 in the males (73:3>5; convergently evolved in Heterochaeta [185]) and in the females (74:3 > 5; convergently evolved in Toxodera denticulata [193] and Hymenopus [213]); meso- and metathoracic femora with lobe-like expansions (90:2>1; convergently gained in clade [133], clade [196], and several OTUs); abdominal tergites in the female with lateral lobe-like expansions (145:2>1; convergently evolved in several OTUs), and the ventral lobe-like expansions of the abdominal sternites in the female (146:2>1; convergently evolved in several OTUs).

\subsubsection{Idolomantis + Empusinae [152]}

This taxon was found in analysis I and II, although it was not resolved in the strict consensus cladogram of analysis I. It is well supported by the lanceolate elongation of the ventral anterodistal apical lobe of the fore coxae $(34: 2>1)$. Furthermore, the postero-ventral femoral spines are strongly elongated in this taxon (50:1>2; convergently evolved in Chaeteessa [2], Stenomantis [36], Sibylla [127] and Euchomenella [129]) and the number of the postero-ventral tibial spines of the males exceeds 22 (77:5>6/3>6; convergently increased in Hymenopus [213] and Acanthops falcataria [236]).

This relationship is also supported by the prothorax being rather short in Blepharodes [149] and Blepharopsis [151], whereas it is distinctly longer in the remaining Empusidae. Also, the forelegs (not including the enormous lamellar enlargements) are very slender in Idolomantis (Figs. 133, 211-213) and resemble the forelegs of Empusinae (Fig. 214) rather than those of 
Blepharopsis (Figs. 132) or Blepharodes. This is also true for the foreleg spination (Figs. 208214). For a discussion see chapter 4.6.2.

\subsubsection{Empusinae: Gongylus + (Empusa + Idolomorphini) [154]}

Empusinae is well supported by the dorsal process of the male subgenital plate $(138: 2>1)$, as has already been mentioned in chapter 2.1.4. Further apomorphies of Empusinae are the bipectinate male antennomeres evolving from the antenna lobata-type (which is apomorphic for Empusidae as a whole) $(20: 5>4)$ and the presence of a ridge on the supra-anal plate (133:2>1; convergently evolved in a part of Tarachodinae except for Iris [94], Rivetinini [115], clade [184], and several OTUs).

Empusinae was found in analysis I (only majority rule) and II. For a discussion of the evolution of the male antennomeres see chapter 4.6.2 and Fig. 401.

\subsubsection{Empusa + Idolomorphini [156]}

This taxon was found in analysis I (only majority rule) and II. In the molecular analysis by Svenson \& Whiting (2009: fig. 3d, clade 197), Idolomorpha came out as the sistergroup of Gongylus + Empusa.

This relationship is supported by the narrowing of the lateral expansions of the pronotum $(29: 2>1)$ and the number of postero-ventral tibial spines of the males counting 18-21 (77:6>5; convergently evolved in Pseudocreobotra wahlbergii [207] and Callibia [234].

\subsubsection{Idolomorphini: Hypsicorypha + Idolomorpha [158]}

The monophyly of Idolomorphini was found in analysis I (strict and majority rule consensus cladograms) and analysis II.

Idolomorphini is supported by the following characters: Claw groove positioned in the distal half of the femur [37:3>4/2>4; convergently evolved in Thespidae [41] (with a reversal in Thrinaconyx + Haaniinae [53]), Angelinae [128] and Ceratocrania [139]]; expansions of the antero- and postero-ventral fore tibial edges lacking (71:2>1); lobes on the meso- and metathoracic femora lacking $(90: 1>2)$; shape of the supra-anal plate in the females nearly triangular (136:3>2; convergently evolved in Tropidomantinae [59] and several OTUs); lateral lobe-like expansions of the abdominal tergites in the females lacking $(145: 1>2)$; ventral lobe-like expansion of the abdominal sternites in the female lacking (146:1>2).

The potential monophyly of Idolomorphini could not be addressed in the molecular analysis by Svenson \& Whiting (2009: fig. 3d) because Hypsicorypha was not included in their dataset.

Roy (2004a: 2) stated that Ehrmann (2002: 378) assigned these two genera to Idolomorphini based on the lack of the lobes on the legs. However, he (Roy) rejected this assumption because the lack of the lobes is a reduction character ("Je rejette ici ce découpage, basé sur un charactère négatif pour les deux premiers genres [Idolomorpha and Hypsicorypha], bien différents par leurs autres charactères, en particulier par les proportions du prolongement $d u$ vertex et la morphologie des pattes antérieures.”). In Roy's new sense Idolomorphini was suggested to 
consist of Chopardempusa + (Idolomorpha + Hemiempusa) (see Roy 2004a: 12 and fig. 24; Fig. 27 herein). The close relationship of Idolomorpha and Hemiempusa is also supported by further characters (Roy 2004a: 2).

Nonetheless, the monophyly of Idolomorphini sensu Ehrmann (2002) is supported by the present dataset. It will be important to include Hemiempusa and Chopardempusa in future studies.

\subsubsection{Clade [161]}

This clade was exclusively found in analysis II. The monophyly is not supported by any unambiguous apomorphies.

Apomorphies found under ACCTRAN alone are the macroptery of the females $(106: 2>1$; convergently evolved in Amorphoscelinae + Perlamantinae [24], Tropidomantinae [59], clade [75], clade [136], clade [142], clade [161], clade [231], and several OTUs), and the DK morphology ("deep groove with knobs") of the metathoracic ear in the females (131:2>1; convergently evolved in Amorphoscelidae including Compsothespis [15], clade [32], Haaniinae [55], Hestiasula + Oxypilinae [79], clade [142] and clade [162]).

The only apomorphy found under DELTRAN assumptions alone is the presence of two ridges on the scutellum $(9: 1>2)$.

\subsubsection{6 (Mantis $+[164])[162]$}

This clade was only found in analysis II. Among others, its monophyly is supported by the following characters: Position of the claw groove between the proximal third of the femur and its middle (37:2>3; convergently evolved in Thrinaconyx + Haaniinae [53] and Stenopyga [132]); organization of discoidal spines (57:1>6; convergently evolved in Hestiasula + Oxypilinae [79], Rivetinini [115], and Idolomantis [153]); fore tibiae being half as long as the femora or shorter (67:2>1; convergently evolved in Amorphoscelidae including Compsothespis [15], Angelinae [128], and several OTUs).

\subsubsection{Clade [164]}

This clade was only found in analysis II. It is supported by the number of antennomeres in the female counting 110-129 (19:3>5; convergently evolved in Metallyticus [4], Humbertiella + Theopompa [65], and Orthoderella [226]) and by the partially sigmoid course of the main veins in the tegmina (111:2>1; convergently evolved in Rivetinini [115], Hymenopus + Theopropus [212], and several OTUs including the species of Metallyticus [4]).

\subsubsection{Tisma $+($ Zoolea $+($ Stagmatoptera + Polyspilotini) $)$ [165]}

This clade was only found in analysis II. It finds support in the organization of the discoidal spines (57:6>4; convergently evolved in Haaniinae [55], Vates [137] and Sphodromantis [144]) and the basitarsomere of the metathoracic legs being longer than the remaining tarsomeres 
taken together (87:2>1; convergently evolved in Paraoxypilinae [16], Amorphoscelinae [28], clade [33], Empusinae [154], and several OTUs including Chaeteessa valida [2]).

\subsubsection{Zoolea + (Stagmatoptera + Polyspilotini) [167]}

This clade was only found in analysis II. It is supported by the converging antero-distal forecoxal lobes (33:1>2; convergently evolved in clade [167], clade [204] within Hymenopodinae and several OTUs) and by the number of cercomeres in the females counting 20-22 (152:4>5; convergently evolved in Parasphendale + Sibylla [125], clade [167], and several OTUs).

\subsubsection{Stagmatoptera + Polyspilotini [169]}

This clade was only found in analysis II. It is supported by the number of branchings of A3 in the alae counting 5 or more in the males (122:2>3; convergently evolved in clade [136], clade [143], Stagmatoptera + Polyspilotini [169], Idolomantis [153], and Ambivia [199]) and in the females (126:2>3; convergently evolved in Rhombodera + Tamolanica [145], Stagmatoptera + Polyspilotini [169], Hymenopus + Theopropus [212], and several OTUs).

\subsubsection{Polyspilotini: Plistospilota + Polyspilota [171]}

Polyspilotini was found in analysis I (majority rule consensus cladogram only) and analysis II. The close relationship of the two genera is supported by molecular data (Svenson \& Whiting 2009: fig. 3e), albeit they also included Prohierodula Bolivar, 1908, which was not sampled in the present dataset.

The monophyly of Polyspilotini is supported by the following characters: Position of the claw groove between the middle of the femur and its proximal third (37:3>2; convergently evolved in Eremiaphilidae [9], Toxoderidae [190] and Oxyopsis [179]); fore tibia being more than half as long as the fore femur (67:1>2; convergently evolved in Toxoderidae [190]); number of postero-ventral fore tibial spines being a third or more lower than the number of antero-ventral fore tibial spines $(79: 1>2$; convergently evolved in clade [110], clade [124], clade [174], and several OTUs); main veins in the tegmina having one point of inflection at most (111:1>2; convergently evolved in clade [180]); Anal veins in the tegmina with 6 or more branches $(114: 2>3$; convergently evolved in several OTUs).

\subsubsection{Deroplatys $+($ Oxyopsis $+($ Archimantis $+($ Heterochaeta $+($ Oxyothespis + Toxoderi- dae)))) [174]}

This taxon was only found in analysis II. It was not supported in the molecular analysis by Svenson \& Whiting (2009).

The monophyly of this taxon is supported by the following characters: Number of posteroventral fore tibial spines being one third or more lower than the number of antero-ventral fore tibial spines (79:1>2; convergently evolved in clade [110], clade [124], Polyspilotini [171], and several OTUs); A3 in the alae being branched once or twice in the male (122:2>1; convergently 
evolved in clade [84], Acanthopidae [227], and several OTUs including Mantoida maya [7]) and in the female (126:2>1; convergently evolved in clade [133], clade [231], and several OTUs including Mantoida maya [7]).

\subsubsection{Oxyopsis + (Archimantis + (Heterochaeta + (Oxyothespis + Toxoderidae $))$ [178]}

This clade was found in analysis II only. There is no support from molecular data.

Its monophyly finds support in the following characters: Bulging eyes protruding the head capsule laterally (15:2>1; convergently evolved in clade [123], and several OTUs); organization of the discoidal spines (57:6>2; convergently evolved in Humbertiella + Theopompa [65], clade [142], and several OTUs); CuA in the alae of the female being simple (124:1>2; convergently evolved in clade [88] and several OTUs); cross veins between the Cubital veins CuA and $\mathrm{CuP}$ in the alae being straight or slightly curved (128:1>2; convergently evolved in Oligonicinae including Haaniinae [43], clade [101], Dystacta + Taumantis [119], clade [124] and several OTUs)

\subsubsection{Archimantis + (Heterochaeta $+($ Oxyothespis + Toxoderidae) $)[180]$}

This clade was only found in analysis II. The molecular data by Svenson \& Whiting (2009) did not find this relationship.

It is supported by the shortened wings in the females $(106: 1>2$; convergently evolved in Gongylus [155]), by the main veins in the tegmina being straight or bent but not sigmoid (111:1>2; convergently evolved in Polyspilotini [171]), by the flattening of the cerci (149:2>1; convergently evolved in clade [22], Tarachodinae except for Iris [89], and several OTUs including Chaeteessa valida [2]), and by the flattening of the distal cercomere without strong enlargement (150:1>2; convergently evolved in Tarachodinae except for Iris [89]).

\subsubsection{Heterochaeta + (Oxyothespis + Toxoderidae) $[184]$}

This relationship was only found in analysis II (Fig. 402) and is not supported by molecular data.

Among its apomorphic characters are the presence of a single ventral cervical sclerite (22:2>1; convergently reduced in Tarachodinae except for Iris [89], Chaeteessa [2], and Blepharopsis [151]), the $\mathrm{CuP}$ in the alae of the male being without branchings (120:1>2; convergently evolved in several OTUs), and the presence of a ridge on the supra-anal plate (133:2>1; convergently evolved in a part of Tarachodinae except for Iris [94], Rivetinini [115], Empusinae [154], and several OTUs).

This relationship was not identically supported by analysis I (Figs. 403, 404). Therein, $\mathrm{He}$ terochaeta and Toxoderidae were found to be sistergroups. In the strict consensus cladogram of analysis I (Fig. 403) Oxyothespis was found in a basal polytomy with together with 50 other clades. In the majority rule cladogram (Fig. 404) it clustered with Compsothespis. These two formed the sistergroup of Heterochaeta + Toxoderidae.

Svenson \& Whiting (2009: fig. 3c, clades 173, 172, respectively) found a sistergroup 
relationship between Oxyothespini and Toxoderidae, together being the sistergroup of Compsothespis. Heterochaeta, however, was found in a closely related clade, being the sistergroup of Iris (Svenson \& Whiting 2009: fig. 3c, clade 168).

A close relationship between Heterochaeta and Toxoderidae was assumed early by Westwood (1845a: 162).

\subsubsection{Oxyothespis + Toxoderidae [188]}

This taxon was only found in analysis II. This relationship is also supported by molecular data (Svenson \& Whiting 2009: fig. 3c, clade 173). In analysis I (majority rule cladogram; Fig. 404),

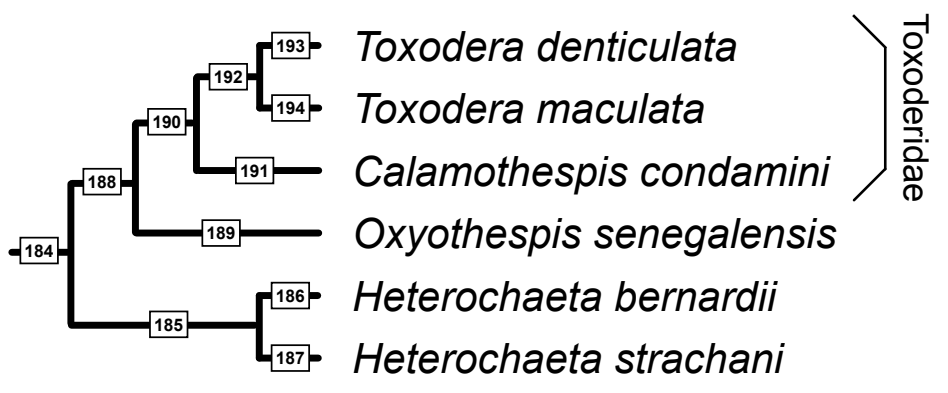

402

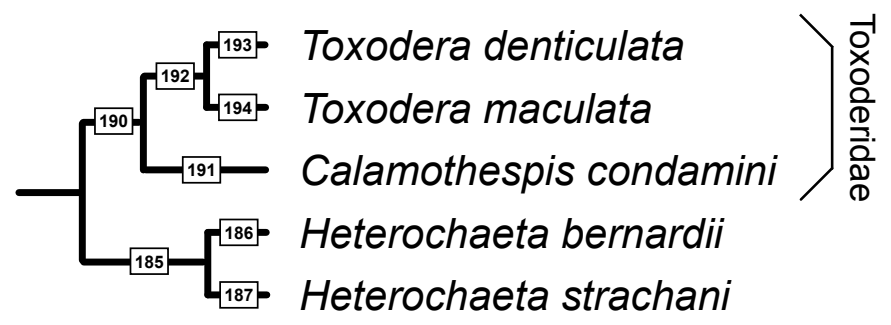

403

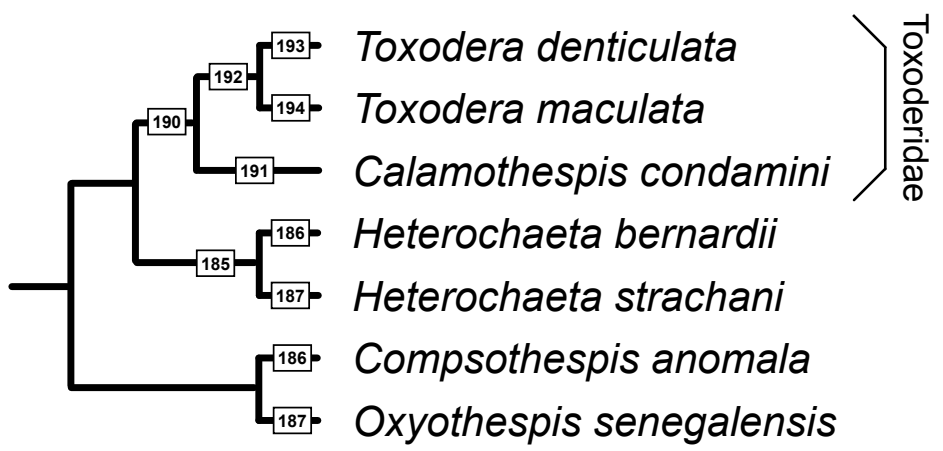

404

Fig. 402-404: Relationships of Toxoderidae, Heterochaeta, Oxyothespis and Compsothespis found in analyses I and II. 402: Relationships found in analysis II. 403: Relationships found in analysis I (strict consensus). 404: Relationships found in analysis I (majority rule, 50\%). See also Amorphoscelidae including Compsothespis (clade [15]) for discussion of the relationships of Compsothespis.
Oxyothespis came out as sistergroup of Compsothespis with this clade forming the sistergroup of Toxoderidae (see also chapter 4.3.85).

Therelationshipbetween Oxyothespis and Toxoderidae is supported by the transverse folding of the clypeus (12:2>1; convergently evolved in Rivetinini [115], clade [196], and several OTUs) and the number of discoidal spines counting 3 (55:4>3; convergently evolved in clade [14], Pogonogaster + Thesprotia [46] and several OTUs).

\subsubsection{Toxoderidae: Calamo- thespis + Toxodera [190]}

Toxoderidae was found to be monophyletic in analysis II and in both consensus cladograms of analysis I (Figs. 402-404). The monophyly is also well-supported in the molecular analysis by Svenson \& Whiting (2009: fig. 3c, clade 175), who also included species of Toxoderidae: Aethalochroaini (the third subgroup of

Toxoderidae, not studied herein) and therefore found even stronger support for the potential monophyly of Toxoderidae. 
The monophyly of Toxoderidae is supported herein by the fore femoral spines being positioned far apart from each other $(43: 1>2)$ and by the short tibial spur being a result of the secondarily elongated tibia $(69: 1>3)$. Further apomorphies are the position of the claw groove in the proximal third of the femur (37:3>2; convergently evolved in Eremiaphilidae [9], Polyspilotini [171] and Oxyopsis [179]), the number of postero-ventral femoral spines counting more than 6 (48:2>4; convergently evolved in Chaeteessa [2] and Gongylus [155]), and the fore tibia being more than half as long as the femur $(67: 1>2$; convergently elongated in Polyspilotini [171]).

\subsubsection{Clade [195]}

This clade was only found in analysis II. It is not supported by any unambiguous apomorphies.

The only apomorphy found under ACCTRAN alone is the presence of two parallel ridges on the scutellum (9:1>2; convergently evolved in clade [162]).

Apomorphies that were found under DELTRAN alone are the presence of a ridge on the clypeus (11:2>1; convergently evolved in Hestiasula + Oxypilinae [79], clade [136], Empusidae [148], and several OTUs) and the lack of the medial euplantula on tarsomere ta5 (97:1>2; convergently evolved in Oligonicinae including Haaniinae [43], clade [73], Tarachodinae except for Iris [89], and several OTUs).

\subsubsection{9 (Acromantis + Ambivia) + Hymenopodinae [196]}

This relationship was only found in analysis II. It is supported by the head process being a small spur $(2: 1>3)$, by the horizontal folding of the clypeus $(12: 2>1$; convergently evolved in Rivetinini [115], Oxyothespis + Toxoderidae [188], and several OTUs), the narrow and laid-down position of the postero-ventral fore tibial spines (76:1>3; convergently evolved in clade [77] and Phyllocrania [140]), and the lobes on the femora of the meso- and metathoracic legs (90:2>1; convergently evolved in clade [133], clade [150] within Empusidae, and several OTUs).

\subsubsection{Acromantis + Ambivia [197]}

This taxon was only found in analysis II. The following characters are apomorphic: Vertical head process present (1:2>1; convergently evolved in Hestiasula + Oxypilinae [79], Ceratocrania + Phyllocrania [138], Empusidae [148], clade [204] within Hymenopodinae, and several OTUs); dorsal edge of the scutellum being elevated into a small spine or knob (7:2>3; convergently evolved in Haaniinae [55], Hestiasula + Oxypilinae [79], and Empusidae [148]); $\mathrm{CuP}$ in the tegmina of the males running in a straight or slightly curved course (104:2>1; convergently evolved in clade [77], clade [92] within Tarachodinae except for Iris, clade [208] within Hymenopodinae, and several OTUs).

Interestingly, Ambivia did not come out as being closely related to Popa (which was found to be more closely related to Vates, Ceratocrania and Phyllocrania in the present dataset, 
clade [133], see chapter 4.3.62). Both genera have a very similar external appearance, exhibiting a strong special resemblance to twigs, with their abdominal tips resembling the ends of broken branches. Ambivia and Popa are assumed to belong to the same subgroup of Vatinae (Danuriini) in the classification by Ehrmann (2002: 378). However, other authors did not assume this relationship but suggested Ambivia belonging to Acromantinae (e.g. Giglio-Tos 1927; Sjöstedt 1930: 13; Lombardo 1995b: 259; Mukherjee et al. 1995: 197; Vyjayandi 2007: 28; for a brief synopsis of the nomenclatural history of the two genera see Schwarz 2004: 12-13). Ambivia was not present in the molecular dataset. If the two genera are actually not sistergroups but are as distantly related to each other as found herein, Ambivia and Popa represent an excellent and impressive further example for a convergent ecomorph in Afrotropical and South-East Asian taxa.

\subsubsection{Hymenopodinae: Galinthias + (Pseudoharpax + (Pseudocreobotra + (Creobroter gemmatus + (Creobroter elongatus + (Hymenopus + Theopropus $))))$ [200]}

Hymenopodinae was found to be monophyletic in analysis I and II (Fig. 405), although the internal relationships in both consensus cladograms of analysis I differed from those in analysis II. The monophyly of the group is supported by the following characters: Cone-shaped eyes that laterally appear to be located on sockets (14:2>1; with a reversal in Theopropus [214]) and that protrude the head capsule dorsally (15:1>3/2>3; with a reduction in Theopropus [214]); metathoracic tibiae being as long as or shorter than the femora in both sexes (males: $88: 1>2$; convergently evolved in Ciulfina + Stenomantis [34], Haaniinae [55], clade [133], and several OTUs; females: 89:1 $>2$; convergently evolved in Amorphoscelinae [28], clade [136], and several OTUs); and the tibiae of the cursorial legs being asymmetrical (93:1>2; convergently evolved in clade [133] and several OTUs).

Svenson \& Whiting (2009: fig. 3c, d; clades 190, 210) found Hymenopodinae to be paraphyletic. While the greater part of the taxa studied formed a monophylum therein (clade 210; encompassing Hymenopus, Theopropus, Creobroter, Pseudocreobotra, Chloroharpax Werner, 1908 and Panurgica Karsch, 1896), the three genera Pseudoharpax, Congoharpax and Galinthias formed a separate monophyletic group (clade 190). Hymenopus and Theopropus were found to be sistergroups (Svenson \& Whiting 2009: fig. 3d, clade 211), whereas Creobroter formed the sistergroup of Pseudocreobotra $+($ Chloroharpax + Panurgica) (clade 213).

\subsubsection{Pseudoharpax + (Pseudocreobotra + (Creobroter gemmatus + (Creobroter elonga- tus + (Hymenopus + Theopropus)))) [202]}

This clade was only found in analysis II. It is supported by the slightly asymmetrical male antennomeres (20:1>2; convergently evolved in clade [136], clade [184], and several OTUs) and the prozona being slightly shorter than up to half as long as the metazona of the pronotum (31:3>2; convergently evolved in clade [94] within Tarachodinae except for Iris). 
4.3.93 Pseudocreobotra + (Creobroter gemmatus + (Creobroter elongatus $+($ Hymenopus + Theopropus))) [204]

This clade was only found in analysis II (Fig. 405). Apomorphies supporting it are: Vertical head process present (1:2>1; convergently evolved in Hestiasula + Oxypilinae [79], Ceratocrania + Phyllocrania [138], Empusidae [148], Acromantis + Ambivia [197], and several OTUs); Pronotum being less than twice as long as wide (28:1 $>2$; convergently evolved in Humbertiella + Theopompa [189], clade [110] within Chroicopterinae, and several OTUs); expansion of the antero- and postero-ventral edges of the fore tibia present $(71: 1>2$; convergently evolved in Empusidae [148]).

Interestingly, all species of this group except for Hymenopus (which is differently adapted in terms of mimesis than the other species in this clade) exhibit conspicuous markings on their tegmina which probably work very well as camouflage in the natural habitats or serve for deimatic displays (e.g. in Pseudocreobotra; see Edmunds 1972: pl. 1, fig. D, 1974: 168; Edmunds \& Brunner 1999: fig. 13.8D). The basic colour of the tegmina in these species is yellowish to bright or dark green, and the markings consist of black and white (or yellowish to pink) lines, markings or circles (see Beier 1934a: pl. 2; Edmunds 1976: fig. 7; see Figs. 405A-F).

Theopropus has nearly parallel lines (a bright one bordered by two black ones; see Beier 1934a: pl. 2, fig. 6, 1964a: fig. 13a; Fig. 405D) on the posterior tegmina and a bright spot, slightly bordered by a black line, near the costal rim on each tegmen. Creobroter has a similar pattern (which shows intrageneric differences; see Beier 1934a: pl. 2, figs. 1, 2) but the parallel lines found in Theopropus are curved in Creobroter and form a closed eye-spot with additional black markings in its centre on each tegmen (Fig. 405C). In Pseudocreobotra, the white markings on the anterior tegmina form a continuous band, whereas the posterior marking forms an eye-spot consisting of spirals of black and white lines including a black and green marking in the centre (Beier 1934a: pl. 2, fig. 11, 1964a: fig. 13c; Edmunds 1972: pl. 1, fig. D; Edmunds 1976: fig. 7; Edmunds \& Brunner 1999: fig. 13.8D; Picker et al. 2002: p. 61, fig. 2; Fig. 405A). Hymenopus and Parymenopus Wood-Mason, 1890 do not have such eye-spots. However, putative remnants of the black lines are still discernible in both genera (Figs. 405E, F). The potential remnants of the eye-spot (two black markings on each tegmen) are positioned in the middle of the wing length, resembling the situation found in the aforementioned taxa (Beier 1934a: pl. 2, fig. 7). The phylogenetic relationships found by me would therefore indicate that either the spiral-shape of the eye-spot found in Pseudocreobotra or the situation found in Creobroter is the plesiomorphic condition. The first scenario would implicate a subsequent detorsion of the eyespot in Creobroter (maintained in a simple form in Theopropus) and a loss of the eye-spot/-line marking in Hymenopus (and probably Parymenopus which is likely to be its sister species). The second scenario would implicate an apomorphic strong torsion of the spot in Pseudocreobotra and a reduction of the spot in Theopropus and Hymenopus as mentioned in the first scenario.

Both hypotheses contradict the assumption by Beier (1964a: 883) who assumed the origin of the situation in Pseudocreobotra to have evolved from simple patterns, such as that of Theopropus: "Auf den Elytren mancher Hymenopodinen (Creobroter Serv., Theopropus 


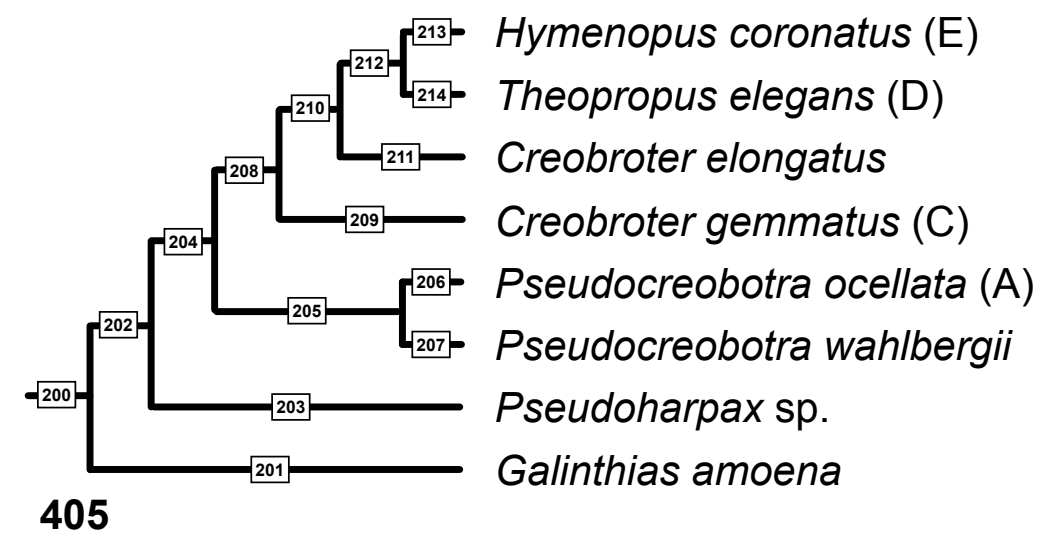

A

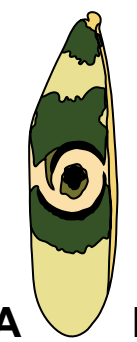

B
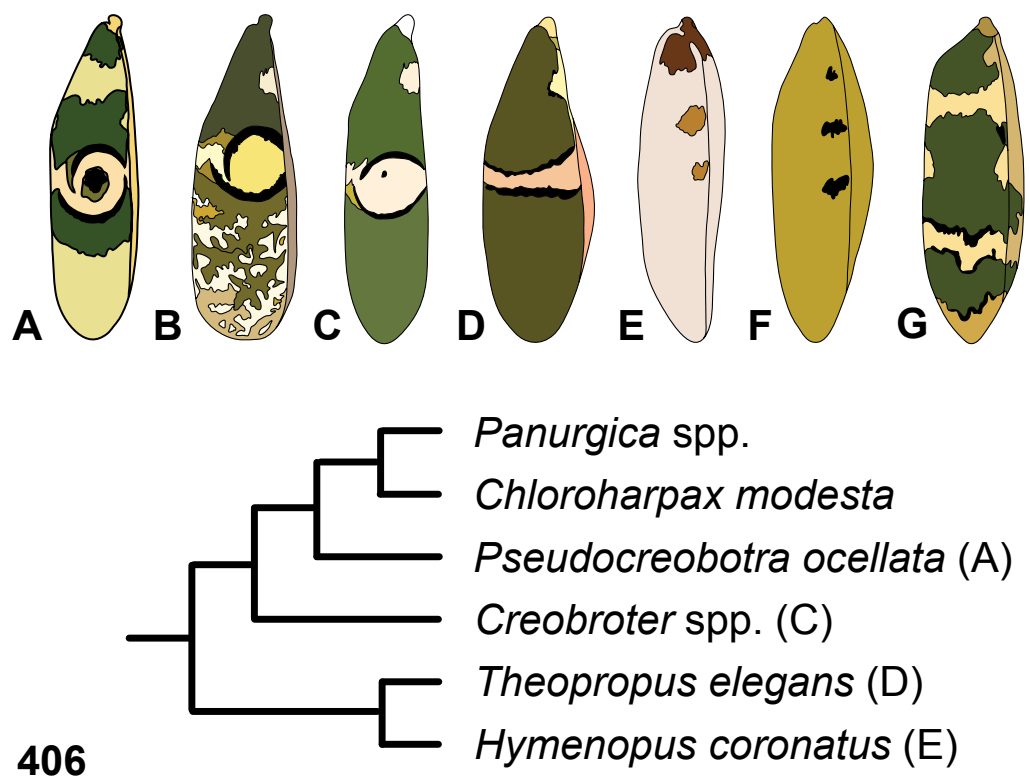

Panurgica spp.

Chloroharpax modesta

Pseudocreobotra ocellata (A)

Creobroter spp. (C)

Theopropus elegans (D)

Hymenopus coronatus (E)

Fig. 405-406: Phylogeny of Hymenopodinae. 405: Relationships of Hymenopodinae found in analysis II. Figs. A-F: Schematic colour patterns and eye spots on mantodean tegmina (in situ from photographs of living specimens, wings not mounted); A-F = Hymeopodinae; $\mathrm{G}=$ Acontistinae. A: Pseudocreobotra ocellata. B: Chlidonoptera sp. (not analysed in present dataset; colour pattern simplified, yellowinsh-green mottled appearance of posterior part of tegmen in specimen). C: Creobroter gemmatus. D: Theopropus elegans. E: Hymenopus coronatus. F: Parymenopus davisoni (not analysed in present dataset). G: Callibia diana. 406:

Relationships found by Svenson \& Whiting (2009: fig. 3D). See text for a discussion of the evolution of eye spots in Hymenopodidae.
Sauss., Anabomistria G.-Tos [= Chlidonoptera, see Ehrmann 2002: 96], Pseudocreobotra Sauss., Chlidonoptera Karsch) bilden diese beiden Farbstoffe primitive Zeichnungsmuster in Form einer hellen, schwarz gerandeten Querbinde, einer gelben proximal und distal von einem schwarzen Bogenstreifen umrahmten Makel oder eines spiraligen Augenflecks, wobei letzterer offensichtlich durch Krümmung ursprünglich gerade Querbinden entstanden ist."

An eye-spot has also been observed in Chlidonoptera Karsch, 1892 (pers. obs. 2010; Beier 1934a: pl. 2, figs. 10, 13, 1964a: fig. 13b, therein named Anabomistria; see above; Edmunds 1976: fig. 7 and plate 2, fig. D; Edmunds \& Brunner 1999: fig. 13.10E; Ehrmann 2002: 389; Fig. 405B). Interestingly, this genus is distributed in Africa (as is Pseudocreobotra) and represents an intermediate between the latter genus and the Asian Creobroter and Theopropus with respect to the colouration of the tegmina. The eye-spot is less twisted, resembles Pseudocreobotra but forms a partial band on the posterior edge of the tegmen. In contrast to Pseudocreobotra, the anterior markings on the tegmina are separate and do not form a continuous band (e.g. Edmunds 1976: fig. 7).

The molecular analysis by Svenson \& Whiting (2009: fig. 3d, clade 210; Fig. 406) allows several different interpretations of the evolutionary history of the wing-patterns in Hymenopodidae. Therein, Hymenopus + Theopropus forms the sistergroup of Creobroter + (Pseudocreobotra $+($ Chloroharpax + Panurgica $))$. It is unclear whether the situation found in Hymenopus, 
in Theopropus or in Creobroter is the ancestral state. It is also possible from the molecular findings that the pattern of Hymenopus + Theopropus evolved convergently to the type found in the sistergroup. The African Chloroharpax and Panurgica as well as the African Harpagomantis also exhibit tegminal colour patterns [Panurgica and Harpagomantis without an eye spot (see Preston-Mafham 1990: pl. 3 and Picker et al. 2002: 61 for Harpagomantis), Chloroharpax apparently with a small eyespot but without a colour pattern)] but were not studied herein. There are also several Neotropical Mantodea (not related to Hymenopodidae) that show similar colourations (e.g. female Callibia diana: Fig. 405G) or even perfect eye spots, as for instance in Stagmatoptera (e.g. Saussure \& Zehntner 1894: pl. 8, fig. 2; Edmunds 1974: 168).

Interestingly, a potentially raptorial Carboniferous "†Prothorthoptera" fossil (†Protodiamphipnoa tertrini Brongniart, 1893) was described to have similar tegminal eye-spots (Carpenter 1971: 1250 and figs. 13, 1992: fig. 75, 14; Edmunds 1976: 32; Rasnitsyn 2002: fig. 357b).

Several other extant insect groups have evolved more or less elaborate eye spots on the dorsal or ventral sides of their fore- and/or hind wings, most commonly and most elaborate in Lepidoptera, but also in Auchenorrhyncha [e.g. Fulgora laternaria (Linnaeus, 1758)], Phasmatodea [described for Metriophasma diocles (Westwood, 1859); e.g. Robinson 1968: 53 and fig. 5; Edmunds 1974: 168; Bedford 1978: 137] and in Ensifera [e.g. Pterochroza ocellata (Linnaeus, 1758) (Badenoch 1899: fig. 24), the genus Tanusia Stål, 1874 (e.g. Beier 1955a: fig. 72), the genus Ommatoptera Pictet, 1888 (e.g. Pictet 1888: pl. 2, figs. 18-20), and Ancylecha fenestrata (Fabricius, 1793)]. In Phasmatodea and Orthoptera there seem to be few species having eye spots (in Ancylecha they rather represent the indication of holes in the wing than eyes). Therefore, the evolution of eye spots in a larger group of closely related genera of Mantodea exhibiting it to varying degrees of "mimetic perfection" is an interesting topic for future studies. A closer look at this character in Mantodea may yield interesting insights into the evolution of Hymenopodinae and the origin of conspicuous display colourations and their potentially protective value for crypsis in case of imperfect eye spots. The latter possibly rather add to the effect of somatolysis than to deimatic display. It is important to compare the wing markings throughout Hymenopodidae displaying them (and closely allied taxa that do not, e.g. the South African Harpagomantis Kirby, 1899; see Picker et al. 2002: 60, fig. 3). Such a study should include the intraspecific variability and the individual lifestyle of the species in order to further investigate the evolutionary scenario implied by the present analysis.

\subsubsection{Creobroter gemmatus $+($ Creobroter elongatus $+($ Hymenopus + Theopropus $))$ [208]}

This taxon was only found in analysis II. It is supported by the straight to slightly curved course of $\mathrm{CuP}$ in the tegmina of the male (104:2>1; convergently evolved in clade [77], clade [92] within Tarachodinae except for Iris, Acromantis + Ambivia [197], and many OTUs) and of the female (107:2>1; convergently evolved in clade [77], Idolomorphini [158], and several OTUs). 


\subsubsection{Creobroter elongatus + (Hymenopus + Theopropus) [210]}

This clade was only found in analysis II. It is only supported by the number of antero-ventral fore tibial spines in the female counting 16-19 (74:3>4; convergently evolved in Idolomorphini [158] and several OTUs).

\subsubsection{Hymenopus + Theopropus [212]}

This sistergroup relationship was found in analysis II and is supported, for instance, by the lack of the clypeal ridge $(11: 1>2)$, by the pronotum being twice as long as wide or longer $(28: 2>1$; convergently evolved in Compsothespis [23]), and by the position of the postero-ventral fore femoral spines on an elevation (49:1>2; convergently evolved in Empusidae [148]).

The sistergroup relationship between the two species was also supported by the molecular data (Svenson \& Whiting 2009: fig. 3d, clade 211). Interestingly, these two genera both live in South-East Asian forest habitats and exhibit extreme male dwarfism (see also chapter 4.6.8). Considering their close relationship, this may represent a further synapomorphic character.

\subsubsection{Choeradodis $+($ Orthodera $+($ Iris $+($ Photina $+($ Orthoderella + Acanthopidae $))))$ [215]} This taxon was only found in analysis II (Fig. 407). It is not supported by the molecular analyses nor by any unambiguous morphological apomorphies.

The following apomorphic traits were found under ACCTRAN alone: Ridge on the clypeus present (11:2>1; convergently evolved in Hestiasula + Oxypilinae [79], clade [136], Empusidae [148], Toxoderidae [190], clade [196], and several OTUs); medial euplantula on the distal tarsomeres ta5 lacking (97:1>2; convergently evolved in Oligonicinae including Haaniinae [43], Tarachodinae except for Iris [89], Oxyothespis + Toxoderidae [188], clade [196], and several OTUs).

The following apomorphies found only under DELTRAN assumptions are: Compound eyes being more or less globular (14:2>3; convergently evolved in clade [143] and several OTUs); A3 in the alae of the male branching 5 or more times (122:2>3; convergently evolved in clade [136], clade [143], Stagmatoptera + Polyspilotini [169], Idolomantis [153], and Ambivia [199]); supra-anal plate nearly triangular (134:3>2; convergently evolved in Oligonicinae including Haaniinae [43] and several OTUs).

\subsubsection{Orthodera $+($ Iris $+($ Photina $+($ Orthoderella + Acanthopidae $)))$ [219]}

This taxon was only found in analysis II. It is supported by the number of antennomeres in the males counting 70-89 (18:4>3; convergently evolved in Amorphoscelinae + Perlamantinae [24], clade [88], clade [178], and several OTUs) and by the number of postero-ventral fore tibial spines in the males counting 13-17 (77:3>4; convergently evolved in clade [92] within Tarachodinae except for Iris, Ceratocrania + Phyllocrania [138], and several OTUs). 


\subsubsection{Iris $+($ Photina $+($ Orthoderella + Acanthopidae $))[221]$}

This clade was found in analysis II and in the majority rule consensus cladogram of analysis I, albeit with slightly different internal relationships. No unambiguous apomorphies were found for this clade.

Apomorphic characters that were found under ACCTRAN assumptions are, for instance, the length pattern 2 of the discoidal spines (57:1>2; convergently evolved in Humbertiella + Theopompa [65], clade [142], clade [178], clade [204] within Hymenopodinae, and in several OTUs) and the straight or slightly curved CuP in the tegmina of the males (104:2>1; convergently evolved in clade [77], clade [92] within Tarachodinae except for Iris, Idolomorphini [158], Toxoderidae [190], Acromantis + Ambivia [197], clade [208] within Hymenopodinae, and in several OTUs).

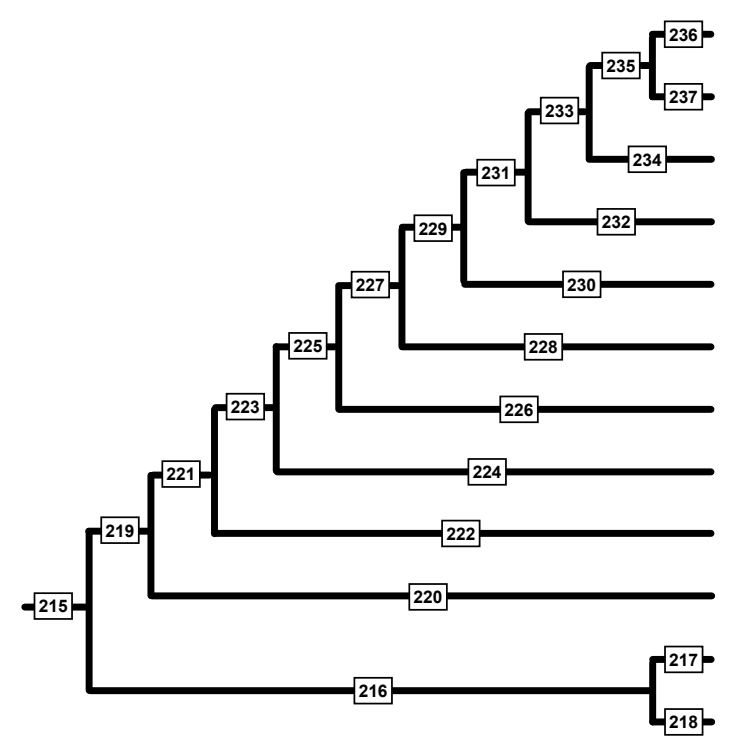

Acanthops falcataria

Acanthops tuberculata

Callibia diana

Tithrone roseipennis

Raptrix fusca

Acontista brevipennis

Orthoderella ornata

Photina laevis

Iris oratoria

Orthodera novaezealandiae

Choeradodis rhombicollis

Choeradodis stalii

Fig. 407: Relationships of clade [215] found in analysis II. Note that Iris is nested within a clade of Neotropical taxa. Note the position of Orthodera and Choeradodis in the same larger clade.

The following apomorphic traits were found among others under DELTRAN: clypeal ridge lacking (11:1>2; convergently evolved in Hymenopus + Theopropus [212]); oval shape of the compound eyes (14:3>2; convergently evolved in Mantodea except for Chaeteessa [3]); 5 postero-ventral femoral spines present (48:2>3; convergently evolved in Empusidae [148] and several OTUs); short wings in the females (106:1>2; convergently evolved in Artimantodea (?) [8], clade [180], and Gongylus [155]); straight or slightly curved $\mathrm{CuP}$ in the tegmina of the female [107:2>1; convergently evolved in clade [77], Idolomorphini [158], clade [208] within Hymenopodinae, and several OTUs); branching of $\mathrm{CuA}$ in the alae of the males lacking $(120: 1>2$; convergently evolved in clade [184] and several OTUs).

\subsubsection{Photina + (Orthoderella + Acanthopidae) [223]}

This clade was only found in analysis II. This relationship of Photinaini and Acanthopidae is not supported by any unambiguous autapomorphies.

The following characters were found to be apomorphic for the group under ACCTRAN 
assumptions: Oval shape of the compound eyes (14:3>2; convergently evolved in Mantodea except for Chaeteessa and Metallyticus [6] and several OTUs); 5 postero-ventral fore femoral spines present (48:2>3; convergently evolved in Empusidae [148], and several OTUs); wings of the females short (106:1>2; convergently evolved in Eremiaphilidae [9], clade [14], clade [180], Gongylus [155] and Iris [222]); supra-anal plate long (132:1>2; convergently evolved in Paraoxypilinae [16], clade [51] and several OTUs).

Apomorphic characters found exclusively under DELTRAN are the reduction of the metathoracic hearing organ from DK ("deep groove with knobs") to MSMT ("meso- and metathoracic segments similar") in the males (130:1>4; 131:1>4).

This South American clade of earless Mantodea was found to be monophyletic in Svenson \& Whiting (2009: fig. 3a, clade 31), although encompassing several additional species not included in the present dataset. Therein, Photina (+ Microphotina Beier, 1935) was the sistergroup of Orthoderella. That taxon (including several other Photinaini) was found to be the sistergroup of Acanthopidae.

\subsubsection{Orthoderella + Acanthopidae [225]}

This sistergroup relationship was only found in analysis II. It is not supported by unambiguous apomorphies.

Apomorphies under ACCTRAN encompass the reduction of the metathoracic ear morphology from DK ("deep groove with knobs") to MSMT ("meso- and metathoracic segments similar") in both sexes (130:1>4, 131:1>4; convergently reduced in Oligonicinae including Haaniinae [43] and Photina [224] in both cases).

Apomorphies found under DELTRAN alone are the presence of 3 discoidal spines $(55: 4>3$; convergently evolved in clade [14], Pogonogaster + Thesprotia [46], Oxyothespis + Toxoderidae [188], and several OTUs) and the supra-anal plate being wider than long (132:2>1; convergently evolved in Mantodea except for Chaeteessa [3]).

This relationship was supported by the molecular data (Svenson \& Whiting 2009: fig. 3a, node 31), although it contained further species that were not included in the present dataset.

A sistergroup relationship between Epaphrodita and Acanthopidae as found by Jantsch (1999) is not corroborated by the present findings.

\subsubsection{Acanthopidae: Acontista + (Raptrix + (Tithrone + (Callibia + Acanthops))) [227]}

The monophyly of Acanthopidae was found in analysis II and the majority rule cladogram of analysis I (Fig. 407). It is supported by A3 in the alae of the males being branched once or twice (122:3>1; convergently evolved in clade [84], clade [174], and several OTUs) and by the cerci of the male counting 9-11 cercomeres (151:3>2; convergently evolved in clade [14], clade [73], clade [101], and several OTUs).

Acanthopidae was found to be monophyletic in the molecular analysis by Svenson \& Whiting (2009: fig. 3a, clade 37). Stenophylla was not included in the present dataset, therefore its relationship with respect to the remaining Acanthopidae could not be addressed. For a discussion of Acanthopidae see chapter 2.1.4. 


\subsubsection{Raptrix + (Tithrone + (Callibia + Acanthops)) [229]}

This taxon was found in analysis I and II with slightly different internal relationships in the strict consensus cladogram of analysis I. There were no unambiguous apomorphies supporting this taxon.

This group finds support under ACCTRAN by the following apomorphies: Ridges on the scutellum present $(8: 2>1$; convergently evolved in Oxypilinae [81], Empusidae [148], clade [196], and several OTUs); transverse folding of the clypeus present (12:2>1; convergently evolved in Chaeteessa [2] and Mantodea except for Chaeteessa [3], Rivetinini [115], clade [124], Oxyothespis + Toxoderidae [188], clade [196], and several OTUs); prozona slightly shorter than up to half as long as metazona (31:3>2; convergently evolved in Oligonicinae including Haaniinae [43], clade [94] within Tarachodinae except for Iris, clade [202] within Hymenopodinae and Acontista [228]); close position of the postero-ventral fore tibial spines and the lied-down position being restricted to the proximal half of the tibia at most $(76: 1>3$; convergently evolved in clade [77], clade [196], and several OTUs); CuA in the alae of the female simple (124:1>2; convergently evolved in Perlamantinae [25], clade [88], Angelinae [128], clade [178], and several OTUs).

Under DELTRAN assumptions this relationship is supported by the following characters: Antero- and postero-ventral fore tibial edges widened $(71: 1>2$; convergently evolved in Empusidae [148], and clade [204] within Hymenopodinae); number of postero-ventral fore tibial spines being larger than of the antero-ventral spines $(79: 1>3$; convergently evolved in clade [75], clade [156] within Empusinae, and Hymenopus + Theopropus [212]); secondary elongation of the wings in the females (106:2>1; convergently evolved in Amorphoscelinae + Perlamantinae [24], Tropidomantinae [59], clade [75], clade [136], clade [141], and several OTUs); styli on the subgenital plate of the males lacking $(142: 1>2$; convergently evolved in Perlamantis [27], Odontomantis [78], and Theopropus [214]).

\subsubsection{Tithrone + (Callibia + Acanthops) $[231]$}

This clade was found in analysis II and the majority rule consensus cladogram of analysis I. It is supported by anal vein A3 in the alae of the female being branched once or twice $(126: 2>1$; convergently evolved in clade [133], clade [174], and several OTUs).

\subsubsection{Callibia + Acanthops [233]}

This relationship was supported in analysis I and II. There are no unambiguous apomorphies for this sistergroup relationship.

It is supported under ACCTRAN, for instance, by the lack of the ventral groove between the proximal postero-ventral fore femoral spines (53:1>2; convergently evolved in Thespidae [41], clade [72], Chroicopterinae [108] several OTUs).

Under DELTRAN, the following characters support this taxon: Alternating position of the proximal antero-ventral fore femoral spines (45:2>1; convergently evolved in Chaeteessa [2], Mantoida [7], and Paraoxypilinae [16]); meso- and metathoracic tibiae asymmetrical (93:1>2; 
convergently evolved in clade [133] and Hymenopodinae [200]); notch in the distal edge of the supra-anal plate present $(135: 2>1)$; lobe-like lateral expansions of the female abdominal tergites present (145:2>1; convergently evolved in Haaniinae [55], clade [150] within Empusidae, and several OTUs).

\subsection{Congruence with taxonomy and molecular data}

\subsubsection{Congruence with taxonomy}

The monophyly of the following taxonomic groups of the traditional classification is supported by the present data (monophyly of genera omitted): Acanthopidae, Artimantodea (?) (see discussion in chapter 4.3.7), Amorphoscelinae, Angelinae (= Angelini), Chroicopterinae (=Chroicopterini), Empusidae, Empusinae, Eremiaphilidae, Haaniinae, Hymenopodinae, Idolomorphini, Oxypilinae (= Oxypilini), Paraoxypilinae, Perlamantinae, Polyspilotini, Rivetinini, Thespidae, Toxoderidae, Toxoderinae, and Tropidomantinae.

The following groups in the typological classification were found to be para- or polyphyletic (genera omitted): Acontistinae, Acontistini, Acromantinae, Acromantini, Amelinae, Amelini, Amorphoscelidae (paraphyletic with respect to Compsothespis, but see discussion of 4.3.10), Blepharodinae, Blepharodini, Danuriini, Epaphroditinae, Hymenopodidae, Liturgusidae, Liturgusinae, Mantidae, Mantinae, Miomantinae, Miomantini, Oligonicinae (paraphyletic with respect to Haaniinae, see discussion of 4.3.23), Paramantini, Photinainae, Photinaini, Phyllocranini, Stagmatopterinae, Stagmatopterini, Tarachodinae (paraphyletic with respect to Iris), Vatinae, and Vatini.

The monophyly of the following groups and their relationships could not addressed by the current taxon sampling or are monogeneric or monospecific: Acanthopinae, Aethalochroaini, Antemninae (monospecific), Archimantini, Caliridinae, Chaeteessidae (monogeneric), Choeradodinae, Compsothespinae (monogeneric), Coptopterygini, Deroplatyinae, Dystactinae, Hapalomantinae, Heterochaetini (monogeneric), Idolomantini (monospecific), Iridopterygidae, Iridopteryginae, Mantini, Mantoididae (monogeneric), Mellierinae (= Mellierini), Metallyticidae (monogeneric), Miopteryginae, Miopterygini, Nanomantinae, Nilomantinae, Orthoderinae, Oxyothespinae, Paraseveriniini, Phyllotheliinae, Pseudomiopteriginae, Pseudomiopterigini, Schizocephalinae (monospecific), Sibyllidae, Sibyllinae, Stenophyllinae (monogeneric), Tarachodidae and Toxoderopsini.

The current taxonomical classification subdivides Mantodea into 15 families, 48 subfamilies and 46 tribus (see Tab. 1). All 15 families (100\%), 34 subfamilies (71\%) and 33 tribus (72\%) were included in the present study.

In conclusion, five families (33\%), 11 subfamilies (32\%) and six tribus (18\%) of those included in the present dataset were found to be monophyletic. Furthermore, four families (27\%), 14 subfamilies ( $41 \%$ ) and 11 tribus (33\%) were found to be para- or polyphyletic. The assumed monophyly of six families (40\%), 20 subfamilies (59\%) and 11 tribus (33\%) could not be 
addressed because of the chosen taxon sample or because they are monogeneric or monospecific (Chaeteessidae, Metallyticidae, Mantoididae, Antemninae, Compsothespinae, Heterochaetini, Idolomantini, Schizocephalinae, Stenophyllinae).

\subsubsection{Congruence with molecular data}

The taxa listed below were also found in the extensive analysis by Svenson \& Whiting (2009: fig. 3). Most of them contained further species that were not studied in the present taxon sample, or the internal relationships among the species were slightly different: Mantodea except for Chaeteessa; Metallyticus; Mantodea except for Chaeteessa, Metallyticus and Mantoida (if Metallyticus falls out of Artimantodea); Eremiaphila; Paraoxypilinae; Amorphoscelinae; Ciulfina + Stenomantis (i.e. “Australian Liturgusidae”); Tropidomantis; Hestiasula + Oxypilinae; Oxypilinae; Pyrgomantis + (Tarachodes + Tarachodula); Chroicopterinae [as sampled in the present dataset; Chroicopterinae was found to be paraphyletic with respect to Chroicoptera in Svenson \& Whiting (2009: fig. 3b); see also Wieland 2008b and chapter 4.6.9]; Ligariella + (Entella + Ligaria $)$; Rivetinini; Leptocola + Stenopyga; Popa; Rhombodera + Tamolanica; Empusidae; Empusinae; Empusa + Idolomorphini; Polyspilotini; Deroplatys; Oxyothespis + Toxoderidae; Toxoderidae; Pseudocreobotra $+($ Creobroter gemmatus $+($ Creobroter elongatus + (Hymenopus + Theopropus))); Hymenopus + Theopropus; Choeradodis; Photina + (Orthoderella + Acanthopidae); Acanthopidae.

\subsection{Homoplasy}

The present study had the aim to test external morphological characters with respect to their significance for a phylogenetic reconstruction of the group. Characters that had previously been assumed to represent convergences without, however, presenting evidence for this, were included here in order to test their potential phylogenetic signal.

As was to be expected from the choice of morphological structures, the degree of homoplasy in the current dataset is high, becoming obvious in the convergent evolution of many structures. This required reweighting the characters in analysis II.

The automatic weighting by Paup* based on the rescaled consistency index (RC) values led to an almost fully resolved phylogeny. In contrast, the consensus cladograms of analysis I showed many polytomies, mainly of the nodes in the middle area of the cladograms. In most cases, the monophyletic terminal groups found in analysis I remained more or less stable and were recovered in analysis II (Figs. 393-395).

The analyses conducted herein provide evidence for extreme convergence in mantodean morphology. The results suggest that vertical head processes have evolved independently at least nine times, whereas processes arising from the area of the lateral ocelli have possibly evolved at least six times (see chapter 4.6.1). Extremely widened lamellar expansions evolved independently at least seven times (see chapter 4.6.3) with at least one subsequent reduction. 
The same is true for a large percentage of the characters discussed herein, as becomes very obvious in the discussion of the relationships in chapter 4.3.

It is likely that these results were strongly influenced by the choice of characters. The definitions of the different character states were formulated with care and respect to variations found among individuals of the same species in the present dataset (e.g. numbers of spines, antennomeres etc.). However, only little is known about how strongly most of the morphological characters vary in nature. Observations on the variability of single structures for small groups of species were published in many taxonomic revisions. A small selection of examples are the variability of the non-visual spines on the eyes of Toxoderini (Roy 2009a) and Heterochaeta (Roy 1976), of the supra-anal plate of Hoplocorypha (Gillon \& Roy 1968), and of the subgenital plate and head processes of Oxypilus pallidus (Gillon \& Roy 1968). Phallomere variability was published for many taxa (e.g. Roy 1962, 1964, 1973, 1996, 2003a, 2004b, 2008c, 2009c; Roy \& Leston 1975; Milledge 1990, 2005). Furthermore, the variability of head processes and lobes on the legs of Sibyllidae (Roy 1996), the variability of pronotum size of Orthodera ministralis (Fabricius, 1775) (Tindale 1923) and the variability of the overall pronotal shape of Parablepharis (Roy 2008b) were published. Variations in the shape of tergal lobes in Nesoxypilus were shown by Milledge (1990), and the variability in fore wing morphology by Béthoux $\&$ Wieland (2009). It is important to summarize the degree of variability that is known to occur in Mantodea and to enrich them with further extensive data for a wide selection of Mantodea in order to more effectively assess the morphological boundaries in which the character states ought to be encoded.

An important result that has crystallized from the high degree of homoplasy found in this study is that, in congruence with the interpretation of the molecular findings (Svenson \& Whiting 2004a, 2009; Yager \& Svenson 2008), Mantodea are a morphologically highly versatile group. Its members adapted in intriguingly similar ways to different habitat types (tree trunks, grass, bushes, savannahs and deserts), apparently many times independently. External similarity is in many cases correlated with special resemblance to plant matter. The selective pressures that had an effect on the resulting, often very similar body shape and structures are most likely strongly related to predator avoidance and preying success. It would be highly interesting to get a deeper insight into the potential combined effect of these two factors for these predators, and to compare them with other highly cryptic insects, for instance Orthoptera sensu stricto or Phasmatodea. It has recently been shown that several Phasmatodea lineages evolved convergently into the charismatic and robust Eurycanthinae ecomorph (Buckley et al. 2009).

\subsection{Observations on selected characters}

The study of the external mantodean morphology revealed many interesting aspects with respect to evolutionary trends, but also with regard to the re-investigation of conspicuous character states that have been stated in the literature for several decades or even centuries but that have since been critically discussed or re-investigated only by very few authors, if by any 
at all. Among the former are, for instance, the head processes (chapter 4.6.1), the spination of the forelegs (parts of chapters chapters 4.6.4, 4.6.7, and 4.6.8) and the female digging devices (chapter 4.6.9), whereas the latter encompass the missing discoidal spines in Metallyticus (chapter 4.6.4), the missing tibial spur in Chaeteessa (chapter 4.6.5), and the reduced number of tarsomeres in Heteronutarsus (chapter 4.6.6).

These characters will be discussed in detail in the following chapters, in addition to the brief statements in the morphological descriptions in chapter 4.1 and in the discussions of the relationships found in the analysis in chapter 4.3.

\subsubsection{Head processes}

Mantodea exhibit numerous shapes of head processes (see characters 1-3). They are not only variously shaped but also originate from different parts of the head capsule. While the head process arises from the dorsal part of the vertex in many taxa [e.g. Ceratocrania (Figs. 64, 64), Ceratomantis (Figs. 58, 59), Empusidae (Figs. 56, 57, 60, 61, 66, 67), Hymenopus (Figs. 75, 76), Phyllocrania (Figs. 41, 63), Pyrgomantis (Fig. 431), Sibyllidae], it is formed by elongated processes arising from the area of the ocelli in other groups (see character 3). While the latter type is rather short in Vates weyrauchi (Fig. 54), the processes are very long and form a pseudouniform process in Zoolea (e.g. Roy \& Ehrmann 2009: figs. 1, 2, 9, 11, 12, 20; Fig. 55).

A further type of head process is present in Didymocorypha lanceolata (not included in the matrix; but see Figs. 428-430). Didymocorypha superficially strongly resembles Pyrgomantis in general appearance. It has a long and slender body, lanceolate and slightly flattened cerci (Fig. 433), and the females are micropterous (Henry 1932: pl. 4; Edmunds 1972: fig. 3b, 1974: fig. 4.22; Edmunds \& Brunner 1999: fig. 13.2b). This is most likely due to a convergent adaptation to living in grasslands. The head processes of these two taxa look very similar at first glance and seem to form long, massive, triangular structures arising from the vertex (compare Wood-Mason 1889: figs. 22, 23 with figs. 24, 25; Figs. 430, 431). However, while this actually is true for Pyrgomantis (e.g. Beier 1954: figs. 12a-c; Fig. 431), the head process in Didymocorypha consists of two separate processes arising from the lateral parts of the vertex (Wood-Mason 1877: 221, 1889: figs. 24, 25; Giglio-Tos 1921: 31; Henry 1932: pl. 4; Ehrmann 2002: 123; Figs. 428430). I was able to confirm separate halves of the head process for the first instar nymph (Figs. 428, 429) and for the adult of Didymocorypha (Fig. 430). Therefore, this character is persistent throughout postembryonic development. This head morphology probably originated from a shape similar to Schizocephala (see Wood-Mason 1889: fig. 34; Ehrmann 2002: fig. 9) or Oxyophthalmus (see Wood-Mason 1889: fig. 28; Henry 1932: pl. 3) and has evolved convergently with regard to the head process of Pyrgomantis. The latter resulted from an elongation of the entire width of the vertex, probably originating from a head shape resembling that of Galepsus (see, for instance, Beier 1954: figs. 7a-c, 12a-c). Interestingly, in the molecular tree by Yager \& Svenson (2008: fig. 6), Schizocephala formed the sistergroup of a clade comprising most of the Tarachodidae of their study. The latter consisted of a basal clade containing Didymocorypha 
and Oxyophthalma, whereas in the sistergroup, Pyrgomantis was nested within a clade comprising Nothogalepsus, Galepsus, Tarachodes and Paragalepsus. Although these taxa formed a larger monophyletic group, the convergent origin of the head process in these two strikingly similar-looking genera is supported therein. Svenson \& Whiting (2009: fig. 3c, node 171) found different results in their analysis, with Didymocorypha coming out as the sistergroup of a clade containing Compsothespis, Oxyothespinae and Toxoderidae. This entire group was part of a larger clade (clade 161) which also contained Eremiaphilidae, Amelinae, some other Tarachodidae and Heterochaeta. Clade 161 formed the sistergroup of another larger clade (clade 150) that contained most of the remaining Tarachodidae including Pyrgomantis. Schizocephala therein came out as the sistergroup of the entire group (clade 148).

Therefore, in addition to the unequivocal morphological evidence for a convergent origin of the head process, molecular data also support a convergent origin of the overall body shape of Didymocorypha and Pyrgomantis, which thus provide a further impressive example of the presence of distinct mantodean ecomorphs as was shown for several taxa Svenson \& Whiting (2009; see chapter 2.3). Interestingly, such grass-dwelling ecomorphs have evolved very similarly in other polyneopteran taxa. The Australian Caelifera Warramunga Rehn, 1952 (Rentz 1991: fig. 24.12c, 1996: fig. 226), Bundinja Key, 1976 (Rentz 1996: fig. 227), and Psednura Burr, 1903 (Rentz 1991: fig. 24.13c, 1996: fig. 230, in the figure mentioned as Cednura) but also the Neotropical Cylindrotettix Bruner, 1906 (e.g. Preston-Mafham 1990: pl. 74) and the African Cannula gracilis (Burmeister, 1838) (e.g. Edmunds 1974: fig. 4.22) show similar morphological adaptations to living on grass blades. They have an elongated, often pointed head (which is widespread in Orthoptera), a slender body and sometimes an elongated ovipositor or pointed wings. These caeliferans cling closely to grass blades in a very similar way as Pyrgomantis does (Rentz 1996: 165, 168). Interestingly, Warramunga and Bundinja are assigned to Eumastacidae: Morabinae (Rentz 1996: 166), whereas Psednura is taxonomically placed in Pyrgomorphidae: Psednurini (Rentz 1996: 168). If these taxonomic assumptions reflect the true phylogenentic relationships, Caelifera, too, has evolved the grass dwelling ecomorph several times independently.

The processes arising from the dorsal vertex of Mantodea may be strongly asymmetrical (see character 2), as can be found in Phyllocrania (Fig. 41, 63). Interestingly, the head process is comparatively short and symmetrical in the first instar nymph of Phyllocrania (Gillon \& Roy 1968: fig. 47; Fig. 408), whereas it becomes longer and more asymmetrical in the subsequent instars.

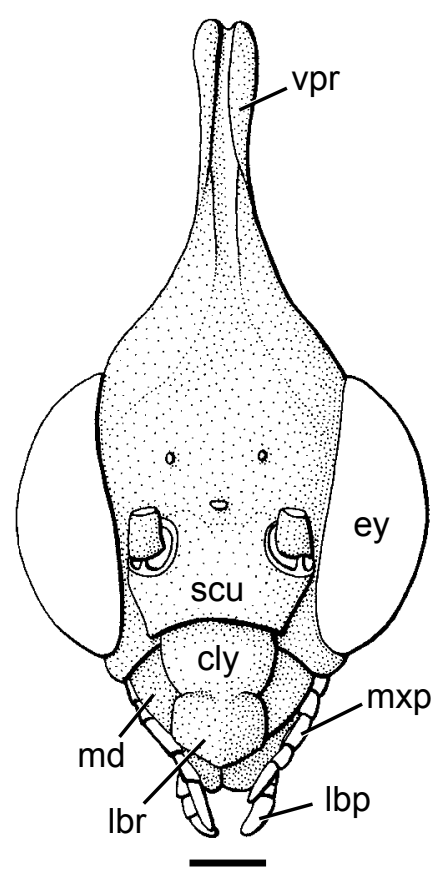

Fig. 408: Phyllocrania paradoxa, first instar nymph. Head in anterior view. Note the symmetrical vertical process which is devoid of lamellar expansions. - Scale bar $=0.25$ $\mathrm{mm}$.

Abbreviations: cly = clypeus; ey = compound eye; lbp = labial palp; lbr = labrum; md $=$ mandible; $\mathbf{m x p}=$ maxillary palp; scu = scutellum; vpr $=$ vertical process (fastigium). 
There is a further type of enormous lobe-like processes which arise from the lateral parts of the vertex. This type is only present in Haania (Chopard 1920: figs. 1, 2; Anisyutkin 2005: figs. 1, 2; Anisyutkin \& Gorochov 2005: figs. 1, 2; Fig. 52).

The present dataset unequivocally supports a multiple convergent origin of head processes arising from the dorsal vertex (character 1:2>1). This type of process evolved independently in Hestiasula + Oxypilinae [79], Amphecostephanus [85], Pyrgomantis [93], Sibylla [127], Ceratocrania + Phyllocrania [138], Empusidae [148], Acromantis + Ambivia [197], clade [204] within Hymenopodinae, and Callibia [234].

Furthermore, head processes arising from the area of the lateral ocelli $(3: 2>1)$ have evolved multiple times convergently, at least in Gyromantis [17], Haaniinae [55], Oxypilinae [81], Vates [137], Zoolea [168], Theopropus [214], and possibly Galinthias [201] and Pseudoharpax [203] (under DELTRAN only).

\subsubsection{The male antennomeres}

The differences between male antennomeres in Mantodea were briefly described in chapter 4.1.1 (character 20) but ought to be discussed in further detail.

The antennomeres are filiform or moniliform in most insects. There are exceptions in which the males exhibit sometimes enormous outgrowths or enlargements of the antennomeres. This was described, for instance, for several Lepidoptera, Coleoptera, Neuroptera (e.g. Dilaridae: Rambur 1842: 445 and pl. 10, fig. 3; Carpenter 1947: fig. 3A), and Diptera (e.g. Tipulidae: Colless \& McAlpine 1991: figs. 39.15A, B). The enlargements of the antennomeres often carry a larger number of sensilla in order to locate females over great distances.

As mentioned in character 20 in chapter 4.1.1, there are several Mantodea of which the males do not have the usual cylindrical or globular antennomeres found in most Dictyoptera. A distinct asymmetry is present in several Hymenopodidae (Figs. 93, 94) and Vatinae (Fig. 95; see Rehn 1935: pl. 7, fig. 26 for Vates; Rivera 2001: fig. 1a for Pseudovates; Orofino et al. 2006: figs. 3, 21, 51, 75, 76 for Phyllovates). In Empusidae, however, the enlargements of the antennomeres are most elaborate.

In Blepharodes, Blepharopsis (Fig. 87) and Idolomantis (Fig. 88) the males show very large, flattened antennomeres (rounded or more or less deeply notched), while those of the females are slightly asymmetrical at most. Idolomorpha (Fig. 90, 91, 97, 98) and Hemiempusa have very slender, single processes that are alternately curved on the successive antennomeres, thus forming a "pseudo-bipectinate" antenna (Wood-Mason 1878a: 261; Edmunds 1972: 16; Roy 2004a: 2 and fig. 7). In the remaining Empusinae, the male antennomeres are truly bipectinate and carry two long, slender processes on each antennomere (e.g. Wood-Mason 1878: fig. 2; Bugnion 1921a: figs. 2, 3; Roy 2004a: 2 and fig. 7; Fig. 89).

The pectinate and bipectinate antennae of Empusidae may add to the cryptic effect when resting in their habitat by "breaking up the praying outline" (Edmunds 1972: 19 with regard to Idolomorpha). However, their role in mate location may be the more important factor to be 
considered. The equipment of sensilla on the empusid antennae has so far only been studied in detail for Gongylus gongylodes by Greven \& Brenner (2009). They found a large number of chemoreceptive sensilla. Although the number of sensilla on the antennomeres of males and females was not studied in detail, the authors argued that a higher number of sensilla on the male antennomeres is likely based on their larger surface (Greven \& Brenner 2009: 71). This is supported by Slifer (1968: 105-107), who recognized four times as many sense organs in the males as in the females of Tenodera angustipennis Saussure, 1869.

The attraction of males towards pheromone-releasing females was described for several taxa (although not for Empusidae) from different mantodean subgroups in the literature [Edmunds 1975: Tarachodes afzelii (Stål, 1871); Robinson \& Robinson 1979: Acanthops falcata Stål, 1877; Hurd et al. 2004: Sphodromantis lineola (Burmeister, 1838); Gemeno et al. 2005: Mantis religiosa and Empusa pennata; Perez 2005: Hierodula patellifera (Serville, 1839); Holwell et al. 2007b: Pseudomantis albofimbriata (Stål, 1860)]. Brief summaries of this topic were published by Maxwell (1999: 71) and Howell et al. (2007b: 308). It is possible that the enlarged surface of the antennomeres increases the sensitivity for the concentration of pheromone molecules in the air and therefore increases the distance from which a "calling" female can still be detected by the males.

The enormous enlargements of the male antennomeres is apomorphic for Empusidae (e.g. Roy 2004a), but the differences found in their shape require a more detailed discussion.

Audinet-Serville (1839) and Stål (1877) described the different shapes of the antennomeres. They pointed out the differences between blepharodine and empusine antennomeres but mentioned that all Empusidae have bipectinate antennae (Audinet-Serville 1839: 140; Stål 1877: 75,76 ). Wood-Mason (1878a) was the first to observe that in Idolomorpha (also present in Hemiempusa) the antennae of the males are pseudo-bipectinate: Each antennomere only carries a single process but the processes are alternately curved (in fact, one is nearly straight whereas the subsequent one is more strongly curved, as pointed out by Wood-Mason 1878a: fig. 1). This creates the superficial impression of a truly bipectinate.

The molecular phylogeny (Svenson \& Whiting 2009) and the mental analysis by Roy (2004a: fig. 15) showed Blepharodinae and Empusinae (each group being monophyletic) to be adelphotaxa. This scenario allows several possible interpretations for the evolution of this character:

1) The enlargement of the antennomeres occurred twice. In this scenario the flattened outgrowths on the antennomeres of Blepharodinae have evolved independently from the slender processes on the antennomeres of Empusinae. Under this assumption it is likely that the common ancestor still had slightly asymmetrical antennomeres at most.

2) A bipectinate antenna is a ground plan feature of Empusidae. The last common ancestor of Empusidae in this scenario would had a bipectinate antenna (it is unclear whether the slender or the flattened condition of the outgrowths represents the plesiomorphic condition). In Blepharodinae, the processes became (remained?) flattened and merged to form a more or less singular plate, whereas they remained (became?) slender in Empusinae. Idolomorpha and Hemiempusa would have synapomorphically lost one process on each antennomere. 
3) A singular process on the male antennomeres belongs to the ground plan of Empusidae. In this scenario the process would have become larger and plate-like in Blepharodinae, whereas Idolomorpha, as sistergroup to the remaining Empusinae, would have retained the plesiomorphic condition. The remaining Empusinae would apomorphically have gained a second, slender process on each antennomere.

The third scenario may gain support from the fact that many Hymenopodidae studied herein (Pseudocreobotra, Hymenopus, Pseudoharpax, Creobroter, Phyllocrania) have asymmetrical antennomeres, which may be the situation from which the characteristic antennomeres of Empusidae evolved. Interestingly, in some species both sexes have asymmetrical antennomeres, for instance in Pseudocreobotra. Nonetheless, hypotheses 1 and 3 do not represent the most parsimonious explanations. They require three evolutionary steps, whereas hypothesis 2 only requires two, no matter which of the two proposed ancestral states is assumed.

The present dataset on the other hand offers a fourth solution which also requires only two steps. Blepharodinae was found to be paraphyletic with respect to Idolomantis [153], which is more closely related to Empusinae [154] than to Blepharopsis and Blepharodes (Figs. 395, 401). This is supported by the enlarged ventral apical lobe of the fore coxa $(34: 2>1)$ in Idolomantis and Empusinae (in Idolomantis embedded in the surrounding ventral lamellar expansion of the coxa, see Fig. 133) and by the strong elongation of the postero-ventral fore femoral spines in these groups $(50: 1>2)$. It might also gain support from the distinctly longer pronotum in the two latter groups (Fig. 117, 124; Blepharopsis not shown) and from the fore legs of Idolomantis which are very slender (in their primordial shape, disregarding the lobes) and much more similar to Empusinae than to Blepharopsis or Blepharodes (Figs. 132, 133, 208-214). Also, the entire foreleg spination of Idolomantis and Empusinae is alike and differs from the aforementioned genera (Figs. 208-214). This analysis and the observations listed above imply that the evolution of asymmetrical male antennomeres from the original symmetrical state $(20: 1>2)$ as present in the outgroup occurred several times independently, for instance in Astape [56], Vates + (Ceratocrania + Phyllocrania) [136], Zoolea [168], clade [202] within Hymenopodinae, Raptrix [230] and Acanthops falcataria [236]. For Empusidae, the results suggest the following evolutionary scenario:

4) The last common ancestor of Empusidae [148] had large, lobe-like antennomeres which originated from the ground-plan of Mantodea (20:1>5). In Empusinae [154] the lobe-like antennomeres were reduced into two slender processes on each antennomere (20:5>4), one of which was finally reduced in Idolomorpha (20:4>3). This hypothesis is as parsimonious as hypothesis 2 mentioned above. It is likely that studies of the postembryonic development of the antennae of various Empusidae may yield further insight into the evolution of these charismatic antennal morphologies (see also chapter 4.6.7 on postembryonic development of the antennae).

\subsubsection{Lamellar expansions of the pronotum}

The pronotum of Mantodea has free lateral lamellar ridges in most species, albeit often very narrow at most (see also character 29 in chapter 4.1.2; Figs. 110-124), as exhibited by all of 
the so-believed "primitive" Mantodea (Mantoida, Chaeteessa, Metallyticus, Amorphoscelidae, Eremiaphilidae). In many taxa, however, the lateral lamellar expansions reach enormous sizes (e.g. Ehrmann 2002: figs. 13-17). The greatest overall size of the pronotum is exhibited by Choeradodis (Fig. 142), Asiadodis, Brancsikia, Deroplatys (Figs. 445, 446), and Idolomantis (Fig. 117). In the four former genera it is always the female that has the largest pronotum while the males have much smaller ones (see e.g. Westwood 1845c: pl. 9, figs. 1-4; Wood-Mason 1884: figs. 6, 7, 10-14; Paulian 1957: figs. 2-4; Ehrmann 2002: figs. 52-54, 55, 56; Roy 2004b: figs. 32, 33; Figs. 445, 446). In Idolomantis, however, both sexes exhibit an extreme enlargement of the pronotum [Saussure 1870a: pl. 5, fig. 36 ()); Westwood 1889: pl. 5, fig. 1 ( đ); Beier 1934b: fig. 4 (ठ); La Greca 1939a: fig. 1 (ð); Schwarz et al. 2006: figure on p. 69 (†); Fig. $117($ đ)].

Many taxa have medium-sized pronota, for instance Blepharopsis, Gongylus (Fig. 124), Parablepharis (Roy 2008b: figs. 1-7), Phyllocrania (Fig. 113), Rhombodera, and Tamolanica (Fig. 126). The greater part of the extant Mantodea only exhibits very narrow lateral lamellar expansions of the pronotum (e.g. Figs. 110-112, 114-116, 119, 120, 122, 123, 125).

It has been suggested that these expansions have evolved independently several times (e.g. Roy 1999: 40). This is supported by the molecular analyses (e.g. Svenson \& Whiting 2009: fig. 3) which provided evidence for the wide phylogenetic separation of, for instance, Deroplatys, Choeradodis and Empusidae.

A multiple convergent origin of the enlarged pronotum also gains strong support from morphology. The widening (in the definition chosen herein, see character 29 in chapter 4.1.2) has occurred many times independently: In Pogonogaster [47] (Fig. 127), Phyllocrania [140] (Fig. 113), Rhombodera + Tamolanica [145] (Fig. 126), Empusidae except for Blepharodes [150] (with a subsequent reduction in Empusa + Idolomorphini [156]; Figs. 117, 124), Deroplatys [175] (Figs. 16, 445, 446), Pseudocreobotra wahlbergii [207] (Fig. 121), and Choeradodis [216] (Fig. 142).

The large pronotum plays an important role in special resemblance to plant matter because it is breaking up the body outline. Therefore, it is part of the primary defence mechanisms (e.g. Edmunds 1972: 10). All taxa with such pronota are highly cryptic, with the most elaborate plant mimics being Choeradodinae, Brancsikia, Deroplatys, Gongylus, Parablepharis, and Phyllocrania. While the latter five genera mimic dry leaves, Choeradodis and Asiadodis very effectively mimic green leaves. Interestingly, Piton (1940: 132 and fig. 12) described a fossil Choeradodinae ( $\uparrow$ Prochaeradodis enigmaticus) from the Palaeocene deposits of Menat in France (statements range from 56 My to 61 My old; see Wedmann et al. 2009: 704). The fossil has conspicuous forewings and strongly enlarged lateral lobes on the pronotum. It was reviewed by Nel \& Roy (1996). Although they were not absolutely convinced that the specimen can actually be assigned to the extant Choeradodinae (Nel \& Roy 1996: 231), they supported Piton's (1940) assignment to Mantodea based on forewing morphology (Nel \& Roy 1996: 230). If this is correct, $\uparrow$ Prochaeradodis enigmaticus represents an approximately 60 million yearold example for elaborate special resemblance to plant matter in Mantodea. A similar case of plant mimesis was published by Wedmann et al. (2007), who described the first fossil phylliine 
phasmatodean from the Eocene deposits of Messel, Germany. The male specimen exhibited special resemblance to leaves similarly elaborate as in extant Phylliinae and was externally nearly indistinguishable from extant specimens (Wedmann et al. 2007: figs. 1-3).

\subsubsection{The "missing" discoidal spines of Metallyticus in view of its behaviour}

The autecology of Metallyticus is virtually unknown (Shelford 1903: 299, 303, 1916: 141; summarized in Wieland 2008a: 147, 157). Only few specimens were collected in the more than 170 years since its taxonomic description, and morphological studies on the genus are rare. Wieland (2008a) published a detailed review of the knowledge of Metallyticus and presented accurate morphological drawings of the
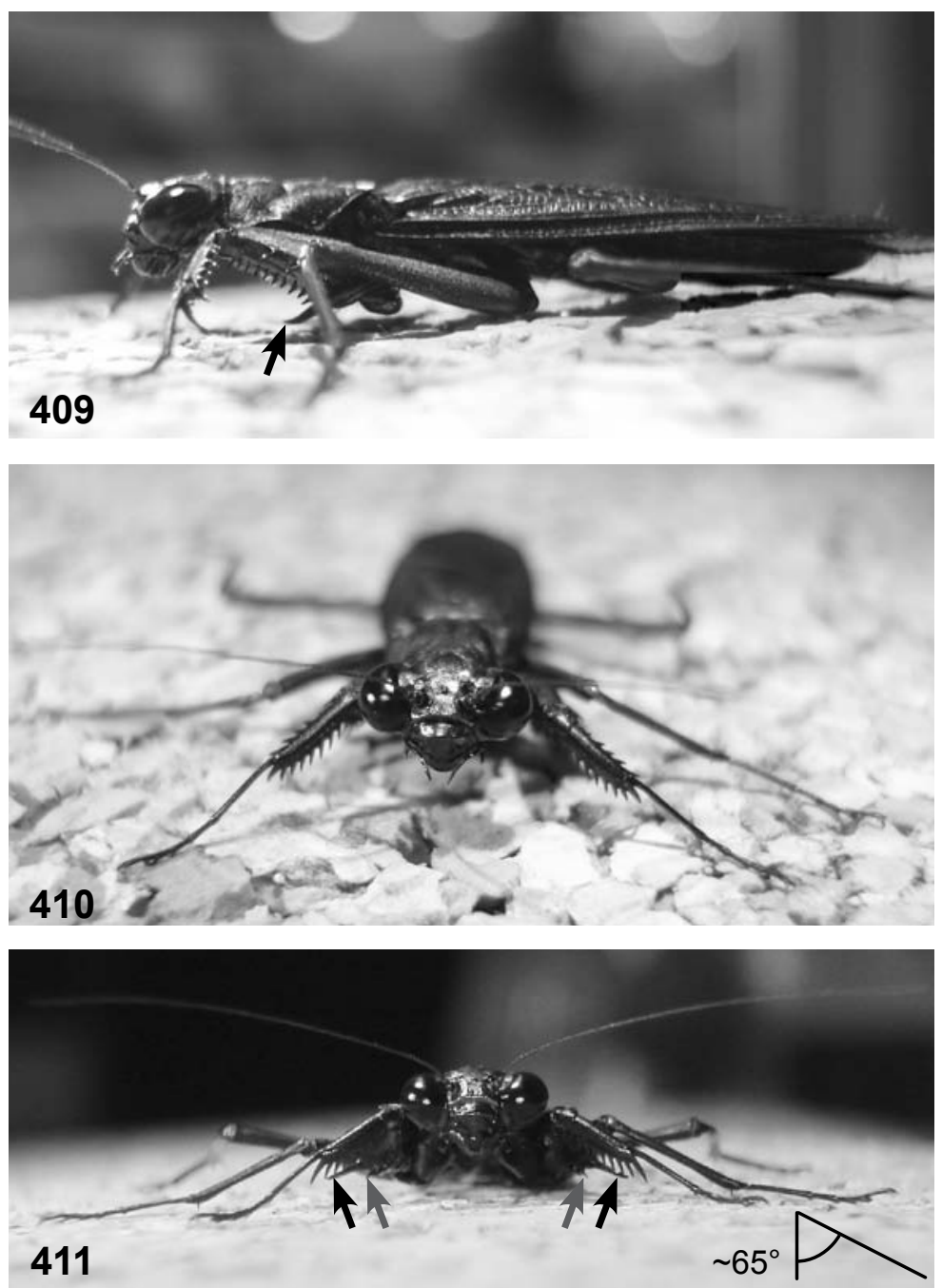

Figs. 409-411: Metallyticus splendidus, $\widehat{\partial}$, foreleg position in life. 409: Lateral view. 410: Anterior view from obliquely above. 411: Low anterior view. Note the oblique position of the forelegs (indicated by angle in Fig. 411), probably owing to the lifestyle of hiding under the bark of trees. Grey arrows indicate persisting discoidal spine, black arrows indicate elongated proximal postero-ventral spine.

Body length is about $26 \mathrm{~mm}$ (head to tip of tegmina).

exoskeleton as well as historical drawings from the literature (Wieland 2008a: figs. 1-30).

Living nymphs and adults of $M$. splendidus were observed in their natural habitat Malaysia in 2007 by S. Materna, J. Mehl, K. Schütte and by the author. Specimens obtained from a local insect trader were used to establish a breeding culture. Captive observations have been conducted over several generations. This allowed first observations on postembryonic development of the raptorial legs, hunting strategy and secondary defence mechanisms. Observations correlated with the morphology of the raptorial legs are discussed herein.

It was mentioned that Metallyticus lives on tree trunks or even under the bark of trees (Shelford 1903: 299, 1916: 142; see Wieland 2008a: 165 for a discussion). This was confirmed for M. splendidus in the natural habitat. While several nymphs were observed sitting on the open bark, adults were rarely seen in the open, and a male was detected scurrying from under a loose piece of bark when the latter was torn off. Subsequent observations in the breeding culture 

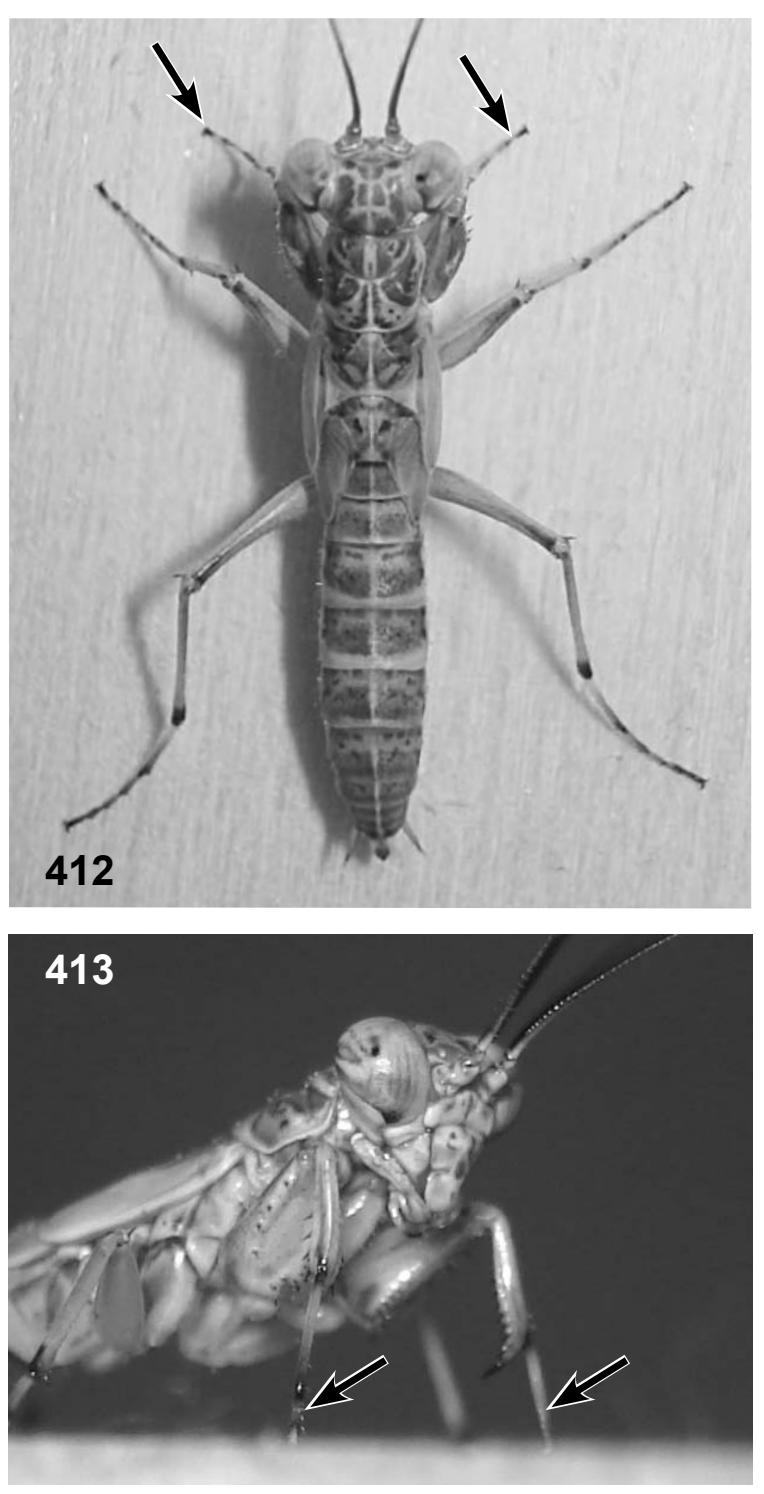

Figs. 412-413: Mantoida sp., subadult female, foreleg position in life. 412: Dorsal view. 413: Oblique anterior view. Note the fore tarsi that are kept on the ground (arrows). Length of animal is approximately $17 \mathrm{~mm}$. strongly support Metallyticus being a bark dweller. Adult Metallyticus regularly hide in narrow crevices under loose bark that sticks out from the surface. They are rarely seen in the open if hideouts are provided in sufficient numbers.

Metallyticus uses all six legs for running and does not lift its fore tarsi from the ground as most other Mantodea do (Figs. 9, 409-411), except for grooming (one tarsus at a time) or feeding purposes. The forelegs are held obliquely (Figs. 409-411). Putting all six legs on the ground instead of taking a "praying" posture was also observed in Mantoida (Deyrup 1986: fig. 1; White, pers. comm. 2008; Schulze, pers. comm. 2009; pers. obs. 2010; Figs. 7, 8, $112,113)$. The same seems to apply to Chaeteessa, as the picture of a living late instar nymph from Ecuador suggests (see Fig. 5). The cryptic colouration of Chaeteessa (see Figs. 5 and 6 and chapter 4.6 .5 below) is indicative of a bark dwelling lifestyle. It is possible that the behaviour of constatly putting all six legs on the ground also applies to this genus. Interestingly, the forelegs of Chaeteessa are held in a similarly oblique position as in Metallyticus, whereas they stand upright in Mantoida (Fig. 412, 413).

One could be inclined to assume that the short prothorax restricts the function of holding the forelegs up. However, several taxa with short prothoraces, e.g. Amorphoscelis, Perlamantis, Eremiaphila and others, regularly fold their legs against the prothorax when at rest, although they may occasionally use them for running (pers. obs.; also see Figs. 10-12). Therefore, it is unlikely that the behaviour of putting all six tarsi on the ground in Mantoida and Metallyticus (and probably Chaeteessa) can solely be explained with the shortness of the prothorax. Metallyticus (pers. obs.), Mantoida and Chaeteessa (Salazar 2005: $270 \mathrm{f}$.) are probably all bark dwellers. The situation is ambiguous in Mantoida, which (at least in the Florida population), may also hunt on the ground or dwell branches and bushes (see Blatchley 1920: 129; Hubbell 1925: 41; Strohecker 1939: 170; Gurney 1951: 359). Therefore, it could be argued that putting all six feet on the ground is a secondary adaptation to this habitat. However, although several bark dwelling Mantodea sometimes 
use their forelegs when moving [e.g. Amorphoscelis (pers. obs.), Liturgusa and Theopompa (Schwarz, pers. comm. 2009)], they regularly hold their legs folded against the prothorax when at rest (see late instar nymph of Liturgusa in Figs. 20, 21 and Amorphoscelidae in Figs. 11, 12). Therefore, it is likely that the habit of putting all feet on the ground represents the plesiomorphic condition within Mantodea.

In Metallyticus, the behaviour of hiding in narrow crevices under loose bark may on the one hand have led to a positive selection of the strong dorso-ventrally flattened body (Shelford 1903: 299, 1916: 142; Wieland 2008a: 165), but it may also have had a strong influence on the hunting behaviour. When spotting prey that runs on the bark and passes the crevice, hiding $\mathrm{Me}$ tallyticus usually make a quick dart for it, catch it and then retreat to their shelter (Mehl, pers. comm.; Wieland, pers. obs.). Narrow space under loose bark may delimit the ability to hold the legs folded against the prothorax because the anterior part of the body cannot be lifted high enough to do so. Therefore, the typical capture strike as exhibited by most extant Mantodea cannot be accomplished by Metallyticus. Instead, it exhibits a unique movement of the raptorial legs in order to grasp its prey. When disturbed (frontal confrontation with a fingertip) and having no chance to escape, Metallyticus strikes towards the source of disturbance (Wieland, pers. obs.; Mehl pers. comm.). During the strike, the ventral surface of the fore femora is held in a 90 degree angle with respect to the surface, therefore their spines are orientated directly towards the aggressor. Meanwhile, the forelegs are held open and the tibiae are positioned in a more or less right angle with respect to the ventral surface of the femora (thus being nearly parallel to the surface). In this position, the forelegs are used to strike forward. The elongated proximal postero-ventral femoral spines face the aggressor and are used like spears during secondary defence. They are orientated nearly parallel to the surface.

In the resting position, the opened forelegs are held in an oblique position. When prey comes into reach, a rapid forward burst is carried out. Although the strike is very quick it looks as if, before the forelegs grasp the prey, the legs are brought into a position resembling that described before for the defence strike. The elongated postero-ventral spines apparently reach below the body of the prey so the latter is captured when the tibia closes against the femur. The capture strike is therefore probably carried out in a quick turning and grasping movement of the forelegs. Use of a high speed camera might yield a better view of the movements.

Two topics concerning the morphology of the raptorial forelegs of Metallyticus have to be addressed: 1) The enormously elongated proximal postero-ventral fore femoral spine, and 2) the lack of discoidal spines. Both phenomena have been taken for a fact and were handed down in the literature for more than one and a half centuries. To my knowledge, no one has ever doubted that the discoidal spines on the fore femora are actually missing in Metallyticus.

The enormously elongated proximal postero-ventral fore femoral spine has been assumed to function as "the prongs of a fork-lift truck" in order to lift prey from the surface or as "a spear or as a pin to winkle cockroaches out of crevices" by Loxton \& Nicholls (1979: $197 \mathrm{ff}$.). The observation of the defence- and prey-capture behaviour supports the former hypothesis, while none of the observations in captivity corroborate the latter. 
The elongated spine is held low and almost parallel to the surface and strikes towards the aggressor or reaches below the body of the prey. The tibia then closes against the femur. The tibial spur meets the elongated spine and closes the ventral, spine-bearing surfaces between femur and tibia of the raptorial leg early, in order to prevent the prey from escaping. This function of a long spine on the ventral side of the femur (usually one strongly elongated discoidal spine), closing the foreleg early by decreasing the closing angle between tibia and femur, is well known and has been described, for instance, by Loxton \& Nicholls (1979). In this respect, the elongated proximal postero-ventral femoral spine of Metallyticus has apparently taken over the very function which is occupied by the longest discoidal spine in other Mantodea (pers. obs.; Schwarz, pers. comm.).

Taking into account the hiding behaviour and the resulting adaptation of the raptorial strike discussed above, a long spine that helps to close the foreleg early will have to be located close to the surface during the strike of the forelegs in order to reach below the prey object. On the other hand, when the forelegs are usually held in an oblique position (as in Metallyticus), possibly due to the narrowness of the crevices, it must be avoided that a long spine gets stuck on the surface, in this case to the bark. A postero-ventral long spine in such a foreleg position is farther away from the surface than a discoidal spine in its ventro-median position of the femur would be (see grey and black arrows in Fig. 411). Therefore, a positive selection of the shortening of the discoidal spine spine with a subsequent elongation of the postero-ventral spine makes sense from a functional point of view. The postero-ventral spine is further away from the surface in "normal" foreleg position and can be brought closer to the surface during the strike described before.

The effectiveness of the elongated postero-ventral femoral spine is furthermore enhanced by the shape of the fore femur, which supports this hypothesis. In adult Metallyticus, the femur has an almost triangular shape in anterior or posterior view (e.g. Giglio-Tos 1921: pl. 1, fig. 1a; Wieland 2008a: 176 and figs. 7, 10, 22; Fig. 226) which is probably autapomorphic. Due to this ventral enlargement, the spine-bearing ventral surface of the femur can more easily be held in a right angle to the surface when striking. It possibly also prevents the spines from getting stuck on the surface within the hiding crevice when the fore tarsi remain on the ground (Figs. 409-411).

In conclusion, the proximal postero-ventral femoral spine has probably been positively selected to become longer and more sturdy. It subsequently took over the function of the discoidal spine due to the secretive lifestyle of Metallyticus which possibly influenced both its hunting and defensive behaviour as well as its entire habitus.

In contrast to this scenario, it has been assumed that the discoidal spines are missing primarily in Metallyticus (implicated, for instance, by Roy 1999: 38). I was able to address this question by studying the first instar nymph of M. splendidus. SEM images (Figs. 414-417) have revealed that the first instar nymph does not exhibit the characteristic spination pattern of the adults. The femoral spines are much more slender in the early nymphs. The femur is more slender in lateral view, and it does not exhibit the strong triangular shape of the femora of the 
adults. Homology between nymph and adult can be ascertained by structures present in both stages, for instance two proximal rows of setae. One of them is in line with the postero-ventral femoral spines, the other one is located medio-ventrally on the femur. Their presence and location will in the following help to address the homology of the femoral spines.

The antero-ventral femoral spines of the first instar nymph exhibit the pattern of most modern Mantodea. There are 13 antero-ventral spines of distinctly alternating length. The proximal four spines have a slightly alternating position (which can also be found to a stronger extent in Mantoida, Chaeteessa and Acanthops, among others; see character 45). The first and third antero-ventral fore femoral spines are comparatively small in the first instar nymph (arrows in Figs. 415-417; similar to nearly all adult Mantodea with few exceptions). They are missing in adult Metallyticus (Fig. 414).

Furthermore, there are five postero-ventral femoral spines in the first instar nymph. The second is longer than the remaining ones, but it does by no means show the relative length with respect to the other spines displayed by adult Metallyticus. All postero-ventral femoral spines are longer than the antero-ventral ones, but only slightly thicker. Based on its position and length, the assumption is justified that the second postero-ventral femoral spine of the first instar nymph is homologous to the strongly elongated spine of the adults, whereas the proximal postero-ventral femoral spine is reduced during postembryonic development.

The oblique, medio-ventral row of slender socketed setae is located between the antero- and postero-ventral femoral spines. As was mentioned earlier, it can also be found in the adults. Its distal seta as well as two of the proximal ones are sturdy and long in the nymph. They resemble the postero-ventral spines in shape and thickness. These setae, or at least some of them, including the distal one, are herein hypothesized to represent the discoidal spines of Metallyticus. This is supported by several morphological observations. The first one is the position of the claw groove in the first instar nymph. When the foreleg is closed, the tibial spur rests in the claw groove. The groove is located between the antero-ventral spines and the medio-ventral row of setae (Figs. 415-417). This is the typical position of the claw groove in all extant Mantodea in which the groove is not strongly reduced due to the aberrant spination pattern (as, for instance, in Amorphoscelinae and some Oligonicinae). The distal medio-ventral spine thus lies posterior to the tibial claw when the leg is closed, which is a distinctive criterion for discoidal spines.

Furthermore, the comparison of the antero-ventral femoral spines (length and position from distal to proximal) of the first instar nymph and the adult of Metallyticus reveals 12 anteroventral femoral spines anterior to the claw groove in adult specimens. 12 spines have been counted in all M. splendidus specimens that were available for study, therefore the number appears to be constant in this species. The first instar nymph, however, has 11 antero-ventral femoral spines (the two very small proximal ones that are reduced in later instars and adults are not counted). This allows to deduce two conclusions. First, the position of claw groove is relocated during postembryonic development and second, one of the discoidal spines (probably the distal one) persists in adult Metallyticus. 

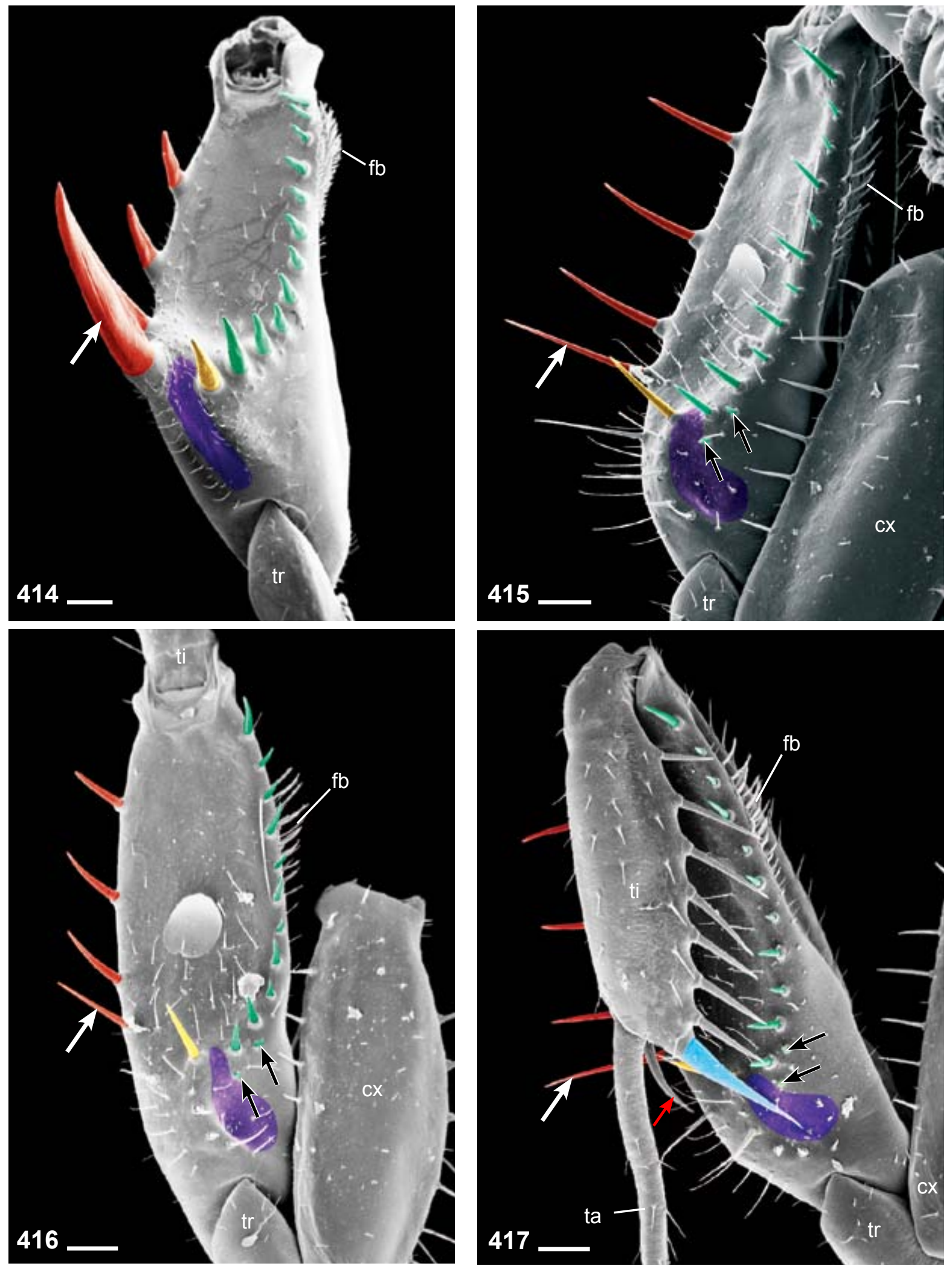

Figs. 414-417: Metallyticus splendidus, spination of fore femur. 414: Adult $q$, right fore femur, oblique ventral view. 415, 416: First instar nymph, left fore femur (mirrored). 417: Right foreleg with tibia closed against femur. Green: Antero-ventral femoral spines; Red: Postero-ventral femoral spines; Yellow: Remnant of discoidal spine; bright Blue: Tibial spur; Violet: Position of claw groove. Black arrows indicate the first and third antero-ventral fore femoral spines in the first instar that are completely reduced in the adult. White arrows indicate enlarged postero-ventral spine in adult and corresponding spine in the nymph. Red arrow in Fig. 417 indicates the long distal postero-ventral fore femoral spine. - Scale bar $=0.5 \mathrm{~mm}(414) ; 0.1 \mathrm{~mm}(415-417)$.

Abbreviations: $\mathbf{c x}=$ coxa; $\mathbf{f b}=$ fore femoral grooming device (femoral brush); $\mathbf{t a}=$ tarsus; $\mathbf{t i}=$ tibia; $\mathbf{t r}=$ trochanter. 
These conclusions can be explained by the evolutionary scenario discussed above. An elongated proximal postero-ventral spine may have been positively selected for the reasons mentioned before. As soon as the spine became longer than the longest discoidal spine, the function of the latter (that may have been similar to that of most other Mantodea) became redundant. Therefore, the long discoidal spine probably was becoming shorter once the selective pressure on its length relaxed. In adult Metallyticus, the only remaining discoidal spine is about as long as the antero-ventral spines and equally sturdy. It functionally has a valuable position on the femur because it continues the curved row of the antero-ventral femoral spines towards the middle of the ventral femur. Therefore, it provides an important function in securing the captured prey, which may be a reason why it has not been entirely reduced.

In consequence, the tibial claw had to get closer to the elongated postero-ventral femoral spine in order to fulfill their joint function the same way it did before with the long discoidal spine. The shift of the claw groove towards the posterior edge of the femur during postembryonic development, thus lying medio-ventrally rather than antero-ventrally in adult Metallyticus, is indicative of this evolutionary step. Therefore, the only remaining discoidal spine in adult Metallyticus lies anterior to the tibial spur when the foreleg is closed.

This scenario gains additional support from the position of the two rows of setae on the proximo-ventral femoral surface of the adult specimens (Fig. 414). The posterior row lies in line with the postero-ventral femoral spines, whereas the anterior row lies in line with the persisting discoidal spine. The claw groove in adult Metallyticus lies between them. In the first instar nymph the two rows are also present in the identical positions (see discussion of nymphal discoidal spines above). The claw groove, however, lies anterior to the "discoidal" row of setae.

To conclude, the study of the postembryonic development of Metallyticus allows to favour an evolutionary scenario which, in combination with observations of living Metallyticus specimens, supports the persistence of one discoidal spine in adult Metallyticus. Selective pressure on an elongated postero-ventral femoral spine likely led to the loss of the primary function of the long discoidal spine (antagonist to the tibial spur) which was probably present in the ancestors of Metallyticus (as in all extant Mantodea). The claw groove shifted from a position anterior to the discoidal spine to a position posterior to it as the primary function of the long discoidal spine was transferred to the elongated postero-ventral femoral spine. This shift is repeated during postembryonic development and is indicative of this evolutionary scenario which may have led to the enigmatic foreleg shape and spination of adult Metallyticus. The raptorial legs of Metallyticus are in no way primitive but highly adapted to a specialized way of living, that potentially is unique among tree-dwelling Mantodea. Unfortunately, fossil relatives of $\mathrm{Me}$ tallyticus that could support such a scenario are unknown.

\subsubsection{The "missing" tibial spur of Chaeteessa}

In all extant Mantodea except for Chaeteessa the apical antero-ventral spine of the tibia is strongly enlarged (“tibial claw” or "tibial spur”) (Beier 1968a: 22; Roy 1999: 38; Klass \& Mei- 
er 2006: 17). Beier (1968a: 22) mentioned that the insertion point of the tarsus was transferred latero-apically due to the presence of the tibial spur.

Klass (1995: 193) argued that the only morphological difference between the tibial claws of Chaeteessa and Mantoida (representing all remaining Mantodea) is merely in its shape (Klass 1995: 193: "Der Unterschied zwischen Mantoida und Chaeteessa dürfte also nur in der etwas abgeleiteteren Ausbildung der Endklaue bei Mantoida bestehen: Sie ist deutlicher von den Dornen der davor gelegenen Reihe verschieden und stärker nach hinten gerichtet als bei Chaeteessa."). The terminal antero-ventral tibial spine of Chaeteessa rests in a claw groove and has consequently been assumed to be homologous to the tibial claw of the remaining Mantodea (Klass 1995: 193). This led to the statement by Klass \& Ehrmann (2003: 186) that the tibial claw of Chaeteessa does not differ from the remaining antero-ventral tibial spines.

Klass \& Meier (2006: 17) stated that the tibial claw of all mantodeans except for Chaeteessa is located on a distal tibial lobe surpassing the base of the tarsus and that such a lobe is missing in Chaeteessa. A further argument of Klass \& Meier (2006: 17) was that the 90 My old $\dagger J e r s i-$ mantis luzzii (Grimaldi 1997: fig. 3; Fig. 31) has a primitive, setae-like spination of the forelegs but also a distinct and enlarged tibial claw. From this, Klass \& Meier (2006: 17) deduced an early origin of the tibial spur and its possibly secondary reduction in Chaeteessa. This seems to find support in much older fossils, e.g. †Cretomantis larvalis (135 My old) which already had a distinct tibial spur (Gratshev \& Zherikhin 1993: figs. 4B-D; Grimaldi 2003: fig. 13; Fig. 29). $†$ Santanmantis axelrodi (115 My old) possibly also had a distinct tibial spur as the $\mu$-CT image presented by Grimaldi (2003: fig. 19, right hand side; Fig. 32A) indicates. The fore tibia of Pseudocreobotra wahlbergii in dorsal view (Fig. 32B) shows a similar organization of the tibial apex as in $†$ Santanmantis (tarsal articulation, potential presence of a lobe carrying the tibial spur), which may indicate the presence of a spur in the fossil.

However, there are also fossil species of about the same age (†Burmantis asiatica, $100 \mathrm{My}$ old) or younger (†Chaeteessites minutissimus Gratshev \& Zherikhin, 1993, 85 My old) that apparently did not have as strongly enlarged tibial spurs on enlarged antero-apical lobes, although the distal antero- and postero-ventral tibial spines are distinctly larger than the remaining spines.

Roy (1999: 38) stated, that Chaeteessa does not possess a tibial spur but that "[...] in its place only a kind of setigerous tubercle is found". Such a tubercle is indeed present in Chaeteessa (also mentioned by Klass 1995: 193 "Bei Chaeteessa findet man median der TarsusArtikulation einen kleinen Stummel mit feinen Borsten [...]"). It is located on the tibial apex, close to the insertion point of the tarsus, as well as distally from the last antero-ventral tibial spine (Figs. 418-420). From the tubercle, a sturdy seta or small spine is directed distad (Figs. $419,421,422)$. There are further but distinctly smaller setae located subapically on the ventral side of the tubercle (Fig. 421). Interestingly, similar setae are also found in Mantodea that have a long tibial spur.

Klass (1995) observed that in Chaeteessa the distal antero-ventral tibial spine rests in the claw groove, i.e. between the proximal antero-ventral femoral spines and the discoidal spines (Fig. 418). However, so far no author has considered the curious overall shape of the fore tibia of this genus. The tibia is strongly curved in dorsal view (e.g. Agudelo Rondón 2004: fig. 3.3; 
Grimaldi \& Engel 2005: fig. 7.91; Figs. 418, 420), with the antero-ventral tibial spines lying on the convex edge and the postero-ventral spines being located on the concave edge of the tibia. This is likely a derived character state and apomorphic for Chaeteessa. Consequently, the distal antero-ventral tibial spines have a position differing from that in other Mantodea when the leg is closed, and the last antero-ventral tibial spine proximal to the former tibial spur (i.e. to the setigerous tubercle) came to lie in the claw groove. This hypothesis gains support from the structure of this spine. Compared to the preceding antero-ventral tibial spines, it is strongly elongated (as is the distal postero-ventral tibial spine; see Fig. 422), thus reaching the anterior margin of the femur when the leg is folded. Therefore, this spine has probably secondarily acquired the function of the reduced tibial spur (see also characters 68, 69). It is likely that the original tibial spur became subsequently reduced until only the small seta remained.

The reason for a curved tibia may lie in an adaptation to the habitat. The only data on living Chaeteessa specimens was published by Salazar (2005: 270 ff.). Several specimens of Chaeteessa (described as C. nigromarginata by Salazar 2004b) were found on the bark of an avocado tree trunk (Persea sp.) together with several Mantoida specimens. The movements of Chaeteessa have been described as quick and agile with a tendency to hide to the opposite side of the trunk (Salazar 2005: 271). This indicates that Chaeteessa is a bark (or canopy) dweller, which also gains support from Dantas et al. (2008: pl. 1), who caught a Chaeteessa specimen with a light trap at 45 metres altitude above ground level in the Brazilian rainforest. Furthermore, Chaeteessa specimens were observed on the bark of living trees in Ecuador in 1986 (McKamey, pers. comm. 2009).

The colouration of living specimens is also indicative of a tree-dwelling lifestyle (Figs. 5 and 6). It strongly resembles the colouration of many Liturgusidae (e.g. Ciulfina, Hagiomantis, Liturgusa, and Stenomantis) which are all bark dwellers. This type of colouration, "of the bark, being grey with patches of green and yellow" (as described for Liturgusa by Hingston 1932: 275; see Preston-Mafham 1990: pl. 48; Figs. 20, 21) is quite typical for these taxa. They resemble the colouration of moss- and lichen-speckled bark. This is also the case in living Chaeteessa specimens, in contrast to the entirely yellowish or brownish condition which is usually found in dried museum specimens. The head and the pronotum are brownish with a yellow pattern (or vice versa), whereas the tegmina are brown with black and yellow parts of venation (Fig. 6). The legs are of a bright green with a parallel pattern of dark brown stripes. Chaeteessa has a flattened body, similar to that of Metallyticus.

All these observations strongly indicate a bark dwelling lifestyle of Chaeteessa. It is unknown whether it also hides in narrow crevices as Metallyticus does. Such an assumption is certainly highly speculative, but would provide one possible explanation for the rare findings of Chaeteessa throughout the past centuries. At least the oblique foreleg position in life is apparently quite similar in the two genera (compare Figs. 5, 9, 409-411). While in Metallyticus the resting position with all six tarsi being generally put on the ground and hiding in crevices may have led to the morphological changes described in the previous chapter, the foreleg morphology of Chaeteessa may have evolved in a different way, resulting in the curved shape of the fore 


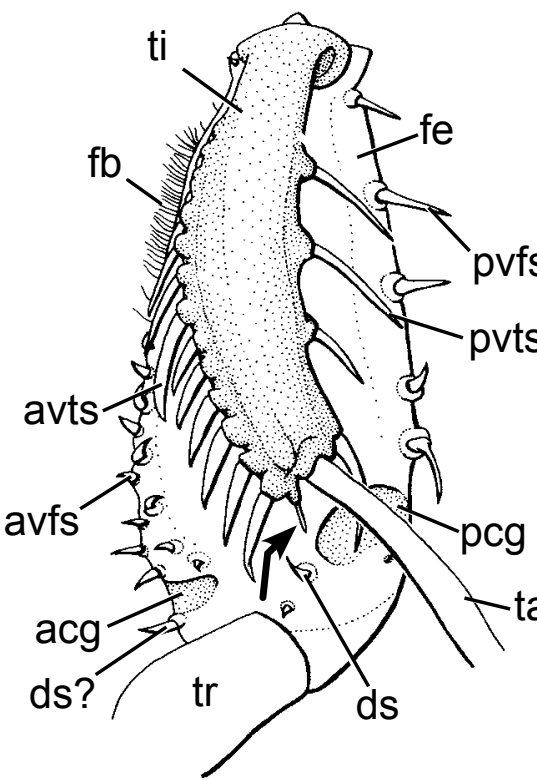

418

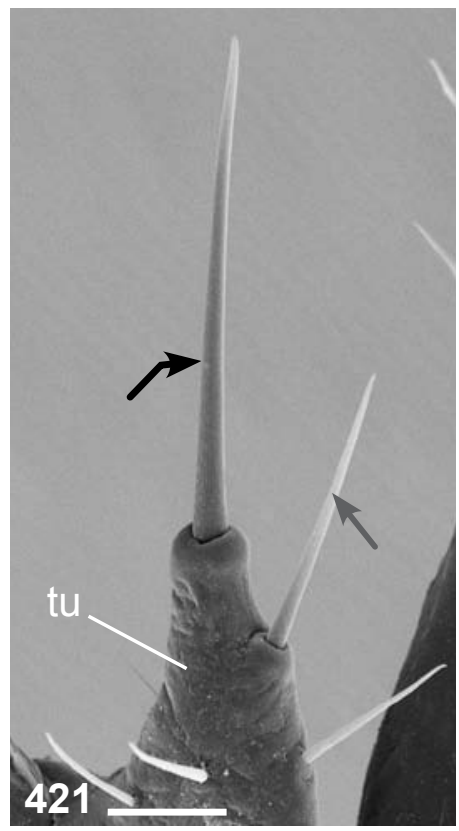

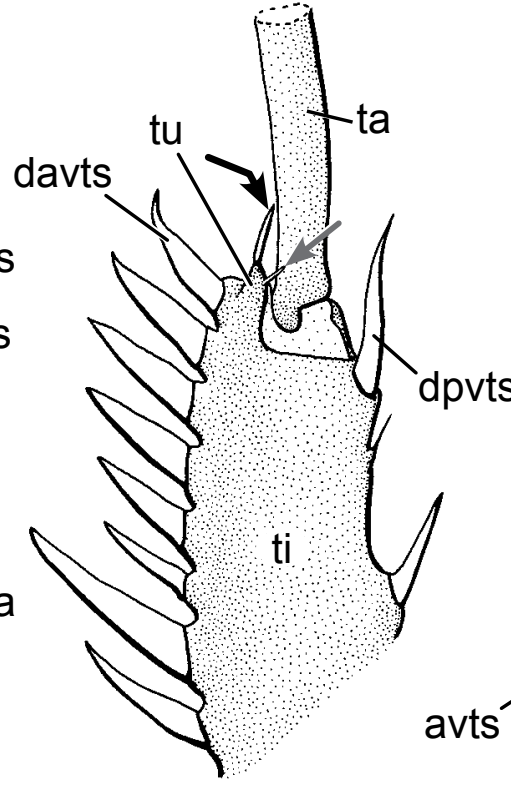

419
422

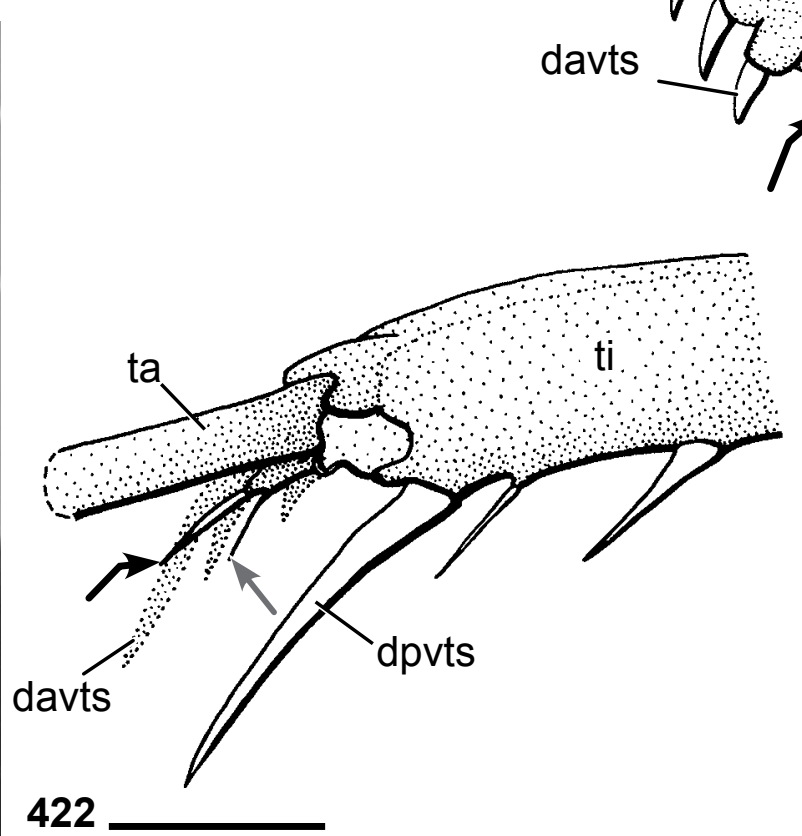

$\mathrm{ti}$

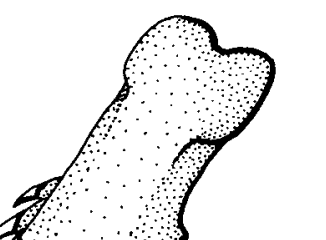

Figs. 418-422: Foreleg morphology of Chaeteessa. 418, 420: Chaeteessa valida, $q$ (NHMW specimen), left foreleg. 419, 421-422: Chaeteessa cf. valida, sex unknown, left foreleg. 418: Entire leg, ventral view, leg slightly opened. 419: Apex of tibia in ventral view. 420: Tibia in dorsal view; note the curved shape. 421: SEM image of detail of reduced tibial spur, ventral view. 422: Apex of tibia in oblique posterior view. Black arrows indicate the reduced tibial spur located on a tubercle. Note the additional bristle (larger than the remaining ones) found proximal to the reduced tibial spur (not recognized in the Vienna specimen), indicated by grey arrows. - Scale bar $=$ $0.5 \mathrm{~mm}$ (418-420, 422); $0.05 \mathrm{~mm}$ (421).

Abbreviations: acg = anterior claw groove; avfs = antero-ventral femoral spine; avts = antero-ventral tibial spine; davts = distal antero-ventral fore tibial spine; $\mathbf{d s}=$ discoidal spine; $\mathbf{d p v t s ~}=$ distal postero-ventral fore tibial spine; $\mathbf{f b}=$ fore femoral grooming device (femoral brush); $\mathbf{f e}=$ femur; $\mathbf{p c g}=$ posterior claw groove; $\mathbf{t a}=$ tarsus; $\mathbf{t i}=$ tibia; $\mathbf{t r}=$ trochanter; $\mathbf{t u}=$ setigerous tubercle.

tibiae, which may have triggered a secondary reduction of the tibial spur.

However, this morphology may also represent an adaptation to special food taxa. These hypotheses will have to be tested by observations of living Chaeteessa. 
To conclude, I assume that the setigerous tubercle, due to its position, is homologous to the tibial spur (and the lobe carrying it) in all other Mantodea (in accordance with Roy 1999). The spur was possibly secondarily reduced (in congruence with the fossil record, Klass \& Meier 2006, and the observations on tibial morphology). Therefore, its lacking rather represents a potential autapomorphy of Chaeteessa than a plesiomorphic trait. The tibial spines became longer during this process (which is very obvious in the distal antero-ventral and postero-ventral tibial spines), possibly in order to compensate for the missing tibial spur. It is utmost important to study the morphology of first instar Chaeteessa under the SEM in order to compare the foreleg morphology of nymph and adult.

\subsubsection{The reduced number of tarsomeres in Heteronutarsus}

Since the description of Heteronutarsus by Lefèbvre in 1835 (501 ff.) it has been repeatedly stated that it is the only genus of Mantodea with a number of tarsomeres other than five (Lefèbvre 1835: 502, 504 and pl. 13B, figs. 7-9; Chopard 1941: 33 and fig. 7; Beier \& Heikertinger 1952: 15; Beier 1964a: 874, 1968a: 22; Roy 1999: 29, 30; Ehrmann 2002: 20; Klass \& Ehrmann 2003: 185; Figs. 423-425). Only Burmeister (1836: 324, 1838: 520, 525) doubted this. According to him, Lefèbvre (1835) had described Heteronutarsus from nymphal specimens, whereas Burmeister claimed that he himself had studied corresponding adult specimens which all had pentamerous tarsi (Burmeister 1838: 525; also discussed by Burmeister 1836 on p. 324: “[...] dass dagegen alle Formen, denen die Normalzahl der Fussglieder fehlt, für Larven gehalten werden müssen, wenn a) ihnen die Nebenaugen und Flügel fehlen, und b) die Fühler zugleich kürzer und dicker sind."). Burmeister (1836: 324, 1838: 525) therefore indicated that it was possible that the nymphs of Eremiaphilidae had lesser tarsomeres, but the adults actually had five. However, a change in tarsomere number during postembryonic development has never been observed. As the presence of only three tarsomeres on the cursorial legs and four on the tarsi of the raptorial legs in Heteronutarsus was confirmed several times (e.g. Chopard 1941: 33 and fig. 7; Wieland, pers. obs.; Figs. 423-425), the description of a lesser number of tarsomeres in Heteronutarsus (no matter what ontogenetic stage) by Lefèbvre (1835) was probably correct.

One further species with a smaller number of tarsomeres is Eremiaphila zolotarevskyi Chopard, 1940 (b: 17; see Eremiaphilidae in chapter 2.1.4). It was later assumed to actually belong to Heteronutarsus by Chopard himself (1941: 34). This species has been described to have five tarsomeres on the fore tarsi and four on the meso- and metathoracic tarsi, while the distal tarsomere is partially segmented (Chopard 1941: 34). It is unclear if the reduction is an individual aberration (possibly due to loss and regeneration of the tarsi on the cursorial legs, see Przibram 1907: 612 and Beier 1964a: 860) or if this character is consistent throughout this species. The assumption that Eremiaphila zolotarevskyi is in fact a species of Heteronutarsus is also supported by the strong difference in the length of the ungues (Chopard 1940b: 17 "griffes très inégales et un peu épaisses"; compare Figs. 332, 424). However, the latter character has also been mentioned for some Eremiaphila species (e.g. Roy 1999: 30) and the number of prothoracic 
tarsomeres in Heteronutarsus is usually four, not five (Fig. 423). Therefore, it can be tentatively assumed that $E$. zolotarevskyi has been described from an Eremiaphila specimen with regenerated tarsi, but this phenomenon requires intensive further study.

It has not often been addressed in detail which tarsomeres are actually fused or possibly missing. Chopard (1941: 34) mentioned for Eremiaphila zolotarevskyi that the last tarsomere is partly segmented ("[...] dont le dernier est partiellement segmenté"), thus indicating that it is the distal segments in some Eremiaphila species and possibly Heteronutarsus that tend to fuse. In polyneopteran insects with a number of tarsomeres less than five (with five probably being a groundplan feature of Dicondylia; e.g. Boudreaux 1979: 168; Ax 1999: 268; Willmann 2003a: 21),

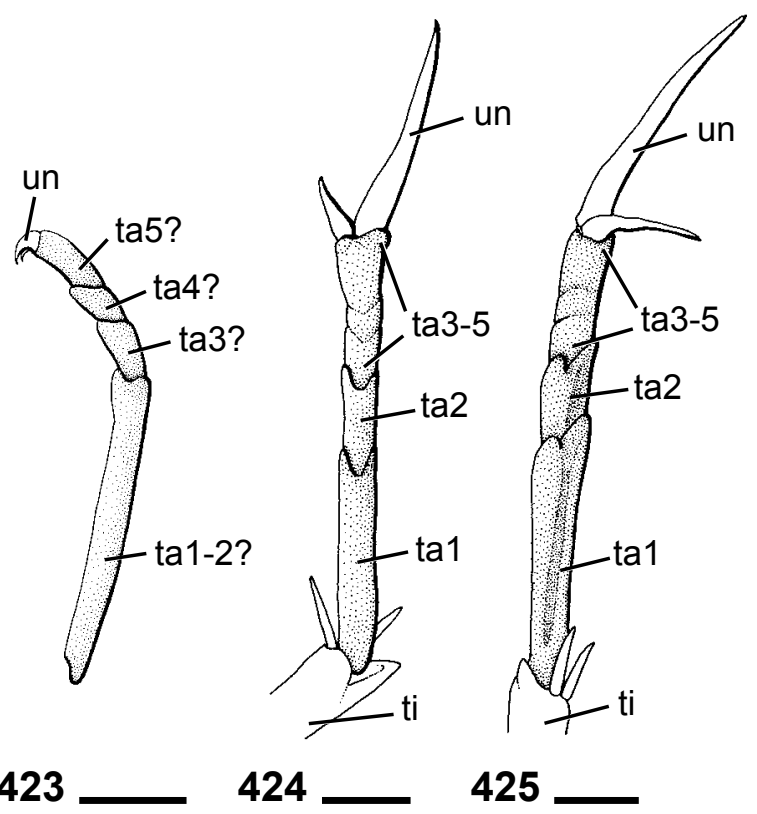

Figs. 423-425: Reduced tarsomeres of Heteronutarsus aegytiacus, 9. 423: Left foreleg, tarsus, posterior view (note the small ungues). 424: Left mesothoracic leg, tarsus, dorsal view. 425: Same leg, posterior view. Scale bar $=0.5 \mathrm{~mm}$.

Abbreviations: $\mathbf{t a 1}-\mathbf{t a 5}=$ tarsomeres $1-5 ; \mathbf{t i}=$ tibia; $\mathbf{u n}$ $=$ unguis. two or more tarsomeres become fused. For instance, Ensifera have four or three tarsomeres, and Caelifera have three, and sometimes only two (e.g. Beier 1955a: 83; Groll \& Günther 2003: 264). In these groups the proximal tarsomeres become fused [Snodgrass 1935: 198; pers. obs. in Hemideina maori (Pictet \& Saussure, 1893), unidentified Gryllacrididae species, and Saga Charpentier, 1825]. Extant Dermaptera also have trimerous tarsi, although in fossil stemgroup representatives they were pentamerous (Hennig 1969: fig. 45; Willmann 1990: figs. 1, 2; Engel 2003: 115; Haas 2003: 173, 178; Haas \& Gorb 2004: 65; Grimaldi \& Engel 2005: 218). To my knowledge it is unknown if two tarsomeres were lost during the evolution of this group or, if not so, which tarsomeres became fused.

In Heteronutarsus, the presence of three tarsomeres likely represents an adaptation to quick running on bare soil by stiffening the tarsi. In the meso- and metathoracic legs tarsomeres ta3-ta5 form a rigid unit. Together with the strongly elongated anterior ungues (see characters 99 and 100 and Figs. 332, 424) the tarsi possibly enable Heteronutarsus to run on soft, sandy surfaces with higher velocity.

The prothoracic tarsi of Heteronutarsus have four tarsomeres (Lefèbvre 1835: 502, 504 and pl. 13B figs. 7-9; Chopard 1941a: 33 and fig. 7; Fig. 423). However, in contrast to the meso- and metathoracic tarsi, no sutures could be detected. Interestingly, the length relations of the three distal tarsomeres are similar to those of the distal three (fused) tarsomeres in the meso- and metathoracic tarsi, whereas the proximal tarsomere is much longer than in the latter (Fig. 423). Therefore it is possible that in the prothoracic tarsi the two proximal tarsomeres are fused. This would imply different selective forces on the prothoracic tarsi and on those of the cursorial 
legs. This scenario is plausible since only the latter are used for running and therefore underlie the selective pressure imposed by living and running around in open terrain at high velocity. On the other hand, it is also possible that one tarsal segment in the fore tarsi was entirely reduced. As mentioned above, tarsi which are amputated during postembryonic development are regenerated in Mantodea, but only with four tarsomeres (e.g. Beier 1964a: 860). However, this is unlikely the case in the forelegs of Heteronutarsus because the tarsi of all specimens studied so far had four tarsomeres in the forelegs. Furthermore, the cursorial legs show distinctly fused tarsomeres, and in the Heteronutarsus female studied herein (fore tarsi missing in male specimen) the tarsomeres of both forelegs exhibited four tarsomeres (Figs. 424, 425).

It is indispensable to study fresh (alcohol-preserved) specimens as well as early instar nymphs under the SEM in order to trace the postembryonic development of the tarsal morphology of Heteronutarsus.

\subsubsection{Ontogenetic observations}

Several publications deal with the postembryonic development of single characters or treat the entire postembryonic development of individual species. Among them are Przibram \& Megušar (1912), Adair (1914), Sztern (1914), Bugnion (1921a), Przibram (1930), Przibram \& Brecher (1930), Roberts (1937a, b), Terra (1980), Nickle (1987), Köchy (1991), Yager (1996b), and Karuppanan (1998). Beier (1964a: 855 ff.) provided an overview of the publications until 1964.

Throughout this study, several significant morphological changes during postembryonic development were observed which will be briefly summarized and discussed in this chapter.

Ontogenetic changes in antennal morphology. Characters 18 and 19 refer to the number of antennomeres of adult Mantodea. About half of the species have more than 70 antennomeres (male sex); however, the material available of many species had damaged antennae and was not encoded, therefore the number in living specimens may have been even higher.

In contrast, the number of antennomeres in first instar nymphs is generally much lower (e.g. Beier 1964a: 858, 1968a: 35; Klass \& Ehrmann 2003: 193). For instance, adult Metallyticus have between 110 and 129 antennomeres, whereas the first instar nymph has no more than 40 (pers. obs., see Figs. 453, 454). All adult Theopompa studied herein had more than 110 antennomeres, the male of T. tosta even more than 130. The first instar nymph of T. servillei (Fig. 458) has little more than 40. The same is true for the first instar nymph of Orthodera novaezealandiae (about 40; Fig. 460), in which all adults had between 70 and 89 antennomeres.

The increasing number of antennomeres during mantodean ontogeny was described, for instance, by Beier (1964a: 858, 1968a: 35). He stated that first instar nymphs of Mantis religiosa have 18 antennomeres, whereas the adults have between 80 and 90. In Empusa the first instar nymphs have 18 antennomeres and the adults between 50 and 80 (Adair 1914: 76; Bugnion 1921a: 118; Chopard 1938: 248 and figs. 267-270; Beier 1964a: 858).

The same postembryonic changes in the number of antennal segments have been described 
for Blattaria (Bugnion 1921b; Schafer 1973: 354; Beier 1974a: 94). In this group, the first antennomere of the flagellum becomes subdivided during postembryonic development (Bugnion 1921b: 60; Schafer 1973: 354; Beier 1974a: 94). This is also true for Mantodea. However, there apparently is a second mechanism by which antennomeres which originated from the first antennal segment become subdivided (Bugnion 1921a: 127).

A curious case of morphological change throughout the postembryonic development of Pseudocreobotra ocellata was

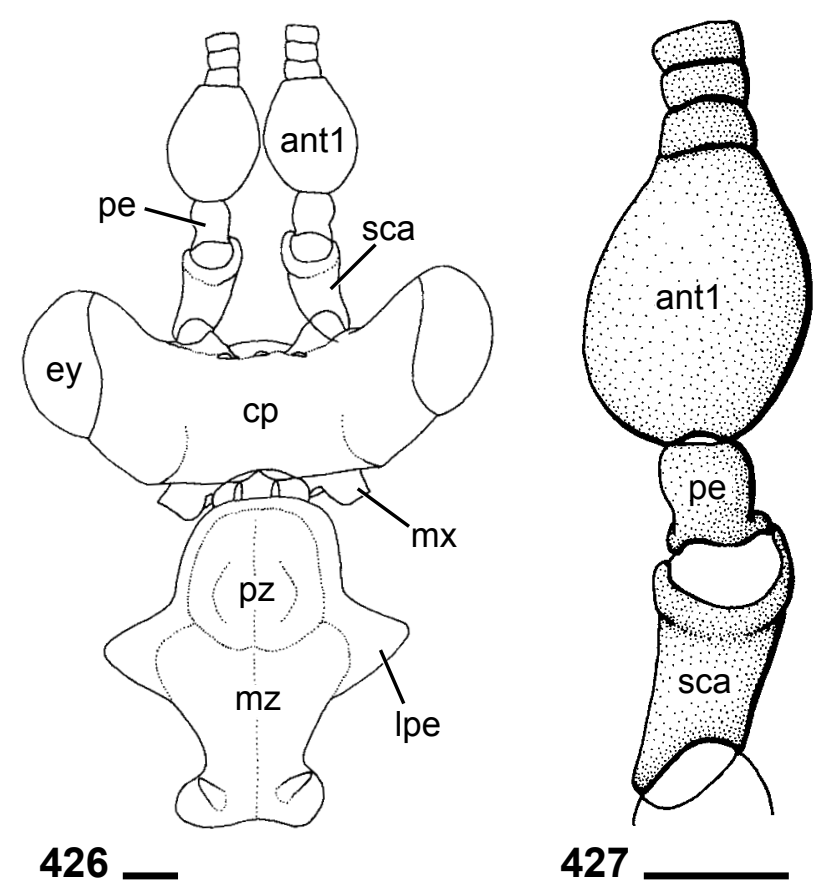

Fig. 426-427: Pseudocreobotra wahlbergii, first instar nymph. 426: Head and pronotum, dorsal view. 427: Bulbous third antennomere, detail. - Scale bar $=0.25 \mathrm{~mm}$.

Abbreviations: ant1 $=$ first antennomere; $\mathbf{c p}=$ caput; $\mathbf{e y}=$ compound eye; lpe = lateral pronotal expansion; $\mathbf{m x}=$ maxilla; $\mathbf{m z}=$ metazona; $\mathbf{p e}=$ pedicellus; $\mathbf{p z}=$ prozona; $\mathbf{s c a}=$ scapus. presented by Roy (1961: 101 and fig. 8). In the first to last instars, the first antennomere of the flagellum is enlarged and globular. This is most distinct in the early instars, as can be seen, for instance, in the second instar of $P$. ocellata shown by Heßler et al. (2008: fig. 90). It is much wider in diameter than the scapus and the pedicellus. Throughout nymphal development, the diameter decreases with respect to the scapus. In the adults, the first antennomere exhibits a "normal", i.e. slender and cylindrical, shape similar to the remaining antennomeres. This could also be confirmed for Pseudocreobotra wahlbergii (Figs. 426, 427), thus supporting the sistergroup relationship of the two species.

The first instars of both Pseudocreobotra species exhibit ant mimicry (e.g. Neumann 2006: 113; Heßler et al. 2008: fig. 90). It is possible that the enlarged first antennomere adds to the mimetic effect.

Ontogenetic changes in the head processes. As mentioned in the description of character 2, the head process of Phyllocrania is small and symmetrical in the first instar nymph (see Gillon \& Roy 1968: fig. 47; Fig. 408), whereas it is much larger, asymmetrical and exhibits large lobe-like lateral expansions in the adult (Figs. 41, 63). A similar situation is present in Phyllothelys (Schütte, pers. comm. 2010) in which the process is symmetrical and shorter in the first instar than in the adults. In Didymocorypha, the (bipartite) head process is much shorter in the first instar than in the adults (see Figs. 429, 430). A comparative study of these postembryonic changes has not been conducted. Studying a wide range of phylogenetically distant species with 


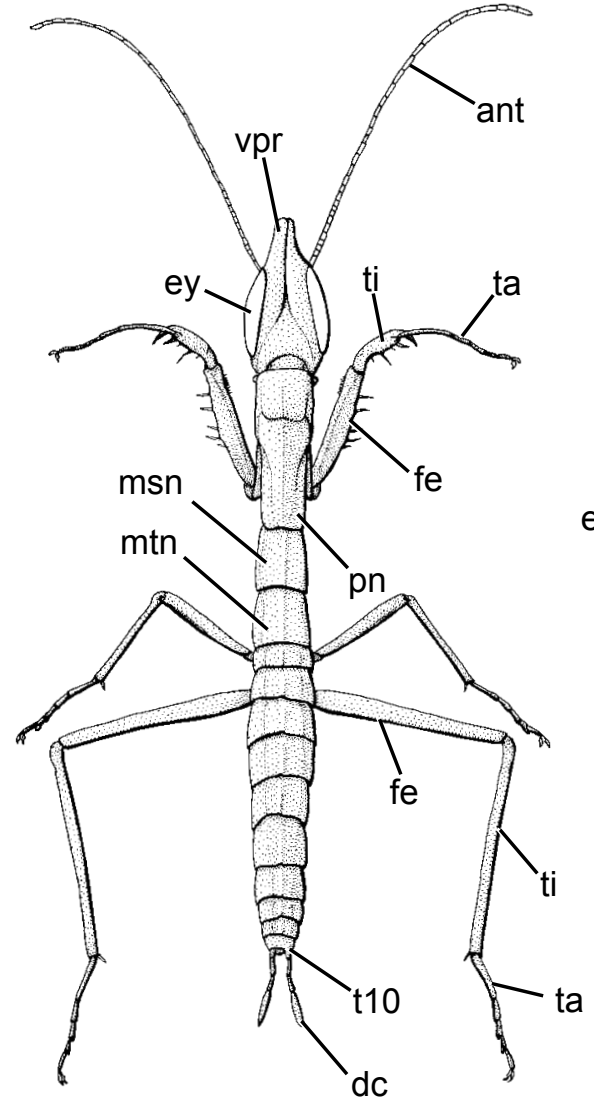

428
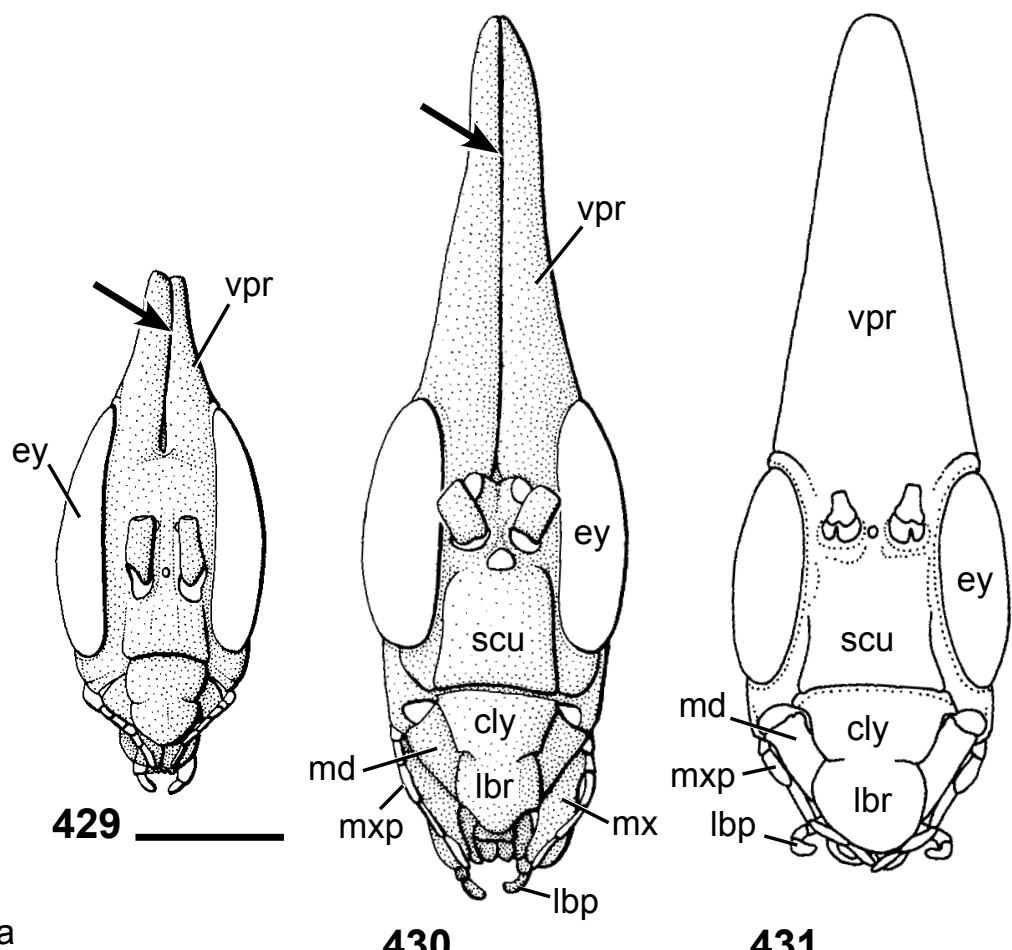

430

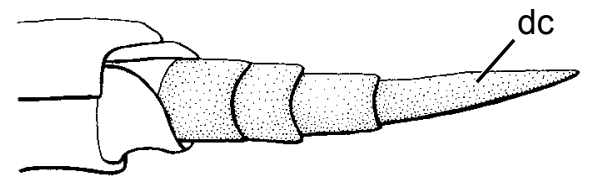

432

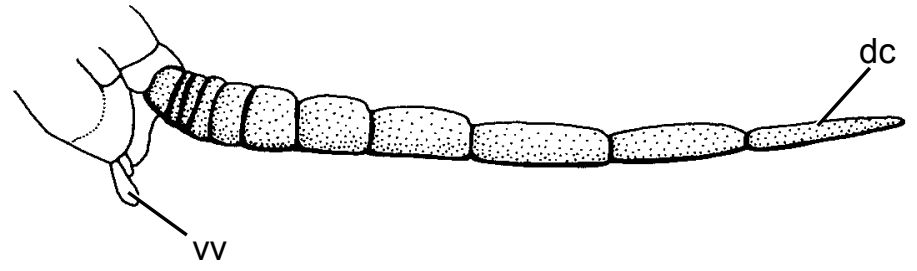

433

Figs. 428-433: Didymocorypha lanceolata and Pyrgomantis jonesi. 428, 429, 432 Didymocorypha lanceolata, first instar nymph; 430, 433: Didymocorypha lanceolata, adult $q$; Fig. 431: Pyrgomantis jonesi, adult ${ }$. 428: Habitus, dorsal view. 429: Head, anterior view; note the cleft vertical process. 430: Head, anterior view; note the cleft vertical process. 431: Head, anterior view; note the undivided vertical process. 432: Left cercus, lateral view. 433: Left cercus, lateral view. Arrows indicate separation between the two halves of the head process. $\uparrow$ cranial (428-431); $\rightarrow$ caudal (432-433). - Scale bar $=0.5 \mathrm{~mm}$.

Abbreviations: ant $=$ antenna; $\mathbf{c l y}=$ clypeus; $\mathbf{d c}=$ distal cercomere; $\mathbf{e y}=$ compound eye; $\mathbf{f e}=$ femur; $\mathbf{l b p}=$ labial palp; lbr = labrum; $\mathbf{m d}=$ mandible; $\mathbf{m s n}=$ mesonotum; $\mathbf{m t n}=$ metanotum; $\mathbf{m x}=$ maxilla; $\mathbf{m x p}=$ maxillary palp; pn = pronotum; t10 $=$ tergite $10($ supraanal plate $) ; \mathbf{t a}=$ tarsus; $\mathbf{t i}=$ tibia; $\mathbf{s c u}=$ scutellum; $\mathbf{v p r}=$ vertical process (fastigium); vv = ventral valvula (gonapophysis VIII).

head processes may yield interesting insights into the convergent evolutionary history of this character. The situation in Phyllocrania and Phyllothelys indicates that their asymmetrical and flattened processes originated from symmetrical processes.

Ontogenetic changes in eyes and head capsule. Head morphology changes sometimes drastically during postembryonic development. For instance, Kaltenbach (1963: fig. 5) showed the development of the head and eyes of Ameles spallanzania (head in frontal view also shown by Bugnion 1922: fig. 2). As shown by Kaltenbach (1963: 532 and fig. 5), the eyes become more 
rounded, and their non-visual elongation becomes almost entirely reduced in the adult. The vertex is bulging in the early instars of this species, whereas it is nearly plane in the adult.

Przibram (1930) and Yamanouti (1937) studied the postembryonic development of the compound eyes and found that the number of ommatidia shows a distinct increase in Sphodromantis viridis (Forskål, 1775) [therein named Sphodromantis bioculata (Burmeister, 1838); see Ehrmann 2002: $321 \mathrm{ff}$.].

The eyes of the first instar nymphs are distinctly larger with respect to the head capsule than in the adults (see Figs. 429, 430 for Didymocorypha; Figs. 453, 454 for Metallyticus; Figs. 455, 457 for Odontomantis).

\section{Ontogenetic changes in the raptorial}

legs. The raptorial legs often undergo radical changes during postembryonic development. In general, it can be observed that the spines are much more slender and often more seta-like in their structure in early instar nymphs.

The highly interesting changes found in Metallyticus splendidus, which are important for the interpretation of the adult spination pattern, have been elaborated in chapter 4.6.4 (Figs. 414-417).

It can be observed, that the distal postero-ventral tibial spine is often much longer than the remaining postero-ventral tibial spines in the first instar nymph of
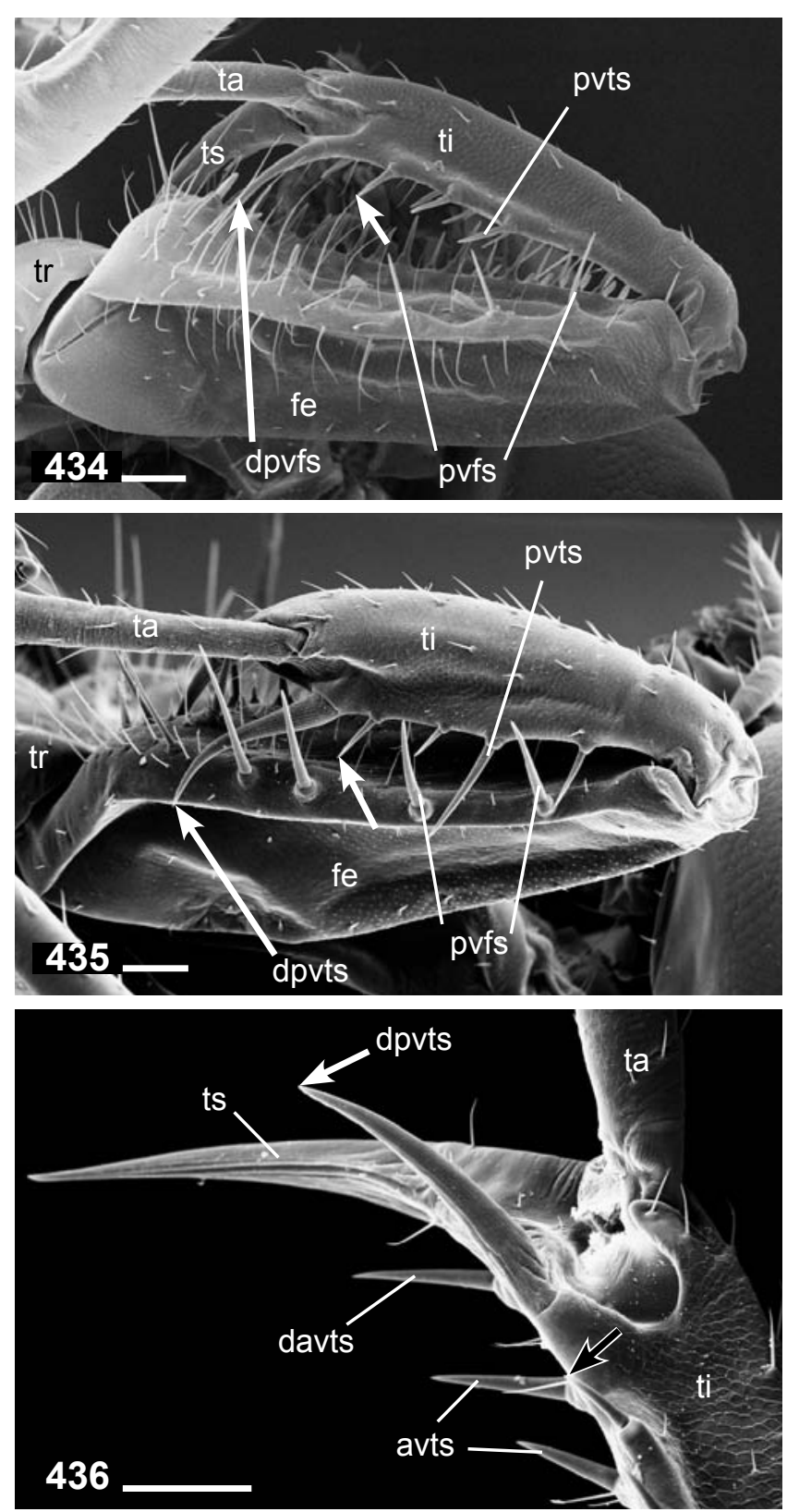

Figs. 434-436: Fore tibiae of first instar nymphs. 434: Mantoida sp., left foreleg, posterior view. 435: Metallyticus splendidus, right foreleg (mirrored), posterior view. 436: Humbertiella sp., left foreleg, detail of tibial apex. Arrows indicate tips of the long distal postero-ventral fore tibial spine and the preceding one. - Scale bar $=0.1 \mathrm{~mm}$. Abbreviations: dpvts $=$ distal postero-ventral fore tibial spine; $\mathbf{f e}=$ femur; davts $=$ distal antero-ventral fore tibial spine; avts $=$ antero-ventral fore tibial spine; $\mathbf{p v f s}=$ postero-ventral fore femoral spine; pvts = postero-ventral fore tibial spine; $\mathbf{t a}=$ tarsus; $\mathbf{t i}=$ tibia; $\mathbf{t r}=$ trochanter; $\mathbf{t s}=$ tibial spur. several species. In the first instar of the undetermined Mantoida specimen this spine is at least two and a half times as long as the preceding spine (Fig. 434). In the adult (observed in photograph) as well as in adult Mantoida maya the spine is one and a half times as long as the preceding spine at most (Fig. 260). This becomes even more obvious in Metallyticus splendidus. 

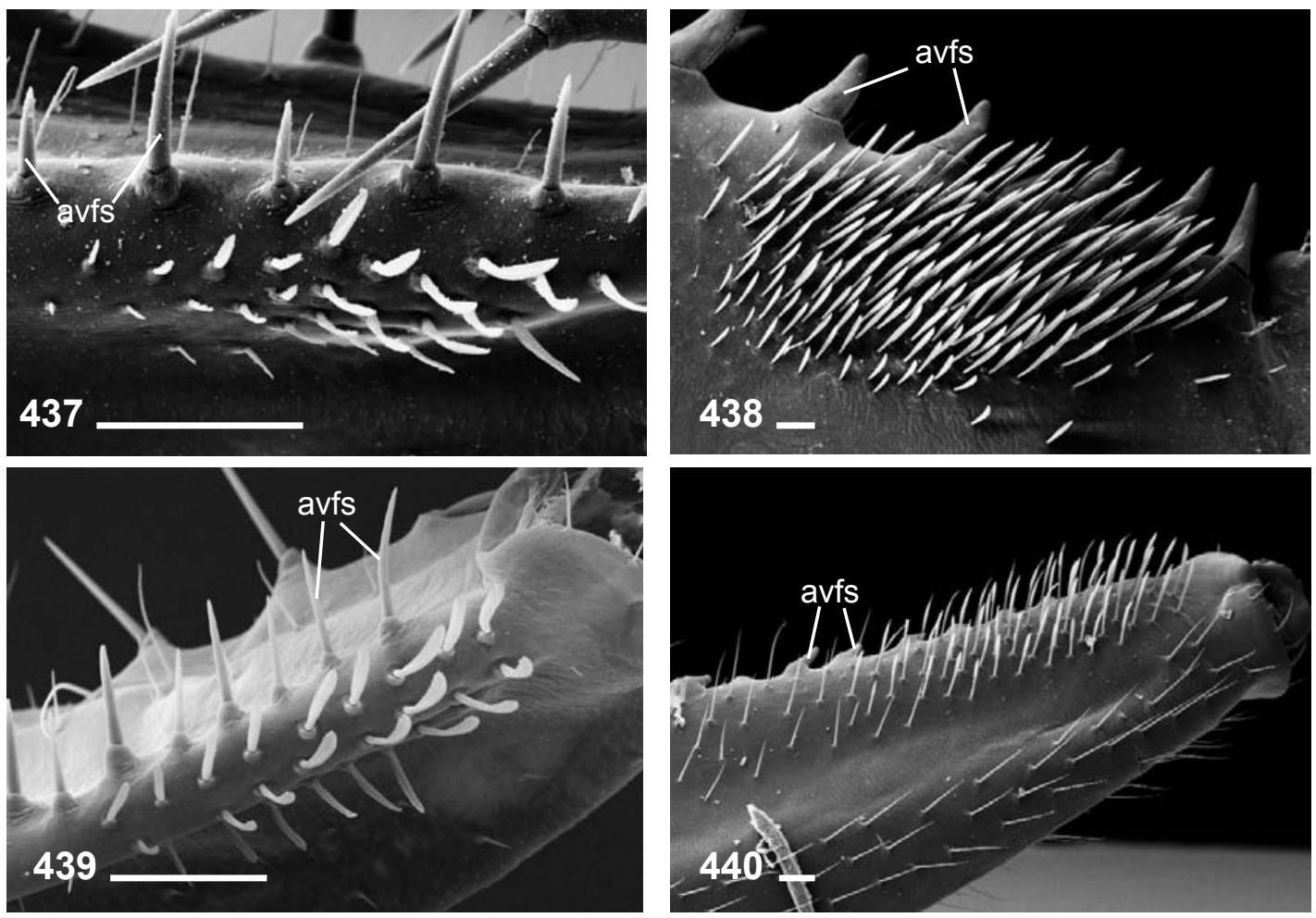

Figs. 437-440: Postembryonic changes of the femoral brush. 437: Metallyticus splendidus, first instar nymph, right fore femur, anterior view. 438: Same species, adult ${ }_{+}$, right fore femur, anterior view. 439: Mantoida sp., first instar nymph, right fore femur, anterior view. 440: Mantoida maya, adult $\overbrace{}^{\partial}$, right fore femur, anterior view. $\rightarrow=$ distal. - Scale bar $=0.1 \mathrm{~mm}$.

Abbreviation: avfs $=$ antero-ventral fore femoral spine.

In the first instar nymph, the spine is almost four times as long as the preceding one (Figs. 417, 435), being nearly as long and sturdy as the tibial spur, whereas in the adult it is only slightly longer than the preceding spine.

A strongly elongated distal postero-ventral tibial spine has also been observed in first instar Humbertiella sp. (Fig. 436), and Phyllocrania paradoxa (pers. obs. 2009). It can frequently be observed in fossil early instars preserved in amber as can be recognized, for instance, in the "Mantoididae" and "Chaeteessidae" specimens in Weitschat \& Wichard (2002: pl. 33, fig. b; fig. 49), $\dagger$ Jersimantis luzzii (Grimaldi 1997: fig. 3; Fig. 31 herein), $\dagger$ Burmantis asiatica (Grimaldi 2003: fig. 8a), $\dagger$ Chaeteessites minutissimus (Grimaldi 2003: fig. 11), $\dagger$ Jersimantis burmiticus (Grimaldi 2003: fig. 15), and also in the three-dimensional reconstruction of the undetermined fossil nymph from Baltic amber (Figs. 33, 34, 37-40). So far it has not been studied what impact this morphology has on the success of prey-capture in early instar Mantodea.

Considerable changes occur in the development of the "femoral brush". The setae of the brush are feather-shaped in all first instar nymphs studied herein, including those of Mantoida and Metallyticus. Although distinctly shorter and stouter, their overall shape is the same as in the adults. Distinct changes occur in their number. In Metallyticus splendidus, the first instar exhibits less than 20 brush setae (Fig. 437). The adult on the other hand has more than 150 of them (Fig. 438). The unidentified Mantoida nymph has about 25 setae (Fig. 439), whereas adult M. maya have approximately 60 (Fig. 440). 


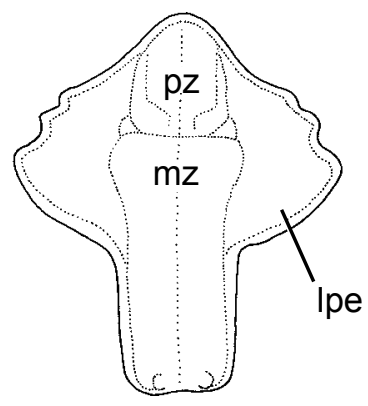

441

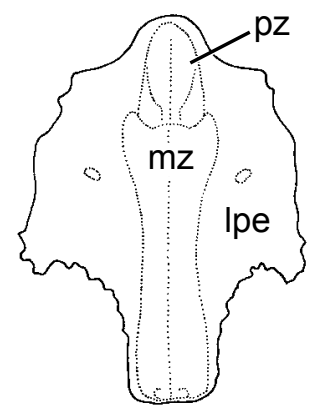

443

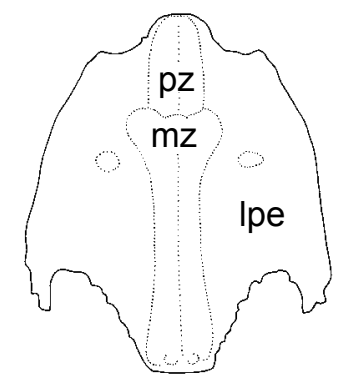

445

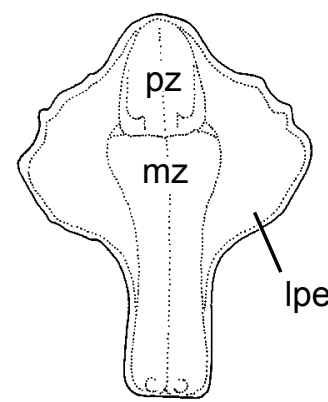

442

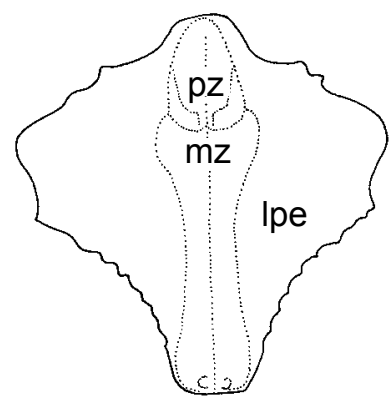

444

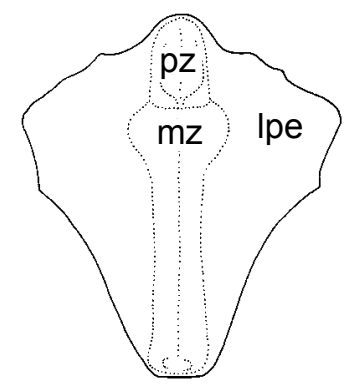

446

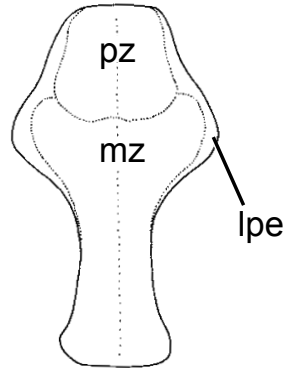

447

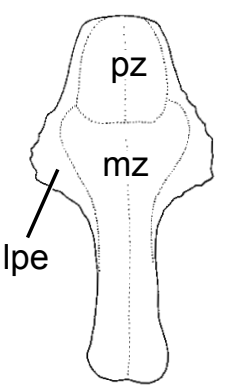

448
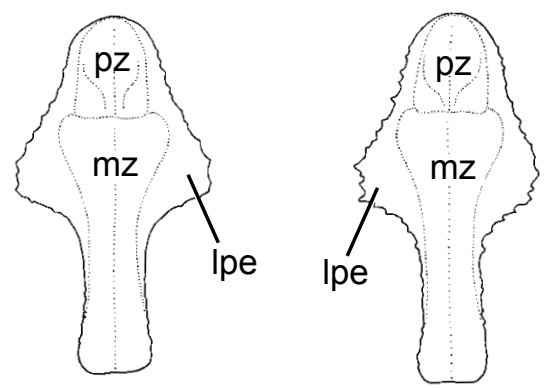

449

450

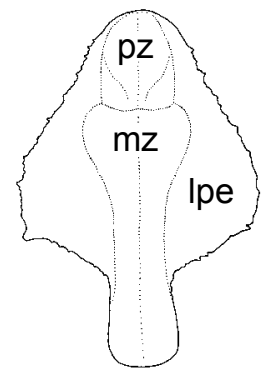

451

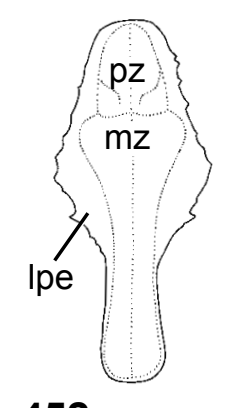

452

Figs. 441-452: Postembryonic development of the pronotal expansions. 441-446: Deroplatys desiccata. 441: First instar nymph. 442: $2^{\text {nd }}$ or $3^{\text {rd }}$ instar nymph. 443: Later instar nymph, $\bigcirc .444:$ Later instar nymph, $O^{\top} .445$ : Adult + . 446: Adult $\overbrace{}^{\lambda}$. 447-452: Phyllocrania paradoxa. 447: First instar nymph. 448: $2^{\text {nd }}$ or $3^{\text {rd }}$ instar nymph.

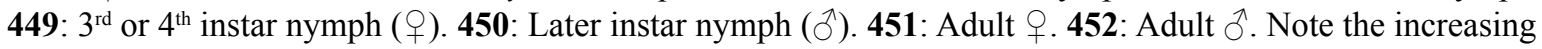
size of the lateral pronotal expansions, the shifting length relations of pro- and metazona during postembryonic development, and the sexually dimorphic shape of the lateral pronotal expansions. - Scale bar $=1.0 \mathrm{~mm}$. Abbreviations: $\mathbf{l p e}=$ lateral pronotal expansion; $\mathbf{m z}=$ metazona; $\mathbf{p z}=$ prozona.

Few ontogenetic observations on the raptorial legs have been published so far. Among them are the postembryonic development of the fore femora and their colouration in Tarachodes afzelii (Gillon \& Roy 1968) and the development of the entire forelegs (Terra 1980). Intensive future studies of the foreleg development may yield further interesting data.

Ontogenetic changes in the pronotum. As has been discussed in the descriptions of characters 28 and 29 and chapter 4.6.3, there are many species that have strongly enlarged lateral expansions of the pronotum. These, too, mostly undergo considerable changes throughout postembryonic development. In Choeradodinae, the differences between first instar nymphs 


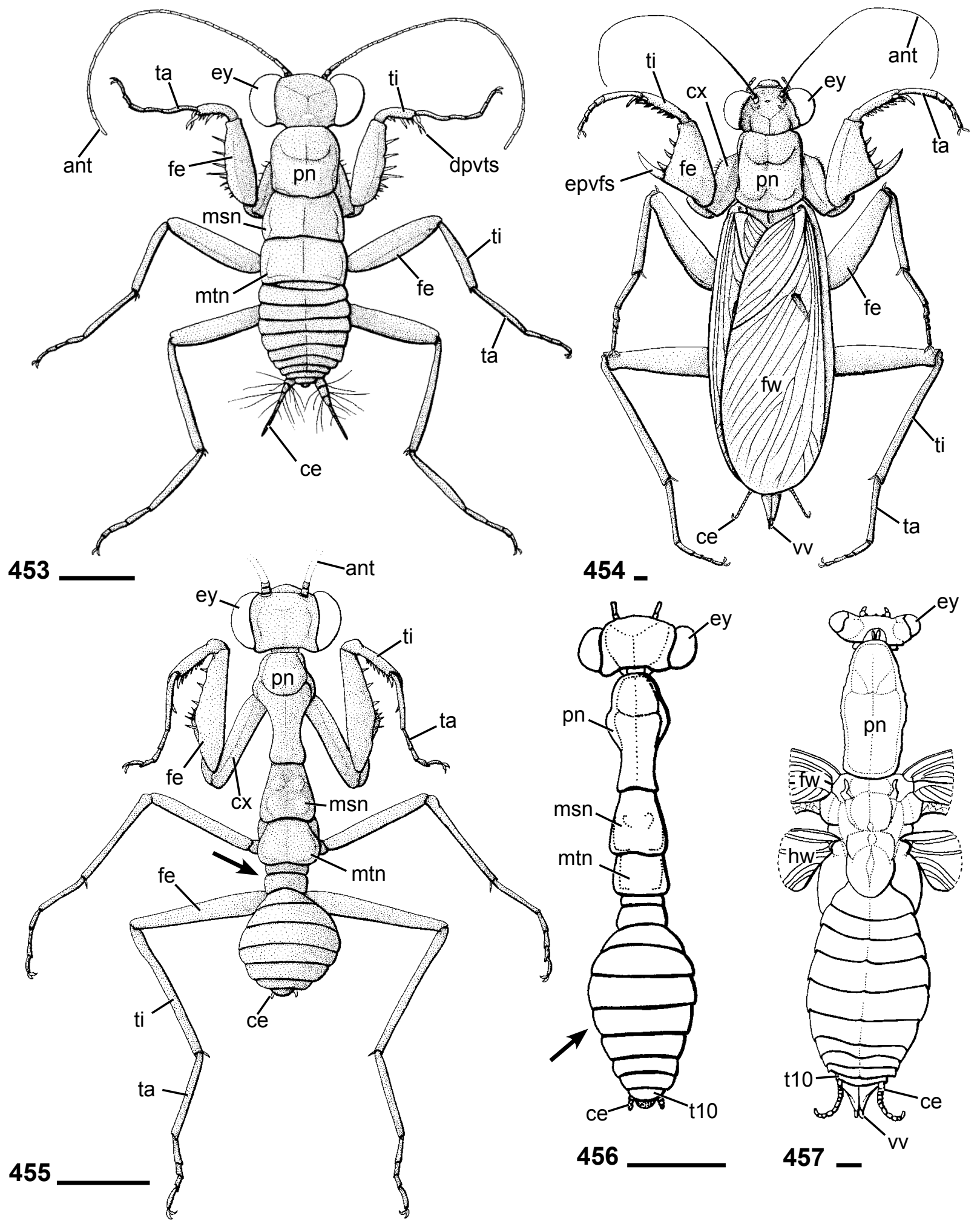

Figs. 453-457: First instar nymphs and selected structures of nymphs and adults. 453: Metallyticus splendidus, first instar nymph, dorsal view; note low number of antennomeres and cerci. 454: Metallyticus fallax, adult $q$, dorsal view. 455: Odontomantis sp., first instar nymph prior to initial feeding, dorsal view; note slender first two abdominal segments mimicking an ant petiolus (arrow) and low number of cercomeres. 456: Odontomantis sp., first instar nymph after initial feeding, dorsal view; note pointed and elongated posterior abdomen mimicking an ant gaster (arrow). 457: Odontomantis sp.,, , dorsal view, wings omitted; note shape of pronotum compared to nymph. - Scale bar $=1.0 \mathrm{~mm}$.

Abbreviations: ant $=$ antenna; $\mathbf{c e}=$ cercus; $\mathbf{c x}=$ coxa; $\mathbf{d p v t s}=$ distal postero-ventral fore tibial spine; epvfs $=$ elongated postero-ventral fore femoral spine; $\mathbf{e y}=$ compound eye; fe = femur; fw = fore wing (tegmen); $\mathbf{h w}=$ hind wing (ala); $\mathbf{m s n}=$ mesonotum; $\mathbf{m t n}=$ metanotum; $\mathbf{p n}=$ pronotum $; \mathbf{t 1 0}=$ tergite $10($ supraanal $\mathbf{p l a t e}) ; \mathbf{t a}=$ tarsus; $\mathbf{t i}=$ tibia; $\mathbf{v v}=$ ventral valvula (gonapophysis VIII). 
and adults become quite evident. WoodMason (1880: 82) wrote that the closest relatives of Choeradodinae are probably the Australian Orthoderinae "which its [= Choeradodis's] young larvae resemble in the form of the pronotum". This is a clear indication that the lamellar expansions in early nymphs are rather narrow and do by no means resemble the enormous shields of adults. Wood-Mason (1884: figs. 1-4) provided drawings of different instars of Choeradodinae. Although he was depicting four different species it becomes obvious that the very early instar (WoodMason 1884: fig. 4) of Asiadodis squilla (Saussure, 1869) has only very narrow pronotal expansions, whereas later instars of A. squilla (Ehrmann 2002: fig. 54) and of other species (Wood-Mason 1884: figs. 1-3; Ehrmann 2002: fig. 54) increasingly resemble the adults.

Another observation with respect to the pronotum is the change of its overall shape by allometric growth. This becomes obvious in some species that exhibit a strong ant mimicry in the early instars. Figs. 455 and 456 show the first instar nymph of Odontomantis sp., whereas Fig. 457 shows the habitus of the corresponding adult female. The pronotum of the first instar nymph strongly enhances its overall similarity to an ant, which in living specimens is supported by a shining black colouration. The greatest width of the pronotum is reached at the supracoxal dilation. The pronotum becomes very slender abruptly in the anterior area of the metazona. Meso- and metathorax and the anterior abdominal segments are also very slender, whereas the subsequent

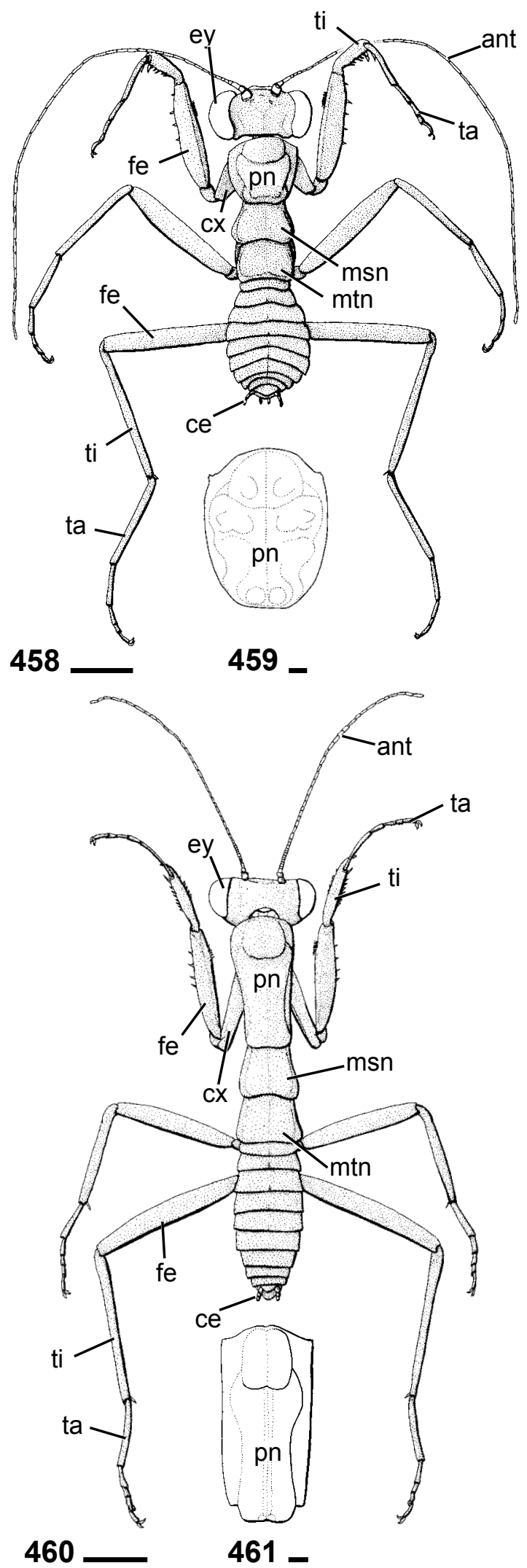

Figs. 458-461: First instar nymphs and pronota of adults. 458: Theopompa servillei, first instar nymph, dorsal view. 459: Same species, $q$, pronotum in dorsal view. 460: Orthodera novaezealandiae, first instar nymph. 461: Same species,, , pronotum in dorsal view. - Scale bar $=1.0 \mathrm{~mm}$. Abbreviations: $\mathbf{a n t}=$ antenna; $\mathbf{c e}=$ cercus; $\mathbf{c x}=$ coxa; $\mathbf{e y}$ $=$ compound eye $; \mathbf{f e}=$ femur; $\mathbf{m s n}=$ mesonotum; $\mathbf{m t n}=$ metanotum; $\mathbf{p n}=$ pronotum $; \mathbf{t a}=$ tarsus; $\mathbf{t i}=$ tibia. 
abdominal segments become much wider, thus imitating the gaster of the ant body. Interestingly, while the posterior abdomen it round in newly hatched nymphs, it becomes pointed after initial feeding, thus further enhancing the effect of an ant abdomen (Fig. 456). In the adult, the pronotum has a completely different shape (Fig. 457). Its sides are almost parallel and the supracoxal dilation is only weak. The abdominal segments, too, resemble other adult Mantodea.

Allometric growth of the pronotum can also be observed in other species, for instance Phyllocrania paradoxa (Fig. 447-452) and Deroplatys desiccata (Figs. 441-446) in which the relations of length and width as well as the relations between pro- and metazona change considerably throughout postembryonic development.

In the first instar of Metallyticus splendidus (Fig. 453), the pronotum is almost square and the sides converge craniad. In the adult is slightly elongated and the sides are almost parallel, therefore rendering the pronotum longer and more rectangular than in the first instar (see Fig. 454 for M. fallax, which is similar to M. splendidus; Wieland 2008a: fig. 1; Fig. 9).

The rectangular shape of the pronotum in adult Orthodera species (Fig. 461; Tindale 1923: fig. 375 and pl. 14, figs. 16-19, 21) is not present, or at least much less distinct, in the first instar nymph (Fig. 460).

On the other hand, the lamellar expansions in the nymph may already be quite similar to the adult in some species, for instance in Theopompa. Fig. 458 shows the first instar nymph of Theopompa servillei, Fig. 459 shows the pronotum of the adult female. The same is true in Deroplatys desiccata (Figs. 441-446). The lamellar expansion is already very distinct in the early instar nymphs, although more restricted to the anterior part of the pronotum, whereas it grows larger and reaches the posterior area of the pronotum during postembryonic development.

For most of the morphological traits studied herein ontogenetic data are still scarce or even missing. It will be an important task in the future to conduct elaborate morphological studies dealing with the nymphal development of individual species of many different groups in order to achieve a better understanding of the evolutionary history of the mantodean morphology. Apparently, in some taxa the first instar nymph already exhibits many of the structures very similar to the adults. For instance, the first instar nymph of Toxodera beieri already exhibits the characteristic structures of the adults in a very similar way (Schütte, pers. comm. 2010). These characters include the lobes on the meso- and metathoracic femora (Roy 2009a: figs. 14, 16E), the characteristic distal ends of the meso- and metathoracic femora (Roy 2009a: fig. 16F, G), the dorsal lobes on the tergites (Roy 2009a: fig. 15; less prominent than in adult) and the posterior edge of the pronotum (Roy 2009a: fig. 16A), as well as the non-visual elongations of the eyes (Roy 2009a: fig. 16D; also compare Fig. 22 for late instar nymph). The presence of such well developed structures in the first instar nymphs may be indicative of comparatively young and highly derived lineages, as Toxodera is positioned far distally on the phylogenetic tree. However, it is important to study such characters in first instar nymphs of many taxa throughout the mantodean phylogeny in order to strengthen this hypothesis. 


\subsubsection{Sexual dimorphism}

Sexual dimorphism is widespread in Mantodea (e.g. Roy 1999: 34). Only few taxa show almost no differences at all among the sexes. This is the case, for instance, in Mantoida maya (pers. obs.), and possibly Chaeteessa and Perlamantinae. In these taxa the females are minimally larger than the males, and have slightly larger heads than the males with respect to body size (pers. obs. in Mantoida maya).

Apart from external intersexual differences related to reproduction (genitalia, female digging structures) there are cases of sexual dimorphism in many aspects of the entire body.

Body size dimorphism. Differences in overall body size can be found in most species (including Mantoida and Metallyticus). The males are generally smaller than the females. Size dimorphism may be considerable, as in Hymenopus coronatus and Theopropus elegans (see Heßler et al. 2008: figs. 17 and 203; Ehrmann 2002: 398, 415). These species exhibit male dwarfism with the males measuring less than half the body length, and probably weighing much less than 50 per cent, possibly as little as 10 per cent of the females (Roy 1999: 34). Interestingly, small males can also be found, for instance, in Deroplatys lobata (see fig. 462 for pronotal size difference), whereas in $D$. desiccata the males are only slightly smaller than the females (Figs. 445, 446). To my knowledge it is unknown why the males in some species are so small and if natural or rather sexual selective pressures drive male body size in Mantodea.

Body shape dimorphism. Females are generally more massive than males, and their abdomina are often much wider. This becomes very obvious, for instance, in Paraoxypilus or Metoxypilus (e.g. Giglio-Tos 1913: figs. 3a, b) and in Cilnia, Oxyopsis, Phyllocrania. In species with micropterous or apterous females the body shape sometimes differs considerably from the male's with regard to lobe-like dorsal expansions of the tergites (characters 145-148), e.g. in Pogonogaster, Astape, Haania, Calopteromantis (Beier 1935a: pl. 1, figs. 17, 18; Lieftinck 1953: figs. 1, 2; Lombardo \& Ayala 1999: pl. 1, figs. 1, 2).

Colour dimorphism. While intraspecific colour polymorphism is widespread among Mantodea (e.g. Mantis religiosa, Iris oratoria, Phyllocrania paradoxa; e.g. di Cesnola 1904, Ergene 1953, Beier 1968a: 24; pers. obs.), sexual colour dimorphism can be found only in few cases. The most spectacular one is certainly exhibited by Metallyticus splendidus. It is, as the name indicates, the most splendid species of Metallyticus, exhibiting the strongest metallic colouration within the genus. While the females have a bright green metallic body with wings that reflect in all kinds of red to yellowish (Fig. 9), the males are darker. Their body is metallic greenish-blue, whereas the wings are of a dark metallic blue (Giglio-Tos 1927: 39; Wieland 2008a: p. 149 and figs. 19, 21; Wieland pers. obs.).

Another case of exceptional colour dimorphism is exhibited by Callibia diana. In this species the males are brownish to greenish-brown, whereas the females are of a brighter green 


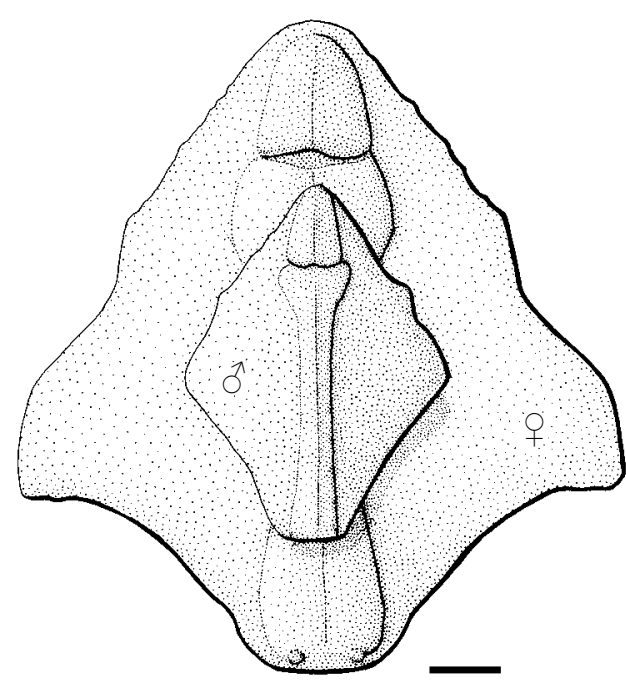

Fig. 462: Deroplatys lobata. Pronotum of $\checkmark$ drawn on top of $q$ to demonstate sexual dimorphism in body size and pronotal shape. $\uparrow$ anterior. - Scale bar $=3.0 \mathrm{~mm}$. to yellowish colour (e.g. Giglio-Tos 1927: 509) and exhibit an elaborate colour pattern resembling that of some many Hymenopodinae (Figs. 406A-G).

Wing dimorphism. Sexual wing dimorphism can be found in many Mantodea. It may occur as dimorphism of wing colouration, wing length, or overall shape.

Wing colour dimorphism was mentioned above for Metallyticus splendidus. The wings of the male are often more or less hyaline or smoky whereas those of the female are opaque and coloured [e.g. Metallyticus fallax (pers. obs. 2009; see species descriptions in Wieland 2008a), Phyllocrania paradoxa, Parasphendale affinis, Stagmatoptera hyaloptera, and Callibia diana].

Sexual dimorphism in wing length is present in many species. Mostly the females have more or less shortened wings or are apterous. This has been discussed in detail in chapter 4.1.2 (characters 101, 105, 106).

The overall shape of the wings may also differ extremely between the sexes. The females become comparatively stationary once they are gravid, whereas the males usually fly around in the search for mates. Therefore, wing size and shape are often less altered in the male than in the female. The latter often have strongly widened costal areas in their tegmina which adds to the resemblance to green or dead leaves. Examples are Choeradodinae (Fig. 142), Deroplatys (Fig. 16), Brancsikia, and Oxyopsis (Fig. 337).

Pronotal dimorphism. A distinct difference between the pronotal shape of male and female is present in many of the species with large lamellar expansions. This is the case in all species of Deroplatys (Ehrmann 2002: 120-121; Figs. 445, 446, 462) and Brancsikia (Paulian 1957: figs. 2-4, 6, 7), many species of Choeradodinae with exception of Asiadodis squilla (Roy 2004b: figs. 9-11), A. yunnanensis (Wang \& Liang, 1995) (Roy 2004b: figs. 15, 16, 27, 28) and Choeradodis strumaria (Linnaeus,

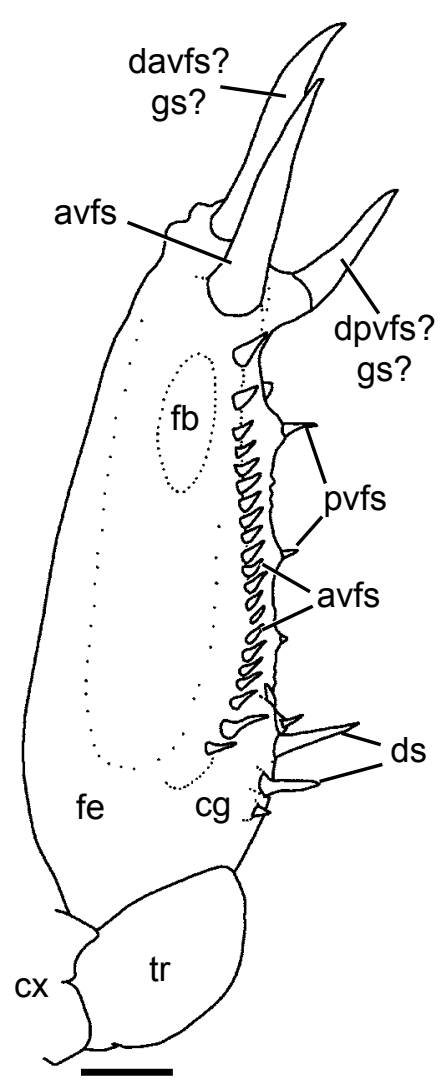

Fig. 463: Eremiaphila berndstiewi, $\widehat{\jmath}$, left fore femur, anterior view. Redrawn from Stiewe (2004: fig. 14). Note the strongly enlarged distal anteroventral and postero-ventral femoral spines (not present in $q$; see Stiewe 2004: fig. 11; distal ones possibly genicular spines). - Scale bar $=1.0 \mathrm{~mm}$.

Abbreviations: avfs $=$ antero-ventral fore femoral spine; $\mathbf{c g}=$ claw groove; $\mathbf{c x}=$ coxa; davfs $=$ distal antero-ventral fore femoral spine; dpvfs = distal postero-ventral fore femoral spine; $\mathbf{d s}=$ discoidal spine; $\mathbf{f b}=$ fore femoral grooming device (femoral brush); fe $=$ femur; $\mathbf{g s}=$ genicular spine; pvfs = postero-ventral fore femoral spine; $\mathbf{t r}=$ trochanter. 
1758). Intersexual shape differences are minimal in the latter species (Ehrmann 2002: 99-100). Differences in pronotal shape are less evident in Phyllocrania (Figs. 451, 452), Empusidae and in Rhombodera, Tamolanica and other Mantidae exhibiting lamellar expansions.

Spination dimorphism. Intersexual differences in the spination of the forelegs have been observed in several species. Among them are several Eremiaphila species in which the males have enormously enlarged distal femoral spines (e.g. Loxton \& Nicholls 1979: fig. 6E.d.). Male Eremiaphila berndstiewi Stiewe, 2004 are among the most impressive examples for this dimorphism (Stiewe 2004: figs. 10, 12, 14; Fig. 463). This character led Werner (1904: 404) to the conclusion that species exhibiting this kind of dimorphism ought to be assigned to a new genus, Centromantis Werner, 1904 which, however, today is considered to be a synonym of Eremiaphila (Ehrmann 2002: 138). This character is also present to a lesser extent in several male Chroicopterinae (e.g. Kaltenbach 1996: figs. 79-92; Roy 1995: 241) and is very conspicuous in male Macracanthopus schoutedeni (Chopard, 1929) (see p. 45 therein for foreleg figure).

A strong sexual dimorphism in spination numbers is present in Toxodera denticulata. While the male exhibits 6-12 antero-ventral fore tibial spines (see character 73), the female has 20-25 spines (see character 74). Interestingly, the males of T. denticulata and the females of T. maculata (male not present in this dataset) have a similar low number of spines.

Cercal dimorphism. Extreme sexual cercal dimorphism was described for several Amorphoscelinae. An extreme enlargement of the distal cercomere of the male is present, for instance, in Caudatoscelis caudata (Giglio-Tos, 1913), C. collarti (Roy, 1964) (both shown in Roy 1964: fig. 9; Figs. 464-467 herein), C. marmorata (Roy, 1965) (shown in Roy 1965a: fig. 6b, c), and Amorphoscelis pulchra Bolivar, 1908 (shown in Gillon \& Roy 1968: fig. 6).

\subsubsection{Evolution of female digging devices}

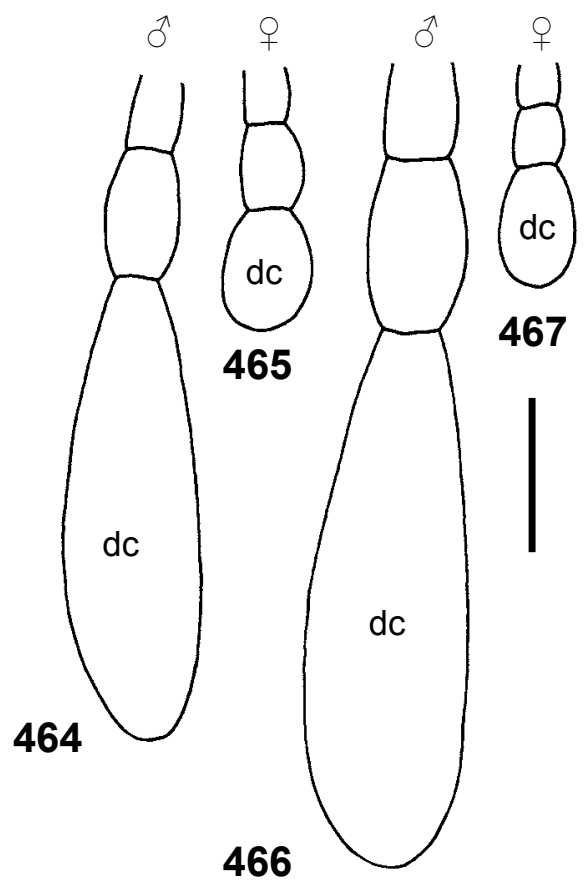

The females of several Mantodea have been observed burying their oothecae in the soil, for instance species of Eremiaphila, Elaea, Humbertiella, Rivetina, Telomantis Giglio-Tos, 1915 (all Ehrmann 2001a: 31, 2002: 26), Sphodropoda Stål, 1871 (Chapman \& Balderson Figs. 464-467: Amorphoscelinae, sexual dimorphism of distal cercomeres. 464-465: Caudatoscelis caudata. 466-467: Caudatoscelis collarti. Redrawn from Roy 1964: fig. 9. - Scale bar $=1.0 \mathrm{~mm}$.

Abbreviation: $\mathbf{d c}=$ distal cercomere. 1984; Milledge 2005), and Rhodomantis Giglio-Tos, 1917 (Chapman \& Balderson 1984: 7; Balderson 1991: 356). In most of them it is unknown if they do so regularly or only occasionally if the climatic conditions (e.g. degree of humidity) 
are not suitable for a proper embryonic development. The latter explanation was mentioned for Humbertiella ceylonica Saussure, 1869 by Müller (2001) and for Sphodropoda tristis (Saussure, 1871) by Milledge (2005: 198).

When ready to lay their eggs, the females dig a furrow into the soil by sweeping it away with the tip of their abdomen. This was described for Eremiaphila, Elaea, Humbertiella, and Rivetina (Ehrmann 2001a: 31, 2002: 26; Müller 2001: 40; Klass \& Ehrmann 2003: 191; Schulze, pers. comm. 2004; pers. obs. 2004). Humbertiella ceylonica and Sphodropoda tristis were observed to use their forelegs for digging in a dog-like manner (Chapman \& Balderson 1984: 5; Müller 2001: 41), at least in case of Humbertiella additionally to the wiping movements of the abdominal apex. The ootheca is then laid into the hole. As soon as the ootheca is built (or sometimes after the formation of smaller subsequent segments, as in Eremiaphila), the hind legs sweep sand from the sides to the middle and so bury it under a layer of sand (Andres 1914: 73; Ehrmann 2002: 27; Schulze, pers. comm. 2004; pers. obs. 2004). The behaviour of covering the ootheca with sand by using the cursorial legs has also been described for Sphodropoda tristis (Chapman \& Balderson 1984).

The oothecae of species that dwell hot and arid savannahs and deserts, if deposited above the ground, would in some habitats have to endure extreme drought and heat during daytime and very low temperatures during the nights. Burying the oothecae beneath the surface may provide protection from such harsh conditions and probably buffers the daily temperature difference and the lack of humidity.

Several species have convergently evolved morphological structures that enhance the digging process, probably positively selected when they were of advantage during the wiping movement. It was mentioned early that these structures are used for digging in Rivetina and Eremiaphila, for instance by Wood-Mason (1878c: 580).

Digging structures of Mantodea were shown in several publications (see discussion of character 141 in chapter 4.1.3) but they have not been compared to each other in detail. Wieland (2008b) presented a first brief overview which is discussed in further detail herein.

Eremiaphilidae-type. Species of Eremiaphila and Heteronutarsus are strict dwellers of deserts and semi-deserts. They occur in northern Africa (Heteronutarsus) and from northern and central Africa and Turkey to India (Eremiaphila). The digging behaviour of Eremiaphila was described by Andres (1914). Chopard (1941: 25) stated that it is likely that the oothecae of Eremiaphilidae are always attached to a stone and covered by sand. This is difficult to observe in captivity as the females do not have the choice between as many different potential deposition locations as in the wild. However, a deposition on underground stones and rock under natural conditions is plausible, not necessarily because of cooler temperatures but because of the increased soilwater content, as has been experimentally shown, for example, for plant-root growth in the Sonoran Desert (Nobel et al. 1992: 96).

The digging device of Eremiaphila and Heteronutarsus (characters 139:2>1, 141:2>1; Figs. 365-368) is autapomorphic for Eremiaphilidae (clade [9]). Sternite 6 is enlarged and extends 
posteriorly, covering the greater part of sternite 7 (the actual subgenital plate). The females carry sturdy spines on the abdominal sternite 6 (often misinterpreted as the subgenital plate by many authors, see also discussion of Eremiaphilidae in 2.1.4). The spines of Eremiaphila are more or less circular in cross section (Saussure 1871: pl. 7, fig. 55a; Fig. 365), whereas they form widened, almost triangular plates in Heteronutarsus (Chopard 1941: fig. 7; Fig. 367). It is possible that the morphological differences between Heteronutarsus and Eremiaphila represent an adaptation to more sandy habitats by the former. The strong elongation of the anterior tarsal ungues (character 99:1>2) and the stiffening of the distal tarsalia by fusion (see chapter 4.6.6; Figs. 424, 425) probably enable Heteronutarsus to run swiftly on fine sands. Furthermore, digging in such a fine substrate is much more effective with shovel-like digging devices than with slender spines.

When Eremiaphilidae are digging, the enlarged sternite 6 together with the long and broad, shovel-like supra-anal plate (tergite 10) possibly encloses the delicate and sensitive genital structures and protects them from injury while the spines protrude posteriorly and dig in the substrate. However, this will have to be studied in detail with regard to abdominal musculature and behavioural observations. Comparative studies of the females of a large number of Eremiaphilidae species are important in order to further elucidate the evolution of the digging spines in this group.

Rivetina-type. Rivetina also buries its oothecae in the soil with similar sweeping movements as Eremiaphila does (e.g. Wood-Mason 1878c: 580; Ehrmann 2001a: 31; Klass \& Ehrmann 2003: 191). In Rivetina, too, there are two sturdy spines forming a digging device (Kaltenbach 1963: fig. 31h, 1982: fig. 65; Ehrmann 2002: 310; Figs. 369, 370). They originate from the proximal part of the subgenital plate (sternite 7). Sternite 6 is not enlarged and does not cover the subgenital plate for protection. Therefore, this digging device has certainly evolved independently from that of Eremiaphilidae. Interestingly, Rivetinula fraterna (Saussure, 1871) from India and Saudi-Arabia carries two pairs of spines, one pair on each sternite 6 and sternite 7. This is one of the characters that led La Greca (1977) to remove it from the genus Rivetina and elevate it to generic rank (La Greca 1977: 24; Ehrmann 2002: 314). To my knowledge, these two genera are the only members of the traditional Miomantinae: Rivetinini exhibiting such digging devices.

Ligaria-type. The females of several Chroicopterinae show characteristic hooks protruding from the genital opening (Figs. 371-375). The hooks are bipartite. Their ventral part is long and bent dorsad, whereas the dorsal part is shorter and points caudad (Fig. 375; see also Wieland 2008b). Such hooks can be found in Ligaria (Beier 1935c: 25; Ehrmann 2002: 202; Figs. 371, 372), Ligariella (Kaltenbach 1998: fig. 35; Ehrmann 2002: 204; Fig. 373), Parentella (Fig. 374; not listed in Giglio-Tos 1915 and Ehrmann 2002), Entella (Entella) (Beier 1935c: 24; Ehrmann 2002: 131) and Entella (Euentella) (Ehrmann 2002: 133). For the other members of the group either no such spines have been mentioned by Beier (1935c) and Ehrmann (2002) [Ligentella Kaltenbach, 1996, Macracanthopus Uvarov, 1940 (which may possibly possess such hooks, see 
Roy 1995: fig. 5), Rhachimantis Giglio-Tos, 1915, Entelloptera Beier, 1942], or the females are unknown (Rogermantis Kaltenbach, 1996, Betamantis Giglio-Tos, 1915, Namamantis Kaltenbach, 1996, Chopardentella Kaltenbach, 1996).

Beier (1935c) and Ehrmann (2002) mentioned such hooks as "not belonging to the subgenital plate". Their morphological origin was studied by Wieland (2008b). The hooks originate from the tip of the ventral valvulae (gonapophyses VIII; Fig. 375; Wieland 2008b). The distal parts of the ventral valvulae including the hooks are strongly sclerotised. Taking into account that the valvulae in Mantodea are usually very sensitive organs that are probably used for sensory purposes when testing the substrate prior to egg deposition (e.g. Kershaw 1910: 136; Beier 1964a: 921) and for quickly stirring the secretion when building the oothecae (Beier 1964a: 922; pers. obs. 2007 in Phyllocrania), it seems rather unlikely that they are used for digging. However, Ligaria actually does deposit its oothecae in the ground (Schütte, pers. comm. 2007, observed while sinking the abdomen into fine gravel) which demands removing ground matter in order to do so. Therefore the hooks probably rather represent a digging device than a structure for manipulating the ootheca.

The hooks are synapomorphic for the Chroicopterinae studied herein (clade [108]). The monophyly of a group encompassing Ligaria, Ligariella, Entella was also found in the molecular analysis by Svenson \& Whiting (2009: fig. 3b, clade 59). It also encompassed Entelloptera, Namamantis, Ligentella and Rogermantis. It will be important to study these genera with respect to the presence of digging devices.

Chroicoptera-type. Female Chroicoptera Stål, 1871 carry two spines on the tip of their subgenital plate that are bent aside (Figs. 376, 377; Beier 1935c: 23; Ehrmann 2002: 103; Wieland 2008b; see also Kaltenbach 1996: fig. 67). These spines distinctly differ from those of the other Chroicopterinae mentioned above. They originate from the distal opening of the genital vestibulum, ventrally with respect to the protruding tips of the valvulae (distal part of sternite 7). The spines are part of the subgenital plate and are possibly autapomorphic for the genus.

Additionally, the females have evolved enormous longitudinal ventral medial ridges on sternites 5 and 6 (e.g. Beier 1935c: 23; Kaltenbach 1996: fig. 67; Fig. 377). They may play a role in the digging process during the wiping movement of the abdomen, but observations are still missing.

The molecular findings by Svenson \& Whiting (2009: fig. 3b, clades 53, 55) have also provided evidence for a closer relationship of Chroicoptera with certain Dystactinae and Amelinae than with Ligaria, Ligariella, Entella and Parentella, although all of them were nested in the same larger clade (Svenson \& Whiting 2009: fig. 3b).

These at least four different types of digging devices (five, if Rivetinula represents an independent type) evolved convergently as becomes evident from their morphological origins [sternite S6, sternite S7 (proximal), sternite S7 (distal), ventral valvulae]. Convergence is also well supported by molecular data (Svenson \& Whiting 2009). 
The biogeographical distribution today is southern Europe and northern Africa to India (Rivetina- and Eremiaphilidae-type) and subsaharan Africa (Chroicoptera- and Ligaria-type). Apart from these two sympatrical occurrences, the two types found north of the Sahara and those found south of it apparently overlap in western Africa (Senegal: Eremiaphilidae-type, Rivetina-type, Ligaria-type) and in eastern Africa (Ethiopia, Kenya, Somalia: Eremiaphilidaetype and Ligaria-type).

To my knowledge, no species from the Central Asian, North- or South American deserts and semi-deserts have been described to have similar morphological structures for laying their oothecae in the soil. However, one Australian species, Trachymantis dentifrons (Stål, 1877), has morphological modifications of the fifth and sixth abdominal sternites. They are posteriorly curved ventrad (see Milledge 2005: 208, therein mentioned as $4^{\text {th }}$ and $5^{\text {th }}$ sternites; Milledge, pers. comm. 2010) and possibly play a role in egg deposition (Milledge 2005: 208). Whether or not this species occasionally deposits its oothecae in the soil is unknown to me.

\subsubsection{Evolutionary trends in the morphology of the raptorial foreleg}

Several evolutionary trends in the evolution of the raptorial forelegs have been observed during this study that are worthwhile to be summarized and discussed in a separate chapter.

The overall foreleg morphology of several taxa is very distinct and unmistakeable. For instance, the foreleg of Perlamantinae (Fig. 220) is highly derived and autapomorphic for the group. Its function has not been studied and putative adaptations for special prey items are unknown.

The same is probably true for the raptorial legs of Paraoxypilinae (Figs. 196, 264, 265), although the interpretations found by Paup* were ambiguous, indicating that Paraoxypilinae might also exhibit the plesiomorphic foreleg morphology with respect to Amorphoscelidae (including Compsothespis) (see discussion in chapter 4.3.10). The foreleg morphology apparently is very similar among the paraoxypiline genera. It is likely that Paraoxypilinae are specialized to some kind of prey. The short and blunt spines standing in small, comb-like arrangements (Figs. 196, 245, 265) as well as the presence of cuticular knobs or ridges on the ventral surface of the fore femora that may serve as a crushing or squeezing area (Fig. 200, 201) indicate a preference for small and hard prey, possibly ants or termites. Some Paraoxypilinae (Nesoxypilus, Myrmecomantis) mimic ants (Milledge 1990: 353; Balderson 1991: 353). Milledge (1990: 353) assumed that Nesoxypilus resembles ants in order to be inconspicuous to predators itself rather than in order to hunt ants. Balderson (1991: 353) stated that Nesoxypilus runs with foraging ants, but that it is unclear whether it preys on ants or uses ant-mimicry as predator defence. Gyromantis has been observed to feed on ants (see photograph by Farhan Bokhari; Fig. 219). The way the mantid holds the tip of the ant's gaster in its raptorial leg suggests that the blunt, comb-like spines are used for securing the mantid against the ant's poison. 
Length relations of foreleg segments and prothorax. The length relations of fore tibia and femur show enormous differences among the species studied. In many of them the tibia is distinctly shorter than the femur. This is reflected by the position of the claw groove (see character 67). It is located in the proximal third (or at the proximal end) of the foreleg in many species, while in others it is located in the distal half of the femur. This is the case when the femur is elongated while the tibia is not, also being reflected by the position of the spine-bearing area. Usually the entire area of spines has been shifted distad when the femur became elongated, indicating a proximal elongation of the femur. A tibia being half as long as the femur or shorter was found in about 40 per cent of the taxa studied. Only in Toxoderidae a distinct elongation of the tibia has been observed. In this group, the claw groove is located more or less at the proximal end of the femur, while the latter and the tibia are extremely elongated (Figs. 22, 207). Secondary elongation of the tibia in this group finds support in the position of the spines along the entire ventral surface of both leg segments and the wide distance between the spines. This indicates that the evolutionary history of the Toxoderidae foreleg is fundamentally different from other Mantodea. The reason for this is obscure, but it is likely that it is an adaptation related to feeding ecology.

The evolutionary history that led to the length relations of the leg segments, including the coxa, and the prothorax is also interesting from a functional perspective. For a mantid, it is indispensable to reach the mouth parts with its fore legs, more precisely with the area between the discoidal spines and the femoro-tibial joint, in order to feed. If a longer femur (or coxa) is positively selected because it allows to catch prey that is farther away from the hunter, this forces other leg segments to be altered, too, in order to maintain the function. The reachability of the mouth parts with the aforementioned area or the foreleg is maintained by the elongation of the fore coxa relative to the elongation of the femur. In many species with short prothoraces the fore coxae reach back beyond the prothorax, including Chaeteessa (Fig. 5), Metallyticus (Fig. 9) and Mantoida (Fig. 8). This is only possible as long as the coxo-femoral joint does not interfere with the mesothoracic legs or are of other hindrance for the individual specimen. Therefore, an extreme elongation of the fore coxae - and the fore femora - is only possible if the prothorax undergoes an elongation, too. Shape and length relation of tibia and femur is functionally not directly affected by the elongation of coxa and femur. Differences in tibial morphology, for instance in Oligonicinae, Haaniinae and others, are therefore likely to be ecological adaptations rather than morphological changes triggered by the changes in coxa and femur.

There are many species with enormously elongated prothoraces in which the coxo-femoral joint does not even reach half of the prothoracic length, for instance in Schizocephala, Euchomenella, Toxodera and others. In many cases the cryptic effect of grass- or stick mimesis has apparently led to an additional elongation of the prothorax that is not directly triggered by foreleg evolution.

Origin of spines. It is very likely that the spines on the mantodean forelegs originated from setae, which becomes obvious if one considers that all of them are separated from the leg by 
sutures. Not all spines are moveable in modern Mantodea (e.g. Rau \& Rau 1913: 3; Loxton \& Nicholls 1979: 186), but those that are function as mechanoreceptors (e.g. Copeland \& Carlson 1977: 1155). From the situation found in many Blattaria (Fig. 285-287) it can be conjectured that the spines on the forelegs of cockroaches and mantodeans are homologous and were already present in the last common ancestor of Dictyoptera.

It is furthermore likely that the spines on the pro-, meso- and metathoracic legs of cockroaches are serially homologous, which finds support in their similar position and surface structure. Interestingly, a similar structure of the spines can be found in the meso- and metathoracic legs of Chaeteessa (see characters 83, 84; Figs. 277-284), which is unique among extant Mantodea (although potentially present in several mantodean fossils; see discussion in characters 83,84 ) and probably a plesiomorphic trait. Chaeteessa alone furthermore exhibits the serrate microstructure of the spines on the forelegs (Figs. 284, 287), resembling the situation in cockroaches. It can therefore be assumed that Mantodea and Blattaria inherited the spination pattern on all three pairs of legs from their last common ancestor. Mantodea subsequently reduced the spines on the meso- and metathoracic legs with the only traces being found in fossils and Chaeteessa. The spination of the fore legs was strengthened due to the raptorial lifestyle. The different patterns we find today likely represent evolutionary adaptations to different habitats or prey.

Function of spines. The foreleg spination in Mantodea has important functions. It serves for being able to hold the prey when catching it. Furthermore, several spines, including some of the discoidal spines, are moveable and have been shown to have mechanoreceptive functions, triggering the prothoracic tibial flexion reflex in Mantodea (Rau \& Rau 1913; Copeland \& Carlson 1977; Loxton \& Nicholls 1979; Prete \& Hamilton 1999). Besides this function they also help to direct the prey deeper between the femur and the tibia and to prevent the prey from escaping (e.g. Loxton \& Nicholls 1979).

Two of the most important spines, however, are the tibial spur and the antagonistic long discoidal spine. The two decrease the angle between tibia and femur through which a caught prey insect could escape (Loxton \& Nicholls 1979: fig. 2), and their amputation has strong influence on the prey-capture success of a mantid (Loxton \& Nicholls 1979: 190 and fig. 3). This antagonistic pair of spines seems to be a successful functional unit because the principle has evolved in many raptorial arthropods, including several Heteroptera, Neuroptera, and Diptera among the insects and, for instance, Stomatopoda among the crustaceans (see also chapters 4.6.4 and 4.6.5).

Reduction of spines. A reduction of several or almost all spines has evolved several times in mantodean evolution. Both tibia and femur of the fore legs undergo reductions of varying degrees in different taxa. The spines in many Mantodea species carry a small, sickle-shaped or circular indentation at their bases (Figs. 204-206). Its function is unknown to me but it might be connected to the likely origin of the spines from mechanoreceptive setae. Interestingly, reduced spines occur under the SEM as very flat sockets, carrying this exact sickle-shaped structure 
(Fig. 195, 205, 246; only weakly discernable in Figs. 185 and 189). Furthermore, a reduction sequence of femoral spines has been observed on a single femur of the Oligonicinae sp. specimen. Four reductive stages neighbour one another (Figs. 202-206), encompassing a fully sized spine and three subsequent reductions including the aforementioned entirely reduced spine which still exhibits the sickle-shaped structure (Fig. 205). These observations suggest that reduced spines can easily be identified if the sickle-shaped structure structure is present.

In Amorphoscelinae and Compsothespis the tibial spines are entirely absent (except for the tibial spur), at least in Amorphoscelis without leaving any trace of their previous existence (Compsothespis could not be studied under the SEM). In Perlamantinae, too, the fore tibial spines are reduced. However, at least the antero-ventral fore tibial spines left comparatively large traces that can be easily recognized in SEM pictures (see character 63 and Figs. 190-192). In Paraoxypilinae, the postero-ventral tibial spines are reduced with few remnants being discernible under the SEM (Figs. 245, 246), whereas the antero-ventral spines are present, albeit reduced into the characteristic blunt spines forming the distal, antero-ventral comb-like structure on the tibia (Figs. 245, 264, 265).

There are strong reductions of the number of tibial spines with a subsequent strong enlargement and partial dorsal shift of others in Oligonicinae and Haaniinae (Figs. 163, 165, 247-254). The lowest number of tibial spines in these groups was recognized in Thesprotia graminis (Figs. 163, 165, 249, 250; Beier 1964a: 874).

Reductions of femoral spines are also common. As in the fore tibia, the fore femur of Amorphoscelis does not carry any spines except for the single discoidal spine. However, remnants of several antero- and postero-ventral femoral spines can be recognized under the SEM (Figs. 184-189). In Compsothespis the fore femoral spines are strongly reduced in size but are still distinctly discernible under the microscope (Fig. 166-168). Perlamantinae have reduced their postero-ventral fore femoral spines entirely with only very inconspicuous remnants being left (Fig. 193-195). Four antero-ventral fore femoral spines are present. The proximal one is normally shaped, whereas the distal three are enlarged and of a plate-like shape (see character 43 and Figs. 220-222).

In Paraoxypilinae, all fore femoral spines are present but are strongly reduced in size (196199, 201). The antero-ventral femoral spines are specialized, blunt structures forming a proximal comb-like structure that interlocks precisely with the corresponding structure on the fore tibia (Figs. 197, 199, 264, 265).

A tendency of reducing fore femoral spines can be found in Oligonicinae, Haaniinae and Eremiaphilidae. In Heteronutarsus, for instance, a strong reduction can be found in the posteroventral fore femoral and fore tibial spines. The reduction trend in Oligonicinae was mentioned in the introduction to this chapter.

The number of discoidal spines differs among the taxa. However, the situation at least in Metallyticus and Amorphoscelinae likely represents a secondary reduction. In Metallyticus, a single one has been shown to persist in adult specimens, whereas the original primary function of the discoidal spines was taken over by the strongly elongated proximal postero-ventral 
femoral spine (see chapter 4.6.4; Figs. 414-417). In Amorphoscelinae, only a single discoidal spine persists (Fig. 178, 184, 186, 188), probably due to its vital function (see introduction to this chapter), whereas all other femoral spines have been reduced. No remnants of discoidal spines other than the remaining one could be identified in Perlamantinae.

The tibial spur is reduced only in Chaeteessa (see chapter 4.6.5; Figs. 418-420). Such a reduction is unexpected considering the importance of the functional unit that consists of the tibial spur and the long discoidal spine (see paragraph on function of spines in this chapter). However, the highly derived foreleg morphology present in Chaeteessa suggests that the distal antero- and postero-ventral fore tibial spines have functionally compensated for the reduction of the tibial spur, which probably evolved in the course of the strong lateral bending of the tibia (see chapter 4.6.5 for a discussion).

Enlargement of spines. A tendency towards an enlargement of spines can be observed in several species. In Metallyticus the proximal postero-ventral spine is enormously enlarged (for a discussion see chapter 4.3.7; Fig. 226). A strong elongation of the discoidal spines (at least of the longest one) occurs, for instance, in Leptocola, Euchomenella (Fig. 240), Hoplocorypha (Figs. 160, 162, 243), and Schizocephala. Both tibial and femoral spines can be partly enlarged in many Oligonicinae and Haaniinae (Figs. 164, 165, 247-254). The enormous enlargement of the distal antero-ventral fore femoral spines in several Eremiaphilidae including Eremiaphila berndstiewi (Stiewe 2004: figs. 12, 14; Fig. 463) have already been discussed in chapter 4.6.8.

A strong elongation of the postero-ventral fore femoral spines occurs in many Hymenopodinae, Empusidae and other taxa. It is achieved partly by the elongation of the spines themselves (as in, for instance, Gongylus and Idolomantis; see Figs. 211-214), whereas it can also be achieved or enhanced by lifting the spines onto ridges (as in some Empusidae; Fig. 225) or sockets (as in Callibia and, more distinctly, Pseudocreobotra; Figs. 223, 224). This trend may indicate a preference to certain prey items, possibly to airborne insects.

\subsubsection{Morphology of the meso- and metathoracic legs}

The meso- and metathoracic legs are unspecialized and similar to each other in many Mantodea, resembling the situation in Blattaria. While the latter often have a great number of articulated spines on the cursorial legs, such spines are missing in all Mantodea except for Chaeteessa, in which they likely represent the plesiomorphic condition as can be deduced from the structure of the spines (see characters 83, 84; Figs. 277-284).

The cursorial legs may be exceptionally long as, for instance, in Eremiaphilidae (Chopard 1938: fig. 25, 1949c: fig. 6, 1941: fig. 7; Beier 1968a: fig. 5; Figs. 10, 276), which is an adaptation to running on hot sand. In other taxa, for instance Leptocola (e.g. Chopard 1949c: fig. 42; Beier 1968a: fig. 10), their enormous length adds to the overall appearance in stick-or grass-mimicking species. The legs may also be shortened in some grass or stick dwelling species which tend to press their bodies against the structure they are sitting on in order to hide 
(see also introduction to the chapter 4.1.2 on cursorial legs). Differences between the taxa can be observed, for instance, in the length relations between tibia and femur of the cursorial legs. Most species have shorter tibiae than femora on the mesothoracic legs (character 86), which is similar in both sexes. The tendency in the metathoracic legs is vice versa, with the tibia being longer than the femur in most species studied. This character showed intersexual differences (see characters 88, 89). Paup* interpreted the shorter tibiae of the mesothoracic legs $(85: 2)$ as the ancestral state with a multiple convergent elongation of the tibia in several taxa, while the the metathoracic tibiae were assumed to be plesiomorphically longer than the femora in both sexes $(88: 1,89: 1)$. These are the states that were also found in cockroaches.

Several species have distinctly elongated metathoracic legs that function as saltatorial legs at least in the nymphs. This has been mentioned, for instance, for Ameles (Wood-Mason 1878d: 268; Chopard 1922: 37, 1938: 267; Kaltenbach 1963: 557; Wieland, pers. obs. 2004, 2006; Fig. 13), Pseudoyersinia (Wiemers 1993: figs. 2, 3, 6; Wieland, pers. obs. 2004; vs. Beier 1935c: 35 and Ehrmann 2002: 301), Yersinia (e.g. Rehn 1907: 26; Rehn \& Hebard 1909a: 118; see Rehn \& Hebard 1908: fig. 1) and Litaneutria (e.g. Rehn \& Hebard 1909b: 415). The early nymphs of many species seem to be well capable of jumping and some species also jump as adults (Wieland, pers. obs.). However, apparently only few species have the hind legs elongated in a way similar to Ameles or Pseudoyersinia. The capability of jumping has not been studied in detail. As the genera mentioned above are ground or low shrub dwellers, jumping might represent an escape strategy against predators. A study including more species adapted to similar lifestyles might yield interesting results.

The tibiae of the cursorial legs of several species have gained an asymmetrical shape if viewed from anteriorly or posteriorly (e.g. Figs. 294, 297). The shape evolved several times independently. Hymenopodinae [200] and clade [133] are two major groups of species for which the asymmetry has been found to have evolved convergently. The curious shape of the tibiae certainly adds to the overall resemblance to plant matter or the cryptic effect in these taxa by breaking up the characteristic leg shape.

The tibiae and femora of the cursorial legs of many species carry lobes that enhance the cryptic effect of the body shape (see characters 90, 92; Figs. 15, 16, 22-24, 294-300). Such lobes on the femora have been found to have originated separately at least eleven times during mantodean evolution. Lobes on the meso- and metathoracic tibiae have evolved independently in clade [133] and in Zoolea [168]. Interestingly, most taxa with lobes have them on the femora while tibial lobes are lacking. Lobes on both femur and tibia can be found only in few species [Ceratocrania, Phyllocrania (Figs. 41, 300), Popa (Fig. 294), Vates, and Zoolea in the present dataset).

With regard to the tarsal euplantulae there are several tendencies in Mantodea. The euplantulae of cockroaches are mostly restricted to the distal part of the tarsomeres whereas in Mantodea they show a trend towards becoming proximally elongated (see character 94 and Figs. 301-330). This can also be found on tarsomere tal in some cockroaches (see discussion of tarsal euplantulae in character 94). Furthermore, while they are rather small and simple in several Mantodea 
(Chaeteessa, Mantoidea, Ciulfina, Thrinaconyx, Eremiaphilidae, Haaniinae, Oligonicinae), the euplantulae of tarsomeres ta1-ta3 tend to become wider and gain a medial notch, thus becoming slightly V-shaped (similar to the situation in ta4; see character 95). Additionally, the euplantulae of ta4 become strongly widened in many Mantodea and reach a size distinctly longer than 1.5 times the length of the previous tarsomere in about 75 per cent of the species studied (see character 96; e.g. Figs. 318-327). Interestingly, the euplantulae of the proximal tarsomeres ta1 and ta 2 are larger than the remaining ones in Pseudoyersinia (Fig. 316). An enlargement of the euplantulae of a single tarsomere is missing in all Blattaria that were available for comparison.

The distal tarsomere ta5 gains a medial euplantula in several Mantodea (character 97; e.g. Figs. 308, 310, 316-319, 323-326, 328-330). This was also confirmed for some cockroaches (e.g. Roth \& Naskrecki 2003: 57; Wieland, pers. obs. in Blaberus craniifer).

It is possible that the enlargement of the euplantulae secondarily compensated for the loss of the arolium in Mantodea (character 98). It could furthermore be hypothesized that the posterior elongation, the spreading and the enlargement of the euplantulae in many Mantodea and some Blattaria may be a result of increasing body size and weight. Many Mantodea grow much larger and are much heavier than most cockroaches. Therefore, it is possible that the additional enlargement of the euplantulae of tarsomere ta 4 evolved because it helped the mantodeans to hold the weight of their bodies on the surfaces they rest on. This leads to the question of the primary habitat of early Mantodea. The improvement of adhesive structures may have been one of the primary evolutionary steps when mantodeans conquered the canopy of trees or other broad-leaved plants because it increased the grip on the smooth surfaces.

\subsubsection{Early evolution of lifestyle}

The split between the lineages of Mantodea and Blattodea probably occurred in the Carboniferous. This is supported by the identification of several members of the "Protorthoptera" from the Upper Carboniferous (e.g. $\uparrow$ Mesoptilus dolloi and $\uparrow$ Homocladus grandis) as potential stemgroup-Mantodea (Béthoux \& Wieland 2009; Béthoux et al. 2010).

The assumed old age of the stem-lineage of Mantodea raises the question of the early lifestyle of the group. It probably resembled the (unknown) lifestyle of the last common ancestor of mantodeans and cockroaches. However, the three potentially earliest branches of Mantodea (Chaeteessa, Mantoida and Metallyticus; see Figs. 5-9, 409-413) are probably bark dwellers. Therefore, it can be argued that this may represent the early lifestyle form in Mantodea.

Angiosperms were not present in the Late Carboniferous, but vast forests existed nonetheless. They were quite different from extant forests and were formed, for instance, by enormous Cordaitales of more than 40 metres height (e.g. Falcon-Lang \& Bashforth 2005) and other, more or less high and tree-like plants (e.g. Lowman \& Rinker 2004: 130 ff) on which early Mantodea could have dwelled.

As has been shown throughout this study, Chaeteessa, Metallyticus and Mantoida show many plesiomorphic traits (mainly in the wing venation but also in the phallomere complex: 
Klass 1995, 1997; Klass \& Meier 2006), but the foreleg morphology rather represents strongly adapted and highly derived states instead of plesiomorphic conditions. Nonetheless, the permanent use of the forelegs for walking is a trait unknown in other extant Mantodea and is likely to represent the plesiomorphic state that was inherited from the last common ancestor with the cockroaches. Although many extant Mantodea use their forelegs from time to time when they are running [e.g. bark dwelling Liturgusidae (e.g. Theopompa, Schwarz, pers. comm. 2009), bark-dwelling Amorphoscelinae (e.g. Amorphoscelis, pers. obs. 2007) and deserticolous Eremiaphila (pers. obs. 2006)], they usually hold them folded against the prothorax at least when at rest (e.g. Figs. 10-12, 20, 21). A primary tree-dwelling lifestyle in combination with the behaviour shown by Chaeteessa, Mantoida and Metallyticus may explain the adaptations of the foreleg morphology in these taxa. If the forelegs are generally used for locomotion, long ventral spines (especially a strongly elongated discoidal spine) on the fore femur may be a hindrance because they can easily get in contact with the surface, which may have led to the different adaptations of the foreleg morphology in these genera (see discussion in chapters 4.6.4 and 4.6.5).

Chaeteessa, Metallyticus and Mantoida do not have strongly elongated forelegs. A barkdwelling lifestyle provides a possible explanation for this. When catching prey on a surface (i.e. in a two-dimensional habitat such as bark or leaf litter on the ground) an elongation of the forelegs (and the prothorax) that would be helpful for reaching out for flying prey would not necessarily be positively selected as strongly as in a three-dimensional habitat (e.g. canopy, bushes or grass) where such an ability would be of great advantage. A potential bark-dwelling lifestyle is also supported by the fossil record of Mantodea as presented by Grimaldi (2003). The Cretaceous taxa have a short prothorax and forelegs that are not strongly elongated (e.g. $\uparrow$ Ambermantis, $\uparrow$ Cretomantis, $\uparrow$ Jersimantis and $\uparrow$ Santanmantis). Long cursorial legs (most distinct in $\uparrow$ Ambermantis, see Grimaldi 2003: 11 and fig. 3a) may also be indicative of dwelling the bark of trees (see pictures of Liturgusa in Preston-Mafham 1990: pl. 48; Figs. 20, 21).

So far, no Cretaceous Mantodea with long prothoraces have been described. This either indicates that all mantodeans at that time had short prothoraces or that those smaller species with shorter prothoraces had a better chance of being preserved due to their size or habitat. Natural selection for elongated forelegs (and an elongated prothorax, see paragraph on length relations of foreleg segments and prothorax in chapter 4.6.10) may have been initiated when new habitats (robust leaves of bushes and treetops, grass blades etc.) could be conquered. In such habitats, the ability of catching flying prey may have been positively selected. If so, this progress probably started after the rise of the angiosperms, which began approximately 217 My ago in the Late Triassic (Smith et al. 2010: 5899). Interestingly, Svenson \& Whiting (2009: fig. 8) found the stem lineage of Artimantodea (most extant descendants of which have elongated prothoraces and often elongated forelegs) to have originated between 140 and 130 My ago. The stemgroup representative of Artimantodea likely had a short prothorax. This is in congruence with the fossil record. It is unknown to date whether elongated prothoraces existed earlier and have just not been discovered yet. It is also possible that the origin of new habitats 
leading to the morphological changes took until the Cretaceous, or that other raptorial insects were more abundant than mantodeans at that time and delayed the rise of morphological diversity in Mantodea as seen in the later fossil record.

Grass-dwelling ecomorphs (e.g. Pyrgomantis: Fig. 25) probably evolved much later, after the stem lineage of the grasses arose as shadow plants (approximately 96 My ago in the Cretaceous of Gondwana, just before the splitting between South America and Africa; see Bouchenak-Khelladi et al. 2010: 554). This is in congruence with the molecular dating in Svenson \& Whiting 2009 (fig. 8) who found the stemgroup of clade 142 (which also includes the mantodeans adapted to grasslands) having originated between 105 and 85 My ago.

\subsubsection{Sound production}

Sound production in Mantodea is a long known phenomenon. Several of the fully winged species simply rub their abdomen between the lifted wings and produce a hissing sound (Edmunds 1972: 7, 9, 11, 25, 28; Ramsay 1990: 45). It has formerly been assumed that during this movement the cerci produce the sound on the wings (e.g. Beier 1964a: 930, 1968a: 16). However, it has been shown that this, at least in Mantis religiosa, is not the case. Hill (2007) presented evidence for a stridulating apparatus in this species consisting of tooth-like structures on the longitudinal veins of the hind wings and fields of small pegs on the abdominal pleura (Hill 2007: 40, 41 and fig. 1A, D, 2A-C). Therefore, the cerci are probably not involved in this process.

Another mechanism has evolved in Choeradodis (see character 29; no data on Asiadodis) which has an extremely enlarged pronotum (Roy 2004b: figs. 9-11, 15-16, 23-28, 32-34). A dorsal row of small, pointed spines (serrate ridge, see Figs. 128, 130) can be found on the fore femora. It has been mentioned that, when disturbed, Choeradodis rubs the dorsal surface of the fore femora against the anterior, thickened edge of the large pronotal shield, thus using the latter as a kind of Sound mirror or amplifying device (Robinson 1969: 297; Ramsay 1990: 45; see discussion of character 29). If actually present, this stridulating mechanism may be unique among Mantodea.

A further stridulatory mechanism was mentioned in the description of character 110. All Empusidae and several Mantidae have ventrally serrate anterior wing margins (Figs. 338-344) against which they rub their hind femora when agitated (Wood-Mason 1878b: fig. 2; Williams \& Sharp 1904: 129; Willey 1906: 226, Shelford 1916: 140; 1918: 357; Giglio-Tos 1912: 59; Hebard 1920: 51 ff.; Hingston 1932: 278; Burtt \& Carpenter 1943: 57; Schwarz et al. 2006: 69 and figures; see summary in Ramsay 1990: 44-45). In the present dataset this character was interpreted to be synapomorphic for all the species possessing it. However, as was discussed in chapter 4.3.66, this is debateable as the character is inconsistent within the Mantidae genera that have it.

It is likely that all these mechanisms of sound production serve as defensive startling noises because they all are produced when specimens are agitated and the primary defence mechanisms (hiding, camouflage) have failed. 


\subsubsection{Metathoracic hearing organ}

The discovery and function of the metathoracic ear have been described in detail in the "metathoracic hearing organ" section in chapter 4.1.2. However, the results found in the present study are contradictory to the molecular (Svenson \& Whiting 2009) and combined findings of morphology and molecular data (Yager \& Svenson 2008).

In these studies, a single origin of the DK-shape ("deep groove with knobs") of the metathoracic hearing organ early in mantodean evolution was postulated. In the present study, however, multiple origins and losses were found. The MSMT-morphology ("meso- and metathoracic segments similar") was interpreted by Paup* to be the ancestral state for males (130:4) and females (131:4).

An independent origin of the male ear in four groups is suggested by the present analysis for Amorphoscelidae including Compsothespis [15], Stenomantis [36], Haaniinae [55] and clade [58]. Furthermore, the situation within clade [37] was ambiguous and two potential scenarios were suggested by the data. Firstly, an origin of the ear in males of clade [37] with a secondary loss in Oligonicinae including Haaniinae [43] and a subsequent re-gain in Haaniinae [55] was found under ACCTRAN. In the second scenario, the male ear evolved independently in Majanga [38], Epaphrodita [40], Hoplocorypha [42] and Haaniinae [55] under DELTRAN, while the remaining taxa in clade [37] retained the ancestral state (130:4).

According to the present analysis, the origin of the ear in female Mantodea was ambiguous. It either occurred in clade [14] or convergently in female Amorphoscelidae including Compsothespis [15] and clade [32]. An unambiguous secondary origin of the female ear within a primarily earless clade was found for Haaniinae [55].

Furthermore, a loss of the ear has been found for both sexes of the south American clade [223] (DELTRAN) or independently for Photina [224] and Orthoderella + Acanthopidae [225] (ACCTRAN).

Many evolutionary changes among the different character states of the metathoracic ear throughout mantodean phylogeny have been found [also found by Yager \& Svenson (2008: 558)], including reductions and regains of the structure.

It is debatable whether or not such a scenario is likely. Many of the structures studied in the present dataset are comparatively simple characters of shape that are prone to changing quickly under selective pressures of predators or preying success. The metathoracic ear on the other hand is a complicated functional unit of which a multiple convergent origin appears less plausible with regard to its morphological and physiological complexity. A multiple reduction on the other hand is rather plausible because it is correlated with the loss of the ability to fly (e.g. Yager \& Svenson 2008: 558; see also notes on Ameles in the description of character 130).

\subsection{Conclusions}

The results of the present study support the monophyly of several groups of the traditional taxonomy of the Mantodea by robust apomorphic morphological characters. However, the greater 
part of the encoded 152 morphological characters have been found to be strongly homoplastic. Especially the major nodes between the basal splits and the more or less well-supported smaller crown groups are weakly supported.

Robust morphological apomorphies have been found for several groups of Mantodea including Empusidae, Chroicopterinae (partim), Amorphoscelinae, Perlamantinae, Paraoxypilinae, Eremiaphilidae and others. Nonetheless, congruence with taxonomy and molecular data is comparatively low.

This study was able to shed light to some of the most well-known morphological enigmata in mantodean evolution, namely the hitherto so-believed "missing" tibial spur of Chaeteessa and the "missing" discoidal spines of Metallyticus. Both have apparently been reduced in size secondarily, but are still present, as becomes obvious from SEM studies of these rare and interesting taxa.

Data has been gathered regarding the lifestyle of Chaeteessa, Metallyticus and Mantoida. Initial observations on living Metallyticus led to hypotheses of the origin of the curious morphology of the forelegs of this genus and Chaeteessa and may, in congruence with the fossil record and behaviour, indicate bark-dwelling as the primary lifestyle of Mantodea.

The study of female digging devices has yielded four well supported, morphologically distinct types that originated independently, with two potential further types that remain to be studied.

For the first time, first instar nymphs of Metallyticus and Mantoida were available for SEM studies, leading to insights into the adult morphology. The first instar nymphs of Theopompa, Phyllocrania, Pseudocreobotra, Humbertiella, and Didymocorypha were studied in comparison with the adults. Throughout this study, the results repeatedly showed that investigating the postembryonic development of Mantodea may be a key factor for many observations in adult morphology. This field has been vastly neglected throughout the recent decades. Future results may make it easier to understand the morphology of many mantodean taxa as well as the fossil record.

\subsection{Future work}

Ontogenetic studies. This study has shown in several cases the value of observations of the postembryonic development. The homologization of the spines on the fore femora of first instar nymph and adult of Metallyticus in combination with behavioural observations of living specimens has led to the refutation of the dogma of missing discoidal spines in this enigmatic genus, and to the rise of a new hypothesis of the evolution of their lifestyle and hunting behaviour.

Another example has been presented in chapter 4.6.1 in the discussion of the head processes. It has been confirmed that the head process in the first instar nymphs of Phyllocrania is strongly differing from that of the adults (also shown by Gillon \& Roy 1968: fig. 47). Further ontogenetic studies of this character in other taxa (Empusidae, Sibyllidae, Vatinae, further Hymenopodidae) may lead to new morphological insights into the evolution of the head processes in Mantodea.

Furthermore, it will be a very important task to study the postembryonic development of 
characteristic structures throughout several different mantodean taxa. It is crucial to know if and to what degree such structures are already present in first instar nymphs in order to deduce evolutionary scenarios. This is indispensable for the study of the evolutionary history of the foreleg spination of many morphologically enigmatic taxa, for instance Chaeteessa, Oligonicinae and Haaniinae, Amorphoscelinae, Paraoxypilinae, Perlamantinae, Compsothespis, Toxoderidae, and others. Therefore breeding such taxa and documenting the changes throughout their development is an important task in order to reconstruct the evolution of many taxa that morphologically differ from the "typical" mantodean foreleg. An especially challenging task is the postembryonic development of the foreleg in Amorphoscelinae and Perlamantinae (and possibly Compsothespis, see 4.3.10) which represent some of the most strongly aberrant foreleg morphologies found in Mantodea.

The knowledge of how foreleg spination, length relations and other characters alter throughout nymphal development may furthermore yield valuable data for accurate interpretations of the palaeontological record, as becomes obvious in the discussion of the $\uparrow$ Cretomantis larvalis specimen in chapter 2.2. Mantodea preserved in amber, for instance, are mostly represented as (often early) instar nymphs. As Ehrmann (1999: 7) pointed out, it is helpful to use first instar nymphs of extant species to determine the affinities of the fossils. However, fossil Mantodea are often assigned to extant lineages without exhibiting apomorphic characters of the latter, thus leading to possibly erroneous identifications. For instance, the affiliation to Chaeteessidae of the specimen presented by Weitschat \& Wichard (2002: fig. 49 and pl. 33, fig. a) in my opinion is doubtful. The tibial spur is apparently well developed and the spines are mostly very short, thus lacking any resemblance to adult Chaeteessa specimens (compare Figs. 418-420 herein). However, as the study of the postembryonic development of Metallyticus revealed, the spination of the first instar nymph and the adult may differ considerably. As the first instar nymphs of Chaeteessa are unknown, we have no information whatsoever on potential characteristics of chaeteessid nymphal forelegs. The definite affinity of fossils like the one mentioned can thus not be ascertained without a proper study of the ontogeny of extant species.

Anatomy of the raptorial legs. Directly connected to the prior tasks is the morphological and anatomical study of the raptorial forelegs of Mantodea. A more detailed comparative study of the spination patterns among the mantodean subgroups as well as between Mantodea and Blattaria may shed light on the evolution of this morphological complex. Ecological observations (feeding habits, food preferences) would certainly be of great value for supporting the morphological data. Studies of the anatomy of the forelegs (musculature, innervation etc.) may yield valuable phylogenetic data that may be able shed further light on the evolutionary history of many Mantodea.

Allometric growth. A point that has not found much attention in this study but may play an important part when behaviour, mimicry or mimesis in different stages of mantodean postembryonic development are concerned, is the documentation of allometric growth in Mantodea. 
It is likely that characters such as the length relations of the pronotum (length, width, length with respect to body length, length relations between pro- and metazona) or other body relations experience a shift throughout postembryonic development. This is the case at least in some species mimicking ants (see Odontomantis in Figs. 455-457; Mantoida in Deyrup 1986: fig. 1; Jackson \& Drummond 1974: fig. 1E, F) but can also be found in other species [e.g. in Gonatista (Nickle 1987: fig. 13.1) and Metallyticus (Figs. 453, 454)]. Terra (1980) studied the foreleg measurements of several Neotropical species throughout their nymphal development and found that more distinct structural changes took place in the ontogeny of long, slender forelegs without enlarged (i.e. widened in lateral view) femora (pattern B in Terra 1980) than in that of stouter and shorter forelegs with enlarged femora (pattern A in Terra 1980). 



\section{References}

Abu-Dannoun, O. \& Katbeh-Bader, A. 2007. Mantodea of Jordan. - Zootaxa 1617: 43-56.

Adair, E.W. 1914. Notes on the early stages in the post-embryonic development of Empusa egena Charp. - Bulletin de la Société Entomologique d’Ègypte 7: 76-80.

Agabiti, B. 2001. Le specie Mediterranee del genere Ameles Burmeister, 1838 (Insecta, Mantodea: Amelinae) con considerazioni biogeografiche e filogenetiche (Tesis di Dottorado). - Dottorado di Ricerca in Biologia Evoluzionistica (Filogenesi e Sistematica). Ciclo 14 (1999-2001): 1-95.

Agudelo Rondón, A.A. 2004. Mántidos de Colombia (Dictyoptera: Mantodea). - Pp. 43-60 in: Fernádez, C., Andrade, C. \& Amat, G. (eds): Insectos de Colombia, vol. 3. - Bogotá, D.C.: Universidad Nacional de Colombia, Facultad de Ciencias. 604 pp.

Agudelo Rondón, A.A. \& Chica E., L.M. 2002. Mantidos - Introducción al conocimiento del orden Mantodea. Bogotá: Universidad Distrial Francisco José de Caldas. 74 pp.

Agudelo Rondón, A.A., Lombardo, F. \& Jantsch, L.J. 2007. Checklist of the neotropical mantids (Insecta, Dictyoptera, Mantodea). - Biota Colombiana 8(2): 105-158.

Ahmad, M. 1950. The phylogeny of termite genera, based on imago-worker mandibles. - Bulletin of the American Museum of Natural History 95(2): 37-86.

Andres, A. 1914. L'oothèque de l'Eremiophila [sic!] Khamsin. (Orthopt. Mantide). - Bulletin de la Société Entomologique d'Ègypte 1913(2): 72-74.

Anisyutkin, L.N. 1998. Notes on the genus Deroplatys Westwood, with description of a new species from Vietnam (Mantodea: Mantidae: Deroplatyinae). - Zoosystematica Rossica 7: 95-99.

Anisyutkin, L.N. 2005. New data on the genus Haania Sauss. (Dictyoptera, Mantidae, Thespinae) of Indochina, with description of a new species. - Proceedings of the Russian Entomological Society 76: 15-20.

Anisyutkin, L.N. \& Gorochov, A.V. 2005. Haania doroshenkoi, a new species of mantises from Cambodia (Mantina: Mantidae: Thespinae) and a case of mirror symmetry in the structure of the male genitalia of mantises. - Russian Entomological Journal 13(3): 119-122.

Annandale, N. 1905. Notes on some oriental geckos in the Indian Museum, Calcutta, with descriptions of new forms. - Annals and Magazine of Natural History including Zoology, Botany and Geology 17(15): 26-32.

Audinet-Serville, J.G. 1831. Revue méthodique des insectes de l'ordre des Orthoptères. - Annales des Sciences Naturelles 22: 28-65.

Audinet-Serville, J.G. 1839. Histoire Naturelle des Insectes. Orthoptères. - Paris: De Fain. 776 pp.

Ax, P. 1999. Das System der Metazoa II. Ein Lehrbuch der phylogenetischen Systematik. - Stuttgart, Jena, Lübeck, Ulm: Gustav Fischer. 383 pp.

Ayala, M. \& Onore, G. 2001. Los mántidos (Insecta: Mantodea) del bosque nublado Otonga y área aledaña. Pp. 243-260 in: Nieder, J. \& Barthlott, W. (eds): Epiphytes and canopy fauna of the Otonga rain forest (Ecuador). Results of the Bonn - Quito ephyphyte project, funded by the Volkswagen Foundation (Vol. 2/2). Books on Demand GmbH. 388 pp.

Baccetti, B. 1967. L'ultrastruttura delle ghiandole della ootheca in Ortotteri Acridoidei, Blattoidei e Mantoidei. Zeitschrift für Zellforschung und mikroskopische Anatomie 77: 64-79.

Badenoch, L.N. 1899. True tales of the insects. - New York: Dutton \& Co.; London: Chapman \& Hall. 255 pp.

Balderson, J. 1978. Reversal of the phallic complex in the genera Ciulfina Giglio-Tos and Stenomantis Saussure (Mantodea: Mantidae: Iridopteryginae). - Australian Journal of Entomology 17: 235-239.

Balderson, J. 1984. Catalogue of Australian Mantodea. - CSIRO Australian Division of Entomology Technical Paper 23: 1-17.

Balderson, J. 1991: Mantodea (Praying Mantids). - Pp. 348-356 in: CSIRO (eds): The insects of Australia. A textbook for students and research workers, vol. 1. - Carlton: Melbourne University Press. 1137 pp. 
Balderson, J., Rentz, D.C.F. \& Roach, A.M.E. 1998. Mantodea. - Pp. 251-277 in: Zoological Catalogue of Australia: Archaeognatha, Thysanura, Blattodea, Isoptera, Mantodea, Dermaptera, Phasmatodea, Embioptera, Zoraptera, vol. 23. - Canberra: CSIRO \& ABRS. 464 pp.

Ball, E.E. \& Stone, R.C. 1982. The cercal receptor system of the praying mantid, Archimantis brunneriana Sauss. I. Cercal morphology and receptor types. - Cell Tissue Research 224: 55-70.

Barabás, S.P. \& Hancock, E.G. 1999. Asymmetrical colour and wing-folding in Tithrone roseipennis (Saussure, 1870) a neotropical praying mantis (Mantodea Hymenopodidae). - Tropical Zoology 12: 325-334.

Battiston, R. \& Fontana, P. 2005. A contribution to the knowledge of the genus Ameles Burmeister, 1838, with the description of a new species from Jordan (Insecta Mantodea). - Atti della Accademia Roveretana degli Agiati, A 255, Ser. VIII, vol. V, B: 173-197.

Battiston, R. \& Massa, B. 2008. The mantids of Caucasus (Insecta: Mantodea). - Atti della Accademia Roveretana degli Agiati, A 258, Ser. VIII, vol. VIII: 5-28.

Battiston, R. \& Picciau, L. 2008. Contribution to the knowledge of the Mantodea of Ecuador with the description of the male of Pseudoxyops perpulchra (Westwood, 1889) (Mantodea Mantidae Stagmatopterinae). - Memoirs on Biodiversity 1: 19-30.

Battiston, R., Picciau, L., Fontana, P. \& Marshall, J. 2010. Mantids of the Euro-Mediterranean area. - WBA Handbooks 2: 1-240.

Beebe, W., Crane, J. \& Hughes-Schrader, S. 1952. An Annotated List of the Mantids (Orthoptera: Mantoidea) of Trinidad, B.W.I. - Zoologica: New York Zoological Society 37(19): 245-258.

Bedford, G.O. 1978. Biology and ecology of the Phasmatodea. - Annual Review of Entomology 23: 125-149.

Beier, M. 1929. Einige neue Mantiden aus der Sammlung des Naturhistorischen Museums in Wien. - Zoologischer Anzeiger 80: 129-139.

Beier, M. 1933. Beiträge zur Fauna sinica. XIII. Die Mantodeen Chinas. - Mitteilungen aus dem Zoologischen Museum in Berlin 18(3): 322-337.

Beier, M. 1934a. Mantodea, Fam. Mantidae, Subfam. Hymenopodinae. - In: Wytsman, P. (ed.): Genera Insectorum, vol. 196. - Brussels: Verteneuil \& Desmet. 37 pp.

Beier, M. 1934b. Mantodea, Fam. Mantidae, Subfam. Sibyllinae, Empusinae. - In: Wytsman, P. (ed.): Genera Insectorum, vol. 197. - Brussels: Verteneuil \& Desmet. 10 pp.

Beier, M. 1934c. Mantodea, Fam. Mantidae, Subfam. Toxoderinae. - In: Wytsman, P. (ed.): Genera Insectorum, vol. 198. - Brussels: Verteneuil \& Desmet. 9 pp.

Beier, M. 1935a. Mantodea, Fam. Mantidae, Subfam. Thespinae. - In: Wytsman, P. (ed.): Genera Insectorum, vol. 200. - Brussels: Verteneuil \& Desmet. 32 pp.

Beier, M. 1935b. Mantodea, Fam. Mantidae, Subfam. Orthoderinae, Choeradodinae, Deroplatynae. - In: Wytsman, P. (ed.): Genera Insectorum, vol. 201. - Brussels: Verteneuil \& Desmet. 10 pp.

Beier, M. 1935c. Mantodea, Fam. Mantidae, Subfam. Mantinae. - In: Wytsman, P. (ed.): Genera Insectorum, vol. 203. - Brussels: Verteneuil \& Desmet. 146 pp.

Beier, M. 1939a. Die geographische Verbreitung der Mantodea. - Internationaler 7. Kongress der Entomologie, Berlin 1: 5-15.

Beier, M. 1939b. Die Mantodea der Ausbeute Klapperich aus Süd-China. - Decheniana 98B: 141-142.

Beier, M. 1940. Über die von Klapperich 1938 in Süd-China erbeuteten Mantodeen. - Decheniana 99B: 91-92.

Beier, M. 1950. Apterameles rammei n. gen. et spec., eine flügellose Mantide aus Mazedonien. - Entomologisches Nachrichtenblatt Österreichischer und Schweizer Entomologen 2: 61-63.

Beier, M. 1952. Die Mantiden des Subtribus Haaniines (Thespinae - Oligonychini) (Orth.). - Treubia 21(1-3): 199-210.

Beier, M. 1954. Mantidea und Pseudophyllinae. - Explorations du Parc National de l'Upemba, Mission G.F. de Witte 20: 1-77. 
Beier, M. 1955a. Ordnung Saltatoptera m. (Saltatoria Latreille, 1817). - Pp. 34-304 in: Weber, H. (ed.): Dr. H.G. Bronns Klassen und Ordnungen des Tierreichs, 5. Band: Arthropoda, 3. Abteilung: Insecta, 6. Buch. Leipzig: Geest \& Portig.

Beier, M. 1955b. Chapter VIII. Mantidea. - Pp. 234-265 in: Hanström, B., Brinck, P. \& Rudebeck, G. (eds): South African animal life. Results of the Lund University Expedition in 1950-1951, vol. 2. - Stockholm: Almqvist \& Wiksell. 576 pp.

Beier, M. 1956. Mantiden aus Ceylon (Orth.). - Verhandlungen der Naturforschenden Gesellschaft Basel 67(1): 33-40.

Beier, M. 1957a. Neues zur Kenntnis der Gattung Pyrgomantis Gerst. (Mantidea). - Revue Zoologique et Botanique Africaine 55(1/2): 90-100.

Beier, M. 1957b. Zur Kenntnis der Gattungen Tarachodes und Galepsus (Mantidea). - Revue Zoologique et Botanique Africaine 56(1/2): 111-146.

Beier, M. 1961. Mantiden aus Südwest-Abessinien der Ausbeute W. Richter 1959/60. - Stuttgarter Beiträge zur Naturkunde 55: 1-4.

Beier, M. 1964a. Blattopteroidea. Mantodea. - Pp. 849-970 in: Weber, H. (ed.): Dr. H.G. Bronns Klassen und Ordnungen des Tierreichs, 5. Band: Arthropoda, 3. Abteilung: Insecta, 6. Buch, 5. Lieferung. Leipzig: Geest $\&$ Portig.

Beier, M. 1964b. Ein neues Mantiden-Genus aus Israel. - Israel Journal of Zoology 13: 184-186.

Beier, M. 1965a. Die Mantodeen Neu-Guineas. - Pacific Insects 7(3): 473-502.

Beier, M. 1965b. Über einige Mantiden von der Insel Prince of Wales. - Pacific Insects 7: 449-452.

Beier, M. 1967. Mantis religiosa L. im Pliozän des Harzvorlandes. - Berichte der Naturhistorischen Gesellschaft Hannover 111: 63-64.

Beier, M. 1968a. Mantodea (Fangheuschrecken). - Pp. 1-47 in: Helmcke, J.-G., Starck, D. \& Wermuth, H. (eds): Handbuch der Zoologie, vol. 4(2), 2/12. - Berlin: Walter de Gruyter.

Beier, M. 1968b. Mantiden von der Insel Rennell. - The Natural History of Rennell Island, British Solomon Islands 5 (74): 79-80.

Beier, M. 1972. Insects of Micronesia. Mantodea. - Insects of Micronesia 5(2): 173-175.

Beier, M. 1974a. Blattariae (Schaben). - Pp. 1-127 in: Handbuch der Zoologie, vol. 4(2), 2/13. - Berlin: Walter de Gruyter.

Beier, M. 1974b. Class Insecta, Order Mantodea. - Pp. 30-31 in: Coaton, W.G.H. (ed.) 1974: Status of the taxonomy of Hexapoda of Southern Africa. - Entomology Memoirs 38, 30-31.

Beier, M. 1976. Zur Kenntnis der Gattungen Toxodera und Paratoxodera (Mantidea). - Revue Suisse de Zoologie 83(2): 393-400.

Beier, M. \& Heikertinger, F. 1952. Fangheuschrecken (Die neue Brehm-Bücherei). - Leipzig: Geest \& Portig. 32 pp.

Beier, M. \& Jaus, J. 1933. Mantodea, Fangheuschrecken. - Pp. 117-168 in: Schulze, P. (ed.): Biologie der Tiere Deutschlands, vol. 36. Lieferung. - Berlin: Borntraeger. 415 pp.

Bell, W.J., Roth, L.M. \& Nalepa, C.A. 2007. Cockroaches - Ecology, Behavior, and Natural History. - Baltimore: Johns Hopkins University Press. 230 pp.

Benton, M.J. 2007. Paläontologie der Wirbeltiere. - München: Pfeil-Verlag. 472 pp.

Béthoux, O. 2005. Wing venation pattern of Plecoptera (Insecta: Neoptera). - Illiesia 1(9): 52-81.

Béthoux, O. 2008. Groundplan, nomenclature, homology, phylogeny, and the question of the insect wing venation pattern. - Alavesia 2: 219-232.

Béthoux, O. 2010. Alteration of sex-related developmental modules: A case of ,feminized“ male wing morphology in Creobroter gemmatus (Mantodea: Hymenopodidae). - European Journal of Entomology 107: 133-135.

Béthoux, O., Beckemeyer, R.J., Engel, M.S. \& Hall, J.D. 2010. New data on Homocladus grandis, a Permian stemMantodean (Polyneoptera: Dictyoptera). - Journal of Paleontology 84(4): 746-753. 
Béthoux, O., Klass, K.-D. \& Schneider, J.W. 2009. Tackle the 'Protoblattoidea problem': revision of Protoblattinopsis stubblefieldi (Dictyoptera; Late Carboniferous). European Journal of Entomology 106: 145-152.

Béthoux, O. \& Wieland, F. 2009. Evidence for Carboniferous origin of the order Mantodea (Insecta: Dictyoptera) gained from forewing morphology. - Zoological Journal of the Linnean Society 156(1): 79-113.

Beutel, R.G. \& Gorb, S.N. 2001. Ultrastructure of attachment specializations of hexapods (Arthropoda): evolutionary patterns inferred from a revised ordinal phylogeny. - Zeitschrift für Zoologische Systematik und Evolutionsforschung/Journal of Zoological Systematics and Evolutionary Research 39: 177-207.

Beutel, R.G. \& Gorb, S.N. 2006. A revised interpretation of the evolution of attachment structures in Hexapoda with special emphasis on Mantophasmatodea. - Arthropod Systematics and Phylogeny 64(1): 3-25.

Beutel, R.G. \& Gorb, S.N. 2008. Evolutionary scenarios for unusual attachment devices of Phasmatodea and Mantophasmatodea (Insecta). - Systematic Entomology 33(3): 501-510.

Blatchley, W.S. 1920. Orthoptera of Northeastern America with especial reference to the faunas of Indiana and Florida. - Indianapolis: The Nature Publishing Company. 784 pp.

Bohn, H. 2003. 14. Ordnung Blattoptera, Schaben. - Pp. 197-223 in: Dathe, H.H. (ed.): Lehrbuch der Speziellen Zoologie, Band I: Wirbellose Tiere, 5. Teil: Insecta. - Heidelberg, Berlin: Spektrum. 961 pp.

Bohn, H. \& Klass, K.-D. 2003. Dictyoptera, Ordnungen 13-15. - Pp. 181-182 in: Dathe, H.H. (ed.): Lehrbuch der Speziellen Zoologie, Band I: Wirbellose Tiere, 5. Teil: Insecta. - Heidelberg, Berlin: Spektrum. 961 pp.

Bouchenak-Khelladi, Y., Verboom, G.A., Savolainen, V. \& Hodkinson, T.R. 2010. Biogeography of the grasses (Poaceae): A phylogenetic approach to reveal evolutionary history in geographical space and geological time. - Botanical Journal of the Linnean Society 162: 543-557.

Boudreaux, H.B. 1979. Arthropod phylogeny with special reference to insects. - New York, Chichester, Brisbane, Toronto: Wiley \& Sons. 320 pp.

Boyan, G.S. \& Ball, E.E. 1986. Wind-sensitive interneurons in the terminal ganglion in praying mantids. - Journal of Comparative Physiology A: Sensory, Neural and Behavioral Physiology 159: 773-789.

Bragg, P.E. 2010. A review of the Liturgusidae of Borneo (Insecta: Mantodea). - Sepilok Bulletin 12: 21-36.

Bravo, F., Pohl, H., Silva-Neto, A. \& Beutel, R.G. 2009. Bahiaxenidae, a "living fossil" and a new family of Strepsiptera (Hexapoda) discovered in Brazil. - Cladistics 25: 614-623.

Brown, W.L. 1982. Mantodea. - Pp. 347-349 in: Synopsis and Classification of the Living Organisms. - Texas: McGraw-Hill. 1119 pp.

Buckley, T.R., Attanayake, D. \& Bradler, S. 2009. Extreme convergence in stick insect evolution: Phylogenetic placement of the Lord Howe Island tree lobster. - Proceedings of the Royal Society B 276: 1055-1062.

Bugnion, E. 1921a. The growth of the antennae of Empusa egena Charp. (Orthop.). - Bulletin de la Société Entomologique d'Egypte 6: 118-132.

Bugnion, E. 1921b. The growth of the antennae and cerci of the cockroach: Periplaneta americana L. - Bulletin de la Société Entomologique d'Egypte 6(3): 56-66.

Bugnion, E. 1922. Note relative a l'Ameles spallanzania. Structure de l'oothèque. Ecolsion des jeunes larves. Bulletin de la Société Zoologique de France 47: 172-180.

Burmeister, H. 1936. Bericht über die Fortschritte der Entomologie. - Archiv für Naturgeschichte 2(2): 293-327.

Burmeister, H. 1838. Fangheuschrecken. Mantodea. Mantides Latr. - Pp. 517-552 in: Handbuch der Entomologie 2.2.1. - Berlin: Reimer. 756 pp.

Burtt, E. \& Carpenter, H 1943. The defensive attitude of the mantid Idolum diabolicum Sauss. (Orthoptera). - Proceedings of the Royal Entomological Society of London (A) 18: 57.

Carpenter, F.M. 1947. Taxonomic notes on the Dilaridae (Neuroptera). - Psyche 54: 100-109.

Carpenter, F.M. 1971. Adaptations among Paleozoic insects. - Proceedings of the North American Paleontological Convention 1969, vol. II, part i: 1236-1251.

Carpenter, F.M. 1992. Superclass Hexapoda. - Pp. 1-655 (= volumes 4/3 and 4/4) in: Kaesler, R.L. (ed.): Treatise on invertebrate paleontology. - Boulder: The Geological Society of America and the University of Kansas. 
Cerdá, F.J. 1993. Mantodea de Venezuela. Generos y lista preliminar de especies parte 1: Familias Mantoididae y Hymenopodidae. - Revista de la Facultad de Agronomía (Maracay) 19: 129-151.

Cerdá, F.J. 1996a. Mantodea de Venezuela. Generos y lista preliminar de especies parte 2: Familia Mantidae (subfamilias Liturgousinae y Thespinae). - Boletín de Entolomogía Venezolana (N.S.) 11(2): 73-87.

Cerdá, F.J. 1996b. Mantodea de Venezuela. Generos y lista preliminar de especies parte 3: Familia Mantidae (subfamilias Oligonychinae, Angelinae y Mantinae). - Boletín de Entolomogía Venezolana (N.S.) 11(2): 89-101.

Cerdá, F.J. 1997a. Mantodea de Venezuela. Generos y lista preliminar de especies parte 4: Familia Mantidae (subfamilia Vatinae). - Boletín de Entolomogía Venezolana (N.S.) 12(1): 17-31.

Cerdá, F.J. 1997b. Mantodea de Venezuela. Generos y lista preliminar de especies parte 5: Familia Mantidae (subfamilia Photininae). - Boletín de Entolomogía Venezolana (N.S.) 12(1): 33-42.

di Cesnola, A.P. 1904. Preliminary note on the protective value of colour in Mantis religiosa. - Biometrika 3: 5859.

Chapman, R.C. \& Balderson (J.) 1984. Ovipositional behaviour of Sphodropoda tristis Saussure (Mantodea: Mantidae). - Australian Entomological Magazine 11(1): 5-7.

Chopard, L. 1911. Contribution a la faune des orthoptères de la Guyane Française. - Annales de la Société entomologique de France 80: 315-350.

Chopard, L. 1916. Étude des mantides Américains de la collection I. Bolivar (Orthoptères). - Annales de la Société entomologique de France 85: 161-187.

Chopard, L. 1920. Description d'un Haania Sauss. (Orth. Mantidae) du Laos. - Bulletin de la Société entomologique de France 3: 55-59.

Chopard, L. 1922. Mantidae. - Pp. 32-40 in: Faune de France 3: Orthoptères et Dermaptères. - Paris: Lechevalier. 212 pp.

Chopard, L. 1929. Description d'un Mantide du Congo Belge appartenant à un genre nouveau. - Revue de Zoologie et de Botanique Africaines 18: 44-45.

Chopard, L. 1938. La Biologie des Orthoptères. - Paris: Lechevalier. 541 pp.

Chopard, L. 1940a. Contribution a l'étude des Orthoptéroïdes du nord de l'Afrique. - Annales de la Société entomologique de France 109: 153-167.

Chopard, L. 1940b. Dictyoptères et Orthoptères récoltés en Mauritanie et dans la région du Tchad par la missin d'études de la biologie des Acridiens. - Revue française d'Entomologie 7: 8-30.

Chopard, L. 1941. Contribution a l'étude des Orthoptéroïdes du nord de 1'Afrique. - Annales de la Société entomologique de France 110: 25-50.

Chopard, L. 1942. Insectes Orthoptéroïdes (Blattidae, Mantidae, Gryllidae, Phasmidae, Dermaptera) racoltés dans les îles atlantiques. - Societas Scientiarum Fennica - Commentationes Biologicae 8(4): 1-13.

Chopard, L. 1943. Faune de 1‘Empire Francais I: Orthoptèroïdes de 1‘Afrique du nord. - Paris: Libraire Larose. $450 \mathrm{pp}$.

Chopard, L. 1949a. Ordre des Dictyoptères, sous-ordre des Mantodea. - Pp. 386-407 in: Grassé (ed.): Traité de Zoologie. Anatomie, Systématique, Biologie. Vol. 9. - Paris: Masson. 799 pp.

Chopard, L. 1949b. Ordre des Dictyoptères, sous-ordre des Blattodea. - Pp. 355-385 in: Grassé (ed.): Traité de Zoologie. Anatomie, Systématique, Biologie. Vol. 9. - Paris: Masson. 799 pp.

Chopard, L. 1949c. Le mimétisme. Colorations animales, dissimulation des formes et déguisements, resemblances mimétiques. - Paris: Payot. 335 pp.

Chopard, L. 1951. Sous-ordre Mantodea. - Pp. 34-49 in: Faune de France 56: Orthoptéroïdes. - Paris: Lechevalier. 359 pp.

Çiplak, B. \& Demirsoy, A. 1997. Mantodea (Insecta) fauna of Malatya vicinity (Turkey) and some remarks on the mantises of Anatolia. - Journal of Orthoptera Research 6: 105-111. 
Cleveland, L.R., Hall, S.R., Sanders, E.P. \& Collier, J. 1934: The wood-feeding roach Cryptocercus, its protozoa, and the symbiosis between protozoa and roach. - Memoirs of the American Academy of Arts and Sciences N.S. 17: 185-342.

Cockerell, T.D.A. 1908. The first American fossil mantis. - Canadian Entomologist 40: 343-344.

Cockerell, T.D.A. 1955. Some fossil insects from Florissant, Colorado. - Proceedings of the United States National Museum 44: 341-346.

Colless, D.H. \& McAlpine, D.K. 1991: Diptera (flies). - Pp. 717-786 in: CSIRO (eds): The insects of Australia. A textbook for students and research workers, vol. 2. - Carlton: Melbourne University Press. 1137 pp.

Copeland, J. \& Carlson, A.D. 1977. Prey capture in mantids: prothoracic tibial flexion reflex. - Journal of Insect Physiology 23: 1151-1156.

Cornet, J. 1894. Le mimétisme dans la faune Africaine. - Le Congo Illustré. Voyages et travaux des Belges dans l'état indépendant du Congo 3(26): 182-184 (part 1); 197-200 (part 2).

Courrent, A., Quennedey, A., Nalepa, C.A., Robert, A., Lenz, M. \& Bordereau, C. 2008. The fine structure of collaterial glands in two cockroaches and three termites, including a detailed study of Cryptocercus punctulatus (Blattaria, Cryptocercidae) and Mastotermes darwiniensis (Isoptera, Mastotermitidae). - Arthropod Structure \& Development 37(1): 55-66.

Crampton, G.C. 1926. A comparison of the neck and prothoracic sclerites throught [sic!] the orders of insects from the standpoint of phylogeny. - Transactions of the American Entomological Society, Philadelphia 52(3): 199-248.

Crane, J. 1952. A comparative study of innate defensive behavior in Trinidad mantids (Orthoptera: Mantoidea). Zoologica (New York) 37(20): 259-294.

Crawford, C.S. 1981. Biology of desert invertebrates. - Berlin, Heidelberg, New York: Springer. 314 pp.

Crosland, M.W.J., Su, N.-Y. \& Scheffrahn, R.H. 2005. Arolia in termites (Isoptera): functional significance and evolutionary loss. - Insectes Sociaux 52: 63-66.

Cumming, G.S. 1996. Mantis movements by night and the interactions of sympatric bats and mantises. - Canadian Journal of Zoology 74: 1771-1774.

Dallwitz, M.J. 1980. A general system for coding taxonomic descriptions. - Taxon 29: 41-46.

Dallwitz, M.J., Paine, T.A. \& Zurcher, E.J. 1999 onwards. User's guide to the DELTA Editor. - http://delta-intkey. com

Dantas, Y.K.L.A., Rafael, J.A., Motta, C. da S., Agudelo Rondón, A.A. \& Xavier Filho, F.F. 2008. Registros de Mantodea (Insecta) coletados à luz no dossel da floresta, na torre do km 14 do núcleo ZF-2, Manaus, Brasil. - Acta Amazonica 38(2): 317-320.

Darwin, C.R. 1859. On the origin of species by means of natural selection, or the preservation of favoured races in the struggle for life. $1^{\text {st }}$ edn. - London: John Murray. 502 pp.

Davis, W.J. \& Liske, E. 1988. Cerci mediate mating movements in the male praying mantis. - Zoologische Jahrbücher Physiologie 92: 47-55.

Deitz, L.L., Nalepa, C. \& Klass, K.-D. 2003. Phylogeny of the Dictyoptera Re-examined (Insecta). - Entomologische Abhandlungen 61(1): 69-91.

Delfosse, E., Bauduin, A. \& Bauduin, C. 2007. Taxinomie, biogéographie et biologie de la mante-orchidée $H y$ menopus coronatus (Olivier, 1792) (Insecta: Mantodea, Hymenopodidae). - Bulletin d‘Arthropoda 34(4): 3-21.

Deyrup, M. 1986. Observations on Mantoida maya (Orthoptera: Mantidae). - Florida Entomologist 69(2): 434435.

Doganlar, M. 2007. A new species of the genus Eremiaphila Lefèbvre, 1835 (Mantodea: Eremiaphilidae) from Turkey. - Australian Journal of Basic and Applied Sciences Research 1(1): 1-6.

Dunger, W. 2003. 2. Ordnung Collembola, Springschwänze. - Pp. 71-86 in: Dathe, H.H. (ed.): Lehrbuch der Speziellen Zoologie, Band I: Wirbellose Tiere, 5. Teil: Insecta. - Heidelberg, Berlin: Spektrum. 961 pp. 
Edmunds, M. 1972. Defensive behaviour in Ghanaian praying mantids. - Zoological Journal of the Linnean Society 51: 1-32.

Edmunds, M. 1974. Defence in Animals. - Harlow: Longman. 357 pp.

Edmunds, M. 1975. Courtship, mating and possible sex pheromones in three species of Mantodea. - Entomologist's Monthly Magazine 111: 53-57.

Edmunds, M. 1976. The defensive behaviour of Ghanaian praying mantids with a discussion of territoriality. Zoological Journal of the Linnean Society 58: 1-37.

Edmunds, M. \& Brunner, D. 1999. 13. Ethology of defenses against predators. - Pp. 276-299 in: Prete, F.R., Wells, H., Wells, P.H. \& Hurd, L.E. (eds): The praying mantids. - Baltimore \& London: Johns Hopkins. 362 pp.

Eggleton, P. 2001. Termites and trees: a review of recent advances in termite phylogenetics. - Insectes Sociaux 48: 187-193.

Ehrmann, R. 1984. Die Bedeutung der Cerci für Bildung der Oothek bei Mantis religiosa. - Articulata 2(4): 79-80.

Ehrmann, R. 1997. Systematik der Ordnung Mantoptera (Insecta: Dictyoptera). - Arthropoda 5(2): 1-19.

Ehrmann, R. 1999. Gottesanbeterinnen in Kopal und Bernstein (Insecta: Mantodea). - Arthropoda 7(3): 2-8.

Ehrmann, R. 2001a. Gottesanbeterinnen (Mantodea), eine Übersicht. Teil 1: Stammesgeschichte, Systematik, Körperbau, Fortpflanzung. - Reptilia 28: 26-32.

Ehrmann, R. 2001b. Gottesanbeterinnen (Mantodea), eine Übersicht. Teil 2: Vorkommen, Lebensweise, Haltung und Zucht, Determination. - Reptilia 29: 62-67.

Ehrmann, R. 2002: Mantodea. Gottesanbeterinnen der Welt. - Münster: Natur und Tier-Verlag. 519 pp.

Ehrmann, R. 2005: Bibliographie zum Thema Mantodea, Band 2. - Keltern: Goecke \& Evers. 432 pp.

Ehrmann, R. \& Koçak, A.Ö. 2009. The neotropical mantids. - Centre for Entomological Studies Ankara-News 49: $1-18$.

Ehrmann, R. \& Roy, R. 2009. Taxonomy and synonymy of Phyllothelys Wood-Mason (Dictyoptera: Mantodea). Annales de la Société Entomologique de France (N.S.) 45(1): 67-76.

Engel, M.S. 2003. The earwigs of Kansas, with a key to genera north of Mexico (Insecta: Dermaptera). - Transactions of the Kansas Academy of Science 106(3/4): 115-123.

Ergene, S. 1953. Homochrome Farbanpassungen bei Mantis religiosa. - Zeitschrift für vergleichende Physiologie 35: 36-41.

Falcon-Lang, H.J. \& Bashforth, A.R. 2005: Morphology, anatomy, and upland ecology of large cordaitalean trees from the Middle Pennsylvanian of Newfoundland. - Review of Palaeobotany and Palynology 135: 223243.

Fenn, J.D., Song, H., Cameron, S.L. \& Whiting, M.F. 2008. A preliminary mitochondrial genome phylogeny ofOrthoptera (Insecta) and approaches to maximizing phylogenetic signal found within mitochondrial genome data. - Molecular Phylogenetics and Evolution 49(1): 59-68.

Frantsevich, L. 1998. The coxal articulation of the insect striking leg: a comparative study. - Journal of Morphology 236: 127-138.

Fritze, A. 1915. Widerstandsfähigkeit der Eikokons der Fangheuschrecken (Mantodeen). - Zeitschrift für Wissenschaftliche Insektenbiologie 11(7): 275-276.

García Becerra, R., de la Nuez Torres, R.I. \& Pérez Sánchez, J.M. 2001. Mantis y cucarachas de Canarias. - Santa Cruz: Servicio de publicaciones de la Caja General de Ahorros de Canarias 252. 160 pp.

Ge, D.-Y. \& Chen, X.-S. 2008. Review of the genus Palaeothespis Tinkham (Mantodea: Thespidae), with description of one new species. - Zootaxa 1716: 53-58.

Gebauer, D., Liske, E., Köchy, K. \& Wolff, H.G. 1987. The praying mantis nervous system: Thoracic and abdominal ganglia. - P. 66 in: Elsner, N. \& Creutzfeldt, O. (eds) 1987: New frontiers in brain research. Proceedings of the $15^{\text {th }}$ Göttingen neurobiology conference. - Stuttgart, New York: Thieme. $340 \mathrm{pp}$.

Gemeno, C., Claramunt, J. \& Dasca, J. 2005. Nocturnal calling behavior in mantids. - Journal of Insect Behavior 18(3): 389-403. 
Germar, E.F. 1851. Die Versteinerungen des Steinkohlengebirges von Wettin und Löbejün im Saalkreise, bildlich dargestellt und beschrieben. - Heft 7: 82-116.

Giebel, C.G.A. 1862. Wirbelthier und Insektenreste im Bernstein. - Zeitschrift für die gesammten Naturwissenschaften 20: 311-321.

Giglio-Tos, E. 1911. Mantidi Esotici III. Gen. Tarachodes Burm.; IV. Gen. Galepsus Stål. - Bollettino della Socièta Entomologica Italiana 42: 93-169.

Giglio-Tos, E. 1912. Mantidi Esotici V. Mantes, Tenoderae, Hierodulae, et Rhomboderae. - Bollettino della Socièta Entomologica Italiana 43: 2-167.

Giglio-Tos, E. 1913. Orthoptera, Fam. Mantidae, Subfam. Perlamantinae. - In: Wytsman, P. (ed.): Genera Insectorum, vol. 144. - Brussels: Verteneuil \& Desmet. 13 pp.

Giglio-Tos, E. 1914a. Mantidi. Raccolti da S.A.R. la Duchessa d'Aosta nella regione dei grandi laghi dell‘Africa equatoriale. Revisione della sottofamiglia dei Toxoderini. - Annuario del Museo Zoologico della Università di Napoli (N.S.) 4(15): 1-17.

Giglio-Tos, E. 1914b. Mantidi Esotici VII. Vatinae. - Bolletino dei Musei di Zoologia ed Anatomia comparata della R. Università di Torino 29(684): 1-87.

Giglio-Tos, E. 1915. Mantidi esotici. Generi e specie nuove. - Bullettino della Società Entomologica Italiana 46: 134-200.

Giglio-Tos, E. 1919. Saggio di una nouva classificazione dei Mantidi. - Bullettino della Società Entomologica Italiana 49: 50-87. [Two dates (1917 and 1919) are mentioned on the title page. The true publication date of this publication is 1919 ("pubblicato il 31 Marzo 1919”). The issue was probably published two years later than planned because of the first World War.]

Giglio-Tos, E. 1921. Mantodea, Fam. Mantidae, Subfam. Eremiaphilinae. - In: Wytsman, P. (ed.): Genera Insectorum, vol. 177. - Brussels: Verteneuil \& Desmet. 36 pp.

Giglio-Tos, E. 1927. Orthoptera Mantidae. - In: Schulze, F.E. \& Kükenthal, W. (eds): Das Tierreich, eine Zusammenstellung und Kennzeichnung der rezenten Tierformen. Fascicle 50. - Berlin \& Leipzig, de Gruyter \& Co. 707 pp.

Gillon, Y. \& Roy, R. 1968. Les mantes de Lamto et des savanes de Côte d'Ivoire. - Bulletin de l'Institute Français d'Afrique Noire, (A) 30(3): 1038-1151.

Gillon, Y. 1983. The invertebrates of the grass layer. - Pp. 289-311 in: Ecosystems of the World (13): Tropical Savannahs. - Amsterdam, Oxford, New York: Elsevier. 730 pp.

Gorb, S.N. \& Beutel, R.G. 2001. Evolution of locomotory attachment pads of hexapods. - Naturwissenschaften 88: 530-534.

Gorochov, A.V. 2006. New and little known Orthopteroid insects (Polyneoptera) from fossil resins: Communication 1. - Paleontological Journal 40(6): 646-654.

Grandcolas, P. \& D‘Haese, C. 2001. The phylogeny of cockroach families: Is the current molecular hypothesis robust? - Cladistics 17: 48-55.

Gratshev, V.G. \& Zherikhin, V.V. 1993. New Fossil Mantids (Insecta: Mantida [sic]). - Paleontological Journal 27(1A): 148-165.

Gray, P.T.A. \& Mill, P.J. 1985. The musculature of the prothoracic legs and its innervation in Hierodula membranacea (Mantidea). - Philosophical Transactions of the Royal Society B. 309: 479-503.

Greven, H. \& Brenner, M. 2009. Zur Morphologie und zum Verhalten der „Wandelnden Geige“ Gongylus gongylodes (Linné, 1758) (Mantodea, Empusidae). - Entomologie heute 21: 63-76.

Grimaldi, D.A. 1990. Insects from the Santana Formation, Lower Cretaceous, of Brazil. - Bulletin of the American Museum of Natural History 195: 1-191.

Grimaldi, D.A. 1997. A fossil mantis (Insecta: Mantodea) in Cretaceous amber of New Jersey, with comments on the early history of the Dictyoptera. - American Museum Novitates 3204: 1-11.

Grimaldi, D.A. 2003. A revision of Cretaceous mantises and their relationships, including new taxa (Insecta: Dictyoptera: Mantodea). - American Museum Novitates 3412: 1-47. 
Grimaldi, D.A. 2008. 11.7 Mantodea: praying mantises. - Pp. 234-238 in: Martill, D.M., Bechly, G. \& Loveridge, R.F. (eds): The Crato Fossil Beds of Brazil. - New York: Cambridge University Press. 624 pp.

Grimaldi, D.A. \& Engel, M.S. 2005. Evolution of the insects. - New York: Cambridge University Press. 772 pp.

Grimaldi, D.A. \& Ross, A.J. 2004. Raphidiomimula, an enigmatic new cockroach in cretaceous amber from Myanmar (Burma) (Insecta: Blattodea: Raphidiomimidae). - Journal of Systematic Paleontology 2(2): 101-104.

Groll, E.K. \& Günther, K.K. 2003. 17. Ordnung Saltatoria (Orthoptera), Heuschrecken, Springschrecken. - Pp. 261-290 in: Dathe, H.H. (ed.): Lehrbuch der Speziellen Zoologie, Band I: Wirbellose Tiere, 5. Teil: Insecta. - Heidelberg, Berlin: Spektrum. 961 pp.

Guerra, P.A. \& Pollack, G.S. 2009. Flight behaviour attenuates the trade-off between flight capability and reproduction in a wing polymorphic cricket. - Biology Letters 5: 229-231.

Gurney, A.B. 1951. Praying mantids of the United States, native and introduced. - Annual Report of the Board of the Regents of the Smithsonian Institution 1950: 339-362.

Guthrie, D.M. \& Tindall, A.R. 1968. The biology of the cockroach. - London: Arnold. 408 pp.

Haan, W. de 1842. Bijdragen tot de Kennis der Orthoptera. - Pp. 45-248 in: Temminck, C.J. (ed.) 1839-1844: Verhandelingen over de natuurlijke Geschiedents der Nederlandsche ozeesche bezittingen, door de Leden der Natuurkundige commissie in Indië en andere Schrijvers. Zoologie, vol. 2. - Leiden: Luchtmans S, Luchtmans J. \& van der Hoek, C.C.

Haas, F. 2003. 12. Ordnung Dermaptera, Ohrwürmer. - Pp. 173-180 in: Dathe, H.H. (ed.): Lehrbuch der Speziellen Zoologie, Band I: Wirbellose Tiere, 5. Teil: Insecta. - Heidelberg, Berlin: Spektrum. 961 pp.

Haas, F. \& Gorb, S. 2004. Evolution of locomotory attachment pads in the Dermaptera (Insecta). - Arthropod Structure \& Development 33: 45-66.

Haas, F. \& Kukalová-Peck, J. 2001. Dermaptera hindwing structure and folding: New evidence for familial, ordinal and superordinal relationships within Neoptera (Insecta). - European Journal of Entomology 98: 445-509.

Handlirsch, A. 1903a. Vorläufige Mitteilung über die Phylogenie der Insekten. - Anzeiger der Kaiserlichen Akademie der Wissenschaften. Mathematisch-naturwissenschaftliche Klasse 40(22): 256-259.

Handlirsch, A. 1903b. Zur Phylogenie der Hexapoden (vorläufige Mitteilung). - Sitzungsberichte der Kaiserlichen Akademie der Wissenschaften 112: 716-738.

Handlirsch, A. 1906-1908. Die fossilen Insekten und die Phylogenie der rezenten Formen. Ein Handbuch für Paläontologen und Zoologen. - Leipzig: Wilhelm Engelmann. 1430 pp.

Handlirsch, A. 1925. Ordnung: Mantodea (Fangheuschrecken, Gottesanbeterinnen). - Pp. 493-502 in: Schröder (ed.): Handbuch der Entomologie, vol. 3. - Jena: Gustav Fischer. 1201 pp.

Handlirsch, A. 1930a. 10. Ordnung der Pterygogenea: Mantodea oder Fangheuschrecken. - Pp. 803-819 in: Krumbach, T. (ed.): Handbuch der Zoologie, vol. 4(1): Progoneata, Chilopoda, Insecta 1. - Berlin \& Leipzig: Walter de Gruyter. 892 pp.

Handlirsch 1930b: 11. Ordnung der Pterygogenea: Blattariae oder Schaben. - Pp. 820-839 in: Krumbach, T. (ed.): Handbuch der Zoologie, vol. 4(1): Progoneata, Chilopoda, Insecta 1. - Berlin \& Leipzig: Walter de Gruyter. $892 \mathrm{pp}$.

Handlirsch, A. 1937. Neue Untersuchungen über die fossilen Insekten mit Ergänzungen und Nachträgen sowie Ausblicken auf phylogenetische, palaeogeographische und allgemein biologische Probleme. 1. Teil. - Annalen des Naturhistorischen Museums in Wien 48: 1-140.

Handlirsch, A. 1938. Neue Untersuchungen über die fossilen Insekten mit Ergänzungen und Nachträgen sowie Ausblicken auf phylogenetische, palaeogeographische und allgemein biologische Probleme. 2. Teil. - Annalen des Naturhistorischen Museums in Wien 49: 1-240.

Hanitsch, R. 1923. On a collection of Blattidae from the Buitenzorg Museum. - Treubia 3(2): 197-221.

Harris, A.C. 2007. Living Statilia maculata Thunberg (Insecta: Mantodea: Mantidae) and other invertebrates, frequently imported into Dunedin on used cars. - Weta 33: 17-19.

Harz, K. 1980. Ein neuer Nachweis von Mantis religiosa L. im Pliozän von Willershausen. - Articulata 1(16): 168. 
Hebard, M. 1920. Studies in Malayan, Papuan, and Australian Mantidae. - Proceedings of the Academy of Natural Sciences of Philadelphia 72(1): 14-82.

Hebard, M. 1923. Studies in the Mantidae and Phasmidae of Panama (Orthoptera). - Transactions of the American Entomological Society 48: 327-362.

Hebard, M. 1935. Studies in the Orthoptera of Arizona. Part 1. New genera, species and geographic races. - Transactions of the American Entomological Society, Philadelphia 61: 111-153.

Helmkampf, M.E., Schwarz, C.J. \& Beck, J. 2007. A first look at the biodiversity of praying mantids (Insecta: Mantodea) in Sabah, Borneo. - Sepilok Bulletin 7: 1-13.

Hennig, W. 1950. Grundzüge einer Theorie der phylogenetischen Systematik. - Berlin: Deutscher Zentralverlag. 370 pp.

Hennig, W. 1965. Phylogenetic systematics. - Annual Review of Entomology 10: 97-116.

Hennig, W. 1966. Einige Bemerkungen über die Typen der von Giebel 1862 angeblich aus dem Bernstein beschriebenen Insektenarten. - Stuttgarter Beiträge zur Naturkunde 162: 1-7.

Hennig, W. 1969. Die Stammesgeschichte der Insekten. - Frankfurt: Kramer. 436 pp.

Hennig, W. 1981. Insect Phylogeny. - Chichester, New York, Brisbane, Toronto: Wiley \& Sons. 514 pp.

Henry, G.M. 1932. Observations on some Ceylonese Mantidae with descriptions of new species. - Spolia Zeylanica 17(1): 1-18.

Heßler, C., Bischoff, I. \& Bischoff, R. 2008. Praxisratgeber: Mantiden, faszinierende Lauerjäger. $2^{\text {nd }}$ edn. - Frankfurt: Chimaira. 207 pp.

Hevers, J. \& Liske, E. 1991. Lauernde Gefahr. Das Leben der Gottesanbeterinnen. Begleitheft zur Ausstellung vom 12. Mai bis 22. September 1991. - Braunschweig: Staatliches Naturhistorisches Museum. 67 pp.

Hill, P.J.B., Holwell, G.I., Göth, A. \& Herberstein, M.E. 2004. Preference for habitats with low structural complexity in the praying mantid Ciulfina sp. (Mantidae). - Acta Oecologica 26: 1-7.

Hill, S.A. 2007. Sound generation in Mantis religiosa (Mantodea: Mantidae): Stridulatory structures and acoustic signal. - Journal of Orthoptera Research 16(1): 35-49.

Hingston, R.W.G. 1932. A naturalist in the Guiana forest. - New York: Longmans, Green \& Co.; London: Arnold \& Co. 384 pp.

Holway, R.T. 1935. Preliminary note on the structure of the pretarsus and its possible phylogenetic significance. - Psyche 42(1): 1-24.

Holwell, G.I. 2007. Spermatophore feeding and mating behaviour in praying mantids (Mantodea: Liturgusidae). Journal of Zoology 271: 255-260.

Holwell, G.I. 2008. Geographic variation in genital morphology of Ciulfina praying mantids. - Journal of Zoology 276: $108-114$.

Holwell, G.I., Ginn, S.G. \& Herberstein, M.E. 2007a. Three new species of Ciulfina Giglio-Tos (Mantodea: Liturgusidae) from north-eastern Australia. - Zootaxa 1583: 23-35.

Holwell, G.I., Barry, K.L. \& Herberstein, M.E. 2007b. Mate location, antennal morphology, and ecology in two praying mantids (Insecta: Mantodea). - Biological Journal of the Linnean Society 91: 307-313.

Hubbell, T.H. 1925. Distributional notes on North American Orthoptera. I. - Florida Entomologist 9(3): 41-45.

Hurd, L.E., Prete, F.R., Jones, T.H., Singh, T.B., Co, J.E. \& Portman, R.T. 2004. First identification of a putative sex pheromone in a praying mantid. - Journal of Chemical Ecology 30(1): 155-166.

Innes Bey, W. 1909. Considérations sur certains cas de mimétisme observés dans la faune entomologique du désert. - Bulletin de la Société Entomologique d'Ègypte 1: 33-40.

Innes Bey, W. 1912. Révision des orthoptères de l'Égypte. - Mémoires de la Société Entomologique d'Ègypte 1(3): 3-78.

Inward, D., Beccaloni, G. \& Eggleton, P. 2007. Death of an order: A comprehensive molecular phylogenetic study confirms that termites are eusocial cockroaches. - Biology Letters 3: 331-335.

Ippolito, S. \& Lombardo, F. 2004. Decimiana clavata, new species from Brazil (Mantidae). - Memorie della Societa Entomologica Italiana 82(2): 373-378. 
Ippolito, S. 2007. A new species of Acanthops Audinette-Serville [sic!], 1831 from Ecuador (Mantodea, Acanthopinae). - Boletín de la Sociedad Entomológica Aragonesa 40: 183-185.

Jackson, J.F. \& Drummond, B.A. 1974. A Batesian ant-mimicry complex from the Mountain Pine Ridge of British Honduras, with an example of transformational mimicry. - American Midland Naturalist 91(1): 248-251.

James, H.G. 1943. Observations on the biology of Mantis religiosa L. - Annual Report of the Entomological Society of Ontario 75: 35-37.

Jantsch, L.J. 1999. Estudos filogenéticos em mantódeos americanos (Insecta: Pterygota: Mantodea). - Rio Grande do Sul: Pontificia Universidade Catolica do (doctoral thesis). $137 \mathrm{pp}$.

Jarvis, K.J., Haas, F. \& Whiting, M.F. 2005. Phylogeny of earwigs (Insecta: Dermaptera) based on molecular and morphological evidence: Reconsidering the classification of Dermaptera. - Systematic Entomology 30: 442-453.

Johnson, C.G. 1969. Migration and dispersal of insects by flight. - London: Methuen \& Co. Ltd. 763 pp.

Jones, G. \& Teeling, E.C. 2006. The evolution of echolocation in bats. - Trends in Ecology and Evolution 21(3): $149-156$.

Kaltenbach, A.P. 1963. Kritische Untersuchungen zur Systematik, Biologie und Verbreitung der europäischen Fangheuschrecken (Dictyoptera - Mantidae). - Zoologische Jahrbücher Systematik 90: 521-598.

Kaltenbach, A. 1964. Orthogenese und geographische Verbreitung bei westpaläarktischen Gottesanbeterinnen (Fam. Mantidae) und Laubheuschrecken (Fam. Tettigoniidae). - Verhandlungen der Zoologisch-Botanischen Gesellschaft in Wien 103/104: 62-81.

Kaltenbach, A.P. 1965. Dictyoptera und Orthopteroidea von Nordost-Griechenland und der Insel Thasos. - Annalen des Naturhistorischen Museums in Wien 68: 465-484.

Kaltenbach, A.P. 1967. Mantodea und Saltatoria aus Griechenland. - Annalen des Naturhistorischen Museums in Wien 70: 183-199.

Kaltenbach, A. 1976. Ordnung/Order Mantodea. - Pp. 129-169 in: Harz, K. \& Kaltenbach, A.: Die Orthopteren Europas vol. 3/The Orthoptera of Europe vol. 3. - Den Haag: Junk. 434 pp.

Kaltenbach, A.P. 1979. Die Mantodea der Kanarischen Inseln. Kritische Übersicht und ergänzende Beschreibungen. - Annalen des Naturhistorischen Museums in Wien 82: 517-531.

Kaltenbach, A. 1980. Ergebnisse einiger Sammelreisen nach Vorderasien. 7. Mantodea und Saltatoria. - Annalen des Naturhistorischen Museums in Wien 83: 575-584.

Kaltenbach, A.P. 1982. Insects of Saudi Arabia. Mantodea. - Fauna of Saudi Arabia 4: 29-72.

Kaltenbach, A.P. 1984. Insects of Saudi Arabia. New species and further records of Mantodea from Saudi Arabia and Oman. - Fauna of Saudi Arabia 6: 203-209.

Kaltenbach, A. 1991. A further contribution to the knowledge of the Mantodea of the Arabian Peninsula. - Fauna of Saudi Arabia 12: 246-255.

Kaltenbach, A.P. 1996. Unterlagen für eine Monographie der Mantodea des südlichen Afrika: 1. Artenbestand, geographische Verbreitung und Ausbreitungsgrenzen (Insecta: Mantodea). - Annalen des Naturhistorischen Museums in Wien (B) 98: 193-346.

Kaltenbach, A.P. 1998. Unterlagen für eine Monographie der Mantodea (Insecta) des südlichen Afrika: 2. Bestimmungstabellen für die höheren Taxa, Nachträge zum Artenbestand. - Annalen des Naturhistorischen Museums in Wien (B) 100: 19-59.

Kambhampati, S. 1995. A phylogeny of cockroaches and related insects based on DNA sequence of mitochondrial ribosomal RNA genes. - Proceedings of the National Academy of Sciences 92: 2017-2020.

Karny, H. 1921. Zur Systematik der orthopteroiden Insekten. - Treubia 1(3): 163-269.

Karuppanan, U. 1987. The role of cerci and ovipositors in the female mantid Euantissa pulchra (Fabricius) (Dictyoptera: Mantidae). - Madras Agricultural Journal 74(12): 504-506.

Karuppanan, U. 1998. Growth rate of the cerci in the nymphal and adult stages of a mantid Euantissa pulchra (Dictyoptera: Mantidae). - Environment \& Ecology 16(4): 891-893.

Kathirithamby, J. 1989. Review of the order Strepsiptera. - Systematic Entomology 14: 41-92. 
Kathirithamby, J. 1991. Strepsiptera. Pp. 684-695 in: CSIRO (eds): The insects of Australia. A textbook for students and research workers, vol. 2. - Carlton: Melbourne University Press. 1137 pp.

Kershaw, J.C. 1910. The formation of the ootheca of a Chinese mantis, Hierodula saussurii. - Psyche 17: 136-141.

Kjer, K.M. 2004. Aligned 18S and insect phylogeny. - Systematic Biology 53(3): 506-514.

Kjer, K.M., Carle, F.L., Litman, J. \& Ware, J. 2006. A molecular phylogeny of Hexapoda. - Arthropod Systematics \& Phylogeny 64(1): 35-44.

Klass, K.-D. 1995. Die Phylogenie der Dictyoptera. (Doctoral thesis, University of Munich). - Göttingen: Cuvillier. $256 \mathrm{pp}$.

Klass, K.-D. 1997. The external male genitalia and the phylogeny of Blattaria and Mantodea. - Bonner Zoologische Monographien 42: 1-341.

Klass, K.-D. 1998a. The Ovipositor of Dictyoptera (Insecta): Homology and Ground-Plan of the Main Elements. - Zoologischer Anzeiger 236: 69-101.

Klass, K.-D. 1998b. The Proventriculus of the Dicondylia, with Comments on Evolution and Phylogeny in Dictyoptera and Odonata (Insecta). - Zoologischer Anzeiger 237(1): 15-42.

Klass, K.-D. 1999. The pregenital abdomen of a mantid and a cockroach: Musculature and nerve topography, with comparative remarks on other Neoptera (Insecta: Dictyoptera). - Deutsche Entomologische Zeitschrift 1: $3-42$.

Klass, K.-D. 2003a. Relationships among the principal lineages of Dictyoptera inferred from morphological data. - Pp. 134-137 in: Klass, K.-D. (ed.): Proceedings of the 1st Dresden Meeting on Insect Phylogeny: „Phylogenetic Relationships within the Insect Orders“ (Dresden, September 19-21, 2003). - Entomologische Abhandlungen 61(2): 119-172.

Klass, K.-D. 2003b. The female genitalic region in basal earwigs (Insecta: Dermaptera: Pygidicranidae s.1.). - Entomologische Abhandlungen 61(2): 173-225.

Klass, K.-D. 2007. Die Stammesgeschichte der Hexapoden: Eine kritische Diskussion neuerer Daten und Hypothesen. - Denisia 20: 413-450.

Klass, K.-D. 2009. A critical review of current data and hypotheses on hexapod phylogeny. - Proceedings of theArthropodan Embryological Society of Japan 43: 3-22.

Klass, K.-D. \& Ehrmann, R. 2003. 13. Ordnung Mantodea, Fangschrecken, Gottesanbeterinnen. - Pp. 182-197 in: Dathe, H.H. (ed.): Lehrbuch der Speziellen Zoologie, Band I: Wirbellose Tiere, 5. Teil: Insecta. - Heidelberg, Berlin: Spektrum. 961 pp.

Klass, K.-D. \& Eulitz, U. 2007. The tentorium and anterior head sulci in Dictyoptera and Mantophasmatodea. Zoologischer Anzeiger 246: 205-234.

Klass, K.-D., Eulitz, U., Schmidt, C. \& Barton, A. 2009. The tibiotarsal articulation and the intertibiotarsal leg sclerite in Dictyoptera (Insecta). - Insect Systematics \& Evolution 40: 361-387.

Klass, K.-D. \& Meier, R. 2006. A phylogenetic analysis of Dictyoptera (Insecta) based on morphological characters. - Entomologische Abhandlungen 63(1-2): 3-50.

Klass, K.-D., Nalepa, C. \& Lo, N. 2008. Wood-feeding cockroaches as models for termite evolution (Insecta: Dictyoptera): Cryptocercus vs. Parasphaeria boleiriana. - Molecular Phylogenetics and Evolution 46: 809-817.

Klebs, R. 1910. Über Bernsteineinschlüsse im allgemeinen und die Coleopteren meiner Bernsteinsammlung. Schriften der Physikalisch-Ökonomischen Gesellschaft zu Königsberg in Preußen 51: 217-242.

Köchy, K. 1991. Untersuchungen zum Abwehrverhalten von Gottesanbeterinnen (Insecta, Mantodea) unter besonderer Berücksichtigung der Postembryonalentwicklung. (Doctoral thesis, University of Braunschweig). Braunschweig: Schmidt. 193 pp.

Kral, K. 1999. 7. Binocular vision and distance estimation. - Pp. 114-140 in: Prete, F.R., Wells, H., Wells, P.H. \& Hurd, L.E. (eds): The praying mantids. - Baltimore, London: Johns Hopkins. 362 pp. 
Kral, K. \& Prete, F.R. 2004. 3. In the mind of a hunter: The visual world of the praying mantis. - Pp. 75-115 in: Prete, F.R. (ed.) 2004: Complex worlds from simpler nervous systems. - Cambridge, London: MIT Press. 436 pp.

Kristensen, N.P. 1975. The Phylogeny of Hexapod “Orders". A Critical Review of Recent Accounts. - Zeitschrift für Zoologische Systematik und Evolutionsforschung/Journal of Zoological Systematics and Evolutionary Research 13: 1-44.

Kristensen, N.P. 1981. Phylogeny of insect orders. - Annual Review of Entomology 26: 135-157.

Kristensen, N.P. 1991. Phylogeny of extant hexapods. - Pp. 125-140 in: CSIRO (eds): The Insects of Australia. A textbook for students and research workers, vol. 1. - Carlton: Melbourne University Press. 1137 pp.

Kristensen, N.P. 1995. Forty years' insect phylogenetic systematics. - Zoologische Beiträge N.F. 36(1): 83-124.

Kristensen, N.P. 1998. The groundplan and basal diversification of the hexapods. - Pp. 283-293 in: Fortey, R.A. \& Thomas, R.H. (eds): Arthropod relationships. - London: Chapman \& Hall. 383 pp.

Kukalová-Peck, J. \& Peck, S.B. 1993. Zoraptera wing structures: Evidence for new genera and relationship with the blattoid orders (Insecta: Blattoneoptera). - Systematic Entomology 18: 333-350.

La Greca, M. 1939a. Su di alcuni mantidi africani. - Bolletino del Laboratorio di Zoologia generale e agraria della Facoltà Agraria in Portici 31: 218-221.

La Greca, M. 1939b. Orthoptera Mantidae. Missione Biologica nel paese dei Borana. Raccolte zoologiche. - Reale Accademia d'Italia 17: 257-266.

La Greca, M. 1966. Su due specie di Mantodei dell'Australia. - Annuario dell'Istituto e Museo di Zoologia della Università di Napoli 17(4): 1-8.

La Greca, M. 1977. Rivetinula n. gen. di Mantodei dell'India, per Rivetina fraterna (Sauss.). - Animalia 4(1/2): 23-33.

La Greca, M. \& Lombardo, F. 1997. A new species of Pseudacanthops Saussure, 1870 from Bolivia (Insecta, Mantodea). - Tropical Zoology 10: 49-55.

La Greca, M. \& Rainone, A. 1949. Il dermascheletro e la muscolatura del'addome di Mantis religiosa. - Annuario dell'Istituto e Museo di Zoologia della Università di Napoli 1(5): 1-43.

La Greca, M. \& Raucci, A. 1949. Il dermascheletro e la muscolatura del torace di Mantis religiosa. - Annuario dell'Istituto e Museo di Zoologia della Università di Napoli 1(3): 1-41.

Lefèbvre, M.A. 1835. Nouveau groupe d'Orthoptères de la famille des mantides. - Annales de la Société Entomologique de France 1(4): 449-508.

Levereault, P. 1936. The morphology of the Carolina mantis. - University of Kansas Science Bulletin 24(13): 205-259.

Levereault, P. 1938. The morphology of the Carolina mantis. Section 2: The musculature. - University of Kansas Science Bulletin 25(21): 577-633.

Liang, J.-H., Vršanský, P., Ren, D. \& Shih, C. 2009. A new Jurassic carnivorous cockroach (Insecta, Blattaria, Raphidiomimidae) from the Inner Mongolia in China. - Zootaxa 1974: 17-30.

Lieftinck, M.A. 1953. Biological and ecological observations on a bark hunting mantid in Java (Orthopt., Mantoidea). - Transactions of the Ninth International Congress of Entomology 2: 125-134.

Lizhong, H. 1984. A new species of the genus Kishinouyeum (Mantodea: Mantidae: Vatinae). - Entomotaxonomia 6(1): 29-30.

Lo, N., Tokuda, G. Watanabe, H. Rose, H. Slaytor, M., Maekawa, K., Bandi, C. \& Noda, H. 2000. Evidence from multiple gene sequences indicates that termites evolved from wood-feeding cockroaches. - Current Biology 10: 801-804.

Lo, N., Beninati, T., Stone, F., Walker, J. \& Sacchi, L. 2007. Cockroaches that lack Blattabacterium endosymbionts: The phylogenetically divergent genus Nocticola. - Biology Letters 3: 327-330.

Lombardo, F. 1988. I. Contributo allo studio dei Mantodei della Somalia. - Animalia 15(1/3): 91-112. 
Lombardo, F. 1995a. Parahestiasula obscura, gen. nov., spec. nov. from Nepal (Insecta, Mantodea, Hymenopodidae). - Spixiana 18(1): 11-14.

Lombardo, F. 1995b. A review of the genus Popa Stål 1865 (Insecta Mantodea). - Tropical Zoology 8: 257-267.

Lombardo, F. 1996. Neotropical mantids. IV. The genera Tithrone Stål and Paratithrone n. gen. (Mantoidea: Mantidae: Acontiothespini). - Journal of Orthoptera Research 5: 45-50.

Lombardo, F. 1997. New and little known Mantodea from Eastern and Central Southern Africa. - Journal of Orthoptera Research 6: 69-81.

Lombardo, F. 2000a. Stenophylla lobivertex, a new species of Stenophyllinae from Amazonia (Insecta, Mantodea). - Studies on Neotropical Fauna and Environment 35: 34-37.

Lombardo, F. 2000b. A review of the genus Decimiana Uvarov, 1940 (Insecta: Mantodea), with description of a new species. - Proceedings of the Academy of Natural Sciences of Philadelphia 150: 159-171.

Lombardo, F. \& Agabiti, B. 2001. The mantids from Ecuador, with some biogeographic considerations. - Journal of Orthoptera Research 10(1): 89-104.

Lombardo, F. \& Ayala, M. 1999. The genus Calopteromantis Terra, 1982 (Insecta, Mantodea) and description of a new species. - Bollettino dell'Accademia Gioenia di Scienze Naturali 31(354): 107-117.

Lombardo, F. \& Ippolito, S. 2004. Revision of the species of Acanthops Serville, 1831 (Mantodea, Mantidae, Acanthopinae) with comments on their phylogeny. - Annals of the Entomological Society of America 97(6): 1076-1102.

Lombardo, F. \& Marletta, A. 2004. Remarks on the genus Raptrix Terra 1995 (Mantodea, Acontithespinae) with a description of two new species. - Journal of Orthoptera Research 13(1): 21-33.

Lombardo, F. \& Perez-Gelabert, D.E. 2004. The mantids of Hispaniola, with the description of two new species (Mantodea). - Boletin Sociedad Entomológica Aragonesa 34: 35-48.

Lowman, M.D. \& Rinker, H.B. 2004. Forest Canopies. - Burlington, London: Elsevier. 517 pp.

Loxton, R.G. \& Nicholls, I. 1979. The functional morphology of the praying mantis forelimb (Dictyoptera: Mantodea). - Zoological Journal of the Linnean Society 66: 185-203.

MacKinnon, J. 1970. Indications of territoriality in mantids. - Zeitschrift für Tierpsychologie 27: 150-155.

Maekawa, K., Kitade, O. \& Matsumoto, T. 1999. Molecular phylogeny of orthopteroid insects based on the mitochondrial Cytochrome Oxidase II gene. - Zoological Science 16: 175-184.

Maekawa, K. \& Matsumoto, T. 2000. Molecular phylogeny of cockroaches (Blattaria) based on mitochondrial COII gene sequences. - Systematic Entomology 25: 511-519.

Marcellini, D.L. \& Keefer, T.E. 1976. Analysis of the gliding behavior of Ptychozoon lionatum [sic!] (Reptilia: Gekkonidae). - Herpetologica 32(4): 362-366.

Marks, E.P. \& Lawson, F.A. 1962. A comparative study of the Dictyopteran ovipositor. - Journal of Morphology 111(2): 139-171.

Martin, J.F. 1916. The thoracic and cervical sclerites of insects. - Annals of the Entomological Society of America 9: $35-83$.

Martynov, A.B. 1925. Über zwei Grundtypen der Flügel bei den Insecten und ihre Evolution. - Zoomorphology 4(3): 465-501.

Matsuda, R. 1970. Morphology and evolution of the insect thorax. - Memoirs of the Entomological Society of Canada 76: 1-431.

Maxwell, M.R. 1999. 5. Mating behaviour. - Pp. 69-89 in: Prete, F. R., Wells, H., Wells, P. H. \& Hurd, L. E. (eds): The praying mantids. - Baltimore: John Hopkins. 362 pp.

Meade-Waldo, G. 1910. Protective attitude of a mantis from Borneo. - Transactions of the Entomological Society of London: L-Lii (= 50-52).

Milledge, G.A. 1990. Revision of the genus Nesoxypilus Beier (Mantodea: Amorphoscelidae: Paraoxypilinae). Memoirs of the Museum of Victoria 50(2): 347-355.

Milledge, G.A. 1997. Revision of the tribe Archimantini (Mantodea: Mantidae: Mantinae). - Memoirs of the Museum of Victoria 56(1): 1-63. 
Milledge, G.A. 2005: Revision of the genera Sphodropoda, Trachymantis and Zopheromantis (Mantodea: Mantidae: Mantinae). - Records of the Australian Museum 57: 191-210.

Miller, L.A. \& Surlykke, A. 2001. How some insects detect and avoid being eaten by bats: Tactics and countertactics of prey and predator. - BioScience 51(7): 570-581.

Morales Agacino, E. 1947. Mántidos de la fauna Ibérica. - Boletín de Patología Vegetal y Entomología Agrícola Madrid 15: 131-164.

Müller, H. 2001. Gräber fürs Gelege. Die Gottesanbeterin Humbertiella ceylonica Saussure, 1869 (Insecta: Mantodea). - Reptilia 28: 38-41.

Mukherjee, T.K., Hazra, A.K. \& Ghosh, A.K. 1995. The mantid fauna of India (Insecta: Mantodea). - Oriental Insects 29: 185-358.

Nalepa, C.A. \& Lenz, M. 2000. The ootheca of Mastotermes darwiniensis Froggat (Isoptera: Mastotermitidae): Homology with cockroach oothecae. - Proceedings of the Royal Society of London 267: 1809-1813.

Nel, A. \& Roy, R. 1996. Revision of the fossil "mantid" and "ephemerid" species described by Piton from the Palaeocene of Menat (France) (Mantodea: Chaeteessidae, Mantidae; Ensifera: Tettigonioidea). - European Journal of Entomology 93: 223-234.

Nesbitt, H.H.J. 1941. A comparative morphological study of the nervous system of the Orthoptera and related orders. - Annals of the Entomological Society of America 34: 51-81.

Neumann, M. 2006. Die Gottesanbeterin Pseudocreobotra wahlbergii Stål, Beobachtungs- und Forschungsergebnisse bei Haltung und Zucht. - Veröffentlichungen des Museums für Naturkunde Chemnitz 29: 105-122.

Nickle, D.A. 1987. 13. Order Mantodea. - Pp. 140-142 in: Stehr, F.W. (ed.): Immature Insects. - Dubuque: Kendall/Hunt. $754 \mathrm{pp}$.

Nobel, P.S., Miller, P.M. \& Graham, E.A. 1992. Influence of rocks on soil temperature, soil water potential, and rooting patterns for desert succulents. - Oecologia 92: 90-96.

Novacek, M.J. 1985. Evidence for echolocation in the oldest known bats. - Nature 315: 140-141.

Olivier, M. 1792. Encyclopédie méthodique. Histoire naturelle. Insectes. Tome septième. - Paris: Panckoucke. $827 \mathrm{pp}$.

Orofino, F., Ippolito, S. \& Lombardo, F. 2006. Remarks on Phyllovates Kirby, 1904 of West South America and description of two new species (Insecta, Mantodea, Vatinae). - Transactions of the American Entomological Society, Philadelphia 132(1-2): 205-222.

Otte, D. \& Spearman, L. 2005. Mantida species file. Catalog of the mantids of the world. - Insect Diversity Association Publication 1: 1-489.

Pantel, J. 1896. Notes orthoptérologiques. Parts IV-V. - Anales de la Sociedad Español de la Historia Natural 5(25): 47-118.

Paulian, R. 1957. Faune de Madagascar. V: Insectes Mantodea. - Publications de l'Institut de Recherche Scientifique Tananarive - Tsimbazaza 5: 1-102.

Perez, B. 2005. Calling behaviour in the female praying mantis, Hierodula patellifera. - Physiological Entomology 30: 42-47.

Picker, M., Griffiths, C. \& Weaving, A. 2002. Field guide to insects of South Africa. - Cape Town: Struik (New Holland Publishers). 444 pp.

Pictet, A. 1888. Locustides nouveaux ou peu connus de Musée de Genève. - Mémoires de la Société de Physique et d'Histoire Naturelle de Genève 3(6): 1-84.

Piton, L. 1940. Paléontologie du gisement Éocène de Menat (Puy-de-Dôme) (flore et faune). - Clermont-Ferrand: Vallier. 306 pp.

Preston-Mafham, K. 1990. Grasshoppers and mantids of the world. - London: Blanford. 192 pp.

Prete, F.R. \& Hamilton, K. 1999. 10. Prey capture. - Pp. 194-223 in: Prete, F. R., Wells, H., Wells, P. H. \& Hurd, L. E. (eds): The praying mantids. - Baltimore \& London: John Hopkins. 362 pp.

Prete, F.R., Wells, H., Wells, P.H. \& Hurd, L.E. (eds.) 1999. The praying mantids. - Baltimore \& London: Johns Hopkins. 362 pp. 
Przibram, H. 1907. Aufzucht, Farbwechsel und Regeneration unsrer europäischen Gottesanbeterin (Mantis religiosa L.). - Archiv für Entwicklungsmechanik der Organismen: Organ für die gesamte kausale Morphologie 23(4): 600-613.

Przibram, H. 1930. Wachstumsmessungen an Sphodromantis bioculata Burm. IV. Zunahme der Facettengrösse und -anzahl. (Zugleich: Aufzucht der Gottesanbeterinnen. XII. Mitteilung). - Wilhelm Roux 'Archiv für Entwicklungsmechanik der Organismen 122(2): 280-299.

Przibram, H. \& Brecher, 1930. Wachstumsmessungen an Tenodera aridifolia Stoll., einer japanischen Fangheuschrecke. (Zugleich: Aufzucht der Gottesanbeterinnen. XI. Mitteilung). - Wilhelm Roux 'Archiv für Entwicklungsmechanik der Organismen 122(2): 251-279.

Przibram, H. \& Megušar, F. 1912. Wachstumsmessungen an Sphodromantis bioculata Burm. I. Länge und Masse. (Zugleich: Aufzucht der Gottesanbeterinnen, IV. Mitteilung). - Archiv für Entwicklungsmechanik der Organismen: Organ für die gesamte kausale Morphologie 34: 680-741.

Ragge, D.R. 1955. The wing-venation of the Orthoptera Saltatoria, with notes on dictyopteran wing-venation. London: Trustees of the British Museum of Natural History. $159 \mathrm{pp}$.

Rambur, P. 1842. Histoire Naturelle des Insectes. Névroptères. - Paris: Libraire Encyclopédique de Roret. XVIII $(=18)+534 \mathrm{pp}$.

Ramsay, G.W. 1990. Mantodea (Insecta) with a review of aspects of functional morphology and biology. - Fauna of New Zealand 19: 1-96.

Ranade, S.P., Mukherjee, S. \& Ghate, H.V. 2004. A note on desert mantis Eremiaphila rotundipennis Kirby (Insecta: Mantodea: Eremiaphilidae) from Rajasthan, India. - Zoos’ Print Journal 19(11): 1694-1695.

Rasnitsyn, A.P. 2002. 2.2.2.0.1. Order Eoblattida Handlirsch, 1906 (= Cacurgida Handlirsch, 1906, = Protoblattodea Handlirsch, 1906). - Pp. 256-260 in: Rasnitsyn, A.P. \& Quicke, D.L.J. (eds): History of Insects. - Dordrecht, Boston, London: Kluwer. 544 pp.

Rau, P. \& Rau, N. 1913. The biology of Stagmomantis carolina. - Transactions of the Academy of Science of St. Louis 22: 1-58.

Rebeur-Paschwitz, E. von 1895. Canarische Insekten (Blepharis mendica und Hypsicorypha Juliae). - Berliner Entomologische Zeitschrift 40(2): 265-276.

Rehn, J.A.G. 1901. The Forficulidae, Blattidae, Mantidae and Phasmidae collected in northeastern Africa by Dr. A. Donaldson Smith. - Proceedings of the Academy of Natural Sciences of Philadelphia 53(2): 273-288.

Rehn, J.A.G. 1907. Notes on the Orthoptera from southern Arizona, with descriptions of new species. - Proceedings of the Academy of Natural Sciences of Philadelphia 59(1): 24-81.

Rehn, J.A.G. 1911. Mantodea, Fam. Mantidae, Subfam. Vatinae. - In: Wytsman, P. (ed.): Genera Insectorum, vol. 119. - Brussels: Verteneuil \& Desmet. 28 pp.

Rehn, J.A.G. 1912. On some African Mantidae and Phasmidae in the collection of the Deutschen Entomologischen Museum. - Archiv für Naturgeschichte 78(6): 106-126.

Rehn, J.A.G. 1913. A contribution to the knowledge of the Orthoptera of Argentina. - Proceedings of the Academy of Natural Sciences of Philadelphia 65(2): 273-379.

Rehn, J.A.G. 1915. A further contribution to the knowledge of the Orthoptera of Argentina. - Proceedings of the Academy of Natural Sciences of Philadelphia 67: 270-292.

Rehn, J.A.G. 1918. Descriptions of one new genus and fifteen new species of Tropical American Orthoptera. Transactions of the American Entomological Society (Philadelphia) 44(4): 321-371.

Rehn, J.A.G. 1935. The Orthoptera of Costa Rica, part I. Mantidae. - Proceedings of the Academy of Natural Sciences of Philadelphia 87: 167-273.

Rehn, J.A.G. 1949. Descriptions of new, and critical notes on previously known, species of African Manteidae (Orthoptera). - Transactions of the American Entomological Society (Philadelphia) 74: 187-224.

Rehn, J.A.G. \& Hebard, M. 1908. An orthopterological reconnoissance of the southwestern United States. Part I: Arizona. - Proceedings of the Academy of Natural Sciences of Philadelphia 60: 365-402. 
Rehn, J.A.G. \& Hebard, M. 1909a. An orthopterological reconnoissance of the southwestern United States. Part II: New Mexico and Western Texas. - Proceedings of the Academy of Natural Sciences of Philadelphia 61: 111-175.

Rehn, J.A.G. \& Hebard, M. 1909b. An orthopterological reconnoissance of the southwestern United States. Part III: California and Nevada. - Proceedings of the Academy of Natural Sciences of Philadelphia 61: 409-483.

Rentz, D.C.F. 1991. Orthoptera (Grasshoppers, locusts, katydids, crickets). - Pp. 369-393 in: CSIRO (eds): The insects of Australia. A textbook for students and research workers, vol. 1. - Carlton: Melbourne University Press. 1137 pp.

Rentz, D.C.F. 1996. Grasshopper Country. The abundant orthopteroid insects of Australia. - Sydney: New South Wales University Press. 284 pp.

Rivera, J.M. 2001. Sobre la identidad de Vates peruviana Rehn, 1911 (Mantodea: Vatidae). - Revista Peruana de Entomologia 42: 93-96.

Rivera, J.M. 2005. A new species of Acanthopinae from Peru: Miracanthops eseejja n. sp. and notes in Acanthops occidentalis Lombardo \& Ippolito, 2004 (Mantodea: Acanthopidae). - Journal of Orthoptera Research 14(2): 255-260.

Roberts, R.A. 1937a. Biology of the bordered mantid, Stagmomantis limbata Hahn (Orthoptera, Mantidae). - Annals of the Entomological Society of America 30: 96-109.

Roberts, R.A. 1937b. Biology of the minor mantid, Litaneutria minor Scudder (Orthoptera, Mantidae). - Annals of the Entomological Society of America 30: 111-121.

Robinson, M.H. 1968. The defensive behaviour of the Javanese stick insect, Orxines macklotti De Haan, with a note on the startle display of Metriotes diocles Westw. (Phasmatodea, Phasmidae). - Entomologist's Monthly Magazine 104: 46-54.

Robinson, M.H. 1969. The defensive behaviour of some orthopteroid insects from Panama. - Transactions of the Royal Entomological Society of London 121(7): 281-303.

Robinson, M.H. \& Robinson, B. 1979. By dawn's early light: Matutinal mating and sex attractants in a Neotropical mantid. - Science 205: 825-827.

Roff, D.A. 1990. The evolution of flightlessness in insects. - Ecological Monographs 60(4): 389-421.

Roth, L.M. 1991: Blattodea, Blattaria (cockroaches). - Pp. 320-329 in: CSIRO (eds): The insects of Australia. A textbook for students and research workers, vol. 1. - Carlton: Melbourne University Press. 1137 pp.

Roth, L.M. \& Naskrecki, P. 2003. A new genus and species of cave cockroach (Blaberidae: Oxyhaloinae) from Guinea, West Africa. - Journal of Orthoptera Research 12(2): 57-61.

Roy, R. 1961. Le Parc National du Niokolo-Koba. VI.: Dictyoptera Mantodea. - Mémoirs de 1'Institute Francais d'Afrique Noire 62(2): 91-103.

Roy, R. 1962. Notes sur le genre Amorphoscelis avec description de quatre espèces nouvelles (Dictyoptera Mantodea). - Bulletin de l'Institute Francais d'Afrique Noire, (A) 24(3): 677-709.

Roy, R. 1963a. Les Amorphoscelis (Mantodea) de l'Afrique du Sud. - Annals of the Transvaal Museum 24(4): 321-325.

Roy, R. 1963b. La reserve naturelle intégrale du Mont Nimba: V. Dictyoptera Mantodea ( $2^{\text {me }}$ note). - Mémoirs de l'Institute Francais d'Afrique Noire 66: 163-206.

Roy, R. 1964. Les Amorphoscelis du groupe d' annulipes (Mantodea Amorphoscelidae). - Bulletin de l'Institute Francais d'Afrique Noire, (A) 26(3): 720-734.

Roy, R. 1965a. Deux nouvelles espèces d'Amorphoscelis en Côte d' Ivoire. - Bulletin de l'Institute Francais d'Afrique Noire, (A) 27(4): 1250-1258.

Roy, R. 1965b: Contribution à la connaissance des Amorphoscelis de la région orientale (Mantodea Amorphoscelidae) - I. Les espèces indo-cingalaises. - Bulletin de la Société entomologique de France 70: 267-273.

Roy, R. 1966. Contribution à la connaissance des Amorphoscelis de la région orientale (Mantodea Amorphoscelidae) - II. Les espèces d'Extrême-Orient. - Bulletin de la Société entomologique de France 71: 261-273. 
Roy, R. 1969. Le Parc National du Niokolo-Koba. VII.: Dictyoptera Mantodea II. - Mémoirs de 1'Institute Francais d'Afrique Noire 84(3): 97-112.

Roy, R. 1970. Contribution à l'étude biologique du Sénégal septentrional. IV. Dictyoptères Mantodea. - Bulletin de 1'Institute Francais d'Afrique Noire, (A) 32(4): 1019-1033.

Roy, R. 1972. Deux nouvelles espèces de Tarachodinae au Sénégal (Mantodea). - Bulletin de l'Institute Francais d'Afrique Noire (A) 34(2): 323-333.

Roy, R. 1973. Premier inventaire des mantes du Gabon. - Biologia Gabonica 3-4: 235-290.

Roy, R. 1976. Mises au point sur le genre Heterochaeta Westwood (Mantodea). - Bulletin de l'Institute Francais d'Afrique Noire, (A) 38(1): 69-111.

Roy, R. 1984. Une nouvelle espèce d'Amorphoscelis, jusqu'à présent confondue avec A. laxeretis Karsch (Mantodea, Amorphoscelidae). - Revue française d'Entomologie (N.S.) 6(2): 75-78.

Roy, R. 1987a. General Observations on the Systematics of Mantodea. - Pp. 483-486 in: Baccetti, B. (ed.): Evolutionary biology of orthopteroid insects. - Chichester: Ellis Horwood. 612 pp.

Roy, R. 1987b. Overview of the biogeography of African mantides. - Pp. 489-495 in: Baccetti, B. (ed.): Evolutionary biology of orthopteroid insects. - Chichester: Ellis Horwood. 612 pp.

Roy, R. 1987c. Le peuplement en mantes des îles situées au large de l'Afrique. - Bulletin de la Société Zoologique de France 112(1-2): 117-125.

Roy, R. 1995. Contribution à la connaissance du genre Macracanthopus Uvarov et description d'une espèce nouvelle, M. seydeli (Mantodea, Mantidae, Amelinae). - Journal of African Zoology 109(3): 239-246.

Roy, R. 1996. Révision des Sibyllinae (Mantodea). - Bulletin du Muséum national d'Histoire naturelle 4(18) Sec A (1-2): 69-138.

Roy, R. 1999. 2. Morphology and taxonomy. - Pp. 19-40 in: Prete, F. R., Wells, H., Wells, P. H. \& Hurd, L. E. (eds): The praying mantids. - Baltimore \& London: John Hopkins. 362 pp.

Roy, R. 2001. Contribution à la connaissance des Angelinae de la région orientale: Les genres Euchomenella, Mythomantis et Tagalomantis (Dictyoptera, Mantidae). - Revue française d'Entomologie (N.S.) 23(1): 79-92.

Roy, R. 2002a. Comments à propos du genre Plesiacanthops Chopard, 1913, et redescription d'Acanthops tuberculata Saussure, 1870 (Dictyoptera, Mantodea). - Revue française d'Entomologie (N.S.) 24(4): 171-177.

Roy, R. 2002b. Une remarkable espèce nouvelle d'Acanthops Audinet-Serville, 1831, en Guyane française (Dictyoptera, Mantodea). - Bulletin de la Société entomologique de France 107(3): 297-300.

Roy, R. 2003a. Répartition, biologie et variabilité de Tarachodella monticola Giglio-Tos, 1917 (Dictyoptera, Mantodea, Tarachodidae). - Bulletin de la Société entomologique de France 108(5): 447-450.

Roy, R. 2003b. Alfredistia reticulata n. gen., n. sp. d'Afrique du Sud (Dictyoptera, Mantodea, Tarachodidae). Bulletin de la Société entomologique de France 108(4): 433-435.

Roy, R. 2004a. Réarrangements critiques dans la famille des Empusidae et relations phylogénétiques [Dictyoptera, Mantodea]. - Revue française d'Entomologie (N.S.) 26(1): 1-18.

Roy, R. 2004b. Révision et phylogénie des Choeradodini Kirby, 1904 (Dictyoptera, Mantidae). - Bulletin de la Société entomologique de France 109(2): 113-128.

Roy, R. 2004c. Lagrecacanthops et Miracanthops, deux nouveaux genres d'Acanthopinae (Dictyoptera, Mantodea, Acanthopidae). - Bulletin de la Société entomologique de France 109(5): 491-498.

Roy, R. 2004d. Acontista Saussure \& Zehntner, 1894, genre valide (Dict. Mantodea). - Bulletin de la Société entomologique de France 109(3): 231-235.

Roy, R. 2005a. Mises au point sur le genre Stenophylla Westwood (Dictyoptera, Acanthopidae). - Bulletin de la Société entomologique de France 110(3): 225-232.

Roy, R. 2005b. Une nouvelle espèce de Galepsus aux Comores (Dictyoptera, Tarachodidae). Bulletin de la Société entomologique de France 110(4/5): 383-386.

Roy, R. 2006. Vue d'ensemble sur les Acontistinae Giglio-Tos, 1919 (Dictyoptera, Acanthopidae). - Bulletin de la Société entomologique de France 111(3): 327-338. 
Roy, R. 2007. Une nouvelle espèce africaine d'Amorphoscelis Stål, 1871 (Dictyoptera Mantodea). - Bulletin de la Société entomologique de France 112(3): 387-388.

Roy, R. 2008a. Vue d'ensemble sur les Tarachodinae malgaches (Dictyoptera, Mantodea). - Bulletin de la Société entomologique de France 113(2): 183-186.

Roy, R. 2008b. Contribution à la connaissance du genre Parablepharis Saussure, 1870 (Mantodea). - Bulletin de la Société entomologique de France 113(1): 53-60.

Roy, R. 2008c. Indomenella, nouveau genre d'Angelinae (Dict. Mantidae). - Bulletin de la Société entomologique de France 113(3): 330.

Roy, R. 2009a. Révision des Toxoderini sensu novo (Mantodea, Toxoderindae). - Revue Suisse de Zoologie 116(1): 93-183.

Roy, R. 2009b. Complément de description pour Amorphoscelis asymmetrica Ingrisch, 1999 (Dict., Mantodea, Amorphoscelidae). - Bulletin de la Société entomologique de France 114(2): 209-210.

Roy, R. 2009c. Nouvelles données sur le genre Junodia Schulthess, 1899 (Mantodea, Hymenopodidae). - Bulletin de la Société entomologique de France 114(1): 119-127.

Roy, R. 2010. Contribution à la connaissance du genre néotropical Mantoida Newman, 1838 (Dict., Mantoididae). - Bulletin de la Société entomologique de France 115(1): 22.

Roy, R. \& Leston, D. 1975. Mantodea of Ghana: new species, further records and habitats. - Bulletin de l'Institute Francais d'Afrique Noire, (A) 37(2): 297-344.

Roy, R. \& Ehrmann, R. 2009. Révision du genre Zoolea Audinet-Serville (Mantodea, Mantidae, Vatinae). - Revue française d'Entomologie (N.S.) 31(1): 1-22.

Roy, R. \& Stiewe, M.B.D. 2009. Contribution to the knowledge of Eastern African Amorphoscelis Stål, 1871, with description of two new species (Dictyoptera, Mantodea, Amorphoscelidae). - Bulletin de la Société entomologique de France 114(2): 195-209.

Roy, R. \& Svenson, G.J. 2007. Revision of the genus Ceratomantis Wood-Mason, 1876 (Dictyoptera, Mantodea). - Bulletin de la Société entomologique de France 112(4): 433-444.

Salazar-E., J.A. 2003. On some mimetic resemblances of Acontista multicolor Saussure, 1870 in Colombia (Insecta: Mantodea). - Lambillionea 103(4): 649-654.

Salazar-E., J.A. 2004a. Epaphrodita Serville, 1831; Stenophylla Westwood, 1843 y Acanthops Serville, 1831, tres géneros de mántioda hoja-seca de la región neotropical (Insecta: Mantodea). - Boletín Cientifico del Museo de Historia Natural Universidad de Caldas 8: 211-221.

Salazar-E., J.A. 2004b. Nuevas especies de Blátidos y Mántidos para Colombia (Insecta: Dictyoptera) y una nota sobre la hembra de Vates festae Giglio-Tos, 1915. - Boletín Cientifico del Museo de Historia Natural Universidad de Caldas 8: 267-286.

Salazar-E., J.A. 2005. Notas sobre Metallyticus Westwood, 1837; Chaeteessa Burmeister, 1838 y Mantoida Newman, 1838. Tres géneros primitivos de mantidos tropicales (Dictyoptera: Mantodea). - Lambillionea 2: 265-276.

Salazar-E., J.A. 2007. Stenophylla lobivertex Lombardo, nuoevo registro de Mántido para la fauna Colombiana (Insecta: Mantodea). - Boletín Cientifico del Museo de Historia Natural Universidad de Caldas 11: 157161.

Salazar-E., J.A. \& Carrejo, N.S. 2002. Nueva especie de Pseudopogonogaster Beier, 1942 para la Cordillera Oriental de Colombia (Insecta: Mantodea). - Boletín Cientifico del Museo de Historia Natural Universidad de Caldas 6: 103-109.

Salt, R.W. \& James H.G. 1947. Low temperature as a factor in the mortality of eggs of Mantis religiosa L. - Canadian Entomologist 79: 33-36.

Saussure, H. de 1869. Essai d'un système des mantides. - Mitteilungen der Schweizerischen Entomologischen Gesellschaft 3(2): 49-73.

Saussure, H. de 1870a. IV. Mantides. - Pp. 149-362 in: Saussure, H. de (ed.): Mélanges orthoptérologiques vol. 1, fascicle III. - Geneva \& Basel: Georg. 
Saussure, H. de 1870b. Additions au système des mantides. - Mittheilungen der Schweizerischen entomologischen Gesellschaft 3(5): 221-244.

Saussure, H. de 1871. Mantides. - Pp. 363-460 in: Saussure, H. de (ed.): Mélanges orthoptérologiques vol. 1, supplement to fascicle III. - Geneva \& Basel: Georg.

Saussure, H. de 1872: I. Mantides. - Pp. 1-164 in: Saussure, H. de (ed.): Mélanges orthoptérologiques vol. 2, fascicle IV (Mantides et Blattides). - Geneva \& Basel: Georg.

Saussure, H. de \& Zehntner, L. 1894. Fam. Mantidae. - Pp. 123-197 in: Saussure, H. de, Zehntner, L. \& Pictet, A. 1893-1899: Biologia Centrali-Americana, Insecta. Orthoptera, vol. 1. - Paris: L'Imprimerie Nationale. 458 pp.

Saussure, H. de \& Zehntner, L. 1895. Histoire naturelle des Blattides et Mantides. - Pp. 1-244 in: Grandidier, A. (ed.): Histoire physique, naturelle et politique de Madagascar, vol. 23: Histoire naturelle des orthoptères. Paris: 1'Imprimerie Nationale. 244 pp.

Schafer, R. 1973. Postembryonic development in the antenna of the cockroach, Leucophaea maderae: Growth, regeneration, and the development of the adult pattern of sense organs. - Journal of Experimental Zoology 183: 353-364.

Schwarz, C.J. 2004. Popa spurca spurca Stål, 1856, eine bemerkenswerte Gottesanbeterin aus Westafrika (Mantodea: Mantidae: Vatinae). - Arthropoda 12(2): 12-20.

Schwarz, C.J., Mehl, J.E. \& Sommerhalder, J. 2006. Die Teufelsblume Idolomantis diabolica (Saussure, 1869), Teil 1. - Terraria 2: 63-70.

Schwarz, C.J., Mehl, J.E. \& Sommerhalder, J. 2007. Die Teufelsblume Idolomantis diabolica (Saussure, 1869), Teil 2. - Terraria 3: 62-69.

Seelinger, G. \& Seelinger, U. 1983. On the social organization, alarm and fighting in the primitive cockroach Cryptocercus punctulatus Scudder. - Zeitschrift für Tierpsychologie 61: 315-333.

Seifert, G. 1995. Entomologisches Praktikum. - Stuttgart, New York: Thieme. 322 pp.

Sereno, P.C. 2007. Logical basis for morphological characters in phylogenetics. - Cladistics 23: 1-23.

Sharov, A.G. 1962. Redescription of Lithophotina floccosa Cock. (Manteodea) with some notes on the manteod wing venation. - Psyche 69: 102-106.

Sharp, D. 1895. Chapter X. Orthoptera continued. Mantidae, soothsayers. - Pp. 242-259 in: Harmer, S.F. \& Shipley, A.E. (eds): The Cambridge Natural History, vol. 5. - London: MacMillan and Co. 584 pp.

Shelford, R.W.C. 1903. Bionomical notes on some Bornean Mantidae. - Zoologist 7: 293-304.

Shelford, R.W.C. 1916. A Naturalist in Borneo. - London: Fisher Unwin. 331 pp.

Simmons, N.B., Seymour, K.L., Habersetzer, J. \& Gunnell, G.F. 2008. Primitive Early Eocene bat from Wyoming and the evolution of flight and echolocation. - Nature 451: 818-822.

Sjöstedt, Y. 1918. Results of Dr. E. Mjöberg's Swedish Scientific Expeditions to Australia 1910-1913. 17: Mantidæ und Phasmidæ. - Arkiv för Zoologi 11(19): 1-61.

Sjöstedt, Y. 1930. Orthopterentypen im Naturhistorischen Reichsmuseum zu Stockholm. I. Mantidae. - Arkiv för Zoologi 21(32): 1-43.

Slifer, E.H. 1968. Sense organs on the antennal flagellum of a praying mantis, Tenodera angustipennis, and of two related species (Mantodea). - Journal of Morphology 124: 105-116.

Smart, J. 1951. The wing-venation of the American cockroach Periplaneta americana Linn. (Insecta: Blattidae). Proceedings of the Zoological Society of London 121: 501-509.

Smart, J. 1956. On the wing-venation of Chaeteessa and other mantids (Insecta: Mantodea). - Proceedings of the Zoological Society of London 127(1): 545-553.

Smith, S.A., Beaulieu, J.M. \& Donoghue, M.J. 2010. An uncorrelated relaxed-clock analysis suggests an earlier origin for flowering plants. - Proceedings of the National Acadademy of Sciences 107(13): 5897-5902.

Smithers, C.N. 1991: Zoraptera. - Pp. 410-411 in: CSIRO (eds): The insects of Australia. A textbook for students and research workers, vol. 1. - Carlton: Melbourne University Press. 1137 pp.

Snodgrass, R.E. 1935. Principles of insect morphology (1993 facsimile reprint). - Ithaca, London: Cornell. 667 pp. 
Stål, C. 1873. Recherches sur le système des mantides. - Bihang till Kongliga Svenska Vetenskaps-Akademiens Handlingar 1(10): 2-26.

Stål, C. 1877. Systema Mantodeorum. Essai d'une systématisation nouvelle des mantodées. - Bihang till Kongliga Svenska Vetenskaps-Akademiens Handlingar 4(10): 1-91.

Steinmann, H. \& Zombori, L. 1985. An atlas of insect morphology. - Budapest: Akadémiai Kiadó. 253 pp.

Stiewe, M.B.D. 2004. Eine neue Art der Gattung Eremiaphila Lefebvre, 1835 aus Ägypten (Mantodea: Eremiaphilidae). - Entomologische Zeitschrift (Stuttgart) 114(5): 199-203.

Stiewe, M.B.D. 2009. Dysaulophthalma nathani gen. et sp. n., A new praying mantis from Southern India (Dictyoptera: Mantodea: Tarachodidae). - Entomologist's Monthly Magazine 145: 51-60.

Stiewe, M.B.D. \& Roy, R. 2010. A new praying mantis species of Junodia Schulthess, 1899 from Kenya (Dictyoptera: Mantodea: Hymenopodidae). - Entomologist's Monthly Magazine 146: 63-70.

Strenger, A. 1942. Funktionelle Analyse des Orthopterenkopfes, eine systematisch-funktionsanatomische Studie. - Zoologische Jahrbücher Systematik, Ökologie und Geographie der Tiere 75: 1-71.

Strohecker, H.F. 1939. Distributional and taxonomic notes on southeastern Dermaptera and Orthoptera, and a new species of Cycloptilum (Gryllidae). - Canadian Entomologist 71: 169-175.

Svatek, S. \& van Duin, S. 2002. Plattschwanzgeckos. Die Gattung Uroplatus. - Banteln: Brämer Verlag. 161 pp.

Svenson, G.J. \& Branham, M.A. 2007. Photinini LeConte, 1881 (Insecta, Coleoptera) and Photininae Giglio-Tos, 1915 (Insecta, Mantodea): Proposed resolution of homonymy between family-group names. - Bulletin of Zoological Nomenclature 64(4): 243-251.

Svenson, G.J. \& Whiting, M.F. 2003. Phylogeny of Mantodea based on molecular data: Evolution of a charismatic predator. - P. 138 in: Klass, K.-D. (ed.) 2003: Proceedings of the 1st Dresden Meeting on Insect Phylogeny: "Phylogenetic Relationships within the Insect Orders" (Dresden, September 19-21, 2003). - Entomologische Abhandlungen 61(2): 119-172.

Svenson, G.J. \& Whiting, M.F. 2004a. Phylogeny of Mantodea based on molecular data: Evolution of a charismatic predator. - Systematic Entomology 29: 359-370.

Svenson, G.J. \& Whiting, M.F. 2004b. Phylogeny of Mantodea based on molecular data: evolution of a charismatic predator. - P. 97 in: Stevenson, D.W. (ed.) 2004: Abstracts of the 22 ${ }^{\text {nd }}$ Annual Meeting of the Willi Hennig Society. - Cladistics 20: 76-100.

Svenson, G.J. \& Whiting, M.F. 2009. Reconstructing the origins of praying mantises (Dictyoptera, Mantodea): The roles of Gondwanan vicariance and morphological convergence. - Cladistics 25(5): 468-514.

Swofford, D.L. 2001. PAUP*. Phylogenetic Analysis Using Parsimony (*and other methods). Version 4.0b10. Sinauer Associates, Sunderland, Massachusetts.

Sztern, H. 1914. Wachstumsmessungen an Sphodromantis bioculata Burm. II. Länge, Breite und Höhe. (Zugleich: Aufzucht der Gottesanbeterinnen. VI. Mitteilung). - Archiv für Entwicklungsmechanik der Organismen: Organ für die gesamte kausale Morphologie 40(3): 430-495.

Teeling, E.C., Springer, M.S., Madsen, O., Bates, P., O‘Brien, S.J. \& Murphy, W.J. 2005. A molecular phylogeny for bats illuminates biogeography and the fossil record. - Science 307: 580-584.

Terra, P.S. 1980. Ontogénese da perna raptória em "Louva-a-deus" (Mantodea): Um estudo comparativo de alometria. - Revista Brasileira de Entomologia 24(2): 117-122.

Terra, P.S. 1995. Revisao sistemática dos gêneros de louva-a-Deus da regiao Neotropical (Mantodea). - Revista Brasileira de Entomologia 39(1): 13-94.

Terry, M.D. \& Whiting, M.F. 2005. Mantophasmatodea and phylogeny of the lower neopterous insects. - Cladistics 21: 240-257.

Thorne, B.L. \& Carpenter, J.M. 1992. Phylogeny of the Dictyoptera. - Systematic Entomology 17: 253-268.

Tillyard, R.J. 1937a. Kansas Permian insects. Part 20. The cockroaches, or order Blattaria. Part 1. - American Journal of Science 34(201): 169-202.

Tillyard, R.J. 1937b. Kansas Permian insects. Part 20. The cockroaches, or order Blattaria. Part 2. - American Journal of Science 34(202): 249-276. 
Tindale, N.B. 1923. Review of Australian Mantidae, Part 1. - Records of the Australian Museum 2(3): 425-457.

Tindale, N.B. 1924. Review of Australian Mantidae, Part 2. - Records of the Australian Museum 2: 547-552.

Toy, S.J. \& Newfield, M.J. 2010. The accidental introduction of invasive animals as hitchhikers through inanimate pathways: A New Zealand perspective. - Revue Scientifique et Technique du Office International des Epizooties 29(1): 123-133.

Travassos Filho, L. 1945. Sôbre a família Acanthopidae Burmeister, 1838, emend. (Mantodea). - Arquivos de Zoologia 4(6): 157-232.

Triblehorn, J.D. \& Yager, D.D. 1997. Near-field sound reception by particle displacement receptors on the cerci of the praying mantis, Sphodromantis aurea. - ARO Abstracts 20: 143.

Triblehorn, J.D. \& Yager, D.D. 2001. Broad versus narrow auditory tuning and corresponding bat-evasive flight behaviour in praying mantids. - Journal of Zoology 254: 27-40.

Triblehorn, J.D. \& Yager, D.D. 2002. Implanted electrode recordings from a praying mantis auditory interneuron during flying bat attacks. - Journal of Experimental Biology 205: 307-320.

Triblehorn, J.D. \& Yager, D.D. 2005. Timing of praying mantis evasive responses during simulated bat attack sequences. - Journal of Experimental Biology 208: 1867-1876.

Triblehorn, J.D. \& Yager, D.D. 2006. Wind generated by an attacking bat: Anemometric measurements and detection by the praying mantis cercal system. - Journal of Experimental Biology 209: 1430-1440.

Uchifune, T. \& Machida, R. 2005. Embryonic development of Galloisiana yuasai Asahina, with special reference to external morphology (Insecta: Grylloblattodea). - Journal of Morphology 266: 182-207.

Vickery, V.R. \& Kevan, D.K.McE. 1983. A monograph of the orthopteroid insects of Canada and adjacent regions. - Memoirs of the Lyman Entomological Museum and Research Laboratory 13: 1-679.

Vršanský, P. 2002. Origin and the early evolution of mantises. - AMBA Projekty 6(1): 1-16.

Vršanský, P. 2003. Umenocoleoidea. An amazing lineage of aberrant insects (Insecta, Blattaria). - AMBA Projekty 7(1): 1-32.

Vršanský, P. 2005. Lower Cretaceous cockroaches and mantids (Insecta: Blattaria, Mantodea) from the Sharin-Gol in Mongolia. - Entomological Problems 35(2): 163-167.

Vršanský, P., Vishniakova, V.N. \& Rasnitsyn, A.P. 2002. 2.2.2.1.1. Order Blattida Latreille, 1810. The cockroaches (= Blattodea Brunner von Wattenvill [sic!], 1882). - Pp. 263-269 in: Rasnitsyn, A.P. \& Quicke, D.L.J. (eds): History of Insects. - Dordrecht, Boston, London: Kluwer. 544 pp.

Vyjayandi, M.C. 2004. On some interesting genera of Mantidae (Insecta: Mantodea) of Kerala. - Perspectives on Biosystematics and Biodiversity: Prof. T.C. Narendran Commemoration Volume March 2004: 567-572.

Vyjayandi, M.C. 2007. Mantid fauna of Kerala. - Records of the Zoological Survey of India: Occasional Paper 267: 1-186.

Vyjayandi, M.C., Rajeesh, R.S., Sajin John, P., Dhanasree, M.M. \& Ehrmann, R. 2009. A new genus of praying mantis Cotigaonopsis from Goa, India (Insecta: Mantodea). - Genus 20(3): 485-492.

Vyjayandi, M.C., Stiewe, M.B.D., Mukherjee, T.K. \& Rajeesh, R.S. 2008. On some variations in Gimantis autheamon (Wood-Mason, 1882) collected from Kerala, India (Dictyoptera: Mantodea: Mantidae). - Genus 19(2): 313-318.

Wagner, D.L. \& Liebherr, J.K. 1992. Flightlessness in insects. - Trends in Ecology and Evolution 7(7): 216-220.

Ware, J.L., Litman, J., Klass, K.-D. \& Spearman, L.A. 2008. Relationships among the major lineages of Dictyoptera: The effect of outgroup selection on dictyopteran tree topology. - Systematic Entomology 33(3): 429-450.

Wedmann, S., Bradler, S. \& Rust, J. 2007. The first fossil leaf insect: 47 million years of specialized cryptic morphology and behavior. - Proceedings of the National Acadademy of Sciences, USA (PNAS) 104(2): 565-569.

Wedmann, S., Wappler, T. \& Engel, M.S. 2009. Direct and indirect fossil records of megachilid bees from the Paleogene of Central Europe (Hymenoptera: Megachilidae). - Naturwissenschaften 96: 703-712.

Weidner, H. 1966: Betrachtungen zur Evolution der Termiten. - Deutsche Entomologische Zeitschrift (Berlin) 13: 323-350. 
Weitschat, W. \& Wichard, W. 2002. Atlas of plants and animals in Baltic amber. - München: Pfeil. 256 pp.

Werner, F. 1904. Über eine neue Gattung aus der Orthopteren-Familie der Mantodeen. - Anzeiger der Akademie der Wissenschaften, Wien 41(27): 403-405.

Werner, F. 1907. Ergebnisse der mit Subvention aus der Erbschaft Treitl unternommenen zoologischen Forschungsreise Dr. Franz Werner's in den ägyptischen Sudan und nach Nord-Uganda. VIII. Orthoptera Blattaeformia (mit einer Revision der Gattung Tarachodes). - Sitzungsberichte der Kaiserlichen Akademie der Wissenschaften 116(2): 165-266.

Werner, F. 1909. Bemerkungen über die geographische Verbreitung der Mantodeen (Fangheuschrecken). - Bericht der Sektion Zoologie (1908): 70-81.

Werner, F. 1915. Verschleppung von Fangheuschrecken (Mantodeen) durch den Schiffer-Verkehr. - Zeitschrift für Wissenschaftliche Insektenbiologie 11(3-4): 98-99.

Werner, F. 1916. Zur Kenntnis afrikanischer und indischer Mantodeen. - Verhandlungen der Zoologisch-Botanischen Gesellschaft zu Wien 66: 254-296.

Werner, F. 1921. Ueber Mantodeen aus Niederländisch-Indien. - Treubia 2(1): 125-135.

Werner, F. 1922. Philippine mantids or praying insects. - Philippine Journal of Science 21(2): 147-157.

Werner, F. 1923. Zweiter Beitrag zur Kenntnis der Mantodeen von Niederländisch-Indien. - Treubia 3(3-4): 387-404.

Werner, F. 1924. Dritter Beitrag zur Kenntnis der Mantodeen von Niederländisch-Indien. - Treubia 5(1-3): 259-266.

Werner, F. 1925. Vierter Beitrag zur Kenntnis der Mantodeen von Niederländisch-Indien. - Treubia 6(3-4): 476-487.

Werner, F. 1928. Zur Kenntnis der Mantodeenfauna des Hinterlandes von Kamerun und des Sepikgebietes von Neuguinea nebst Beschreibung einiger interessanter Arten aus anderen Ländern. - Mitteilungen aus dem Zoologischen Museum in Berlin 14(1): 12-41.

Werner, F. 1931. Neue Geradflügler, gesammelt auf einer im Jahre 1930 mit Unterstützung der Akademie unternommenen Reise nach Marokko. - Anzeiger der Akademie der Wissenschaften Wien 68: 201-204.

Westwood, J.O. 1845a. Description of a new mantideous insect with pointed eyes. - Pp. 161-162 in: Arcana Entomologica or illustrations of new, rare and interesting insects, vol. 1. - London: Smith. 192 pp.

Westwood, J.O. 1845b. Description of some new species of soothsayers (Mantidae). - Pp. 51-52 in: Arcana Entomologica or illustrations of new, rare and interesting insects, vol. 2. - London: Smith. 191 pp.

Westwood, J.O. 1845c. Description of a new genus of mantideous Orthoptera. - Pp. 33-34 in: Arcana Entomologica or illustrations of new, rare and interesting insects, vol. 1. - London: Smith. 192 pp.

Westwood, J.O. 1889. Revisio Insectorum. Familiae Mantidarum, speciebus novis aut minus congnitis, descriptis et delineatis. - London: Gurney \& Jackson. $53+4$ pp.

Wheeler, W.C. 1998. Sampling, groundplans, total evidence and the systematics of arthropods. - Pp. 87-96 in: Fortey, R.A. \& Thomas, R.H. (eds): Arthropod relationships. - London: Chapman \& Hall. 383 pp.

Wheeler, W.C., Whiting, M.F., Wheeler, Q.D. \& Carpenter, J.M. 2001a. The Phylogeny of Extant Hexapod Orders. - Cladistics 17: 113-169.

Wheeler, W.C., Whiting, M.F., Wheeler, Q.D. \& Carpenter, J.M. 2001b. The Phylogeny of Extant Hexapod Orders (Erratum). - Cladistics 17: 403-404.

Whiting, M.F., Bradler, S. \& Maxwell, T. 2003. Loss and Recovery of Wings in Stick Insects. - Nature 421: 264-267.

Wieland, F. 2003: Evolution of prothoracic structures in Mantodea (Insecta: Polyneoptera: Dictyoptera) and their significance for mantodean phylogeny. - P. 26 in: Melzer, R. \& Schrödl, M. (eds) 2003: Abstracts of the 5th Annual Meeting of the Gesellschaft für Biologische Systematik (Society for Biological Systematics). Organisms, Diversity \& Evolution 3(1), Electronic Supplement 2. 31 pp.

Wieland, F. 2006. The cervical sclerites of Mantodea discussed in the context of dictyopteran phylogeny (Insecta: Dictyoptera). - Entomologische Abhandlungen 63(1-2): 51-76. 
Wieland, F. 2008a. The genus Metallyticus reviewed (Insecta: Mantodea). - Species, Phylogeny and Evolution 1(3): 147-170.

Wieland, F. 2008b. Digging for the offspring, or how to bury an ootheca underground. - P. 344 in Gradstein, S.R., Klatt, S., Normann, F., Weigelt, P., Willmann, R. \& Wilson, R. (eds): Systematics 2008 (Göttingen 7-11 April 2008). Göttingen, Universitätsverlag. 425 pp.

Wiemers, M. 1993. Eine neue Gottesanbeterin der Gattung Pseudoyersinia von den Kanarischen Inseln (Mantodea, Mantidae). - Nachrichten des entomologischen Vereins Apollo (N.F.) 14(3): 261-269.

Willey, A. 1906. Stridulation of Gongylus gongylodes. - Spolia Zeylanica 3: 226-227.

Willey, A. 1918. Popular and practical entomology. Insect Tropisms. - Canadian Entomologist 50(11): 357-360.

Williams, C.E. \& Sharp, D. 1904. Notes on the life history of Gongylus gongyloides, a mantis of the tribe Empusides and a floral simulator. - Transactions of the Entomological Society of London 1904(1): 125-137.

Willmann, R. 1990. Die Bedeutung paläontologischer Daten für die zoologische Systematik. - Verhandlungen der Deutschen Zoologischen Gesellschaft 83: 277-289.

Willmann, R. 2003a: Phylogenese und System der Insecta. - Pp. 1-65 in: Dathe, H.H. (ed.): Lehrbuch der Speziellen Zoologie, Band I: Wirbellose Tiere, 5. Teil: Insecta. - Heidelberg, Berlin: Spektrum. 961 pp.

Willmann, R. 2003b: Die phylogenetischen Beziehungen der Insecta: Offene Fragen und Probleme. - Verhandlungen des Westdeutschen Entomologentages 2001: 1-64.

Willmann, R. 2004: Phylogenetic relationships and evolution of insects. - Pp. 330-344 in: Cracraft, J. \& Donoghue, M.J. 2004: Assembling the Tree of Life. - New York: Oxford University Press. 576 pp.

Wipfler, B. \& Hörnschemeyer, T. 2008. The head morphology of Hymenopus coronatus (Insecta, Mantodea) reconstructed from micro computer tomography. - P. 349 in: Gradstein, S.R., Klatt, S., Normann, F., Weigelt, P., Willmann, R. \& Wilson, R. (eds): Systematics 2008 (Göttingen 7-11 April 2008). Göttingen, Universitätsverlag. $425 \mathrm{pp}$.

Wood-Mason, J. 1877. Descriptions of two new genera and species of Indian Mantidae. - Annals and Magazine of Natural History, Zoology, Botany and Geology 4(19): 219-222.

Wood-Mason, J. 1878a. On the difference in the form of the antennae between the males of Idolomorpha and those of other genera of Empusidae, a subfamily of Mantidae. - Transactions of the Entomological Society 1878(4): 259-262.

Wood-Mason, J. 1878b. On the presence of a stridulating apparatus in certain Mantidæ. - Transactions of the Entomological Society 1878(4): 263-267.

Wood-Mason, J. 1878c. On new and little known Mantidæ. - Proceedings of the Zoological Society of London 38: 580-587.

Wood-Mason, J. 1878d. On a saltatorial 'Mantis'. - Transactions of the Entomological Society 1878(4): 268-269.

Wood-Mason, J. 1880. Synopsis of the species of Choeradodis, a remarkable genus of Mantodea common to India and Tropical America. - Journal of the Asiatic Society of Bengal 47(1): 82-84.

Wood-Mason, J. 1884. Revised synopsis of the species of Choeradodis, a remarkable genus of Mantodea common to India and Tropical America. - Journal of the Asiatic Society of Bengal 53(3): 238-244.

Wood-Mason, J. 1889. A catalogue of the Mantodea, with descriptions of new genera and species, and an enumeration of the specimens, in the collection of the Indian Museum, Calcutta, vol. 1. - Calcutta: Trustees of the Indian Museum. 48 pp.

Wood-Mason, J. 1891. A catalogue of the Mantodea, with descriptions of new genera and species, and an enumeration of the specimens, in the collection of the Indian Museum, Calcutta, vol. 2. - Calcutta: Trustees of the Indian Museum. 18 pp.

Xu, J. \& Mao, B. 2004. A new species of the genus Kishinouyeum Ôchi (Mantodea: Vatidae) from Hengduang mountain region of western Yunnan, China. - Entomotaxonomia 26(1): 8-10.

Yager, D.D. 1990. Sexual dimorphism of auditory function and structure in praying mantises (Mantodea: Dictyoptera). - Journal of Zoology 221: 517-537. 
Yager, D.D. 1996a. Serially homologous ears perform frequency range fractionation in the praying mantis, Creobroter (Mantodea, Hymenopodidae). - Journal of Comparative Physiology A: Sensory, Neural and Behavioral Physiology 178: 463-475.

Yager, D.D. 1996b. Nymphal development of the auditory system in the praying mantis Hierodula membranacea Burmeister (Dictyoptera, Mantidae). - Journal of Comparative Neurology 364: 199-210.

Yager, D.D. 1999a. Structure, development, and evolution of insect auditory systems. - Microscopic Research and Technique 47: 380-400.

Yager, D.D. 1999b. 6. Hearing. - Pp. 93-113 in: Prete, F. R., Wells, H., Wells, P. H. \& Hurd, L. E. (eds): The praying mantids. - Baltimore \& London: John Hopkins. 362 pp.

Yager, D.D. 2005. Cockroach homologs of praying mantis peripheral auditory components. - Journal of Morphology 265: 120-139.

Yager, D.D. \& Hoy, R.R. 1986. The cyclopean ear: A new sense for the praying mantis. - Science 231: 727-729.

Yager, D.D. \& Hoy, R.R. 1987. The midline metathoracic ear of the praying mantis, Mantis religiosa. - Cell Tissue Research 250: 531-541.

Yager, D.D. \& Hoy, R.R. 1989. Audition in the praying mantis, Mantis religiosa L.: identification of an interneuron mediating ultrasonic hearing. - Journal of Comparative Physiology A: Sensory, Neural and Behavioral Physiology 165: 471-493.

Yager, D.D., May, M.L. \& Fenton, M.B. 1990. Ultrasound-triggered, flight-gated evasive maneuvers in the praying mantis Parasphendale agrionina. 1. Free flight. - Journal of Experimental Biology 152: 17-39.

Yager, D.D. \& May, M.L. 1990. Ultrasound-triggered, flight-gated evasive maneuvers in the praying mantis Parasphendale agrionina. 2. Tethered flight. - Journal of Experimental Biology 152: 41-58.

Yager, D.D. \& Svenson, G.J. 2008. Patterns of praying mantis auditory system evolution based on morphological, molecular, neurophysiological, and behavioural data. - Biological Journal of the Linnean Society 94 : 541-568.

Yamanouti, T. 1937. Wachstumsmessungen an Sphodromantis bioculata Burm. V. Bestimmungen der absoluten Zunahmewerte der Facetten-Größe und -Anzahl (Zugleich: Aufzucht der Gottesanbeterinnen. XIII. Mitteilung). - Biologia generalis, 12: 126-141.

Yoshizawa, K. 2007. The Zoraptera problem: evidence for Zoraptera + Embiodea from the wing base. - Systematic Entomology 32: 197-204.

Yoshizawa, K. \& Johnson, K.P. 2005. Aligned 18S for Zoraptera (Insecta): Phylogenetic position and molecular evolution. - Molecular Phylogenetics and Evolution 37: 572-580.

Zeuner, F. 1931. Die Insektenfauna des Böttinger Marmors. - Fortschritte der Geologie und Paläontologie 9(28): I-VIII (=1-8), 1-406.

Zherikhin, V.V. 2002. Order Mantida Latreille, 1802. The Mantises (= Mantodea Burmeister 1838). - Pp. 273-276 in: Rasnitsyn, A.P. \& Quicke, D.L.J. (eds): History of Insects. - Dordrecht, Boston, London: Kluwer. 544 pp.

Zhou, X. \& Zhou, W. 2004. A new species of the genus Kishinouyeum (Mantodea: Vatidae). - Entomotaxonomia 26(3): 161-162.

Zompro, O. 2005. Inter- and intra-ordinal relationships of the Mantophasmatodea, with comments on the phylogeny of polyneopteran orders (Insecta: Polyneoptera). - Mitteilungen des Geologisch-Paläontologischen Instituts der Universität Hamburg 89: 85-116. 



\section{Acknowledgements}

This project was funded by the German Science Foundation (DFG Wi-599/12). It has been kindly supported by many people to whom I am very grateful.

I would like to thank Prof. Dr. Rainer Willmann and PD Dr. Thomas Hörnschemeyer for supervising my thesis and for valuable discussions.

I am grateful to Thomas Hörnschemeyer for helping me with the three-dimensional reconstruction of the mantid fossil, the use of Amira, and for his endless patience whenever it comes to questions about Paup*.

I want to thank Carsten Gröhn for providing the amber fossil for my studies. For generating the $\mu$-CT data of the amber fossil I would like to thank Manuel Dierick from the Subatomic and Radiation Physics department of Gent University, Belgium.

I am endlessly grateful to the members of the Interessengemeinschaft Mantodea (IGM) who never hesitate to support my studies with specimens, photographs, data, helpful discussions and the exchange of observations. My special thanks in alphabetical order go to Robert Bäuml, Matthias Borer, Rob Byatt, Reinhard Ehrmann, Markus Löwe, Thomas Maag, Sören Materna, Jürgen Mehl, Kai Schütte, Tobias Schulze, Christian Schwarz, Karin Sieber, Jürg Sommerhalder, Andreas Steiger, Alexander Windisch, and Andreas Ziegler. At this point I want to thank Sören Materna for always being an amazing and selfless host for the biannual IGM meetings.

I received specimens, literature and data from many colleagues and friends from all over the world. I would like to thank (in alphabetical order) Nancy Adams $\dagger$, Pieter Baas, Roberto Battiston, George Beccaloni, Jan Beck, Olivier Béthoux, Sven Bradler, Birgit \& Matthias Braun, Jerome Constant, José Correas, Mark Deyrup, Didier Drugmand, Klaus Fabian, Julia Goldberg, Hartmut Greven, Benny de Groof, Thomas Hörnschemeyer, Gregory Holwell, Alfred Kaltenbach $\uparrow$, Charlotte Klank, Klaus Klass, Kristian Köchy, Christoph Losemann, Ryuichiro Machida, Darren Mann, Thomas Marent, Stuart McKamey, Graham Milledge, Gerald Moser, Reinhard Predel, Niels Raes, Roger Roy, Julián Salazar, Susanne Schulmeister, Arne Schulze, Christoph Seiler, Zoe Simmons, Martin Stiewe, Gavin Svenson, Jan van Tol, Steve Trewick, Gisela Vogel, Sonja Wedmann, Francisco Welter-Schultes, Peter van Welzen, Dave Williams, Benjamin Wipfler, and of course all members of the IGM (see above).

For providing me with their amazing photographs and allowing me to publish them I would like to thank Farhan Bokhari, Geoffrey Gallice, Sören Materna, Tobias Schulze, Karin Sieber, and Machele White.

I am very grateful to Ulrike Aspöck, Susanne Randolf (Naturhistorisches Museum Wien, NHMW) and Michael Ohl (Zoologisches Museum der Humboldt-Universität Berlin, MNHUB) who granted access to the Mantodea collections of the respective museums.

I would like to thank Verena Feuerstein for her helpful comments on the manuscript.

I want to thank my friends and colleagues for supporting me throughout the recent years: Sven Bradler, Sebastian Büsse, Oliver Eikel, Verena Feuerstein, Christina Förster, 
Ina Frambach, Stephanie \& Timm Georg, Thomas Hörnschemeyer, Agnes Holm-Petersen, Christine Höttermann, Beatrice Hoffmann, Rebecca Klug, Kathrin Luhn, Alexander Neuhäuser, Nicole \& Ralf Pahling, Regina \& Markus Pauly, Christian Richter, Gert Tröster, Gisela Vogel, Jana Willkommen, and Carsten Wortmann.

I am endlessly grateful to my loving family who has unreservedly supported me throughout the long and sometimes tough times. Thank you all for being there for me and for your confidence in me.

Finally I want to thank Jule for being in my life. Thank you for always supporting me, for being there for me when I need you and for just being yourself. 


\section{Appendix}

The appendix contains the following tables:

Tab. 2

Species studied in the present analysis including their generic distribution and the collections they were obtained from.

Tab. 3

Morphological data matrix generated in this study (122 Mantodea + Periplaneta; 152 characters).

Tab. 4

Character diagnostics for analysis I.

Tab. 5

Character diagnostics for analysis II.

Tab. 6

Apomorphies found in analysis II.

Tab. 7

xxvi

Alphabetical list of genera used in this study and their current taxonomical affiliations (Ehrmann 2002).

Tab. 8

xxviii

Alphabetical list of the current taxonomical groups (Ehrmann 2002) and the corresponding genera used in this study. 

Tab. 2: Species studied in the present analysis. Grey markings indicate species that are shown in some figures but were not included in the analysis.

Collections: CGS = Gavin Svenson; CW = Wieland; CKS = Kai Schütte; CSB = Sven Bradler; CTS = Tobias Schulze; MNHUB = Museum für Naturkunde der Humboldt-Universität zu Berlin, Germany; NHWM = Naturhistorisches Museum Wien, Austria; ZMUG = Zoologisches Museum der Universität Göttingen.

Biogeographical affinities: $\mathrm{NR}=$ Neotropical region; $\mathrm{PR}=$ Palaearctic region; $\mathrm{NAR}=$ Nearctic region; $\mathrm{ATR}=$ Afrotropical region; $\mathrm{OR}=$ Oriental region; $\mathrm{AR}=$ Australian region.

\begin{tabular}{|c|c|c|c|c|c|}
\hline $\begin{array}{l}\text { Classification sensu } \\
\text { Ehrmann (2002) }\end{array}$ & Taxon & $\begin{array}{l}\text { Generic distribution / } \\
\text { Biogeographical affinity }\end{array}$ & Preservation & Collection & Remarks \\
\hline Mantoididae & Mantoida maya Saussure \& Zehntner, 1894 & $\begin{array}{l}\text { Florida, Central and South } \\
\text { America / NR }\end{array}$ & dry, softened in $80 \%$ EtOH & CW & \\
\hline Mantoididae & Mantoida sp. & South America / NR & $\begin{array}{l}\text { fresh material, preserved in } \\
80 \% \text { EtOH }\end{array}$ & $\mathrm{CW}$ & 1 first instar nymph \\
\hline Chaeteessidae & Chaeteessa valida (Perty, 1833) & Central and South America / NR & dry & NHMW & \\
\hline Chaeteessidae & Chaeteessa cf. valida & Central and South America / NR & dry, softened in $100 \%$ EtOH & CGS & \\
\hline Metallyticidae & Metallyticus splendidus Westwood, 1835 & $\begin{array}{l}\text { South-East Asia (see also } \\
\text { Wieland 2008) / OR }\end{array}$ & $\begin{array}{l}\text { dried material, fresh material, } \\
\text { preserved in } 80 \% \mathrm{EtOH}\end{array}$ & $\mathrm{CW}$ & \\
\hline Metallyticidae & Metallyticus fallax Giglio-Tos, 1917 & $\begin{array}{l}\text { South-East Asia (see also } \\
\text { Wieland 2008) / OR }\end{array}$ & $\begin{array}{l}\text { dried specimen, softened in } \\
80 \% \text { EtOH }\end{array}$ & $\mathrm{cW}$ & \\
\hline $\begin{array}{l}\text { Amorphoscelidae: } \\
\text { Perlamantinae }\end{array}$ & $\begin{array}{l}\text { Paramorphoscelis gondokorensis Werner, } \\
1907\end{array}$ & Subsaharan Africa / ATR & dried material & NHMW & \\
\hline $\begin{array}{l}\text { Amorphoscelidae: } \\
\text { Perlamantinae }\end{array}$ & Perlamantis allibertii Guérin-Méneville, 1843 & $\begin{array}{l}\text { southern Europe, northern } \\
\text { Africa / PR }\end{array}$ & $\begin{array}{l}\text { fresh material, preserved in } \\
80 \% \text { EtOH }\end{array}$ & $\mathrm{CW}$ & \\
\hline $\begin{array}{l}\text { Amorphoscelidae: } \\
\text { Amorphoscelinae }\end{array}$ & Amorphoscelis sp. (Malaysia) & Southeast Asia / OR & $\begin{array}{l}\text { fresh material, preserved in } \\
80 \% \text { EtOH }\end{array}$ & CW & \\
\hline $\begin{array}{l}\text { Amorphoscelidae: } \\
\text { Amorphoscelinae }\end{array}$ & Caudatoscelis annulipes (Karsch, 1892) & Subsaharan Africa / ATR & dry & NHMW & $\begin{array}{l}\text { labelled as } \\
\text { Amorphoscelis }\end{array}$ \\
\hline $\begin{array}{l}\text { Amorphoscelidae: } \\
\text { Amorphoscelinae }\end{array}$ & Maculatoscelis sp. & Subsaharan Africa / ATR & $\begin{array}{l}\text { fresh material, preserved in } \\
80 \% \text { EtOH }\end{array}$ & $\mathrm{CW}$ & \\
\hline $\begin{array}{l}\text { Amorphoscelidae: } \\
\text { Paraoxypilinae }\end{array}$ & Paraoxypilus sp. 1 & Australia / AR & $\begin{array}{l}\text { fresh material, preserved in } \\
80 \% \text { EtOH }\end{array}$ & $\mathrm{CW}$ & \\
\hline $\begin{array}{l}\text { Amorphoscelidae: } \\
\text { Paraoxypilinae }\end{array}$ & Paraoxypilus sp. 2 & Australia / AR & $\begin{array}{l}\text { fresh material, preserved in } \\
80 \% \text { EtOH }\end{array}$ & $\mathrm{CW}$ & \\
\hline $\begin{array}{l}\text { Amorphoscelidae: } \\
\text { Paraoxypilinae }\end{array}$ & Paraoxypilinae sp. & Australia / AR & $\begin{array}{l}\text { fresh material, preserved in } \\
80 \% \text { EtOH }\end{array}$ & $\mathrm{CW}$ & \\
\hline $\begin{array}{l}\text { Amorphoscelidae: } \\
\text { Paraoxypilinae }\end{array}$ & Gyromantis sp. & Australia / AR & $\begin{array}{l}\text { fresh material, preserved in } \\
80 \% \text { EtOH }\end{array}$ & $\mathrm{CW}$ & i partly decomposed \\
\hline Eremiaphilidae & Eremiaphila sp. 1 (Pakistan) & $\begin{array}{l}\text { Turkey, northern Africa, to } \\
\text { Pakistan, India / PR, OR }\end{array}$ & $\begin{array}{l}\text { fresh material, preserved in } \\
80 \% \text { EtOH }\end{array}$ & $\mathrm{CW}$ & \\
\hline Eremiaphilidae & Eremiaphila sp. 2 (Ägypten) & $\begin{array}{l}\text { Turkey, northern Africa, to } \\
\text { Pakistan, India / PR, OR }\end{array}$ & $\begin{array}{l}\text { fresh material, preserved in } \\
80 \% \text { EtOH }\end{array}$ & $\mathrm{CW}$ & \\
\hline Eremiaphilidae & Heteronutarsus aegyptiacus Lefèbvre, 1835 & $\begin{array}{l}\text { north-eastern to central Saharan } \\
\text { Africa / PR, AFT }\end{array}$ & dry & NHMW & \\
\hline Acanthopidae: Acanthopinae & Acanthops falcataria (Goeze, 1778) & South and Central America / NR & dry & MNHUB & \\
\hline Acanthopidae: Acanthopinae & Acanthops tuberculata Saussure, 1870 & South and Central America / NR & $\begin{array}{l}\text { fresh material, preserved in } \\
80 \% \text { EtOH }\end{array}$ & $\mathrm{cW}$ & \\
\hline Acanthopidae: Acontistinae & Acontista brevipennis Saussure, 1872 & South and Central America / NR & dry & MNHUB & $\begin{array}{l}\text { labelled as } \\
\text { Aconthiothespis } \\
\text { brevipennis }\end{array}$ \\
\hline Acanthopidae: Acontistinae & Callibia diana (Stoll, 1813) & South America / NR & dry & MNHUB & \\
\hline Acanthopidae: Acontistinae & Raptrix fusca (Olivier, 1792) & South and Central America / NR & dry & MNHUB & $\begin{array}{l}\text { labelled as Acontista } \\
\text { fusca }\end{array}$ \\
\hline Acanthopidae: Acontistinae & Tithrone roseipennis (Saussure, 1870) & $\begin{array}{l}\text { northern South America, Central } \\
\text { America, North America / NR, } \\
\text { NAR }\end{array}$ & dry & MNHUB & \\
\hline $\begin{array}{l}\text { Hymenopodidae: } \\
\text { Epaphroditinae }\end{array}$ & Amphecostephanus rex Rehn, 1912 & $\begin{array}{l}\text { southern Subsaharan Africa } \\
\text { / ATR }\end{array}$ & dry & MNHUB & \\
\hline $\begin{array}{l}\text { Hymenopodidae: } \\
\text { Epaphroditinae }\end{array}$ & Epaphrodita dentifrons Saussure, 1872 & Greater Antilles / NR & dry & MNHUB & \\
\hline $\begin{array}{l}\text { Hymenopodidae: } \\
\text { Epaphroditinae }\end{array}$ & Phyllocrania paradoxa Burmeister, 1838 & $\begin{array}{l}\text { Subsaharan Africa and Mada- } \\
\text { gascar / ATR }\end{array}$ & $\begin{array}{l}\text { fresh material, preserved in } \\
80 \% \text { EtOH }\end{array}$ & $\mathrm{cW}$ & \\
\hline $\begin{array}{l}\text { Hymenopodidae: } \\
\text { Acromantinae }\end{array}$ & Acromantis sp. & $\begin{array}{l}\text { South-East Asia to Australia } \\
\text { IOR, AR }\end{array}$ & $\begin{array}{l}\text { fresh material, preserved in } \\
80 \% \text { EtOH }\end{array}$ & $\mathrm{cW}$ & \\
\hline $\begin{array}{l}\text { Hymenopodidae: } \\
\text { Acromantinae }\end{array}$ & Hestiasula brunneriana Saussure, 1871 & South-East Asia & dry & CTS & \\
\hline
\end{tabular}


Tab. 2 (continued): Species studied in the present analysis.

\begin{tabular}{|c|c|c|c|c|c|}
\hline $\begin{array}{l}\text { Hymenopodidae: } \\
\text { Acromantinae }\end{array}$ & Odontomantis sp. & South-East Asia / OR & $\begin{array}{l}\text { fresh material, preserved in } \\
80 \% \text { EtOH }\end{array}$ & $\mathrm{cW}$ & \\
\hline Hymenopodidae: Oxypilinae & Ceratomantis saussurii Wood-Mason, 1876 & South-East Asia / OR & $\begin{array}{l}\text { dry, re-softened and preserved in } \\
80 \% \text { EtOH }\end{array}$ & $\mathrm{CW}$ & \\
\hline Hymenopodidae: Oxypilinae & Oxypilus distinctus Beier, 1930 & Subsaharan Africa / ATR & $\begin{array}{l}\text { fresh material, preserved in } \\
80 \% \text { EtOH }\end{array}$ & $\mathrm{CW}$ & \\
\hline $\begin{array}{l}\text { Hymenopodidae: } \\
\text { Hymenopodinae }\end{array}$ & Creobroter elongata Beier, 1929 & South-East Asia / OR & $\begin{array}{l}\text { fresh material, preserved in } \\
80 \% \text { EtOH }\end{array}$ & $\mathrm{CW}$ & \\
\hline $\begin{array}{l}\text { Hymenopodidae: } \\
\text { Hymenopodinae }\end{array}$ & Creobroter gemmatus (Stoll, 1813) & South-East Asia / OR & dry & MNHUB & \\
\hline $\begin{array}{l}\text { Hymenopodidae: } \\
\text { Hymenopodinae }\end{array}$ & Galinthias amoena (Saussure, 1871) & Subsaharan Africa / ATR & $\begin{array}{l}\text { fresh material, preserved in } \\
80 \% \text { EtOH }\end{array}$ & $\mathrm{cW}$ & \\
\hline $\begin{array}{l}\text { Hymenopodidae: } \\
\text { Hymenopodinae }\end{array}$ & Hymenopus coronatus (Olivier, 1792) & South-East Asia / OR & $\begin{array}{l}\text { fresh material, preserved in } 80 \% \\
\text { EtOH, some specimens dried and } \\
\text { softened in EtOH }\end{array}$ & $\mathrm{CW}$ & \\
\hline $\begin{array}{l}\text { Hymenopodidae: } \\
\text { Hymenopodinae }\end{array}$ & $\begin{array}{l}\text { Pseudocreobotra ocellata (Palisot de } \\
\text { Beauvois, 1805) }\end{array}$ & Subsaharan Africa / ATR & $\begin{array}{l}\text { fresh material, preserved in } \\
80 \% \text { EtOH }\end{array}$ & $\mathrm{CW}$ & \\
\hline $\begin{array}{l}\text { Hymenopodidae: } \\
\text { Hymenopodinae }\end{array}$ & Pseudocreobotra wahlbergii Stål, 1871 & Subsaharan Africa / ATR & $\begin{array}{l}\text { fresh material, preserved in } \\
80 \% \text { EtOH }\end{array}$ & $\mathrm{CW}$ & \\
\hline $\begin{array}{l}\text { Hymenopodidae: } \\
\text { Hymenopodinae }\end{array}$ & Pseudoharpax sp. & Subsaharan Africa / ATR & $\begin{array}{l}\text { fresh material, preserved in } \\
80 \% \text { EtOH }\end{array}$ & $\mathrm{cW}$ & \\
\hline $\begin{array}{l}\text { Hymenopodidae: } \\
\text { Hymenopodinae }\end{array}$ & Theopropus elegans (Westwood, 1832) & South-East Asia / OR & $\begin{array}{l}\text { fresh material, preserved in } \\
80 \% \text { EtOH }\end{array}$ & $\mathrm{CW}$ & \\
\hline Liturgusidae: Liturgusinae & Ciulfina biseriata (Westwood, 1889) & Australia / AR & dry & MNHUB & \\
\hline Liturgusidae: Liturgusinae & Humbertiella sp. & South-East Asia / OR & $\begin{array}{l}\text { fresh material, preserved in } \\
80 \% \text { EtOH }\end{array}$ & $\mathrm{CW}$ & \\
\hline Liturgusidae: Liturgusinae & Majanga basilaris (Westwood, 1889) & Madagascar / ATR & $\begin{array}{l}\text { fresh material, preserved in } \\
80 \% \text { EtOH }\end{array}$ & CKS & \\
\hline Liturgusidae: Liturgusinae & Stenomantis novaeguineae (De Haan, 1842) & Australia / AR & dry & MNHUB & \\
\hline Liturgusidae: Liturgusinae & Theopompa sp. 1 & South-East Asia / OR & $\begin{array}{l}\text { fresh material, preserved in } \\
80 \% \mathrm{EtOH}\end{array}$ & CKS & \\
\hline Liturgusidae: Liturgusinae & Theopompa sp. 2 & South-East Asia / OR & $\begin{array}{l}\text { fresh material, preserved in } \\
80 \% \text { EtOH }\end{array}$ & CKS & \\
\hline Liturgusidae: Liturgusinae & Theopompa tosta Stål, 1877 & South-East Asia / OR & dry & СTS & \\
\hline Liturgusidae: Liturgusinae & Theopompa serviellei (De Haan, 1842) & South-East Asia / OR & $\begin{array}{l}\text { fresh material, preserved in } \\
80 \% \mathrm{EtOH}\end{array}$ & CTS & $\begin{array}{l}1 \text { first instar nymph, } \\
1 \text { adult }+\end{array}$ \\
\hline Tarachodidae: Tarachodinae & Iris oratoria (Linnaeus, 1758) & $\begin{array}{l}\text { southern Europe, northern Africa } \\
\text { up to India, South-East Asia, } \\
\text { northern South America, North } \\
\text { America (introduced) / PR, NAR } \\
\text { (introduced), NR, OR }\end{array}$ & $\begin{array}{l}\text { fresh material, preserved in } \\
80 \% \text { EtOH }\end{array}$ & $\mathrm{CW}$ & \\
\hline Tarachodidae: Tarachodinae & Paralygdamia sp. & Madagascar, Comoros / ATR & $\begin{array}{l}\text { fresh material, preserved in } \\
80 \% \text { EtOH }\end{array}$ & $\mathrm{cW}$ & \\
\hline Tarachodidae: Tarachodinae & Pseudogalepsus nigricoxa (Sjöstedt, 1909) & Subsaharan Eastern Africa / ATR & $\begin{array}{l}\text { fresh material, preserved in } \\
80 \% \text { EtOH }\end{array}$ & $\mathrm{CW}$ & \\
\hline Tarachodidae: Tarachodinae & Pyrgomantis jonesi Kirby, 1904 & Subsaharan Africa / ATR & $\begin{array}{l}\text { fresh material, preserved in } \\
80 \% \text { EtOH }\end{array}$ & $\mathrm{CW}$ & \\
\hline Tarachodidae: Tarachodinae & Tarachodes sp. 1 ("slender") & Subsaharan Africa / ATR & $\begin{array}{l}\text { fresh material, preserved in } \\
80 \% \text { EtOH }\end{array}$ & $\mathrm{CW}$ & \\
\hline Tarachodidae: Tarachodinae & Tarachodes sp. 2 ("robust") & Subsaharan Africa / ATR & $\begin{array}{l}\text { fresh material, preserved in } \\
80 \% \text { EtOH }\end{array}$ & CW & \\
\hline Tarachodidae: Tarachodinae & Tarachodes sp. 3 ("grey”) & Subsaharan Africa / ATR & $\begin{array}{l}\text { fresh material, preserved in } \\
80 \% \text { EtOH }\end{array}$ & $\mathrm{CW}$ & \\
\hline Tarachodidae: Tarachodinae & Tarachodula pantherina (Gerstaecker, 1869) & Central Eastern Africa / ATR & $\begin{array}{l}\text { fresh material, preserved in } \\
80 \% \text { EtOH }\end{array}$ & cW & \\
\hline Tarachodidae: Tarachodinae & Didymocorypha lanceolata (Fabricius, 1798) & India, Nepal, Sri Lanka & $\begin{array}{l}\text { fresh material, preserved in } \\
80 \% \mathrm{EtOH}\end{array}$ & CW & \\
\hline Thespidae: Hoplocoryphinae & Hoplocorypha sp. & $\begin{array}{l}\text { Subsaharan Africa and Mada- } \\
\text { gascar / ATR }\end{array}$ & $\begin{array}{l}\text { fresh material, preserved in } \\
80 \% \text { EtOH }\end{array}$ & $\mathrm{cW}$ & \\
\hline Thespidae: Oligonicinae & $\begin{array}{l}\text { Bactromantis tolteca (Saussure \& Zehntner, } \\
\text { 1894) }\end{array}$ & $\begin{array}{l}\text { Central America, southern North } \\
\text { America / NR, NAR }\end{array}$ & dry & MNHUB & \\
\hline Thespidae: Oligonicinae & Oligonicinae sp. & Central America / NR & & $\mathrm{CW}$ & \\
\hline Thespidae: Oligonicinae & Pogonogaster tristani Rehn, 1918 & $\begin{array}{l}\text { northern South America and } \\
\text { Central America / NR }\end{array}$ & dry & MNHUB & \\
\hline Thespidae: Oligonicinae & Thesprotia graminis (Scudder, 1877) & $\begin{array}{l}\text { Central and South America, } T \text {. } \\
\text { graminis also in North America } \\
\text { / NR, NAR }\end{array}$ & $\begin{array}{l}\text { fresh material, preserved in } \\
80 \% \text { EtOH }\end{array}$ & $\mathrm{CW}$ & \\
\hline
\end{tabular}


Tab. 2 (continued): Species studied in the present analysis.

\begin{tabular}{|c|c|c|c|c|c|}
\hline Thespidae: Oligonicinae & $\begin{array}{l}\text { Thesprotia macilenta Saussure \& Zehntner, } \\
1894\end{array}$ & $\begin{array}{l}\text { Central and South America, } T \text {. } \\
\text { graminis also in North America } \\
\text { I NR, NAR }\end{array}$ & dry & MNHUB & \\
\hline Thespidae: Oligonicinae & $\begin{array}{l}\text { Thrinaconyx kirschianus Saussure \& } \\
\text { Zehntner, } 1894\end{array}$ & $\begin{array}{l}\text { South and Central America, } \\
\text { North America / NR, NAR }\end{array}$ & dry & MNHUB & \\
\hline Thespidae: Haaniinae & Astape denticollis Stảl, 1877 & South-East Asia / OR & dry & NHMW & \\
\hline Thespidae: Haaniinae & Haania lobiceps (De Haan, 1842) & South-East Asia / OR & dry & NHMW & \\
\hline $\begin{array}{l}\text { Iridopterygidae: Tropido- } \\
\text { mantinae }\end{array}$ & Enicophlebia pallida Westwood, 1889 & Madagascar / ATR & dry & MNHUB & \\
\hline $\begin{array}{l}\text { Iridopterygidae: Tropido- } \\
\text { mantinae }\end{array}$ & Tropidomantis (Eomantis) sp. & South-East Asia / OR & $\begin{array}{l}\text { fresh material, preserved in } \\
80 \% \mathrm{EtOH}\end{array}$ & $\mathrm{CW}$ & \\
\hline $\begin{array}{l}\text { Iridopterygidae: Tropido- } \\
\text { mantinae }\end{array}$ & Tropidomantis tenera (Stål, 1860) & South-East Asia / OR & $\begin{array}{l}\text { fresh material, preserved in } \\
80 \% \mathrm{EtOH}\end{array}$ & $\mathrm{cW}$ & \\
\hline Mantidae: Orthoderinae & Orthodera novaezealandiae (Colenso, 1882) & Australia, New Zealand / AR & $\begin{array}{l}\text { fresh material, preserved in } \\
80 \% \mathrm{EtOH}\end{array}$ & CW & \\
\hline Mantidae: Oxyothespinae & Oxyothespis senegalensis Saussure, 1870 & Africa up to Iran / ATR, PR & dry & MNHUB & \\
\hline Mantidae: Angelinae & Euchomenella sp. & South-East Asia / OR & $\begin{array}{l}\text { fresh material, preserved in } \\
80 \% \mathrm{EtOH}\end{array}$ & CW & \\
\hline Mantidae: Angelinae & Leptocola phthisica (Saussure, 1869) & Subsaharan Africa / ATR & dry & MNHUB & \\
\hline Mantidae: Angelinae & Stenopyga ziela Roy, 1963 & Subsaharan Africa / ATR & dry & MNHUB & \\
\hline Mantidae: Compsothespinae & Compsothespis anomala Saussure, 1872 & Subsaharan Africa / ATR & dry & MNHUB & \\
\hline Mantidae: Chroicopterinae & Entella sp. & Subsaharan Africa / ATR & $\begin{array}{l}\text { fresh material, preserved in } \\
80 \% \text { EtOH }\end{array}$ & CKS & \\
\hline Mantidae: Chroicopterinae & Ligaria sp. & Subsaharan Africa / ATR & $\begin{array}{l}\text { fresh material, preserved in } \\
80 \% \mathrm{EtOH}\end{array}$ & CW/ CKS & \\
\hline Mantidae: Chroicopterinae & Ligariella trigonalis (Saussure, 1899) & Subsaharan Africa / ATR & dry & MNHUB & \\
\hline Mantidae: Chroicopterinae & Parentella major Giglio-Tos, 1915 & Subsaharan Africa / ATR & dry & MNHUB & \\
\hline Mantidae: Chroicopterinae & Chroicoptera Ionga Giglio-Tos, 1915 & southern Africa / ATR & dry & MNHUB & o holotype \\
\hline Mantidae: Dystactinae & Dystacta alticeps (Schaum, 1853) & Subsaharan Africa / ATR & dry & MNHUB & \\
\hline Mantidae: Amelinae & Ameles decolor (Charpentier, 1825) & $\begin{array}{l}\text { northern Africa, southern } \\
\text { Europe / PR }\end{array}$ & $\begin{array}{l}\text { fresh material, preserved in } \\
80 \% \mathrm{EtOH}\end{array}$ & $\mathrm{CW}$ & \\
\hline Mantidae: Amelinae & Ameles gracilis (Brullé, 1840) & $\begin{array}{l}\text { northern Africa, southern } \\
\text { Europe / PR }\end{array}$ & $\begin{array}{l}\text { fresh material, preserved in } \\
80 \% \mathrm{EtOH}\end{array}$ & CW & \\
\hline Mantidae: Amelinae & Elaea marchali (Reiche \& Fairmaire, 1847) & Africa up to Iran / PR, ATR & $\begin{array}{l}\text { fresh material, preserved in } \\
80 \% \mathrm{EtOH}\end{array}$ & CW & \\
\hline Mantidae: Amelinae & Gimantis sp. & South-East Asia / OR & $\begin{array}{l}\text { fresh material, preserved in } \\
80 \% \mathrm{EtOH}\end{array}$ & $\mathrm{CW}$ & \\
\hline Mantidae: Amelinae & Gonypeta brigittae Kaltenbach, 1994 & South-East Asia & $\begin{array}{l}\text { fresh material, preserved in } \\
80 \% \mathrm{EtOH}\end{array}$ & $\mathrm{CW}$ & \\
\hline Mantidae: Amelinae & Pseudoyersinia betancuriae Wiemers, 1993 & Fuerteventura / PR & $\begin{array}{l}\text { fresh material, preserved in } \\
80 \% \mathrm{EtOH}\end{array}$ & CW & \\
\hline Mantidae: Mantinae & Archimantis gracilis Milledge, 1997 & Australia / AR & $\begin{array}{l}\text { fresh material, preserved in } \\
80 \% \mathrm{EtOH}\end{array}$ & CW & \\
\hline Mantidae: Mantinae & Archimantis sobrina Saussure, 1872 & Australia / AR & $\begin{array}{l}\text { fresh material, preserved in } \\
80 \% \mathrm{EtOH}\end{array}$ & $\mathrm{CW}$ & \\
\hline Mantidae: Mantinae & Mantis religiosa (Linnaeus, 1758) & $\begin{array}{l}\text { cosmopolitic / PR, NAR, ATR, } \\
\text { OR, AR }\end{array}$ & $\begin{array}{l}\text { fresh material, preserved in } \\
80 \% \mathrm{EtOH}\end{array}$ & $\mathrm{CW}$ & \\
\hline Mantidae: Mantinae & Plistospilota guineensis Roy, 1965 & Subsaharan Africa / ATR & $\begin{array}{l}\text { fresh material, preserved in } \\
80 \% \mathrm{EtOH}\end{array}$ & $\mathrm{cW}$ & \\
\hline Mantidae: Mantinae & Polyspilota aeruginosa (Goeze, 1778) & $\begin{array}{l}\text { Subsaharan Africa including } \\
\text { Madagascar, the Seychelles and } \\
\text { the Cape Verde / ATR }\end{array}$ & $\begin{array}{l}\text { fresh material, preserved in } \\
80 \% \mathrm{EtOH}\end{array}$ & $\mathrm{CW}$ & \\
\hline Mantidae: Mantinae & Rhombodera basalis (De Haan, 1842) & $\begin{array}{l}\text { South-East Asia reaching Austra- } \\
\text { lian Region / OR, AR }\end{array}$ & $\begin{array}{l}\text { fresh material, preserved in } \\
80 \% \mathrm{EtOH}\end{array}$ & $\mathrm{CW}$ & \\
\hline Mantidae: Mantinae & $\begin{array}{l}\text { Sphodromantis baccetti La Greca \& } \\
\text { Lombardo, } 1987\end{array}$ & Spain, Africa to Iran / PR, ATR & $\begin{array}{l}\text { fresh material, preserved in } \\
80 \% \text { EtOH }\end{array}$ & CW & \\
\hline Mantidae: Mantinae & Tamolanica tamolana (Brancsik, 1897) & $\begin{array}{l}\text { Australia and eastern South-East } \\
\text { Asia / AR, OR }\end{array}$ & $\begin{array}{l}\text { fresh material, preserved in } \\
80 \% \mathrm{EtOH}\end{array}$ & $\mathrm{cW}$ & \\
\hline Mantidae: Mantinae & Tisma pauliani Roy, 2005 & Madagascar / ATR & $\begin{array}{l}\text { fresh material, preserved in } \\
80 \% \text { EtOH }\end{array}$ & CW & \\
\hline
\end{tabular}


Tab. 2 (continued): Species studied in the present analysis.

\begin{tabular}{|c|c|c|c|c|c|}
\hline Mantidae: Miomantinae & Bolivaria sp. & $\begin{array}{l}\text { South-Eastern Europe up to } \\
\text { India, Kazakhstan and Mongolia } \\
\text { / PR }\end{array}$ & $\begin{array}{l}\text { fresh material, preserved in } \\
80 \% \text { EtOH }\end{array}$ & $\mathrm{CW}$ & \\
\hline Mantidae: Miomantinae & Parasphendale affinis Giglio-Tos, 1915 & Subsaharan Africa / ATR & $\begin{array}{l}\text { fresh material, preserved in } \\
80 \% \text { EtOH }\end{array}$ & $\mathrm{CW}$ & \\
\hline Mantidae: Miomantinae & Rivetina sp. & $\begin{array}{l}\text { northern Africa and southern } \\
\text { Europe, up to Pakistan and } \\
\text { Tajikistan / PR }\end{array}$ & $\begin{array}{l}\text { fresh material, preserved in } \\
80 \% \text { EtOH }\end{array}$ & $\mathrm{CW}$ & \\
\hline Mantidae: Miomantinae & Taumantis sigiana (Giglio-Tos, 1911) & Subsaharan Africa / ATR & $\begin{array}{l}\text { fresh material, preserved in } \\
80 \% \text { EtOH }\end{array}$ & $\mathrm{CW}$ & \\
\hline Mantidae: Stagmatopterinae & Oxyopsis gracilis Giglio-Tos, 1914 & South America / NR & $\begin{array}{l}\text { fresh material, preserved in } \\
80 \% \text { EtOH }\end{array}$ & $\mathrm{CW}$ & \\
\hline Mantidae: Stagmatopterinae & Stagmatoptera hyaloptera (Perty, 1832) & South and Central America / NR & $\begin{array}{l}\text { fresh and dry material, preserved } \\
\text { (softened) in 80\% EtoH }\end{array}$ & $\mathrm{CW}$ & \\
\hline Mantidae: Vatinae & Ambivia undata (Fabricius, 1793) & South-East Asia / OR & $\begin{array}{l}\text { fresh material, preserved in } \\
80 \% \text { EtOH }\end{array}$ & $\mathrm{CW}$ & \\
\hline Mantidae: Vatinae & Heterochaeta strachani (Kirby, 1904) & Africa to Saudi Arabia / PR, ATR & $\begin{array}{l}\text { fresh material, preserved in } \\
80 \% \mathrm{EtOH}\end{array}$ & $\operatorname{CSB}$ & \\
\hline Mantidae: Vatinae & Heterochaeta bernardii Roy, 1973 & Africa to Saudi Arabia / PR, ATR & $\begin{array}{l}\text { dry, softened and stored in } 80 \% \\
\text { EtoH }\end{array}$ & $\mathrm{CW}$ & \\
\hline Mantidae: Vatinae & Popa spurca spurca Stăl, 1856 & $\begin{array}{l}\text { Subsaharan Africa and Mada- } \\
\text { gascar / ATR }\end{array}$ & $\begin{array}{l}\text { fresh material, preserved in } \\
80 \% \text { EtOH }\end{array}$ & $\mathrm{CW}$ & \\
\hline Mantidae: Vatinae & Popa spurca crassa Giglio-Tos, 1917 & $\begin{array}{l}\text { Subsaharan Africa and Mada- } \\
\text { gascar / ATR }\end{array}$ & $\begin{array}{l}\text { fresh material, preserved in } \\
80 \% \text { EtOH }\end{array}$ & $\mathrm{CW}$ & \\
\hline Mantidae: Vatinae & Vates weyrauchi Beier, 1958 & Central and South America / NR & $\begin{array}{l}1 \text { pair fresh material, preserved } \\
\text { in } 80 \% \text { EtOH; } 1 \text { pair dry, softened } \\
\text { and stored in } 80 \% \text { EtOH }\end{array}$ & $\mathrm{CW}$ & \\
\hline Mantidae: Vatinae & Zoolea lobipes (Olivier, 1792) & South America / NR & dry & MNHUB & \\
\hline Mantidae: Photininae & Orthoderella ornata Giglio-Tos, 1897 & South America / NR & dry & MNHUB & \\
\hline Mantidae: Photininae & Photina laevis Giglio-Tos, 1915 & northern South America / NR & dry & MNHUB & \\
\hline Mantidae: Choeradodinae & Choeradodis rhombicollis (Latreille, 1833) & Central and South America / NR & $\begin{array}{l}\text { fresh material, preserved in } \\
80 \% \mathrm{EtOH}\end{array}$ & $\mathrm{CW}$ & \\
\hline Mantidae: Choeradodinae & Choeradodis stalii Wood-Mason, 1880 & Central and South America / NR & dry, softened in $80 \%$ EtOH & $\mathrm{CW}$ & \\
\hline Mantidae: Deroplatyinae & Deroplatys desiccata Westwood, 1839 & South-East Asia / OR & $\begin{array}{l}\text { fresh material, preserved in } \\
80 \% \mathrm{EtOH}\end{array}$ & $\mathrm{CW}$ & \\
\hline Mantidae: Deroplatyinae & Deroplatys lobata (Guérin-Méneville, 1838) & South-East Asia / OR & $\begin{array}{l}\text { fresh material, preserved in } \\
80 \% \text { EtOH }\end{array}$ & $\mathrm{CW}$ & \\
\hline Mantidae: Deroplatyinae & Brancsikia sp. & Madagascar & $\begin{array}{l}\text { fresh material, preserved in } \\
80 \% \mathrm{EtOH}\end{array}$ & ZMUG & 10 late instar nymph \\
\hline Mantidae: Phyllotheliinae & Ceratocrania macra Westwood, 1889 & South-East Asia & $\begin{array}{l}\text { fresh material, preserved in } \\
80 \% \mathrm{EtOH}\end{array}$ & $\mathrm{CW}$ & \\
\hline Toxoderidae: Toxoderinae & Calamothespis condamini Roy, 1969 & Subsaharan Africa / ATR & dry & MNHUB & \\
\hline Toxoderidae: Toxoderinae & Toxodera denticulata Serville, 1837 & South-East Asia / OR & dry & MNHUB & \\
\hline Toxoderidae: Toxoderinae & Toxodera maculata Ouwens, 1913 & South-East Asia / OR & $\begin{array}{l}\text { dry, softened and stored in } 80 \% \\
\text { EtOH }\end{array}$ & & \\
\hline Sibyllidae: Sibyllinae & Sibylla pretiosa Stål, 1856 & Subsaharan Africa / ATR & $\begin{array}{l}\text { fresh material, preserved in } \\
80 \% \mathrm{EtOH}\end{array}$ & $\mathrm{CW}$ & \\
\hline Empusidae: Blepharodinae & Blepharodes sudanensis Werner, 1907 & eastern Central Africa / ATR & dry & MNHUB & \\
\hline Empusidae: Blepharodinae & Blepharopsis mendica (Fabricius, 1775) & $\begin{array}{l}\text { northern Africa up to India / } \\
\mathrm{PR}, \mathrm{OR}\end{array}$ & $\begin{array}{l}\text { fresh material, preserved in } 80 \% \\
\text { EtoH // dried pair }\end{array}$ & $\begin{array}{l}\text { CW // } \\
\text { MNHUB }\end{array}$ & \\
\hline Empusidae: Blepharodinae & Idolomantis diabolica (Saussure, 1869) & eastern Central Africa / ATR & $\begin{array}{l}\text { fresh material, preserved in } \\
80 \% \text { EtOH }\end{array}$ & $\mathrm{CW}$ & \\
\hline Empusidae: Empusinae & Empusa pennata (Thunberg, 1815) & $\begin{array}{l}\text { southern Europe, Africa up to } \\
\text { India / PR, ATR, OR }\end{array}$ & $\begin{array}{l}\text { fresh material, preserved in } \\
80 \% \mathrm{EtOH}\end{array}$ & $\mathrm{cW}$ & \\
\hline Empusidae: Empusinae & Gongylus gongylodes (Linnaeus, 1758) & South-East Asia / OR & $\begin{array}{l}\text { fresh material, preserved in } \\
80 \% \mathrm{EtOH}\end{array}$ & $\mathrm{CW}$ & \\
\hline Empusidae: Empusinae & Hypsicorypha gracilis (Burmeister, 1838) & $\begin{array}{l}\text { northern Africa to Saudi-Arabia } \\
\text { and Oman / PR }\end{array}$ & $\begin{array}{l}\text { fresh material, preserved in } \\
80 \% \mathrm{EtOH}\end{array}$ & $\mathrm{CW}$ & \\
\hline Empusidae: Empusinae & Idolomorpha lateralis Burmeister, 1838 & $\begin{array}{l}\text { Subsaharan Africa and Mada- } \\
\text { gascar / ATR }\end{array}$ & dry, softened in $80 \%$ EtOH & $\mathrm{CW}$ & \\
\hline Blattaria: Blattidae & 'eriplaneta americana (Linnaeus, 1758) & $\begin{array}{l}\text { cosmopolitic today, usually } \\
\text { Africa / ATR }\end{array}$ & $\begin{array}{l}\text { fresh material, preserved in } \\
80 \% \text { EtOH }\end{array}$ & CW & \\
\hline
\end{tabular}


Tab. 3: Character matrix (characters 1-90: Acanthops - Parentella). 152 morphological characters from the entire exoskeleton were encoded for 122 Mantodea species and Periplaneta americana.

Taxon/Node

111111111122222222223333333333444444444455555555556666666666777777777788888888889 Acanthops falcat Acanthops tuberc Acontista brevip Acromantis sp. Ambivia sp. Ameles decolor Ameles gracilis Amorphoscelis sp. Amphecostephanus Archimantis grac Archimantis sobr Astape denticoll Bactromantis tol Blepharodes suda Blepharopsis men Bolivaria sp. Calamothespis co Callibia diana Caudatoscelis an Ceratocrania mac Ceratomantis sau Chaeteessa valid Choeradodis rhom Choeradodis stal Ciulfina biseria Compsothespis an Creobroter elong Creobroter gemma Deroplatys lobat Deroplatys desic Dystacta alticep Elaea marchali Empusa pennata Enicophlebia pal Entella sp. Epaphrodita dent Eremiaphila sp.1 Eremiaphila sp.2 Euchomenella sp. Galinthias amoen Gimantis sp. Gongylus gongylo Gonypeta brigitt Gyromantis sp. Haania lobiceps Hestiasula brun Heterochaeta ber Heterochaeta str Heteronutarsus a Hoplocorypha sp. Humbertiella sp. Hymenopus corona Hypsicorypha gra Idolomantis diab Idolomorpha dent Iris oratoria Leptocola phthis Ligaria sp.

Ligariella trigo Maculatoscelis Majanga basilari Mantis religiosa Mantoida maya Metallyticus spl Metallyticus fal Odontomantis sp. Oligonicinae sp. Orthodera novaez Orthoderella orn Oxyopsis gracili oxyothespis sene oxypilus distinc Paralygdamia sp. Paramorphoscelis Paraoxypilus sp.1 2?211112?221121222112?2212321122121131211134132211112132?2?2?2123111111122????211222221212 Paraoxypilus sp.2 2?211112?22112122?1?2?22123211221211312111341?2211112132?2?2?21231111111?2????211222221?12 Paraoxypilinae sp.2?211112?22112122???2?22123211221211312111341?3211112132?2?2?21231111111?2????211222221?12 Parasphendale af 2?211112?222221224411211123111321211211111122332111111421?2?2?1111211112332122212122122112 Parentella major 2?211112?2222222???11211123111221211211111122222111221422?1?2?1111111112222122112122222112

$2 ? 211122 ? 221222122221211123111322211211111121334211121421 ? 2 ? 1 ? 1111211112442266312122122112$ 2?211122?22122212???1211123111322211211111121?34211121421?2?1?1111211112?422?6312122122?12 ?2111212221222222?1???212311122121121111112222311122132?1?2?11111211112322344112122222112 (3211131221122122??11211123111321211211111122332111121421?2?2?1111211112222333312122222111 13211132?211231222?112111231113222112121111223?211111142??2?2?11111111122?224?1121222221?] 2?211112?222222223212?11123111221211211111122222111111427?1?2?1111211112222133122122222112 2?211112?222222222212?11123111221211211111122222111111427?2?2?1111211112222133112122222112 2?211122?221121223?12?221132112212114111224????????2111??????224?11111???????112222112?2 11211112 2221222???1????12311122121??????1?????3?????14????????1???1?1?????4?????12222???2 2?211122?222221226511211123111322211311111122332111111425?2?2?1111111112332133212122221112 2?211122?22222122??11211123111322211311111122332111111425?2?2?1111111112432133212122221112 2?111132?22112122112??11123211221211312111122222111221424?2?2?1122111114114?11211222211222 2?211112?22112122??11?121231112232114111111222?2111221426?2?2?11221111121?4?1?211?22??1??2 11211131121222222325 ??11123111323211211111132333211111422?2?1?1111211122332133112122222112 112111311211222224351112123121323211212111132333211111422 ? ? 1 ?1111211122552254112122222111 2?211122?221231222211211123111221211211111122332111111426?1?2?1111211112232133112122221112 2?211122?2??12212???2?11123111321211211111222?3411121132?2?2?21111213112?321?2222222222?12 11211121222122222 ? 21 ?? 1112311122121111111112133331112132 ? 1 ??21111211122342355312122222211 2?211122?2211212?3?12?221132112212114111224????????2111??????224?11111????????112222?1?22 122111313212222222 ?212111231113212114111111223?2111111422?2?2?11111111124?214?1??1222222?1 11111131121212222 ? 311212123211121211211111122222111211421 ?2?2?1111111112222233312122212112 2?211122?22123121?3?112?112211221211111111121?3412111122??????111121?115?331?2222111221?12 2?211122?21223222??112111231213212112111111223?2111111421?2?2?11111111123?224?1121222221?2 2?211122?21223222??11211123121321211211111122332111211421?2?2?1111111112332133112222222112 2?211122?221221224?11?121231113212113111111223?211111132?2?2?211111111122?212?12112222?2?2 2?211112?222221121112?111231113212114111116?211211122132?2?1?2224?11111???????11122122112 13211121221121322 ?221211123211222211211111122332111111422?2?2?1111211122342344112122222221 132111212211213223 ?21211123211222211211111122332111111422?2?2?1111211122332244112122222221 2?211112?22222222??11211123121323211311111122332111111426?2?2?1111111112442133212122222111 2?211212?222222225511211123121323211311111122332111111426?2?2?1111111112332133212122222111 2?211112?222222225?11211123111321211211111122332111111421?2?2?1111211112222133112122221112 2?211112?222122215411211123211221211211111122332111121423?2?2?1111211112222133222122222112 112111311212122223241211123111321111312111132333221111422 ? ? 1 ?111111122452255312122121111 2?211121122212122?3???11123111221211211111122?3211111132?1?2?21111211112?322?4312222221?12 2?211112?22222222???1211123211221211211111122?22111121427?2?2?1111211112?221?2222122222?22 2?211112?2211212???1??111231113212112111111223?3111111421?2?1?11111111122?224?3??2222111?1 2?211112?222222223312?22113211221211212111112442111221423?1?2?1111211112332522212122222112 2?211112?22222222?212?22113211221211212111112?42111221423?2?2?1111211112?325?2212122222?12 2?211122?221231224?11211123111322211411111122332121111426?2?2?1111111112332132212122221112 ?11121221121312??11211123111321211211111122222111121421?2?2?111121111233234411212222222 2?211112?222222225411211123211221211211111122222111221422?2?1?1111211112222133311122211112 112111311212222224241211123121322111211111132554221111422 ? 1 ? ?11112111226622661?2122211221 2?211112?22212222???1211123111221211211111122332111121427?2?2?1111211112222133112122222112 2?111112?22112122??12?2112321122121131211134133211112132?2?2?2123111111123????211222211112 2?111132?22112122111??11123111321211312111122?21111221424?2?2?1122111114114511212222211222 11211132 ?21212222???1211123211122211211111122222111121426?2?1?1111211112222334312122222221 2?211122?222221124?111111231113212113121111223?3131221422?2?2?11111111125?253?2121222221?1 2?211122?222221224311111123111321211312111122323131221422?2?2?1111111112542522212122222111 2?211122?222222222?1????113211221211212111112332111221423?1?2?1111211112342512212122222222 2?2111???22112122111121112311132121141111112222211122132?2?1?21111111112222122212222211112 2?211122?221121225511212123211321221211111122332111111422?2?2?1111211112222133112122222112 132111212222213122321211123111223211212111122332211111422 ? ? 1 ? 1111211122452266312122222221 11211131121222222 ?241211123111321111411111132?43221111422?2?1?1111111112?422?6312122211?22 11211131121222222 ? 351211123121323111212111132553221111426 ? ? 1 ? 111111122552166312122122111 112111311212222224331211123111321111411111132443221111421 ?2?1?1111111112442256312122221112 2?211122?222222223311211123111321211211111122333131111422?2?2?1111211112332144112122222112 2?211112?22122122???1212123111321211411111122?32111221426?1?2?1111111112?321?2222122211?12 2?211112?222222224211211123211221211211111122222111121427?2?2?1111211112222122222122222112 2?211112?22222222?2?????123211221211211111122?22111121427?2?2?1111211112?221?2212122221?12 2?211122?22112122?4?2?221133112212114111224????????2111??????224 ?11111 ???????11222211?22 ? 211122?22112122??11211123111321211211111122332111111421?2?2?1111211112223122212122221112 2?211122?221221212312?2211221122121111121111133211122131?3?2?21111211112221122211122222112 2?211122?121221225512?2111221122121111211111222211212112????? 1111211112222122122122221112 2?211122?12122122?5?2?21112211221211112111112?3211212112??????1111211112?221?2122122221?12 2?211122?22112122??11212123111221211211111122222111121427?2?2?1111211112222344312122221222 2?211122?2212312????1112123111221211211111122222111221426?2?2?1122111114114?11211222211112 2?211112?212232223312?11123111321211211111122332111111422?2?2?1111211112332144112122222222 2?211122?22212122551121112311132121121111112233311111132?2?2?21111111112442144112222222112 2?211112?222221223211211123111321211211111122332111111422?2?2?1111111112332133212222222112 2?211112?22122112??1??121231113212113111111222?211111132?2?2?211111111122?213?1211221221?2 11111131121212222 ? 311211123211121211211111122222111221426?2?2?1111211112222422112122222112 (1) ?211122?2211212?3?12?2211221122121131211255?11?????2112??????224?11221????????21222222112 
Tab. 3 (continued): Character matrix (characters 1-90: Perlamantis - Zoolea / Periplaneta).

Taxon/Node

Perlamantis alli Photina laevis Phyllocrania par Plistospilota gu Pogonogaster tri Polyspilota aeru Popa spurca cras Popa spurca spur Pseudocreobotra 0 Pseudocreobotra w Pseudogalepsus $n$ Pseudoharpax sp.

Pseudoyersinia be Pyrgomantis jone Raptrix fusca

Rhombodera basal Rivetina sp. Sibylla pretiosa Sphodromantis ba Stagmatoptera hy Stenomantis nova Stenopyga ziela Tamolanica tamol Tarachodes sp. 1 Tarachodes sp. 2 Tarachodes sp. 3 Tarachodula pant Taumantis sigian Theopompa sp.1 Theopompa sp. 2 Theopompa tosta Theopropus elega Thesprotia grami Thesprotia macil Thrinaconyx kirs Tisma pauliani Tithrone roseipe Toxodera denticu Toxodera maculat Tropidomantis sp. Tropidomantis te Vates weyrauchi zoolea lobipes

Periplaneta amer

1111111111222222222233333333334444444444555555555566666666667777777777788888888889 123456789012345678901234567890123456789012345678901234567890123456789012345678901234567890

2?211122?221121223?12?2211221122121131211255?1??????2112??????224?11221???????212222221?2 2?211112?2212222????1211123111321211211111122?33111111422?2?2?1111211112?322?4112122222?12 12211122 ?212222224221211123121321211212111122333111111421?2?2?1111211112342344312122222221 2?211122?212222226611211123111323211211111122332111111424?2?2?1111211112332133212122221112 2?211112?22122122?1?12121231213212114121111?2?2211122132?2?2?21122111113?14??1211222111?12 2?211112?222222225511211123111322211211111122332111111421?2?2?1111211112322133212122221112 2?211122?222131224311211123111322211212111122332111111421?2?2?1111211112223133112122222211 2?211122?22213122?311211123111322211212111122332111111421?2?2?1111211112223133112122222211 3211121221111322222121112321112221121211112222231121132 ? ? 2?11111211122332345312122222221 13211121221111322222121112322112221121211112222231121132 ? 1 ? ? 11111211122332354312122222211 2?211112?222222223?121221231113212212111111222?2111121422?2?2?11112111122?213?1221222221?2 2?111121221221312212??11123111221211211111122222111111421?2?2?1111211112232333112122222221 2?211112?222222222212?11123111221211211111122222131121422?2?2?1111211112222133122122122112 11211312 ?222222223312?22123111321221211111112232111121421?2?2?1111211112332144112122222112 2?2111212221222223?2??1?1231112212111111111223331111?132?1?1?21111211122332344312122222112 2?211122?22223222??11211123121322211211111122332111111422?2?2?1111211112332133112122222112 2?211122?21122222?3?1211123111221211211111122?22111111426?2?2?1111111112?221?32121222???12 11211122 ?22112122?311211123111321211211111122332121111427?2?2?1111211112333122212122211111 2?211122?222232224411211123111322211211111122332111111424?2?2?1111211112332133112122222112 2?211122?22222222???1211123111322211311111122332111211424?2?2?1111111112332133112222222112 2?211112?22222122441??1112311132121131111112233212121132?2?1?21111111112223122222122211222 2?211112?22122122??112121231113212113111111223?2111111421?2?2?11111111123?213?1121222212?2 2?211222?212232225?11211123121322211211111122332111111422?2?2?1111211112332133112122222112 2?211112?222222224412?22123111321221211111112222111111427?2?2?1111211112332144122122222112 2?211112?222222224312?22123111221221211111112332111111427?2?2?1111211112332144122122222112 2?211112?22222222?312122123111221221211111112222111111427?2?2?1111211112332244122122222112 2?211112?222222225412?22123111221221211111112222111111427?2?2?1111211112332144122122222112 2?211122?22222222???1211123111321211211111122?321111?1421?2?1?1111211112?221?3212122222?12 2?211122?221121225?112111232112212212111111123?2111111422?2?2?11112111122?213?1121222221?2 2?211112?221121225?112111232112212212111111123?2111111422?2?2?11112111122?213?1121222221?2 2?211112?22112122651??11123211221221211111112332111111422?2?2?1111211112222133112122222112 11111121222122222 ?21121112311122321121111112233221121132?2?2?11111111122442356312122222221 2?211112?221121224?112121231113232114111111?222111??2132?1?2?21122111114114?11211222111112 2?211112?221121222?1??121231113232114111111222?11???2132?1?2?211221111141?4?1?211?22??1??? 2?211122?221121223?1??111231112212113111111222?2111221421?2?2?11221111131?4?1?2112222111?2 2?211112?222222225?11211123111321211311111122332111111424?2?2?1111111112332133112122221112 2?211112?2211212???112121231112212112111111223331111?132?1?2?21111211122332144312122221112 2?2111212211221123?1111212311132121121211122233412121132?1?2?21111213113252523222122122111 2?211121221122112?2?1112123111321211212111222?341?121132?1?2?21111213112?225?2222122122?11 2?211112?22222122431??1112311122121121111112222211111132?2?2?11111211112223133112222221112 2?211112?22222122331121112311122121121111112233211111132?2?2?11111211112223133112222221112 2?111132?21112122?221211123111323211211111122332111211424?2?2?1111211112332134112122222221 2?111122?21122222??22211123111322211311111122332111111424?2?2?11111111124422331?2122211111 
Tab. 3 (continued): Character matrix (characters 91-152: Acanthops - Parentella).

11111111111111111111111111111111111111111111111111111 99999999900000000001111111111222222222233333333334444444444555 Taxon/Node 12345678901234567890123456789012345678901234567890123456789012

Acanthops falcat Acanthops tuberc Acontista brevip Acromantis sp. Ambivia sp. Ameles decolor Ameles gracilis Amorphoscelis sp. Amphecostephanus Archimantis grac Archimantis sobr Astape denticoll Bactromantis tol Blepharodes suda Blepharopsis men Bolivaria sp. Calamothespis co Callibia diana Caudatoscelis an Ceratocrania mac Ceratomantis sau Chaeteessa valid Choeradodis rhom Choeradodis stal Ciulfina biseria Compsothespis an Creobroter elong Creobroter gemma Deroplatys lobat Deroplatys desic Dystacta alticep Elaea marchali Empusa pennata Enicophlebia pal Entella sp. Epaphrodita dent Eremiaphila sp. Eremiaphila sp.2 Euchomenella sp. Galinthias amoen Gimantis sp.

Gongylus gongylo Gonypeta brigitt Gyromantis sp.

Haania lobiceps Hestiasula brunn Heterochaeta ber Heterochaeta st Heteronutarsus Hoplocorypha sp. Humbertiella sp. Hymenopus corona Hypsicorypha gra Idolomantis diab Idolomorpha dent Iris oratoria Leptocola phthis Ligaria sp.

Ligariella trigo Maculatoscelis sp. Majanga basilari Mantis religiosa Mantoida maya Metallyticus spl Metallyticus fal Odontomantis sp. Oligonicinae sp. Orthodera novaez Orthoderella orn Oxyopsis gracili Oxyothespis sene Oxypilus distinc Paralygdamia sp. Paramorphoscelis Paraoxypilus sp.1 ?21112121111112??1?2211121112122????12213213232222211212212122 Paraoxypilus sp.2 ?21112121111??2???2??11???1?????????21321??3??222?12122121?2 Paraoxypilinae sp.?21112121111??2??????11????1?????????21321??3??222?12222121?2 Parasphendale af ?2111112111112122122211222112132213212212123232222211222222145 Parentella major ?2111212111112122122211221?1????????21312??3??222?1122222???

?22111?2111111111122211221212121212111244114142222211212222312 ?221111211111?11112221122121????21211124411??4?2222?12122223?3 ?2111122111111121122211222112221????1224412??32?22211222222122 32111122111111111122111221112 ?32213211211123232222211222222133 3211111211111111?122211222112133????122111132?22???11?????213? ?2111212111112122122211222112121221112213223232222211222222122 ?2111212112112122122211222112221221112223223232222211222222122 ?21112221111?111?122211211112132????122111232?12?22?12????133? ?21????2??1111???122211121?12121????1121?1132?22???11?????233? ?2111112111111121122211222112132223212211123232222211222221244 ?2111112111111121122211222112132223212211123232222211222221244 ?212212211111212?222211121?1???1??????21121??31?22211212112122 ?21111221111122??1222111221121?1????122443122?2????11?????2?3? ?2111212111112112121111222?1?????????2111???32222211222222133 3211121211111211212111122211 ? 132213211211123232222211211122133 ?21112121121?212212211112211212111211?223112232222211222222122 ?211112211111?12212211122211????2?3?1?2?111??3??222?12222212?2 32211122111111111122111322?12121????122441241?2222221212122111 ?21112221111??11112221131111????21321221112??3??222?12222213?2 4121112211111211 ?122211222112133????1121?1132?22???11?????213? ?2111122111111111122211222112122212211211123232222211212122133 ?211212211112?11121221122221??????????44 ??????222?12222211?6 ?211112211111211?122111222112133????112111222?22???11?????214? ?211112211111211212211122211213321221121112223222221122222215? ?211212211211112?12211112111??????????24?1232?22???11?????213? ?2111222112111121122211222?1?????????211123232222211222221211 32211122111111111122211222112132213211211123232222211222222123 3221112211111111112221122211?????????2111?3?322222112222221?3 32211112111111111122111122112121212111212123232222211212222145 32111112111111111122111122112121212111212123232222211212222144 ?211111211111212212221122111213221211221211??32?22211222222133 ?2111212111112122122211222112121211111213123232222211222222133 321112121111121121211112221121 ?2213212211113232122211211122133 ?21?112211111?111122211?2??1?????????2?13???2?????11?????2??? ?21111121111??12112221122211????22211221312??3??222?11222221?3 321111121111121??122111221112121????1121?3????22??11?????2?? ?21222221221??12?1?2?????21??1???1???2??113232212111222222111 ?21222221221??12?1?2??????21?????:12??2??11??3??121?12222221?1 ?2111112111112122122211222112132222?12212113232222211222222133 32211122111112112122211322112231223112211122222222211222222123 ?2111122111112112122111221112132212211212113232222211222222123 32111112111112122121211222112132212211211113232122211211222134 ?2111122111112122122211221112122????11212113232222211222222122 ?2111212111111122122111221112122212211212313232222211212212132 ?212212211111212?12221122121?????????21121??31?22211212212121 1211112211111111112221122211 ?????????2111????2?222112222221?? 3221111211211212?122211222112231????122112122?22???11?????124? 32211112112112121122211222112231223112211212222222211222221244 ?21222222221??12???2???????1?????????2??11321?2121?122222 111 ?21112121121122??122111221112221????11233313212222211222222122 ?211111211111112112221122111????212111212123232222211222122145 42211122111112112122111222112132213311211113232222211222222134 ?211121211111?11112121122211????21321121111??2?1222?12222221?3 32111212111111111121211222111133213311211123232222211211222144 ?2111212111111111121211222112132213211211112222122211222222133 ?2111112111111121122211222112231223112212222232222211222222123 ?21111121121??12112221112?11?????????2121??23??222?12222221?? ?21112121111121221222112212121212??112212123232?22211122222122 ?21112?21111??1221?2????21?1?????????21312??3??222?11222221?1 ?21112221111??11112221121111????21321121112??3??222?12222213?2 ?2111112111111112122211222112132213312211123232222211222222144 ?2112222111121111222211122212121212111244123232222211222222122 ?2111222111122112212112311211132112211?44121212222211222222144 ?211122211112?11221211231121????112211?4412??1??222?12222221?4 ?2111122111111111122211222112122212211212123232222221222222123 ?21221221111112??122211121212121????122442122222222?12222221?2 ?2111122111111112122211222112133213311211112222222211222222133 ?2111??211111?122122?11222?1?????????2441????2???11 ????1 ??? ?2111112111112112122111221112?31223112211123232222211222222144 ?211112211?11212?122211222?1??????????2111????????11 ?????1??? ?21111221111112??122211121212121????11213123232222211222212122 ?21112221121??1221?2?1???211?????????2?312??3??222?12222212?3 
Tab. 3 (continued): Character matrix (characters 91-152: Perlamantis - Zoolea / Periplaneta).

11111111111111111111111111111111111111111111111111111 99999999900000000001111111111222222222233333333334444444444555 12345678901234567890123456789012345678901234567890123456789012

Taxon/Node ?211122211111111?122211121112122????122111232?22???21?????222? Perlamantis alli ?211122211111111? Photina laevis ?21111?21111??121122211222?1?????????24421??2??222?1222222??? Phyllocrania par 41111112111111111122211221111131213112212113232222211212222133
Plistospilota gu Pogonogaster tri ?21111221111??2???????????1?????????2?431??3??222?12112121?2 Polyspilota aeru ?2111112111112112122211322112133213312211122222222211222222145 Popa spurca cras 41211112111112122122211222112131213111212113232222211222222133 Popa spurca spur 41211112111112122122211122112131223111212113232222211222222133 Pseudocreobotra o 32211112111112112122111221112132213311211123232222211211122133 Pseudocreobotra w 32211112111112112122111221112132213211211123232222211211122134 Pseudogalepsus $n$ ?211112211111212?122211222112121????1121?1122?22???11????123? Pseudoharpax sp. 32211122111112112122211222112132213212211123232222211222222133 Pseudoyersinia be ?21112121121??12???????????????????23312323222221122222 122 Pyrgomantis jone ?2111122111111122122211121112121221?11212123232222211222221233 Raptrix fusca ?211112211111111?122211221212231223?11244123232222221222222122 Rhombodera basal ?2111122111112112121211222112133213311211123232222211222222155 Rivetina sp. ?21????211?11?12212211122211????22211221231??2??212?12222221?2 Sibylla pretiosa 42111112111112112122211122112132213212211123231222211211122145 Sphodromantis ba ?2111112111112112121211222112133213211211123232222211222222144 Stagmatoptera hy ?211111211111211212211122211213321331221112??322222112222221?5 Stenomantis nova ?2111122112121121122211221?1?????????211222232222211222222133 Stenopyga ziela ?211112211211212?122211222?1?????????2121?3?32?????1???????? Tamolanica tamol ?211111211111211212121122211213321331121112323222221122222214? Tarachodes sp.1 ?2111222111111122122211222112121221111213113232222211222221233 Tarachodes sp.2 ?21112221111111221222112221121212?11112131132322222112222112?3 Tarachodes sp. 3 ?2111222111111122122211222112121??1?12213113232222211222211233 Tarachodula pant ?211112211111112212221122?112121221111213123232222211222221233 Taumantis sigian ?211111211111?12112211122111????21221221222??2??222?12222221?5 Theopompa sp.1 ?211111211111211?122111221112122????112111232?22???11?????216? Theopompa sp.2 ?211111211111211?122111221112132????112111232?22???11?????216? Theopompa tosta ?21111121111121111221112211111222122112111????2?22211212122166 Theopropus elega 32211112111111111122111222112132213311211123232222221221222124 Thesprotia grami ?21111221121122??122211222212121????12244312222222211222222133 Thesprotia macil ?211112211?1122??122211222?1?121????12244312?2??????1???????? Thrinaconyx kirs ?21?212211111?2??122?1?1???1?????????244????22???11????11?? Tisma pauliani ?2111112111112112122111222112132213211211123232?22211222222144 Tithrone roseipe ?2111122111112112122211221212121212111244123232222221222222122 Toxodera denticu 2211111211211112212221122211223122311221111??3??222?12222113?5 Toxodera maculat 2211111211211?12212221122211????22311221111??3??222?12222113?5 Tropidomantis sp. ?211112211111111112221112111212?212212211312222222211222222133 Tropidomantis te ?2111122111111111122211121112122212211211312222222211222222133 Vates weyrauchi 41211112111112112122211222112123212311211123232222211222222144 Zoolea lobipes 311112121111111121221112221121322132112111232?22222?1211122145

Periplaneta amer ?21222211112111111?222232222?133?133221??224142222212222222155 
Tab. 4: Character diagnostics for analysis I. All characters were of weight 1.0, 19 characters were parsimony uninformative. Tree length $=1347$ steps; Consistency Index $(\mathrm{CI})=0.1878$; Homoplasy Index $(\mathrm{HI})=0.8122$; CI excluding uninformative characters $=0.1762$; HI excluding uninformative characters $=0.8238$; Retention Index $(\mathrm{RI})=0.6191$; Rescaled Consistency Index $(\mathrm{RC})=0.1163$. Analysis I resulted in 888 equally parsimonious trees after 100 replicates. See Fig. 393 for strict consensus tree and Fig. 394 for maximum likelihood consensus tree.

\begin{tabular}{|c|c|c|c|c|c|c|c|c|c|}
\hline Character & Range & $\begin{array}{c}\text { Min } \\
\text { steps }\end{array}$ & $\begin{array}{l}\text { Tree } \\
\text { steps }\end{array}$ & $\begin{array}{c}\text { Max } \\
\text { steps }\end{array}$ & CI & RI & $\mathrm{RC}$ & HI & $\begin{array}{l}\text { G- } \\
\text { fit }\end{array}$ \\
\hline 1 & 1 & 1 & 10 & 24 & 0.100 & 0.609 & 0.061 & 0.900 & 0.250 \\
\hline 2 & 2 & 2 & 4 & 9 & 0.500 & 0.714 & 0.357 & 0.500 & 0.600 \\
\hline 3 & 1 & 1 & 7 & 10 & 0.143 & 0.333 & 0.048 & 0.857 & 0.333 \\
\hline 4 & 1 & 1 & 1 & 1 & 1.000 & $0 / 0$ & $0 / 0$ & 0.000 & 1.000 \\
\hline 5 & 1 & 1 & 1 & 1 & 1.000 & $0 / 0$ & $0 / 0$ & 0.000 & 1.000 \\
\hline 6 & 2 & 2 & 3 & 3 & 0.667 & 0.000 & 0.000 & 0.333 & 0.750 \\
\hline 7 & 2 & 2 & 24 & 64 & 0.083 & 0.645 & 0.054 & 0.917 & 0.120 \\
\hline 8 & 1 & 1 & 9 & 25 & 0.111 & 0.667 & 0.074 & 0.889 & 0.273 \\
\hline 9 & 2 & 2 & 4 & 11 & 0.500 & 0.778 & 0.389 & 0.500 & 0.600 \\
\hline 10 & 1 & 1 & 1 & 2 & 1.000 & 1.000 & 1.000 & 0.000 & 1.000 \\
\hline 11 & 1 & 1 & 9 & 30 & 0.111 & 0.724 & 0.080 & 0.889 & 0.273 \\
\hline 12 & 1 & 1 & 18 & 57 & 0.056 & 0.696 & 0.039 & 0.944 & 0.150 \\
\hline 13 & 1 & 1 & 16 & 40 & 0.063 & 0.615 & 0.038 & 0.938 & 0.167 \\
\hline 14 & 3 & 3 & 11 & 21 & 0.273 & 0.556 & 0.152 & 0.727 & 0.273 \\
\hline 15 & 2 & 2 & 17 & 63 & 0.118 & 0.754 & 0.089 & 0.882 & 0.167 \\
\hline 16 & 1 & 1 & 7 & 11 & 0.143 & 0.400 & 0.057 & 0.857 & 0.333 \\
\hline 17 & 1 & 1 & 3 & 4 & 0.333 & 0.333 & 0.111 & 0.667 & 0.600 \\
\hline 18 & 5 & 5 & 32 & 53 & 0.156 & 0.438 & 0.068 & 0.844 & 0.100 \\
\hline 19 & 5 & 5 & 28 & 47 & 0.179 & 0.452 & 0.081 & 0.821 & 0.115 \\
\hline 20 & 4 & 4 & 11 & 20 & 0.364 & 0.563 & 0.205 & 0.636 & 0.300 \\
\hline 21 & 1 & 1 & 8 & 28 & 0.125 & 0.741 & 0.093 & 0.875 & 0.300 \\
\hline 22 & 1 & 1 & 5 & 9 & 0.200 & 0.500 & 0.100 & 0.800 & 0.429 \\
\hline 23 & 1 & 1 & 4 & 23 & 0.250 & 0.864 & 0.216 & 0.750 & 0.500 \\
\hline 24 & 1 & 1 & 14 & 36 & 0.071 & 0.629 & 0.045 & 0.929 & 0.188 \\
\hline 25 & 1 & 1 & 1 & 1 & 1.000 & $0 / 0$ & $0 / 0$ & 0.000 & 1.000 \\
\hline 26 & 1 & 1 & 2 & 12 & 0.500 & 0.909 & 0.455 & 0.500 & 0.750 \\
\hline 27 & 1 & 1 & 2 & 7 & 0.500 & 0.833 & 0.417 & 0.500 & 0.750 \\
\hline 28 & 2 & 2 & 13 & 34 & 0.154 & 0.656 & 0.101 & 0.846 & 0.214 \\
\hline 29 & 1 & 1 & 9 & 12 & 0.111 & 0.273 & 0.030 & 0.889 & 0.273 \\
\hline 30 & 1 & 1 & 1 & 1 & 1.000 & $0 / 0$ & $0 / 0$ & 0.000 & 1.000 \\
\hline 31 & 2 & 2 & 14 & 57 & 0.143 & 0.782 & 0.112 & 0.857 & 0.200 \\
\hline 32 & 1 & 1 & 1 & 1 & 1.000 & $0 / 0$ & $0 / 0$ & 0.000 & 1.000 \\
\hline 33 & 2 & 2 & 17 & 32 & 0.118 & 0.500 & 0.059 & 0.882 & 0.167 \\
\hline 34 & 1 & 1 & 1 & 5 & 1.000 & 1.000 & 1.000 & 0.000 & 1.000 \\
\hline 35 & 1 & 1 & 2 & 11 & 0.500 & 0.900 & 0.450 & 0.500 & 0.750 \\
\hline 36 & 1 & 1 & 1 & 1 & 1.000 & $0 / 0$ & $0 / 0$ & 0.000 & 1.000 \\
\hline 37 & 3 & 3 & 18 & 44 & 0.167 & 0.634 & 0.106 & 0.833 & 0.167 \\
\hline 38 & 1 & 1 & 1 & 1 & 1.000 & $0 / 0$ & $0 / 0$ & 0.000 & 1.000 \\
\hline 39 & 1 & 1 & 14 & 28 & 0.071 & 0.519 & 0.037 & 0.929 & 0.188 \\
\hline 40 & 1 & 1 & 1 & 1 & 1.000 & $0 / 0$ & $0 / 0$ & 0.000 & 1.000 \\
\hline 41 & 1 & 1 & 1 & 3 & 1.000 & 1.000 & 1.000 & 0.000 & 1.000 \\
\hline 42 & 1 & 1 & 1 & 5 & 1.000 & 1.000 & 1.000 & 0.000 & 1.000 \\
\hline 43 & 5 & 5 & 5 & 13 & 1.000 & 1.000 & 1.000 & 0.000 & 1.000 \\
\hline 44 & 4 & 4 & 7 & 28 & 0.571 & 0.875 & 0.500 & 0.429 & 0.500 \\
\hline 45 & 1 & 1 & 4 & 9 & 0.250 & 0.625 & 0.156 & 0.750 & 0.500 \\
\hline 46 & 4 & 4 & 16 & 37 & 0.250 & 0.636 & 0.159 & 0.750 & 0.200 \\
\hline 47 & 4 & 4 & 19 & 40 & 0.211 & 0.583 & 0.123 & 0.789 & 0.167 \\
\hline 48 & 3 & 3 & 15 & 30 & 0.200 & 0.556 & 0.111 & 0.800 & 0.200 \\
\hline 49 & 2 & 2 & 5 & 14 & 0.400 & 0.750 & 0.300 & 0.600 & 0.500 \\
\hline 50 & 2 & 2 & 9 & 14 & 0.222 & 0.417 & 0.093 & 0.778 & 0.300 \\
\hline 51 & 1 & 1 & 1 & 2 & 1.000 & 1.000 & 1.000 & 0.000 & 1.000 \\
\hline 52 & 1 & 1 & 16 & 30 & 0.063 & 0.483 & 0.030 & 0.938 & 0.167 \\
\hline 53 & 1 & 1 & 14 & 48 & 0.071 & 0.723 & 0.052 & 0.929 & 0.188 \\
\hline 54 & 1 & 1 & 1 & 1 & 1.000 & $0 / 0$ & $0 / 0$ & 0.000 & 1.000 \\
\hline 55 & 3 & 3 & 14 & 35 & 0.214 & 0.656 & 0.141 & 0.786 & 0.214 \\
\hline 56 & 1 & 1 & 2 & 4 & 0.500 & 0.667 & 0.333 & 0.500 & 0.750 \\
\hline 57 & 6 & 6 & 32 & 60 & 0.188 & 0.519 & 0.097 & 0.813 & 0.103 \\
\hline 58 & 2 & 2 & 6 & 12 & 0.333 & 0.600 & 0.200 & 0.667 & 0.429 \\
\hline 59 & 1 & 1 & 8 & 8 & 0.125 & 0.000 & 0.000 & 0.875 & 0.300 \\
\hline 60 & 1 & 1 & 4 & 4 & 0.250 & 0.000 & 0.000 & 0.750 & 0.500 \\
\hline 61 & 1 & 1 & 7 & 14 & 0.143 & 0.538 & 0.077 & 0.857 & 0.333 \\
\hline 62 & 1 & 1 & 3 & 6 & 0.333 & 0.600 & 0.200 & 0.667 & 0.600 \\
\hline 63 & 1 & 1 & 1 & 6 & 1.000 & 1.000 & 1.000 & 0.000 & 1.000 \\
\hline 64 & 1 & 1 & 1 & 10 & 1.000 & 1.000 & 1.000 & 0.000 & 1.000 \\
\hline 65 & 3 & 3 & 3 & 18 & 1.000 & 1.000 & 1.000 & 0.000 & 1.000 \\
\hline 66 & 1 & 1 & 1 & 8 & 1.000 & 1.000 & 1.000 & 0.000 & 1.000 \\
\hline 67 & 1 & 1 & 15 & 50 & 0.067 & 0.714 & 0.048 & 0.933 & 0.176 \\
\hline 68 & 1 & 1 & 1 & 1 & 1.000 & $0 / 0$ & $0 / 0$ & 0.000 & 1.000 \\
\hline 69 & 2 & 2 & 2 & 5 & 1.000 & 1.000 & 1.000 & 0.000 & 1.000 \\
\hline 70 & 1 & 1 & 1 & 2 & 1.000 & 1.000 & 1.000 & 0.000 & 1.000 \\
\hline 71 & 1 & 1 & 5 & 14 & 0.200 & 0.692 & 0.138 & 0.800 & 0.429 \\
\hline 72 & 4 & 4 & 7 & 13 & 0.571 & 0.667 & 0.381 & 0.429 & 0.500 \\
\hline 73 & 5 & 5 & 22 & 59 & 0.227 & 0.685 & 0.156 & 0.773 & 0.150 \\
\hline
\end{tabular}


Tab. 4 (continued): Character diagnostics of analysis I.

\begin{tabular}{|c|c|c|c|c|c|c|c|c|c|}
\hline Character & Range & $\begin{array}{r}\text { Min } \\
\text { steps }\end{array}$ & $\begin{array}{r}\text { Tree } \\
\text { steps }\end{array}$ & $\begin{array}{r}\text { Max } \\
\text { steps }\end{array}$ & CI & RI & RC & HI & $\begin{array}{l}\text { G- } \\
\text { fit }\end{array}$ \\
\hline 74 & 5 & 5 & 27 & 61 & 0.185 & 0.607 & 0.112 & 0.815 & 0.120 \\
\hline 75 & 3 & 3 & 8 & 17 & 0.375 & 0.643 & 0.241 & 0.625 & 0.375 \\
\hline 76 & 4 & 4 & 25 & 40 & 0.160 & 0.417 & 0.067 & 0.840 & 0.125 \\
\hline 77 & 5 & 5 & 28 & 53 & 0.179 & 0.521 & 0.093 & 0.821 & 0.115 \\
\hline 78 & 5 & 5 & 24 & 60 & 0.208 & 0.655 & 0.136 & 0.792 & 0.136 \\
\hline 79 & 2 & 2 & 23 & 63 & 0.087 & 0.656 & 0.057 & 0.913 & 0.125 \\
\hline 80 & 1 & 1 & 9 & 24 & 0.111 & 0.652 & 0.072 & 0.889 & 0.273 \\
\hline 81 & 1 & 1 & 7 & 21 & 0.143 & 0.700 & 0.100 & 0.857 & 0.333 \\
\hline 82 & 1 & 1 & 9 & 26 & 0.111 & 0.680 & 0.076 & 0.889 & 0.273 \\
\hline 83 & 1 & 1 & 1 & 2 & 1.000 & 1.000 & 1.000 & 0.000 & 1.000 \\
\hline 84 & 1 & 1 & 1 & 2 & 1.000 & 1.000 & 1.000 & 0.000 & 1.000 \\
\hline 85 & 1 & 1 & 9 & 12 & 0.111 & 0.273 & 0.030 & 0.889 & 0.273 \\
\hline 86 & 1 & 1 & 12 & 19 & 0.083 & 0.389 & 0.032 & 0.917 & 0.214 \\
\hline 87 & 1 & 1 & 20 & 45 & 0.050 & 0.568 & 0.028 & 0.950 & 0.136 \\
\hline 88 & 1 & 1 & 14 & 27 & 0.071 & 0.500 & 0.036 & 0.929 & 0.188 \\
\hline 89 & 1 & 1 & 13 & 22 & 0.077 & 0.429 & 0.033 & 0.923 & 0.200 \\
\hline 90 & 1 & 1 & 12 & 30 & 0.083 & 0.621 & 0.052 & 0.917 & 0.214 \\
\hline 91 & 3 & 3 & 6 & 10 & 0.500 & 0.571 & 0.286 & 0.500 & 0.500 \\
\hline 92 & 1 & 1 & 3 & 6 & 0.333 & 0.600 & 0.200 & 0.667 & 0.600 \\
\hline 93 & 1 & 1 & 5 & 18 & 0.200 & 0.765 & 0.153 & 0.800 & 0.429 \\
\hline 94 & 1 & 1 & 3 & 7 & 0.333 & 0.667 & 0.222 & 0.667 & 0.600 \\
\hline 95 & 1 & 1 & 4 & 11 & 0.250 & 0.700 & 0.175 & 0.750 & 0.500 \\
\hline 96 & 1 & 1 & 11 & 37 & 0.091 & 0.722 & 0.066 & 0.909 & 0.231 \\
\hline 97 & 1 & 1 & 14 & 58 & 0.071 & 0.772 & 0.055 & 0.929 & 0.188 \\
\hline 98 & 1 & 1 & 1 & 1 & 1.000 & $0 / 0$ & $0 / 0$ & 0.000 & 1.000 \\
\hline 99 & 1 & 1 & 1 & 1 & 1.000 & $0 / 0$ & $0 / 0$ & 0.000 & 1.000 \\
\hline 100 & 1 & 1 & 1 & 3 & 1.000 & 1.000 & 1.000 & 0.000 & 1.000 \\
\hline 101 & 1 & 1 & 10 & 18 & 0.100 & 0.471 & 0.047 & 0.900 & 0.250 \\
\hline 102 & 1 & 1 & 1 & 1 & 1.000 & $0 / 0$ & $0 / 0$ & 0.000 & 1.000 \\
\hline 103 & 1 & 1 & 3 & 5 & 0.333 & 0.500 & 0.167 & 0.667 & 0.600 \\
\hline 104 & 1 & 1 & 21 & 47 & 0.048 & 0.565 & 0.027 & 0.952 & 0.130 \\
\hline 105 & 1 & 1 & 4 & 11 & 0.250 & 0.700 & 0.175 & 0.750 & 0.500 \\
\hline 106 & 1 & 1 & 9 & 50 & 0.111 & 0.837 & 0.093 & 0.889 & 0.273 \\
\hline 107 & 1 & 1 & 19 & 40 & 0.053 & 0.538 & 0.028 & 0.947 & 0.143 \\
\hline 108 & 1 & 1 & 3 & 5 & 0.333 & 0.500 & 0.167 & 0.667 & 0.600 \\
\hline 109 & 1 & 1 & 1 & 3 & 1.000 & 1.000 & 1.000 & 0.000 & 1.000 \\
\hline 110 & 1 & 1 & 2 & 10 & 0.500 & 0.889 & 0.444 & 0.500 & 0.750 \\
\hline 111 & 1 & 1 & 18 & 31 & 0.056 & 0.433 & 0.024 & 0.944 & 0.150 \\
\hline 112 & 1 & 1 & 1 & 1 & 1.000 & $0 / 0$ & $0 / 0$ & 0.000 & 1.000 \\
\hline 113 & 1 & 1 & 3 & 4 & 0.333 & 0.333 & 0.111 & 0.667 & 0.600 \\
\hline 114 & 2 & 2 & 20 & 28 & 0.100 & 0.308 & 0.031 & 0.900 & 0.143 \\
\hline 115 & 1 & 1 & 2 & 5 & 0.500 & 0.750 & 0.375 & 0.500 & 0.750 \\
\hline 116 & 1 & 1 & 19 & 41 & 0.053 & 0.550 & 0.029 & 0.947 & 0.143 \\
\hline 117 & 1 & 1 & 5 & 16 & 0.200 & 0.733 & 0.147 & 0.800 & 0.429 \\
\hline 118 & 1 & 1 & 1 & 1 & 1.000 & $0 / 0$ & $0 / 0$ & 0.000 & 1.000 \\
\hline 119 & 1 & 1 & 4 & 4 & 0.250 & 0.000 & 0.000 & 0.750 & 0.500 \\
\hline 120 & 1 & 1 & 6 & 9 & 0.167 & 0.375 & 0.063 & 0.833 & 0.375 \\
\hline 121 & 2 & 2 & 9 & 38 & 0.222 & 0.806 & 0.179 & 0.778 & 0.300 \\
\hline 122 & 2 & 2 & 18 & 47 & 0.111 & 0.644 & 0.072 & 0.889 & 0.158 \\
\hline 123 & 1 & 1 & 2 & 3 & 0.500 & 0.500 & 0.250 & 0.500 & 0.750 \\
\hline 124 & 1 & 1 & 8 & 19 & 0.125 & 0.611 & 0.076 & 0.875 & 0.300 \\
\hline 125 & 2 & 2 & 12 & 37 & 0.167 & 0.714 & 0.119 & 0.833 & 0.231 \\
\hline 126 & 2 & 2 & 19 & 41 & 0.105 & 0.564 & 0.059 & 0.895 & 0.150 \\
\hline 127 & 1 & 1 & 1 & 1 & 1.000 & $0 / 0$ & $0 / 0$ & 0.000 & 1.000 \\
\hline 128 & 1 & 1 & 18 & 39 & 0.056 & 0.553 & 0.031 & 0.944 & 0.150 \\
\hline 129 & 1 & 1 & 1 & 1 & 1.000 & $0 / 0$ & $0 / 0$ & 0.000 & 1.000 \\
\hline 130 & 3 & 3 & 11 & 23 & 0.273 & 0.600 & 0.164 & 0.727 & 0.273 \\
\hline 131 & 3 & 3 & 19 & 57 & 0.158 & 0.704 & 0.111 & 0.842 & 0.158 \\
\hline 132 & 2 & 2 & 13 & 26 & 0.154 & 0.542 & 0.083 & 0.846 & 0.214 \\
\hline 133 & 1 & 1 & 17 & 48 & 0.059 & 0.660 & 0.039 & 0.941 & 0.158 \\
\hline 134 & 3 & 3 & 14 & 22 & 0.214 & 0.421 & 0.090 & 0.786 & 0.214 \\
\hline 135 & 1 & 1 & 2 & 3 & 0.500 & 0.500 & 0.250 & 0.500 & 0.750 \\
\hline 136 & 3 & 3 & 16 & 22 & 0.188 & 0.316 & 0.059 & 0.813 & 0.188 \\
\hline 137 & 1 & 1 & 5 & 6 & 0.200 & 0.200 & 0.040 & 0.800 & 0.429 \\
\hline 138 & 1 & 1 & 1 & 4 & 1.000 & 1.000 & 1.000 & 0.000 & 1.000 \\
\hline 139 & 1 & 1 & 1 & 3 & 1.000 & 1.000 & 1.000 & 0.000 & 1.000 \\
\hline 140 & 1 & 1 & 1 & 1 & 1.000 & $0 / 0$ & $0 / 0$ & 0.000 & 1.000 \\
\hline 141 & 1 & 1 & 1 & 3 & 1.000 & 1.000 & 1.000 & 0.000 & 1.000 \\
\hline 142 & 1 & 1 & 5 & 6 & 0.200 & 0.200 & 0.040 & 0.800 & 0.429 \\
\hline 143 & 1 & 1 & 1 & 1 & 1.000 & $0 / 0$ & $0 / 0$ & 0.000 & 1.000 \\
\hline 144 & 1 & 1 & 1 & 4 & 1.000 & 1.000 & 1.000 & 0.000 & 1.000 \\
\hline 145 & 1 & 1 & 14 & 23 & 0.071 & 0.409 & 0.029 & 0.929 & 0.188 \\
\hline 146 & 1 & 1 & 7 & 10 & 0.143 & 0.333 & 0.048 & 0.857 & 0.333 \\
\hline 147 & 1 & 1 & 10 & 12 & 0.100 & 0.182 & 0.018 & 0.900 & 0.250 \\
\hline 148 & 1 & 1 & 6 & 12 & 0.167 & 0.545 & 0.091 & 0.833 & 0.375 \\
\hline 149 & 1 & 1 & 8 & 23 & 0.125 & 0.682 & 0.085 & 0.875 & 0.300 \\
\hline 150 & 2 & 2 & 9 & 23 & 0.222 & 0.667 & 0.148 & 0.778 & 0.300 \\
\hline 151 & 5 & 5 & 26 & 55 & 0.192 & 0.580 & 0.112 & 0.808 & 0.125 \\
\hline 152 & 5 & 5 & 34 & 63 & 0.147 & 0.500 & 0.074 & 0.853 & 0.094 \\
\hline
\end{tabular}


Tab. 5: Character diagnostics for analysis II. 39 characters have weight 1.0, 113 were reweighted (reweight $=$ RC), 19 characters were parsimony uninformative. Tree length $=175.41238$; Consistency Index $(\mathrm{CI})=0.4411$; Homoplasy Index $(\mathrm{HI})=0.5589 ; \mathrm{CI}$ excluding uninformative characters $=0.3732 ; \mathrm{HI}$ excluding uninformative characters $=0.6268$; Retention Index $(\mathrm{RI})=0.7391$; Rescaled Consistency Index $(\mathrm{RC})=0.3260$. Analysis II resulted in 10 equally parsimonious trees after 1,000 replicates. Strict consensus and maximum likelihood consensus trees were identical (see Fig. 395).

\begin{tabular}{|c|c|c|c|c|c|c|c|c|c|}
\hline Character & Range & $\begin{array}{l}\text { Min } \\
\text { steps }\end{array}$ & $\begin{array}{l}\text { Tree } \\
\text { steps }\end{array}$ & $\begin{array}{c}\text { Max } \\
\text { steps }\end{array}$ & CI & RI & RC & HI & $\begin{array}{l}\text { G- } \\
\text { fit }\end{array}$ \\
\hline 1 & 1 & 1 & 9 & 24 & 0.111 & 0.652 & 0.072 & 0.889 & 0.273 \\
\hline 2 & 2 & 2 & 3 & 9 & 0.667 & 0.857 & 0.571 & 0.333 & 0.750 \\
\hline 3 & 1 & 1 & 8 & 10 & 0.125 & 0.222 & 0.028 & 0.875 & 0.300 \\
\hline 4 & 1 & 1 & 1 & 1 & 1.000 & $0 / 0$ & $0 / 0$ & 0.000 & 1.000 \\
\hline 5 & 1 & 1 & 1 & 1 & 1.000 & $0 / 0$ & $0 / 0$ & 0.000 & 1.000 \\
\hline 6 & 2 & 2 & 3 & 3 & 0.667 & 0.000 & 0.000 & 0.333 & 0.750 \\
\hline 7 & 2 & 2 & 28 & 64 & 0.071 & 0.581 & 0.041 & 0.929 & 0.103 \\
\hline 8 & 1 & 1 & 10 & 25 & 0.100 & 0.625 & 0.063 & 0.900 & 0.250 \\
\hline 9 & 2 & 2 & 2 & 11 & 1.000 & 1.000 & 1.000 & 0.000 & 1.000 \\
\hline 10 & 1 & 1 & 1 & 2 & 1.000 & 1.000 & 1.000 & 0.000 & 1.000 \\
\hline 11 & 1 & 1 & 11 & 30 & 0.091 & 0.655 & 0.060 & 0.909 & 0.231 \\
\hline 12 & 1 & 1 & 19 & 57 & 0.053 & 0.679 & 0.036 & 0.947 & 0.143 \\
\hline 13 & 1 & 1 & 17 & 40 & 0.059 & 0.590 & 0.035 & 0.941 & 0.158 \\
\hline 14 & 3 & 3 & 12 & 21 & 0.250 & 0.500 & 0.125 & 0.750 & 0.250 \\
\hline 15 & 2 & 2 & 14 & 63 & 0.143 & 0.803 & 0.115 & 0.857 & 0.200 \\
\hline 16 & 1 & 1 & 7 & 11 & 0.143 & 0.400 & 0.057 & 0.857 & 0.333 \\
\hline 17 & 1 & 1 & 3 & 4 & 0.333 & 0.333 & 0.111 & 0.667 & 0.600 \\
\hline 18 & 5 & 5 & 34 & 53 & 0.147 & 0.396 & 0.058 & 0.853 & 0.094 \\
\hline 19 & 5 & 5 & 29 & 47 & 0.172 & 0.429 & 0.074 & 0.828 & 0.111 \\
\hline 20 & 4 & 4 & 10 & 20 & 0.400 & 0.625 & 0.250 & 0.600 & 0.333 \\
\hline 21 & 1 & 1 & 7 & 28 & 0.143 & 0.778 & 0.111 & 0.857 & 0.333 \\
\hline 22 & 1 & 1 & 5 & 9 & 0.200 & 0.500 & 0.100 & 0.800 & 0.429 \\
\hline 23 & 1 & 1 & 3 & 23 & 0.333 & 0.909 & 0.303 & 0.667 & 0.600 \\
\hline 24 & 1 & 1 & 17 & 36 & 0.059 & 0.543 & 0.032 & 0.941 & 0.158 \\
\hline 25 & 1 & 1 & 1 & 1 & 1.000 & $0 / 0$ & $0 / 0$ & 0.000 & 1.000 \\
\hline 26 & 1 & 1 & 2 & 12 & 0.500 & 0.909 & 0.455 & 0.500 & 0.750 \\
\hline 27 & 1 & 1 & 2 & 7 & 0.500 & 0.833 & 0.417 & 0.500 & 0.750 \\
\hline 28 & 2 & 2 & 12 & 34 & 0.167 & 0.688 & 0.115 & 0.833 & 0.231 \\
\hline 29 & 1 & 1 & 8 & 12 & 0.125 & 0.364 & 0.045 & 0.875 & 0.300 \\
\hline 30 & 1 & 1 & 1 & 1 & 1.000 & $0 / 0$ & $0 / 0$ & 0.000 & 1.000 \\
\hline 31 & 2 & 2 & 15 & 57 & 0.133 & 0.764 & 0.102 & 0.867 & 0.188 \\
\hline 32 & 1 & 1 & 1 & 1 & 1.000 & $0 / 0$ & $0 / 0$ & 0.000 & 1.000 \\
\hline 33 & 2 & 2 & 18 & 32 & 0.111 & 0.467 & 0.052 & 0.889 & 0.158 \\
\hline 34 & 1 & 1 & 1 & 5 & 1.000 & 1.000 & 1.000 & 0.000 & 1.000 \\
\hline 35 & 1 & 1 & 2 & 11 & 0.500 & 0.900 & 0.450 & 0.500 & 0.750 \\
\hline 36 & 1 & 1 & 1 & 1 & 1.000 & $0 / 0$ & $0 / 0$ & 0.000 & 1.000 \\
\hline 37 & 3 & 3 & 19 & 44 & 0.158 & 0.610 & 0.096 & 0.842 & 0.158 \\
\hline 38 & 1 & 1 & 1 & 1 & 1.000 & $0 / 0$ & $0 / 0$ & 0.000 & 1.000 \\
\hline 39 & 1 & 1 & 16 & 28 & 0.063 & 0.444 & 0.028 & 0.938 & 0.167 \\
\hline 40 & 1 & 1 & 1 & 1 & 1.000 & $0 / 0$ & $0 / 0$ & 0.000 & 1.000 \\
\hline 41 & 1 & 1 & 1 & 3 & 1.000 & 1.000 & 1.000 & 0.000 & 1.000 \\
\hline 42 & 1 & 1 & 1 & 5 & 1.000 & 1.000 & 1.000 & 0.000 & 1.000 \\
\hline 43 & 5 & 5 & 5 & 13 & 1.000 & 1.000 & 1.000 & 0.000 & 1.000 \\
\hline 44 & 4 & 4 & 7 & 28 & 0.571 & 0.875 & 0.500 & 0.429 & 0.500 \\
\hline 45 & 1 & 1 & 4 & 9 & 0.250 & 0.625 & 0.156 & 0.750 & 0.500 \\
\hline 46 & 4 & 4 & 16 & 37 & 0.250 & 0.636 & 0.159 & 0.750 & 0.200 \\
\hline 47 & 4 & 4 & 17 & 40 & 0.235 & 0.639 & 0.150 & 0.765 & 0.188 \\
\hline 48 & 3 & 3 & 14 & 30 & 0.214 & 0.593 & 0.127 & 0.786 & 0.214 \\
\hline 49 & 2 & 2 & 5 & 14 & 0.400 & 0.750 & 0.300 & 0.600 & 0.500 \\
\hline 50 & 2 & 2 & 9 & 14 & 0.222 & 0.417 & 0.093 & 0.778 & 0.300 \\
\hline 51 & 1 & 1 & 1 & 2 & 1.000 & 1.000 & 1.000 & 0.000 & 1.000 \\
\hline 52 & 1 & 1 & 17 & 30 & 0.059 & 0.448 & 0.026 & 0.941 & 0.158 \\
\hline 53 & 1 & 1 & 15 & 48 & 0.067 & 0.702 & 0.047 & 0.933 & 0.176 \\
\hline 54 & 1 & 1 & 1 & 1 & 1.000 & $0 / 0$ & $0 / 0$ & 0.000 & 1.000 \\
\hline 55 & 3 & 3 & 13 & 35 & 0.231 & 0.688 & 0.159 & 0.769 & 0.231 \\
\hline 56 & 1 & 1 & 2 & 4 & 0.500 & 0.667 & 0.333 & 0.500 & 0.750 \\
\hline 57 & 6 & 6 & 32 & 60 & 0.188 & 0.519 & 0.097 & 0.813 & 0.103 \\
\hline 58 & 2 & 2 & 6 & 12 & 0.333 & 0.600 & 0.200 & 0.667 & 0.429 \\
\hline 59 & 1 & 1 & 8 & 8 & 0.125 & 0.000 & 0.000 & 0.875 & 0.300 \\
\hline 60 & 1 & 1 & 4 & 4 & 0.250 & 0.000 & 0.000 & 0.750 & 0.500 \\
\hline 61 & 1 & 1 & 7 & 14 & 0.143 & 0.538 & 0.077 & 0.857 & 0.333 \\
\hline 62 & 1 & 1 & 3 & 6 & 0.333 & 0.600 & 0.200 & 0.667 & 0.600 \\
\hline 63 & 1 & 1 & 1 & 6 & 1.000 & 1.000 & 1.000 & 0.000 & 1.000 \\
\hline 64 & 1 & 1 & 1 & 10 & 1.000 & 1.000 & 1.000 & 0.000 & 1.000 \\
\hline 65 & 3 & 3 & 3 & 18 & 1.000 & 1.000 & 1.000 & 0.000 & 1.000 \\
\hline 66 & 1 & 1 & 1 & 8 & 1.000 & 1.000 & 1.000 & 0.000 & 1.000 \\
\hline 67 & 1 & 1 & 17 & 50 & 0.059 & 0.673 & 0.040 & 0.941 & 0.158 \\
\hline 68 & 1 & 1 & 1 & 1 & 1.000 & $0 / 0$ & $0 / 0$ & 0.000 & 1.000 \\
\hline 69 & 2 & 2 & 2 & 5 & 1.000 & 1.000 & 1.000 & 0.000 & 1.000 \\
\hline 70 & 1 & 1 & 1 & 2 & 1.000 & 1.000 & 1.000 & 0.000 & 1.000 \\
\hline 71 & 1 & 1 & 5 & 14 & 0.200 & 0.692 & 0.138 & 0.800 & 0.429 \\
\hline 72 & 4 & 4 & 6 & 13 & 0.667 & 0.778 & 0.519 & 0.333 & 0.600 \\
\hline 73 & 5 & 5 & 19 & 59 & 0.263 & 0.741 & 0.195 & 0.737 & 0.176 \\
\hline
\end{tabular}


Tab. 5 (continued): Character diagnostics of analysis II.

\begin{tabular}{|c|c|c|c|c|c|c|c|c|c|}
\hline Character & Range & $\begin{array}{l}\text { Min } \\
\text { steps }\end{array}$ & $\begin{array}{l}\text { Tree } \\
\text { steps }\end{array}$ & $\begin{array}{c}\text { Max } \\
\text { steps }\end{array}$ & CI & RI & $\mathrm{RC}$ & $\mathrm{HI}$ & $\begin{array}{l}\text { G- } \\
\text { fit }\end{array}$ \\
\hline 74 & 5 & 5 & 26 & 61 & 0.192 & 0.625 & 0.120 & 0.808 & 0.125 \\
\hline 75 & 3 & 3 & 8 & 17 & 0.375 & 0.643 & 0.241 & 0.625 & 0.375 \\
\hline 76 & 4 & 4 & 24 & 40 & 0.167 & 0.444 & 0.074 & 0.833 & 0.130 \\
\hline 77 & 5 & 5 & 24 & 53 & 0.208 & 0.604 & 0.126 & 0.792 & 0.136 \\
\hline 78 & 5 & 5 & 22 & 60 & 0.227 & 0.691 & 0.157 & 0.773 & 0.150 \\
\hline 79 & 2 & 2 & 23 & 63 & 0.087 & 0.656 & 0.057 & 0.913 & 0.125 \\
\hline 80 & 1 & 1 & 9 & 24 & 0.111 & 0.652 & 0.072 & 0.889 & 0.273 \\
\hline 81 & 1 & 1 & 7 & 21 & 0.143 & 0.700 & 0.100 & 0.857 & 0.333 \\
\hline 82 & 1 & 1 & 10 & 26 & 0.100 & 0.640 & 0.064 & 0.900 & 0.250 \\
\hline 83 & 1 & 1 & 1 & 2 & 1.000 & 1.000 & 1.000 & 0.000 & 1.000 \\
\hline 84 & 1 & 1 & 1 & 2 & 1.000 & 1.000 & 1.000 & 0.000 & 1.000 \\
\hline 85 & 1 & 1 & 9 & 12 & 0.111 & 0.273 & 0.030 & 0.889 & 0.273 \\
\hline 86 & 1 & 1 & 11 & 19 & 0.091 & 0.444 & 0.040 & 0.909 & 0.231 \\
\hline 87 & 1 & 1 & 18 & 45 & 0.056 & 0.614 & 0.034 & 0.944 & 0.150 \\
\hline 88 & 1 & 1 & 14 & 27 & 0.071 & 0.500 & 0.036 & 0.929 & 0.188 \\
\hline 89 & 1 & 1 & 14 & 22 & 0.071 & 0.381 & 0.027 & 0.929 & 0.188 \\
\hline 90 & 1 & 1 & 12 & 30 & 0.083 & 0.621 & 0.052 & 0.917 & 0.214 \\
\hline 91 & 3 & 3 & 4 & 10 & 0.750 & 0.857 & 0.643 & 0.250 & 0.750 \\
\hline 92 & 1 & 1 & 2 & 6 & 0.500 & 0.800 & 0.400 & 0.500 & 0.750 \\
\hline 93 & 1 & 1 & 6 & 18 & 0.167 & 0.706 & 0.118 & 0.833 & 0.375 \\
\hline 94 & 1 & 1 & 3 & 7 & 0.333 & 0.667 & 0.222 & 0.667 & 0.600 \\
\hline 95 & 1 & 1 & 4 & 11 & 0.250 & 0.700 & 0.175 & 0.750 & 0.500 \\
\hline 96 & 1 & 1 & 11 & 37 & 0.091 & 0.722 & 0.066 & 0.909 & 0.231 \\
\hline 97 & 1 & 1 & 17 & 58 & 0.059 & 0.719 & 0.042 & 0.941 & 0.158 \\
\hline 98 & 1 & 1 & 1 & 1 & 1.000 & $0 / 0$ & $0 / 0$ & 0.000 & 1.000 \\
\hline 99 & 1 & 1 & 1 & 1 & 1.000 & $0 / 0$ & $0 / 0$ & 0.000 & 1.000 \\
\hline 100 & 1 & 1 & 1 & 3 & 1.000 & 1.000 & 1.000 & 0.000 & 1.000 \\
\hline 101 & 1 & 1 & 11 & 18 & 0.091 & 0.412 & 0.037 & 0.909 & 0.231 \\
\hline 102 & 1 & 1 & 1 & 1 & 1.000 & $0 / 0$ & $0 / 0$ & 0.000 & 1.000 \\
\hline 103 & 1 & 1 & 3 & 5 & 0.333 & 0.500 & 0.167 & 0.667 & 0.600 \\
\hline 104 & 1 & 1 & 22 & 47 & 0.045 & 0.543 & 0.025 & 0.955 & 0.125 \\
\hline 105 & 1 & 1 & 4 & 11 & 0.250 & 0.700 & 0.175 & 0.750 & 0.500 \\
\hline 106 & 1 & 1 & 12 & 50 & 0.083 & 0.776 & 0.065 & 0.917 & 0.214 \\
\hline 107 & 1 & 1 & 20 & 40 & 0.050 & 0.513 & 0.026 & 0.950 & 0.136 \\
\hline 108 & 1 & 1 & 3 & 5 & 0.333 & 0.500 & 0.167 & 0.667 & 0.600 \\
\hline 109 & 1 & 1 & 1 & 3 & 1.000 & 1.000 & 1.000 & 0.000 & 1.000 \\
\hline 110 & 1 & 1 & 1 & 10 & 1.000 & 1.000 & 1.000 & 0.000 & 1.000 \\
\hline 111 & 1 & 1 & 21 & 31 & 0.048 & 0.333 & 0.016 & 0.952 & 0.130 \\
\hline 112 & 1 & 1 & 1 & 1 & 1.000 & $0 / 0$ & $0 / 0$ & 0.000 & 1.000 \\
\hline 113 & 1 & 1 & 3 & 4 & 0.333 & 0.333 & 0.111 & 0.667 & 0.600 \\
\hline 114 & 2 & 2 & 22 & 28 & 0.091 & 0.231 & 0.021 & 0.909 & 0.130 \\
\hline 115 & 1 & 1 & 2 & 5 & 0.500 & 0.750 & 0.375 & 0.500 & 0.750 \\
\hline 116 & 1 & 1 & 19 & 41 & 0.053 & 0.550 & 0.029 & 0.947 & 0.143 \\
\hline 117 & 1 & 1 & 5 & 16 & 0.200 & 0.733 & 0.147 & 0.800 & 0.429 \\
\hline 118 & 1 & 1 & 1 & 1 & 1.000 & $0 / 0$ & $0 / 0$ & 0.000 & 1.000 \\
\hline 119 & 1 & 1 & 4 & 4 & 0.250 & 0.000 & 0.000 & 0.750 & 0.500 \\
\hline 120 & 1 & 1 & 6 & 9 & 0.167 & 0.375 & 0.063 & 0.833 & 0.375 \\
\hline 121 & 2 & 2 & 10 & 38 & 0.200 & 0.778 & 0.156 & 0.800 & 0.273 \\
\hline 122 & 2 & 2 & 17 & 47 & 0.118 & 0.667 & 0.078 & 0.882 & 0.167 \\
\hline 123 & 1 & 1 & 2 & 3 & 0.500 & 0.500 & 0.250 & 0.500 & 0.750 \\
\hline 124 & 1 & 1 & 9 & 19 & 0.111 & 0.556 & 0.062 & 0.889 & 0.273 \\
\hline 125 & 2 & 2 & 12 & 37 & 0.167 & 0.714 & 0.119 & 0.833 & 0.231 \\
\hline 126 & 2 & 2 & 19 & 41 & 0.105 & 0.564 & 0.059 & 0.895 & 0.150 \\
\hline 127 & 1 & 1 & 1 & 1 & 1.000 & $0 / 0$ & $0 / 0$ & 0.000 & 1.000 \\
\hline 128 & 1 & 1 & 22 & 39 & 0.045 & 0.447 & 0.020 & 0.955 & 0.125 \\
\hline 129 & 1 & 1 & 1 & 1 & 1.000 & $0 / 0$ & $0 / 0$ & 0.000 & 1.000 \\
\hline 130 & 3 & 3 & 10 & 23 & 0.300 & 0.650 & 0.195 & 0.700 & 0.300 \\
\hline 131 & 3 & 3 & 20 & 57 & 0.150 & 0.685 & 0.103 & 0.850 & 0.150 \\
\hline 132 & 2 & 2 & 14 & 26 & 0.143 & 0.500 & 0.071 & 0.857 & 0.200 \\
\hline 133 & 1 & 1 & 21 & 48 & 0.048 & 0.574 & 0.027 & 0.952 & 0.130 \\
\hline 134 & 3 & 3 & 14 & 22 & 0.214 & 0.421 & 0.090 & 0.786 & 0.214 \\
\hline 135 & 1 & 1 & 2 & 3 & 0.500 & 0.500 & 0.250 & 0.500 & 0.750 \\
\hline 136 & 3 & 3 & 16 & 22 & 0.188 & 0.316 & 0.059 & 0.813 & 0.188 \\
\hline 137 & 1 & 1 & 5 & 6 & 0.200 & 0.200 & 0.040 & 0.800 & 0.429 \\
\hline 138 & 1 & 1 & 1 & 4 & 1.000 & 1.000 & 1.000 & 0.000 & 1.000 \\
\hline 139 & 1 & 1 & 1 & 3 & 1.000 & 1.000 & 1.000 & 0.000 & 1.000 \\
\hline 140 & 1 & 1 & 1 & 1 & 1.000 & $0 / 0$ & $0 / 0$ & 0.000 & 1.000 \\
\hline 141 & 1 & 1 & 1 & 3 & 1.000 & 1.000 & 1.000 & 0.000 & 1.000 \\
\hline 142 & 1 & 1 & 5 & 6 & 0.200 & 0.200 & 0.040 & 0.800 & 0.429 \\
\hline 143 & 1 & 1 & 1 & 1 & 1.000 & $0 / 0$ & $0 / 0$ & 0.000 & 1.000 \\
\hline 144 & 1 & 1 & 1 & 4 & 1.000 & 1.000 & 1.000 & 0.000 & 1.000 \\
\hline 145 & 1 & 1 & 15 & 23 & 0.067 & 0.364 & 0.024 & 0.933 & 0.176 \\
\hline 146 & 1 & 1 & 7 & 10 & 0.143 & 0.333 & 0.048 & 0.857 & 0.333 \\
\hline 147 & 1 & 1 & 10 & 12 & 0.100 & 0.182 & 0.018 & 0.900 & 0.250 \\
\hline 148 & 1 & 1 & 7 & 12 & 0.143 & 0.455 & 0.065 & 0.857 & 0.333 \\
\hline 149 & 1 & 1 & 7 & 23 & 0.143 & 0.727 & 0.104 & 0.857 & 0.333 \\
\hline 150 & 2 & 2 & 8 & 23 & 0.250 & 0.714 & 0.179 & 0.750 & 0.333 \\
\hline 151 & 5 & 5 & 28 & 55 & 0.179 & 0.540 & 0.096 & 0.821 & 0.115 \\
\hline 152 & 5 & 5 & 34 & 63 & 0.147 & 0.500 & 0.074 & 0.853 & 0.094 \\
\hline
\end{tabular}


Tab. 6: Analysis II, apomorphic character states. Column 1 refers to the clade numbers given in the cladogram (Fig. 395). The corresponding taxon names are listed in column 2. Underlined names are the traditional groups of the current classification or previous phylogenetic hypotheses that were herein recovered to be monophyletic (with slight differences in Amorphoscelidae and Tarachodidae). They are followed by the taxa they comprise (in the present dataset). Columns "IS" and „IM" are marked "X" if the taxon was identical in the strict consensus and/or majority rule cladogram, respectively, of analysis I. Markings in the coloum SW refer to the molecular support for the corresponding taxon as found by Svenson \& Whiting (2009: fig. 3). If the marking is set in parentheses " $(\mathrm{X})$ ", the group of taxa was found to be monophyletic in the respective analyses but the internal relationships were not identically resolved (same for column SW; therein other species that were not included in the present analysis may be nested within the taxon and the taxon may be paraphyletic therein due to the larger taxon sample, but corroborates the relations as found in the present dataset). A question mark in column SW indicates that taxa were missing therein to address the assumed monophyly of the corresponding taxon. Mantodea as a whole is not marked because it was defined by the outgroup settings. Character state transformations that were recovered under both ACCTRAN and DELTRAN assumptions are considered to be apomorphies (column "apomorphies"). The columns "ACCTRAN only" and "DELTRAN only" additionally list those transformations that were found only under one or the other assumption. Transformations that are set in bold type and underlined indicate unique transformations $(\mathrm{CI}=1.0)$. In cases where the apomorphic character state was found to have evolved from different previous states under ACCTRAN and DELTRAN both scenarios are listed in the "apomorphies" column, separated by a slash (e.g. 123:1>2/3>2).

\begin{tabular}{|c|c|c|c|c|c|c|c|}
\hline Clade & Taxon & I S & I M & SW & Apomorphies & ACCTRAN only & DELTRAN only \\
\hline 1 & $\underline{\text { Mantodea }}$ & & & & & & 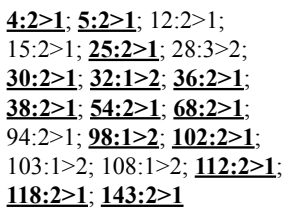 \\
\hline 2 & Chaeteessa valida & l & / & / & $\begin{array}{l}14: 4>3 ; 19: 6>3 ; 22: 2>1 ; \\
44: 1>2 ; 45: 2>1 ; 48: 1>4 ; \\
50: 1>2 ; 53: 2>1 ; 87: 2>1 ; \\
96: 2>1 ; 113: 2>1 ; 114: 3>2 ; \\
149: 2>1 ; 152: 5>6\end{array}$ & 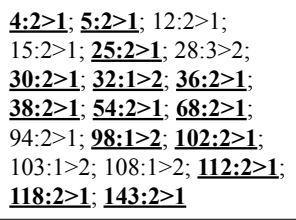 & \\
\hline 3 & $\begin{array}{l}\text { Mantodea except for } \\
\underline{\text { Chaeteessa }}\end{array}$ & & $(\mathrm{X})$ & $(\mathrm{X})$ & & $\begin{array}{l}\underline{\mathbf{4}: \mathbf{2}>\mathbf{1}} ; \underline{\mathbf{5}: \mathbf{2}>\mathbf{1}} ; 12: 2>1 ; \\
15: 2>1 ; \underline{\mathbf{2 5}: \mathbf{2}>\mathbf{1}} ; 28: 3>2 ; \\
\underline{\mathbf{3 0}: \mathbf{2}>\mathbf{1}} ; \underline{\mathbf{3 2 : 1}} ; \underline{\mathbf{3 6 : 2}} ; \underline{\mathbf{3 8}: \mathbf{2}>\mathbf{1}} ; \underline{\mathbf{5 4}: \mathbf{2}>\mathbf{1}} ; \underline{\mathbf{6 8 : 2}>\mathbf{1}} ; \\
73: 1>2 ; 77: 1>2 ; 87: 2>1 ; \\
94: 2>1 ; \underline{\mathbf{9 8 : 1}>\mathbf{2}} ; \underline{\mathbf{1 0 2}: \mathbf{2}>\mathbf{1}} ; \\
103: 1>2 ; 108: 1>2 ; \underline{\mathbf{1 1 2}: \mathbf{2}>\mathbf{1}} ; \\
\underline{\mathbf{1 1 8 : 2}>\mathbf{1}} ; \underline{\mathbf{1 4 3}: \mathbf{2}>\mathbf{1}}\end{array}$ & $\begin{array}{l}14: 4>2 ; 21: 1>2 ; 48: 1>2 ; \\
72: 5>2 ; 74: 3>2 ; \underline{\mathbf{8 3}: 1>\mathbf{2}} ; \\
\underline{\mathbf{8 4}: \mathbf{1}>\mathbf{2}} ; 125: 3>2 ; \underline{\mathbf{1 2 7}: \mathbf{2}>\mathbf{1}} ; \\
128: 2>1 ; 132: 2>1 ; 135: 1>2\end{array}$ \\
\hline 4 & Metallyticus & $\mathrm{X}$ & $\mathrm{X}$ & $\mathrm{X}$ & $\begin{array}{l}\underline{\mathbf{1 0}: 2>1} ; 17: 1>2 ; 19: 6>5 \\
24: 2>1 ; 39: 1>2 ; \underline{\mathbf{5 1}} \mathbf{1}>\mathbf{2} \\
55: 2>1 ; 75: 3>2 ; 79: 2>1 \\
95: 2>1 ; 107: 1>2 ; 111: 2>1 \\
115: 2>1 ; 116: 2>1 ; 126: 3>2 \\
136: 4>1 ; 152: 5>4\end{array}$ & $\begin{array}{l}14: 4>2 ; 18: 6>5 ; 21: 1>2 ; \\
46: 3>2 ; 48: 1>2 ; 72: 5>2 ; \\
74: 3>2 ; \underline{\mathbf{8 3}: 1>\mathbf{2}} ; \underline{\mathbf{8 4}: \mathbf{1}>\mathbf{2}} ; \\
104: 1>2 ; 122: 3>2 ; 125: 3>2 \\
\underline{\mathbf{1 2 7}: \mathbf{2}>\mathbf{1}} ; 128: 2>1 ; 132: 2>1 ; \\
134: 4>1 ; 135: 1>2 ; 151: 5>4\end{array}$ & $87: 2>1$ \\
\hline 5 & Metallyticus splendidus & / & / & l & $47: 3>2$ & & $\begin{array}{l}18: 6>5 ; 46: 3>2 ; 104: 1>2 ; \\
122: 3>2 ; 134: 4>1 ; 151: 5>4\end{array}$ \\
\hline 6 & $\begin{array}{l}\text { Mantodea except for Cha- } \\
\underline{\text { eteess } a \text { and Metallyticus }}\end{array}$ & & $\mathrm{X}$ & & $19: 6>3 ; 58: 1>3$ & $\begin{array}{l}14: 4>2 ; 21: 1>2 ; 48: 1>2 ; \\
60: 1>2 ; 62: 1>2 ; 72: 5>2 ; \\
74: 3>2 ; \underline{\mathbf{8 3}: 1>\mathbf{2}} ; \underline{\mathbf{8 4}: 1>\mathbf{2}} ; \\
125: 3>2 ; \underline{\mathbf{1 2 7}: \mathbf{2}}>\mathbf{\mathbf { 1 }} ; 128: 2>1 ; \\
132: 2>1 ; 135: 1>2\end{array}$ & 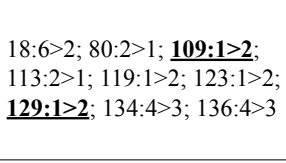 \\
\hline 7 & Mantoida maya & / & I & l & $\begin{array}{l}\underline{\mathbf{4 0}: 1>2} ; 45: 2>1 ; 52: 1>2 \\
55: 2>3 ; 56: 2>1 ; 75: 3>1 ; \\
81: 2>1 ; 114: 3>1 ; 121: 3>2 \\
122: 3>1 ; 126: 3>1 ; 151: 5>2 \\
152: 5>2\end{array}$ & $\begin{array}{l}18: 6>2 ; 80: 2>1 ; 87: 1>2 ; \\
\underline{\mathbf{1 0 9}: \mathbf{1}>\mathbf{2}} ; 113: 2>1 ; 119: 1>2 \\
123: 1>2 ; \underline{\mathbf{1 2 9}}: \mathbf{1}>\mathbf{2} ; 134: 4>3 \\
136: 4>3\end{array}$ & \\
\hline 8 & $\begin{array}{l}\text { Mantodea except for } \\
\text { Chaeteessa, Metallyticus } \\
\text { and Mantoida (Artiman- } \\
\text { todea?) }\end{array}$ & & $\mathrm{X}$ & $(\mathrm{X})$ & $57: 1>3 ; 75: 3>2 ; 126: 3>2$ & $\begin{array}{l}18: 6>2 ; 52: 1>2 ; 61: 1>2 ; \\
80: 2>1 ; 87: 1>2 ; \underline{\mathbf{1 2 9}: \mathbf{1}>\mathbf{2}} \\
134: 4>3 ; 136: 4>1\end{array}$ & $\begin{array}{l}17: 1>2 ; 27: 2>3 ; 106: 1>2 ; \\
108: 2>1 ; 133: 2>1\end{array}$ \\
\hline 9 & $\begin{array}{l}\text { Eremiaphilidae } \\
\text { Heteronutarsus } \\
\text { miaphila }\end{array}$ & $\mathrm{X}$ & $\mathrm{X}$ & $?$ & 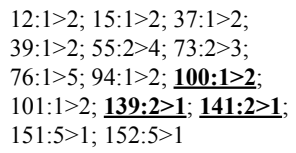 & $\begin{array}{l}17: 1>2 ; 27: 2>3 ; 74: 2>3 \\
106: 1>2 ; 108: 2>1 ; 121: 3>1 \\
125: 2>1 ; 133: 2>1\end{array}$ & $52: 1>2$ \\
\hline
\end{tabular}


Tab. 6 (continued): Apomorphy lists.

\begin{tabular}{|c|c|c|c|c|c|c|c|}
\hline Clade & Taxon & I S & I M & SW & Apomorphies & ACCTRAN only & DELTRAN only \\
\hline 10 & $\begin{array}{l}\text { Heteronutarsus aegyp- } \\
\text { tiacus }\end{array}$ & / & / & / & $\begin{array}{l}77: 2>1 ; 88: 1>2 ; 89: 1>2 \\
\underline{\mathbf{9 9 : 1}} ; \mathbf{2} ; 74: 3>4 / 2>4\end{array}$ & & $136: 3>1$ \\
\hline 11 & Eremiaphila & $\mathrm{X}$ & $\mathrm{X}$ & $\mathrm{X}$ & $7: 2>1 ; 47: 3>4$ & $18: 2>3 ; 46: 3>4 ; 136: 1>3$ & $74: 2>3 ; 125: 2>1$ \\
\hline 12 & Eremiaphila sp. 1 & I & I & I & & & $18: 2>3 ; 46: 3>4 ; 121: 3>1$ \\
\hline 13 & Eremiaphila sp. 2 & 1 & 1 & 1 & $19: 3>2 ; 59: 1>2$ & & \\
\hline 14 & $\begin{array}{l}\text { Mantodea except for } \\
\text { Chaeteessa, Metallyticus, } \\
\text { Mantoida and Eremi- } \\
\text { aphilidae }\end{array}$ & & $\mathrm{X}$ & & $\begin{array}{l}55: 2>3 ; 121: 3>2 ; 122: 3>2 \\
151: 5>2 ; 152: 5>2\end{array}$ & $\begin{array}{l}17: 1>2 ; 27: 2>3 ; 106: 1>2 ; \\
108: 2>1 ; \underline{\mathbf{1 0 9}: 1>2} ; 113: 2>1 ; \\
119: 1>2 ; 123: 1>2 ; 136: 1>3\end{array}$ & $\begin{array}{l}13: 2>1 ; 19: 3>1 ; 26: 1>2 ; \\
37: 1>3 ; 58: 3>2 ; 95: 2>1 ; \\
103: 2>1 ; 114: 3>2 ; 116: 2>1 ; \\
117: 2>1 ; 131: 4>1\end{array}$ \\
\hline 15 & $\begin{array}{l}\text { Amorphoscelidae (incl. } \\
\text { Compsothespis) } \\
\text { (Compsothespis + (Amor- } \\
\text { phoscelinae + Perlamanti- } \\
\text { nae) + Paraoxypilinae }\end{array}$ & & & & $\frac{\mathbf{6 4 : 1}>\mathbf{2}}{130: 4>1} ; 67: 2>1 ; 81: 2>1$ & $\begin{array}{l}7: 2>1 ; 13: 2>1 ; 19: 3>1 ; \\
26: 1>2 ; 37: 1>3 ; \underline{\mathbf{4 3}: 1>\mathbf{3}} \\
44: 1>4 ; 58: 3>2 ; \underline{\mathbf{6 5}: \mathbf{1}>\mathbf{3}} \\
72: 2>1 ; 82: 1>2 ; 95: 2>1 ; \\
103: 2>1 ; 114: 3>2 ; 116: 2>1 \\
117: 2>1 ; 128: 1>2 ; 131: 4>1\end{array}$ & \\
\hline 16 & $\begin{array}{l}\text { Paraoxypilinae } \\
\text { Gyromantis }+ \text { (Paraoxypi- } \\
\text { linae sp. }+ \text { Paraoxypilus }) \\
\end{array}$ & $\mathrm{X}$ & $\mathrm{X}$ & $(\mathrm{X})$ & $\begin{array}{l}39: 1>2 ; 45: 2>1 ; 87: 2>1 \\
97: 2>1 ; 148: 2>1\end{array}$ & $\begin{array}{l}52: 2>1 ; 107: 1>2 ; 131: 1>2 \\
132: 1>2 ; 133: 2>1 ; 145: 2>1\end{array}$ & $\begin{array}{l}7: 2>1 ; \underline{\mathbf{4 3}: \mathbf{1}>\mathbf{3}} ; 44: 1>4 ; \\
\underline{\mathbf{6 5}: \mathbf{1}>\mathbf{3}} ; 72: 2>1 ; 82: 1>2\end{array}$ \\
\hline 17 & Gyromantis sp. & / & / & l & $\begin{array}{l}3: 2>1 ; 24: 2>1 ; 74: 2>3 \\
86: 2>1 ; 111: 2>1 ; \\
132: 2>3 / 1>3 ; 151: 2>3\end{array}$ & $128: 2>1$ & $107: 1>2 ; 131: 1>2 ; 145: 2>1$ \\
\hline 18 & $\begin{array}{l}\text { Paraoxypilinae sp. }+ \\
\text { Paraoxypilus }\end{array}$ & $\mathrm{X}$ & $\mathrm{X}$ & & $105: 1>2 ; 131: 2>3 / 1>3$ & $88: 1>2 ; 114: 2>1$ & $132: 1>2$ \\
\hline 19 & Paraoxypilinae sp. & / & / & I & & $145: 1>2$ & \\
\hline 20 & Paraoxypilus & I & I & I & $47: 3>2$ & & $145: 2>1$ \\
\hline 21 & Paraoxypilus sp. 1 & / & l & l & & & $88: 1>2 ; 114: 2>1 ; 128: 1>2$ \\
\hline 22 & $\begin{array}{l}\text { Compsothespis }+ \\
\text { (Amorphoscelinae }+ \\
\text { Perlamantinae) }\end{array}$ & & & & $\begin{array}{l}46: 3>1 ; 47: 3>1 ; \underline{\mathbf{6 3 : 1}}>\mathbf{2} ; \\
\underline{\mathbf{6 5 : 3}} ; \mathbf{4} / \mathbf{1}>\mathbf{4} ; 149: 2>1\end{array}$ & $\begin{array}{l}18: 2>1 ; 37: 3>4 ; \underline{\mathbf{4 3 : 3}>\mathbf{4}} \\
44: 4>5 ; 60: 2>1 ; 150: 1>2\end{array}$ & $133: 1>2$ \\
\hline 23 & Compsothespis anomala & / & l & / & 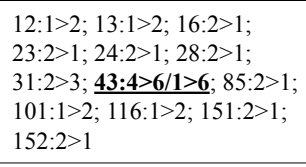 & $82: 2>1$ & $\begin{array}{l}7: 2>1 ; 18: 2>1 ; 37: 3>4 \\
52: 1>2 ; 60: 2>1 ; 150: 1>2\end{array}$ \\
\hline 24 & $\begin{array}{l}\text { Amorphoscelinae + Perla- } \\
\text { mantinae }\end{array}$ & $\mathrm{X}$ & $\mathrm{X}$ & $?$ & $\begin{array}{l}18: 1>3 / 2>3 ; 26: 2>1 ; \underline{\mathbf{4 2 : 1}>\mathbf{2}} \\
55: 3>1 ; 106: 2>1\end{array}$ & $\begin{array}{l}7: 1>2 ; 19: 1>4 ; 137: 2>1 \\
150: 2>1\end{array}$ & $82: 1>2 ; 128: 1>2 ; 150: 1>3$ \\
\hline 25 & $\begin{array}{l}\text { Perlamantinae } \\
\begin{array}{l}\text { Perlamantis }+ \text { Paramor }- \\
\text { phoscelis }\end{array}\end{array}$ & $\mathrm{X}$ & $\mathrm{X}$ & $?$ & $\begin{array}{l}27: 3>2 ; 39: 1>2 ; \underline{\mathbf{4 3 : 4}>\mathbf{5} / \mathbf{1}>\mathbf{5}} \\
\underline{\mathbf{6 9}: \mathbf{1}>\mathbf{2}} ; \underline{\mathbf{7 0}: \mathbf{1}>\mathbf{2}} ; 80: 1>2 \\
114: 2>1\end{array}$ & $37: 4>3 ; 124: 1>2$ & $44: 1>5$ \\
\hline 26 & $\begin{array}{l}\text { Paramorphoscelis gondo- } \\
\text { korensis }\end{array}$ & / & / & l & & & $124: 1>2 ; 137: 2>1$ \\
\hline 27 & Perlamantis allibertii & / & / & / & $142: 1>2 ; 149: 1>2 ; 150: 3>2$ & $137: 1>2$ & \\
\hline 28 & $\begin{array}{l}\text { Amorphoscelinae } \\
\text { Maculatoscelis } \mathrm{sp} .+ \\
\text { Amorphoscelis } \mathrm{sp} .+ \\
\text { Caudatoscelis } \\
\end{array}$ & $\mathrm{X}$ & $\mathrm{X}$ & $\mathrm{X}$ & $\begin{array}{l}\underline{\mathbf{4 1 : 1}: \mathbf{2}} ; 56: 2>1 ; 86: 2>1 ; \\
87: 2>1 ; 89: 1>2 ; 115: 2>1 \\
125: 2>3\end{array}$ & $88: 1>2 ; 121: 2>3 ; 151: 2>3$ & $37: 3>4 ; \underline{\mathbf{4 3 : 1}>\mathbf{4}}$ \\
\hline 29 & Maculatoscelis sp. & I & / & / & $28: 2>3 ; 128: 2>1$ & & $19: 1>4$ \\
\hline 30 & Amorphoscelis sp. & / & l & / & & & $\begin{array}{l}88: 1>2 ; 121: 2>3 ; 137: 2>1 ; \\
151: 2>3\end{array}$ \\
\hline 31 & Caudatoscelis annulipes & I & l & l & $114: 2>3$ & & \\
\hline 32 & $33+58$ & & & & $91: 1>3$ & $\begin{array}{l}19: 3>1 ; 26: 1>2 ; 37: 1>2 \\
58: 3>2 ; 67: 2>1 ; 95: 2>1 \\
103: 2>1 ; 114: 3>2 ; 116: 2>1 \\
117: 2>1 ; 131: 4>1\end{array}$ & $\begin{array}{l}18: 2>4 ; 21: 2>1 ; 23: 2>1 ; \\
24: 2>1 ; 28: 2>1 ; 44: 1>2 ; \\
53: 2>1 ; 59: 1>2 ; 96: 2>1 ; \\
151: 2>3 ; 152: 2>3\end{array}$ \\
\hline 33 & $\begin{array}{l}\text { (Stenomantis }+ \text { Ciulfina }) \\
+(\text { Majanga }+(\text { Epaphro- } \\
\text { dita }+(\text { Hoplocorypha }+ \\
(\text { Oligonicinae }+ \text { Haani- } \\
\text { inae })))\end{array}$ & & & & $31: 2>3 ; 87: 2>1$ & $\begin{array}{l}18: 2>1 ; 21: 2>1 ; 23: 2>1 \\
24: 2>1 ; 28: 2>1 ; 44: 1>2 \\
52: 2>1 ; 53: 2>1 ; 57: 3>1 \\
59: 1>2 ; 96: 2>1 ; 122: 2>1 \\
126: 2>1 ; 151: 2>3 ; 152: 2>3\end{array}$ & \\
\hline 34 & Ciulfina + Stenomantis & & & $\mathrm{X}$ & $80: 1>2 ; 88: 1>2 ; 101: 1>2$ & $\begin{array}{l}18: 1>4 ; 19: 1>4 ; 37: 2>3 ; \\
89: 1>2\end{array}$ & $13: 1>2 ; 67: 2>1 ; 133: 1>2$ \\
\hline 35 & Ciulfina biseriata & / & / & / & $\begin{array}{l}24: 1>2 ; 79: 2>1 ; 81: 2>1 ; \\
95: 1>2 ; 111: 2>1 ; 114: 2>1\end{array}$ & & \\
\hline
\end{tabular}


Tab. 6 (continued): Apomorphy lists.

\begin{tabular}{|c|c|c|c|c|c|c|c|}
\hline Clade & Taxon & I S & I M & SW & Apomorphies & ACCTRAN only & DELTRAN only \\
\hline 36 & $\begin{array}{l}\text { Stenomantis novaegui- } \\
\text { neae }\end{array}$ & / & / & l & $\begin{array}{l}7: 2>1 ; 12: 1>2 ; 50: 1>2 \\
52: 1>2 ; 60: 2>1 ; 75: 2>3 \\
86: 2>1 ; 103: 1>2 ; 130: 4>1 \\
132: 1>2 ; 134: 3>2\end{array}$ & & $19: 1>4 ; 89: 1>2$ \\
\hline 37 & $\begin{array}{l}\text { Majanga }+(\text { Epaphrodita } \\
+(\text { Hoplocorypha }+(\text { Oli- } \\
\text { gonicinae }+ \text { Haaniinae })))\end{array}$ & & $(\mathrm{X})$ & & $55: 3>4 ; 97: 2>1 ; 131: 1>3$ & $13: 2>1 ; 130: 4>3 ; 133: 2>1$ & $37: 3>2 ; 57: 3>1 ; 122: 2>1$ \\
\hline 38 & Majanga basilaris & / & / & l & $\begin{array}{l}75: 2>3 ; 116: 1>2 ; 137: 2>1 \\
145: 2>1 ; 147: 2>1\end{array}$ & $67: 1>2$ & $126: 2>1 ; 130: 4>3$ \\
\hline 39 & $\begin{array}{l}\text { Epaphrodita }+(\text { Hoploco- } \\
\text { rypha }+(\text { Oligonicinae }+ \\
\text { Haaniinae }))\end{array}$ & & $(\mathrm{X})$ & & $\begin{array}{l}7: 2>1 ; 82: 1>2 ; 86: 2>1 \\
104: 1>2 ; 132: 1>3\end{array}$ & $47: 3>2 ; 111: 2>1 ; 152: 3>2$ & $67: 2>1$ \\
\hline 40 & Epaphrodita dentifrons & l & / & l & $\begin{array}{l}48: 2>3 ; 61: 2>1 ; 76: 1>2 \\
77: 2>4 ; 79: 2>3 ; 90: 2>1 \\
130: 3>1 / 4>1\end{array}$ & & $111: 2>1$ \\
\hline 41 & $\begin{array}{l}\text { Thespidae } \\
\text { Hoplocorypha }+ \text { Oligoni- } \\
\text { cinae (incl. Haaniinae) }\end{array}$ & $(\mathrm{X})$ & $(\mathrm{X})$ & & $\begin{array}{l}37: 2>4 ; 46: 3>2 ; 52: 1>2 \\
53: 1>2 ; 105: 1>2\end{array}$ & $57: 1>6$ & $47: 3>2 ; 152: 3>2$ \\
\hline 42 & Hoplocorypha sp. & l & l & l & $\begin{array}{l}55: 4>3 ; 60: 2>1 ; 96: 1>2 \\
101: 1>2 ; 120: 1>2 ; 136: 3>1 \\
151: 3>2\end{array}$ & & $18: 4>1 ; 111: 2>1 ; 130: 4>3$ \\
\hline 43 & $\begin{array}{l}\text { Oligonicinae incl. } \\
\text { Haaniinae } \\
\text { Bactromantis }+((\text { Pogo- } \\
\text { nogaster }+ \text { Thesprotia })+ \\
(\text { Oligonicinae sp. }+(\text { Thri- } \\
\text { naconyx }+ \text { Haaniinae })))\end{array}$ & $(\mathrm{X})$ & $(\mathrm{X})$ & & 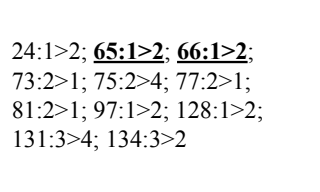 & $\begin{array}{l}31: 3>2 ; 74: 2>1 ; 76: 1>5 \\
78: 2>1 ; 111: 1>2 ; 114: 2>1 \\
116: 1>2 ; 130: 3>4\end{array}$ & \\
\hline 44 & Bactromantis tolteca & l & / & l & $33: 1>3$ & & $\begin{array}{l}31: 3>2 ; 57: 1>6 ; 114: 2>1 \\
116: 1>2\end{array}$ \\
\hline 45 & $\begin{array}{l}\text { (Pogonogaster }+ \\
\text { Thesprotia })+(\text { Oligonici- } \\
\text { nae sp. }+(\text { Thrinaconyx }+ \\
\text { Haaniinae }))\end{array}$ & & & & $72: 2>4 ; 117: 1>2$ & & $74: 2>1 ; 78: 2>1$ \\
\hline 46 & $\begin{array}{l}\text { Pogonogaster }+ \\
\text { Thesprotia }\end{array}$ & $\mathrm{X}$ & $\mathrm{X}$ & & $55: 4>3 ; 85: 2>1$ & $18: 1>2 ; 31: 2>3 ; 114: 1>2$ & \\
\hline 47 & Pogonogaster tristani & / & / & l & $\begin{array}{l}13: 1>2 ; 29: 1>2 ; 39: 1>2 \\
72: 4>3 ; 145: 2>1 ; 146: 2>1 \\
148: 2>1\end{array}$ & & \\
\hline 48 & Thesprotia & $\mathrm{X}$ & $\mathrm{X}$ & & $\begin{array}{l}33: 1>3 ; 48: 2>1 ; 58: 2>1 \\
136: 3>2\end{array}$ & $101: 1>2 ; 152: 2>3$ & $116: 1>2$ \\
\hline 49 & Thesprotia graminis & I & 1 & 1 & & $18: 2>4$ & $101: 1>2 ; 152: 2>3$ \\
\hline 50 & Thesprotia macilenta & 1 & 1 & 1 & & & $18: 4>2$ \\
\hline 51 & $\begin{array}{l}\text { Oligonicinae sp. }+(\text { Thri- } \\
\text { naconyx }+ \text { Haaniinae })\end{array}$ & $\mathrm{X}$ & $\mathrm{X}$ & & $\begin{array}{l}7: 1>2 ; 94: 1>2 ; 95: 1>2 \\
132: 3>2\end{array}$ & $\begin{array}{l}22: 2>1 ; 37: 4>2 ; 116: 2>1 ; \\
151: 3>2\end{array}$ & $31: 2>1 ; 114: 2>1$ \\
\hline 52 & Oligonicinae sp. & / & / & l & $\begin{array}{l}13: 1>2 ; 14: 2>3 ; 104: 2>1 ; \\
136: 3>2\end{array}$ & & $22: 2>1 ; 37: 4>2 ; 57: 1>6$ \\
\hline 53 & Thrinaconyx + Haaniinae & $\mathrm{X}$ & $\mathrm{X}$ & & $24: 2>1 ; 37: 2>3 / 4>3$ & $\begin{array}{l}57: 6>1 ; 89: 1>2 ; 145: 2>1 ; \\
148: 2>1\end{array}$ & \\
\hline 54 & Thrinaconyx kirschianus & l & l & l & $72: 4>3 ; 149: 2>1 ; 18: 1>3 / 4>3$ & & \\
\hline 55 & $\frac{\text { Haaniinae }}{\text { Haania }+ \text { Astape }}$ & $\mathrm{X}$ & $\mathrm{X}$ & $?$ & $\begin{array}{l}3: 2>1 ; 7: 2>3 ; 39: 1>2 ; 57: 1>4 \\
88: 1>2 ; 105: 2>1 ; 130: 4>1 \\
131: 4>1 ; 137: 2>1\end{array}$ & & $\begin{array}{l}18: 4>1 ; 89: 1>2 ; 145: 2>1 ; \\
148: 2>1 ; 151: 3>2\end{array}$ \\
\hline 56 & Astape denticollis & I & / & I & $\begin{array}{l}20: 1>2 ; 28: 1>2 ; 108: 1>2 \\
147: 2>1\end{array}$ & & \\
\hline 57 & Haania lobiceps & l & I & I & $\begin{array}{l}31: 2>3 ; 48: 2>1 ; 81: 1>2 \\
114: 1>2 ; 152: 2>1\end{array}$ & & $76: 1>5$ \\
\hline 58 & $59+64$ & & & & $130: 4>1$ & $\begin{array}{l}18: 2>4 ; 21: 2>1 ; 23: 2>1 \\
24: 2>1 ; 28: 2>1 ; 44: 1>2 \\
52: 2>1 ; 53: 2>1 ; 87: 2>1 \\
96: 2>1 ; 133: 2>1 ; 151: 2>3 \\
152: 2>3\end{array}$ & $\begin{array}{l}37: 3>2 ; 77: 2>3 ; 78: 2>3 \\
79: 2>1\end{array}$ \\
\hline 59 & $\begin{array}{l}\text { Tropidomantinae } \\
\text { Enicophlebia }+ \text { Tropido- } \\
\text { mantis }\end{array}$ & $\mathrm{X}$ & $\mathrm{X}$ & & $\begin{array}{l}12: 1>2 ; 19: 1>3 ; 82: 1>2 \\
106: 2>1 ; 132: 1>3 ; 136: 3>2\end{array}$ & $\begin{array}{l}67: 1>2 ; 77: 2>3 ; 78: 2>3 \\
79: 2>1 ; 114: 2>1 ; 134: 3>2\end{array}$ & $87: 2>1$ \\
\hline
\end{tabular}


Tab. 6 (continued): Apomorphy lists.

\begin{tabular}{|c|c|c|c|c|c|c|c|}
\hline Clade & Taxon & I S & I M & SW & Apomorphies & ACCTRAN only & DELTRAN only \\
\hline 60 & Enicophlebia pallida & / & / & l & $\begin{array}{l}8: 2>1 ; 58: 2>1 ; 74: 2>3 \\
76: 1>2 ; 78: 3>4 ; 79: 1>3\end{array}$ & $13: 2>1$ & \\
\hline 61 & Tropidomantis & $\mathrm{X}$ & $\mathrm{X}$ & $(\mathrm{X})$ & $7: 2>1 ; 62: 2>1 ; 75: 2>3$ & & $13: 1>2 ; 114: 2>1 ; 134: 3>2$ \\
\hline 62 & $\begin{array}{l}\text { Tropidomantis (Eomantis) } \\
\text { sp. }\end{array}$ & / & / & / & $46: 3>2 ; 47: 3>2 ; 128: 1>2$ & & \\
\hline 63 & Tropidomantis tenera & / & / & l & $18: 4>3$ & & \\
\hline 64 & $65+71$ & & & & & $\begin{array}{l}13: 2>1 ; 59: 1>2 ; 67: 1>2 \\
77: 2>3 ; 78: 2>3 ; 79: 2>1\end{array}$ & $18: 4>5 ; 55: 3>4 ; 97: 2>1$ \\
\hline 65 & $\begin{array}{l}\text { Humbertiella }+ \text { Theo- } \\
\text { pompa }\end{array}$ & $(\mathrm{X})$ & $\mathrm{X}$ & $?$ & $\begin{array}{l}19: 1>5 ; 28: 1>2 ; 35: 1>2 ; \\
57: 3>2 ; 133: 1>2 ; 147: 2>1\end{array}$ & $\begin{array}{l}18: 4>5 ; 55: 3>4 ; 87: 1>2 ; \\
97: 2>1 ; 151: 3>4 ; 152: 3>5\end{array}$ & \\
\hline 66 & Humbertiella sp. & / & l & / & $\begin{array}{l}24: 1>2 ; 31: 2>3 ; 126: 2>1 ; \\
131: 1>2\end{array}$ & & $151: 3>4 ; 152: 3>5$ \\
\hline 67 & Theopompa & $(\mathrm{X})$ & $\mathrm{X}$ & $?$ & $\begin{array}{l}44: 2>1 ; 104: 1>2 ; 106: 2>1 ; \\
111: 2>1 ; 151: 4>6 / 3>6\end{array}$ & $145: 2>1 ; 152: 5>6$ & \\
\hline 68 & $\begin{array}{l}\text { Theopompa } \mathrm{sp} .2+\text { Theo- } \\
\text { pompa tosta }\end{array}$ & & $\mathrm{X}$ & $?$ & $7: 2>1$ & & \\
\hline 69 & Theopompa sp. 2 & / & / & l & $121: 2>3$ & & \\
\hline 70 & Theopompa tosta & l & l & l & $18: 5>6 ; 119: 2>1$ & & $145: 2>1 ; 152: 3>6$ \\
\hline 71 & $72+118$ & & & & $19: 1>3$ & $18: 4>5 ; 55: 3>4 ; 87: 1>2$ & $\begin{array}{l}12: 1>2 ; 15: 1>2 ; 104: 1>2 ; \\
107: 1>2 ; 131: 1>2\end{array}$ \\
\hline 72 & $73+84$ & & & & $7: 2>1$ & $\begin{array}{l}12: 1>2 ; 15: 1>2 ; 53: 1>2 ; \\
57: 3>7 ; 91: 3>1 ; 104: 1>2 \\
107: 1>2 ; 131: 1>2\end{array}$ & \\
\hline 73 & $\begin{array}{l}\text { Gonypeta }+(\text { Gimantis }+ \\
(\text { Odontomantis }+(\text { Hestia }- \\
\text { sula }+ \text { Oxypilinae })))\end{array}$ & & & & $151: 3>2$ & & $53: 1>2 ; 57: 3>7 ; 97: 1>2$ \\
\hline 74 & Gonypeta brigittae & / & l & l & $152: 3>2$ & & \\
\hline 75 & $\begin{array}{l}\text { Gimantis }+(\text { Odonto }- \\
\text { mantis }+(\text { Hestiasula }+ \\
\text { Oxypilinae }))\end{array}$ & & & & $\begin{array}{l}46: 3>2 ; 47: 3>2 ; 79: 1>3 ; \\
106: 2>1\end{array}$ & $28: 2>1 ; 87: 2>1$ & \\
\hline 76 & Gimantis sp. & / & / & / & $\begin{array}{l}13: 1>2 ; 19: 3>4 ; 52: 1>2 ; \\
57: 7>2 ; 61: 2>1 ; 81: 2>1 ; \\
86: 2>1 ; 111: 2>1 ; 121: 2>3\end{array}$ & & $28: 1>2 ; 87: 2>1$ \\
\hline 77 & $\begin{array}{l}\text { Odontomantis }+(\text { Hestia- } \\
\text { sula }+ \text { Oxypilinae })\end{array}$ & & $\mathrm{X}$ & & $\begin{array}{l}76: 1>3 ; 104: 2>1 ; 107: 2>1 ; \\
116: 1>2 ; 133: 1>2\end{array}$ & $\begin{array}{l}7: 1>2 ; 78: 3>4 ; 88: 1>2 \\
89: 1>2\end{array}$ & \\
\hline 78 & Odontomantis sp. & / & l & / & $\begin{array}{l}12: 2>1 ; 15: 2>1 ; 24: 1>2 \\
77: 3>4 ; 142: 1>2\end{array}$ & $28: 2>1$ & $\begin{array}{l}7: 1>2 ; 78: 3>4 ; 87: 2>1 \\
88: 1>2 ; 89: 1>2\end{array}$ \\
\hline 79 & Hestiasula + Oxypilinae & $\mathrm{X}$ & $\mathrm{X}$ & $(\mathrm{X})$ & $\begin{array}{l}1: 2>1 ; 7: 2>3 / 1>3 ; 11: 2>1 \\
31: 2>1 ; 57: 7>6 ; 131: 2>1\end{array}$ & $87: 1>2$ & $28: 1>2$ \\
\hline 80 & Hestiasula brunneriana & / & / & / & $33: 1>2 ; 61: 2>1 ; 90: 2>1$ & & $\begin{array}{l}78: 3>4 ; 88: 1>2 ; 89: 1>2 \\
91: 3>1\end{array}$ \\
\hline 81 & $\frac{\text { Oxypilinae }}{\text { Ceratomantis }}+$ Oxypilus & $\mathrm{X}$ & $\mathrm{X}$ & $(\mathrm{X})$ & $3: 2>1 ; 8: 2>1 ; 52: 1>2$ & $\begin{array}{l}76: 3>2 ; 78: 4>2 ; 88: 2>1 \\
89: 2>1\end{array}$ & \\
\hline 82 & Ceratomantis saussurii & / & l & l & $\begin{array}{l}24: 1>2 ; 53: 2>1 ; 57: 6>1 ; \\
67: 2>1 ; 86: 2>1 ; 145: 2>1 \\
147: 2>1 ; 151: 2>3\end{array}$ & $78: 2>3$ & $76: 3>2$ \\
\hline 83 & Oxypilus distinctus & / & l & I & $\begin{array}{l}76: 2>4 / 3>4 ; 77: 3>2 ; 79: 3>1 \\
105: 1>2 ; 114: 2>1 ; 116: 2>1 \\
117: 1>2 ; 122: 2>1 ; 131: 1>3 \\
148: 2>1 ; 152: 3>2\end{array}$ & & $78: 3>2$ \\
\hline 84 & $\begin{array}{l}\text { Amphecostephanus }+ \\
\text { (Elaea }+ \text { (Tarachodinae } \\
\text { except Iris }+ \text { Ameles }+ \\
\text { Rivetinini }+ \text { Chroicop- } \\
\text { terinae })\end{array}$ & & & & $122: 2>1$ & $\begin{array}{l}80: 1>2 ; 96: 1>2 ; 97: 2>1 ; \\
125: 2>1 ; 126: 2>1 ; 131: 2>3\end{array}$ & \\
\hline 85 & Amphecostephanus rex & / & / & l & $\begin{array}{l}1: 2>1 ; 48: 2>3 ; 76: 1>4 \\
104: 2>1 ; 114: 2>1 ; 150: 1>3\end{array}$ & & \\
\hline 86 & $\begin{array}{l}\text { Elaea }+ \text { Tarachodinae } \\
\text { except Iris }+(\text { Ameles }+ \\
\text { Rivetinini }+ \text { Chroicop- } \\
\text { terinae })\end{array}$ & $(\mathrm{X})$ & $(\mathrm{X})$ & & $116: 1>2 ; 133: 1>2$ & & $\begin{array}{l}80: 1>2 ; 96: 1>2 ; 125: 2>1 ; \\
126: 2>1 ; 131: 2>3\end{array}$ \\
\hline 87 & Elaea marchali & I & / & l & $\begin{array}{l}17: 2>1 ; 19: 3>4 ; 28: 1>2 ; \\
79: 1>2\end{array}$ & $57: 7>3$ & $53: 1>2$ \\
\hline
\end{tabular}


Tab. 6 (continued): Apomorphy lists.

\begin{tabular}{|c|c|c|c|c|c|c|c|}
\hline Clade & Taxon & I S & I M & SW & Apomorphies & ACCTRAN only & DELTRAN only \\
\hline 88 & $\begin{array}{l}\text { Tarachodinae except Iris } \\
+ \text { (Ameles + Rivetinini }+ \\
\text { Chroicopterinae })\end{array}$ & $(\mathrm{X})$ & $(\mathrm{X})$ & & $\begin{array}{l}13: 1>2 ; 18: 5>3 ; 21: 1>2 \\
46: 3>2 ; 124: 1>2\end{array}$ & $53: 2>1$ & $57: 3>7$ \\
\hline 89 & $\begin{array}{l}\text { Tarachodinae (excluding } \\
\text { Iris) } \\
\text { Paralygdamia }+ \text { Pseu- } \\
\text { dogalepsus }+(\text { Pyrgo- } \\
\text { mantis }+(\text { Tarachodes }+ \\
\text { Tarachodula }))\end{array}$ & $(\mathrm{X})$ & $(\mathrm{X})$ & $(\mathrm{X})$ & $\begin{array}{l}22: 2>1 ; 23: 1>2 ; 24: 1>2 ; \\
31: 2>3 ; 35: 1>2 ; 97: 1>2 \\
149: 2>1 ; 150: 1>2\end{array}$ & & \\
\hline 90 & Paralygdamia sp. & / & / & / & $48: 2>3 ; 101: 1>2$ & & \\
\hline 91 & Pseudogalepsus nigricoxa & / & / & / & $\begin{array}{l}53: 1>2 ; 57: 7>2 ; 96: 2>1 \\
133: 2>1 ; 134: 3>2\end{array}$ & & \\
\hline 92 & $\begin{array}{l}\text { Pyrgomantis }+(\text { Taracho- } \\
\text { des }+ \text { Tarachodula })\end{array}$ & $(\mathrm{X})$ & $(\mathrm{X})$ & $(\mathrm{X})$ & $\begin{array}{l}44: 2>1 ; 73: 2>3 ; 74: 2>3 \\
77: 3>4 ; 78: 3>4 ; 104: 2>1\end{array}$ & & \\
\hline 93 & Pyrgomantis jonesi & l & l & / & $\begin{array}{l}1: 2>1 ; 6: 1>3 ; 53: 1>2 ; 57: 7>1 \\
80: 2>1 ; 96: 2>1 ; 114: 2>1 \\
116: 2>1 ; 131: 3>2\end{array}$ & & \\
\hline 94 & $\begin{array}{l}\text { Tarachodes sp. } 2+ \\
\text { (Tarachodes sp. } 3+ \\
\text { (Tarachodes sp. } 1+ \\
\text { Tarachodula) }\end{array}$ & $(\mathrm{X})$ & $(\mathrm{X})$ & & $18: 3>4 ; 31: 3>2 ; 133: 2>1$ & $148: 2>1$ & \\
\hline 95 & Tarachodes sp. 2 & l & / & / & $46: 2>3$ & & $148: 2>1$ \\
\hline 96 & $\begin{array}{l}\text { Tarachodes sp. } 3+ \\
(\text { Tarachodes sp. } 1+ \\
\text { Tarachodula) }\end{array}$ & & & & $47: 3>2$ & & \\
\hline 97 & Tarachodes sp. 3 & / & l & / & $76: 1>2 ; 128: 1>2$ & & $148: 2>1$ \\
\hline 98 & $\begin{array}{l}\text { Tarachodes sp. } 1+ \\
\text { Tarachodula }\end{array}$ & & & & $19: 3>4$ & $148: 1>2$ & \\
\hline 99 & Tarachodes sp. 1 & l & / & / & $31: 2>3$ & & \\
\hline 100 & Tarachodula pantherina & l & / & / & $18: 4>5 ; 96: 2>1 ; 133: 1>2$ & & \\
\hline 101 & $\begin{array}{l}\text { Ameles decolor }+(\text { Pseu- } \\
\text { doyersinia }+(\text { Ameles } \\
\text { gracilis }+((\text { Bolivaria }+ \\
\text { Rivetina })+(\text { Parentella }+ \\
(\text { Ligariella }+(\text { Entella }+ \\
\text { Ligaria })))))\end{array}$ & $\mathrm{X}$ & $\mathrm{X}$ & & $\begin{array}{l}19: 3>2 ; 47: 3>2 ; 128: 1>2 \\
151: 3>2 ; 152: 3>2\end{array}$ & & \\
\hline 102 & Ameles decolor & / & / & / & $59: 2>1 ; 132: 1>2$ & & \\
\hline 103 & $\begin{array}{l}\text { Pseudoyersinia }+(\text { Ameles } \\
\text { gracilis }+((\text { Bolivaria }+ \\
\text { Rivetina })+(\text { Parentella }+ \\
(\text { Ligariella }+(\text { Entella }+ \\
\text { Ligaria }))))\end{array}$ & $\mathrm{X}$ & $\mathrm{X}$ & & $18: 3>2 ; 101: 1>2$ & & \\
\hline 104 & $\begin{array}{l}\text { Pseudoyersinia betan- } \\
\text { curiae }\end{array}$ & / & / & l & $\begin{array}{l}50: 3>1 ; 53: 1>2 ; 57: 7>2 \\
85: 2>1 ; 130: 1>3\end{array}$ & & \\
\hline 105 & $\begin{array}{l}\text { Ameles gracilis }+ \\
((\text { Bolivaria }+ \text { Rivetina })+ \\
(\text { Parentella }+(\text { Ligariella } \\
+(\text { Entella }+ \text { Ligaria }))))\end{array}$ & $\mathrm{X}$ & $\mathrm{X}$ & & $80: 2>1$ & & \\
\hline 106 & Ameles gracilis & / & I & / & $120: 1>2 ; 130: 1>2 ; 132: 1>2$ & & \\
\hline 107 & $\begin{array}{l}(\text { Bolivaria }+ \text { Rivetina })+ \\
(\text { Parentella }+(\text { Ligariella } \\
+(\text { Entella }+ \text { Ligaria })))\end{array}$ & $\mathrm{X}$ & $\mathrm{X}$ & $?$ & $21: 2>1 ; 125: 1>2$ & & \\
\hline 108 & $\begin{array}{l}\text { Chroicopterinae } \\
\text { Parentella }+(\text { Ligariella }+ \\
(\text { Entella }+ \text { Ligaria }))\end{array}$ & $\mathrm{X}$ & $\mathrm{X}$ & $(\mathrm{X})$ & $\begin{array}{l}53: 1>2 ; 77: 3>2 ; 78: 3>2 ; \\
101: 2>1 ; 116: 2>1 ; \underline{144: 2>1}\end{array}$ & $18: 2>4$ & \\
\hline 109 & Parentella major & / & / & / & $\begin{array}{l}52: 1>2 ; 57: 7>2 ; 59: 2>1 \\
67: 2>1\end{array}$ & & \\
\hline 110 & $\begin{array}{l}\text { Ligariella }+(\text { Entella }+ \\
\text { Ligaria })\end{array}$ & $\mathrm{X}$ & $\mathrm{X}$ & $(\mathrm{X})$ & $28: 1>2 ; 79: 1>2$ & & \\
\hline 111 & Ligariella trigonalis & / & / & / & $87: 2>1 ; 152: 2>1$ & & \\
\hline 112 & Entella + Ligaria & $\mathrm{X}$ & $\mathrm{X}$ & & $80: 1>2$ & & \\
\hline
\end{tabular}


Tab. 6 (continued): Apomorphy lists.

\begin{tabular}{|c|c|c|c|c|c|c|c|}
\hline Clade & Taxon & I S & I M & SW & Apomorphies & ACCTRAN only & DELTRAN only \\
\hline 113 & Entella sp. & / & / & l & $\begin{array}{l}89: 1>2 ; 96: 2>1 ; 107: 2>1 \\
116: 1>2 ; 152: 2>3\end{array}$ & & \\
\hline 114 & Ligaria sp. & l & I & I & $117: 1>2 ; 131: 3>2$ & & $18: 2>4$ \\
\hline 115 & 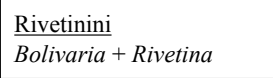 & $\mathrm{X}$ & $\mathrm{X}$ & $(\mathrm{X})$ & $\begin{array}{l}7: 1>2 ; 12: 2>1 ; 57: 7>6 \\
111: 2>1 ; 133: 2>1\end{array}$ & $46: 2>3 ; 87: 2>1 ; 134: 3>2$ & \\
\hline 116 & Bolivaria sp. & / & / & / & $\begin{array}{l}14: 2>3 ; 15: 2>1 ; 47: 2>3 \\
59: 2>1 ; 74: 2>3 ; 114: 2>1 \\
123: 2>1 ; 124: 2>1 ; 130: 1>2\end{array}$ & & $46: 2>3 ; 87: 2>1 ; 134: 3>2$ \\
\hline 117 & Riventina $\mathrm{sp}$ & I & / & / & $\begin{array}{l}11: 2>1 ; 19: 2>3 ; 67: 2>1 \\
79: 1>2 ; 131: 3>2 ; 132: 1>3 \\
136: 3>2 ; \underline{\mathbf{1 4 0 : 2}} \mathbf{1}\end{array}$ & & \\
\hline 118 & $119+122$ & & & & $57: 3>1$ & $\begin{array}{l}12: 1>2 ; 15: 1>2 ; 97: 2>1 \\
104: 1>2 ; 131: 1>2\end{array}$ & $13: 1>2 ; 31: 2>3 ; 121: 2>3$ \\
\hline 119 & Dystacta + Taumantis & & & & $128: 1>2$ & $13: 1>2 ; 31: 2>3 ; 121: 2>3$ & \\
\hline 120 & Dystacta alticeps & I & 1 & I & $7: 2>1 ; 87: 2>1 ; 126: 2>1$ & $107: 1>2$ & \\
\hline 121 & Taumantis sigiana & / & / & / & $\begin{array}{l}61: 2>1 ; 79: 1>2 ; 111: 2>1 ; \\
132: 1>2 ; 133: 1>2 ; 136: 3>2 \\
152: 3>5\end{array}$ & & $107: 2>1$ \\
\hline 122 & $123+141$ & & & & & $31: 2>3 ; 107: 1>2 ; 121: 2>3$ & $\begin{array}{l}18: 5>4 ; 73: 2>3 ; 74: 2>3 \\
116: 1>2 ; 125: 2>3\end{array}$ \\
\hline 123 & $\begin{array}{l}((\text { Parasphendale }+ \\
\text { Sibylla })+(\text { Eucho }- \\
\text { menella }+(\text { Leptocola }+ \\
\text { Stenopyga }))+(\text { Popa }+ \\
(\text { Vates }+(\text { Ceratocrania }+ \\
\text { Phyllocrania })))\end{array}$ & & & & $15: 2>1 ; 91: 3>4$ & $\begin{array}{l}\mathbf{9 : 1 > 3} ; 18: 5>4 ; 73: 2>3 ; \\
74: 2>3 ; 116: 1>2 ; 125: 2>3\end{array}$ & \\
\hline 124 & $\begin{array}{l}\text { (Parasphendale }+ \text { Sibyl- } \\
\text { la })+(\text { Euchomenella }+ \\
(\text { Leptocola }+ \text { Stenopyga })) \\
\end{array}$ & & & & $78: 3>2 ; 79: 1>2 ; 128: 1>2$ & $12: 2>1 ; 13: 1>2 ; 87: 2>1$ & \\
\hline 125 & Parasphendale + Sibylla & & & & $\begin{array}{l}77: 3>2 ; 133: 1>2 ; 151: 3>4 \\
152: 3>5\end{array}$ & & \\
\hline 126 & Parasphendale affinis & / & / & / & $7: 2>1 ; 19: 3>4 ; 85: 2>1$ & $12: 1>2 ; 87: 1>2$ & \\
\hline 127 & Sibylla pretiosa & / & / & l & $\begin{array}{l}1: 2>1 ; 13: 2>1 ; 50: 1>2 ; \\
57: 1>7 ; 75: 2>3 ; 86: 2>1 ; \\
90: 2>1 ; 106: 2>1 ; 114: 2>1 ; \\
131: 2>1 ; 137: 2>1 ; 145: 2>1 \\
146: 2>1 ; 147: 2>1\end{array}$ & & $12: 2>1 ; 87: 2>1$ \\
\hline 128 & $\begin{array}{l}\text { Angelinae } \\
\text { Euchomenella }+(\text { Lepto- } \\
\text { cola }+ \text { Stenopyga })\end{array}$ & & & & $37: 2>4 ; 67: 2>1$ & $57: 1>6 ; 124: 1>2 ; 125: 3>2$ & $12: 2>1 ; 87: 2>1$ \\
\hline 129 & Euchomenella sp. & I & / & I & $14: 2>3 ; 33: 1>2 ; 50: 1>2$ & & $57: 1>6 ; 124: 1>2 ; 125: 3>2$ \\
\hline 130 & Leptocola + Stenopyga & & & $\mathrm{X}$ & $7: 2>1 ; 24: 1>2 ; 101: 1>2$ & $88: 1>2 ; 107: 2>1$ & \\
\hline 131 & Leptocola phthisica & / & I & I & $\begin{array}{l}52: 1>2 ; 53: 1>2 ; 59: 2>1 \\
80: 1>2 ; 86: 2>1 ; 114: 2>1\end{array}$ & & $57: 1>6 ; 107: 2>1$ \\
\hline 132 & Stenopyga ziela & I & I & I & $37: 4>3 ; 79: 2>1 ; 97: 1>2$ & $57: 6>1$ & $88: 1>2$ \\
\hline 133 & $\begin{array}{l}\text { Popa }+(\text { Vates }+(\text { Cerato }- \\
\text { crania }+ \text { Phyllocrania }))\end{array}$ & & & & $\begin{array}{l}88: 1>2 ; 90: 2>1 ; 92: 2>1 \\
93: 1>2 ; 126: 2>1\end{array}$ & $2: 1>2 ; 122: 2>1$ & \\
\hline 134 & Popa & $\mathrm{X}$ & $\mathrm{X}$ & $(\mathrm{X})$ & $\begin{array}{l}14: 2>3 ; 33: 1>2 ; 39: 1>2 \\
73: 3>2 ; 74: 3>2 ; 75: 2>3\end{array}$ & & $13: 2>1 ; 122: 2>1$ \\
\hline 135 & Popa spurca spurca & / & / & / & $114: 2>1 ; 124: 1>2$ & & \\
\hline 136 & $\begin{array}{l}\text { Vates }+(\text { Ceratocrania }+ \\
\text { Phyllocrania })\end{array}$ & & $(\mathrm{X})$ & & $\begin{array}{l}11: 2>1 ; 19: 3>2 ; 20: 1>2 \\
78: 3>4 ; 89: 1>2 ; 106: 2>1 \\
122: 1>3 / 2>3\end{array}$ & $7: 2>3$ & \\
\hline 137 & Vates weyrauchi & / & I & l & $\begin{array}{l}3: 2>1 ; 12: 2>1 ; 33: 1>3 ; \\
52: 1>2 ; 57: 1>4 ; 121: 3>2 \\
125: 3>2 ; 126: 1>3 ; 131: 2>1 \\
133: 1>2 ; 151: 3>4 ; 152: 3>4\end{array}$ & & $7: 2>3 ; 13: 2>1$ \\
\hline 138 & $\begin{array}{l}\text { Ceratocrania }+ \text { Phyllo- } \\
\text { crania }\end{array}$ & & & & $1: 2>1 ; 15: 1>2 ; 77: 3>4$ & $\begin{array}{l}13: 1>2 ; 74: 3>4 ; 107: 2>1 \\
145: 2>1\end{array}$ & $2: 1>2$ \\
\hline 139 & Ceratocrania macra & I & / & I & $\begin{array}{l}8: 2>1 ; 18: 4>2 ; 37: 2>4 \\
57: 1>2 ; 67: 2>1 ; 73: 3>4 \\
97: 1>2\end{array}$ & & $7: 2>3 ; \underline{\mathbf{9}: \mathbf{1}>\mathbf{3}}$ \\
\hline
\end{tabular}


Tab. 6 (continued): Apomorphy lists.

\begin{tabular}{|c|c|c|c|c|c|c|c|}
\hline Clade & Taxon & I S & I M & SW & Apomorphies & ACCTRAN only & DELTRAN only \\
\hline 140 & Phyllocrania paradoxa & l & / & l & $\begin{array}{l}29: 1>2 ; 39: 1>2 ; 48: 2>3 \\
76: 1>3 ; 79: 1>3 ; 93: 2>1 \\
104: 2>1 ; 116: 2>1 ; 119: 2>1 \\
122: 3>1 ; 128: 1>2\end{array}$ & $7: 3>2$ & $74: 3>4 ; 107: 2>1 ; 145: 2>1$ \\
\hline 141 & $142+161$ & & & & & $\begin{array}{l}13: 1>2 ; 18: 5>4 ; 73: 2>3 ; \\
74: 2>3 ; 116: 1>2 ; 125: 2>3\end{array}$ & $106: 2>1 ; 131: 2>1 ; 133: 1>2$ \\
\hline 142 & $\begin{array}{l}\text { (Sphodromantis }+(\text { Rhom- } \\
\text { bodera }+ \text { Tamolanica }))+ \\
\text { Empusidae }\end{array}$ & & & & $57: 1>2 ; \underline{110: 2>1}$ & $\begin{array}{l}33: 1>2 ; 106: 2>1 ; 131: 2>1 \\
133: 1>2\end{array}$ & \\
\hline 143 & $\begin{array}{l}\text { Sphodromantis }+(\text { Rhom- } \\
\text { bodera }+ \text { Tamolanica })\end{array}$ & $\mathrm{X}$ & $\mathrm{X}$ & & $14: 2>3 ; 122: 2>3 ; 151: 3>4$ & $19: 3>4 ; 152: 3>4$ & $33: 1>2$ \\
\hline 144 & Sphodromantis baccetti & 1 & 1 & 1 & $57: 2>4$ & & $19: 3>4 ; 152: 3>4$ \\
\hline 145 & $\begin{array}{l}\text { Rhombodera + Tamo- } \\
\text { lanica }\end{array}$ & $\mathrm{X}$ & $\mathrm{X}$ & $(\mathrm{X})$ & $29: 1>2 ; 126: 2>3$ & $18: 4>5 ; 152: 4>5$ & \\
\hline 146 & Rhombodera basalis & I & I & 1 & $97: 1>2 ; 151: 4>5$ & & $152: 3>5$ \\
\hline 147 & Tamolanica tamolana & / & I & 1 & $6: 1>2 ; 11: 2>1$ & & $18: 4>5$ \\
\hline 148 & $\begin{array}{l}\text { Empusidae } \\
\text { Blepharodes }+(\text { Ble- } \\
\text { pharopsis }+(\text { Idolomantis } \\
+ \text { Empusinae }))\end{array}$ & $(\mathrm{X})$ & $\mathrm{X}$ & $(\mathrm{X})$ & $\begin{array}{l}1: 2>1 ; 7: 2>3 ; 8: 2>1 ; 11: 2>1 \\
20: 1>5 ; 33: 2>3 / 1>3 ; 44: 2>3 \\
48: 2>3 ; 49: 1>2 ; 61: 2>1 \\
71: 1>2 ; 96: 1>2\end{array}$ & $111: 2>1$ & \\
\hline 149 & Blepharodes sudanensis & I & / & 1 & $18: 4>3 ; 19: 3>2$ & & $111: 2>1$ \\
\hline 150 & $\begin{array}{l}\text { Blepharopsis }+(\text { Idolo- } \\
\text { mantis }+(\text { Gongylus }+ \\
(\text { Empusa }+(\text { Hypsicory- } \\
\text { pha }+ \text { Idolomorpha })))\end{array}$ & $(\mathrm{X})$ & $\mathrm{X}$ & & $\begin{array}{l}29: 1>2 ; 73: 3>5 ; 74: 3>5 \\
90: 2>1 ; 145: 2>1 ; 146: 2>1\end{array}$ & $\begin{array}{l}39: 1>2 ; 76: 1>2 ; 77: 3>5 \\
78: 3>4\end{array}$ & \\
\hline 151 & Blepharopsis mendica & / & / & / & $\begin{array}{l}12: 2>1 ; 22: 2>1 ; 24: 1>2 \\
147: 2>1\end{array}$ & & $\begin{array}{l}39: 1>2 ; 76: 1>2 ; 77: 3>5 \\
78: 3>4 ; 111: 2>1\end{array}$ \\
\hline 152 & $\begin{array}{l}\text { Idolomantis }+(\text { Gongylus } \\
+(\text { Empusa }+(\text { Hypsicory- } \\
\text { pha }+ \text { Idolomorpha })))\end{array}$ & $(\mathrm{X})$ & $\mathrm{X}$ & & $\underline{\mathbf{3 4}}: \mathbf{2}>\mathbf{1} ; 50: 1>2 ; 77: 5>6 / 3>6$ & $\begin{array}{l}46: 3>5 ; 47: 3>5 ; 67: 2>1 ; \\
78: 4>6 ; 79: 1>3 ; 111: 1>2 \\
152: 3>4\end{array}$ & $78: 3>6$ \\
\hline 153 & Idolomantis diabolica & l & / & l & $\begin{array}{l}57: 2>6 ; 85: 2>1 ; 104: 2>1 \\
107: 2>1 ; 119: 2>1 ; 122: 2>3 \\
126: 2>3 ; 151: 3>4\end{array}$ & $76: 2>1$ & $\begin{array}{l}39: 1>2 ; 46: 3>5 ; 47: 3>5 \\
67: 2>1 ; 79: 1>3 ; 152: 3>4\end{array}$ \\
\hline 154 & $\begin{array}{l}\text { Empusinae } \\
\text { Gongylus }+(\text { Empusa }+ \\
(\text { Hypsicorypha }+ \text { Idolo- } \\
\text { morpha }))\end{array}$ & & $\mathrm{X}$ & $(\mathrm{X})$ & $\begin{array}{l}19: 3>2 ; 20: 5>4 ; 87: 2>1 ; \\
133: 2>1 ; \underline{\mathbf{1 3 8 : 2}}>\mathbf{1}\end{array}$ & $33: 3>1 ; 39: 2>1 ; 73: 5>4$ & $76: 1>2$ \\
\hline 155 & Gongylus gongylodes & l & l & l & $\begin{array}{l}33: 1>2 / 3>2 ; 48: 3>4 ; 59: 2>1 \\
73: 4>6 / 5>6 ; 74: 5>6 ; 86: 2>1 \\
88: 1>2 ; 89: 1>2 ; 96: 2>1 \\
106: 1>2 ; 125: 3>2\end{array}$ & $67: 1>2 ; 79: 3>1$ & $46: 3>5 ; 47: 3>5 ; 152: 3>4$ \\
\hline 156 & $\begin{array}{l}\text { Empusa }+(\text { Hypsicorypha } \\
+ \text { Idolomorpha })\end{array}$ & & $\mathrm{X}$ & & $29: 2>1 ; 77: 6>5$ & $\begin{array}{l}37: 2>3 ; 46: 5>3 ; 47: 5>3 \\
152: 4>3\end{array}$ & $\begin{array}{l}33: 3>1 ; 67: 2>1 ; 73: 5>4 \\
79: 1>3\end{array}$ \\
\hline 157 & Empusa pennata & / & I & I & $\begin{array}{l}13: 2>1 ; 18: 4>3 ; 39: 1>2 \\
78: 6>5 ; 85: 2>1 ; 111: 2>1 \\
128: 1>2 ; 147: 2>1\end{array}$ & & $37: 2>3$ \\
\hline 158 & $\begin{array}{l}\text { Idolomorphini } \\
\text { Hypsicorypha }+ \text { Idolo- } \\
\text { morpha }\end{array}$ & $\mathrm{X}$ & $\mathrm{X}$ & $?$ & $\begin{array}{l}37: 3>4 / 2>4 ; 47: 3>4 ; 71: 2>1 \\
74: 5>4 ; 90: 1>2 ; 107: 2>1 \\
136: 3>2 ; 145: 1>2 ; 146: 1>2\end{array}$ & $46: 3>4 ; 104: 2>1 ; 134: 3>2$ & \\
\hline 159 & Hypsicorypha gracilis & I & / & I & $86: 2>1 ; 89: 1>2$ & & \\
\hline 160 & Idolomorpha dentifrons & / & / & / & $19: 2>3 ; 20: 4>3 ; 57: 2>1$ & & $46: 3>4 ; 104: 2>1 ; 134: 3>2$ \\
\hline 161 & $162+195$ & & & & & $106: 2>1 ; 131: 2>1 ; 133: 1>2$ & $\underline{9: 1>2}$ \\
\hline 162 & Mantis +164 & & & & $\begin{array}{l}37: 2>3 ; 57: 1>6 ; 67: 2>1 \\
151: 3>4 ; 152: 3>4\end{array}$ & $\underline{\mathbf{9 : 1}>\mathbf{2}} ; 18: 4>3$ & \\
\hline 163 & Mantis religiosa & I & / & / & $\begin{array}{l}59: 2>1 ; 82: 1>2 ; 88: 1>2 ; \\
89: 1>2 ; 104: 2>1 ; 126: 2>3 ; \\
128: 1>2\end{array}$ & & $18: 4>3$ \\
\hline 164 & $165+174$ & & & & $19: 3>5 ; 111: 2>1$ & $7: 2>1 ; 18: 3>5$ & $18: 4>5$ \\
\hline 165 & $\begin{array}{l}\text { Tisma }+(\text { Zoolea }+(\text { Stag- } \\
\text { matoptera }+ \text { Polyspi- } \\
\text { lotini }))\end{array}$ & & & & $57: 6>4 ; 87: 2>1$ & & \\
\hline 166 & Tisma pauliani & 1 & 1 & 1 & & & $7: 2>1$ \\
\hline
\end{tabular}


Tab. 6 (continued): Apomorphy lists.

\begin{tabular}{|c|c|c|c|c|c|c|c|}
\hline Clade & Taxon & I S & I M & SW & Apomorphies & ACCTRAN only & DELTRAN only \\
\hline 167 & $\begin{array}{l}\text { Zoolea }+(\text { Stagmatoptera } \\
+ \text { Polyspilotini })\end{array}$ & & & & $33: 1>2 ; 152: 4>5$ & $7: 1>2$ & \\
\hline 168 & Zoolea lobipes & / & / & l & $\begin{array}{l}3: 2>1 ; 11: 2>1 ; 12: 2>1 \\
20: 1>2 ; 21: 1>2 ; 73: 3>4 \\
74: 3>4 ; 76: 1>2 ; 86: 2>1 \\
90: 2>1 ; 92: 2>1 ; 96: 1>2 \\
104: 2>1 ; 145: 2>1 ; 146: 2>1 \\
147: 2>1\end{array}$ & & \\
\hline 169 & $\begin{array}{l}\text { Stagmatoptera + Polyspi- } \\
\text { lotini }\end{array}$ & & & & $122: 2>3 ; 126: 2>3$ & $128: 1>2$ & \\
\hline 170 & Stagmatoptera hyaloptera & I & / & / & $\begin{array}{l}52: 1>2 ; 82: 1>2 ; 87: 1>2 ; \\
128: 1>2\end{array}$ & & \\
\hline 171 & $\begin{array}{l}\text { Polyspilotini } \\
\text { Plistospilota }+ \text { Polyspi- } \\
\text { lota }\end{array}$ & & $\mathrm{X}$ & $(\mathrm{X})$ & $\begin{array}{l}37: 3>2 ; 67: 1>2 ; 79: 1>2 \\
111: 1>2 ; 114: 2>3\end{array}$ & & \\
\hline 172 & Plistospilota guineensis & / & / & l & $\begin{array}{l}11: 2>1 ; 18: 5>6 ; 19: 5>6 \\
33: 2>3 ; 104: 2>1 ; 107: 2>1 \\
113: 1>2 ; 151: 4>5 ; 152: 5>6\end{array}$ & $128: 2>1$ & \\
\hline 173 & Polyspilota aeruginosa & / & / & / & $\begin{array}{l}7: 2>1 ; 57: 4>1 ; 74: 3>2 \\
134: 3>2 ; 136: 3>2\end{array}$ & & $128: 1>2$ \\
\hline 174 & $\begin{array}{l}\text { Deroplatys }+(\text { Oxyopsis }+ \\
(\text { Archimantis }+(\text { Hetero- } \\
\text { chaeta }+(\text { Oxyothespis }+ \\
\text { Toxoderidae })))\end{array}$ & & & & $79: 1>2 ; 122: 2>1 ; 126: 2>1$ & $107: 2>1$ & \\
\hline 175 & Deroplatys & $\mathrm{X}$ & $\mathrm{X}$ & $(\mathrm{X})$ & $\begin{array}{l}29: 1>2 ; 33: 1>3 ; 90: 2>1 ; \\
104: 2>1 ; 114: 2>1 ; 121: 3>2 \\
125: 3>2 ; 131: 1>2 ; 145: 2>1\end{array}$ & & $7: 2>1 ; 107: 2>1$ \\
\hline 176 & Deroplatys lobata & / & l & / & $\begin{array}{l}73: 3>4 ; 74: 3>4 ; 93: 1>2 \\
152: 4>5\end{array}$ & & \\
\hline 177 & Deroplatys desiccata & / & l & l & $6: 1>2$ & & \\
\hline 178 & $\begin{array}{l}\text { Oxyopsis }+(\text { Archimantis } \\
+(\text { Heterochaeta }+(\text { Oxyo- } \\
\text { thespis }+ \text { Toxoderidae })))\end{array}$ & & & & $\begin{array}{l}15: 2>1 ; 18: 5>3 ; 57: 6>2 \\
124: 1>2 ; 128: 1>2\end{array}$ & $19: 5>2$ & \\
\hline 179 & Oxyopsis gracilis & / & l & l & $37: 3>2 ; 82: 1>2 ; 116: 2>1$ & $107: 1>2$ & $7: 2>1 ; 19: 5>2$ \\
\hline 180 & $\begin{array}{l}\text { Archimantis }+(\text { Hetero- } \\
\text { chaeta }+(\text { Oxyothespis }+ \\
\text { Toxoderidae }))\end{array}$ & & & & $\begin{array}{l}106: 1>2 ; 111: 1>2 ; 149: 2>1 \\
150: 1>2\end{array}$ & $7: 1>2$ & \\
\hline 181 & Archimantis & $\mathrm{X}$ & $\mathrm{X}$ & $?$ & $\begin{array}{l}33: 1>2 ; 57: 2>5 ; 87: 2>1 \\
104: 2>1 ; 122: 1>2 ; 126: 1>2\end{array}$ & $18: 3>6 ; 19: 2>5$ & $107: 2>1$ \\
\hline 182 & Archimantis gracilis & I & l & l & & & $18: 3>6$ \\
\hline 183 & Archimantis sobrina & I & l & l & $73: 3>4$ & & \\
\hline 184 & $\begin{array}{l}\text { Heterochaeta }+(\text { Oxyo- } \\
\text { thespis }+ \text { Toxoderidae })\end{array}$ & & & & $\begin{array}{l}22: 2>1 ; 78: 3>2 ; 120: 1>2 ; \\
133: 2>1\end{array}$ & $\begin{array}{l}16: 2>1 ; 52: 1>2 ; 73: 3>2 \\
101: 1>2 ; 134: 3>2\end{array}$ & \\
\hline 185 & Heterochaeta & $\mathrm{X}$ & $\mathrm{X}$ & & $\begin{array}{l}18: 3>4 ; 39: 1>2 ; 48: 2>3 \\
50: 1>3 ; 53: 1>2 ; 73: 2>5 / 3>5 \\
76: 1>5 ; 90: 2>1 ; 93: 1>2 \\
132: 1>2\end{array}$ & $\begin{array}{l}19: 2>3 ; 47: 3>2 ; 74: 3>4 \\
136: 3>2\end{array}$ & $52: 1>2 ; 101: 1>2 ; 134: 3>2$ \\
\hline 186 & Heterochaeta bernardii & / & / & l & & & $16: 2>1$ \\
\hline 187 & Heterochaeta strachani & / & / & l & $77: 3>2$ & $16: 1>2$ & $\begin{array}{l}19: 5>3 ; 47: 3>2 ; 74: 3>4 \\
107: 2>1 ; 136: 3>2\end{array}$ \\
\hline 188 & $\begin{array}{l}\text { Oxyothespis + Toxode- } \\
\text { ridae }\end{array}$ & & & $(\mathrm{X})$ & $12: 2>1 ; 55: 4>3 ; 80: 1>2$ & $\begin{array}{l}24: 1>2 ; 85: 2>1 ; 91: 3>2 \\
97: 1>2 ; 107: 1>2 ; 152: 4>2\end{array}$ & $16: 2>1 ; 73: 3>2$ \\
\hline 189 & Oxyothespis senegalensis & & & & $\begin{array}{l}7: 2>1 ; 46: 3>2 ; 79: 2>1 \\
81: 2>1\end{array}$ & $52: 2>1$ & $24: 1>2 ; 85: 2>1 ; 97: 1>2$ \\
\hline 190 & $\begin{array}{l}\text { Toxoderidae } \\
\text { Calamothespis }+ \\
\text { Toxodera }\end{array}$ & $\mathrm{X}$ & $\mathrm{X}$ & $(\mathrm{X})$ & $\begin{array}{l}37: 3>2 ; \underline{\mathbf{4 3}: \mathbf{1}>\mathbf{2}} ; 48: 2>4 \\
67: 1>2 ; \underline{\mathbf{6 9 : 1}>\mathbf{3}}\end{array}$ & $11: 2>1 ; 77: 3>2 ; 104: 2>1$ & $52: 1>2$ \\
\hline 191 & Calamothespis condamini & / & / & / & $\begin{array}{l}13: 2>1 ; 15: 1>2 ; 21: 1>2 \\
82: 1>2 ; 111: 2>1\end{array}$ & $24: 2>1 ; 85: 1>2 ; 101: 2>1$ & $97: 1>2 ; 152: 4>2$ \\
\hline 192 & Toxodera & $\mathrm{X}$ & $\mathrm{X}$ & & $\begin{array}{l}8: 2>1 ; 39: 1>2 ; 58: 2>1 \\
76: 1>5 ; 90: 2>1 ; 148: 2>1 \\
150: 2>3 ; 152: 2>5 / 4>5\end{array}$ & $50: 1>2 ; 74: 3>2 ; 97: 2>1$ & $\begin{array}{l}11: 2>1 ; 24: 1>2 ; 85: 2>1 ; \\
91: 3>2 ; 101: 1>2\end{array}$ \\
\hline
\end{tabular}


Tab. 6 (continued): Apomorphy lists.

\begin{tabular}{|c|c|c|c|c|c|c|c|}
\hline Clade & Taxon & I S & I M & SW & Apomorphies & ACCTRAN only & DELTRAN only \\
\hline 193 & Toxodera denticulata & I & / & / & $72: 2>3 ; 74: 2>5 / 3>5 ; 78: 2>3$ & & $50: 1>2 ; 77: 3>2 ; 104: 2>1$ \\
\hline 194 & Toxodera maculata & l & l & I & & & $19: 5>2 ; 74: 3>2$ \\
\hline 195 & $196+215$ & & & & & $\underline{9: 1>2}$ & $11: 2>1 ; 97: 1>2$ \\
\hline 196 & $\begin{array}{l}(\text { Acromantis }+ \text { Ambivia })+ \\
\text { Hymenopodinae }\end{array}$ & & & & $\begin{array}{l}2: 1>3 ; 12: 2>1 ; 18: 4>2 \\
76: 1>3 ; 90: 2>1\end{array}$ & $\begin{array}{l}8: 2>1 ; 11: 2>1 ; 15: 2>1 \\
19: 3>1 ; 62: 2>1 ; 77: 3>4 \\
97: 1>2 ; 128: 1>2\end{array}$ & \\
\hline 197 & Acromantis + Ambivia & & & & $\begin{array}{l}1: 2>1 ; 7: 2>3 ; 73: 3>2 \\
104: 2>1\end{array}$ & $74: 3>2 ; 107: 2>1$ & $15: 2>1$ \\
\hline 198 & Acromantis sp. & l & / & l & $\begin{array}{l}53: 1>2 ; 79: 1>3 ; 111: 2>1 \\
116: 2>1\end{array}$ & $77: 4>3 ; 128: 2>1$ & $8: 2>1 ; 74: 3>2 ; 107: 2>1$ \\
\hline 199 & Ambivia sp. & / & / & / & $\begin{array}{l}14: 2>3 ; 33: 1>2 ; 39: 1>2 \\
67: 2>1 ; 76: 3>2 ; 97: 2>1 ; \\
122: 2>3 ; 133: 2>1\end{array}$ & $8: 1>2$ & $77: 3>4 ; 128: 1>2$ \\
\hline 200 & $\begin{array}{l}\text { Hymenopodinae } \\
\text { Galinthias }+(\text { Pseudohar }- \\
\text { pax }+(\text { Pseudocreobotra } \\
+(\text { Creobroter gemmatus } \\
+(\text { Creobroter elongatus } \\
+(\text { Hymenopus }+ \text { Theop }- \\
\text { ropus }))))\end{array}$ & $(\mathrm{X})$ & $(\mathrm{X})$ & & $\begin{array}{l}14: 2>1 ; 15: 1>3 / 2>3 ; 46: 3>2 \\
47: 3>2 ; 88: 1>2 ; 89: 1>2 \\
93: 1>2\end{array}$ & $3: 2>1 ; 16: 2>1 ; 78: 3>4$ & $8: 2>1$ \\
\hline 201 & Galinthias amoena & l & I & / & $\begin{array}{l}53: 1>2 ; 114: 2>3 ; 120: 1>2 \\
122: 2>1 ; 124: 1>2 ; 126: 2>1 \\
134: 3>2 ; 136: 3>2 ; 151: 3>2\end{array}$ & & $\begin{array}{l}3: 2>1 ; 16: 2>1 ; 77: 3>4 \\
78: 3>4 ; 128: 1>2\end{array}$ \\
\hline 202 & $\begin{array}{l}\text { Pseudoharpax }+(\text { Pseudo- } \\
\text { creobotra }+(\text { Creobroter } \\
\text { gemmatus }+(\text { Creobroter } \\
\text { elongatus }+(\text { Hymenopus } \\
+ \text { Theopropus }))))\end{array}$ & & & & $20: 1>2 ; 31: 3>2$ & & \\
\hline 203 & Pseudoharpax sp. & I & I & I & $12: 1>2 ; 73: 3>2$ & $77: 4>3 ; 78: 4>3$ & $\begin{array}{l}3: 2>1 ; 16: 2>1 ; 19: 3>1 \\
128: 1>2\end{array}$ \\
\hline 204 & $\begin{array}{l}\text { Pseudocreobotra }+ \\
\text { (Creobroter gemmatus } \\
+(\text { Creobroter elongatus } \\
+(\text { Hymenopus }+ \text { Theop }- \\
\text { ropus })))\end{array}$ & & & $(\mathrm{X})$ & $\begin{array}{l}1: 2>1 ; 19: 1>2 / 3>2 ; 28: 1>2 \\
33: 1>2 ; 71: 1>2\end{array}$ & $\begin{array}{l}3: 1>2 ; 16: 1>2 ; 57: 1>2 \\
128: 2>1\end{array}$ & $62: 2>1 ; 77: 3>4 ; 78: 3>4$ \\
\hline 205 & Pseudocreobotra & $\mathrm{X}$ & $\mathrm{X}$ & $?$ & $\begin{array}{l}13: 2>1 ; 31: 2>1 ; 39: 1>2 \\
49: 1>3 ; 52: 1>2 ; 55: 4>3 \\
58: 2>1 ; 79: 1>3 ; 97: 2>1 \\
111: 2>1 ; 116: 2>1 ; 145: 2>1 \\
146: 2>1 ; 147: 2>1\end{array}$ & & \\
\hline 206 & Pseudocreobotra ocellata & / & / & / & $78: 4>5 ; 126: 2>3$ & & \\
\hline 207 & $\begin{array}{l}\text { Pseudocreobotra wahl- } \\
\text { bergii }\end{array}$ & I & / & / & $\begin{array}{l}29: 1>2 ; 77: 4>5 ; 89: 2>1 ; \\
152: 3>4\end{array}$ & & \\
\hline 208 & $\begin{array}{l}\text { Creobroter gemmatus }+ \\
(\text { Creobroter elongatus }+ \\
(\text { Hymenopus }+ \text { Theop }- \\
\text { ropus }))\end{array}$ & & & & $\begin{array}{l}46: 2>3 ; 47: 2>3 ; 104: 2>1 ; \\
107: 2>1\end{array}$ & $151: 3>2$ & $57: 1>2$ \\
\hline 209 & Creobroter gemmatus & I & I & I & $18: 2>3 ; 76: 3>2$ & & \\
\hline 210 & $\begin{array}{l}\text { Creobroter elongatus }+ \\
\text { (Hymenopus }+ \text { Theop- } \\
\text { ropus) }\end{array}$ & & & & $74: 3>4$ & & \\
\hline 211 & Creobroter elongatus & I & / & I & & & $151: 3>2$ \\
\hline 212 & Hymenopus + Theopropus & & & $\mathrm{X}$ & $\begin{array}{l}11: 1>2 ; 28: 2>1 ; 33: 2>3 ; \\
49: 1>2 ; 73: 3>4 ; 78: 4>6 ; \\
79: 1>3 ; 111: 2>1 ; 126: 2>3 \\
152: 3>4\end{array}$ & $61: 2>1 ; 77: 4>5$ & \\
\hline 213 & Hymenopus coronatus & l & / & / & $\begin{array}{l}12: 1>2 ; 16: 2>1 ; 19: 2>3 \\
39: 1>2 ; 74: 4>5 ; 76: 3>2 \\
77: 5>6 / 4>6 ; 91: 3>4 ; 104: 1>2 \\
107: 1>2 ; 133: 2>1\end{array}$ & $151: 2>3$ & $61: 2>1$ \\
\hline 214 & Theopropus elegans & l & I & I & $\begin{array}{l}2: 3>1 ; 3: 2>1 ; 14: 1>2 ; 15: 3>2 \\
20: 2>1 ; 52: 1>2 ; 55: 4>3 \\
67: 2>1 ; 97: 2>1 ; 142: 1>2 \\
146: 2>1\end{array}$ & & $77: 4>5 ; 151: 3>2$ \\
\hline
\end{tabular}


Tab. 6 (continued): Apomorphy lists.

\begin{tabular}{|c|c|c|c|c|c|c|c|}
\hline Clade & Taxon & I S & I M & SW & Apomorphies & ACCTRAN only & DELTRAN only \\
\hline 215 & $\begin{array}{l}\text { Choeradodis }+(\text { Ortho- } \\
\text { dera }+(\text { Iris }+(\text { Photina }+ \\
(\text { Orthoderella }+ \text { Acantho- } \\
\text { pidae })))\end{array}$ & & & & & $11: 2>1 ; 97: 1>2$ & $14: 2>3 ; 122: 2>3 ; 134: 3>2$ \\
\hline 216 & Choeradodis & $\mathrm{X}$ & $\mathrm{X}$ & $(\mathrm{X})$ & $29: 1>2 ; 67: 2>1 ; 111: 2>1$ & $\begin{array}{l}14: 2>3 ; 122: 2>3 ; 125: 3>2 \\
134: 3>2 ; 151: 3>4\end{array}$ & \\
\hline 217 & Choeradodis rhombicollis & / & / & / & $76: 1>2 ; 77: 3>4$ & & $151: 3>4$ \\
\hline 218 & Choeradodis stalii & / & / & / & $52: 1>2 ; 82: 1>2 ; 151: 4>5 / 3>5$ & & $125: 3>2$ \\
\hline 219 & $\begin{array}{l}\text { Orthodera }+(\text { Iris }+ \\
(\text { Photina }+(\text { Orthoderella } \\
+ \text { Acanthopidae })))\end{array}$ & & & & $18: 4>3 ; 77: 3>4$ & $14: 2>4 ; 122: 2>3 ; 134: 3>2$ & $57: 1>2 ; 78: 3>4 ; 104: 2>1$ \\
\hline 220 & $\begin{array}{l}\text { Orthodera novaezeal- } \\
\text { andiae }\end{array}$ & l & / & / & $\begin{array}{l}7: 2>1 ; 21: 1>2 ; 88: 1>2 ; \\
89: 1>2 ; 126: 2>3 ; 133: 2>1 ; \\
136: 3>2\end{array}$ & $57: 1>2 ; 78: 3>4 ; 104: 2>1$ & \\
\hline 221 & $\begin{array}{l}\text { Iris }+(\text { Photina }+(\text { Ortho }- \\
\text { derella }+ \text { Acanthopidae }))\end{array}$ & & $(\mathrm{X})$ & & & $57: 1>2 ; 78: 3>4 ; 104: 2>1$ & $\begin{array}{l}11: 1>2 ; 14: 3>2 ; 48: 2>3 ; \\
106: 1>2 ; 107: 2>1 ; 120: 1>2 \\
132: 1>2\end{array}$ \\
\hline 222 & Iris oratoria & l & l & I & $\begin{array}{l}50: 1>3 ; 97: 2>1 ; 122: 3>1 \\
124: 1>2 ; 126: 2>1 ; 128: 1>2 \\
131: 1>2 ; 151: 3>2\end{array}$ & $\begin{array}{l}11: 1>2 ; 14: 3>2 ; 48: 2>3 \\
106: 1>2 ; 107: 2>1 ; 120: 1>2 \\
132: 1>2\end{array}$ & \\
\hline 223 & $\begin{array}{l}\text { Photina }+(\text { Orthoderella } \\
+ \text { Acanthopidae })\end{array}$ & & & $(\mathrm{X})$ & & $\begin{array}{l}11: 1>2 ; 14: 3>2 ; 48: 2>3 \\
106: 1>2 ; 107: 2>1 ; 132: 1>2\end{array}$ & $130: 1>4 ; 131: 1>4$ \\
\hline 224 & Photina laevis & l & l & / & $\begin{array}{l}7: 2>1 ; 12: 2>1 ; 76: 1>2 \\
133: 2>1 ; 136: 3>2\end{array}$ & $130: 1>4 ; 131: 1>4$ & \\
\hline 225 & $\begin{array}{l}\text { Orthoderella + Acan- } \\
\text { thopidae }\end{array}$ & & & & & $130: 1>4 ; 131: 1>4$ & $55: 4>3 ; 132: 2>1$ \\
\hline 226 & Orthoderella ornata & / & l & l & $\begin{array}{l}13: 2>1 ; 15: 2>1 ; 18: 3>5 \\
19: 3>5 ; 67: 2>1 ; 73: 3>4 \\
74: 3>4 ; 82: 1>2 ; 107: 1>2 \\
149: 2>1\end{array}$ & $55: 4>3 ; 132: 2>1$ & \\
\hline 227 & $\begin{array}{l}\text { Acanthopidae } \\
\text { Acontista }+(\text { Raptrix }+ \\
(\text { Tithrone }+(\text { Callibia }+ \\
\text { Acanthops })))\end{array}$ & & $\mathrm{X}$ & $(\mathrm{X})$ & $122: 3>1 ; 151: 3>2$ & $\begin{array}{l}55: 4>3 ; 120: 1>2 ; 128: 1>2 ; \\
132: 2>1\end{array}$ & $\begin{array}{l}12: 2>1 ; 31: 3>2 ; 53: 1>2 \\
58: 2>1 ; 76: 1>3 ; 152: 3>2\end{array}$ \\
\hline 228 & Acontista brevipennis & / & / & / & $\begin{array}{l}8: 2>1 ; 18: 3>2 ; 24: 1>2 ; \\
46: 3>2 ; 47: 3>2 ; 52: 1>2 \\
62: 2>1 ; 74: 3>2 ; 121: 3>2\end{array}$ & $\begin{array}{l}12: 2>1 ; 31: 3>2 ; 53: 1>2 \\
58: 2>1 ; 76: 1>3 ; 152: 3>2\end{array}$ & $128: 1>2$ \\
\hline 229 & $\begin{array}{l}\text { Raptrix }+(\text { Tithrone }+ \\
(\text { Callibia }+ \text { Acanthops }))\end{array}$ & $(\mathrm{X})$ & $\mathrm{X}$ & & & $\begin{array}{l}8: 2>1 ; 12: 2>1 ; 31: 3>2 \\
58: 2>1 ; 76: 1>3 ; 124: 1>2 \\
152: 3>2\end{array}$ & $\begin{array}{l}71: 1>2 ; 79: 1>3 ; 106: 2>1 ; \\
116: 2>1 ; 117: 1>2 ; 134: 2>3 \\
142: 1>2\end{array}$ \\
\hline 230 & Raptrix fusca & / & / & / & $20: 1>2 ; 37: 2>1 ; 60: 2>1$ & $\begin{array}{l}71: 1>2 ; 79: 1>3 ; 106: 2>1 \\
116: 2>1 ; 117: 1>2 ; 128: 2>1 \\
134: 2>3 ; 142: 1>2\end{array}$ & $8: 2>1 ; 124: 1>2$ \\
\hline 231 & $\begin{array}{l}\text { Tithrone }+(\text { Callibia }+ \\
\text { Acanthops })\end{array}$ & & $\mathrm{X}$ & & $126: 2>1$ & $\begin{array}{l}24: 1>2 ; 71: 1>2 ; 79: 1>3 ; \\
106: 2>1 ; 116: 2>1 ; 117: 1>2 \\
128: 2>1 ; 134: 2>3 ; 142: 1>2\end{array}$ & $120: 2>1 ; 121: 3>2$ \\
\hline 232 & Tithrone roseipennis & / & / & / & $\begin{array}{l}7: 2>1 ; 13: 2>1 ; 15: 2>1 \\
76: 3>1 ; 87: 2>1 ; 104: 1>2 \\
107: 1>2 ; 125: 3>2\end{array}$ & $\begin{array}{l}8: 1>2 ; 120: 2>1 ; 121: 3>2 \\
124: 2>1\end{array}$ & $24: 1>2$ \\
\hline 233 & Callibia + Acanthops & $\mathrm{X}$ & $\mathrm{X}$ & & & $53: 1>2 ; 120: 2>1 ; 121: 3>2$ & $\begin{array}{l}45: 2>1 ; 74: 3>4 ; 93: 1>2 ; \\
135: 2>1 ; 145: 2>1\end{array}$ \\
\hline 234 & Callibia diana & / & / & / & $\begin{array}{l}1: 2>1 ; 19: 3>2 ; 37: 2>1 ; \\
49: 1>3 ; 77: 4>5 ; 78: 4>5 \\
88: 1>2 ; 90: 2>1 ; 11: 2>1 \\
114: 2>3 ; 116: 1>2 ; 128: 1>2 \\
134: 3>4 ; 147: 2>1 ; 151: 2>1 \\
152: 2>1\end{array}$ & $\begin{array}{l}24: 2>1 ; 45: 2>1 ; 74: 3>1 ; \\
93: 1>2 ; 135: 2>1 ; 145: 2>1\end{array}$ & $8: 2>1$ \\
\hline 235 & Acanthops & $\mathrm{X}$ & $\mathrm{X}$ & & $125: 3>2$ & $\begin{array}{l}24: 2>1 ; 45: 2>1 ; 74: 3>4 \\
93: 1>2 ; 124: 2>1 ; 145: 2>1\end{array}$ & $\begin{array}{l}16: 2>1 ; 31: 2>3 ; 33: 1>2 ; \\
48: 3>4 ; 49: 1>2 ; 55: 3>4 ; \\
57: 2>161: 2>1 ; 71: 2>1 ; \\
76: 3>2 ; 78: 4>6 ; 85: 2>1 ; \\
133: 2>1 ; 136: 3>4 ; 150: 1>3\end{array}$ \\
\hline
\end{tabular}


Tab. 6 (continued): Apomorphy lists.

\begin{tabular}{|c|c|c|c|c|c|c|c|}
\hline Clade & Taxon & I S & I M & SW & Apomorphies & ACCTRAN only & DELTRAN only \\
\hline 236 & Acanthops falcataria & l & / & 1 & $\begin{array}{l}18: 3>2 ; 19: 3>2 ; 20: 1>2 \\
73: 3>4 ; 77: 4>6 ; 134: 3>4 \\
142: 2>1 ; 151: 2>1\end{array}$ & $\begin{array}{l}8: 1>2 ; 16: 2>1 ; 31: 2>3 \\
33: 1>2 ; 48: 3>4 ; 49: 1>2 \\
55: 3>4 ; 57: 2>1 ; 61: 2>1 ; \\
71: 2>1 ; 76: 3>2 ; 78: 4>6 ; \\
85: 2>1 ; 133: 2>1 ; 135: 2>1 ; \\
136: 3>4 ; 150: 1>3\end{array}$ & \\
\hline 237 & Acanthops tuberculata & / & / & 1 & $97: 2>1 ; 152: 2>3$ & $\begin{array}{l}8: 1>2 ; 16: 2>1 ; 31: 2>3 \\
33: 1>2 ; 48: 3>4 ; 49: 1>2 \\
55: 3>4 ; 57: 2>1 ; 61: 2>1 \\
71: 2>1 ; 76: 3>2 ; 78: 4>6 ; \\
85: 2>1 ; 133: 2>1 ; 136: 3>4 \\
150: 1>3\end{array}$ & \\
\hline
\end{tabular}


Tab. 7: Classification of the genera used in this study, sorted alphabetically by genus names (Ehrmann 2002).

\begin{tabular}{|c|c|c|c|}
\hline Genus & Family & Subfamily & Tribus \\
\hline Acanthops & Acanthopidae & Acanthopinae & Acanthopini \\
\hline Acontista & Acanthopidae & Acontistinae & Acontistini \\
\hline Acromantis & Hymenopodidae & Acromantinae & Acromantini \\
\hline Ambivia & Mantidae & Vatinae & Danuriini \\
\hline Ameles & Mantidae & Amelinae & Amelini \\
\hline Amorphoscelis & Amorphoscelidae & Amorphoscelinae & - \\
\hline Amphecostephanus & Hymenopodidae & Epaphroditinae & Phyllocranini \\
\hline Archimantis & Mantidae & Mantinae & Archimantini \\
\hline Astape & Thespidae & Haaniinae & - \\
\hline Bactromantis & Thespidae & Oligonicinae & Oligonicini \\
\hline Blepharodes & Empusidae & Blepharodinae & Blepharodini \\
\hline Blepharopsis & Empusidae & Blepharodinae & Blepharodini \\
\hline Bolivaria & Mantidae & Miomantinae & Rivetinini \\
\hline Calamothespis & Toxoderidae & Toxoderinae & Toxoderopsini \\
\hline Callibia & Acanthopidae & Acontistinae & Acontistini \\
\hline Caudatoscelis & Amorphoscelidae & Amorphoscelinae & - \\
\hline Ceratocrania & Mantidae & Phyllotheliinae & - \\
\hline Ceratomantis & Hymenopodidae & Oxypilinae & Oxypilini \\
\hline Chaeteessa & Chaeteessidae & - & - \\
\hline Choeradodis & Mantidae & Choeradodinae & - \\
\hline Ciulfina & Liturgusidae & Liturgusinae & Liturgusini \\
\hline Compsothespis & Mantidae & Compsothespinae & - \\
\hline Creobroter & Hymenopodidae & Hymenopodinae & Hymenopodini \\
\hline Deroplatys & Mantidae & Deroplatyinae & - \\
\hline Dystacta & Mantidae & Dystactinae & Dystactini \\
\hline Elaea & Mantidae & Amelinae & Amelini \\
\hline Empusa (Empusa) & Empusidae & Empusinae & Empusini \\
\hline Enicophlebia & Iridopterygidae & Tropidomantinae & Tropidomantini \\
\hline Entella (Entella) & Mantidae & Chroicopterinae & Chroicopterini \\
\hline Epaphrodita & Hymenopodidae & Epaphroditinae & Phyllocranini \\
\hline Eremiaphila & Eremiaphilidae & - & \\
\hline Euchomenella & Mantidae & Angelinae & Angelini \\
\hline Galinthias & Hymenopodidae & Hymenopodinae & Hymenopodini \\
\hline Gimantis & Mantidae & Amelinae & Amelini \\
\hline Gongylus & Empusidae & Empusinae & Empusini \\
\hline Gonypeta & Mantidae & Amelinae & Amelini \\
\hline Gyromantis & Amorphoscelidae & Paraoxypilinae & - \\
\hline Haania & Thespidae & Haaniinae & - \\
\hline Hestiasula & Hymenopodidae & Acromantinae & Acromantini \\
\hline Heterochaeta & Mantidae & Vatinae & Heterochaetini \\
\hline Heteronutarsus & Eremiaphilidae & - & \\
\hline Hoplocorypha & Thespidae & Hoplocoryphinae & Hoplocoryphini \\
\hline Humbertiella & Liturgusidae & Liturgusinae & Liturgusini \\
\hline Hymenopus & Hymenopodidae & Hymenopodinae & Hymenopodini \\
\hline Hypsicorypha & Empusidae & Empusinae & Idolomorphini \\
\hline Idolomantis & Empusidae & Blepharodinae & Idolomantini \\
\hline Idolomorpha & Empusidae & Empusinae & Idolomorphini \\
\hline Iris & Tarachodidae & Tarachodinae & - \\
\hline Leptocola & Mantidae & Angelinae & Angelini \\
\hline Ligaria & Mantidae & Chroicopterinae & Chroicopterini \\
\hline Ligariella & Mantidae & Chroicopterinae & Chroicopterini \\
\hline Maculatoscelis & Amorphoscelidae & Amorphoscelinae & - \\
\hline Majanga & Liturgusidae & Liturgusinae & Liturgusini \\
\hline
\end{tabular}


Tab. 7 (continued): Classification of the genera used in this study, sorted alphabetically by genus names (Ehrmann 2002).

\begin{tabular}{|c|c|c|c|}
\hline Mantis & Mantidae & Mantinae & Mantini \\
\hline Mantoida & Mantoididae & - & - \\
\hline Metallyticus & Metallyticidae & - & - \\
\hline Odontomantis & Hymenopodidae & Acromantinae & Acromantini \\
\hline Oligonicinae sp. & Thespidae & Oligonicinae & Oligonicini \\
\hline Orthodera & Mantidae & Orthoderinae & - \\
\hline Orthoderella & Mantidae & Photinainae $^{1}$ & Photinaini $^{1}$ \\
\hline Oxyopsis & Mantidae & Stagmatopterinae & Stagmatopterini \\
\hline Oxyothespis & Mantidae & Oxyothespinae & Oxyothespini \\
\hline Oxypilus (Oxypilus) & Hymenopodidae & Oxypilinae & Oxypilini \\
\hline Paralygdamia & Tarachodidae & Tarachodinae & - \\
\hline Paramorphoscelis & Amorphoscelidae & Perlamantinae & - \\
\hline Paraoxypilinae sp. & Amorphoscelidae & Paraoxypilinae & \\
\hline Paraoxypilus & Amorphoscelidae & Paraoxypilinae & - \\
\hline Parasphendale & Mantidae & Miomantinae & Miomantini \\
\hline Parentella & Mantidae & Chroicopterinae & Chroicopterini \\
\hline Perlamantis & Amorphoscelidae & Perlamantinae & - \\
\hline Photina & Mantidae & Photinainae $^{1}$ & Photinaini $^{1}$ \\
\hline Phyllocrania & Hymenopodidae & Epaphroditinae & Phyllocranini \\
\hline Plistospilota & Mantidae & Mantinae & Polyspilotini \\
\hline Pogonogaster & Thespidae & Oligonicinae & Pogonogasterini \\
\hline Polyspilota & Mantidae & Mantinae & Polyspilotini \\
\hline Popa & Mantidae & Vatinae & Danuriini \\
\hline Pseudocreobotra & Hymenopodidae & Hymenopodinae & Hymenopodini \\
\hline Pseudogalepsus & Tarachodidae & Tarachodinae & - \\
\hline Pseudoharpax & Hymenopodidae & Hymenopodinae & Hymenopodini \\
\hline Pseudoyersinia & Mantidae & Amelinae & Amelini \\
\hline Pyrgomantis & Tarachodidae & Tarachodinae & - \\
\hline Raptrix & Acanthopidae & Acontistinae & Acontistini \\
\hline Rhombodera & Mantidae & Mantinae & Paramantini \\
\hline Rivetina & Mantidae & Miomantinae & Rivetinini \\
\hline Sibylla (Sibylla) & Sibyllidae & Sibyllinae & - \\
\hline Sphodromantis & Mantidae & Mantinae & Paramantini \\
\hline Stagmatoptera & Mantidae & Stagmatopterinae & Stagmatopterini \\
\hline Stenomantis & Liturgusidae & Liturgusinae & Liturgusini \\
\hline Stenopyga & Mantidae & Angelinae & Angelini \\
\hline Tamolanica & Mantidae & Mantinae & Paramantini \\
\hline Tarachodes & Tarachodidae & Tarachodinae & - \\
\hline Tarachodula & Tarachodidae & Tarachodinae & - \\
\hline Taumantis & Mantidae & Miomantinae & Miomantini \\
\hline Theopompa & Liturgusidae & Liturgusinae & Liturgusini \\
\hline Theopropus & Hymenopodidae & Hymenopodinae & Hymenopodini \\
\hline Thesprotia & Thespidae & Oligonicinae & Pogonogasterini \\
\hline Thrinaconyx & Thespidae & Oligonicinae & Oligonicini \\
\hline Tisma & Mantidae & Mantinae & Paramantini \\
\hline Tithrone & Acanthopidae & Acontistinae & Acontistini \\
\hline Toxodera & Toxoderidae & Toxoderinae & Toxoderini \\
\hline Tropidomantis (Eomantis) & Iridopterygidae & Tropidomantinae & Tropidomantini \\
\hline Tropidomantis (Tropidomantis) & Iridopterygidae & Tropidomantinae & Tropidomantini \\
\hline Vates & Mantidae & Vatinae & Vatini \\
\hline Zoolea & Mantidae & Vatinae & Vatini \\
\hline
\end{tabular}


Tab. 8: Classification of the genera used in this study, sorted alphabetically by taxonomical subgroups (Ehrmann 2002).

\begin{tabular}{|c|c|c|c|}
\hline Family & Subfamily & Tribus & Genus \\
\hline Acanthopidae & Acanthopinae & Acanthopini & Acanthops \\
\hline Acanthopidae & Acontistinae & Acontistini & Acontista \\
\hline Acanthopidae & Acontistinae & Acontistini & Callibia \\
\hline Acanthopidae & Acontistinae & Acontistini & Raptrix \\
\hline Acanthopidae & Acontistinae & Acontistini & Tithrone \\
\hline Amorphoscelidae & Amorphoscelinae & - & Amorphoscelis \\
\hline Amorphoscelidae & Amorphoscelinae & - & Caudatoscelis \\
\hline Amorphoscelidae & Amorphoscelinae & - & Maculatoscelis \\
\hline Amorphoscelidae & Paraoxypilinae & - & Gyromantis \\
\hline Amorphoscelidae & Paraoxypilinae & - & Paraoxypilus \\
\hline Amorphoscelidae & Paraoxypilinae & & Paraoxypilinae sp. \\
\hline Amorphoscelidae & Perlamantinae & - & Paramorphoscelis \\
\hline Amorphoscelidae & Perlamantinae & - & Perlamantis \\
\hline Chaeteessidae & - & - & Chaeteessa \\
\hline Empusidae & Blepharodinae & Blepharodini & Blepharodes \\
\hline Empusidae & Blepharodinae & Blepharodini & Blepharopsis \\
\hline Empusidae & Blepharodinae & Idolomantini & Idolomantis \\
\hline Empusidae & Empusinae & Empusini & Empusa (Empusa) \\
\hline Empusidae & Empusinae & Empusini & Gongylus \\
\hline Empusidae & Empusinae & Idolomorphini & Hypsicorypha \\
\hline Empusidae & Empusinae & Idolomorphini & Idolomorpha \\
\hline Eremiaphilidae & - & & Eremiaphila \\
\hline Eremiaphilidae & - & & Heteronutarsus \\
\hline Hymenopodidae & Acromantinae & Acromantini & Acromantis \\
\hline Hymenopodidae & Acromantinae & Acromantini & Hestiasula \\
\hline Hymenopodidae & Acromantinae & Acromantini & Odontomantis \\
\hline Hymenopodidae & Epaphroditinae & Phyllocranini & Amphecostephanus \\
\hline Hymenopodidae & Epaphroditinae & Phyllocranini & Epaphrodita \\
\hline Hymenopodidae & Epaphroditinae & Phyllocranini & Phyllocrania \\
\hline Hymenopodidae & Hymenopodinae & Hymenopodini & Creobroter \\
\hline Hymenopodidae & Hymenopodinae & Hymenopodini & Galinthias \\
\hline Hymenopodidae & Hymenopodinae & Hymenopodini & Hymenopus \\
\hline Hymenopodidae & Hymenopodinae & Hymenopodini & Pseudocreobotra \\
\hline Hymenopodidae & Hymenopodinae & Hymenopodini & Pseudoharpax \\
\hline Hymenopodidae & Hymenopodinae & Hymenopodini & Theopropus \\
\hline Hymenopodidae & Oxypilinae & Oxypilini & Ceratomantis \\
\hline Hymenopodidae & Oxypilinae & Oxypilini & Oxypilus (Oxypilus) \\
\hline Iridopterygidae & Tropidomantinae & Tropidomantini & Enicophlebia \\
\hline Iridopterygidae & Tropidomantinae & Tropidomantini & Tropidomantis (Eomantis) \\
\hline Iridopterygidae & Tropidomantinae & Tropidomantini & Tropidomantis (Tropidomantis) \\
\hline Liturgusidae & Liturgusinae & Liturgusini & Ciulfina \\
\hline Liturgusidae & Liturgusinae & Liturgusini & Humbertiella \\
\hline Liturgusidae & Liturgusinae & Liturgusini & Majanga \\
\hline Liturgusidae & Liturgusinae & Liturgusini & Stenomantis \\
\hline Liturgusidae & Liturgusinae & Liturgusini & Theopompa \\
\hline Mantidae & Amelinae & Amelini & Ameles \\
\hline Mantidae & Amelinae & Amelini & Elaea \\
\hline Mantidae & Amelinae & Amelini & Gimantis \\
\hline Mantidae & Amelinae & Amelini & Gonypeta \\
\hline Mantidae & Amelinae & Amelini & Pseudoyersinia \\
\hline Mantidae & Angelinae & Angelini & Euchomenella \\
\hline Mantidae & Angelinae & Angelini & Leptocola \\
\hline
\end{tabular}


Tab. 8 (continued): Classification of the genera used in this study, sorted alphabetically by taxonomical subgroups (Ehrmann 2002).

\begin{tabular}{|c|c|c|c|}
\hline Mantidae & Angelinae & Angelini & Stenopyga \\
\hline Mantidae & Choeradodinae & - & Choeradodis \\
\hline Mantidae & Chroicopterinae & Chroicopterini & Entella (Entella) \\
\hline Mantidae & Chroicopterinae & Chroicopterini & Ligaria \\
\hline Mantidae & Chroicopterinae & Chroicopterini & Ligariella \\
\hline Mantidae & Chroicopterinae & Chroicopterini & Parentella \\
\hline Mantidae & Compsothespinae & - & Compsothespis \\
\hline Mantidae & Deroplatyinae & - & Deroplatys \\
\hline Mantidae & Dystactinae & Dystactini & Dystacta \\
\hline Mantidae & Mantinae & Archimantini & Archimantis \\
\hline Mantidae & Mantinae & Mantini & Mantis \\
\hline Mantidae & Mantinae & Paramantini & Rhombodera \\
\hline Mantidae & Mantinae & Paramantini & Sphodromantis \\
\hline Mantidae & Mantinae & Paramantini & Tamolanica \\
\hline Mantidae & Mantinae & Paramantini & Tisma \\
\hline Mantidae & Mantinae & Polyspilotini & Plistospilota \\
\hline Mantidae & Mantinae & Polyspilotini & Polyspilota \\
\hline Mantidae & Miomantinae & Miomantini & Parasphendale \\
\hline Mantidae & Miomantinae & Miomantini & Taumantis \\
\hline Mantidae & Miomantinae & Rivetinini & Bolivaria \\
\hline Mantidae & Miomantinae & Rivetinini & Rivetina \\
\hline Mantidae & Orthoderinae & - & Orthodera \\
\hline Mantidae & Oxyothespinae & Oxyothespini & Oxyothespis \\
\hline Mantidae & Photinainae $^{1}$ & Photinaini $^{1}$ & Orthoderella \\
\hline Mantidae & Photinainae $^{1}$ & Photinaini $^{1}$ & Photina \\
\hline Mantidae & Phyllotheliinae & - & Ceratocrania \\
\hline Mantidae & Stagmatopterinae & Stagmatopterini & Oxyopsis \\
\hline Mantidae & Stagmatopterinae & Stagmatopterini & Stagmatoptera \\
\hline Mantidae & Vatinae & Danuriini & Ambivia \\
\hline Mantidae & Vatinae & Danuriini & Popa \\
\hline Mantidae & Vatinae & Heterochaetini & Heterochaeta \\
\hline Mantidae & Vatinae & Vatini & Vates \\
\hline Mantidae & Vatinae & Vatini & Zoolea \\
\hline Mantoididae & - & - & Mantoida \\
\hline Metallyticidae & - & - & Metallyticus \\
\hline Sibyllidae & Sibyllinae & - & Sibylla (Sibylla) \\
\hline Tarachodidae & Tarachodinae & - & Iris \\
\hline Tarachodidae & Tarachodinae & - & Paralygdamia \\
\hline Tarachodidae & Tarachodinae & - & Pseudogalepsus \\
\hline Tarachodidae & Tarachodinae & - & Pyrgomantis \\
\hline Tarachodidae & Tarachodinae & - & Tarachodes \\
\hline Tarachodidae & Tarachodinae & - & Tarachodula \\
\hline Thespidae & Haaniinae & - & Astape \\
\hline Thespidae & Haaniinae & - & Haania \\
\hline Thespidae & Hoplocoryphinae & Hoplocoryphini & Hoplocorypha \\
\hline Thespidae & Oligonicinae & Oligonicini & Bactromantis \\
\hline Thespidae & Oligonicinae & Oligonicini & Oligonicinae sp. \\
\hline Thespidae & Oligonicinae & Oligonicini & Thrinaconyx \\
\hline Thespidae & Oligonicinae & Pogonogasterini & Pogonogaster \\
\hline Thespidae & Oligonicinae & Pogonogasterini & Thesprotia \\
\hline Toxoderidae & Toxoderinae & Toxoderini & Toxodera \\
\hline Toxoderidae & Toxoderinae & Toxoderopsini & Calamothespis \\
\hline
\end{tabular}

${ }^{1}$ see Svenson \& Branham 2007 



\title{
Curriculum Vitae
}

\author{
Name \\ Frank Wieland \\ Date of birth \\ 03 December 1975 in Oldenburg (OLDB) \\ Marital status \\ unmarried \\ Nationality \\ German
}

\section{School}

1982-1986

Primary school (Grundschule Jägerstraße), Hude

1986-1988

"Orientierungsstufe", Hude

1988-1995

Grammar school (Graf-Anton-Günther-Gymnasium), Oldenburg

\section{National Service}

1995-1996 Social service (Sozialstation Hude/Hatten), Hude

\section{Scientific Education}

1996-1998 Basic courses (biology), Georg-August-University Göttingen.

1998 Intermediate diploma (German Vordiplom) in zoology, microbiology, anorganic chemistry and physical chemistry .

1998-2001 Advanced study courses (biology) in zoology (main course), microbiology and biochemistry, Georg-August-University Göttingen.

1999-2002 Student assistant in the Department of Morphology, Systematics \& Evolutionary Biology; assistance in zoological basic courses, extensive assistance in the Zoological Museum of the University.

2001-2002 Diploma thesis "Morphologische Untersuchungen am Prothorax der Mantodea (Gottesanbeterinnen)" at the Institute of Zoology \& Anthropology under supervision of Prof. Dr. R. Willmann.

July 2002 Diploma

2002-2004 Scientific assistant in the Department of Morphology, Systematics \& Evolutionary Biology; preparation of project for doctoral thesis; assistance in zoological basic and main courses and in the Zoological Museum of the University.

2004-2005 Scientific employee in the department of Morphology, Systematics \& Evolutionary Biology; doctoral candidate, thesis: "The phylogenetic system of Mantodea (Insecta: Dictyoptera)"; funded by the German Science Foundation (DFG Wi599/12).

2005-2006 Continuation of the doctoral thesis; scientific assistant in in the Department of Morphology, Systematics \& Evolutionary Biology; assistance in zoological basic and main courses and in the Zoological Museum of the University.

2006-2007 Continuation of the doctoral thesis; scientific employee in the department of Morphology, Systematics \& Evolutionary Biology.

since 2007 Continuation of the doctoral thesis; scientific assistant in in the Department of Morphology, Systematics \& Evolutionary Biology; assistance in zoological basic and main courses and in the Zoological Museum of the University; assistance in the AnimalBase-Project of the University of Göttingen (since 2009). 